European

Thyroid Journal

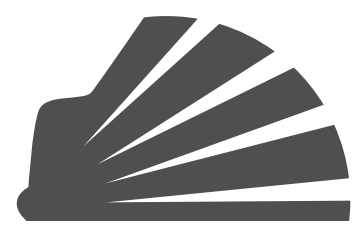

ETA2@14

SANTIAGO DE COMPOSTELA I SPAIN
Eur Thyroid J 2014:3(suppl 1):73-226

DOI: $10.1159 / 000365244$

\title{
38th Annual Meeting of the European Thyroid Association
}

\section{Abstracts}

Santiago de Compostela, Spain

September 6-10, 2014

Guest Editors

Furio Pacini, Siena, Italy

Clara V. Alvarez, Santiago de Compostela, Spain 


\section{OP1 Topic Highlights}

\section{OP01 \\ A ZEBRAFISH MODEL OF NETRIN1 DEFICIENCY REVEALS ABNORMAL THYROID MORPHOGENESIS LINKED TO DEFECTIVE CARDIOVASCULAR DEVELOPMENT \\ Robert Opitz ${ }^{1}$, Achim Trubiroha ${ }^{1}$, Isabelle Vandernoot ${ }^{1}$, Marc-Philip Hitz ${ }^{2}$, Rasha Abu-Khudir², Valérie Désilets ${ }^{2}$, Johnny Deladoëy ${ }^{2}$, Gregor Andelfinger ${ }^{2}$, Sabine Costagliola ${ }^{1}$ ${ }^{1}$ Iribhm, Université Libre de Bruxelles, Brussels, Belgium, ${ }^{2}$ Department of Pediatrics, Centre Hospitalier Universitaire Sainte Justine, Montréal, Qué., Canada}

Mutual interactions between mesoderm and pharyngeal endoderm are vital for cardiovascular development and morphogenesis of endodermderived organs such as the thyroid. In fact, thyroid development represents one exciting model to study this interrelationship given the close coordination of thyroid and cardiovascular development and a high prevalence of thyroid anomalies in mouse and zebrafish models with defective cardiovascular development. Since congenital cardiac defects represent the most frequent extrathyroidal anomalies in patients with congenital thyroid disorders, we set out to examine patients with combined thyroid and cardiac defects for rare chromosomal aberrations. In one patient presenting thyroid ectopy and congenital cardiac defects, we detected a de novo deletion at the Netrin1 locus. Netrin1 is an axon guidance molecule with additional functions in vascular development and endodermal organ morphogenesis. To examine a potential role of zebrafish netrinl in thyroid organogenesis, we analyzed developmental expression patterns of $n t n l a$ and $n t n l b$ in the thyroid/pharyngeal region and injected zebrafish embryos with a ntn $1 a$ splice-blocking morpholino to study thyroid development in ntnla-deficient embryos. Early steps of thyroid development (i.e. thyroid anlage specification, formation of thyroid primordium and thyroid budding), were not affected in ntnla morphants despite the presence of early cardiac laterality defects. However, the tight spatiotemporal coordination of subsequent heart descent, pharyngeal vessel morphogenesis and thyroid relocalization into the subpharyngeal mesenchyme, as seen in controls, was disrupted in ntnla morphant embryos which frequently displayed laterally misplaced thyroid tissue. Confocal analyses of transgenic tg (tg: $m$ Cherry; $k d r l: E G F P$ ) embryos injected with ntn $1 a$ morpholino revealed that ectopic localization of thyroid tissue occurred concurrent with specific pharyngeal vasculature anomalies including aortic arch artery defects and abnormal hypobranchial artery morphogenesis. Collectively, results from our studies indicate a novel role of netrin1 function in the regulation of pharyngeal vessel morphogenesis and thyroid organogenesis.

\section{OP02 \\ ANALYSES OF A PHASE 3, MULTICENTER, DOUBLE-BLIND, PLACEBO-CONTROLLED TRIAL OF LENVATINIB (E7080) IN PATIENTS WITH 131-I-REFRACTORY DIFFERENTIATED THYROID CANCER (SELECT)}

Steven I. Sherman ${ }^{1}$, Makoto Tahara ${ }^{2}$, Lori Wirth ${ }^{3}$, Bruce Robinson ${ }^{4}$, Marcia F. Brose ${ }^{5}$, Rossella Elisei ${ }^{6}$, Corina E. Dutcus ${ }^{7}$,

Begoña de las Heras ${ }^{8}$, Junming Zhu' ${ }^{7}$, Mouhammed Amir Habra ${ }^{1}$, Kate Newbold ${ }^{9}$, Manisha H. Shah ${ }^{10}$, Ana O. Hoff ${ }^{11}$,

Andrew G. Gianoukakis ${ }^{12}$, Naomi Kiyota ${ }^{13}$, Matthew H. Taylor ${ }^{14}$ Sung-Bae Kim ${ }^{15}$, Monika K. Krzyzanowska ${ }^{16}$, Martin Schlumberger ${ }^{17}$

${ }^{1}$ Department of Endocrine Neoplasia and Hormonal Disorders, Division of Internal Medicine, The University of Texas M. D. Anderson Cancer Center, Houston, Tex., USA, ${ }^{2}$ Department of Head and Neck Medical Oncology National Cancer Center Hospital East, Kashiwa, Japan, ${ }^{3}$ Department of Medicine, Massachusetts General Hospital, Boston, Mass., USA, ${ }^{4}$ Kolling Institute of Medical Research, University of Sydney, N.S.W., Australia, ${ }^{5}$ Department of Otorhinolaryngology: Head and Neck Surgery, Abramson Cancer Center of the University of Pennsylvania, Philadelphia, Pa., USA, ${ }^{6}$ Endocrine Unit, Department of Clinical and Experimental Medicine, University of Pisa, Pisa, Italy, ${ }^{7}$ Eisai Inc, Woodcliff Lake, N.J., USA, ${ }^{8}$ Eisai Ltd, Hatfield, England, UK, ${ }^{9}$ The Royal Marsden Hospital National Health Service Trust, London, England, UK, ${ }^{10}$ Department of Internal Medicine, The Ohio State University Comprehensive Cancer Center, Columbus, Ohio, USA, ${ }^{11}$ Instituto Do Cancer de Sao Paulo, Universidade de Sao Paulo, Sao Paulo, Brazil, ${ }^{12}$ Division of Endocrinology and Metabolism, HarborUcla Medical Center, Torrance, Calif., USA, ${ }^{13}$ Department of Medical Oncology and Hematology, Kobe University Hospital, Kobe, Japan, ${ }^{14}$ Knight Cancer Institute, Oregon Health and Science University, Portland, Oreg., USA, ${ }^{15}$ Department of Oncology, Asan Medical Center, University of Ulsan College of Medicine, Seoul, Korea, ${ }^{16}$ Division of Medical Oncology \& Hematology, Princess Margaret Cancer Centre, Uhn 610 University Avenue, Toronto, Ont., Canada, ${ }^{17}$ Department of Nuclear Medicine and Endocrine Oncology, Gustave Roussy and Université Paris-Sud, Villejuif, France

Objectives: Lenvatinib is an oral multikinase inhibitor of VEGFR1-3, FGFR1-4, PDGFR $\alpha$, RET and KIT. This analysis reports detailed outcomes from the phase 3 Study of (E7080) LEnvatinib in Differentiated Cancer of the Thyroid (SELECT).

Methods: Patients with documented progressive ${ }^{131}$ I-refractory differentiated thyroid cancer (RR-DTC) within 13 months were randomized (2:1) in this double-blind, multicenter study to lenvatinib or placebo $(24 \mathrm{mg} / \mathrm{d} ; 28-\mathrm{d}$ cycle). Primary endpoint was progression-free survival (PFS); secondary endpoints included overall response rate (ORR; complete response [CR] + partial response $[\mathrm{PR}])$, OS, and safety.

Results: 392 Patients (median age 63.0 y; 51.0\% male) were randomized. PFS was significantly prolonged with lenvatinib vs placebo; median PFS 18.3 months vs 3.6 months (hazard ratio [HR] $0.21 ; 95 \%$ confidence interval [CI] $0.14-0.31 ; P<0.0001)$. Lenvatinib PFS benefit was maintained in all predefined subgroups; median lenvatinib PFS for patients with prior vs no prior anti-VEGF pathway therapy was 15.1 months $(95 \%$ CI 8.8 -not reached [NR]) and 18.7 months (95\% CI 16.4-NR), respectively. Exploratory analyses suggest lenvatinib PFS benefit is also maintained in subgroups including patients with lung metastasis (median PFS: lenvatinib, 18.7 months; placebo, 3.6 months; HR $0.21 ; 95 \%$ CI $0.15-0.29$ ) and bone metastasis only (median PFS: lenvatinib, NR; placebo, 7.4 months; HR 0.65 ; 95\% CI 0.11-4.07). Rates of CRs for lenvatinib: $1.5 \%$; placebo: 0 ; PR for lenvatinib: $63.2 \%$; pla-

\begin{tabular}{ll}
\hline KARGER & $\begin{array}{l}\text { (c) 2014 European Thyroid Association } \\
\text { Published by S. Karger AG, Basel } \\
2235-0640 / 14 / 0035-0073 \$ 39.50 / 0\end{array}$ \\
$\begin{array}{l}\text { E-Mail karger@karger.com } \\
\text { www.karger.com }\end{array}$ & $\begin{array}{l}\text { Accessible online at: } \\
\text { www.karger.com } / \text { etj }\end{array}$
\end{tabular}


cebo: $1.5 \%$. Median OS has not been reached. Deaths in the lenvatinib group: 27.2\%; placebo: $35.9 \%$. $78.5 \%$ Of patients required dose reduction; $14.2 \%$ discontinued treatment due to AEs. The 5 most common lenvatinib treatment-related adverse events (AEs) were (any Grade; Grade $\geq 3$ ) hypertension $(67.8 \% ; 41.8 \%)$, diarrhea $(59.4 \% ; 8.0 \%)$, decreased appetite $(50.2 \% ; 5.4 \%)$, decreased weight $(46.4 \% ; 9.6 \%)$, and nausea $(41.0 \% ; 2.3 \%)$.

Conclusion: Lenvatinib significantly improved PFS compared with placebo in patients with RR-DTC, with toxicities and AEs that were expectantly managed.

\section{OP03 \\ TARGETED MUTATIONS IN MCT10 EXPAND THE SUBSTRATE SPECTRUM TO INCLUDE T4 \\ Jörg Johannes ${ }^{0}$, Ulrich Schweizer ${ }^{1}$, Doreen Braun ${ }^{2}$, Kleinau Gunnar ${ }^{3}$ \\ ${ }^{1}$ Rheinische Friedrich-Wilhelms-Universität Bonn; Institut Für \\ Biochemie und Molekularbiologie, ${ }^{2}$ Rheinische Friedrich-Wilhelms- \\ Universität Bonn; Institut Für Biochemie und Molekularbiologie, \\ ${ }^{3}$ Charité - Universitätsmedizin Berlin; Institut Für Experimentelle \\ Pädiatrische Endokrinologie}

The crucial turning point changing our understanding from passive diffusion of thyroid hormones $(\mathrm{TH})$ towards a transmembrane transport facilitated by transporter proteins, was the finding of mutations in the monocarboxylate transporter 8 (MCT8), in patients suffering from the Allan-Herndon-Dudley syndrome (AHDS)

Because of the lack of a crystallized or empirical structure of any TH transporter, little is known about how TH transporters recognize their specific substrate and which structural changes they undergo to perform a substrate translocation.

The MCT8/MCT10 couple is an ideal model to investigate the molecular aspects of structure function relationship in transmembrane TH transport. Despite their high homology, both transporters differ in their substrate specificity: both are capable of T3 transport, but only MCT8 accepts T4 as a substrate, whereas MCT10 is an aromatic amino acid transporter (a.k.a. TAT1).

Based on our MCT8 homology model, we identified 8 amino acids, which differ between MCT8 and MCT10 and line the presumed substrate translocation channel. Our hypothesis is that these AAs play a major role in substrate specificity. Consequently, the exchange of these target AAs should result in an altered substrate specificity of MCT10.

Indeed, T4 and Tyrosine Uptake Assays performed in transfected MDCK-1 cells and Xenopus Oocytes, expressing the resulting single and compound MCT $10^{\mathrm{MCT} 8}$ chimeras, show a clear impact on substrate specificity: The 8 -fold MCT $10^{\mathrm{MCT}}$ chimera gains the ability to transport T4, acting more like MCT8. We already could achieve the same effect in the 6-fold MCT10 $10^{\text {MCT8 }}$ chimera. Currently we are addressing the question, if there is a single pivotal amino acid responsible for substrate specificity, or if it is rather a combination of different AAs.

Our findings contribute to a deeper inside into the structure function relationship of MCT10 and proof the feasibility of structure based targeted mutations guided by our MCT8 homology model.

\section{OP04 \\ THE TARGETED INACTIVATION OF MEN1 GENE EXACERBATES RET/PTC3-INDUCED THYROID NEOPLASTIC TRANSFORMATION}

Samia Selmi-Ruby ${ }^{1}$, Maria-Jésus Obregon ${ }^{2}$, Juliette Dupayrat ${ }^{3}$, Françoise Borson-Chazot ${ }^{3}$, Chang Zhang ${ }^{3}$

${ }^{1}$ Faculté de Médecine Rth-Laennec Crcl Umr 1052, ${ }^{2}$ Instituto de Investigations Biomedicas, ${ }^{3} \mathrm{Crcl}-\mathrm{Umr} 1052$

Multiple Endocrine Neoplasia type1 is an inherited predisposition syndrome characterized by an increased risk of endocrine tumors. In a previous study, we found that menin acts as a negative regulator controlling the thyroid growth. To explore the tumor suppressor function of menin, we analyzed whether thyroid-selective inactivation of Men1 gene affects Ret/PTC3driven neoplastic transformation. Mice with Men1-disruption selectively in thyrocytes (Men1flox/floxThyrCre) were generated by crossing transgenic ThyrCre mice (expressing the Cre selectively in thyrocytes) with mice carrying Men1floxalleles. Men1flox/floxThyrCre mice were then crossed with mice expressing the Ret-PTC3 oncogene selectively in the thyroid (RP3 mice). Thyroid phenotype of mice was analyzed at 4,8 and 12 months of age. RP3-induced thyroid hypertrophy was transiently reduced and followed by a significant increase in aging Men1flox/floxThyrCre/RP3 mice in comparison with aged-matched RP3 littermates. At 4, 8 and 12 months of age, hormonal status analyses disclosed a decrease of serum T4 and T3 associated with a marked increase in serum TSH concentrations. The functional activity of the thyroid assessed by measurements of the expression level of thyroid-specific genes appeared reduced in Men1flox/floxThyrCre/RP3 mice compared to that of age-matched RP3 littermates. In aging Men1flox/floxThyrCre/RP3 mice, morphological examinations of the thyroid showed a profound tissue remodeling associated with the occurrence of mesenchymal structures. This observation was evidenced by a 2-fold-increase in vimentin and fibronectin 1 without significant decrease in E-cadherin mRNA levels. However, a 3-fold-increase in Sip1 and Snail mRNA levels known to be E-cadherin repressors involved in the epithelial-to-mesenchymal transition (EMT) was observed in Men 1flox/ floxThyrCre/RP3 mice compared to that of RP3 littermates. Our data indicate that tumors developped following Ret/PTC3 expression are susceptible to undergo EMT in response to the targeted-inactivation of the Men1 gene. In conclusion, we show for the first time that Men1 gene acts as a tumor suppressor in Ret/PTC3-driven thyroid neoplastic transformation.

\section{OP05 \\ NEXT GENERATION SEQUENCING (NGS) REVEALS A HIGHLY FREQUENT MULTIGENIC INVOLVEMENT IN THE PATHOGENESIS OF CONGENITAL HYPOTHYROIDISM}

Luca Persani', Tiziana de Filippis ${ }^{2}$, Giulia Gelmini², Federica Marelli ${ }^{2}$ Maria Cristina Vigone ${ }^{3}$, Mirella Moro ${ }^{2}$, Maria Carolina Salerno ${ }^{4}$,

Giorgio Radetti ${ }^{5}$, Giovanna Weber ${ }^{6}$, Fabiana Guizzardi ${ }^{2}$

${ }^{1}$ University of Milan and Irccs Istituto Auxologico Italiano, ${ }^{2}$ Irccs Istituto

Auxologico Italiano, ${ }^{3}$ San Raffaele Hospital, ${ }^{4}$ University of Naples,

${ }^{5}$ Hospital of Bolzano, ${ }^{6}$ San Raffaele Hospital and Vita-Salute University, Italy

The pathogenesis of Congenital Hypothyroidism $(\mathrm{CH})$ is still undetermined in most of the affected patients. Several candidate genes have been associated either with dysgenetic or functional thyroid defects. However, the involvement of individual candidate genes has been so far assessed on series of patients selected by their clinical phenotype (eg, dyshormonogenic goiter vs dysgenesis, or isolated vs syndromic $\mathrm{CH}$ ). By following this approach, variations in these candidate genes do not account for $>10 \%$ of the investigated patients. Here we report a novel approach based on the systematic analysis of a panel of 11 known candidate genes (NKX2.1, PAX8, FOXE1, GLIS3, JAG1, TSHR, SLC26A4, TG, TPO, DUOX2, DUOXA2) by Next Generation Sequencing (NGS, variants confirmed by automated sequencing) in a series of 33 patients with $\mathrm{CH}$. In contrast with current knowledge, only 9/33 patients $(27 \%)$ had no rare (defined as Minor Allele Frequency, MAF, <0.01) or novel non-synonymous variants in these candidate genes, whereas carriers of at least $1 \mathrm{rare} /$ novel variant were $7 / 33(21 \%)$, and 2 or more novel/rare variants were detected in 17/33 (51\%) of the patients. All the examined genes, except DUOXA2, were involved in at least one case. The genes most frequently affected by rare/novel non-synonymous variations are DUOX2 $(\mathrm{n}=$ 12) and TG $(n=12)$. Interestingly, heterozygous FOXE1 rare/novel variants were detected in $2 \mathrm{CH}$ cases with an apparently normal gland-in-situ, and no other midline defect. These data indicate that, in contrast with current understanding, variations in the currently known candidate genes can account for the $\mathrm{CH}$ pathogenesis in a large number of affected patients. The systematic NGS approach reveals a highly frequent multigenic origin of $\mathrm{CH}$, including unexpected variations in genes that would have been excluded a priori from screening on the basis of the current knowledge.

Partially supported by research fund: RF-2010-2309484 


\section{OP06}

SERUM MIRNAS AS A NON INVASIVE TOOL IN THE DIFFERENTIAL DIAGNOSIS OF THYROID NODULES IN A CAUCASIAN POPULATION

\section{Tania Pilli', Silvia Cantara', Sebastiano Guidi ${ }^{2}$, Cevenini Gabriele ${ }^{3}$,} Giulia Busonero ${ }^{1}$, Sandro Cardianale ${ }^{1}$, Francesco Dotta ${ }^{1}$, Furio Pacini ${ }^{1}$

${ }^{1}$ Department of Medicine, Surgery and Neuroscience, University of Siena, Siena, Italy, ${ }^{2}$ Fondazione Umberto DI Mario Onlus, Toscana Life Sciences, Siena, Italy, ${ }^{3}$ Department of Medical Biotechnologies, University of Siena, Siena, Italy

Background: MicroRNA are non-protein encoding RNAs that regulate gene expression and allow the distinction of benign from malignant tissues in human cancers.

Aim: We investigated the role of circulating miRNA in the differential diagnosis of thyroid nodules in a cohort of Caucasian patients.

Methods: Our study is composed of several steps. Step 1: we assessed microRNAs profile, by TaqMan ${ }^{\circledR}$ Array Human MicroRNA A Cards v2.0, in pooled sera from 12 healthy subjects (HS), 12 nodular goiter (NG) and 12 papillary thyroid cancer (PTC) patients (first cohort). Step 2: 8 miRNAs, found to be differentially expressed in PTC patients in Step 1, were validated, by qRT-PCR, in each sample of the first cohort of patients. Four out of 8 miRNAs were confirmed to be differentially expressed in PTC. Step 3: the 4 miRNAs identified in Step 2 were analyzed by qRT-PCR in a second cohort of subjects including 79 PTC, $80 \mathrm{NG}$ and $41 \mathrm{HS}$.

Results: In step 1 we identified 8 miRNAs differentially expressed in PTC compared to NG and HS. In particular, miRNA579, $-95,-29 b,-501-3 p$ and miRNA-548d-5p were down-regulated while miR190, -362-3p, -518a-5p were up-regulated. In step 2 , the results were confirmed only for 4 miRNAs (miRNA579, miRNA95, miRNA29b and miRNA190). In step 3 these findings were further validated in a second cohort of sera. MiRNA95 showed a sensitivity of $94.9 \%$ that was improved up to $98.7 \%$ by its combination with miRNA190 in a multivariate risk model.

Conclusion: In this study we identified 4 circulating miRNAs differentially expressed in PTC patients compared to NG and HS. The combination of 2 of them (miRNA190 and miRNA95), by a multivariate risk model, is a potential, novel, non invasive, powerful tool in the differential diagnosis of thyroid nodules.

\section{OP2 Young Investigator-Clinical}

\section{OP07 \\ TELOMERASE PROMOTER MUTATIONS, EXPRESSION AND LOCALIZATION IN DIFFERENTIATED THYROID CANCER}

Marina Muzza ${ }^{1}$, Carla Colombo ${ }^{1}$, Stefania Rossi ${ }^{2}$, Valentina Cirello', Michela Perrino', Simone De Leo ${ }^{1}$, Elisa Pignatt ${ }^{3}$, Manuela Simoni ${ }^{3}$, Gaetano Bulfamante ${ }^{2}$, Laura Fugazzola ${ }^{1}$

${ }^{1}$ Department of Clinical Sciences and Community Health, University of Milan; Endocrine Unit, Fondazione Irccs Ca' Granda, Milan, ${ }^{2}$ Department Health Sciences, University of Milan; Division of Pathology, San Paolo Hospital, Milan, ${ }^{3}$ Unit and Chair of Endocrinology and Metabolism, Department of Biomedical, Metabolic and Neural Sciences, University of Modena and Reggio Emilia; Department of Medicine, Endocrinology, Metabolism and Geriatrics, Azienda Usl of Modena, Italy

Telomerase reverse transcriptase (TERT), the catalytic subunit of telomerase, maintains telomeres at the end of chromosomes. TERT is normally repressed in somatic tissues and its reactivation has been implicated in human tumorigenesis. TERT promoter mutations were found to up-regulate the protein expression and were recently reported in thyroid cancer. Aim of the present study was to investigate the prognostic value of these alterations and the molecular mechanisms implicated in the progression of TERT mutated (TERT $^{\mathrm{MUT}}$ ) thyroid tumors. TERT, BRAF and RAS mutations have been explored in a series of 240 DTCs by PCR and sequencing and correlated with full clinical data. Furthermore, the effect of these mutations on TERT expression and localization was studied by Western blot and immunohistochemistry studies. The prevalence of TERT mutations was of $12 \%$ in papillary thyroid cancers (PTCs) and of 14\% in follicular thyroid cancers (FTCs). A significant correlation $(\mathrm{P}<0.0001)$ was found between $T E R T^{\mathrm{MUT}}$, older age at diagnosis and poorer outcome. The prevalence of $B R A F^{\mathrm{V} 600 \mathrm{E}}$ was of $35 \%$ in PTCs, and was higher in TERT ${ }^{\mathrm{MUT}}$ than in TERT ${ }^{\mathrm{WT}}$ cases. Coexisting TERT and BRAF mutations were associated with a poorer outcome. $R A S$ mutations were found in $24 \%$ of FTCs, without differences between TERT $T^{\mathrm{MUT}}$ and TER $T^{\mathrm{WT}}$ cases. At Western blot analysis, a higher expression of TERT was found in tumors with respect to normal samples. Moreover, TERT ${ }^{\mathrm{MUT}}$ tumors displayed a mildly increased expression of the protein with respect to $T E R T^{\mathrm{WT}}$ cases. At immunohistochemistry, TERT was found to be excluded from the nucleus in neoplastic tissues, suggesting a shuttling of the enzyme to mitochondria. In conclusion, the prognostic value of TERT mutations was shown in a large series of DTCs. Moreover, TERT mutations were demonstrated to promote a higher protein expression in thyroid tumors that might contribute to cancer progression through a mechanism independent from telomeres elongation.

\section{OP08 \\ DUAL GS-GQ VERSUS GQ SELECTIVE SIGNALLING DEFECTS IN CARBOXY-TERMINAL TSHR MUTANTS: IN VITRO AND CLINICAL CORRELATES}

Marta Garcia ${ }^{1}$, Jacqueline van Sande ${ }^{2}$, Ainhoa Iglesias ${ }^{1}$,

Helène Bony-Trifunovic ${ }^{3}$, Maria Chueca ${ }^{4}$, Erica van den Akker ${ }^{5}$, Isolina Riaño ${ }^{6}$, Cristina Luzuriaga ${ }^{7}$, Patricia Crock ${ }^{8}$, Isabel González-Casado ${ }^{9}$, Jacques E. Dumont ${ }^{2}$, José C. Moreno ${ }^{1}$ ${ }^{1}$ Thyroid Molecular Laboratory, Institute for Medical and Molecular Genetics (Ingemm), La Paz University Hospital, Madrid, Spain, ${ }^{2}$ Institute of Interdisciplinary Research, University of Brussels, Brussels, Belgium, ${ }^{3}$ Pediatric Endocrinology and Diabetes, Nord Hospital, Amiens, France, ${ }^{4}$ Pediatric Endocrinology, Virgen del Camino Hospital, Pamplona, Spain, ${ }^{5}$ Pediatric Endocrinology, Sophia Children's Hospital, Erasmus Medical Center, Rotterdam, The Netherlands, ${ }^{6}$ Pediatric, Carmen and Severo Ochoa Hospital, Cangas del Narcea, Spain, ${ }^{7}$ Endocrinology, Marqués de Valdecilla University Hospital, Santander, Spain, ${ }^{8}$ Pediatric Diabetes and Endocrinology, John Hunter Children's Hospital, Newcastle, Australia, ${ }^{9}$ Pediatric Endocrinology, La Paz University Hospital, Madrid, Spain

TSHR is a G protein-coupled receptor mediating the effects of TSH on thyroid development and hormonosynthesis. The signal transduction involves Gs and Gq/11 proteins. The Gs-cAMP-PKA cascade controls growth and differentiation whereas the Gq-IP-Ca ${ }^{2+}$ regulates iodination. TSHR biallelic inactivating mutations lead to severe thyroid hypoplasia while monoallelic defects cause TSH-resistance and variable mild hypoplasia. No patients with TSHR defects are described with positive perchlorate discharge test (PDT).

Objective: To dissect the signalling cascades affected by carboxy-terminal TSHR mutations identified in patients with hyperthyrotropinemia, and explore phenotype-genotype correlations.

Patients and Methods: 72 patients with isolated, permanent hyperthyrotropinemia were screened for mutations in TSHR. TSHR cDNA was mutagenized with defects identified and transfected in Cos-7 cells. TSHR cell-surface expression was quantified by flow-immunocytofluorometry and cAMP and IP production capacity was determined under TSH-stimulation.

Results: Five different heterozygous mutations in 7 patients were identified in exon 10 of TSHR: 4 missense (p.L436P, p.R450H, p.R534C, p.R519H), and a 2-bp deletion, (p.T655CfsX656). Mutations disrupt the transport to the cell membrane fully (L436P, p.T655CfsX656), partially (40-60\%: p.R450H, p.R519H) or have intact cell-surface expression (p.R534C). Those with partial surface expression decrease both Gs-cAMP/Gq-IP signalling to $75 \% / 26 \%$ and $34 \% / 17 \%$, respectively. However, p.R534C selectively impairs Gq-IP-Ca ${ }^{2+}$ cascade $(75 \%)$. Patients carrying mutations with no surface expression show thyroid hypoplasia $(\mathrm{n}=2)$ and positive $(30 \%)$ PDT $(\mathrm{n}=1)$. Those with mutations partially impairing membrane transport show isolated TSH elevation with normal thyroid size. The patient with selective impairment of Gq signalling belongs to a family with hereditary goiter.

Conclusions: Monoallelic TSHR mutations with absent surface expression damage Gs and Gq signalling and may lead to a poorly recognised "mixed" dysgenetic-dyshormonogenetic phenotype. Selective Gq-IP-Ca2+ 
signal impairment lead to "purely dyshormonogenic" features, including hyperthyrotropinemic goitrogenesis. Our work suggests a critical role of Arg534 located at the 2nd intracellular loop of TSHR in specific Gq activation.

\section{OP09 \\ IMAGE-GUIDED, TUMOR STROMA-TARGETED I-131 THERAPY OF HEPATOCELLULAR CARCINOMA (HCC) USING A HIF-1-SPECIFIC SODIUM IODIDE SYMPORTER GENE SYSTEM \\ Kerstin Knoop ${ }^{1}$, Andrea M. Mueller ${ }^{1}$, Kathrin A. Schmohl', Nathalie Schwenk', Janette Carlsen ${ }^{2}$, Marcus Hacker², Burkhard Goeke ${ }^{1}$, Ernst Wagner ${ }^{3}$, Peter J. Nelson ${ }^{4}$, Christine Spitzweg ${ }^{1}$ ${ }^{1}$ Department of Internal Medicine II, University Hospital of Munich, Germany, ${ }^{2}$ Department of Nuclear Medicine, University Hospital of Munich, Germany, ${ }^{3}$ Department of Pharmacy, Center of Drug Research, Pharmaceutical Biology-Biotechnology, Ludwig-Maximilians- University, Munich, Germany, ${ }^{4}$ Clinical Biochemistry Group, Medical Policlinic, University Hospital of Munich, Germany}

The tumor-homing property of mesenchymal stem cells (MSCs) has lead to their use as delivery vehicles for therapeutic genes. The application of the sodium iodide symporter (NIS) as reporter and therapy gene allows non-invasive imaging of MSC biodistribution and functional transgene expression by ${ }^{123} \mathrm{I}$-scintigraphy or PET-imaging, as well as therapeutic application of ${ }^{131} \mathrm{I}$. Hypoxia-inducible factor 1 (HIF-1) is a key mediator of the cellular response to hypoxia and therefore highly expressed in solid malignancies. Placing NIS under the control of the HIF-1 promoter should consequently allow tumorspecific NIS expression after MSC-mediated delivery.

We stably transfected human MSCs with NIS driven by the HIF-1 promoter (HIF-1-NIS-MSCs) and analyzed functional NIS expression by iodide uptake assay, Western blot and FACS analyses. We further investigated distribution and tumor recruitment of HIF-1-NIS-MSCs by ${ }^{123}$ I-scintigraphy and evaluated their therapeutic potential for systemic NIS-based radioiodine therapy in a HCC xenograft mouse model.

After hypoxia was simulated in vitro by treatment of HIF-1-NIS-MSCs with $300 \mu \mathrm{M}$ of the hypoxia mimetic agent $\mathrm{CoCl}_{2}$, a 48 -fold increase in perchlorate-sensitive iodide uptake was observed compared to HIF-1-NIS-MSCs under normal conditions. Western blot and FACS analyses confirmed $\mathrm{CoCl}_{2}-$ induced NIS expression in HIF-1-NIS-MSCs. After establishment of subcutaneous HCC xenografts in nude mice, HIF-1-NIS-MSCs were injected i.v. and MSC distribution was analyzed by $\gamma$-camera imaging. Injection of $18.5 \mathrm{MBq}$ ${ }^{123} \mathrm{I}$ resulted in a tumor-selective iodide accumulation showing active MSC recruitment and tumor-specific promoter activation which was confirmed by immunohistochemistry and ex vivo $\gamma$-counter analysis. Administration of a therapeutic dose of ${ }^{131} \mathrm{I}(55.5 \mathrm{MBq})$ in HIF-1-NIS-MSC-treated mice resulted in a significant delay of tumor growth and improved survival.

Our results demonstrate selective recruitment of HIF-1-NIS-MSCs into HCC tumors resulting in tumor-specific iodide accumulation, opening the exciting prospect of NIS-mediated radionuclide therapy of extrathyroidal tumors after MSC-mediated gene delivery.

\section{OP10 \\ THE COACTIVATOR TAZ MODULATES THE PHENOTYPIC EXPRESSION OF NKX2.1 MUTANTS IN THE BRAIN-THYROID-LUNG SYNDROME}

Amanda Herranz ${ }^{1}$, Christian Martín Moya ${ }^{1}$, Lucía Garzón ${ }^{2}$, Carmen Luna ${ }^{3}$, Noemí Núñez ${ }^{4}$, Rogelio Simón ${ }^{4}$, Pilar Santisteban ${ }^{5}$, José Carlos Moreno ${ }^{1}$

${ }^{1}$ Thyroid Molecular Laboratory, Institute for Medical and Molecular Genetics (Ingemm), La Paz University Hospital, Madrid, Spain,

${ }^{2}$ Paediatric Endocrinology, 12 de Octubre University Hospital, Madrid, Spain, ${ }^{3}$ Paediatric Pneumology and Allergy, 12 de Octubre University Hospital, Madrid, Spain, ${ }^{4}$ Paediatric Neurology, 12 de Octubre

University Hospital, Madrid, Spain, ${ }^{5}$ Physiopathology of Endocrine and Nervous System, Biomedical Research Institute (lib), Spanish Council for Scientific Research (Csic), Madrid, Spain

NKX2.1 is a homeobox transcription factor involved in development of the thyroid gland, basal ganglia and lungs. NKX2.1 defects cause the BrainThyroid-Lung syndrome (BTLS) presenting with congenital hypothyroidism, coreoathetosis and respiratory distress. BTLS shows a largely unexplained clinical variability, especially for the presence and severity of the lung disease. TAZ is a nuclear co-activator capable to interact with NKX2.1 and highly expressed in lungs.

Objective: To investigate whether TAZ modifies the transactivation capacity of NKX2.1 mutants and the clinical expression of the lung disease in BTLS.

Patient \& Methods: A 5 year old boy with congenital hypothyroidism (detected at screening), chorea, chronic lung disease and developmental delay, suggestive of BTLS. PCR and sequencing of the NKX2.1 coding region. Luciferase-reporter assay in HeLa cells of wildtype and NKX2.1 mutants over 3 gene promoters from tissues affected in BTLS: Thyroglobulin (thyroid), Lim-homeobox-6 (basal ganglia) and Surfactant-protein-B (lung), cotransfected or not with TAZ.

Results: A novel missense NKX2.1 mutation was identified (p.N211S), inherited from a mother with moderate phenotype and located at the DNAbinding-domain. This mutant was experimentally compared with a de novo p.V75fsX 408 at the amino-terminal-domain associated with severe lung emphysema and with a yet described mutation (p.A276fsX350) at the carboxy-terminal tail (BTLS patient with normal lung function).

All mutations dramatically decreased the transcriptional activity of NKX2.1 on the three promoters, showing no or residual activity compared to wildtype. Co-transfection with TAZ did not increase transactivation of neither p.N211S nor p.V75fsX408 (whose carriers showed severe lung disease) on any of the promoters. In contrast, co-transfection with TAZ completely rescued the transactivation activity of p.A276fsX350, selectively on the pulmonary promoter.

Conclusion: TAZ rescues transactivation capacity of mutants associated with normal lung function but not that of mutants with severe lung disease. TAZ represents a modifier gene of the lung phenotypic variability of BLTS.

\section{OP11 \\ EFFICACY AND TOXICITY OF VANDETANIB FOR ADVANCED MEDULLARY THYROID CANCER TREATMENT, THE FRENCH EXPERIENCE \\ Cecile Chougnet ${ }^{1}$, Martin Schlumberger ${ }^{2}$, Borget Isabelle ${ }^{3}$, TUTHYREF Network ${ }^{4}$ \\ ${ }^{1}$ Hopital Saint Louis, ${ }^{2}$ Department of Nuclear Medicine and Endocrine Oncology, Gustave Roussy and Université Paris-Sud, Villejuif, France, \\ ${ }^{3}$ Gustave Roussy, Université Paris-Sud, Villejuif, France, ${ }^{4}$ Tuthyref}

A randomized phase III trial in metastatic medullary thyroid cancer (MTC) demonstrated that vandetanib treatment is effective, leading to Regulatory Approvals, but its use may be associated with notable toxicities. The objective of the study was to describe the toxicity profile and response rate of vandetanib treatment in France, outside a trial.

Methods: 68 patients were treated with vandetanib in the frame of a temporary use authorization (ATU) in France from 08/2010 to 02/2012, where the drug was available on request for locally advanced or metastatic MTC, with patient's agreement for safety data collection. Patient characteristics, treatment 
parameters, toxicity profile and efficacy were retrospectively collected. Eight patients were excluded from the analysis: vandetanib was not started $(n=3)$ or had been given in a trial before ATU $(n=3)$, non-MTC cancer $(n=2)$.

Results: Data from the 60 MTC patients were analyzed. Mean age was 58 [range 11-83] years, 39 patients were male, and 6 had hereditary MTC. 56 $(93 \%)$ had metastatic disease: in the mediastinum $(82 \%)$, bones $(65 \%)$, liver $(53 \%)$ or lung $(53 \%)$, and 4 had locally advanced disease. Median follow-up was 20 months, median duration of treatment was 10 (range: 0.3-36) months and 15 patients are continuing vandetanib (range: $18-36$ months). 25 patients discontinued treatment for disease progression (range: 0.3-29 months). Best tumor response was a complete response in one patient, a partial response in $12(20 \%)$, stable disease in $33(53 \%)$ and progression in 12 patients $(20 \%)$. All patients had at least one AE during treatment: main AEs were skin toxicity, diarrhea and asthenia. Grade 3 and 4 AEs occurred in 21 and 6 patients, respectively, and 16 patients discontinued treatment for toxicity.

Vandetanib is an efficacious option for patients with MTC, but AEs should be monitored carefully and can be minimized by educating both patients and care providers.

\section{OP12 \\ METHYLATION PROFILING REVEALS MARKERS SPECIFIC TO DISTINCT RET MUTATIONS IN MEDULLARY THYROID CARCINOMA}

Veronika Mancikova ${ }^{1}$, Esmeralda Castelblanco ${ }^{2}$,

Agustín F. Fernández ${ }^{3}$, Lucía Inglada Pérez ${ }^{4}$, Aguirre De Cubas ${ }^{5}$,

Salud Borrego ${ }^{6}$, Xavier Matias-Guiu ${ }^{7}$, Mario Encinas $^{2}$, Mario F. Fraga ${ }^{3}$,

Mercedes Robledo ${ }^{4}$

${ }^{1}$ Hereditary Endocrine Cancer Group, Spanish National Cancer

Research Centre (Cnio), ${ }^{2}$ Hospital Universitari Arnau de Vilanova,

Lleida, ${ }^{3}$ Instituto Universitario de Oncología del Principado de

Asturias, Oviedo, ${ }^{4}$ Hereditary Endocrine Cancer Group, Spanish

National Cancer Research Centre (Cnio); Isciii Center for Biomedical

Research on Rare Diseases (Ciberer), ${ }^{5}$ Hereditary Endocrine Cancer

Group, Spanish National Cancer Research Centre (Cnio), Madrid,

Spain, ${ }^{6}$ Hospital Virgen del Rocío, Sevilla, ${ }^{7}$ Department of Pathology,

University Hospital Arnau de Vilanova, University of Lleida, Irblleida

Medullary thyroid carcinoma (MTC) accounts for $2-5 \%$ of thyroid malignancies. Around $75 \%$ of them are sporadic, and the remaining $25 \%$ are hereditary and related to Multiple Endocrine Neoplasia type 2 syndrome. Although a genotype-phenotype correlation related to specific germline RET mutations is well established, the disease etiology specifically associated with each mutation still remains largely unknown. Here, we investigated the role of aberrant DNA methylation in the development of MTC. We performed genome-wide DNA methylation profiling in the largest MTC series reported to date, comprising 48 primary tumors, using the $27 \mathrm{~K}$ Illumina platform. We observed significant differences between the methylation patterns among the samples bearing the $R E T^{\mathrm{M} 918 \mathrm{~T}}, R E T^{\mathrm{C} 634 \mathrm{X}}$ mutation and "wild-type" (WT) tumors; those $R E T^{\mathrm{M} 918 \mathrm{~T}}$ - related had a larger number of hypomethylation events when compared to $R E T^{\mathrm{C} 634 \mathrm{X}}$ - positive and WT tumors. Moreover, through the integration of methylation and transcriptional expression data of the same tumors, we identified genes whose expression is associated with the methylation status of their promoters. Finally, we validated the aberrant methylation events of three of the genes showing negative correlation between gene expression and methylation in an independent set of 35 MTCs by bisulfite pyrosequencing. Currently, we are working with MZ-CRC-1 and TT cell lines in order to elucidate the functional effect of these aberrant events to add new insights into MTC carcinogenesis.

\section{OP13 \\ SPONTANEOUS ABORTION AND STILLBIRTH ARE COMMON IN BOTH HYPERTHYROIDISM AND HYPOTHYROIDISM: A DANISH POPULATION-BASED STUDY OF 1,028,045 PREGNANCIES}

Stine Linding Andersen ${ }^{1}$, Jørn Olsen ${ }^{2}$, Chun Sen Wu ${ }^{2}$, Peter Laurberg ${ }^{1}$

${ }^{1}$ Aalborg University Hospital, ${ }^{2}$ Aarhus University

Objectives: Pregnancy loss in women with thyroid dysfunction has been described in case reports, but the risk of pregnancy loss caused by maternal hyperthyroidism or hypothyroidism in a population is unknown.

Methods: All pregnancies in Denmark, 1997-2008, leading to hospital in- or outpatient visit $(n=1,028,045)$ were identified plus information on maternal thyroid dysfunction up to two years after the pregnancy (hospital diagnosis/drug prescription). Cox proportional hazards model was used to estimate adjusted (e.g. maternal age, smoking) hazard ratio (aHR) with $95 \%$ confidence interval (CI) for spontaneous abortion $(<22$ weeks) and stillbirth ( $\geq 22$ weeks), reference: no maternal thyroid dysfunction.

Results: Outcomes studied were: spontaneous abortion $(\mathrm{n}=111,942)$, stillbirth $(\mathrm{n}=2,934)$, and live birth $(\mathrm{n}=730,359)$, excluding induced abortion $(16.6 \%)$ and molar/ectopic pregnancies $(1.1 \%)$. When maternal hyperthyroidism $(\mathrm{n}=5,229)$ or hypothyroidism $(\mathrm{n}=5,430)$ were diagnosed before/during the pregnancy, spontaneous abortion occurred more often, both in women treated for thyroid dysfunction before the pregnancy alone (hyperthyroidism: aHR 1.28 (95\% CI: 1.16-1.41)/hypothyroidism: 1.31 (1.09-1.57)), and in women treated medically for thyroid dysfunction in early pregnancy (hyperthyroidism: 1.18 (1.06-1.32)/hypothyroidism: 1.16 (1.08-1.25)). No change in the risk of stillbirth was observed. When maternal hyperthyroidism ( $\mathrm{n}=$ $2,361)$ or hypothyroidism $(n=2,900)$ were diagnosed and treated for the first time in the two-year period after the pregnancy, there was a high risk that the pregnancy under study had terminated with stillbirth (hyperthyroidism: 2.21 (1.35-3.61)/hypothyroidism (excluding treatment the first year only): 1.81 (1.07-3.06)). No change in the risk of spontaneous abortion was observed.

Conclusions: Both early (spontaneous abortion) and late (stillbirth) pregnancy loss were common in women suffering from thyroid dysfunction. Inadequately treated thyroid dysfunction in early pregnancy may have been involved in spontaneous abortion, and undetected abnormal maternal thyroid hormone levels present in late pregnancy may have attributed to an increased risk of stillbirth. Results are in support of routine thyroid function testing in pregnancy.

\section{OP14 \\ INCREASED FAT MASS RESULTS IN HIGHER FREE T3 LEVELS IN CHILDREN}

Pete N. Taylor ${ }^{1}$, Nicholas Timpson ${ }^{2}$, Jonathan Tobias ${ }^{2}$, Colin Dayan ${ }^{1}$

${ }^{1}$ Institute of Molecular \& Experimental Medicine; School of Medicine;

Cardiff University, ${ }^{2}$ University of Bristol, UK

Objective: Thyroid status is intimately associated with body composition, but the impact of common variation in childhood thyroid hormone levels has not been extensively studied. We therefore explored the relationship between thyroid status and body composition in a population based birth cohort.

Methods: Thyroid function was measured in 3,634 children at age 7 who also underwent DXA scans at ages 9 and 15 in a subset of the Avon Longitudinal Study of Parents and Children. Ordinary least squares linear regression of standardized variables were used to examine the relationship between thyroid stimulating hormone (TSH) free tri-iodothyronine $\left(\mathrm{FT}_{3}\right)$ and free thyroxine $\left(\mathrm{FT}_{4}\right)$ on fat mass and lean mass. Analyses were adjusted for age, sex, social class, height and other thyroid hormone parameters. Using variation in FTO robustly associated with fat mass we sought to address if changes in thyroid function were a cause or a consequence of differences in fat mass.

Results: At age 9 no association between TSH and body composition was observed. $\mathrm{FT}_{3}$ was positively associated with fat mass $(\mathrm{B}=0.1295 \% \mathrm{CI}$ $0.09,0.15 \mathrm{P}<0.001)$ and negatively associated with lean mass $(\mathrm{B}=-0.03$ $95 \% \mathrm{CI}-0.05,-0.01 \mathrm{p}=0.01)$. In contrast $\mathrm{FT}_{4}$ was negatively associated with fat mass $(\mathrm{B}=-0.0695 \% \mathrm{CI}-0.09,0.03 \mathrm{P}<0.001)$ and lean mass $(\mathrm{B}=-0.02$ $95 \% \mathrm{CI}-0.04,0.01 \mathrm{p}=0.06)$. Similar associations were also present at age 15 . Instrumental variable analysis using rs1558902 in FTO, revealed a positive 
association between higher levels of fat mass and $\mathrm{FT}_{3}$. $(\mathrm{B}=0.0695 \% \mathrm{CI} 0.02$, $0.11 \mathrm{p}=0.006$ ).

Conclusion: Variation in thyroid status even within the normal population is associated with key changes in body composition. Intriguingly $\mathrm{FT}_{3}$ is positively associated with fat mass, with instrumental variable analysis indicating increased $\mathrm{FT}_{3}$ levels are a consequence rather than a cause of increased fat mass. Given rising childhood obesity levels, this relationship could have important cardiovascular and metabolic consequences.

\section{OP3 Medullary Thyroid Cancer \& Anaplastic Carcinoma}

\section{OP15 \\ ARE DIFFERENCES IN GENE EXPRESSION PROFILE BETWEEN TYPE OF RET MUTATION IN MEDULLARY THYROID CANCER PRESENT? \\ Malgorzata Oczko-Wojciechowska ${ }^{1}$, Michal Swierniak ${ }^{2}$, \\ Malgorzata Kowalska', Tomasz Tyszkiewicz', Agnieszka Pawlaczek ${ }^{1}$, \\ Michal Jarzab ${ }^{3}$, Dagmara Rusinek $k^{4}$, Jadwiga Zebracka-Gala ${ }^{1}$, \\ Agnieszka Czarniecka ${ }^{5}$, Jolanta Krajewska ${ }^{6}$, Barbara Jarzab ${ }^{1}$ \\ ${ }^{1}$ Msc Memorial Cancer Center and Institute of Oncology, Gliwice Branch, Department of Nuclear Medicine and Endocrine Oncology, ${ }^{2}$ Msc Memorial Cancer Center and Institute of Oncology, Gliwice Branch, Department of Nuclear Medicine and Endocrine Oncology; Genomic Medicine; Department of Genenal Transplant and Liver Surgery; Medical University of Warsaw, ${ }^{3}$ Msc Memorial Cancer Center and Institute of Oncology, Gliwice Branch, III Radiotherapy Clinic, ${ }^{4} \mathrm{Msc}$ Memorial Cancer Center and Institute of Oncology, Gliwice Branch, Poland; Department of Nuclear Medicine and Endocrine Oncology, ${ }^{5}$ Centre of Oncology M.Sklodowska-Curie Memorial Institute Gliwice Branch, ${ }^{6}$ M.Sklodowska-Curie Memorial Cancer Center and Institute of Oncology, Gliwice Branch}

Aim of the Study: Medullary thyroid cancer (MTC) arises from parafollicular C and occurs as hereditary (20\% of all cases) and sporadic form. Hereditary type is a consequence of RET proto-oncogene germline mutations. Strong genotype-phenotype manifestation is correlated with different sites of RET mutation. The aim of our study was to evaluate whether the differences in gene expression profile between hereditary and sporadic MTC are detectable and related to the particular RET mutation.

Methods: Fresh-frozen tumor samples from $60 \mathrm{MEN} 2$ patients and 21 RET negative MTC patients were collected. Germline mutation screening was performed according to standard diagnostic algorithm. RET somatic mutations were analyzed among sporadic MTC patients and twenty one exons of $R E T$ gene were directly sequenced. Gene expression profile was analyzed in 34 MTC samples using Gene Chip 1.0 ST Arrays (Affymetrix). An independent set of 26 MTC samples was used for QPCR validation.

Results: Hierarchical clustering did not show any global differences in gene expression profile regarding to the type of RET mutation, however, supervised analysis revealed 10 genes which expression differed tumor samples with mutation at RET codon 634 or and 918 . The most significant gene was NTRK3. Samples with mutation at codon 918 were characterized by higher level of expression of NTRK3 gene. However, verification by QPCR on separate group of samples did not show a statistical significance. Gene PROM1, proposed in the literature as gene related to RET 918 mutation, was also validated but did not show any differences in expression, both in microarray and QPCR analysis.

Conclusion: RET mutation status was more important for overall transcriptome profile rather than the heredity aspect. The differences in gene expression profile between tumors induced by RET cysteine codons 634 and RET 918 mutations are rather marginal.

Supported by NCN, grant no NN401 410639

\section{OP16 \\ MILD HYPERCALCITONINEMIA IN PATIENTS WITH BENIGN DISEASES OF THE THYROID AND CALCIUM HOMEOSTASIS}

Juergen Kratzsch ${ }^{1}$, Stefan Karger ${ }^{1}$, Friedhelm Raue ${ }^{2}$, Joachim Feldkamp ${ }^{3}$, Sandra Scheeß ${ }^{3}$, Tanja Diana ${ }^{4}$, Nina Mattheis ${ }^{4}$, George J. Kahaly'

${ }^{1}$ University Hospital, Leipzig, ${ }^{2}$ Praxis Prof. Raue \& Colleagues, Heidelberg, ${ }^{3}$ Klinikum Bielefeld, Bielefeld, ${ }^{4}$ Johannes Gutenberg University Medical Center, Mainz, Germany

Objective: Calcitonin (CT) is a biomarker for diagnostic and therapeutic monitoring of patients with medullary thyroid carcinoma. We systematically evaluated a new immunoassay for the determination of CT in sera from patients with different benign diseases of the thyroid and of the calcium homeostasis.

Methods: Serum was withdrawn from the following groups of patients in a multi-centric study: apparently healthy adult subjects (females: $\mathrm{n}=166$; males: $n=160$ ), patients with thyroid nodules (PTN; $n=181)$, Graves disease (GD; $\mathrm{n}=54$ ); Hashimoto thyreoiditis (HT; $\mathrm{n}=87$ ); non-Graves hyperthyroidism (NGHT; $\mathrm{n}=18$ ) and diseases of the calcium homeostasis (DCH; $\mathrm{n}=$ 25). CT was measured by the new Cobas assay (Roche). Intra- and inter-assay coefficients of variation were below $6.2 \%$ for concentrations between 1-20 $\mathrm{pg} / \mathrm{mL}$.

Results: The upper reference limit (97.5 percentile) was defined at 6.40 $\mathrm{pg} / \mathrm{mL}$ for females and $9.52 \mathrm{pg} / \mathrm{mL} \mathrm{CT}$ for males by data of apparently healthy subjects. The same statistical limit for patients with PTN was found at $13.3 \mathrm{pg} /$ $\mathrm{mL}$. Maximal CT concentrations of patients with GD, HT, NGHT and DCH were $19.9,10.1 ; 13.3$ and $19.1 \mathrm{pg} / \mathrm{mL}$.

Conclusion: Benign diseases of the thyroid and calcium homeostasis may cause a mild hypercalcitoninemia if values are compared with reference limits of apparently healthy subjects. The detailed clinical history of patients has to be considered for the correct interpretation of CT results to avoid unwarranted further diagnostic efforts.

\section{OP17 \\ DIVERGENT ROLES OF FORKHEAD FAMILY TRANSCRIPTION FACTOR PARALOGS IN MEDULLARY THYROID CARCINOMA}

Mikael Nilsson', Shawn Liang ${ }^{2}$, Camilla Ingeson ${ }^{2}$, Therese Carlsson², Jakob Dahlberg ${ }^{3}$, Svante Jansson ${ }^{3}$, Akif Demir ${ }^{4}$, Henrik Fagman ${ }^{4}$ ${ }^{1}$ Sahlgrenska Cancer Center, Sahlgrenska Academy, University of Gothenburg, Gothenburg, Sweden, ${ }^{2}$ Sahlgrenska Cancer Center, University of Gothenburg, ${ }^{3}$ Department of Surgery, Sahlgrenska University Hospital, ${ }^{4}$ Department of Pathology, Sahlgrenska University Hospital

Forkhead transcription factor A1 and A2 (Foxa1 and Foxa2) are closely related regulators of embryonic development that probably evolved by duplication of a common ancestral gene. Both factors are implicated in lung and prostate cancer particularly in tumors with a neuroendocrine phenotype. We recently identified Foxa1 and Foxa2 expression in embryonic thyroid $\mathrm{C}$ cells and that this also distinguished $\mathrm{C}$ cell precursors from the follicular cell lineage in mice. The aim of this study was to elucidate a potential role of Foxa1/2 in medullary thyroid carcinoma (MTC).

FOXA1 and FOXA2 expression were determined employing public microarray databases obtained from human thyroid tumors and by RT-PCR analysis of established thyroid cancer cell lines. The influence of FOXA1/2 knockdown by siRNA on $\left[{ }^{3} \mathrm{H}\right]$ thymidine incorporation was examined in TT cells; qRT-PCR and Western blotting confirmed efficient knockdown. Expression and localization of FOXA1, FOXA2, Ki67, E-cadherin and calcitonin were investigated in human MTC samples by immunofluorescence microscopy.

Foxa1 and Foxa2 were detected in MTC but not in PTC or ATC tumors. Nuclear localization of Foxal was prominent in MTC tumor areas with a high Ki67 labeling index. Knockdown of Foxa1 reduced the proliferation rate of TT cells. Foxa2 was nuclear in most MTC cells independently of Ki67. However, infiltrating tumor cells at the invasive front showed no expression or cytoplasmic staining of Foxa2. Nuclear exclusion/down-regulation of Foxa2 correlated with loss of E-cadherin. Both Foxa1 and Foxa2 were co-expressed with calcitonin and E-cadherin in MTC metastases. 
The observations infer Foxa1 and Foxa2 as novel biomarkers of neuroendocrine thyroid cancer. While Foxal seems to participate in MTC tumor cell proliferation Foxa2 may promote the tumor epithelial phenotype that is transiently lost in invasive MTC cells. Distinct functions of Foxa1 and Foxa2 in tumors may be related to the evolutionary determined diversification of these two gene paralogs.

\section{OP18 \\ EXOME SEQUENCING REVEALS THAT SOS1 GENE MUTATIONS CAUSE A SYNDROME WITH PHENOTYPIC FEATURES OF MULTIPLE ENDOCRINE NEOPLASIA TYPE 2B BUT NO ENDOCRINOPATHY}

Bijay Vaidya ${ }^{1}$, Martina Owens ${ }^{2}$, Emma Kivuva ${ }^{3}$, Ali Chakera ${ }^{4}$, Peter Turnpenny ${ }^{3}$, Andrew Hattersley ${ }^{5}$, Anthony Quinn ${ }^{6}$,

Richard Caswell , Hana Lango Allen ${ }^{7}$, Sian Ellard ${ }^{8}$

${ }^{1}$ Department of Endocrinology; Royal Devon \& Exeter Hospital, ${ }^{2}$ Department of Molecular Genetics, Royal Devon \& Exeter Hospital, Exeter, UK, ${ }^{3}$ Department of Clinical Genetics, Royal Devon \& Exeter Hospital, Exeter, UK, ${ }^{4}$ Department of Endocrinology, Royal Devon and Exeter Hospital, Exeter, UK, ${ }^{5}$ Department of Endocrinology, Royal Devon and Exeter Hospital, Exeter, Uk; Institute of Biomedical and Clinical Science, University of Exeter Medical School, Exeter, UK, ${ }^{6}$ Department of Ophthalmology, Royal Devon and Exeter Hospital, Exeter, UK, ${ }^{7}$ Institute of Biomedical and Clinical Science, University of Exeter Medical School, Exeter, UK, ${ }^{8}$ Department of Molecular Genetics, Royal Devon \& Exeter Hospital, Exeter, UK; Institute of Biomedical and Clinical Science, University of Exeter Medical School, Exeter, UK

Objective: Multiple Endocrine Neoplasia type 2B (MEN2B) is characterized by medullary thyroid carcinoma (MTC), phaeochromocytoma, and typical physical features (including mucosal neuromas, Marfanoid habitus and prominent corneal nerves). Rarely, patients present with physical features of MEN2B without endocrinopathy or RET gene mutations, thought to represent a distinct subgroup termed 'mucosal neuroma syndrome'. We aimed to investigate genetic basis of this syndrome.

Methods: We studied two families. Family 1: 13-year-old girl presented with photophobia. She had prominent corneal nerves, mucosal neuromas and Marfanoid habitus (Spyer et al., 2006). Urinary catecholamines were normal but pentagastrin-stimulated calcitonin levels were equivocal (baseline $0.09 \mu / 1$ (normal $<0.08 \mu / \mathrm{L}$ ), peak $0.14 \mu / \mathrm{L}$ ). She underwent prophylactic thyroidectomy; histology was normal. Subsequent genetic testing showed no RET gene mutations. Her parents are unaffected. Family 2: 37-year-old man also presented with eye symptoms and was found to have prominent corneal nerves, mucosal neuromas and Marfanoid habitus. Plasma metanephrines and pentagastrinstimulated calcitonin levels were normal. No RET mutations were detected. His 64-year-old mother has similar clinical features and no biochemical evidence of endocrinopathy. We undertook exome sequencing in the probands to detect a shared gene with a rare or novel non-synonymous variant.

Results: We identified a heterozygous SOS1 frameshift mutation, c.3248dup or c.3266dup, in each family. Sanger sequencing showed that the c.3248dup mutation had arisen de-novo in the proband of Family 1 and the c.3266dup mutation was inherited from the proband's affected mother in Family 2. SOS1 is a RAS-specific guanine nucleotide exchange factor which catalyses the activation of the RAS-MAPK pathway. The c.3248dup mutation has previously been reported in patients with Hereditary Gingival Fibromatosis, and generates a truncated protein lacking functional domains that maintain the down-regulated state.

Conclusions: Our results demonstrate the existence of mucosal neuroma syndrome as a clinical entity distinct from MEN2B that can now be diagnosed by genetic testing.

\section{OP19 \\ EFFICACY AND SAFETY OF VANDETANIB IN AGGRESSIVE AND SYMPTOMATIC MEDULLARY THYROID CANCER (MTC) - POST-HOC ANALYSIS FROM THE ZETA TRIAL (NCT00410761)}

Michael Kreiss I', Ole Hauch ${ }^{2}$, Alan Webster ${ }^{2}$, Martin Schlumberger ${ }^{3}$

${ }^{1}$ Department of Nuclear Medicine, Central Hospital of Augsburg,

Augsburg, Germany, ${ }^{2}$ Astrazeneca, Macclesfield, UK, ${ }^{3}$ Institut Gustave Roussy, Villejuif, France

In the Phase III ZETA trial (JCO 2012;30:134-141), vandetanib conferred a statistically significant progression-free survival (PFS) benefit versus placebo for patients with measurable, unresectable locally advanced or metastatic, hereditary or sporadic MTC. Based on ZETA, vandetanib was approved in the EU for the treatment of a smaller cohort: aggressive and symptomatic MTC.

Objectives: This analysis determined PFS, objective response rate (ORR), and adverse events (AEs) for vandetanib versus placebo among a sub-population of ZETA patients with symptomatic and progressive disease, reflecting EU approval.

Methods: Patients with symptomatic and progressive disease at baseline were identified. 'Progressive' was defined as documented progression $\leq 12$ months prior to enrolment and 'symptomatic' defined as at least one of the following symptoms at baseline: pain score $>4, \geq 10 \mathrm{mg} /$ day opioid use, diarrhoea, flushing, fatigue, pain, nausea, dysphagia, dysphonia, respiratory symptoms, weight loss. Endpoints: Central Read PFS, investigator-assessed PFS, ORR, AEs grade $\geq 3$.

Results: Of the 331 ZETA patients, 186 had symptomatic and progressive disease at baseline (126 vandetanib; 60 placebo). After discontinuation of randomized drug open-label vandetanib was started in 38 (88\%; placebo arm) and $26(38 \%$; vandetanib arm) patients. PFS and ORR are presented in the table below. Seventy-seven (61\%) of vandetanib-treated patients and $14(24 \%)$ placebo-treated patients had AEs grade $\geq 3$.

Conclusions: Vandetanib is well tolerated and offers clinical benefit over a wide spectrum of patients with MTC, with a statistically significant prolonged PFS among 'symptomatic and progressive disease' patients from ZETA, in line with the EU label.

Table 1. (for Abstract OP19)

\begin{tabular}{|c|c|c|c|c|}
\hline & \multicolumn{2}{|l|}{ Median PFS (months) } & \multirow[t]{2}{*}{ Hazard ratio } & \multirow[t]{2}{*}{$\mathrm{P}$ value } \\
\hline & Vandetanib $(\mathrm{n}=126)$ & Placebo $(n=60)$ & & \\
\hline Central Read, excluding open-label vandetanib & $30.1^{\dagger}$ & 11.1 & 0.32 & $<0.0001$ \\
\hline & \multicolumn{2}{|l|}{ ORR, n (\%) } & & \\
\hline & Vandetanib $(n=126)$ & Placebo $(n=60)$ & Odds ratio & P value \\
\hline
\end{tabular}

Median not reached. The reported median is estimated based on a Weibull model 


\section{OP20 \\ CLINICAL IMPACT OF RET GENETIC SCREENING OF IN THE MANAGEMENT OF MEDULLARY THYROID CARCINOMA (MTC) PATIENTS: 20 YEARS OF EXPERIENCE}

Francesca Casella ${ }^{1}$, Alessia Tacito ${ }^{1}$, Raffaele Ciampi ${ }^{1}$ Eleonora Molinaro ${ }^{1}$, Laura Agate ${ }^{1}$, Valeria Bottici' ${ }^{1}$, Antonio Matrone', Agnese Biagini ${ }^{1}$, Rossella Elisei ${ }^{1}$, Cristina Romei ${ }^{1}$

${ }^{1}$ Section of Endocrinology, Department of Clinical and Experimental Medicine, University of Pisa, Pisa, Italy

During the last 20 years, we performed RET genetic screening in 1556 subjects: 1007 arrived as affected by an apparently sporadic form of MTC and 95 were clearly affected by a hereditary form. The remaining 454 were relatives of RET positive MTC patients.

An unsuspected germline RET mutation was found in 69/1007(6.7\%) apparently sporadic MTC patients and were reclassified as hereditary: 60 FMTC and 8 MEN 2A syndromes were identified. The remaining 939 patients were $R E T$ negative thus their sporadic nature was confirmed. Ninety-five patients, 71 index cases and 24 relatives, arrived at our first clinical observation as affected by a hereditary MTC. A germline RET mutation was found in $69 / 71$ index cases. The 71 families were classified in FMTC $(\mathrm{n}=33)$, MEN $2 \mathrm{~A}(\mathrm{n}=26)$, MEN 2B $(\mathrm{n}=12)$. A total of 140 MEN 2 families (94 FMTC, 34 MEN 2A, 12 MEN 2B) have been diagnosed.

Following the identification of a germline RET mutations in the index cases, 454 relatives performed the RET screening and 137 were found to be gene carriers (GC).

RET screening allowed us to identify 33 different mutations. The V804M (exon 14) is the most prevalent mutation (22.4\%), but codon 634 (exon 11) is the most frequent $(\mathrm{n}=34)$. MEN $2 \mathrm{~A}$ syndromes are mainly associated to the C634R mutation while the MEN 2B to the M918T mutation. Among all mutations we found rare variants (R833C, A883T, M848T, M918V, E632K, S904F, T338I, V648I) whose biological significance has to be proven.

On the basis of our experience, the clinical impact of the RET genetic screening can be summarized as follows: 1) identification of hereditary cases (7-8\%) that would be clinically considered as sporadic; 2 ) identification of GC unaware of their condition thus allowing their early treatment; 3 ) identification of new RET mutations not necessarily pathogenetic for the disease.

\section{OP21 \\ REFERENCE RANGE OF SERUM CALCITONIN IN CHILDREN \\ Maria Grazia Castagna1, Laura Fugazzola², Fabio Maino', \\ Danila Covelli ${ }^{3}$, Fausta Sestini', Chiara Ferraris Fusarini", \\ Carlo Scapellato $^{5}$, Francesco Macchini ${ }^{6}$, Gabriele Cevenini ${ }^{7}$, \\ Furio Pacini ${ }^{1}$ \\ ${ }^{1}$ Section of Endocrinology, University of Siena, Siena, Italy, \\ ${ }^{2}$ Department of Clinical Sciences and Community Health, University of Milan, Milan, Italy; Endocrine Unit, Fondazione Policlinico Irccs, Milan, Italy, ${ }^{3}$ Section of Endocrinology and Diabetology, University of Milan, Italy, ${ }^{4}$ Laboratory of Clinical Pathology, University of Milan, Italy, ${ }^{5}$ Laboratory of Clinical Pathology, University of Siena, Siena, Italy, ${ }^{6}$ Section of Pediatrics, University of Milan, Italy, ${ }^{7}$ Section of Surgery Bioengineering, University of Siena, Siena, Italy}

MEN2 children carrying germline RET mutations are candidate to prophylactic thyroidectomy, whose timing is based on the mutation associated risk status and on the calcitonin (CT) levels. However the reference range for serum CT in children and adolescent is not clearly defined.

Aim of this study was to establish the reference range for serum CT in newborn, children and adolescents up to 16 years. Serum samples were available in a total of 2006 children ( 940 girls and 1066 boys) equally distributed in each age-range. Samples were collected in the out-patient pediatric clinic in children undergoing blood testing for any medical condition not affecting serum CT levels. We also evaluated 32 cord blood samples. Serum CT levels were measured by a commercially available two-site chemiluminescence immunometric assay (analytic sensitivity $2.0 \mathrm{pg} / \mathrm{ml}$ ).

Serum CT was undetectable $(<2.0 \mathrm{pg} / \mathrm{ml})$ in $1152 / 2006(57.4 \%)$ samples. Undetectable samples were more frequent starting from the second years of age. Mean $( \pm \mathrm{SD}$ ) serum $\mathrm{CT}$ in children $\leq 1$ year was $9.0 \pm 8.8 \mathrm{pg} / \mathrm{ml}$ (range
0.7-48.9) with a significant trend to decrease in subsequent years $(\mathrm{P}<0.0001)$. At age 16, mean serum CT was $1.22 \pm 1.31 \mathrm{pg} / \mathrm{ml}$ (range 0-5.6) The same trend was found in males and females. CT levels in cord blood samples were similar to the first year-levels $(6.3 \pm 4.7 \mathrm{pg} / \mathrm{ml}$, range $0.43-17.8)$.

In conclusion, serum $\mathrm{CT}$ levels in children progressively decrease during the first 3 years of life. Thereafter, serum CT are comparable with those of adults. These data may be of help in the screening of young RET-mutation carriers.

\section{OP22 \\ MOLECULAR PROFILING OF SPORADIC MEDULLARY THYROID CARCINOMA PATIENTS BY NEXT GENERATION SEQUENCING}

Vera Tiedje $^{1}$, Saskia Ting ${ }^{2}$, Thomas Herold ${ }^{3}$, Kurt Werner Schmid ${ }^{2}$, Dagmar Führer-Sake/ ${ }^{4}$

${ }^{1}$ Department of Endocrinology and Metabolism; University of Duisburg-

Essen, ${ }^{2}$ Institute of Pathology; University Hospital Essen, ${ }^{3}$ Institute of

Pathology; University Hospital Essen; German Cancer Consortium

(Dktk) Heidelberg, ${ }^{4}$ Department of Endocrinology and Metabolism;

University Hospital Essen

Objectives: Sporadic medullary thyroid carcinoma (MTC) is often diagnosed in an advanced stage with lymph node or distant metastases. Treatment for aggressive metastatic disease has recently become available in form of TKI. However, prognostic factors and biomarkers for response to therapy are still lacking. The aim of this study was to use next generation sequencing in order be able to identify molecular profiles correlating to tumor biology and response to targeted treatment.

Methods: Paraffin embedded tissue from 36 sporadic MTC patients (13 male/23 female) treated at the Department of Endocrinology and Metabolism (University Hospital Essen) were available. After DNA isolation, next generation sequencing of 15 different oncogenes was performed.

Results: Seventeen (47\%) patients were cured by surgery and in $19(53 \%)$ patients metastatic disease was diagnosed (10 treated with vandetanib). Most frequently detected, mutations occurred in $\mathrm{PI} 3 \mathrm{KCa}(25 / 36 ; 69 \%)$, ERBB2 $(14 / 36 ; 39 \%)$ and NRAS $(7 / 36 ; 19 \%)$. In $15 / 17$ (88\%) patients without metastasis and in 10/19 (53\%) patients with metastatic disease PI3KCa mutations were detected. ERBB2 mutations were predominately detected in patients with metastatic disease versus patients with local disease. The same pattern was observed for NRAS mutations.

Conclusion: We present preliminary data indicating that aggressive MTC has a distinct molecular profile from surgically cured MTC. Expression studies are planned to elucidate the role of ERBB2, NRAS and PI3KCa mutations. 


\section{OP4 Thyroid Autoimmunity}

\section{OP23 \\ FIRST INTERNATIONAL STANDARDISATION OF A TSH-RECEPTOR STIMULATING AUTOANTIBODY BIOASSAY}

Tanja Diana ${ }^{1}$, Markus Lehmann ${ }^{1}$, Jasmin Franken ${ }^{1}$, Michael Kanitz ${ }^{1}$, George J. Kahaly

${ }^{1}$ Johannes Gutenberg University Medical Center, Mainz, Germany

Objective: Reproducibility of bioassays varies between laboratories. To reduce assay variation, TSH-R stimulating autoantibody (TSAb) levels were calibrated against an international standard (IS) or patient sera using an FDAcleared TSAb bioassay.

Methods: TSAb was measured using $\mathrm{CHO}$ cells expressing a chimeric TSH-R. The second IS (NIBSC 08/204) was applied for calibration. Four users performed all experiments in one laboratory. TSAb was determined in triplicate. Results were reported as percentage of specimen-to-reference ratio (SRR\%) and converted into $\mathrm{mIU} / \mathrm{L}$.

Results: Assay precision was evaluated in 1:11 diluted negative serum and ten dilutions were assayed from 1:704 to 1:360448. Intra-assay precision was determined by one user and two plates were repeated within the same day. Interassay precision was measured using the same dilution samples during 20 consecutive days. For intra- and interassay precision, coefficient of variation values were $\leq 7 \%$ for patient dilutions and IS. A total of 102 dilution experiments were performed using 12 high TSAb-positive sera of patients with Graves' disease. Two-fold dilutions were prepared from 1:352 to 1:360448. Mean \pm SD values were $559 \pm 52,441 \pm 46,442 \pm 47,424 \pm 52,365 \pm 52,268 \pm 51,174 \pm 43,113 \pm 29,80 \pm 20$, $63 \pm 15$ and $56 \pm 15$, respectively. The IS dose-response curve was generated using concentrations of $0.3125-200 \mathrm{mIU} / \mathrm{L}$. A linear calibration curve was obtained between 10 and $100 \mathrm{mIU} / \mathrm{L}$. When diluting TSAb-positive sera, the IS concentrations $10,20,40$ and $80 \mathrm{mIU} / \mathrm{L}$ were used as a four-point calibration curve. Values were $419 \pm 43,348 \pm 40,229 \pm 31$ and $139 \pm 23$, respectively. All standard curves had $\mathrm{R}^{2}$ values $>0.95$. Dilution experiments using IS and TSAb sera showed high correlation and excellent reproducibility. The corresponding international unit measured value for the assay cut-off (SRR $<140 \%$ ) was $10.14 \pm 1.3 \mathrm{mIU} / \mathrm{L}$.

Conclusions: For the first time, standardization of a bioassay converting $\mathrm{TSAb}$ SRR\% to IU/L was achieved. These results will standardize TSAb levels among laboratories and enable more accurate comparison of TSAb studies.

\section{OP24 \\ MECHANISM OF TSHR ACTIVATION BY TSH AND THYROID STIMULATING ANTIBODIES}

Jane Sanders', Ricardo Nunez Miguel', Paul Sanders', Stuart Young', Jadwiga Furmaniak', Bernard Rees Smith ${ }^{1}$

${ }^{1}$ Firs Laboratories, Rsr Ltd, Cardiff, UK

The crystal structure of the FSHR ectodomain (ECD) in complex with FSH was used to build a comparative model of the TSHR ECD in complex with TSH. The coordinates of the TSHR leucine rich repeat domain (LRD) in the predicted TSH-TSHR ECD model were replaced by those of the LRD from the crystal structure of the K1-70-TSHR LRD complex, after coordinate superimposition. The structures of the M22-TSHR ECD and K1-70-TSHR ECD complexes were produced by replacing TSH coordinates with M22 and K1-70 coordinates from the crystal structures of their complexes with the TSHR LRD. The new structures allowed us to analyse hormone and antibody interactions with the receptor beyond the TSHR LRD and activation mechanisms.

The structure of the FSH-FSHR ECD complex suggests that the second step in FSHR activation is a movement of the receptor hinge loop driven by an electrostatic interaction between a sulfated Tyr in the hinge loop and a positively charged pocket on FSH. Analysis of the interactions in the TSH-TSHR ECD model indicated that receptor induced conformational changes involving TSHR sulfated Tyr385 can occur between unbound TSH and receptor-bound TSH similar to those observed between FSH and the FSHR ECD. However, receptor induced conformational changes involving Tyr385 cannot occur in interactions with M22. The surface of M22, which could potentially be in contact with Tyr385, is not charged, whereas that of the blocking antibody K1-70 is negatively charged. Furthermore, the K1-70 surface is too distant from Tyr385 in the complex to interact.
Our model of the structure of the TSH-TSHR ECD complex indicates that activation of the TSHR by TSH occurs in a similar way to FSHR activation by FSH. However, the interactions observed in the M22-TSHR ECD model suggest that the mechanisms of TSHR activation by TSH and TSHR stimulating antibodies are different.

\section{OP25 \\ CHANGES OF SERUM TSH RECEPTOR ANTIBODIES (TRAB) LEVELS IN PATIENTS WITH GRAVES' DISEASE (GD) SUBMITTED TO RADIOIODINE THERAPY (RAI)}

Irene Campi ${ }^{1}$, Guia Vannucchi ${ }^{1}$, Danila Covelli', Silvia Tirelli ${ }^{2}$ Rodari Marcello ${ }^{3}$, Giovanna Pepe ${ }^{4}$, Arturo Chiti', Mario Salvi' ${ }^{1}$ Department of Clinical Sciences and Community Health, Fondazione Irccs Ca' Granda Ospedale Maggiore Policlinico, '2Laboratory Medicine Unit, Fondazione Irccs Ca' Granda Ospedale Maggiore Policlinico, ${ }^{3}$ Department of Nuclear Medicine, Humanitas Clinical and Research Center, Rozzano, ${ }^{4}$ Department of Nuclear Medicine, Humanitas Clinical and Research Center, Rozzano, Italy

Aims of the present study were to analyze serum TRAb in patients submitted to RAI with steroid prophilaxis in relation to the therapeutic outcome and to compare two different assays. We measured serum TRAb in 119 patients treated with RAI, of whom 46 with oral (OGC) and 46 with i.v. (IVGC) steroid prophylaxis (cumulative dose $1.5 \mathrm{gr}$ ). Serum TRAb were measured at baseline and at 45, 90 and 180 days after RAI by Brahms TRAk human RIA and Brahms TRAk Kryptor (Thermo). Mean basal \pm SE serum TRAb was $9.6 \pm 2.2$ $\mathrm{U} / \mathrm{L}$ and $7.4 \pm 0.9 \mathrm{U} / \mathrm{L}$ with Kryptor and RIA methods, respectively. In all groups serum TRAb increased significantly after RAI $(P<0.01)$, but in those without prophylaxis they peaked at 45 days and were more elevated when compared to patients receiving either OGC or IVGC $(\mathrm{P}<0.01)$, with both assays. Interestingly, we observed four different patterns of serum TRAb changes after RAI: a) a late increase at 180 days; b) a decrease at 45 days and subsequent progressive marked increase during follow-up; c) a progressive increase until 90 days followed by a slow decrease; $d$ ) a progressive decrease from 45 days until the end of follow-up. These different patterns had no relationship with either basal TRAb and TSH $(\mathrm{P}=\mathrm{NS})$, the restoration of euthyroidism and the persistence/relapse of hyperthyroidism ( $\mathrm{n}=13)$, as well as the modality of steroid prophylaxis administration. At the end of follow-up serum TRAb measured with both assays became negative in four patients. The two assays were comparable $\left(\mathrm{r}^{2}=0.54, \mathrm{P}<0.0001\right)$ and the correlation was more significant after exclusion of Kryptor values higher than $200 \mathrm{U} / \mathrm{L}\left(\mathrm{r}^{2}=0.7, \mathrm{P}<0.0001\right)$. In this study, beside confirming an increase of serum TRAb after RAI, it is suggested that TRAB could have many different pattern of variation and that steroid prophylaxis is useful probably because it blunts serum TRAb elevation.

\section{OP26 \\ THYROID AUTOIMMUNITY IS PREVALENT IN PATIENTS WITH CELIAC DISEASE AND THEIR FIRST DEGREE RELATIVES - A PROSPECTIVE, CONTROLLED STUDY}

Detlef Schuppan ${ }^{1}$, Martin Rosenthal', Mareike Roth ${ }^{1}$, Jessica Willim ${ }^{1}$, Elisa Kolbe ${ }^{1}$, Tanja Diana ${ }^{1}$, George J. Kahaly ${ }^{1}$

'Johannes Gutenberg University Medical Center, Mainz, Germany

Objective: Autoimmune thyroid diseases (AITD) may cluster with several autoimmune disorders. We aimed to prospectively evaluate the close relationship between celiac disease (CD) and AITD.

Methods: In an academic referral center for autoimmune diseases with a joint thyroid-gastrointestinal outpatient clinic, demographic and clinical data were obtained from 101 consecutive patients with $\mathrm{CD}$ and their 132 firstdegree relatives. Serological and genetic investigations were performed and thyroid-related hormones and autoantibodies were measured in all 233 subjects. Also, three internationally validated psychometric tests were applied.

Results: Median age of the patients with CD (78 female) and their relatives $(78 / 132$ female or $59 \%)$ was 29 years (2-76 yrs.) and 33 yrs. (1-76 yrs.), respectively. AITD was present in 55/101 (54\%) and 40/132 (30\%) of CD patients and their relatives, respectively. Hashimoto's thyroiditis (HT) and Graves' disease (GD) were present in 41 and $14 \mathrm{CD}$ pts., respectively. HT was also more prevalent in the relatives $(33 / 132,25 \%)$ than GD $(7 / 132,5 \%)$. 
The following HLA-haplotypes (Xeligen RT, Eurospital) were noted in the CD pts.: DQ2.5 heterozygous (Cis+Trans) 39\%, DQ2.5 homozygous 20\%, DQ2.5/ DQ2.2 17\%, DQ2.5/DQ8 11\%, DQ2.2 heterozygous 6\%, heterozygous DQ8 $3 \%$, DQ2.2/DQ8 3\%, and homozygous DQ8 1\%. AITD was present in $80 \%$ and $57 \%$ in the DQ2.5/DQ8 and in the heterozygous DQ2.5 carriers, respectively. $83 \%$ of relatives with the DQ2.5/DQ8 had AITD. Both the Short Form36 Health Survey (all eight scales) and the Giessen Complaint List (GBB-24) showed markedly impaired scores in CD pts. with AITD vs. a large German healthy collective $(\mathrm{P}<0.001)$, whereas pts. with combined CD+AITD had higher fatigue scores vs. $\mathrm{CD}$ alone $(\mathrm{P}<0.044)$. The Hospital Anxiety and Depression Scales were not significantly impaired in CD.

Conclusions: This innovative prospective controlled study highlights the close relationship between $\mathrm{CD}$ and AITD and recommends immune genetic and thyroidal screening for pts. with $\mathrm{CD}$ and their relatives.

\section{OP27 \\ INCREASED REGULATORY B LYMPHOCYTES (BREG) IN PATIENTS WITH HASHIMOTO'S THYROIDITIS AND ASSOCIATED AUTOIMMUNE DISORDERS}

Ilenia Gatto ${ }^{1}$, Maria Giulia Santaguida ${ }^{2}$, Giorgio Mangino ${ }^{1}$,

Susanna Carlotta Del Duca², Camilla Virili2 Lucilla Gargano $^{3}$,

Giovanna Romeo ${ }^{1}$, Marco Centanni4

${ }^{1}$ Dept of Medico-Surgical Sciences and Biotechnologies, "sapienza" University of Rome, Latina, Italy, ${ }^{2}$ Dept of Experimental Medicine "sapienza" University of Rome, Latina, Italy, ${ }^{3}$ Endocrinology Unit, Santa Maria Goretti Hospital, AusI Latina, Latina, Italy, ${ }^{4}$ "sapienza" University of Rome Dept of Medico-Surgical Sciences and Biotechnologies Latina

Hashimoto's thyroiditis (HT) may occur with non-endocrine autoimmune disorders such as celiac disease (CD), chronic atrophic gastritis (CAG) and non-segmental vitiligo (NSV). The path of cellular immunity has been long ignored but it has been recently described a predominant Th1 and/or Th17 pattern in isolated HT, CD and CAG. However, data about the behaviour of regulatory $\mathrm{T}$ and B cells (Treg and Breg) in HT isolated or associated with other autoimmune disorders are scanty and the analysis of their involvement in these disorders represents the aim of our study.

Fourteen healthy donors (HD), 17 patients with isolated $\mathrm{HT}$, 7 with $\mathrm{HT}+\mathrm{CAG}$, 6 with $\mathrm{HT}+\mathrm{CD}$ and 6 with $\mathrm{HT}+\mathrm{NSV}$ to a total of 50 patients (44 women, 6 men, median age: 44 years) were selected for the study using tight inclusion criteria. Freshly PBMCs, collected from these patients, were assessed by flow cytometry to characterize Th17, Treg and Breg lymphocytes specific phenotype.

The median percentage of Th17 lymphocytes in HD was $1.4 \%$ while in patients with isolated HT it was significantly higher $(1.8 \% ; \mathrm{p}=0.0267)$. On the contrary, both B and T regulatory cells were similar in HD and isolated HT (both $\mathrm{p}=\mathrm{ns})$. As far as concerns patients with HT and concurrent autoimmune disorders, we observed a 2.5 times increase of Th17 lymphocytes $(3.2 \% ; p=0.0142)$ only when HT was concomitant with NSV. Again, Treg cells were similar in all patients while Breg phenotype was more expressed when thyroiditis was concurrent with $\mathrm{CAG}$ or $\mathrm{CD}(3.8 \%$ and $3.7 \% \mathrm{vs} 1.7 \% ; \mathrm{p}=0.0231$ and $\mathrm{p}=0.0330$ respectively).

In conclusion, Th17 cells were higher in HT isolated or associated with further autoimmune disorders. Treg cells were quantitatively similar in all conditions while Breg cells were clearly upregulated when HT was associated with gastrointestinal autoimmunity. These preliminary findings represent the first focus on Breg lymphocytic phenotype in HT.

\section{OP28 \\ GRAVES` DISEASE IS ASSOCIATED WITH A DEFECTIVE EXPRESSION OF THE IMMUNE REGULATORY MOLECULE GALECTIN-9 IN ANTIGEN-PRESENTING DENDRITIC CELLS Ana Maria Ramos Levi ${ }^{1}$, Miguel A. Sampedro-Núñez ${ }^{1}$, Sandra Campos ${ }^{1}$, Susana Leskela ${ }^{1}$, Ana Serrano ${ }^{1}$ \\ Ana Rodriguez-Muñoz ${ }^{1}$, Rebeca Martínez ${ }^{1}$, Hortensia de la Fuente ${ }^{1}$, Roberto Gonzalez-Amaro ${ }^{2}$, Monica Marazuela ${ }^{1}$ \\ ${ }^{1}$ Hospital Universitario Princesa. Instituto de Investigación Princesa, \\ ${ }^{2}$ Universidad San Luis Potosi}

Background: Patients with autoimmune thyroid disease (AITD) exhibit aberrant immune-regulatory mechanisms. Galectins (Gal) may play an impor- tant role. This study evaluates the expression and function of Gal-1 and Gal-9 in dendritic cells (DCs) from patients with AITD.

Materials and Methods: Peripheral blood samples from 25 patients with Graves' disease (GD), 11 Hashimoto's thyroiditis (HT), and 24 healthy subjects were studied. Thyroid tissue samples from 44 patients with AITD and 22 patients with goiter were also analyzed. Expression and function of Gal-1 and Gal-9 was assessed by quantitative RT-PCR, immunofluorescence and flow cytometry.

Results: A diminished expression of Gal-9, but not of Gal-1, by peripheral blood DCs was observed in GD patients (MFI 1.20 in EG vs 1.51 in controls, $p=0.0176$ ), especially in those with Graves' ophthalmopathy (GO). A negative correlation between GO severity and Gal-9 expression was observed $(\mathrm{r}=-0.679, \mathrm{p}=0.031)$, and Gal-9 expression was diminished in patients with severe GD ( $\mathrm{p}=0.043)$. No differences were found in Gal-1 or in Gal-9's ligand, TIM-3, expression. Thyroid hormone and antibody levels were not correlated with Gal expression in DCs. mRNA levels of Gal-9 and TIM-3 were increased in thyroid tissue from AITD patients and its expression was associated with levels of Th1/Th12/Th17 cytokines. Immunofluorescence studies proved that intrathyroidal Gal-9 expression was confined to DCs and macrophages. In vitro functional assays showed that exogenous Gal-9 had a suppressive effect on the release of Th1/Th2/Th17 cytokines by DC/lymphocyte autologous co-cultures from both AITD patients and healthy controls.

Conclusions: The altered pattern of expression of Gal-9 in peripheral blood DCs from GD patients, its correlation with disease severity and its ability to suppress cytokine release suggest that Gal-9 could be involved in the pathogenesis of AITD.

\section{OP29 \\ FETAL CELL MICROCHIMERISM AND HLA-G STATUS IN A LARGE SERIES OF AUTOIMMUNE THYROID DISEASES}

Valentina Cirello ${ }^{1}$, Roberta Rizzo ${ }^{2}$, Milena Crippa ${ }^{3}$, Irene Campi ${ }^{4}$, Carla Colombo $^{1}$, Guia Vannucchit, Antonia Maffini ${ }^{1}$, Stefano Ferrero ${ }^{5}$, Palma Finelli ${ }^{6}$, Laura Fugazzola ${ }^{7}$

${ }^{1}$ Department of Clinical Sciences and Community Health, University of Milan, Milan, Italy, ${ }^{2}$ Department of Medical Sciences, Section of Microbiology and Medical Genetics, University of Ferrara, Ferrara, Italy, ${ }^{3}$ Laboratory of Medical Cytogenetics and Molecular Genetics, Istituto Auxologico Italiano, Milan, Italy, ${ }^{4}$ Endocrine Unit, Fondazione Policlinico Irccs, Milan, Italy, ${ }^{5}$ Division of Pathology, Fondazione Policlinico Irccs, Milan, Italy; Department of Biomedical, Surgical and Dental Sciences, University of Milan, Medical School, Milan, Italy, ${ }^{6}$ Laboratory of Medical Cytogenetics and Molecular Genetics, Istituto Auxologico Italiano, Milan, Italy; Department of Medical Biotechnology and Translational Medicine, University of Milan, Milan, Italy,

${ }^{7}$ Department of Clinical Sciences and Community Health, University of Milan, Milan, Italy; Endocrine Unit, Fondazione Policlinico Irccs, Milan, Italy

The persistence of fetal cells in maternal organs and circulation after pregnancy is called fetal cell microchimerism (FCM) and has been hypothesized to elicit the development of autoimmune diseases. It can take place because the maternal immune system tolerates genetically different fetal cells. Moreover, embryo implantation takes place due to the expression of membrane-bound and soluble HLA-G molecules with a tolerogenic function. Genetic polymorphisms of the 3' UTR of this gene (14 bp insertion/deletion and $+3142 \mathrm{C}>G$ ) seem to be involved in the modulation of HLA-G expression.

Since scanty data are available in autoimmune thyroid diseases (AITD), we analysed circulating FCM in a large series of Graves's (GD, n =69) and Hashimoto's (HT, $n=33$ ) patients compared to healthy controls $(n=66)$, by amplification of the Y-chromosome-specific gene SRY. Thus, all the women enrolled had a previous male pregnancy. At the tissue level, the Y chromosome was identified by Fluorescence in situ hybridization (FISH). To verify if a given HLA-G profile could favor the passage of fetal cells, the 2 HLA-G polymorphisms were assessed by Real-time PCR. A significantly lower frequency of FCM was found in GD (33.3\%) and HT (21.2\%) women with respect to controls $(63.6 \%)(P=0.0004$ and $P<0.0001$, respectively). Microchimeric cells were found in tissues as part of thyroid follicles or in blood vessels. The 14bp deletion HLA-G profile was found to associate, though not significantly, with a lower presence of FCM and with HT $(P=0.068)$.

In conclusion, FCM is significantly lower in AITD patients than in healthy controls, indicating a protective role of FCM towards the onset of these diseases. Consistently, the HLA-G profile associated with low FCM was more 
frequent in HT cases with respect to controls. Finally, the tissue localization of microchimeric cells demonstrates their ability in migrating to damaged tissues, with a possible protective or repairing function.

\section{OP30}

\section{GLYCOSYLATION PATTERN ANALYSIS OF GLYCOPROTEIN HORMONES AND THEIR RECEPTORS}

Ricardo Nunez Miguel' ${ }^{1}$, Jane Sanders ${ }^{1}$, Paul Sanders ${ }^{1}$, Stuart Young ${ }^{1}$, Jadwiga Furmaniak ${ }^{1}$, Bernard Rees Smith ${ }^{1}$

${ }^{1}$ Firs Laboratories, Rsr Ltd, Cardiff, UK

We have studied glycosylation patterns in glycoprotein hormones (GPHs) and glycoprotein hormone receptor (GPHR) ectodomains (ECD) from different species in order to identify areas on the surfaces of the hormones and receptors that are not glycosylated in any of the species studied. These nonglycosylated surfaces may be important in ligand-receptor interactions and interactions between different domains of the receptors.

Comparative models of the structure of the TSHR ECD in complex with TSH, M22 and K1-70 were obtained using the crystal structures of the FSHFSHR ECD, M22-TSHR LRD and K1-70-TSHR LRD complexes. The amino acid sequences of the FSHR, TSHR and FSH, CG, LH and TSH from the species studied (in all 78 receptor sequences, 62 alpha subunit and 202 beta subunit sequences) were aligned and glycosylation sites identified. From the alignments, TSH and TSHR equivalent residues that are glycosylated in any of the sequences were mapped onto the molecular surface of the TSH-TSHR ECD complex. Further, the areas that are not involved in currently known interactions and do not contain glycosylation sites were identified on the molecular surfaces of TSH and TSHR.

The study indicated that the areas on the surfaces of GPHs that interact with their respective receptors do not bear sugar residues. Two areas on GPHs free from glycosylation, that are not involved in currently known interactions, have been identified. The concave faces of GPHRs leucine rich repeats 3 to 7 are free from glycosylation consistent with the interactions with the hormones. Four further non-glycosylated areas are identified on the surface of the receptors. Two areas are located on the convex surface, one in the long hinge loop and one at the $\mathrm{C}$ terminus of the ectodomain. These non-glycosylated areas on GPHs and GPHRs are good candidates for forming intra-molecular and intermolecular interactions.

\section{OP5 Tissue Specific Actions of Thyroid Hormone}

\section{OP31}

ANALYSIS OF TRANSCRIPTOMIC LANDSCAPE ENLIGHTENS THE IMPACT OF HYPOTHYROIDISM ON NEUROGENESIS

Ghislaine Morvan Dubois ${ }^{0}$, Jean David Gothié1,

Marie-Stephanie Clerget-Froidevaux ${ }^{2}$, Evelyne Duvernois-Berthet ${ }^{3}$,

Sylvie Remaud ${ }^{3}$, Barbara Demeneix ${ }^{3}$

${ }^{1} U m r 7221$ Cnrs Mnhn, ${ }^{2} U m r$ 7221cnrs Mnhn, ${ }^{3} U m r 7221$ Mnhn Cnrs

Objective: The processes of neural stem cell (NSC) self-renewal and commitment in the adult mammalian brain are controlled by several intrinsic and extrinsic signals, including thyroid hormones (TH). We have previously demonstrated that TH signaling, through liganded TR $\alpha$, is required for full activation of NSC proliferation and that TR $\alpha 1$ expression in progenitors is essential to neuronal commitment. Our goal is now to identify the pathways controlling this outcome.

Methods: We used RNA-seq and real time PCR to investigate the consequences of hypothyroidism in a laser micro-dissected area of in the adult mouse subventricular zone (SVZ), one of the two neurogenic areas in the adult brain.. As expression of individual genes is context dependent, we analysed both differentially regulated mRNA and microRNA, notably examining potential miRNA regulators of target mRNA. Data sets were also cross-analysed with published information on putative TREs.
Results: This large-scale study on mRNA and microRNA expression in the dorsal SVZ identified several regulated networks, notably chromatin remodelling and cell cycle control. Furthermore, thyroid status regulated a number of target genes indirectly through miRNA regulation, whereas others were directly regulated. Among the newly identified putative direct $\mathrm{T} 3$ targets, DCX and Ki67 are of particular interest, given their respective roles in NSC commitment and proliferation. Most of the regulated microRNAs are upregulated in hypothyroid conditions. Among the few inversely regulated, miR-696 expression is nearly abolished in hypothyroid SVZ. Its potential targets are to be characterized. Most of the miRNA up-regulated in the hypothyroid SVZ, such as miR-222, miR-210 and miR-27b, have been associated with stemness in other systems. Examining expression of miR-222 potential targets, revealed to be regulated by thyroid status.

In conclusion, the large-scale transcriptomic analysis is providing valuable insights into the mechanisms and signal pathways controlling the different stages of neurogenesis, notably proliferation and commitment.

\section{OP32 \\ NF-KB SIGNALING IN THE HYPOTHALAMUS IS ESSENTIAL FOR THE RESPONSE OF THE CENTRAL HPT AXIS TO ACUTE INFLAMMATION \\ Emmely de Vries ${ }^{1}$, Ronny Haenold ${ }^{2}$, Sigrun Horn ${ }^{2}$, Eric Fliers ${ }^{1}$, \\ Falk Weih', Heike Heuer ${ }^{3}$, Anita Boelen ${ }^{1}$ \\ ${ }^{1}$ Academic Medical Center, ${ }^{2}$ Leibniz Institute for Age Research - Fritz Lipmann Institute (Fli), ${ }^{3}$ Leibniz Institute for Age Research - Fritz Lipmann Institute (Fli), Jena; Leibniz Research Institute for Environmental Medicine, Dusseldorf, Germany}

Introduction: The nonthyroidal illness syndrome (NTIS) occurs in a large proportion of critically ill patients and is characterized by low serum TH levels and a suppressed central hypothalamus-pituitary-thyroid (HPT) axis. The main source of $T_{3}$ in the brain is type 2 deiodinase (D2) which is predominantly expressed in tanycytes. During acute inflammation in rodents, induced by bacterial endotoxin (LPS) administration, D2 mRNA expression is increased in tanycytes and this is hypothesized to suppress TRH secretion from the PVN via increased local $\mathrm{T}_{3}$ availability. We have shown before that NF-kB p65 (RelA) is essential for the LPS-mediated increase of D2 in tanycyte cell cultures. The aim of the current study verify this role in vivo.

Methods: Tanycyte specific RelA deficient mice were created by crossing $\operatorname{Rel}^{f l f l}$ animals with mice expressing a tamoxifen inducible CRE recombinase under the glutamate aspartate transporter (GLAST)-promoter as a transgene

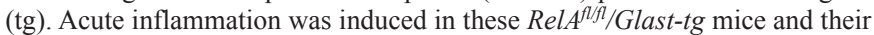
$\operatorname{Rel}^{\mathrm{fl} / \mathrm{fl}}$ littermates by intraperitoneal LPS injection, saline injection was given as a control. Hypothalamic TRH and D2 mRNA expression were analyzed 8 hours later by in situ hybridization. Serum $\mathrm{T}_{3}$ and $\mathrm{T}_{4}$ levels were measured by RIA.

Results: LPS administration led to a 2-fold increase in D2 mRNA expression in the mediobasal hypothalamus within $8 \mathrm{~h}$ in the RelA $A^{f l f l}$ mice but not in the RelA $A^{f / f l} /$ Glast-tg mice. The decrease in TRH expression in RelA $A^{f l / f l}$ mice upon LPS injection was completely prevented in RelAtlff/Glast-tg mice. Interestingly TRH expression in the PVN was lower in saline injected RelAtlft/ Glast-tg mice compared to Rel $A^{f l f l}$ mice. However, the LPS-induced decrease in serum $\mathrm{T}_{3}$ and $\mathrm{T}_{4}$ levels was similar in both genotypes.

Conclusion: NF-kB signalling in tanycytes is essential for the LPS induced changes in TH metabolism in the hypothalamus, but it is not necessary for the changes in serum TH levels.

\section{OP33}

\section{RE-EXPRESSION OF TYPE 1 IODOTHYRONINE} DEIODINASE REDUCES PROLIFERATION IN RENAL CANCER CELLS

Piotr Popławski, Agnieszka Piekiełko-Witkowska

Centre of Postgraduate Medical Education, Department of

Biochemistry and Molecular Biology, Warsaw, Poland

Objectives: Iodothyronine deiodinases catalyze deiodination of iodothyronines and thus contribute to final concentrations of thyroid hormones.

Expression of deiodinases is disturbed in cancers. Manipulations with levels of deiodinases may inhibit neogenesis, as shown by previous studies on colon cancer and basal cell carcinoma. In clear cell renal cell carci- 
noma (ccRCC), the expression of type 1 deiodinase (DIO1) is dramatically decreased and intracellular T3 concentration is lowered by approximately $50 \%$. To our knowledge, there are currently no functional studies exploring the role of DIO1 in tumorigenesis and cancer progression.

The aim of this study was to investigate how re-expression of $\mathrm{DIOI}$ affects proliferation in ccRCC.

Methods: We created a ccRCC cell line (KIJ-265T-pcDNA3-DIO1) with stable re-expression of DIO1 and a control cell line (KIJ-265T-pcDNA3) stably transfected with empty pcDNA3 vector. Cell proliferation was analyzed using Cell Proliferation ELISA, BrdU (Roche). The expression of DIOI and 14 cell cycle related genes was measured using qPCR.

Results: DIO1 re-expression resulted in statistically significant $40 \%$ decrease in proliferation. The expression of MKI67 proliferation marker was decreased by about $37 \%$ in KIJ-265T-pcDNA3-DIO1 when compared with control cell line. Furthermore, the expression of cyclins E1 and E2 and cyclin dependent kinase cdk 2 was also decreased by $55 \%, 25 \%$, and $43 \%$, respectively. Re-expression of $\mathrm{DIOI}$ resulted also in $35 \%$ decrease in expression of proproliferative $\mathrm{E} 2 \mathrm{~F} 2$ transcription factor and $54 \%$ increase in antiproliferative E2F5.

Conclusions: We show, for the first time, that type I deiodinase plays crucial role in proliferation control in ccRCC. DIO1 re-expression in ccRCC results in decreased proliferation, possibly due to repressed expression of cyclins E, cdk2 and changed levels of transcription factors involved in cell cycle control. Therefore, the role of DIO1 in regulation of proliferation and other processes disturbed in cancer cells needs further investigation.

Supported by National Science Centre grant: 2012/05/B/NZ5/01541.

\section{OP34 \\ EFFECTS OF THYROID HORMONE DERIVATIVES ON IN VITRO CHONDROGENESIS \\ Janine Martitz ${ }^{1}$, Peter Hofmann ${ }^{2}$, Alexandra DeichseP ${ }^{2}$, \\ Petra Seemann ${ }^{2}$, Lutz Schomburg \\ ${ }^{1}$ Institute for Experimental Endocrinology, ${ }^{2}$ Charite Berlin, Germany}

Thyronamines (TAM) and 3,5,3'-triiodothyroacetic acid (Triac) are endogenous signaling molecules sharing high similarities to the classical thyroid hormones (TH). In rodent experiments, TAM elicited biological effects on oppose to classical TH, e.g. by reducing heart rate, cardiac output or systolic pressure. Despite their physiological roles, the modes of action remain elusive.

To identify putative human TAM target genes, HepG2 cells were used as a robust in vitro model and analyzed by gene chip analyses. Hereby, transcriptome profiling identified 108 genes differentially expressed from control in experiments with two different dosages of $\mathrm{T}_{1} \mathrm{AM}(10 \mathrm{nM}$ and $10 \mu \mathrm{M}$, f.c., for $6 \mathrm{~h}$ each), some of which overlapped with T3-responsive genes. In order to test direct effects on $\mathrm{TH}$ receptors, transcriptional effects were studied with a classical TH reporter assay in vitro. Significant concentration-dependent effects on a thyroid hormone response element (DR4) were observed for 10-100 nM $\mathrm{T}_{3} \mathrm{AM}$ and Triac, but not for $\mathrm{T}_{0} \mathrm{AM}$ and $\mathrm{T}_{1} \mathrm{AM}$. As some of the most strongly regulated genes are involved in bone maturation, we hypothesized that $T_{1} A M$ may also affect skeletal development. Primary chicken micromass (chMM) stem cell differentiation experiments were thus performed in order to further test whether the TH derivatives affect chondrogenesis directly. Significant effects on chondrogenic differentiation were observed for $T_{3} A M, T_{3}$ and Triac using Alcian Blue staining. These effects on chondrogenesis are in agreement with the results of BMP reporter gene assays in which these hormones affected SMAD reporter gene activity in a similar concentration range. We conclude that our in vitro chondrogenesis model represents a promising in vitro system for identifying and characterizing molecular activities, target genes and endocrine effects of TAM.

Supported by Deutsche Forschungsgemeinschaft DFG, Priority Program SPP1629 (SCHO849/4-1), BSRT, GraKo1208, and an Elsa-NeumannScholarship of Berlin.

\section{OP35 \\ DIFFERENTIAL EFFECTS OF CHRONIC, INTRAHYPOTHALAMIC THYROID HORMONE ADMINISTRATION IN THE VENTROMEDIAL AND PARAVENTRICULAR NUCLEUS}

Zhi Zhang ${ }^{1}$, Ewout Foppen ${ }^{1}$, Peter Bisschop ${ }^{1}$, Anita Boelen ${ }^{1}$, Andries Kalsbeek ${ }^{2}$, Eric Fliers ${ }^{1}$

${ }^{1}$ Academic Medical Centre, ${ }^{2}$ Academic Medical Centre; Netherlands Institute for Neuroscience (Nin)

In addition to peripheral effects of triiodothyronine (T3), recent studies have demonstrated that acute administration of $\mathrm{T} 3$ in the paraventricular nucleus (PVN) and the ventromedial nucleus $(\mathrm{VMH})$ of the hypothalamus increases hepatic glucose production and energy expenditure, respectively, via the autonomic nervous system. However, the effects of chronic intrahypothalamic $\mathrm{T} 3$ administration on energy metabolism are still unknown.

We aimed to develop a technique for chronic administration of T3 in the PVN and VMH region using slow-release beeswax pellets. T3-containing or blank pellets were implanted bilaterally in either the PVN or VMH region. We assessed plasma T3, T4 and TSH concentrations 2 days before and 3, 7, 14, and 28 days after placement of the pellets by tail cuts. Liver, brown adipose tissue (BAT), pituitary and hypothalamic tissue blocks were collected at 28 days for analysis of gene expressions using qPCR.

Implanting T3-containing pellets in the PVN resulted in increased D3 mRNA in the PVN, but unchanged preproTRH mRNA. Gene expression in the VMH was not affected. Pituitary TSH $\beta$ and D1 mRNA expression decreased significantly. The observed changes were accompanied by temporarily lower plasma T3 (for 20 days) and T4 (for 7 days) concentrations. UCP1 mRNA in BAT and D1 mRNA expression in liver were lower in the T3 group compared to controls.

Implanting T3-containing pellets in the VMH resulted in increased D3 mRNA and hairless mRNA in the VMH but not in the PVN. PreproTRH mRNA did not change in either the PVN or the VMH region. No changes in pituitary and peripheral thyroid hormone metabolism were observed.

Our pilot experiments provide a promising technique to selectively deliver $\mathrm{T} 3$ for 4 weeks to specific hypothalamic nuclei. This will enable future studies to dissect the effects of chronic thyrotoxicosis in various hypothalamic nuclei on energy metabolism.

\section{OP36 \\ CARDIAC REGENERATION AND THYROID HORMONE: A STUDY IN METAMORPHOSING XENOPUS LAEVIS}

Céline Vivien ${ }^{1}$, Lindsey Marshall ${ }^{1}$, Louise Péricard ${ }^{1}$, Fabrice Girardot ${ }^{1}$, Barbara Demeneix ${ }^{2}$, Laurent Coen ${ }^{1}$

${ }^{1} U$ mr Cnrs 7221, Département Régulations, Développement Et

Diversité Moléculaire, Muséum National D'histoire Naturelle - Paris,

${ }^{2} U m r$ Cnrs 7221, Département Régulations, Développement Et

Diversité Moléculaire, Muséum National D’histoire Naturelle

Ischemic heart disease is the leading cause of mortality worldwide, incurring enormous medical and economical impact. As there is no cardiac muscle regeneration in the injured human heart after myocardial infarction increasing research focuses on facilitating cardiac regeneration to overcome the pathological consequences. Interestingly, the heart can regenerate up to seven days after birth in mice, thereafter the capacity is lost. Conversely, urodele amphibians or teleosts, such as axolotl and zebrafish respectively, possess lifelong robust cardiac regenerative capacity. Although cardiac regeneration is unexplored in the anuran amphibian Xenopus, the regenerative capacity of limbs, retina and nervous tissue is impaired after metamorphosis. Interestingly, both metamorphosis in anuran amphibians and the neonatal period of mammals are stages of thyroid hormone (TH) dependent intense tissue remodelling. This loss of regenerative capacity, correlated with a peak of $\mathrm{TH}$, in both groups, suggests a putative causative link.

We are exploring Xenopus laevis as a model for characterising cardiac regeneration and for analysing the impact of $\mathrm{TH}$ on the process. Adapting methods used in zebrafish and mice, to Xenopus, we studied a global view of cardiac regeneration relative to TH-dependent metamorphosis, through ventricular apex resections before, during and after metamorphosis. Regenerative capacity is assessed at different time points before and after metamorphosis. 
To determine whether regenerated cardiac muscle is formed histology, immunohistochemistry (for regenerative muscle or fibrosis) and qPCR are applied.

We observed that the strong cardiac regenerative capacity in Xenopus before metamorphosis was impaired after the TH peak. Notably, post metamorphosis increased scar tissue is formed, characterised by fibrosis. A number of TH-dependent genes are differentially modulated by cardiac resection.

Besides introducing a new model for cardiac regeneration, our data suggest a link between thyroid hormone and the capacity of cardiac regeneration in Xenopus.

\section{OP37}

THYROID HORMONE T3 IS A MITOGENIC AND ANTIAPOPTOTIC FACTOR IN RAT GRANULOSA CELLS AND FOLLICLES

Cecilia Verga Falzacappa ${ }^{1}$, Virginia Di Paolo ${ }^{2}$, Claudia Mangialardo ${ }^{3}$ Alessandra Scamporrino ${ }^{1}$, Valentina Andriollo ${ }^{1}$, Ilenia Cammarata ${ }^{1}$, Silvia Misiti ${ }^{1}$, Rita Canipari ${ }^{2}$, Marco Centanni ${ }^{4}$

${ }^{1}$ Experimental Medicine Department, Sapienza, University of Rome, ${ }^{2}$ Department of Anatomy, Histology, Forensic Medicine and Orthopedic, Section of Histology, Sapienza, ${ }^{3}$ Dept of Medico-Surgical Sciences and Biotechnologies, "sapienza" University of Rome, Latina, Italy, 4"sapienza" University of Rome Dept of Medico-Surgical Sciences and Biotechnologies Latina

Clinical evidence suggests that thyroid disorders are associated with impaired fertility in women and these abnormalities may be improved by restoring euthyroid homeostasis. The exact mechanisms are not well known; however, follicle development and function might play a key role, so that it is conceivable that thyroid hormones could exert their action via receptors in granulosa cells. Since these cells are essential regulator of follicle fate, we evaluated the effect of thyroid hormone T3 on proliferation, apoptosis and survival of spontaneously immortalized rat granulosa cells (rGROV) and freshly isolated rat follicles. Cells and follicles were treated with T3 (100 nM). Cell growth and viability were evaluated by cell counting and MTT assay, whether follicle growth was evaluated by volume measurement. T3 induced cell growth and cell metabolism in rGROV (40\% at $48 \mathrm{~h}$ ), furthermore hormone treatment increased follicle volumes of $40 \%$ (after 7 days). In accordance, as shown by cytofluorimetry, T3 induced cell cycling in rGROV and upregulated cyclins A, B and D together with cdk1 protein levels. Conversely, p21 and p27 mRNAs were downregulated by hormone treatment. As demonstrated by Tunel assay, when apoptosis was induced by serum deprivation, T3 decreased the cell apoptotic rate of $40 \%$ and the proapoptotic molecules Casp 3 and Bax were downregulated. In addition Akt activation was upregulated specifically involving PI3K. These findings strongly suggest that T3 acts on follicle growth by influencing cell survival of ovarian granulosa cells.

\section{OP38}

\section{A NEW GENE PREDISPOSING TO CONGENITAL HYPOTHYROIDISM}

Angela Pascarella', Giuseppe Ferrandino ${ }^{2}$, Maria Basile ${ }^{2}$,

Olga Spadaro' , Fulvio D’Angelo², Chiara D'Ambrosio ${ }^{3}$,

Andrea Scaloni ${ }^{3}$, Mario De Felice ${ }^{2}$, Roberto Di Lauro ${ }^{1}$,

Gabriella De Vita ${ }^{1}$, Elena Amendola ${ }^{1}$

${ }^{1}$ Dipartimento DI Medicina Molecolare E Biotecnologie Mediche,

Universita' Federico II, Napoli, Italia, ${ }^{2}$ Istituto DI Ricerche Genetiche

G.Salvatore, Biogem S.C.Ar.L, Ariano Irpino, Avellino, Italia,

${ }^{3}$ Proteomics \& Mass Spectrometry Laboratory, Ispaam, National

Research Council, Naples, Italy

Congenital hypothyroidism $(\mathrm{CH})$ is the most frequent endocrinological congenital disorder frequently due to thyroid dysgenesis (TD). It is known that mutations in genes involved in thyroid development such as Nxk2.1, Pax8, Foxe1, and Tshr cause TD in animal models, and loss of function mutations in the same genes are associated with TD in patients. This demonstrated that TD can be a genetic disorder. However, mutations in these genes have been identified only in few patients affected by $\mathrm{CH}$ with $\mathrm{TD}$, suggesting that other loci could be involved in the genesis of TD. A polygenic mouse model of $\mathrm{CH}$ with TD allowed us to identify Dnajc17, a member of the type III heat-shock protein-40 (Hsp40) family, as a potential modifier gene for TD. Furthermore we demonstrated that homozygous null mutation in the Dnajc17 gene are early embryo lethal. Taken together, our findings highlight a gene essential in very early embryogenesis, that is likely to play an important role in thyroid.

Currently, molecular mechanisms of DNAJC17 function are unknown. In Hela cells DNAJC17 localizes predominantly in the nucleus. Co-IP and mass spectrometry suggest that DNAJC17 interacts with proteins involved in splicing. Some of these proteins are essential for embryonic development. Moreover, gene expression profile of Hela cells knocked-down for Dnajc17 show alterations in the balance between alternatively spliced isoforms of many genes.

To elucidate the functional role of DNAJC17 in thyroid we generated a conditional knockout mouse in which Dnajc17 has been disrupted specifically in the thyroid. In addition, we will search for the presence of mutations in the Dnajc17 gene in patients with TD.

Furthermore, we will perform knock-down of Dnajc17 in mouse ES cells to identify genes affected by its absence to shed light on the mechanisms causing early embryonic lethality in the absence of a functional Dnajc17 gene.

\section{OP6 Thyroid Cancer Diagnostics}

\section{OP39 \\ IS AN INCREASE IN THYROID NODULE VOLUME A PREDICTOR OF MALIGNANCY? \\ Hirotoshi Nakamura ${ }^{1}$, Mitsuyoshi Hirokawa1 ${ }^{1}$, Minoru Kihara ${ }^{1}$, \\ Akihiro Miwa ${ }^{1}$, Akira Miyauchi ${ }^{1}$ \\ ${ }^{1}$ Kuma Hospital, Kobe, Japan}

Background: Most benign thyroid nodules on fine-needle aspiration cytology (FNAC) can be followed up clinically with periodic ultrasonography (US) examinations. During follow-up, when a nodule volume significantly increases, re-FNA biopsy or even surgical resection for histological examination is recommended by international guidelines. However, there are little data about the risk of malignancy of nodule growth.

Method: We retrospectively reviewed the records of 951 patients with suspicious of (s/o) adenomatous nodule (AN) or s/o follicular tumor (FT) on FNAC who underwent surgery between 2011 and 2013 at Kuma Hospital and extracted 196 patients with s/o AN and 82 patients with s/o FT who had surgical resection because of nodule growth during the observation period $(47+/-23$ months). The nodule growth was measured by US and expressed as percent ratio of final volume/baseline volume per year.

Results: The mean nodule growth rate was $91 \%$ per year in patients with s/o AN and $105 \%$ per year in patients with s/o FT. Histological diagnoses in patients with s/o AN were as follows; $158 \mathrm{ANs}, 32$ follicular adenomas (FA), 4 follicular tumors of uncertain malignant potential (FT-UMP), one papillary carcinoma (PC), one follicular carcinoma (FC). This shows that increased "benign" nodules contained only $6(3 \%)$ candidates for surgery (FT-UMP + malignancy). On the other hand, histological diagnoses were 44 FA or AN, 23 FT-UMP, 1 PC and 14 FC in 82 patients with increased FT. Malignancy plus FT-UMP consisted of $46.3 \%$, which was significantly higher than that in 327 patients with s/o FT operated shortly after FNAC (30.0\%).

Conclusion: This is the first demonstration that the risk of malignancy is very low in solid nodule of s/o AN on FNAC, even if the volume significantly has increased. By contrast, nodules of s/o FT have high risk, especially when nodule volumes become enlarged. 


\section{OP40 \\ MOLECULAR TESTING OF ROUTINE AIR-DRIED \\ FINE NEEDLE ASPIRATION (FNA) SMEARS \\ IMPROVES PRE-SURGICAL DIAGNOSIS AND SUPPORTS THE HISTOLOGICAL IDENTIFICATION OF FOLLICULAR CARCINOMAS}

Markus Eszlinger ${ }^{1}$, Simonetta Piana ${ }^{2}$, Anja Moll' ${ }^{1}$, Eileen Boesenberg ${ }^{1}$,

Ralf Paschke ${ }^{1}$

${ }^{1}$ Division of Endocrinology and Nephrology, University of Leipzig,

Leipzig, Germany, ${ }^{2}$ Pathology Unit, Arcispedale Santa Maria Nuova-

Irccs, Reggio Emilia, Italy

Objectives: Recently we described the feasibility of molecular testing of routine air-dried FNA smears. Now we analyzed the impact of molecular testing for FNA smears, which were rated in a five category scheme according to the British Thyroid Association guidelines from 2007.

Methods: RNA and DNA was extracted from 354 consecutive FNAs (8 non-diagnostic, 108 benign, 166 follicular lesion, 39 suspicious, and 33 malignant). PAX8/PPARG and RET/PTC rearrangements were detected by qPCR, while BRAF and RAS mutations were detected by high resolution melting $\mathrm{PCR} /$ pyrosequencing.

Results: On average $2 \%$ and $6 \%$ of FNA samples were non-satisfactory for the DNA and RNA based analysis, respectively. The presence of a BRAF mutation was associated with cancer in $100 \%$ of samples, whereas the presence of a RAS mutation was associated with cancer in 50\% of samples. Moreover, histologic re-evaluation of 10 RAS positive follicular adenomas revealed the diagnosis of 3 additional minimally invasive follicular carcinomas. $49 \%$ of carcinomas were identified by molecular testing in the group of follicular lesions, which increased the sensitivity from $59 \%$ (cytology alone) to $78 \%$ (cytology + molecular testing). While FNAs with the cytological diagnosis of a follicular lesion alone had a $25 \%$ risk of malignancy, the risk of malignancy significantly increased up to $71 \%$ for mutation positive follicular lesions, and decreased to $18 \%$ for mutation negative follicular lesions.

Conclusion: Our data suggest that molecular testing improves pre-surgical diagnosis: while the detection of BRAF mutations can result in more primary total thyroidectomies, the detection of RAS mutations in the cytological group of follicular lesions strongly suggests diagnostic surgery. Moreover, the knowledge of the molecular testing (especially the presence of RAS mutations) might support the histologic identification of follicular carcinomas, which is characterized by high interobserver variation.

\section{OP41 \\ RE-EVALUATIONG THE MALIGNANCY RISK OF THYROID FOLLICULAR NEOPLASMS: A TWO- YEAR STUDY ON 598 CASES}

Bénédicte Royer ${ }^{1}$, Claude Bigorgne ${ }^{2}$, Gilles Russ ${ }^{3}$, Agnès RouxeP,

Marie Bienvenu ${ }^{4}$

${ }^{1}$ Centre de Pathologie Et D'imagerie, ${ }^{2}$ Centre de Pathologie Et D'imagerie; La Pitié-Salpêtrière Hospital, ${ }^{3}$ Centre de Pathologie Et D'imagerie; La Pitié-Salpêtrière Hospital; Pierre and Marie Curie University, ${ }^{4}$ Centre de Pathologie Et D'imagerie; Cochin Hospital

Objectives: to determine the risk of malignancy of thyroid follicular neoplasms (FN).

Methods: retrospective study on 9970 thyroid ultrasound-guided fine needle aspirations (US-FNAs) performed during 2011 and 2012. All results were read by two specialized cytopathologists, according to the Bethesda System for Reporting Thyroid Cytopathology (BSRTC). Histological reports concerning FNs were obtained after a request by mail to the corresponding physicians and analyzed to check concordance with the nodule initially referred for FNA. Results were compared with literature data.

Results: among 9970 US-FNAs there were 598 follicular neoplasms (including 21 oncocytic type), representing $6 \%$ of all cytological results. Histological results were obtained in 128 cases. They corresponded to: 109 benign lesions (colloid nodule/nodular hyperplasia, 10; follicular adenoma, 70; oncocytic adenoma, 29), 17 malignant lesions, 1 undeterminated malignant potential tumor and 1 entirely necrotic tumor. Within the malignant lesions there were 11 papillary carcinomas including 8 follicular variants, 5 follicular carcinomas and 1 medullary carcinoma. The malignancy rate was $14 \%$. It was slightly inferior to the lower range of values reported with the BSRTC (15-30\%). Two different reasons could be found to explain this discrepancy:

- The study periods of the reports that estimated the average risk of cancer at $25 \%$ in FNs are highly variable and took place before the Bethesda's terminology was adopted. Hence, the authors of these reports were forced to reclassify retrospectively the lesions.

- It has been shown that inter-observer expert agreement for the diagnostic FN can be as low as $36 \%$.

Conclusions: the malignancy rate of FNs may be lower than expected according to the standard range given by the Bethesda system. Thus, a more conservative approach could be considered. These findings should lead to reevaluate the malignancy rate of FNs by multi-center prospective studies and to harmonize the inclusion criteria of this diagnostic category.

\section{OP42 \\ DIAGNOSTIC PERFORMANCE OF ELASTOGRAPHY IN CYTOLOGICALLY INDETERMINATE THYROID NODULES}

Francesca Garino ${ }^{1}$, Maurilio Deandrea ${ }^{1}$, Federico Ragazzoni', Alberto Mormile ${ }^{1}$, Nicola Palestini ${ }^{2}$, Milena Freddi ${ }^{2}$, Guido Gasparri ${ }^{2}$, Manuela Motta ${ }^{3}$, Donatella Pacchioni ${ }^{4}$, Paolo Piero Limone ${ }^{1}$

${ }^{1}$ Department of Endocrinology, Diabetes and Metabolism, Ao Mauriziano, ${ }^{2}$ Department of Surgery, Scu General Surgery III, Aou Città Della Salute E Della Scienza, ${ }^{3}$ Department of Diagnostic and Clinical Pathology, Ao Mauriziano, ${ }^{4}$ Department of Laboratory Medicine, Scu Diagnostic Anatomy and Histopathology, Aou Città Della Salute E Della Scienza

Objectives: Cytological examination of material from fine needle aspiration biopsy is the mainstay of diagnosis of thyroid nodules, thanks to its accuracy and scarcity of complications. Follicular lesions (or indeterminate lesions or Thy3) represent its main limit. Elastography has been proposed as a potential diagnostic tool to define the risk of malignancy in this group, but at present conclusive data are lacking due to the small number and discordance of specifically addressed studies. Our objective was to evaluate the real-time elastography (RTE) role for refining diagnosis of Thy 3 nodules, by integrating information provided by traditional ultrasound (US).

Methods: The study included 108 patients with Thy 3 nodules awaiting for surgery, which were evaluated by US (considering hypoecogenicity, irregular margins, microcalcifications, halo sign and intranodular vascularization) and RTE. Nodules were classified at RTE using a four-class color scale.

Results: At histology, 75 nodules were benign and 33 malignant. None of the ultrasound parameters alone was adequate in predicting malignancy of the nodules; in presence of at least two US risk factors $83 \%$ specificity and $77 \%$ accuracy were obtained with 6.8 OR (95\%CI 2.4-20.4). RTE scores 3 and 4 showed $76 \%$ sensitivity, $88 \%$ specificity, $74 \%$ PPV and $89 \%$ NPV with diagnostic accuracy of $84 \%$ with a 21.9 OR $(95 \%$ CI $7.1-76, \mathrm{P}<0.001)$. By combining RTE with US parameters, the presence of at least 2 characters of suspicion had $88 \%$ sensitivity and $94 \%$ NPV with 23.8 OR (95\% CI $7-106.3$ ).

Conclusions: The use of combined RTE and US leads to the identification of two patients subpopulations with a significantly different malignancy risk ( $6 \%$ vs $63 \%$ ); further studies are needed to verify if it's possible to send only the first group to thyroidectomy and the other to follow-up

\section{OP43 \\ PREDICTIVE VALUE OF HIGH SENSITIVE THYROGLOBULIN ASSAY (USTG) AND NECK ULTRASONOGRAPHY (US) AT THE TIME OF REMNANT ABLATION ON LT-4 THERAPY IN PATIENTS WITH LOW AND INTERMEDIATE RISK DIFFERENTIATED THYROID CANCER (DTC)}

Antonio Matrone $^{1}$, Carla Gambale ${ }^{1}$, Eleonora Molinaro ${ }^{1}$, Laura Agate ${ }^{1}$,

Valeria Bottici ${ }^{1}$, Agnese Biagini ${ }^{1}$, David Viola', Paolo Vitti ${ }^{1}$,

Rossella Elisei ${ }^{1}$

${ }^{1}$ University of Pisa, Endocrine Unit - Department of Clinical and

Experimental Medicine, Pisa, Italy

Background: Differentiated thyroid carcinoma (DTC) is a malignant tumor of epithelial origin. Initial treatment consists of total thyroidectomy and 
radioiodine ablation with ${ }^{131}$ I. The follow-up is based on clinical, laboratoristic and neck ultrasound findings. The gold standard to define disease remission is rhTSH-Tg test. The recent introduction of UsTg and high resolution US seem to be able to modify the algorithm of follow-up of these patients.

Methods: We evaluated data of 505 patients with DTC at the time of remnant ablation ( $30 \mathrm{mCi}$ of ${ }^{131} \mathrm{I}$ after rhTSH) and during the following 12 months, with anti-thyroglobulin antibodies $(\mathrm{TgAb})<20 \mathrm{U} / \mathrm{ml}$. We excluded from our study "very low" and "high" risk DTC patients. The aim was to evaluate which parameters could have a predictive value for remission/persistence of disease in a short-term follow-up.

Results: In a multivariate analysis, only values of UsTg-LT4 $>1 \mathrm{ng} / \mathrm{ml}$ $(\mathrm{P}<0.0001)$ and the presence of lymphnodes metastases at the US examinations $(\mathrm{P}<0.0001)$, at the moment of remnant ablation, correlated significantly with the persistence of the disease after 12 months. At the contrary, low UsTg-LT4 values $(<0.1 \mathrm{ng} / \mathrm{ml})$ and absence of lymphnode metastases showed a positive predictive value for the remission of disease after 1 year. All other epidemiological and clinicopathological parameters considered (age, initial histology, staging, lymph node metastases at the histology, prophylactic dissection of the central compartment, multifocality, TNM, staging) showed no statistical significance.

Conclusions: 1) LT4-UsTg values and neck US at the time of remnant ablation, are prognostic factors of remission/persistence of disease in a short term follow-up; 2) Other parameters analyzed don't have a prognostic role in our series; 3) In the near future, low and intermediate risk patients with these features might avoid radioiodine ablation and be followed with clinical, laboratoristic and ultrasonographic controls.

\section{OP44 \\ LOCALLY INVASIVE PAPILLARY THYROID CARCINOMA INVOLVING A FUNCTIONING RECURRENT LARYNGEAL NERVE EXCLUSIVELY MAY BE TREATED BY SHAVING TECHNIQUE WITH MACROSCOPIC RESIDUAL TUMOR FOLLOWED BY HIGH-DOSE RADIOIODINE THERAPY}

Hyoung Shin Lee ${ }^{1}$, Kang Dae Lee ${ }^{1}$, Jun Woong Song ${ }^{2}$, Gi Yun Nam², Sung Won Kim², Seon Mi Baek ${ }^{3}$, Hyo Sang Park ${ }^{3}$

${ }^{1}$ Department of Otolaryngology-Head and Neck Surgery, Kosin

University College of Medicine, ${ }^{2}$ Kosin University College of Medicine,

${ }^{3}$ Sharing and Happiness Hospital

Objective: We sought to validate the feasibility of preserving a functioning recurrent laryngeal nerve (RLN) involved by papillary thyroid carcinoma (PTC) with shaving technique followed by high-dose radioiodine therapy.

Methods: A retrospective review of 41 patients with locally invasive PTC who had tumor invasion exclusively to the RLN was done. All patients underwent total thyroidectomy with or without central neck dissection (CND) and high-dose radioiodine therapy. Patients with concomitant invasion to nonRLN sites were excluded. Shaving technique with macroscopic residual tumor was conducted by leaving as little amount of residual tumor as possible but still attempting to preserve the function of the nerve. Clinicopathologic factors and oncologic outcomes of the patients in group A (resected RLN, $n=19$ ) and group B (preserved RLN, $\mathrm{n}=22$ ) were analyzed and compared between the two groups.

Results: The two groups showed no difference in factors such as sex, age, maximal tumor size, multifocality, bilaterally, lymph node metastasis, CND, radioiodine therapy, thyroid-stimulation hormone suppression and followup period. Mean dose of radioiodine therapy was $245.0 \pm 140.3 \mathrm{mCi}$ (range 100-740 mCi). Permanent postoperative vocal cord paralysis occurred in two patients of group B (10.5\%). Only one patient $(4.5 \%)$ in group B had local recurrence at the thyroid bed where residual tumor was located, while others were regional or distant metastasis. Overall recurrence rate was $26.3 \%(5 / 19)$ and $27.3 \%(6 / 22)$ in two groups, respectively showing no significant difference $(p=0.945)$ with no death due to PTC during the median follow-up of 75 months (range 36-150 months).

Conclusion: The short to medium term outcome of this study showed that patients with locally invasive PTC involving a functioning RLN exclusively, may be treated by shaving the nerve with macroscopic residual tumor followed by high-dose radioiodine therapy.

\section{OP45}

MOLECULAR TESTING OF RESIDUAL THINPREP MATERIAL INCREASES SENSITIVITY AND

\section{ALLOWS MOLECULAR DIAGNOSTICS FOR} CYTOLOGICALLY NON-DIAGNOSTIC SAMPLES

Jeffrey Krane ${ }^{1}$, Markus Eszlinger ${ }^{2}$, Edmund Cibas ${ }^{1}$, Ralf Paschke ${ }^{2}$

${ }^{1}$ Department of Pathology, Brigham and Women's Hospital, Harvard Medical School, Boston, Mass., USA, ${ }^{2}$ Division of Endocrinology and Nephrology, University of Leipzig, Leipzig, Germany

Objectives: Molecular testing of routinely available thyroid nodule fine needle aspiration (FNA) material has many advantages. Recently, we described the molecular testing of air-dried smears. Now, we performed molecular testing on liquid based FNA material and analyzed the remaining CytoLyt-FNA material for BRAF, RAS, RET/PTC and PAX8/PPARG mutations.

Methods: Thyroid FNAs were classified according to the Bethesda System for Reporting Thyroid Cytopathology after routine cytological examination. The remaining material from all available cases was used for blinded analysis of BRAF, NRAS, HRAS, KRAS point mutations and RET/PTC and PAX8/PPARG rearrangements by high resolution melting PCR/pyrosequencing, and qPCR, respectively.

Results: A total of 597 consecutive cases were collected and stored at $8^{\circ} \mathrm{C}$ for up to 6 months. Molecular analysis was possible for 391 cases. Higher volumes of remaining material $(>2 \mathrm{ml})$ were associated with higher diagnostic success rates ( $23 \%$ vs. $79 \%, \mathrm{P}<0.001$, chi-squared test). Mutations and rearrangements were detected in $11.5 \%$ of atypias of undetermined significance, $33 \%$ suspicious cases, $47.1 \%$ malignant cases, $7.1 \%$ follicular neoplasms, $9.3 \%$ benign cases, and $2.5 \%$ non-diagnostic (molecular analysis possible for $63.2 \%$ ) samples. Compared to histology, which was available for 211 samples, the combination of cytology and molecular analysis increased sensitivity, specificity, PPV, and NPV compared to cytology alone to $86.7 \%, 98.1 \%$, $98.7 \%$, and $81.5 \%$, respectively, considering 13 mutation-positive but histologically benign cases as true positives.

Conclusion: Mutational analysis is feasible on residual ThinPrep material and has the advantage of not requiring additional FNA procedures or dedicated passes, special preservation, or storage. Its reflex use can be valuable in the preoperative determination of the extent of surgery for mutation-positive cases, help to clarify $12 \%$ of atypias of undetermined significance, decrease the rate of false negatives for benign cases, and allow molecular diagnostics for $63.2 \%$ of the non-diagnostic samples

\section{OP46 \\ PREDICTIVE VALUE OF ULTRASENSITIVE SERUM TG AT ABLATION IN DIFFERENTIATED THYROID CANCER PATIENTS}

$\underline{S i l v i a ~ M e m m o}^{1}$, Maria Grazia Castagna ${ }^{1}$, Valentina Belardini',

Fabio Maino ${ }^{1}$, Carla Fioravanti ${ }^{1}$, Fausta Sestini ${ }^{1}$, Gabriele Cevenini ${ }^{2}$,

Furio Pacini

${ }^{1}$ Section of Endocrinology, University of Siena, Siena, Italy, ${ }^{2}$ Section of

Surgery Bioengineering, University of Siena, Siena, Italy

Several studies reported a very high negative predictive value (NPV) of hypo-Tg obtained at the time of 131I ablative therapy in patients with differentiated thyroid carcinoma (DTC). Less clear data are available regarding the predictive value of Tg on levo-thyroxine (LT4) therapy before ablation.

To evaluate if Tg on LT4 therapy before ablation has a value in predicting outcome in DTC, we studied 250 patients with negative $\mathrm{TgAb}$ treated with surgery and thyroid remnant ablation after rhTSH. Serum $\mathrm{Tg}$ was performed using an assay with a functional sensitivity of $0.1 \mathrm{ng} / \mathrm{ml}$ (Abl-US-Tg). AblUS-Tg was correlated with the clinical status of DTC patients at the short and long term follow-up (median 4.5 years).

ROC curve analysis demonstrated that Abl-US-Tg was predictive of disease free status one year later ( $\mathrm{AUC}=0.897$ ) and a cut-off value was established at $0.22 \mathrm{ng} / \mathrm{ml}$ (sensitivity of $96.3 \%$, specificity of $66.1 \%$, positive predictive value (PPV) of $44.5 \%$ and NPV of $98.5 \%$ ). ROC curve analysis demonstrated also that Abl-US-Tg of $0.22 \mathrm{ng} / \mathrm{ml}$ was predictive of disease free status at last follow-up (AUC $=0.880$ ) with a sensitivity of $97.2 \%$, specificity of $61.0 \%$, PPV of $30.2 \%$ and NPV of $99.2 \%$. In patients with values of AblUS-Tg $<0.22 \mathrm{ng} / \mathrm{ml}$, the probability of having persistent disease was $1.5 \%$ at the time of the first control and of $0.5 \%$ during follow-up. 
In conclusion, serum Abl-US-Tg is a reliable indicator of disease status during follow-up and should be measured before ablation. Abl-US-Tg measurement before 131I remnant ablation may contribute to the definition of risk categories in DTC patients.

\section{OP7 Thyroid Hormone Transporters and Deiodinases}

\section{OP47 \\ STRONG INDUCTION OF DEIODINASES BY NOVEL SELENOCOMPOUNDS \\ Mette Stoedter ${ }^{1}$, Elena Ibanez ${ }^{2}, K_{\text {ostja Renko }}^{3}$, Janine Martitz ${ }^{1}$ Eddy Rijntjes ${ }^{4}$, Nicole Pietschmann ${ }^{3}$, Alfonso Calvo ${ }^{2}$, Juan A. Palop ${ }^{2}$, Sanmartin Grijalba Carmen², Lutz Schomburg ${ }^{1}$ \\ ${ }^{1}$ Institute for Experimental Endocrinology, ${ }^{2}$ Universidad de Navarra, ${ }^{3}$ Charite Berlin, ${ }^{4}$ Institut Für Experimentelle Endokrinologie, Charité - Universitätsmedizin Berlin, Germany}

Introduction: Deiodinases (DIO1-3) are highly specific selenoenzymes involved in thyroid hormone metabolism. Recent studies suggest an important role in tumorigenesis and cancer growth. Potent modulators of DIO expression are therefore needed. We have synthesized and studied a series of selenocompounds with a methyl- and benzyl-imidoselenocarbamate backbone, which proved as potent chemotherapeutic substances in a murine tumor model (PMID 22455594).

Methods: HepG2 and HEK293T cells were cultivated, and DIO1-3 mRNA expressions were quantified by qRT-PCR. Enzymatic activities were determined with a non-radioactive DIO assay. Western blot was used for protein quantification. Stable expression of DIO enzymes was achieved in HEK293T cells after transfection with DIO-specific expression vectors.

Results: In HepG2 cells, DIO1 activity was strongly (up to 10-fold) increased by methyl- but not by benzyl-imidoselenocarbamates $(1 \mu \mathrm{M}, 48 \mathrm{~h}$ incubation). Notably, mRNA levels remained unaltered. These results were confirmed and followed-up in stably transfected DIO1, DIO2 and DIO3 expressing HEK293T cells. DIO1 activity and protein concentrations were again positively affected by the methyl-imidoselenocarbamates. The inducing effects on DIO2 and DIO3 activities were even stronger in DIO2 and DIO3 expressing HEK293T cells. Among our novel selenocompounds, especially the methyl-imidoselenocarbamate EI201 proved as a very potent stimulator of DIO1-3 activities, while the benzyl-series remained again without effects in the stably transfected cells.

Conclusion: We conclude that the anticancer effects of the methyl-imidoselenocarbamates as observed in the murine tumor model may be related to their strong modulating effects on DIO activities. Further studies are needed to verify this notion. Collectively, our data confirm the qualification of these novel selenocompounds as highly interesting and promising pharmacological candidates.

Supported by the Deutsche Forschungsgemeinschaft (DFG; GraKo 1208, SCHO849/4-1).

\section{$0 P 48$}

\section{THR92ALA D2-POLYMORPHISM DETERMINES} REDUCED T4-INDUCED APOPTOSIS IN MUSCLE STEM CELLS

Monica Dentice $^{1}$, Cristina Luongo ${ }^{1}$, Raffaele Ambrosio ${ }^{1}$

Tommaso Porcelli ${ }^{1}$, Daniela Di Girolamo ${ }^{1}$, Fausta Alfano ${ }^{1}$,

Maria Angela De Stefano ${ }^{1}$, Domenico Salvatore ${ }^{1}$

${ }^{1}$ Department of Clinical Medicine and Surgery, University of Naples

"Federico II"

Thyroid hormones (TH) are important regulators of growth, development, and metabolism. Most of the active TH is generated by peripheral metabolism mediated by the iodothyronine deiodinases. We demonstrated that D2 is expressed in muscle precursor cells, and its over-expression is essential for myoblast differentiation.
A body of evidence suggests an association between the single-nucleotide polymorphism in D2 (T92A) and impaired tissue activity of TH. In clinical settings, the mutant form D2 T92A has been associated with widespread pathological conditions such as insulin resistance and impaired glucose metabolism, altered bone metabolism and alterations in circulating $\mathrm{TH}$ parameters. Nevertheless, based on published studies reporting no differences between patients carrying either the normal or the T92A D2 gene, the relevance of the D2 mutant isoform yet remains a matter of debate. Studies conducted by our group demonstrated that T4 excess causes apoptosis in muscle stem cells. The same effect was observed in cells overexpressing type 2 deiodinase by increased intracellular T3 concentrations.

In order to investigate the consequences of D2 T92A polymorphism in a physiological setting muscle cells were transfected with the D2 wild type (WT) and D2 T92A variant. Strikingly, while no differences were observed between the two isoforms in terms of mRNA, protein expression levels and protein half-life, transfection with the T92A gene caused a reduced cell apoptosis compared to the D2-WT gene. This result suggests that the mutant D2 isoform presents a lower capacity to convert thyroxine into the active hormone $\mathrm{T} 3$ in vivo.

In conclusion, this work provides the first evidence that in proliferating muscle stem cells the mutated T92A polymorphism of type 2 deiodinase causes a functional impairment in the physiologic activity of the protein, and thus a reduced pro-apoptotic response in T3-sensitive cells.

\section{OP49}

FIRST INSIGHTS INTO LAT2 AS T2 TRANSPORTER AND ITS STRUCTUREFUNCTION RELATIONSHIPS

Anita Kinne ${ }^{1}$, Katrin Hinz ${ }^{2}$, Melanie Wittner ${ }^{2}$, Josef Köhrle ${ }^{3}$,

Gerd Krause ${ }^{2}$

${ }^{1}$ Leibniz-Institut Für Molekulare Pharmakologie (Fmp), Berlin,

Germany, ${ }^{2}$ Leibniz-Institut Für Molekulare Pharmakologie

(Fmp), ${ }^{3}$ Institut Für Experimentelle Endokrinologie, Charité -

Universitätsmedizin Berlin, Germany

Transmembrane transporter proteins are required for the influx and efflux of thyroid hormones (TH) across cell membranes such as monocarboxylate transporters (MCTs) but also by L-type amino acid transporters (Lats). However, the uptake of TH by Lat 2 and the underlying transport mechanisms have to be investigated to understand the role of Lat 2 in transport of these hormones.

For this purpose TH substrate specificity as well as transport sensitive residues of Lat2 were determined. TH uptake studies in Xenopus laevis oocytes by Lat 2 identified 3,3'-T2 as preferred substrate and its strong competition by TH metabolites such as 3-T1 and other T2 isomers. In contrast, for T3 only a limited uptake and for rT3 and T4 even none uptake by Lat 2 was observed.

Insight into structure-function relationships for TH uptake by Lat 2 was obtained by homology modelling and site-directed mutagenesis, which revealed structural determinants of Lat 2 involved in TH binding site and transport. The Lat 2 model is based on crystal structure of the arginine/agmantine antiporter (AdiC). We utilized the bound arginine at AdiC to predict the substrate binding site at Lat2. Using model-guided mutagenesis of Lat 2 residues we identified by in vitro studies positions in transmembrane helices 1, 3 and 5 to be sensitive for TH transport. Furthermore, the cell membrane expression of the Lat 2 variants was analyzed and compared with the wild type protein.

In summary we report for Lat 2 a) that it is involved in the uptake of 3,3'$\mathrm{T} 2$, b) that it likely has a similar substrate binding site as AdiC and c) that we localized residues involved in transport mechanisms of Lat2. Thus, we conclude Lat 2 might contribute to termination of $\mathrm{TH}$ action by mediating influx and/or efflux of 3,3'-T2, which is generated either by T3 inactivation or by rapid Dio1-mediated rT3 degradation. 


\section{OP50 \\ NOVEL THYROID HORMONE TRANSPORTERS: \\ LAT3 AND LAT4 MEDIATE CELLULAR IODOTHYRONINE EFFLUX}

Chantal Zevenbergen ${ }^{1}$, Edward Visser ${ }^{1}$, Robin Peeters ${ }^{1}$, Anita Kinne ${ }^{2}$, Gerd Krause ${ }^{2}$, Marcel Meima ${ }^{1}$, Theo Visser ${ }^{1}$

${ }^{1}$ Erasmus University Medical Center, Rotterdam, The Netherlands,

${ }^{2}$ Leibniz-Institut Für Molekulare Pharmakologie (Fmp)

Introduction: Thyroid hormone $\mathrm{TH}$ ) transporters facilitate cellular $\mathrm{TH}$ influx and efflux, which is paramount for normal physiology. Several TH transporters have been characterized, but others remain to be identified, and it is still unclear how they cooperate in cellular TH transport. The L-type amino acid transporters LAT1-2 facilitate TH transport, but the role of LAT3-5 is as yet unknown.

Materials and Methods: FLAG-tagged constructs of LAT1-5 were transiently transfected into COS1 cells. LAT1 and LAT2 were co-transfected with the CD98 heavy chain. Cellular uptake of ${ }^{125}$ I-labeled 3,3'-T2, T3 and T4 was measured after $30 \mathrm{~min}$ incubations. Type 3 deiodinase (D3) was co-transfected with LAT1-4 to assess cellular metabolism of T2, T3 and T4 during 2-4 h incubations.

Results: LAT1 and LAT2 induced modest T4 and T3 uptake, but stimulated T2 uptake 2 to 3-fold. With all iodothyronines, LAT1 was more active than LAT2. LAT3 and LAT4 did not affect transport of T4 and T3 but decreased cellular accumulation of T2. LAT5 did not facilitate TH transport. Co-transfection with LAT3 or LAT4 prevented cellular T2 accumulation induced by LAT1 and LAT2. Metabolism of T4, T3 and T2 was enhanced in cells co-expressing D3 and LAT1 or LAT2, whereas T2 metabolism was decreased in cells co-expressing D3 and LAT3 or LAT4.

Discussion: Our findings demonstrate that LAT1 and LAT2 strongly facilitate T2 uptake, whereas LAT3 and LAT4 specifically facilitate T2 efflux. Co-expression of these transporters suggests that $\mathrm{T} 2$ is transported into the cell by LAT1 or LAT2, and subsequently exported by LAT3 or LAT4. Together, our findings suggest that different sets of transporters with specific influx or efflux capacity may cooperate to influence cellular thyroid state.

\section{OP51 \\ NEW INSIGHTS INTO THE MECHANISM OF DEIODINASE3}

Ulrich Schweizer ${ }^{1}$, Doreen Braun ${ }^{2}$, Christine Schlicker ${ }^{3}$, Josef Köhrle ${ }^{4}$

Clemens Steegborn ${ }^{5}$

${ }^{1}$ Rheinische Friedrich-Wilhelms-Universität Bonn; Institut Für

Biochemie und Molekularbiologie, ${ }^{2}$ Rheinische Friedrich-Wilhelms-

Universität Bonn; Institut Für Biochemie und Molekularbiologie,

${ }^{3}$ Ruhr-University Bochum, ${ }^{4}$ Institut Für Experimentelle Endokrinologie,

Charité - Universitätsmedizin Berlin, Germany, ${ }^{5}$ University of Bayreuth,

Germany

The reaction mechanism of selenium-dependent deiodination of iodothyronines by deiodinases is incompletely understood. Three questions have not been clearly answered: How is the proton transferred to the iodothyronine after abstraction of the iodine? How is the iodide liberated from the proposed selenenyl-iodide intermediate? How is the enzyme regenerated and by which physiological electron donor? A major limitation answering these questions represents the lack of an experimental deiodinase structure. Based on a model for this Trx-fold protein, we mutated several conserved amino acids in and adjacent to the loop harboring the catalytic selenocysteine 170 and connecting the $\beta$-sheet and $\alpha$-helix. The proteins were expressed in COS cells and analyzed by selective affinity labeling of selenols and activity assays in the presence of reducing cofactors. These biochemical experiments with mutant proteins indicate that hydroxyls from so far unrecognized conserved residues are important for activity. A reconstituted reducing system comprising thioredoxin, thioredoxin reductase, and NADPH or glutaredoxin, glutathione, glutathione reductase, and NADPH supported Dio3 activity in an NADPHdependent manner at physiological concentrations. A thioredoxin mutant Cys35Ser was trapped with Dio3 in non-reducing SDS-PAGE suggesting interaction of Dio3 and thioredoxin. Mutation of conserved cysteines to alanine reduced interaction with thioredoxin and selenol labeling, supporting a disulfide cascade ultimately reduced by thioredoxin.

\section{OP52 \\ FUNCTIONAL CHARACTERISTICS OF MCT8 ORTHOLOGS: INSIGHTS FROM HUMAN, MOUSE AND ZEBRAFISH}

Simone Kersseboom $^{0}$, Ramona E.A. van Heerebeek ${ }^{1}$, Francisco J. Arjona ${ }^{2}$, W. Edward Visser ${ }^{1}$, Robin P. Peeters ${ }^{1}$, Theo J. Visser ${ }^{1}$

${ }^{1}$ Erasmus University Medical Center, ${ }^{2}$ Radboud University Nijmegen

The monocarboxylate transporter 8 (MCT8) mediates transport of thyroid hormone (TH) across the plasma membrane. Mutations in MCT8 are associated with the Allan-Herndon-Dudley syndrome which comprises severe psychomotor retardation and an altered serum $\mathrm{TH}$ profile. The underlying pathogenic mechanism has been addressed using Mct8 ko mice and knockdown experiments in zebrafish. However, little is known about the functional characteristics of the MCT8 orthologs in these species. Therefore, we studied substrate specificity and transport characteristics of zebrafish (dr), mouse (mm) and human (hs) MCT8 in transfected COS1 and JEG3 cells.

All 3 MCT8 orthologs demonstrated transport of T3 $>$ T4 $>$ rT3, with drMCT 8 being more efficient than mmMCT8 and hsMCT8 in COS1 cells but not in JEG3 cells. Interestingly, low but significant Triac uptake by drMCT8 was observed in COS1 cells. Saturation of T3 transport showed lowest IC50 values for drMCT8, i.e. $0.58 \mu \mathrm{M}$ in COS1 cells and $0.25 \mu \mathrm{M}$ in JEG3 cells. T3 transport was inhibited more potently by iodothyronines than by analogs with propionic or acetic acid side chains, and inhibition was also greater for drMCT8 than for mmMCT8 or hsMCT8.

This study demonstrates similar characteristics of TH transport by human, mouse and zebrafish MCT8, although drMCT8 shows higher affinities for its substrates than mmMCT8 and hsMCT8. Characteristics of TH transport by the MCT8 orthologs are dependent on the cells in which they are expressed, suggesting the involvement of different interacting proteins in the formation of functional transporters in these cells.

\section{OP53 \\ HYPOTHALAMIC THYROID HORMONE SENSING REGULATES TORPOR INDUCTION \\ Eva K. Wirth ${ }^{1}$, Monja Willershäuser ${ }^{2}$, Franziska Meyer ${ }^{1}$, Eddy Rijntjes $^{3}$ Marc Klein ${ }^{4}$, Arnaud Agin ${ }^{5}$, Martin Hrabě de Angelis ${ }^{6}$, Josef Köhrle ${ }^{3}$, Martin Klingenspor ${ }^{2}$, Jan Rozman ${ }^{7}$, Ulrich Schweizer ${ }^{8}$ \\ ${ }^{1}$ Institut Für Experimentelle Endokrinologie, Charite Universitätsmedizin Berlin, ${ }^{2}$ Molecular Nutritional Medicine, Else-Kröner Fresenius Center, Technische Universität München, ${ }^{3}$ Institut Für Experimentelle Endokrinologie, Charité - Universitätsmedizin Berlin, Germany, ${ }^{4}$ Service D'endocrinologie, Centre Hospitalier Et Universitaire de Nancy, ${ }^{5}$ Icube Umr 7357, Université de Strasbourg/Cnrs, ${ }^{6}$ German Mouse Clinic, Institute of Experimental Genetics, Helmholtz Zentrum München; Center of Life and Food Sciences Weihenstephan, Technische Universität München,; German Center for Diabetes Research, Neuherberg, ${ }^{7}$ Molecular Nutritional Medicine, Else-Kröner Fresenius Center, Technische Universität München; German Mouse Clinic, Institute of Experimental Genetics, Helmholtz Zentrum München; German Center for Diabetes Research, Neuherberg, ${ }^{8}$ Rheinische Friedrich-Wilhelms- Universität Bonn; Institut Für Biochemie und Molekularbiologie, Germany}

A variety of hormones and neuroendocrine factors have been implicated in the induction of torpor, a reversible lowering of energy expenditure and body temperature during times of scarce food. Thyroid hormones $(\mathrm{TH})$ belong to this regulatory network of torpor induction. Access of $\mathrm{TH}$ to the hypothalamus depends on transmembrane transporters like monocarboxylate transporter 8 (Mct8). Mct8 is the most specific TH transmembrane transporter and is expressed in hypothalamus, pituitary and other organs. We have taken advantage of our Mct8-deficient mouse model to study torpor. Based on hypothalamic deiodinase gene expression, male and female Mct8-deficient mice represent two different models distinguished by hypothalamic hypo- vs. hyperthyroidism. Accordingly, $T s h \beta$ expression is high in $\mathrm{Mct}^{-/ \mathrm{y}}$ and low in $M c t 8^{-/-}$pituitary possibly associated with diverging circulating $\mathrm{T}_{4}$ levels. Interestingly, 24 hours of fasting invariably induces torpor only in the hypothalamic hyperthyroid situation. Induction of torpor in female Mct8-deficient mice occurs independent of previously identified changes in leptin and Fgf21. These findings underscore the basal role of TH handling and sensing for the regulation of energy metabolism not only in peripheral tissues, but also in the hypothalamus. 


\section{$0 P 54$ \\ THYROID HORMONE DEGRADING ENZYME TYPE 3 DEIODINASE (D3) IS PRESENT IN HUMAN NEUTROPHILS}

Anne van der Spek ${ }^{1}$, Monica Dentice ${ }^{2}$, Domenico Salvatore ${ }^{3}$, Eric Fliers ${ }^{1}$, Anita Boelen ${ }^{1}$

${ }^{1}$ Academic Medical Centre, ${ }^{2}$ Department of Clinical Medicine and Surgery, University of Naples "Federico II", ${ }^{3}$ Università DI Napoli "Federico II"

Illness and infection induce profound changes in thyroid hormone metabolism at the cellular level. We previously reported in experimental animals that type 3 deiodinase (D3) is strongly upregulated in infiltrating polymorphonuclear leukocytes (PMNs) during acute infection. D3 inactivates the thyroid hormones $T_{4}$ and $T_{3}$ into the inactive metabolites reverse $T_{3}$ and $T_{2}$, respectively. PMNs are deeply involved in the innate immune response induced by bacterial pathogens. Mice that lack D3 have impaired bacterial clearance following infection suggesting that D3 plays an important role in the bacterial killing capacity of PMNs.

To further elucidate this role, freshly isolated human neutrophils were isolated from venous blood of healthy volunteers. We subsequently assessed D3 protein using Western Blot. Furthermore we developed an in vitro model using differentiated NB4 cells, a neutrophil-like human cell line, to analyse the subcellular localization of D3 using confocal microscopy.

The presence of D3 was confirmed in freshly isolated human neutrophils and differentiated NB4 cells using Western Blot. In differentiated NB4 cells, D3 appeared to be present in intracellular vesicles, with a morphology resembling granules involved in bacterial killing. In contrast to previous reports in other cell types, D3 in NB4 cells was not present in early endosomes. Staining was checked for specificity by preincubation of the D3 antibody with the corresponding immunizing peptide.

In conclusion, D3 is present in human neutrophils and its subcellular localization in differentiated NB4 cells differs from that previously reported in the literature, possibly indicating a unique role for D3 in the bacterial killing machinery of these cells.

\section{OP8 Hyperthyroidism and Orbitopathy}

\section{OP55 \\ DIAGNOSIS AND MANAGEMENT OF GRAVES' DISEASE IN EUROPE - RESULTS OF A SURVEY AMONG MEMBERS OF THE EUROPEAN THYROID ASSOCIATION (ETA)}

Luigi Bartalena $^{1}$, Henry B. Burch ${ }^{2}$, George J. Kahaly ${ }^{3}$

${ }^{1}$ University of Insubria, Varese, Italy, ${ }^{2}$ Uniformed Services University of the Health Sciences, ${ }^{3}$ Johannes Gutenberg University Medical Center, Dept. of Medicine I, Mainz, Germany

Aims and Design: To assess clinical practice patterns in diagnosis and management of Graves' disease (GD) in Europe. ETA members were asked to complete an online questionnaire modified from a recent American survey. Respondents were 147 , accounting for $38 \%$ of members with clinical interests

Results: Most respondents requested FT4 (82\%) and TSH (81\%) measurements for hyperthyroidism diagnosis, while free $(61 \%)$ and total T3 (14\%) determinations were less frequently obtained. Confirmation of etiology required TSH-receptor antibody determination $(86 \%)$, while anti- thyroperoxidase $(64 \%)$ and/or anti-thyroglobulin $(30 \%)$ antibodies were requested less commonly. Thyroid ultrasound $(71 \%)$ was used more than scanning $(33 \%)$. Together with beta-blockers, antithyroid drugs (ATDs), radioiodine (RAI), and thyroidectomy were applied in $84 \%, 14 \%$ and $2 \%$, respectively, as first-line treatment for uncomplicated GD. ATDs were used in preparation for RAI in $61 \%$ and withdrawn 3-7 days prior to RAI. Reinstitution of ATDs after RAI was advised by $19 \%$. Methimazole was most used $(79 \%)$ followed by carbimazole $(18 \%)$ and propylthiouracil (3\%). The titration method was preferred by $36 \%$, the block-and-replace regimen by $26 \%$, while the remaining $38 \%$ would use the latter only in selected cases. ATDs were given for $12(16 \%)$, $18(51 \%)$ or $24(17 \%)$ months. In patients with mild orbitopathy, ATDs were preferred (75\%); if RAI was administered (19\%), concurrent low-dose oral steroids were generally used. In women planning pregnancy, $>30 \%$ of respondents advised thyroid surgery, $10 \%$ RAI, and $60 \%$ ATDs. During pregnancy, $76 \%$ of respondents use propylthiouracil during the first trimester, most of whom $(59 \%)$ then switch to methimazole/carbimazole. Compared with North American endocrinologists, Europeans used significantly more ultrasound and TSH-receptor antibodies and less scanning. ATDs remain the first-line treatment in Europe, while RAI is still preferred in North America.

Conclusions: Significant differences between Europe and between Europe and North America remain both in diagnostic procedures and therapeutic approach.

\section{OP56}

THYROID STIMULATING AUTOANTIBODIES ARE CLINICALLY USEFUL AND PREDICTIVE IN GRAVES' DISEASE - A PROSPECTIVE TRIAL George J. Kahaly ${ }^{1}$, Michael Kanitz ${ }^{1}$, Elisa Kolbe ${ }^{1}$, Nina Matheis ${ }^{1}$, Tanja Diana ${ }^{1}$

'Johannes Gutenberg University Medical Center, Mainz, Germany

Purpose: We hypothesized that serum thyroid stimulating autoantibodies (TSAb) levels are clinically useful and predictive in Graves' disease (GD).

Methods: Hundred consecutive untreated hyperthyroid GD patients received Methimazole for 24 weeks. Response vs. non-response to treatment was defined as biochemical euthyroidism at weeks 24 and 36 vs. persistent hyperthyroidism at week 24 and/or relapse at week 36 . Serum TSAb levels were sequentially measured with a bioassay that uses a chimeric TSH-R and a CRE-dependent luciferase. TSAb results are expressed as percentage of specimen-to-reference ratio (SRR\%).

Results: Forty three patients (median age 42 years, 13 smokers or $31 \%$ ) responded to Methimazole (2.5-30 mg/day) of whom 19 (45\%) had Graves' orbitopathy (GO). In contrast, 57 were non-responders (43.5 years, 28 smokers or $50 \%$ and 36 or $64 \%$ with GO, P $<0.04)$. At week 0 , responders demonstrated marked differences in diluted TSAb titers compared with nonresponders $(\mathrm{P}<0.001)$. Also, 12 weeks after starting Methimazole, differences of TSAb levels and Methimazole dose (both $\mathrm{P}<0.001$ ) were noted between responders vs. non-responders. At week 24 and compared with baseline, serum TSAb levels decreased markedly in responders (median SRR\% 420 vs. 271, $\Delta-60 \%, \mathrm{P}<0.001)$ but increased in non-responders ( 422 vs. $465, \Delta+10 \%)$. In contrast, serum levels of TSH-R binding inhibiting immunoglobulins (TBII, ECLIA) decreased in non-responders at week 24 (14.9 vs. $9.27 \mathrm{IU} / \mathrm{L}, \Delta-40 \%$, $\mathrm{P}=0.002)$. TSAb and TBII levels positively correlated $(\mathrm{r}=0.66, \mathrm{P}<0.001)$ however TSAB but not TBII values differentiated between GD and GD/GO $(\mathrm{P}<0.001)$. Median thyroid volume was $15.85 \mathrm{ml}$ (range 3-35) and $23.5 \mathrm{ml}$ (5-64) in responders and non-responders, respectively $(\mathrm{P}=0.002)$.

Conclusions: Serum TSAb levels mirror the severity of GD. Their increase during medical treatment is a marker for on-going disease activity. TSAb dilution analysis is additionally predictive. Thus, TSAb levels are a prognostic parameter for GD and reliably predict response to medical therapy.

\section{OP57 \\ EFFECTS OF TRIIODOTHYRONINE ON BONE MARROW AND SPLENIC GRANULOCYTES AND MONOCYTES POPULATIONS \\ Flavia Bloise $^{1}$, Felipe Leite Oliveira ${ }^{2}$, Huila Fonseca², \\ Samuel dos Santos Valença ${ }^{2}$, Radovan Borojevic'², \\ Carmen Cabanelas Pazos-Moura ${ }^{3}$, Valéria de Mello-Coelho ${ }^{2}$ \\ ${ }^{1}$ Institute of Biomedical Sciences; Institute of Biophysics Carlos \\ Chagas Filho. Federal University of Rio de Janeiro; Academic Medical \\ Central. University of Amsterdam; Capes, ${ }^{2}$ Institute of Biomedical \\ Sciences, ${ }^{3}$ Institute of Biophysics Carlos Chagas Filho}

Bone marrow hematopoietic stem cells and myeloid cells express thyroid hormone $(\mathrm{TH})$ receptors. However, studies on the effects of $\mathrm{TH}$ regulating myeloid cell population are controversial in the literature. In this study, we analyzed the influence of triiodothyronine $\left(\mathrm{T}_{3}\right)$ subcutaneously administrated in C57BL/6 mice for 14 days on the frequency of bone marrow myeloid 
precursors as well as the influence of $\mathrm{T}_{3}$ on the activity of splenic granulocytes and monocytes. Our results show that serum $\mathrm{T}_{3}$ was three times higher in $\mathrm{T}_{3}$-treated group than control, as verified by radioimmunoassay for $\mathrm{T}_{3}$. The in vivo $\mathrm{T}_{3}$ treatment promoted a $60 \%$ increase in the absolute number of bone marrow cells. By flow cytometry, we observed that the frequency of $\mathrm{CD} 117^{+} \mathrm{Sca}^{+}{ }^{+}$bone marrow immature progenitor cells increased $75 \%$ after $\mathrm{T}_{3}$ treatment. On the other hand, high-circulating levels of $\mathrm{T}_{3}$ induced a decrease of $21 \%$ in the frequency of $\mathrm{CD} 117^{+} \mathrm{CD} 11 \mathrm{~b}^{+} \mathrm{Grl}^{+}$myeloblasts and of $9 \%$ in the total $\mathrm{CD} 117^{-} \mathrm{CD} 11 \mathrm{~b}^{+} \mathrm{Gr} 1^{+}$neutrophil granulocytic population in the bone marrow. In the periphery, we found an increase of $21 \%$ in the frequency of CD45R ${ }^{-}$GR $1^{1 / \mathrm{int}}$ granulocytes and of $18 \%$ or $23 \%$ in the frequency of $\mathrm{CD} 11 \mathrm{~b}^{+}$ or $\mathrm{F} 4 / 80^{+}$myeloid cells in the spleen of $\mathrm{T}_{3}$-treated mice, respectively. When splenic cell cultures were treated with $10^{-11} \mathrm{M} \mathrm{T}_{3}$, we found an increase in the amount of anion peroxide production by these cells, as detected by nitroblue tetrazolium assay. Our data suggest that high-circulating levels of $\mathrm{T}_{3}$ regulates the differentiation of bone marrow monocytic and granulocytic myeloid cells and might stimulate the activity of macrophages and granulocytes to produce oxidative reactive species in the periphery.

\section{OP58 \\ THYROXINE-INDUCED CHANGES OF THE PLASMA PROTEOME - A STUDY OF EXPERIMENTAL HYPERTHYROIDISM IN HUMANS}

Beatrice Engelmann ${ }^{1}$, Anna-Luise Dirk², Elke Hammer ${ }^{3}$,

Alexander Iwen" ${ }^{2}$, Henri Wallaschofski ${ }^{4}$, Uwe Völker ${ }^{3}$, Georg Homuth ${ }^{3}$, Georg Brabant ${ }^{2}$

${ }^{1}$ Interfaculty Institute for Genetics and Functional Genomics, University of Greifwald, ${ }^{2}$ Experimental and Clinical Endocrinology, University of Luebeck, ${ }^{3}$ Interfaculty Institute for Genetics and Functional Genomics, University of Greifswald, ${ }^{4}$ Interfaculty Institute for Genetics and Functional Genomics

Objective: Effects of experimentally induced hyperthyroidism on the human plasma proteome were tested using exogenous levothyroxine. Using an unsupervised proteomic approach we assessed alterations in the amounts of potential new marker proteins in comparison to well known parameters changed in thyrotoxicosis.

Materials and Methods: 16 healthy male subjects (median age $28 \mathrm{yrs}$, no clinical, biochemical or sonographical of thyroid problems) participated in the study following informed consent. Blood was sampled at baseline, 4 and 8 weeks under a daily dose of $250 \mu \mathrm{g}$ thyroxine as well as 4 and 8 weeks after discontinuation of thyroxine. Thyroid function was measured and subjectively assessed by questionnaire. In addition we assessed cardiovascular function (24 $\mathrm{h}$ recording) at baseline and after 8 weeks under thyroxine. Individual plasma protein profiles were recorded by label-free tandem mass spectrometry and subjected to relative quantification of time-dependent alterations of protein abundances.

Results: All subjects developed biochemical hyperthyroidism after 8 weeks with completely suppressed TSH and increased of fT4 and fT3 levels but no hyperthyroid symptoms in the questionnaire. Systolic but not diastolic blood pressure increased significantly $(4 \mathrm{mmHg}$ ) as did pulse rate ( 9 beats/ min; $\mathrm{P}<0.02$ each). In parallel, SHBG and cystatin $\mathrm{C}$ levels increased under thyroxine and returned quickly to pretreatment levels whereas total, LDL- and HDL-cholesterol significantly decreased in the therapeutic period and rapidly re-increased thereafter $(\mathrm{P}<0.001$, each). The plasma proteome analysis confirmed these changes but additionally identified a further 76 proteins exhibiting significant alterations in abundance during treatment. Interestingly, these included numerous coagulation factors as well as complement system proteins exhibiting positive correlations with thyroxine, thereby indicating a procoagulatory condition associated with hyperthyroidism.

Conclusion: Combining an experimental model of hyperthyroidism with an unsupervised proteomic approach we were able to identify new molecular markers of hyperthyroidism (Supported by DFG SPP 1629).

\section{OP59 \\ DECREASE OF GLUT4 AND T3 AND INCREASE OF NOX2 ARE INVOLVED IN THE OXIDATIVE STRESS OF EYE MUSCULAR CELLS AND ORBITAL ADIPOCYTES IN GRAVES' ORBITOPATHY}

Van Regemorter Elliott ${ }^{1}$, Julie Craps ${ }^{2}$, Joris Virginie ${ }^{1}$,

Behets Catherine ${ }^{1}$, Benoit Lengelé2 ${ }^{2}$, Baldeschi Lelio ${ }^{1}$

Chantal Daumerie ${ }^{3}$, Marie-Christine Many ${ }^{2}$

${ }^{1}$ Ucl, ${ }^{2}$ Université Catholique de Louvain, Morphologie Expérimentale,

${ }^{3}$ Cliniques Universitaires Saint-Luc; Université Catholique de Louvain

To further explore the mechanisms underlying the oxidative stress (OS) in Graves' orbitopathy (GO), we have hypothesized a cell deprivation in glucose and T3. Therefore, we analyzed the expression of the glucose transporter, GLUT4, whose translocation is regulated by Caveolin-1 (Cav1), and the T3 cell content regulated by the balance between type II (D2) and type III (D3) deiodinases, D3 being increased during hyperthyroidism and inactivating T4 and T3. We also analyzed the expression of NOX2 which, in GLUT4-/- mice, is a source of reactive oxygen species (ROS) in cardiac muscular cells ( $\mathrm{Li}$ et al., FEBS J, 279: 599-611, 2012)

Orbital muscles and adipose tissues were obtained from GO patients ( $\mathrm{n}=$ $8)$ and control patients operated either for strabismus or at autopsy $(n=8)$. They were then processed for detection of Cav1, GLUT4, D2, D3, T3 and NOX2, by immunofluorescence (IF) or immunohistochemistry (IHC).

Results: First, by double IF, we showed the colocalization of Cav1 and GLUT4 in control eye muscular cells and adipocytes.

Second, by IHC, we demonstrated a reduction of GLUT4 and Cav1 in muscular cells and adipocytes from GO patients, as compared to controls. T3 was also dramatically decreased in the cytoplasm of both cell types, and this decrease was correlated with D2 downregulation and D3 upregulation in GO patients.

Third, by IHC, we demonstrated an increase of the expression of NOX2 in GO muscular cells and adipocytes, as compared to controls.

In conclusion, the oxidative stress in GO muscular cells and adipocytes could be explained by glucose deprivation due to caveolin-1 and GLUT4 downregulation, as well as by T3 deprivation due to D3 upregulation. Moreover, GLUT4 decrease is associated with an overproduction of cytosolic ROS by NOX2, which could thus be an interesting therapeutic target to prevent OS-related damages.

\section{OP60 \\ ROLE OF ALTERNATE TSHR LIGANDS AND BINDING PROTEINS (TSHR-BP) IN GRAVES' ORBITOPATHY (GO)}

Mohd Shazli Draman ${ }^{1}$, Fiona Grennan-Jones ${ }^{1}$, Lei Zhang ${ }^{1}$

Pete N. Taylor ${ }^{1}$, Daniel Morris ${ }^{2}$, Carol Lane ${ }^{2}$, Colin Dayan ${ }^{1}$, Marian Ludgate

${ }^{1}$ Institute of Molecular \& Experimental Medicine; School of Medicine; Cardiff University, ${ }^{2}$ Department of Ophthalmology; University Hospital of Wales

Introduction: Thyroid stimulating autoantibodies (TSAB) contribute to GO pathogenesis. We hypothesise that expression of TSHR-BP (TSHRV, exons $1-8)$ and/or thyrostimulin ( $\alpha 2 \beta 5)$ might protect against or exacerbate GO. We measured their expression and function using primary human orbital fibroblasts (OF) from $\mathrm{GO}(\mathrm{n}=5)$ and non-GO $(\mathrm{n}=5)$.

Methods: Adipogenic medium was added to confluent OF and RNA extracted (days $0 \& 15$ ) for QPCR measurement of $\alpha 2, \beta 5$, TSHR and TSHRV transcripts. cAMP induced by TSH $5 \mathrm{mu} / \mathrm{ml}$ or monoclonal TSAB (M22) $0.2 \mathrm{ng} / \mathrm{ul}$ was measured directly in OF using RIA. CHO-cells expressing TSHR were treated with TSH/M22 in conditioned medium (CM) from OF or control human fibroblasts, and cAMP measured indirectly (luciferase bioassay) to determine whether the TSHRV is secreted as a TSHR-BP. Data are presented as mean \pm SEM.

Results: $\alpha 2$ transcripts were present on D0 but reduced during adipogenesis $(\mathrm{P}<0.005)$ with no difference between $\mathrm{GO} /$ non-GO; $\beta 5$ transcripts were at the limit of detection. There was no difference in TSHRV levels between $\mathrm{GO} /$ non-GO on D0 but on D15 levels in GO $(0.029 \pm 0.009$ vs. $0.011 \pm 0.002$ $\mathrm{TCN} / 1000$ APRT, $\mathrm{p}=0.039$ ) were significantly higher. TSH and M22 induced 
4-fold and 2-fold increases in cAMP in non-GO (D15). In GO, responses to TSH and M22 were substantially lower, and this was inversely correlated with the ratio of TSHRV to TSHR transcripts (Spearman correlation: TSH r $=$ $-0.55, \mathrm{p}=0.23, \mathrm{M} 22 \mathrm{r}=0.87, \mathrm{p}=0.03$ ). In the CHO-TSHR cells, TSH and M22 induced 5-fold and 4-fold increases in light; these were reduced in control fibroblast $\mathrm{CM}$ but no additional reduction was apparent in $\mathrm{CM}$ from $\mathrm{GO} /$ non-GO OF.

Conclusions: There is no evidence that thyrostimulin contributes to GO pathogenesis. TSHRV transcripts are more abundant than full length TSHR in OF and during adipogenesis are significantly higher in GO than non-GO. TSHRV is not secreted but may potentially alter intracellular TSHR signalling.

\section{OP61 \\ THE EFFECT OF RITUXIMAB (RTX) AND INTRAVENOUS METHYLPREDNISOLONE (IVMP) ON TSH-RECEPTOR ANTIBODIES (TRAB) AND THE CXCL-10 CHEMOKINE IN PATIENTS WITH ACTIVE GRAVES' ORBITOPATHY (GO) \\ Guia Vannucchi ${ }^{1}$, Irene Campi ${ }^{2}$, Danila Covelli ${ }^{2}$, P. Fallahi ${ }^{3}$, \\ S. Tirelli' , A. Di Domenicoantonio ${ }^{3}$, Nicola Currò ${ }^{5}$, Davide Dazzi ${ }^{6}$, Alessandro Antonelli ${ }^{3}$, Mario Salvi ${ }^{2}$ \\ ${ }^{1}$ Endocrine Unit, Fondazione Policlinico Irccs, Milan, Italy, ${ }^{2}$ Endocrine Unit, Fondazione Irccs Cà Granda, Milan, ${ }^{3}$ Department of Clinical and Experimental Medicine, University of Pisa, ${ }^{4}$ Laboratory Medicine Unit, Fondazione Ca' Granda Irccs, ${ }^{5}$ Ophthalmology4, Fondazione Ca' Granda Irccs, ${ }^{6}$ Internal Medicine5, Ospedale DI Fidenza, Fidenza, Italy}

Increased proinflammatory chemokines and circulating TRAb are associated with GO activity and severity. We conducted a double blind, randomized, controlled study (EUDRACT 2007-003910-33) in which patients with active moderate-severe GO were treated with either RTX $(n=16)$ or IVMP $(\mathrm{n}=16)$. We measured serum TRAb in both IVMP and RTX patients and CXCL-10 at baseline, at 8 weeks or at the time of CD20 B cell depletion and at 24 weeks. GO activity was assessed by the clinical activity score (CAS). We observed a progressive, significant decrease of TRAb from 18.1 \pm 21.6 and $10.7 \pm 9.1$ to $17.5 \pm 24.3$ and $4.0 \pm 4.5$ at 24 weeks after IVMP $(P=0.05)$ and RTX $(\mathrm{P}=0.005)$, respectively, but no differences between the two arms $(\mathrm{P}=$ NS). Mean basal \pm SE serum CXCL10 was $178.2 \pm 22.4 \mathrm{pg} / \mathrm{ml}$ and significantly decreased to $75.4 \pm 18.6$ at 24 weeks after IVMP $(\mathrm{P}=0.001)$ but increased from $135.5 \pm 14.5 \mathrm{pg} / \mathrm{ml}$ to $139.1 \pm 12$ after RTX (P = NS). The decrease of CXCL-10 correlated with that of the CAS in only the IVMP group $(\mathrm{P}=0.005)$. In RTX patients, we observed a significant increase of CD16+CD3- natural killer cells (NK) from 16 weeks after therapy $(\mathrm{P}=0.028)$, and this inversely correlated with the decrease of the CAS $(\mathrm{P}=0.045)$ in patients who had a more progressive GO inactivation. These latter patients also showed CXCL-10 significantly higher when compared to those who had very rapid inactivation $(\mathrm{P}=0.005)$. Overall, we did not find significant correlation between the number of NK cells and CXCL-10 ( $\mathrm{P}=\mathrm{NS})$. In conclusion, the decrease of TRAb after RTX does not differ from that after IVMP. As previously reported, the decrease of CXCL10 after IVMP is associated to GO inactivation. We hypothesize that serum CXCL-10 do not change after RTX due to an overwhelming chemokine production resulting from massive B-cell lysis induced by the drug. Changes of NK cells might be potentially useful for the prediction of RTX efficacy.

\section{OP62 \\ FORKHEAD TRANSCRIPTION FACTORS; POSSIBLE TARGETS FOR THERAPY IN GO?}

Lei Zhang ${ }^{1}$, Fiona Ruge ${ }^{2}$, Mohd Shazli Draman ${ }^{1}$

Fiona Grennan-Jones ${ }^{1}$, Carol Lane ${ }^{3}$, Colin Dayan ${ }^{1}$, Marian Ludgate ${ }^{1}$

${ }^{1}$ Institute of Molecular \& Experimental Medicine; School of Medicine; Cardiff University, ${ }^{2}$ Cardiff-Peking Cancer Institute; School of Medicine; Cardiff University, ${ }^{3}$ Department of Ophthalmology; University Hospital of Wales

Objectives: Graves' Orbitopathy (GO) is known as an autoimmune condition caused by expansion of the orbital contents by excess adipogenesis and overproduction of hyaluronan (HA) from preadipocyte/fibroblasts, (PF). We previously revealed an orbit specific cell signalling network regulating hyaluronan synthase 2 (HAS2, major source of HA), leading to adipogenesis and
HA accumulation being linked in the orbit but not other depots, where adipogenesis leads to HA reduction. Further investigation revealed the importance of PI3K signalling on regulation of HAS2/HA. Forkhead box protein (FOXO) is essential in adipogenesis, we hypothesized that FOXO, the down-stream target of PI3K, is involved in the regulation of HAS2 transcription.

Methods: Confluent human orbital $(n=7)$ or subcutaneous $(n=5) P F$, obtained with appropriate approvals/consent, were cultured in adipogenicmedium for 15 days. Nuclear and cytosolic proteins were extracted at different time points. Levels of FOXO1, FOXO3a \& FOXO4 protein were analysed by Western blotting. A promoter-luciferase construct containing FOXO binding sites was introduced into PF. Luciferase activity was analysed through adipogenesis to assess FOXOs nuclear location. siRNA for FOXO1, FOXO3a or scrambled control were transfected into PF followed by QPCR measurement of FOXO1, FOXO3a and HAS2 transcripts.

Results: Levels of FOXO1, FOXO3a \& FOXO4 proteins (relative to actin control) were lower in orbital PF throughout adipogenesis, compared with subcutaneous in both nuclear and cytosolic fractions. FOXO-binding promoter-luciferase assay confirmed lower binding activity in orbital compared with subcutaneous PF through adipogenesis. Treatment with siRNA for FOXO1 or FOXO3a ( 60\% knock-down FOXO1 or FOXO3a transcripts) significantly increased HAS2 transcripts.

Conclusions: FOXO1 and FOXO3a showed a negative impact on HAS2 transcript levels. Our results suggest that low level FOXO expression might explain the increased HA unique to orbital adipogenesis. This significant depot specific difference may contribute to the development of GO, and suggests possible nuclear targets for therapies of GO.

\section{OP9 Thyroid Cancer Pathogenesis}

\author{
OP63 \\ A NOVEL MODULATOR OF CELL INVASION AND \\ METASTASIS \\ Rachel Watkins ${ }^{1}$, Vicki Smith ${ }^{1}$, Neil Sharma ${ }^{1}$, Martin Read ${ }^{1}$ \\ Perkin Kwan ${ }^{1}$, Rachel Fletcher ${ }^{1}$, Vikki Poole ${ }^{1}$, \\ Waraporn Imruetaicharoenchoke ${ }^{1}$, Kristien Boelaert', Jayne Franklyn', \\ Christopher McCabe ${ }^{1}$ \\ ${ }^{1}$ University of Birmingham, UK
}

Metastasis is a multistep process responsible for the majority of endocrine cancer deaths. PBF is a proto-oncogene which is upregulated in differentiated thyroid cancer; patients with high PBF expression show significantly decreased disease-specific survival. Recently, PBF expression has been correlated with distant thyroid cancer metastasis at diagnosis. We now show that PBF promotes cellular invasion in thyroid carcinoma SW1736 cells compared to vector only $(\mathrm{VO})(\mathrm{P}<0.01)$. In keeping with $\mathrm{PBF}$ 's role in cell invasion, overexpression of $\mathrm{PBF}$ in $\mathrm{HCT} 116$ cells induced $\sim 60 \%$ greater migration compared to VO-transfected controls $(\mathrm{P}<0.001)$, implying a role for PBF in promoting cell movement. These data were supported by wound healing assays in TPC cells, which revealed that GFP-tagged PBF cells migrate further than GFP-VO cells $(\mathrm{VO}=11.0 \mathrm{mM} /$ hour, $\mathrm{PBF}=15.8 \mathrm{mM} /$ hour, $\mathrm{P}<0.01)$. To unravel the mechanism by which PBF promotes cell migration and invasion we used IP-MS to discover PBF binding partners, and identified the cortical actin binding protein, cortactin. Interaction was confirmed through co-IP, immunofluorescence and Proximity Ligation Assays. The latter indicated that this interaction occurs within or close to the plasma membrane, and particularly at the leading edge of migrating TPC and MDA-MB-231 cells. To determine if the interaction between PBF and cortactin promotes cellular invasion we overexpressed PBF and simultaneously knocked down cortactin expression. Our preliminary data reveal that knockdown of cortactin attenuates PBF induction of cell invasion. The colocalisation of PBF and cortactin was narrowed down to invadopodia-like structures and adhesion rings, which promote recruitment of vesicles carrying the proteases required for degradation of the ECM. Taken together these data suggest that PBF induces cellular invasion and migration across a range of thyroid and other cell lines via its interaction with cortactin, supporting its in vivo association with thyroid tumour metastasis. 


\section{OP64}

AN INTRONIC DELETION IN PHOSPHOLIPASE-C $\beta 1$ (PLC $\beta 1$ ) ASSOCIATED WITH EUTHYROID MULTINODULAR GOITRE (MNG) AND RISK OF PROGRESSING TO PAPILLARY THYROID CANCER (PTC)

loannis Ladas ${ }^{1}$, Fiona Grennan-Jones ${ }^{1}$, Ameen Bakhsh' ${ }^{1}$, Lei Zhang ${ }^{1}$, Anna de Lloyd ${ }^{1}$, Denise Zwanziger ${ }^{2}$, Dagmar Fuhrer-Sake ${ }^{2}$, Marian Ludgate

${ }^{1}$ Cardiff University, Thyroid Research Group, Institute of Molecular and Experimental Medicine, Cardiff, UK, ${ }^{2}$ Klinik Fur Endokrinologie, Universitatsklinikum, Essen, Germany

Objectives: A novel InDel (1076 bp deletion, ATAA insertion at junction) was identified in the 3rd intron of plc $\beta 1$ (Chr 20) by genome-wide linkage study. The InDel has a prevalence of $\sim 1 \%$ in healthy Caucasians and $\sim 5 \%$ in patients with euthyroid multi-nodular goiter (MNG). We hypothesize that the InDel could be a marker for MNG patients most likely to develop papillary thyroid cancer (PTC). We aimed to develop a genotyping technique to facilitate high-throughput screening. Since patients carrying the InDel have increased thyroidal PLC $\beta 1$ transcript levels, we also investigated the effects of modifying PLC $\beta 1$ expression on thyrocyte proliferation.

Methods: A qPCR based screening tool was optimized, using primers within and flanking the InDel, to distinguish normal individuals and InDel heterozygotes, based on differences in Ct value. Transcript levels in FRTL5 cells were reduced using siRNA specific for rat PLC $\beta 1$, their proliferation was compared (direct counting) with control scrambled siRNA treated cells.

Results: Optimal results were obtained using primers flanking the InDel and were applied to screen 208 adults plus 36 with varying thyroid proliferative disease (TPD). Four were InDel heterozygotes; (all sequences identical) 3 men (aged 37 to 39) and 1 woman (aged 47). One male (TPD) had follicular adenoma, a second male was noted to have a goitre, and the woman who has a family history of cancer is due to undergo a thyroid scan. Preliminary data using PLC $\beta 1$ knockdown in FRTL5 indicate that their proliferation is reduced by about $20 \%$ compared with control cells.

Conclusions: The optimized protocol will facilitate screening large cohorts to determine the potential of the InDel as a marker for TPD most likely to progress to cancer. Further experiments are required to confirm the effects of variations in PLC $\beta 1$ expression levels on thyroid growth.

\section{OP65 \\ SELECTIVE KILLING AND SUBSEQUENT SENESCENCE INDUCTION: A DOUBLE ACTION FOR ANAPLASTIC THYROID CANCER THERAPY Elisa Stellaria Grassi ${ }^{1}$, Valeria Vezzoli ${ }^{1}$, Irene Negri ${ }^{1}$, Giovanni Vitale ${ }^{2}$, Luca Persani \\ ${ }^{1}$ Dept. of Clinical Sciences \& Community Health, University of Milan, ${ }^{2}$ Dept. of Clinical Sciences \& Community Health, University of Milan; Division of Endocrine and Metabolic Diseases \& Lab. of Endocrine and Metabolic Research, Irccs Istituto Auxologico Italiano}

Anaplastic thyroid cancer therapy has been storically concentrated on apoptosis induction; undifferentiated cells, anyway, are often able to escape cell-cycle checkpoints and apoptosis with clonogenic survival, while normal cells retains full sensibility. This accounts for the lack of efficacy and, on the other side, for the considerably side effects of cytotoxic drugs. For these reasons new mechanisms of selective cell death are promising targets for anticancer therapy. The purpose of our study was to achieve a selective killing of undifferentiated thyroid cancer cells with minor effects on differentiated ones. In our study we evaluated the effects of promising agents on the induction of different cell death mechanisms in thyroid cancer-derived cell lines by western-blotting, confocal microscopy, apoptosis and senescence assays. The results shown that treatment with low doses $(5-10 \mu \mathrm{M})$ of a small compound, SP600125, avoided unspecific massive apoptosis induction while it was able to induce early cell death by mitotic catastrophe only in undifferentiated cell lines $(\mathrm{P}<0.01)$. Moreover the small fraction of treated cells that escaped death was unable to form colonies but underwent an accelerated senescence transformation with typical morphological changes, alterations in lysosomal structure and SA- $\beta$-gal expression $(\mathrm{P}<0.01)$. All these effects were accompanied by sustained p21 pathway activation.
No significant effects were detected in differentiated thyroid cancer cells. In conclusion, our treatment was able to sequentially activate mitotic catastrophe and cell senescence processes in anaplastic thyroid cancer cells with abnormal DNA damage control that are usually more resistant to cytotoxic therapy. Mitotic catastrophe represents a selective mechanism of cell death activation in undifferentiated neoplasms while the induction of accelerated senescence in drug-resistant clones may increase therapy efficacy, inhibiting mass growth and metastatic spreading. For these reasons SP600125 represents a valid candidate for the therapy of undifferentiated thyroid cancers not responsive to currently available therapies.

\section{OP66 \\ THE FIRST REPORTED MUTATIONS IN THE PITUITARY TUMOR-TRANSFORMING GENE (PTTG) BINDING FACTOR (PBF)}

Waraporn Imruetaicharoenchoke ${ }^{1}$, Martin Read ${ }^{1}$, Joel Smith ${ }^{1}$

Vicki Smith ${ }^{1}$, Bhavika Modasia ${ }^{1}$, Vikki Poole ${ }^{1}$, Rachel Watkins ${ }^{1}$,

Jayne Franklyn ${ }^{1}$, Kristien Boelaert ${ }^{1}$, Christopher McCabe ${ }^{1}$

${ }^{1}$ University of Birmingham, UK

PBF is a ubiquitous glycoprotein which is over-expressed in thyroid and breast cancer, and modulates both radioiodine uptake and thyroid hormone efflux. Papillary thyroid cancer patients with high PBF expression show decreased disease specific survival than those with lower expression. Previously classified as a proto-oncogene, 11 substitution-missense mutations of $\mathrm{PBF}$ have been reported in tumours from patients with various cancers via the COSMIC database, suggesting PBF may be an oncogene. We have therefore examined the biological implications of all mutations in thyroid and breast cells. Substitution mutations were predominantly clustered within the PSI domain and close to the nuclear localisation signal of the protein. Modelling of all mutants using I-TASSER identified potentially severe alterations in the 3D structure of mutants W59F, R87C, S103L, G106R, G106W and R140W. SIFT predictions identified that all mutations except G106V should result in significant changes in protein function. Western blotting revealed that mutations C51R and G106R inhibited PBF dimerisation and glycosylation in vitro. Anisomycin half life studies in SW1736 thyroid and MCF7 breast cancer cells revealed that the $\mathrm{C} 51 \mathrm{R}$ mutation resulted in increased protein stability compared to wild type (half life approximately 22 hours), whereas V55I, W59F, R87C and S103L, G106R, G106V, G106W and R146W mutants were less stable, ranging from approximately 6 hours to 12 hours. BrdU proliferation assays revealed that $\mathrm{C} 51 \mathrm{R}$ and $\mathrm{V} 55 \mathrm{I}$ induced a significant proliferative advantage in TPC-1 thyroid cells compared with WT, whilst R140W significantly repressed proliferation. This is the first description of mutations in PBF, a gene implicated both in thyroid cancer and thyroid hormone metabolism. Based on our biochemical characterisation of three-dimensional protein structure, glycosylation, dimerisation, stability and proliferative influence, we suggest that mutations in PBF might represent a novel aetiological event in human cancer.

\section{OP67 \\ CLM29 AND CLM3, TWO TYROSINE KINASE INHIBITORS, HAVE ANTINEOPLASTIC ACTIVITY IN VITRO IN PRIMARY CULTURES FROM ANAPLASTIC THYROID CANCER, OBTAINED FROM FINE NEEDLE ASPIRATION \\ Alda Corrado ${ }^{1}$, Poupak Fallahi ${ }^{1}$, Silvia Martina Ferrari ${ }^{1}$, \\ Guido Bocci' ${ }^{1}$, Concettina La Motta ${ }^{1}$, Gabriele Materazzi', \\ Andrea Di Domenicantonio ${ }^{1}$, Romano Danesi', Federico Da Settimo ${ }^{1}$, \\ Paolo Miccoli ${ }^{1}$, Alessandro Antonelli ${ }^{\circ}$ \\ ${ }^{1}$ University of Pisa, Italy}

Objective: Anaplastic Thyroid Cancer (ATC) is one of the most lethal malignancies with very short survival and extremely poor treatment outcome and the mean survival is approximately 6 months.

The most effective therapy for ATC is multimodal treatment protocol that include surgery, chemotherapy (doxorubicin and cisplatin), and hyperfractionated accelerated external beam radiotherapy, with a median patient survival of 10 months.

Testing the sensitivity of "primary anaplastic thyroid cancer cells" (pATC) obtained from each subject, to different drugs, could determinate an increase 
in the effectiveness of the treatment avoiding the administration of inactive therapeutics.

Methods: Here, we tested the antineoplastic effect of two new "pyrazolo[3,4- $d]$ pyrimidine" compounds (CLM3, CLM29) in pATC obtained both from biopsy (biop-pATC), or from fine needle aspiration (FNA-pATC) both established from 5 patients.

We tested different concentrations of CLM29 and CLM3 in the in vitro experiments $(1,10,30,50 \mu \mathrm{M})$.

Results: WST-1 assay shows a significant reduction of proliferation in FNA-pATC, or biop-pATC cells respect to the control, in particular with CLM29, and slightly with CLM3, while both compounds increased the percentage of apoptotic cells in FNA-pATCs, or biop-pATC, dose-dependently.

No significant differences in sensitivity to CLM29 or CLM3 between the tested ATC cells from FNA, or biopsy were demonstrated in our study.

Conclusion: Our results show that: 1) primary FNA-pATC cells have a sensitivity to tyrosine kinase inhibitors (TKIs) agents quite similar to that observed in primary cells from biopsy (biop-pATC), and for this reason the use of primary FNA-pATC cells for the tests can reduce the time needed for biopsy; 2) CLM29 and CLM3 reduce cell growth, increasing apoptosis in ATC; 3) the possibility to test senitivity to different TKIs in each patient is able to increase the efficacy of treatments, avoiding the administration of ineffective drugs.

\section{OP68 \\ ESTROGEN AND PROGESTERON RECEPTORS EXPRESSION IN DIFFERENTIATED THYROID CANCER: A ROLE IN THE RISK STRATIFICATION OF THYROID TUMORS}

Simone De Leo ${ }^{1}$, Michela Perrino ${ }^{1}$, Carla Colombo', Stefania Rossi ${ }^{2}$, Delfina Tosi', Leonardo Vicentini', Gaetano Bulfamante ${ }^{2}$,

Laura Fugazzola', Guia Vannucchi

${ }^{1}$ Endocrinology, Fondazione Ca' Granda Irccs, University of Milan,

${ }^{2}$ Pathology Unit, Ospedale San Paolo, University of Milan, and

${ }^{3}$ Endocrine Surgery Unit, Fondazione Irccs Cà Granda, Milan, Italy

A possible role of sex hormones in the pathogenesis and development of thyroid tumors has been suggested. We studied the expression of Estrogen receptor $\alpha(E R \alpha)$ and Progesteron receptor (PR) in $190(146 \mathrm{~F} / 44 \mathrm{M})$ patients with differentiated thyroid cancer (DTC) in relation to clinical and molecular features. Tumors were incidental in $39 \%$ of cases, while $61 \%$ had a preoperative diagnosis. The $81 \%$ of cases were genotyped for BRAFV600E mutation. $\mathrm{ER} \alpha$ ?and PR expression was scored as negative, and weakly positive or positive according to the degree of staining. ER $\alpha$ and PR expression was found in 91/146 (62.3\%), 110/146 (75.3\%), 34/44 (77\%) and 31/44 (70.3\%) tumor specimens in female and male, respectively. Overall, ER $\alpha$ and PR expression significantly correlated with tumor size $(E R: P=0.006, P R: P=0.005)$ and with the presence of metastatic neck lymph-nodes at diagnosis $(\mathrm{P}=0.02, \mathrm{P}=$ 0.04). In women, a higher $E R \alpha$ and $P R$ expression correlated with tumor size, particularly for the $\mathrm{T} 1$ subgroup $(\mathrm{P}=0.03)$, without differences in pre- and postmenopausal age. T3 and T4 tumors had a higher PR expression in both sexes. Moreover, ER and PR expression was significantly higher in clinically evident tumors compared to incidental cases $(\mathrm{P}=0.0001)$. The BRAF V600E mutation was found in 35/154 tissues (22.7\%) and mutated cases had a higher, though not statistically significant, ER and PR expression compared to nonmutated. No significant differences in the outcome were recorded between neither ER $\alpha$ positive/negative nor $\mathrm{PR}$ positive/negative cases $(\mathrm{P}=0.8$ and $\mathrm{P}=0.95$, respectively). In conclusion, present data indicate that ER and PR expression is higher in more aggressive DTCs. Consistently, clinically evident and BRAFV600E positive tumors had a higher ER $\alpha$ and PR expression. Although an impact on the outcome was not found in the present series, these data confirm a possible role of ER $\alpha$ and PR expression in the risk stratification of thyroid tumors.

\section{OP69 \\ INSULIN RESISTANCE: ANOTHER RISK FACTOR THAT INCREASES THE RISK OF RECURRENCE IN PATIENTS WITH THYROID CANCER?}

Fabian Pitoia $^{1}$, Erika Abelleira ${ }^{1}$, Fernanda Bueno ${ }^{1}$, Carolina Urciuoli ${ }^{1}$

${ }^{1}$ Hospital de Clinicas - University of Buenos Aires

Insulin resistance (IR) has been implicated as an important factor for cell proliferation. We have previously shown that patients with IR have larger thyroid glands.

Objective: To evaluate the initial response to treatment and long-term outcome of patients with papillary thyroid cancer (PTC) according to the risk of recurrence of the ATA 2009 (RR) and the presence or absence of IR.

Subjects and Methods: We reviewed our database of patients with PTC who had been followed-up from 2001 to 2013 in the Hospital de Clínicas. We included 38 patients with classic PTC, stable body mass index 5 years previously to PTC diagnosis and during the entire time of follow-up and low and intermediate RR. The IR was evaluated through the HOMA index. When $H O M A \geq 2.5$, IR was diagnosed. The initial response to treatment was classified as remission or persistent disease (biochemical (B) and/or structural (S)). The clinical status at final follow-up was defined as no evidence of disease, $\mathrm{B}$ or S persistence, or recurrence. Median age of included patients was 37 y. (range 18-64 y). RR was: low: $\mathrm{n}=15$, intermediate: $\mathrm{n}=23$. The median follow-up was 63.5 months (range 22-264 m).

Results: Remission was more frequently found in those patients without IR when the intermediate RR was considered ( $50 \%$ vs. $22 \%, \mathrm{P}<0.01)$. When the status at final follow-up was considered, we found more frequency of $\mathrm{S}$ persistent disease in low RR, $(10 \%$ vs $0 \%$ with or without IR, respectively; $\mathrm{P}<$ $0.02)$ and intermediate RR ( $45 \%$ vs $7 \%$ with or without IR; $\mathrm{P}<0.01)$.

Conclusions: in this series of patients with PTC the state of IR was associated with increased probability of S persistent disease at final follow-up. The IR could have a deleterious effect on the outcome of patients with PTC.

\section{OP70 \\ GENETIC PREDISPOSITION TO PAPILLARY THYROID CANCER}

Dorota Kula ${ }^{1}$, Daria Handkiewicz-Junak ${ }^{1}$, Michal Kalemba ${ }^{1}$,

Zbigniew Puch ${ }^{1}$, Malgorzata Kowalska ${ }^{1}$, Monika Kowal' ${ }^{1}$,

Tomasz Tyszkiewicz'1, Renata Cyplinska', Joanna Polanska²,

Barbara Jarzab ${ }^{1}$

${ }^{1}$ Maria Sklodowska-Curie Memorial Cancer Center and Institute of Oncology, ${ }^{2}$ Silesian University of Technology

Genetic predisposition to papillary thyroid cancer (PTC) is well known. It is family occurring and it is estimated, that approximately $5 \%$ of differentiated thyroid cancers (where PTC is the most frequent) are hereditary. PTC predisposition is expected to be multigenetic with low- to moderate-penetrance genes. Susceptibility genes are poorly known, however, SNPs located on chromosome 9 in FOXE1 locus (rs965513) and on chromosome 14 (rs944289) close to NKX2-1 locus have been confirmed to be associated with PTC in different populations.

The aim of our study was to analyze the association of five SNPs with PTC in Polish population located in FOXE1 locus on chromosome 9 (rs965513, rs1867277, rs1443434) and in other loci: close to NKX2-1 gene on chromosome 14 (rs944289) and in Chek2 gene on chromosome 22 (rs17879961, known as I157T).

The whole material consisted of 2251 DNA samples from PTC patients and 1168 controls. rs 965513 was analyzed in 833 cases and 845 controls, rs 1867277 in 1724 cases and 869 controls, rs1443434 in 1779 cases and 788 controls, rs944289 and rs17879961 in all samples. SNPs were analyzed by allelic discrimination technique (7900HT Fast Real-Time PCR System, Applied Biosystems).

All inestigated SNPs were significantly associated with PTC. OR values were significantly increased: it was $1.43\left(95 \%\right.$ CI: $\left.1.4-2.11, \mathrm{p}=2.5 \times 10^{-7}\right)$ for rs $965513,1.59\left(95 \%\right.$ CI: $\left.1.33-1.89, \mathrm{p}=3.4 \times 10^{-7}\right)$ for $\mathrm{rs} 1867277,1.53(95 \% \mathrm{CI}$ : $\left.1.28-1.84, \mathrm{p}=4.4 \times 10^{-6}\right)$ for rs $1443434,1.52\left(95 \% \mathrm{CI}: 1.26-1.83, \mathrm{p}=9.6 \times 10^{-6}\right)$ for rs944289. For rs 17879961 OR was 1.97 (95\% CI: $\left.1.47-2.63 ; \mathrm{p}=2.7 \times 10^{-6}\right)$.

Conclusion: All analyzed SNPs (rs965513, rs1867277, rs1443434, rs944289, rs17879961) were significantly associated with papillary thyroid cancer in Polish population.

The study was supported Polish National Science Center (grant number: N N402 193740).

Eur Thyroid J 2014;3(suppl 1):73-226 


\section{OP10 Clinical Thyroidology}

\section{OP71 \\ SUBCLINICAL HYPOTHYROIDISM AND THE RISK OF NON-FATAL AND FATAL STROKE: AN INDIVIDUAL PARTICIPANT ANALYSIS}

Layal Chaker ${ }^{1}$, Christine Baumgartner ${ }^{2}$, Wendy J.P. den Elzen ${ }^{3}$, M. Arfan Ikram ${ }^{4}$, Manuel R. Blum², Stephan J.L. Bakker ${ }^{5}$, Abbas Dehghan ${ }^{6}$, Christiane Drechsler ${ }^{7}$, Robert N. Luben ${ }^{8}$, Albert Hofman ${ }^{6}$, Marileen L.P. Portegies ${ }^{6}$, Marco Medici', Giorgio lervasi ${ }^{9}$, Tinh-Hai Collet ${ }^{2}$, Alexandra Brenmer ${ }^{10}$, Christoph Wanner ${ }^{11}$, Massimo lacoviello ${ }^{12}$, Robin P. Dullaart ${ }^{5}$, José A. Sgarbi ${ }^{13}$, Graziano Ceresini14, Rudi G. Westendorp ${ }^{15}$, J. Wouter Jukema ${ }^{16}$, Misa Imaizumi ${ }^{17}$, Jayne A. Franklyn ${ }^{18}$, Douglas C. Bauer ${ }^{19}$, Anne R. Cappola ${ }^{20}$, John P. Walsh ${ }^{21}$, Salman Razvi22, Kay-Tee Khaw ${ }^{8}$, Henry Völzke ${ }^{23}$, Oscar H. Franco ${ }^{6}$, Jacobijn Gussekloo ${ }^{3}$, Nicolas Rodondi ${ }^{2}$, Robin P. Peeters ${ }^{1}$ ${ }^{1}$ Department of Internal Medicine, Thyroid Center, Erasmus Medical Center, Rotterdam, The Netherlands, ${ }^{2}$ Department of Ambulatory Care and Community Medicine, University of Lausanne, Lausanne, Switzerland, ${ }^{3}$ Department of Epidemiology Departments of Public Health and Primary Care; Leiden University Medical Center, Leiden, the Netherlands, ${ }^{4}$ Department of Neurology, Erasmus University Medical Center, Rotterdam, The Netherlands; Department of Radiology, Erasmus University Medical Center, Rotterdam, The Netherlands; Department of Epidemiology, Erasmus University Medical Center, Rotterdam, The Netherlands, ${ }^{5}$ Department of Internal Medicine, University of Groningen, University Medical Center Groningen, The Netherlands, ${ }^{6}$ Department of Epidemiology, Erasmus University Medical Center, Rotterdam, The Netherlands, ${ }^{7}$ Department of Medicine, Division of Nephrology, University Hospital of Würzburg, Germany, ${ }^{8}$ Department of Public Health and Primary Care, University of Cambridge, Cambridge, UK, ${ }^{9}$ National Council Research Institute of Clinical Physiology, Pisa, Italy, ${ }^{10}$ School of Population Health, University of Western Australia, Crawley, ${ }^{11}$ Department of Medicine, Division of Nephrology and Scientific Director, Clinical Trial Unit, University Hospital of Würzburg, Germany, ${ }^{12}$ Cardiology Unit, Department of Emergency and Organ Transplantation, University of Bari, Italy, ${ }^{13}$ Division of Endocrinology, Department of Medicine, Federal University of Sao Paulo, Brazil; Division of Endocrinology, Faculdade de Medicina de Marília, Marília, Brazil, ${ }^{14}$ Facolta DI Medicina E Chirurgia, Department of Clinical and Experimental Medicine, Parma, Italy, ${ }^{15}$ Department of Epidemiologynd Gerontology and Geriatrics, Leiden University Medical Center, Leiden, the Netherlands; Netherlands Consortium for Healthy Ageing, Leiden, ${ }^{16}$ Department of Cardiology, Leiden University Medical Centre, Leiden, The Netherlands, ${ }^{17}$ Department of Clinical Studies, Radiation Effects Research Foundation, Nagasaki, Japan, ${ }^{18} \mathrm{School}$ of Clinical and Experimental Medicine, College of Medical and Dental Sciences, University of Birmingham, Birmingham, UK, ${ }^{19}$ Departments of Medicine, Epidemiology, and Biostatistics and Medicineuniversity of California, San Francisco, Calif., USA, ${ }^{20}$ Division of Endocrinology, Diabetes, and Metabolism, Department of Medicine, School of Medicine, University of Pennsylvania, Pa., USA, ${ }^{21}$ Department of Endocrinology and Diabetes, Sir Charles Gairdner Hospital, Nedlands, Western Australia, ${ }^{22}$ Department of Endocrinology, Gateshead Health Foundation NHS Trust, Gateshead, UK, ${ }^{23}$ Institute for Community Medicine, Clinical-Epidemiological Research, University of Greifswald, Greifswald, Germany

Background: The association between subclinical hypothyroidism and the risk of stroke has been evaluated in only few studies with conflicting results.

Aim: To determine the risk of non-fatal and fatal stroke in adults with subclinical hypothyroidism compared to euthyroid subjects, and explore possible subgroups at risk.

Data Sources: Published studies were identified through a systematic literature search without language restriction, in electronic databases from inception to November 2013, and reviewing reference lists. Additional studies were sought within the Thyroid Studies Collaboration (TSC).
Study Selection and Data Extraction: Studies were eligible if they provided baseline thyroid function and longitudinal stroke outcome data. Individual Participant Data (IPD) were extracted and an IPD analysis was conducted. Euthyroidism was defined as TSH level between 0.45 and 4.49 $\mathrm{mIU} / \mathrm{L}$. Subclinical hypothyroidism was defined as TSH level between 4.5 and $20 \mathrm{mIU} / \mathrm{L}$ with normal (free) thyroxin level.

Results: We included 47901 adults with a follow-up of 489616 personyears from 18 cohorts, representing participants from Europe, United States, Australia, Brazil and Japan. 3490 participants had subclinical hypothyroidism. During follow-up 2321 stroke events, of which 918 fatal, and 10721 overall deaths occurred. In overall age- and sex-adjusted analyses, subclinical hypothyroidism was not significantly associated with an increased risk for stroke events, fatal stroke or total mortality. There was an increased risk of stroke events with a pooled hazard ratio (HR) of 3.32 (95\% Confidence Interval [CI]; $1.25-8.80)$ for those with subclinical hypothyroidism in the age group 18 to 50 years. Also, we found an increased risk of fatal stroke in the age groups 18 to 50 and 50 to 65 with HRs of 4.22 (CI; $1.08-16.55)$ and 2.86 (CI; $1.31-6.26)$ respectively. Conclusions: Subclinical hypothyroidism is associated with a higher risk of non-fatal and fatal stroke events in adults younger than 65 years.

\section{OP72 \\ MILD HYPERTHYROIDISM LEADS TO INCREASED CONNECTIVITY IN TEMPORAL LOBE STRUCTURES: A RESTING STATE FMRI STUDY}

Anna-Luise Dirk', Martin Göttlich², Marcus Heldmann², Anna Goebel2, Georg Brabant ${ }^{1}$, Thomas F. Muente ${ }^{2}$

${ }^{1}$ Experimental and Clinical Endocrinology, University of Luebeck,

${ }^{2}$ Department of Neurology, University of Luebeck, Germany

Objective: The effect of experimentally induced hyperthyroidism on the human brain was tested using exogenous levothyroxine. Changes in grey matter structure and in functional imaging were matched with a psychological assessment of the volunteers under baseline conditions and during hyperthyroidism.

Materials and Methods: 20 healthy, male, right-handed subjects (age range 21 to 49 years, median 30 years) in good general health without mood or cognitive disorders and normal thyroid state gave their informed consent to participate. High resolution 3D T1-weighted MRI scans were acquired by 3T MRI from the subjects before and after taking $250 \mu \mathrm{g} \mathrm{L}$-thyroxin for a period of 8 weeks. Thyroid function was measured by standard methods and subjectively assessed by questionnaire. During scanning a working memory task (n-back task) was conducted. High-resolution 3D T1-weighted images were acquired by 3 T MRI. Voxel based morphometry (VBM) analysis was performed using Statistical Parametric Mapping 8 (SPM8).

Results: Despite a lack of significant subjective changes assessed by questionnaire all subjects developed biochemical hyperthyroidism after 8 weeks with a 1,7 fold increase in baseline fT3, a 2,1 fold increase in fT4 levels and suppressed TSH levels. In the hyperthyroid condition the subjects showed slower reaction times, but a higher accuracy in 0-back memory tasks. Grey matter volumes were higher in right posteriocerebellum (lobule VI) and lower in the bilateral visual cortex and anteriocerebellum (lobules I-IV) compared to the euthyroid condition. The cerebellar regions are associated with learning and working memory tasks in the literature.

Conclusion: Our study provides evidence that short periods of hyperthyroidism induce distinct alterations in brain structure and function of regions associated with working memory. (Supported by DFG SPP 1629) 


\section{OP73 \\ EUTHYROID AND HYPERTHYROID HAVE INCREASED RISK OF CEREBROVASCULAR EVENTS AFTER 131IODINE TREATMENT - A COHORT STUDY OF MORE THAN 5000 TREATED PATIENTS}

Jeppe Lerche la Cour ${ }^{1}$, Lars Thorbjoern Jensen ${ }^{2}$, BIrte Nygaard ${ }^{3}$ ${ }^{1}$ Section of Clinical Physiology, Department of Diagnostics, Glostrup Hospital, University of Copenhagen, ${ }^{2}$ Department of Clinical Physiology and Nuclear Medicine, Herlev Hospital, University of Copenhagen; Section of Clinical Physiology, Department of Diagnostics, Glostrup Hospital, University of Copenhagen, ${ }^{3}$ Department of Endocrinology, Herlev Hospital

Background/Aim: External radiotherapy of cancer increases the risk of arteriosclerosis and cerebrovascular events. The lower limit is about two Gy. Radioiodine therapy (RAI) for benign thyroid diseases exposes the carotid arteries to an average of 2-25 Gy pr. treatment. RAI treated hyperthyroid patients have an increased cerebrovascular morbidity. Therefore we tested whether some of the increased morbidity arises from radiation induced atherosclerosis.

Methods: A cohort of 5,027 RAI treated patients (4,001 hyperthyroid, 1,026 euthyroid (goiter), was age and gender matched 1:4 with controls through National Danish Patient Register. Data on occurrence of cerebrovascular events (stroke, amaurosis fugax and TIA) and death where collected. The cohort was followed from date of treatment until cerebrovascular event, death or 20 years follow up. A Cox regression model was applied to compare controls with both hyperthyroid and euthyroid, thereby testing whether thyroid disease and/or radiation contribute to increased cerebrovascular events. All analyses where adjusted for age and gender, and later for Charlson's comorbidity score (CCS) and atrial fibrillation (AF) at time of treatment.

Results: Mean follow up time was 11.3 years (sd 6.1), mean age 61 (sd 14.2), $14.3 \%$ were men. Mean administrated amount of ${ }^{131}$ I were $498 \mathrm{MBq}$ (sd 312). Total number of events was 4,159 .

Compared with controls all RAI-treated had increased risk of cerebrovascular events HR 1.32; (CI 1.22-1.42). The risk was increased both among hyperthyroid (HR 1.33; CI 1.23-1.44) and euthyroid (HR 1.26; CI 1.08-1.47). Adding CCS and AF did not change the overall picture (hyperthyroid HR 1.28; CI 1.18-1.38; euthyroid HR 1.24 CI 1.06-1.44).

Conclusion: We found an increased risk of cerebrovascular events among both hyperthyroid (HR 1.33) and euthyroid (HR 1.26) treated with RAI for benign thyroid disorders. This points to the possibility that RAI can induce clinically relevant atherosclerosis leading to cerebrovascular events.

\section{$0 P 74$ \\ LOW SERUM THYROTROPIN LEVEL AND DURATION OF SUPPRESSION AS A PREDICTOR OF MAJOR OSTEOPOROTIC FRACTURES - THE OPENTHYRO REGISTER COHORT}

Anne Sofie Laulund ${ }^{1}$, Bo Abrahamsen ${ }^{2}$, Henrik Løvendahl Jørgensen ${ }^{3}$, Mads $\mathrm{Nybo}^{4}$, Thomas Heiberg Brix ${ }^{1}$, Laszlo Hegedüs ${ }^{1}$

${ }^{1}$ Department of Endocrinology and Metabolism, Odense University Hospital, ${ }^{2}$ Research Centre for Aging and Osteoporosis, Odense University Hospital, ${ }^{3}$ Department of Clinical Biochemistry, Bispebjerg Hospital, ${ }^{4}$ Department of Clinical Biochemistry and Pharmacology, Odense University Hospital

Background: The relationship between thyrotoxicosis and osteoporotic fractures remains controversial, particularly in men.

Methods: Register-based cohort study including all patients with a serum thyrotropin (TSH) measurement in the region of Funen 1996-2010. All TSH determinations were done in the same laboratory, which served all hospitals and GP practices in the region. Persons with raised TSH or a history of thy$\mathrm{roid} /$ pituitary disease or use of thyroid medications were excluded.

Results: The study population consisted of $222,138(96 \%)$ persons with normal and 9,217 (4\%) with low TSH $(<0.3 \mathrm{mIU} / 1)$. A single low TSH at baseline was associated with increased risk of hip fractures (adj. HR 1.16, 95\% CI 1.07-1.26, P < 0.001) but not major osteoporotic fractures (MOF, adj. HR $1.06,95 \%$ CI $0.99-1.12, \mathrm{p}=0.058$ ) over a median follow-up of 7.5 years.
When men were analyzed separately, results did not reach statistical significance. We found a significant association between duration of thyrotoxicosis and fracture. For each six months in which the mean TSH value was decreased $(<0.3 \mathrm{mIU} / \mathrm{L})$, hip fracture risk increased by a factor 1.07 (adj. HR, 95\% CI $1.04-1.10, \mathrm{P}<0.001$ ) and MOF by 1.05 (adj. HR, 95\% CI $1.03-1.07, \mathrm{P}<$ 0.001 ). Overt thyrotoxicosis was associated with an increased risk of hip fractures but not MOF. In euthyroid patients, the risk of fractures increased significantly with each SD unit of TSH decrease: Hip fracture (HR 1.45, 95\% CI 1.22-1.71, P < 0.001) and MOF (HR 1.32, 95\% CI 1.19-1.46, P<0.001).

Conclusion: In a population-based cohort, a single, first measurement of decreased TSH in a patient without known thyroid disease was associated with an increased long-term risk of hip fracture, which remained significant in women but not in men after adjusting for confounders. Moreover, the risk of both hip fracture and MOF increased exponentially by the length of time during which TSH had remained low.

\section{OP75 \\ RANDOMISED CONTROLLED PRE-CLINICAL TRIAL OF TRI-IODOTHYRONINE (T3) AND HYDROCORTISONE REPLACEMENT IN SEPTIC SHOCK \\ Matthew Maiden ${ }^{1}$, Marianne Chapman ${ }^{1}$, Tim Kucher ${ }^{2}$, David Torpy ${ }^{1}$, lain Clarke $^{3}$, Guy Ludbrook ${ }^{1}$ \\ ${ }^{1}$ Royal Adelaide Hospital; University of Adelaide, ${ }^{2}$ Sahmri, ${ }^{3}$ Monash University}

Background: Plasma T3 levels fall during septic shock in proportion to the severity of disease. It is unclear if this is an adaptive or pathological endocrine response. Controversy exists as to whether T3 replacement (with and without hydrocortisone) during septic shock is beneficial or harmful.

Methods: Following animal ethics approval, 32 ewes were anaesthetised for insertion of carotid arterial line, pulmonary artery catheter, tracheostomy and insertion of cannulae in the coronary sinus, renal vein, hepatic vein, and femoral vein. Animals were studied individually and received protocol-guided management of sedation, ventilation, fluids and noradrenaline. Sepsis was induced by intravenous E.coli. After 2 hours of sepsis, sheep received continuous infusion of (i) T3+placebo, (ii) hydrocortisone (HC)+placebo, (iii) $\mathrm{T} 3+\mathrm{HC}$, or $(i v)$ placebo+placebo. Primary outcome was 24-hour total dose of noradrenaline (NorA). Secondary outcomes included haemodynamics, oxygen extraction and organ function.

Results: Study group baseline demographics were evenly matched. All animals developed a hyperdynamic septic response. Plasma T3 levels fell in placebo animals $(\mathrm{P}<0.01)$ while animals that received $\mathrm{T} 3 \mathrm{had}$ increased plasma hormone levels ( $\mathrm{P}<0.001)$. The 24-hr NorA dose was no different between any of the study groups $(\mathrm{mg} / \mathrm{kg}$, T3 group, $501 \pm 131$; T3+HC group, $466 \pm 175$; HC group, $167 \pm 101$; placebo group, $208 \pm 160$; $\mathrm{p}=0.20)$. Two animals in the placebo group died before 24-hours $(p=0.09)$. There was no treatment effect on any haemodynamic variable, temperature, $\mathrm{pH}$, lactate or organ-specific oxygen extraction.

Conclusion: In a 24-hour model of septic shock, T3 infusion (with or without HC) did not reduce NorA requirement nor alter any other measured physiological variable.

\section{OP76 \\ BIRTH DEFECTS AFTER THE USE OF THE ANTITHYROID DRUGS (ATDS) METHIMAZOLE/CARBIMAZOLE (MMI/CMZ) AND PROPYLTHIOURACIL (PTU) IN PREGNANCY: HIGH RISK IS CONFINED TO A BRIEF TIME WINDOW OF PREGNANCY}

Peter Laurberg ${ }^{1}$, Stine Linding Andersen ${ }^{1}$

${ }^{1}$ Aalborg University Hospital

ATDs may be teratogenic with excess cases of birth defects after MMI/ CMZ (3\% extra) and PTU (2\% extra) use in pregnancy. Types of defects differ after $\mathrm{MMI} / \mathrm{CMZ}$ and PTU.

Objective: To study the association between the time period of ATD exposure in pregnancy and birth defects using two approaches. 
Methods: A Danish nationwide register-based cohort study of 817,093 children identified 149 children born to mothers who had shifted from MMI/ CMZ to PTU in pregnancy weeks 1-10. 13/149 children had defects. Types were MMI associated $(\mathrm{n}=5)($ upper airway strictures and VSD)/PTU associated $(\mathrm{n}=2)$ (facial sinus and cyst)/MMI OR PTU associated $(\mathrm{n}=1)$ (hydronephrosis)/types NOT MMI and PTU associated $(\mathrm{n}=5)$. B: A systematic literature search identified publications on birth defects after the use of ATD $(\mathrm{n}=92)$, and we identified additional cases $(\mathrm{n}=24)$ with maternal shift in ATD therapy. We analyzed correlation between ATD exposure period and the presence of birth defect.

Results: Study A: In the five pregnancies leading to MMI associated birth defects, mothers had been shifted from MMI to PTU significantly later (mean 20 days) than in the 136 pregnancies with no birth defects $(p=0.016)$, and 23 days later than pregnancies with NOT MMI and PTU associated birth defects $(\mathrm{p}=0.04)$. All children with MMI and PTU associated defects had been exposed after week five of pregnancy, which is compatible with the main teratogenic period of pregnancy being the 'organogenic period' weeks 6-10. Study B: Among the 24 cases from literature, 22 (omphalocele, mesenteric duct, gastroschisis, esophageus atresia, choanal atresia, aplasia cutis, dysmorphia, kidney agenesis) had been exposed to $\mathrm{MMI} / \mathrm{CMZ}$ during gestational weeks 6-10.

Conclusion: Our study suggests that the risk of birth defects can be minimized if pregnant women terminate ATD intake before gestational week six. We propose how to minimize the risk of birth defects in fertile women treated with ATDs.

\section{OP77 \\ EVALUATION THE ASSOCIATION OF POLYMORPHISMS IN DIO1 AND DIO2 GENES WITH MENTAL, BIOCHEMICAL PARAMETERS AND QUALITY OF LIFE IN WOMEN WITH PRIMARY HYPOTHYROIDISM}

\section{Valentin Fadeyev ${ }^{1}$, Meruert Madiyarova ${ }^{1}$, Tatyana Morgunova ${ }^{1}$}

${ }^{1}$ I.M.Sechenov First Moscow State Medical University, Department of Endocrinology

Objective: To evaluate the association of polymorphisms in DIOI and $\mathrm{DIO} 2$ genes with mental, biochemical parameters, QoL in patients with compensated autoimmune $(\mathrm{AIH})$ and postoperative $(\mathrm{PH})$ hypothyroidism.

Methods: 115 young women with hypothyroidism on L-T4 (64 with AIH, 51 with PH). 40 women with persistent symptoms (score $\geq 10$, TSQ) or dyslipidemia were switched to L-T4+T3 (L-T4 25 mcg were replaced with $12.5 \mathrm{~T} 3$ ). Evaluation at baseline and after 3 months of L-T4+T3 included: QoL, HADS, TSQ, cognitive functions, TSH; polymorphism in DIO1 (rs2235544), DIO2 (rs225014) genes (using PCR-RT).

Results: TSH levels didn't differ between carriers of $A A, C A, C C$ genotypes in $D I O 1$ and TT, $C T, C C$ in $D I O 2$ genes. The fT3/fT4 ratio was lower in patients with $\mathrm{PH}$ and $A A$ genotype compared to $C C$ genotype in $D I O 1$ ( $p=$ 0.04); fT4, fT3, TC, LDL levels didn't differ between the genotype carriers in DIO1. When patients were switched to L-T4+T3 the TC, LDL levels decreased, regardless of genotype in DIO1 $(P<0.02)$. There were no differences in QoL, HADS, TSQ, cognitive functions between carriers of $C C, C T$, $T T$ in DIO2. Patients were grouped on the basis of genotype in DIO1: Group I- $C C$; Group II- $C A$, Group III- $A A$ genotype with any genotype (TT, $C T, C C$ ) in $D I O 2$ gene. In patients with PH the fT3/fT4 ratio was lower in Group III $(C C / x)$ vs. Group I $(A A / x)(p=0.04)$. Gene-gene interactions were analyzed with MDR. $A A / C T, C A / C T, C C / T T$ genotypes in $D I O 1$ and $D I O 2$ genes were associated with higher risk of dislipidemia (TC: $\chi^{2}=7.853, p=0.005$; LDL $\chi^{2}=3.627, p=0.05$ )

Conclusions: In patients with hypothyroidism $C C$ genotype in $D I O 1$ gene regardless of genotype in $\mathrm{DIO} 2$ was associated with lower fT3/fT4 ratio vs. $A A$; and wasn't accompanied by dislipidemia. Improvement in lipid profile was shown in patients switched on L-T4+T3 regardless of genotype in DIOI. The polymorphism in DIO2 wasn't associated with psychoemotional features, cognitive functions, QoL.

\section{OP78 \\ LONG-TERM SELENIUM SUPPLEMENTATION AND THYROTROPIN IN DANISH SENIORS. RESULTS FROM A RANDOMISED CONTROLLED TRIAL}

Kristian Winther $^{1}$, Frederik Cold ${ }^{2}$, Steen Joop Bonnema ${ }^{1}$, Søren Cold ${ }^{2}$, Birgit Debrabant ${ }^{3}$, Mads $\mathrm{Nybo}^{4}$, Laszlo Hegedüs ${ }^{1}$

${ }^{1}$ Department of Endocrinology and Metabolism, Odense University Hospital, Denmark, ${ }^{2}$ Department of Oncology, Odense University Hospital, Denmark, ${ }^{3}$ Department of Epidemiology, Biostatistics and Biodemography, University of Southern Denmark, Odense, Denmark, ${ }^{4}$ Department of Clinical Biochemistry, Odense University Hospital, Denmark

Context: Selenium is at the active centre of enzymes that catalyse the deiodination of thyroxine to triiodothyronine. Selenium intake in Denmark is acceptable but low, and supplementation may impact thyroid hormone conversion and thus thyroid function.

Objectives: The primary objective is to investigate the effect of longterm selenium supplementation in different doses on serum thyrotropin (TSH) concentrations.

Design and Setting: The Danish PREvention of Cancer by Intervention with SElenium trial (DK-PRECISE) was a non-stratified, randomised, doubleblinded, placebo-controlled, one-centre, multi-arm trial with four groups (allocation ratio $1: 1: 1: 1$ )

Participants and Intervention: 491 males and females aged 60-74 years were randomised to $100 \mu \mathrm{g}(\mathrm{N}=124), 200 \mu \mathrm{g}(\mathrm{N}=122)$ or $300 \mu \mathrm{g}(\mathrm{N}=$ 119) selenium-enriched yeast or matching yeast-based placebo tablets $(\mathrm{N}=$ 126) daily for five years. 361 participants, equally distributed across treatment groups, completed the five-year intervention period.

Main Outcome Measure: Serum TSH and plasma selenium concentrations were measured in blood samples collected at baseline, and after six months and five years intervention.

Results: Plasma selenium concentrations increased significantly and dose dependently in treatment groups receiving selenium $(P<0.001)$. In the intention-to-treat analysis, changes in serum TSH, did not differ significantly between any treatment groups after six months or five years intervention.

Conclusions: In this elderly Danish population we found no indication that selenium supplementation, short-term or long-term, impacts thyrotropin concentrations.

\section{OP11 Young Investigator-Basic}

\author{
OP79 \\ BIOSYNTHESIS OF 3-IODOTHYRONAMINE \\ FROM THYROXINE IN INTESTINAL TISSUE \\ Carolin Höfig ${ }^{1}$, Tilo Wuensch ${ }^{2}$, Ina Lehmphuß ${ }^{3}$ Hannelore Danie/4, \\ Ulrich Schweizer ${ }^{5}$, Jens Mittag ${ }^{6}$, Josef Köhrle ${ }^{3}$ \\ ${ }^{1}$ Karolinska Institutet, Department of Cell \& Molecular Biology, \\ Sweden; Institut Für Experimentelle Endokrinologie, Charité - \\ Universitätsmedizin Berlin, Germany, ${ }^{2}$ Karolinska Institutet, Department \\ of Cell \& Molecular Biology, Sweden; Ziel Research Center of Nutrition \\ and Food Science, Abteilung Biochemie, Technische Universität \\ München, Germany, ${ }^{3}$ Institut Für Experimentelle Endokrinologie, \\ Charité - Universitätsmedizin Berlin, Germany, ${ }^{4}$ Ziel Research Center \\ of Nutrition and Food Science, Abteilung Biochemie, Technische \\ Universität München, Germany, ${ }^{5}$ Institut Für Biochemie und \\ Molekularbiologie, Rheinische Friedrich-Wilhelms-Universität Bonn, \\ Germany, ${ }^{6}$ Karolinska Institutet, Department of Cell \& Molecular \\ Biology, Sweden
}

Objectives: 3 -Iodothyronamine $\left(3-\mathrm{T}_{1} \mathrm{AM}\right)$ is an endogenous signaling molecule, which at pharmacological doses induces a rapid drop in body temperature and heart rate. Although the biosynthesis of $3-\mathrm{T}_{1} \mathrm{AM}$ from thyroid hormone precursors appears likely, the sequence of reactions and site(s) of biosynthesis are still controversial: our previous study in thyroxine $\left(\mathrm{T}_{4}\right)$ - 
substituted thyroid cancer patients lacking functional thyroid tissue provided evidence for extrathyroidal 3-T $\mathrm{AM}$ production, while other studies using labeled thyroid hormone precursors in mice suggested intrathyroidal formation. However, as patients received $\mathrm{T}_{4}$ orally whereas mice were injected i.p., we hypothesized that intestinal passage might be an important step in $3-\mathrm{T}_{1} \mathrm{AM}$ biosynthesis.

Methods: We studied uptake and metabolism of $\mathrm{T}_{4}$ as a potential precursor of 3-T $\mathrm{T}_{1} \mathrm{AM}$ biosynthesis using the ex vivo everted mouse gut sac model and liquid chromatography - tandem mass spectrometry for hormone analysis. To dissect the sequence of reactions involved in 3- $\mathrm{T}_{1} \mathrm{AM}$ biosynthesis, we used real-time PCR of mouse intestinal tissues and an in vitro decarboxylation assay with purified human ornithine decarboxylase (ODC) enzyme.

Results: Here, we demonstrate that mouse intestinal tissue produces 3- $\mathrm{T}_{1} \mathrm{AM}$ from $\mathrm{T}_{4}$ via several deiodination and decarboxylation steps, with $3,5-\mathrm{T}_{2}$ and 3,5- $\mathrm{T}_{2} \mathrm{AM}$ as likely intermediates. In vitro experiments with ODC revealed that this enzyme decarboxylates $3,5-\mathrm{T}_{2}$ as well as $\mathrm{T}_{4}$ to the respective thyronamines (TAM). Subsequent gene expression analysis showed that all three deiodinases as well as the required ODC are expressed in mouse intestinal tissue.

Conclusions: Our data provide the first evidence that intestinal $3-\mathrm{T}_{1} \mathrm{AM}$ biosynthesis from $T_{4}$ is possible. This observation could explain elevated 3- $\mathrm{T}_{1} \mathrm{AM}$ serum levels in patients substituted orally with $\mathrm{T}_{4}$ compared to healthy controls, and identifies ODC as the first enzyme catalyzing decarboxylation of thyroid hormones to TAM. These findings open the path to further investigations of TAM metabolism on the molecular and cellular level.

\section{OP80 \\ REGULATION OF EMBRYONIC THYROID GROWTH BY MESENCHYMAL FGF10 COINCIDING WITH FUNCTIONAL DIFFERENTIATION}

Shawn Liang ${ }^{1}$, Henrik Fagman ${ }^{2}$, Mikael Nilsson ${ }^{1}$

${ }^{1}$ Sahlgrenska Cancer Center, Sahlgrenska Academy, University of Gothenburg, Gothenburg, Sweden, ${ }^{2}$ Department of Clinical Pathology

and Genetics, Sahlgrenska University Hospital, Gothenburg, Sweden

Fibroblast growth factors (FGFs) derived from embryonic mesenchyme orchestrate the development of many organs. Apart from FGF8 that contributes to the generation of thyroid progenitors in the anterior endoderm, the involvement of FGFs in thyroid organogenesis is largely unknown. A previous study reported agenesis of the thyroid gland in FgflO null mouse. Here we reinvestigate this mutant phenotype and provide evidence that FGF10 is essential for parenchymal growth at late stages of organogenesis but has no role in early thyroid development.

Wild type (WT) and Fgfl0 null mouse embryos at different developmental stages (E9.5-18.5) were analysed. Thyroid precursor cells were identified by immunostaining for Nkx2.1, Pax8, thyroglobulin and calcitonin. Microvessels were identified by CD31 staining. The embryonic thyroid gland was 3D reconstructed based on serial sections. Cell proliferation was monitored by quantification of Ki-67 positive nuclei. In addition, expression of $N k x 2.1, F g f 10$ and FGFR2b in WT embryos was analysed by in situ hybridisation.

The midline thyroid bud appeared in the pharyngeal floor of $F g f 10$ deficient embryos. The size of the growing and migrating thyroid primordium did not differ between mutant and WT embryos and a bi-lobed gland in the correct position was also established. Subsequent thyroid growth was markedly retarded leading to severely hypoplastic gland with reduction of parenchyme volume by $90 \%$ in E18.5 mutant embryos. However, thyroid vascularisation, folliculogenesis and functional differentiation were not affected. Fgf10 transcripts were detected in mesenchyme surrounding the thyroid at both E15.5 and E18.5. In addition, Fgfr $2 b$ encoding the receptor to FGF10 was ubiquitously expressed in the follicular epithelium at these developmental stages.

We identify FGF 10 produced by adjacent mesenchyme as a fundamentally important regulator of embryonic thyroid growth at late glandular development when precursors differentiate to follicular cells. Fgflo deficiency leads to hypoplasia of the orthotopic gland but no loss of thyroid function.

\section{OP81 \\ IDENTIFICATION OF MIR-146B-3P AS A KEY PLAYER IN THYROID CANCER DEDIFFERENTIATION}

\section{León Wert-Lamas ${ }^{0}$, Garcilaso Riesco ${ }^{1}$, Pilar Santisteban ${ }^{2}$}

${ }^{1}$ lib Alberto Sols. Csic-Uam, ${ }^{2}$ Instituto de Investigaciones Biomédicas (Csic-Uam)

Next-generation sequencing expression analysis of papillary thyroid cancer performed in our laboratory, revealed the existence of a set of abundant miRNAs differentially expressed in this pathology. MiR-146b-3p was unveiled as one of the most upregulated microRNAs (miRs) in this neoplasm, surpassing the minimum threshold amount to repress target mRNAs. Furthermore, our previous results showed that this miR binds to the 3'UTR of NIS, inhibiting its expression and consequently its activity. As NIS is a gene regulated by thyroid transcription factors, the aim of this work was to study the effect of miR-146b-3p on the thyroid cancer dedifferentiation process, focusing on the most relevant event for the clinical outcome: the loss of NIS function that leads to radioiodide therapy refractority.

By computational predictions, we identified PAX8 and FOXE1 as potential targets of miR-146b-3p according to miRanda algorithm. We validated these predictions through functional studies in PCCl3, NThy-ori and, hNISMDCK cells. Mir-146b expression vector was transfected and reporter constructs containing $3^{\circ} \mathrm{UTR}$, miR specific binding sites, and NIS promoter were generated. The levels of mRNA and protein were determined by RT-qPCR and Western Blot; direct mRNA destabilizing effect was measured in luciferase assays.

Overexpression of miR-146b-3p resulted in NIS, PAX8, and FOXE1 mRNA and protein silencing. Furthermore, we observed that as NIS, the PAX8 inhibition is specifically exerted through a direct binding to their $3^{\text {'UTRs. }}$ Also, preliminary data suggest that FoxE1 and PAX8 silencing induced by miR-146b-3p overexpression was sufficient to repress NIS promoter activation. The general outcome was a significant decrease in iodide uptake in miR$146 \mathrm{~b}-3 \mathrm{p}$ overexpressing cells.

These data suggest that tumor overexpression of miR-146b-3p widely contributes to thyroid dedifferentiation and especially to NIS loss of function through at least two complementary mechanisms: (i) direct postranscriptional level, and (ii) through PAX8 and FOXE1 silencing.

\section{OP82 \\ IN VIVO DISSECTION OF THE ROLE OF GLIS3 TRANSCRIPTION FACTOR IN THE HYPOTHALAMIC-PITUITARY-THYROID AXIS}

Federica Marelli ${ }^{1}$, Franco Cotelli ${ }^{2}$, Luca Persani ${ }^{3}$

${ }^{1}$ Irccs Istituto Auxologico Italiano, ${ }^{2}$ University of Milan, ${ }^{3}$ Irccs Istituto Auxologico Italiano; University of Milan, Italy

Introduction: GLIS3 is a nuclear zinc-finger protein that could function as an activator or repressor of transcription. Mutations in the GLIS3 gene cause Neonatal Diabetes Mellitus associated with Congenital Hypothyroidism and polycystic kidneys. Recently, a GWAS-study associated GLIS3 with circulating TSH levels but the action of GLIS3 in the hypothalamic-pituitarythyroid (HPT) axis is presently unknown. In this study, we took advantage of the zebrafish model to clarify the role of GLIS3 in the onset and regulation of HPT-axis.

Methods and Results: The spatio-temporal expression of GLIS3 during development was determined by RQ-PCR and in situ hybridization in zebrafish embryos from zygote through larvae stage. The GLIS3 transcript is detectable starting from the prim5-stage with a specific localization in the pharyngeal endoderm and pronephric-ducts. From the hatching-period onward, GLIS3 was more abundant in liver, endocrine pancreas, kidneys and particular brain regions, suggesting a possible involvement in the regulation of HPT-axis. To understand the role of GLIS3, we generated transient knock-down embryos by the microinjection of specific antisense-morpholinos. The GLIS3-morphants displayed severe hypothyroid features: thyroid volume and T4-content were decreased by $50 \%$, while TSHb was increased 5 -fold compared to controls by day 2 . To test the sensitivity of GLIS3-morfants to T4 administration, we treated the injected-embryos with growing doses of L-T4 and compared them with a similar hypothyroid condition of PTU-treated embryos. Thirty nM L-T4 was sufficient to restore normal TSH $\beta$ levels in PTU-treated embryos, 
whereas a significant decrease of TSH $\beta$ expression could be seen in GLIS3morphants only after treatment with $100 \mathrm{nM}$ of L-T4.

Conclusions: The obtained data represent the first in vivo approach to explore the key role of GLIS3 in the regulation of HPT-function. The experiments conducted in GLIS3-morphants indicate a likely role for this transcription factor in thyroid morphogenesis and in the regulation of central thyroid hormone sensitivity.

\section{OP83}

\section{THE EFFECT OF DEIODINASE TYPE 3 KNOCKDOWN ON ZEBRAFISH EYE DEVELOPMENT}

Anne M. Houbrechts ${ }^{1}$, Enise Bagci ${ }^{2}$, Marjolein Heijlen ${ }^{1}$, Dries Knapen ${ }^{3}$, Veerle M. Darras ${ }^{1}$

${ }^{1}$ Laboratory of Comparative Endocrinology, Ku Leuven, Belgium,

${ }^{2}$ Systemic Physiological \& Ecotoxicological Research, Ua, Belgium,

${ }^{3}$ Systemic Physiological \& Ecotoxicological Research, Ua, Belgium;

Veterinary Physiology and Biochemistry, Ua, Belgium

Objective: Thyroid hormones (THs) are of great importance during the development of vertebrates and appropriate levels have to be present at the right place at the right time. One of the key regulators in this process is the TH-inactivating enzyme deiodinase type 3 (D3), which protects embryonic tissues from an excess of active $\mathrm{T}_{3}$. We investigated the effect of $\mathrm{D} 3$ deficiency at the level of the eye during embryonic and early larval development of zebrafish.

Methods: Knockdown was accomplished by morpholino (MO) injection, into the yolk of 2-cell stage embryos, blocking the translation of the D3b enzyme. Animals were collected at 1, 3 and 4 days post fertilisation (dpf). The eye phenotype was studied, using morphometric and micro-array analysis and immunohistochemical staining.

Results: D3bMO almost completely abolished D3 enzyme activity as tested at $3 \mathrm{dpf}$. Morphometric analysis of the eye at $1 \mathrm{dpf}$ showed a smaller eye size and aberrant layer formation in knockdown embryos. To further elucidate these abnormalities, additional experiments were performed. Behaviour tracking at $3 \mathrm{dpf}$ demonstrated an impaired response to light in knockdown embryos. Studies of the transcriptome in the head at $3 \mathrm{dpf}$ revealed a striking impact of D3b knockdown on the expression of several genes involved in eye development, phototransduction and visual perception. We also performed immunohistochemical staining with a zpr-1 antibody. This antibody specifically recognizes the gene arrestin $3 a$, which was strongly downregulated in the micro-array, and codes for red/green double cones in the retina. Both at 3 and $4 \mathrm{dpf}$ there was a significant reduction in the number of cones per retinal surface in knockdown compared to control animals.

Conclusion: D3b knockdown strongly impedes normal eye development in zebrafish through altered gene expression, resulting in morphological and functional deficiencies, emphasizing the importance of correct TH regulation in vertebrate development.

\section{OP84}

\section{CHARACTERIZATION OF CHICKEN THYROID HORMONE TRANSPORTERS}

Nele Bourgeois ${ }^{1}$, Stijn L.J. Van Herck', Joke Delbaere',

Chantal Zevenbergen ${ }^{2}$, Simone Kersseboom², Veerle M. Darras ${ }^{1}$, Theo J. Visser ${ }^{2}$

${ }^{1}$ Laboratory of Comparative Endocrinology, Ku Leuven, Belgium,

${ }^{2}$ Department of Internal Medicine, Erasmus University Medical Center, Rotterdam, The Netherlands

Objectives: Thyroid hormones (THs) are important for vertebrate brain development. To better understand the multiple roles/functions of THs in early development, we study TH transport and metabolism in chicken embryonic brain. Until now, the only TH transporter characterized in chicken was the organic anion transporting polypeptide (OATP) 1C1 (Nakao et al., 2006). We therefore tried to identify and functionally characterize the other TH transporters present in chicken.

Methods: Based on mammalian sequence information, we cloned the chicken TH transporters and performed in vitro transport studies. In addition their expression pattern was studied by in situ hybridisation (ISH).
Results: We successfully cloned full length coding sequences for chicken monocarboxylate transporters (MCT) 8 and 10 and L-type amino acid transporters (LAT) 1. The LAT2 gene, however, seems absent from the avian genome. Chicken OATP1C1, MCT8, MCT10 and LAT1 share 72, 67, 76 and $88 \%$ amino acid homology with the human orthologs, respectively. Chicken OATP1C1, MCT8 and MCT10 also have similar substrate specificities as human transporters. Our in vitro studies showed $\mathrm{T}_{4}$ uptake by OATP1C1, $\mathrm{T}_{3}$ and $\mathrm{T}_{4}$ uptake by MCT8 and $\mathrm{T}_{3}>>\mathrm{T}_{4}$ uptake by MCT10, although the chicken MCT10 showed a higher export rate than the human ortholog. LAT1 was coexpressed with the human 4F2 heavy chain, and showed only $\mathrm{T}_{2}$ uptake (no $\mathrm{T}_{3}$ or $\mathrm{T}_{4}$ ). ISH revealed high and widespread MCT8 expression in the embryonic brain while OATP1C1 was highly expressed in the choroid plexus. MCT10 expression was limited in brain, but was found in the developing liver.

Conclusions: The chicken OATP1C1, MCT8 and MCT10 are efficient TH transporters and based on their expression pattern, MCT8 and OATP1C1 seem vital for the regulation of intracellular TH levels in developing brain.

\section{OP85 \\ IMPACT OF THYROID HORMONES ON ER $\alpha$ - DEPENDENT ACTIONS IN THE HYPOTHALAMUS}

Larissa Faustino ${ }^{1}$, Khatuna Gagnidze ${ }^{2}$, Tania M. Ortiga-Carvalho ${ }^{3}$,

Donald Pfaff ${ }^{2}$

${ }^{1}$ Universidade Federal Do Rio de Janeiro; The Rockefeller University,

${ }^{2}$ The Rockefeller University, ${ }^{3}$ Universidade Federal Do Rio de Janeiro

Interactions between thyroid hormones (TH) and estradiol (E2) on ventromedial hypothalamus (VMH) influence reproduction and sexual behavior. Here, we investigated the impact of TH and E2 interactions on early molecular events, such as histone modifications and receptors recruitment, associated with progesterone receptor $(\mathrm{Pgr})$ and oxytocin $(\mathrm{Oxtr})$ gene regulation within VMH. Ovariectomized mice were induced to hypothyroidism (diet containing $0.15 \%$ PTU for 2 weeks) or hyperthyroidism by a injection of T3 $(50 \mathrm{mg} / 100$ g BW). All groups were further divided into 2 different groups, one injected with $20 \mathrm{mg}$ of $\mathrm{E} 2$ and the other one with vehicle. First, we observed that hyperthyroidism abolished E2-induction of Pgr and Oxtr mRNA 6 hours after hormonal treatment, indicating that high levels of $\mathrm{T} 3$ were able to interfere with E2 signaling. Hyperthyroidism was also able to suppress the up-regulation of the co-factors steroid-receptor coactivator 1 and integrator complex $\mathrm{p} 300 / \mathrm{CBP}$ by E2. We evaluated by ChIP the association of H3Ac (acetylation of histone $\mathrm{H} 3$ ) and $\mathrm{H} 3 \mathrm{~K} 4 \mathrm{me} 3$ (trimethylation of $\mathrm{H} 3$ ) with the promoters of these genes 3 hours after injections. Influences of TH status on the association of H3Ac and H3K 4me3 were gene and brain-region specific. Next, we analyzed the recruitment of ERa and TRa?to regulatory sequences within Pgr and Oxtr promoters. Interestingly, hypothyroidism and hyperthyroidism affected E2-induced ERa recruitment to $P g r$ promoter in a similar fashion. E2 was able to recruit TRa to Pgr promoter, mimicking T3, however, when T3 was in excess and therefore TRa recruitment was high, E2 inhibited T3-induced TRa recruitment by $44 \%$ $(\mathrm{P}<0.05)$. Finally, to Oxtr promoter, E2 and T3 worked together to increase $\mathrm{ERa}$ and TRa recruitment in a synergic fashion. Our data indicate, for the first time, a cross-regulation between $\mathrm{E} 2$ and $\mathrm{TH}$ at early molecular stages that could be involved in TH previously reported interferences on E2-dependent sex behavior. 


\section{OP86}

ROLE OF THE NADPH OXIDASE NOX4 IN BRAFV600E-DEPENDENT DOWNREGULATION

\section{OF NIS}

Naïma Azouzi ${ }^{1}$, Jérémy Cailloux', Amélie Boichard², Abir Al Ghuzlan ${ }^{3}$, Dana Hart ${ }^{3}$, Martin Schlumberger ${ }^{4}$, Ameziane El Hassani Rabii ${ }^{5}$, Corinne Dupuy ${ }^{0}$

${ }^{1}$ Université Paris-Sud 11; Umr 8200 Cnrs; Institut Gustave Roussy, ${ }^{2}$ Institut Gustave Roussy, ${ }^{3}$ Gustave Roussy, Université Paris-Sud, Villejuif, France, ${ }^{4}$ Department of Nuclear Medicine and Endocrine Oncology, Gustave Roussy and Université Paris-Sud, Villejuif, France, ${ }^{5}$ Ubrm, Centre National de L'energie, des Sciences Et des Techniques Nucléaires, Rabat,

The loss of expression of the basolateral sodium iodide symporter (NIS) is an early event in thyroid carcinogenesis. Molecular mechanisms of the control of its expression are still poorly understood. On a clinical level, some thyroid cancers, in particular those having a BRAF mutation, insufficiently concentrate iodine 131 to benefit of the metabolic radiotherapy. In vitro, experiments have confirmed the involvement of BRAF V600E in the extinction of the expression of NIS gene. The effect of reactive oxygen species (ROS) on the expression of NIS is unknown. However, several studies suggest that the promoter activity of NIS gene is dependent on the redox state of the cell. Cells can produce ROS through activation and/or induction of NADPH oxidases. Since it has been shown that the NADPH oxydase NOX4 is overexpressed in papillary cancers we hypothesized that this NADPH oxidase could be involved in redox mechanisms responsible for the altered expression of NIS in thyroid tumor cells carrying the BRAF (V600E) mutation.

Using the papillary thyroid cancer cell line BCPAP, homozygous for the BRAF V600E mutation, we show that a treatment of cells with antioxidants such as N-acetylcysteine (NAC) or Tiron increases NIS expression at the transcriptional level indicating that ROS have a negative regulatory role on NIS gene expression. BCPAP cells express NOX4 and its functional partner p22phox. Knockdown of NOX4 expression by siRNAs leads to NIS expression in BCPAP cells highlighting that NOX4-dependent ROS are involved in redox mechanisms controlling expression of the iodide symporter. In addition, using pharmacological inhibitors and siRNAS we show that mutated BRAF controls both NOX4 and p22phox expression via the TGFB pathway, which has been recently shown to have a key role in the negative regulation of NIS expression and in the epithelial-mesenchymal transition (EMT) induced by mutated BRAF.

\section{OP12 Thyroid Cancer Therapy}

\begin{abstract}
OP87
EXPLORATORY ANALYSIS OF OUTCOMES FOR PATIENTS WITH LOCALLY ADVANCED OR METASTATIC RADIOACTIVE IODINEREFRACTORY DIFFERENTIATED THYROID CANCER (RAI-RDTC) RECEIVING OPEN-LABEL SORAFENIB POST-PROGRESSION ON THE PHASE III DECISION TRIAL

Martin Schlumberger ${ }^{1}$, Christopher Nutting ${ }^{2}$, Barbara Jarzab ${ }^{3}$,

Rossella Elisei ${ }^{4}$, Salvatore Siena ${ }^{5}$, Lars Bastholt ${ }^{6}$,

Christelle de la Fouchardiere ${ }^{7}$, Furio Pacini ${ }^{8}$, Ralf Paschke ${ }^{9}$ Young Kee Shong ${ }^{10}$, Steven I. Sherman ${ }^{11}$, Johannes W.A. Smit ${ }^{12}$ John Chung ${ }^{13}$, Christian Kappeler ${ }^{14}$, Istvan Molnar ${ }^{13}$, Marcia S. Brose ${ }^{15}$ ${ }^{1}$ Gustave Roussy, ${ }^{2}$ Royal Marsden Hospital, ${ }^{3}$ Maria Sklodowska-Curie Memorial Cancer Centre and Institute of Oncology, ${ }^{4}$ Department of Clinical and Experimental Medicine, University of Pisa, ${ }^{5}$ Ospedale Niguarda Ca' Granda, ${ }^{6}$ Odense University Hospital, ${ }^{7}$ Consortium Cancer Thyroïdien, Hospices Civils-Centre Anticancéreux, ${ }^{8}$ Unit of Endocrinology, University of Siena, ${ }^{9}$ Department for Endocrinology and Nephrology, Leipzig University, ${ }^{10}$ Asan Medicine Center, University of Ulsan College of Medicine, ${ }^{11}$ The University of Texas MD Anderson Cancer Center, ${ }^{12}$ Department of Internal Medicine, Radboud University Medical Center, ${ }^{13}$ Bayer Healthcare Pharmaceuticals, ${ }^{14}$ Bayer Pharma AG, ${ }^{15}$ Department of Otorhinolaryngology: Head and Neck Surgery, Abramson Cancer Center of the University of Pennsylvania
\end{abstract}

Background: RAI-rDTC patients treated with sorafenib (SOR) in the phase III placebo (PLC)-controlled DECISION trial had significantly improved progression-free survival (PFS). Patients who progressed in the double-blind (DB) period were unblinded and at the investigator's discretion allowed to receive open-label (OL) SOR and then followed for subsequent disease progression (PFS2).

Methods: PFS2, an exploratory endpoint, was defined as time from new baseline until centrally assessed progression or death during or after OL-SOR treatment.

Results: A total of 207 patients were randomized to sorafenib (DB-SOR) and 210 to placebo (DB-PLC). 150 PLC patients crossed over to OL-SOR at progression; 137 evaluable for efficacy (PLC-SOR). 55 DB-SOR patients continued OL-SOR at progression; 46 evaluable for efficacy (SOR-SOR). The PLC-SOR and SOR-SOR patients had poorer risk features at enrollment compared to patients not assessed for PFS2.

Partial responses in the DB-SOR and PLC-SOR patients were $12.2 \%$ and $9.5 \%$, respectively. Among SOR-SOR patients with progression events in both periods, PFS2 was more than two-fold longer than PFS1 in $22 \%$. The median treatment duration for SOR patients receiving DB and OL treatment was 56.9 weeks. Adverse events were similar for PLC-SOR and DB-SOR patients. For SOR-SOR patients, diarrhea and hand-foot skin reactions were reduced compared to the DB-SOR treatment period.

Conclusions: PFS2 for the subset of PLC patients who crossed over to OL-SOR was longer (9.6 months) than PFS1 (5.3 months). Continuation of SOR after progression needs to be explored further.

Table 1. (for Abstract OP87)

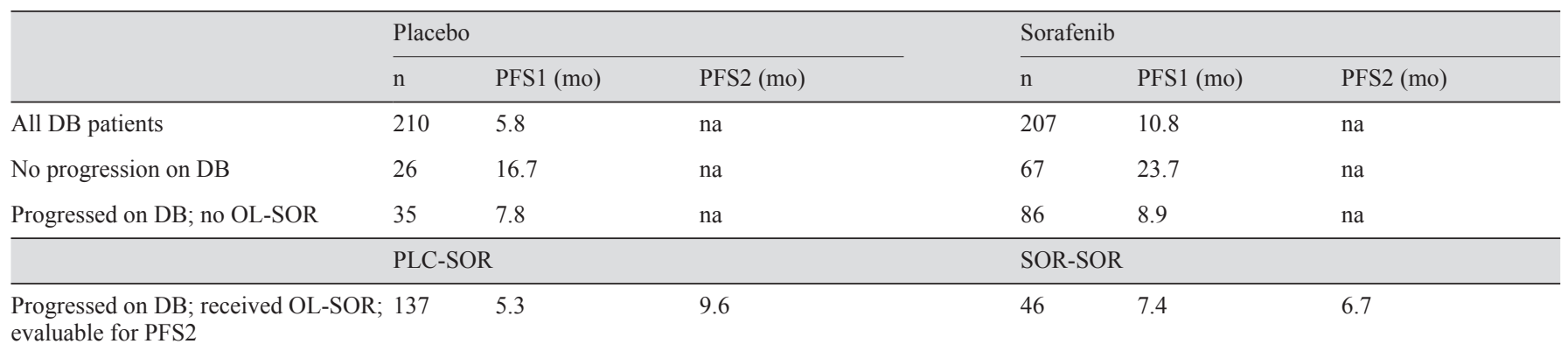

na: not applicable. 


\section{OP88 \\ FOLLICULAR (FVPTC) VS CLASSICAL (CVPTC) VARIANT OF PAPILLAR THYROID CANCER: CORRELATION OF THE OUTCOME AFTER 12 YEARS OF FOLLOW UP}

Carlotta Giani ${ }^{1}$, Liborio Torregrossa ${ }^{2}$, Eleonora Molinaro', Andrea Cacciato Insilla ${ }^{2}$, Laura Agate ${ }^{1}$, David Viola',

Luciana Puleo ${ }^{1}$, Gabriele Materazzi ${ }^{2}$, Ivo Marchetti ${ }^{3}$, Paolo Miccoli², Giancarlo Di Coscio ${ }^{3}$, Paolo Vitti ${ }^{1}$, Fulvio Basolo ${ }^{2}$, Rossella Elisei ${ }^{1}$

${ }^{1}$ Endocrine Unit, Department of Clinical and Experimental Medicine, University of Pisa, Pisa, Italy, ${ }^{2}$ Department of Surgical, Medical and Molecular Pathology of the Clinical Area, University of Pisa, Pisa, Italy, ${ }^{3}$ Department of Translational Research and New Technologies in Medicine and Surgeons, University of Pisa, Pisa, Italy

Objectives: Papillary thyroid carcinoma (PTC) is the most frequent histotype of thyroid cancer and accounts for more than $70 \%$ of primary thyroid cancer. CVPTC is the most common variant followed by FVPTC whose prevalence has been increasing in the last decades. It is known that the prognosis of both CVPTC and FVPTC is good even if FVPTC seems to have a better prognosis than CVPTC. However, at the present there are no correlative studies of long term follow-up of FVPTC vs CVPTC.

Aim: 1) to analyze the epidemiological, clinical and pathological features of FVPTC including the outcome after 12 years of follow-up. 2) to correlated the outcome of FVPTC and CVPTC.

Methods: we selected 449 FVPTC and 248 CVPTC diagnosed from 2000 and 2003. Their clinical, epidemiological and pathological features were analyzed. In particular the outcome after 12 year of follow-up was considered.

Results: 418/449 of FVPTC (93\%) were in clinical remission; 31/449 FVPTC(7\%) had a persistent disease [14/449(3.1\%) biochemical disease; 17/449(3.7\%) structural disease (latero-cervical lymphnode, lung and/or bone metastases)]. The persistence correlated with: De Groot and ATA risk class, absence of tumor capsule, infiltration of thyroid capsule, extension to perithyroid soft tissues, multifocality, presence of metastatic lymphnodes and pre-surgical cytological diagnosis. 210/248(85\%) of CVPTC were in clinical remission; $38 / 248(15 \%)$ had a persistent disease [23/248(9\%) biochemical disease; $15 / 248(6 \%)$ structural disease (latero-cervical and mediastinum lymphnode and lung metastases)]. We found a statistically significant difference in the outcome of FVPTC and CVPTC $(p=0.0004)$ after 12 years of follow-up.

Conclusions: A) the outcome of FVPTC after 12 years of follow-up is statistically better than the outcome of CVPTC; B) however, 7\% of FVPTC were still not cured after a 12 years follow-up with $3.7 \%$ of cases showing a structural disease involving lymphnodes, lung and bone.

\section{OP89 \\ CONSERVATIVE VS RADICAL SURGICAL MANAGEMENT IN CHILDREN WITH DIFFERENTIATED THYROID CANCER: MULTICENTRIC STUDY}

Claudio Spinelli', Paolo Miccoli' ${ }^{1}$, Alessandro Inserra², Gianni Bisogno ${ }^{3}$, Giovanni Cecchetto ${ }^{3}$, Poupak Fallahi', Alessandro Antonelli ${ }^{\circ}$

${ }^{1}$ University of Pisa, ${ }^{2 " B a m b i n o ~ G e s u ̀ " ~ P e d i a t r i c ~ H o s p i t a l, ~}{ }^{3}$ University of

Padova, Italy

Objective: In children there is a particular attention about the extension of thyroid surgery in (non medullary) differentiated thyroid cancer (DTC). In case of clinical evidence of nodal involvement the most common treatment is total thyroidectomy, with/without central neck dissection, and laterocervical lymphadenectomy. Radioiodine therapy and lifelong hormone replacement therapy are usually used after thyroidectomy. The radical surgical approach are correlated with a higher percentage of postsurgical complications but also with substantial decrease of DTC recurrences. In this work, we report the results of a multicentric Italian study in 180 patients (TREP Project; Tumori Rari in Età Pediatrica).

Methods: We analyzed the data of 180 DTC patients (median age 13 years, range 4-18 years) who underwent surgery from January 2001 to December 2011. One hundred and fifty-four patients underwent total thyroidectomy (TT) associated in 74 cases with a cervical lymphadenectomy (CL); 26 patients considered low-risk DTC with carefully preoperative staging under- went hemithyroidectomy (HT). The median follow-up was 5.6 years (range $1-11$ years).

Results and Conclusions: The study evidenced $22 \%$ cases of postoperative complications that included hypoparathyroidism ( $76 \%$ transient and $24 \%$ permanent) and recurrent laryngeal nerve injury in $2.4 \%(1.6 \%$ unilateral and $0.8 \%$ bilateral) in the TT group.

There were clinical persistent disease (presence of neoplastic tissue uptaking radioiodine) in 3 patients with lymphnode metastases, 2 cases of local recurrence and 4 cases of lung metastases in the TT group. In these group recurrences occurred in 17 patients; 11 cases of lymphnode metastases, 4 cases of lung metastases, and 2 cases of local recurrence (between the 6th and 24th months after surgery). In the HT group no complications and local recurrences was observed.

In conclusion, in children it is necessary a careful preoperative staging of DTCs to identify low-risk cases that can be treated with a conservative surgery.

\section{OP90 \\ PROGNOSTIC VALUE OF MICROSCOPIC LYMPH NODE INVOLVEMENT IN PATIENTS WITH PAPILLARY THYROID CANCER}

Stephane Bardet ${ }^{1}$, Renaud Ciappuccini ${ }^{1}$, Elske Quak', Jean-Pierre Rame ${ }^{1}$, David Blanchard'1 ${ }^{1}$ Dominique De Raucourt', Emmanuel Babin' ${ }^{2}$, Jean-Jacques Michels ${ }^{1}$, Dominique Vaur ${ }^{1}$, Natacha Heutte ${ }^{3}$

${ }^{1}$ Centre Francois Baclesse, ${ }^{2} \mathrm{Chu},{ }^{3}$ Université de Basse-Normandie

Objective: Macroscopic nodal involvement increases the risk of persistent/recurrent disease (PRD) in patients with PTC. In contrast, the impact of microscopic nodal involvement remains controversial. The aim of the study was to assess the risk of PRD and the outcome in PTC patients according to their initial nodal status ( $\mathrm{pNx}, \mathrm{pN} 0, \mathrm{pN} 1$ microscopic or $\mathrm{pN} 1$ macroscopic).

Methods: We conducted a retrospective cohort study on 308 consecutive PTC patients referred for radioiodine ablation from 2006 to 2011. The main outcome measures were the risk of PRD and the disease status at last follow-up.

Results: Of 308 patients, 129 (42\%) were pNx, 84 (27\%) pN0, 45 (15\%) pN1 microscopic and $50(16 \%)$ pN1 macroscopic. After a 4-yr follow-up, 53 $(17 \%)$ patients presented PRD (neck only, $\mathrm{n}=33$; distant sites only, $\mathrm{n}=11$; neck and distant sites, $\mathrm{n}=9$ ) which was detected on post-radioiodine ablation SPECT-CT in $34(64 \%)$ and on FDG PET-CT in $16(30 \%)$. The 4 yrcumulative risk of PRD was higher in N1 macroscopic than in N1 microscopic patients $(48 \%$ vs. $24 \%, \mathrm{P}=0.02)$, and higher in $\mathrm{N} 1$ microscopic than in N0 ( $24 \%$ vs. $12 \%, \mathrm{P}=0.01)$ or Nx patients $(6 \%, \mathrm{P}<0.001)$. On multivariate analysis tumor size $\geq 20 \mathrm{~mm}(\mathrm{RR}=3.4 ; \mathrm{P}<0.0001)$, extrathyroid extension $(\mathrm{RR}=$ $2.7 ; \mathrm{P}=0.001), \mathrm{N} 1$ macroscopic $(\mathrm{RR}=4.2 ; \mathrm{P}<0.0001)$ and $\mathrm{N} 1$ microscopic $(\mathrm{RR}=2.5 ; \mathrm{P}<0.02)$ were independent risk factors for PRD. At last visit, the proportion of disease-free patients decreased from Nx $(98 \%)$, N0 $(93 \%)$, N1 microscopic $(87 \%)$ to $\mathrm{N} 1$ macroscopic patients $(68 \%)(\mathrm{P}<0.0001$, CochranArmitage trend test). Extrathyroid extension $(\mathrm{OR}=9.8 ; \mathrm{P}<0.0001)$ and $\mathrm{N} 1$ macroscopic $(\mathrm{OR}=4.4 ; \mathrm{P}<0.001)$ independently predicted active disease or detectable Tg level at last visit, but N1 microscopic did not.

Conclusions: Patients with microscopic lymph-node involvement present an intermediate outcome between that observed in N0-Nx patients and $\mathrm{N} 1$ macroscopic patients. These data may justify modifications to the risk recurrence staging systems. 


\section{OP91 \\ METASTATIC THYROID CANCER \\ UNRESPONSIVE TO CONVENTIONAL THERAPY \\ TREATED WITH SORAFENIB "OFF-LABEL": AN UPDATE OF OUR 6 YEARS OF EXPERIENCE}

Virginia Cappagli ${ }^{1}$, Valeria Bottici ${ }^{1}$, Eleonora Molinaro ${ }^{1}$, Laura Agate ${ }^{1}$, David Viola', Laura Valerio ${ }^{1}$, Paolo Piaggi², Salvatore Mazzeo ${ }^{3}$, Valentina Battaglia ${ }^{3}$, Benedetta Pontillo Contillo ${ }^{3}$, Paolo Vitti ${ }^{1}$, Rossella Elisei ${ }^{1}$

${ }^{1}$ Endocrine Unit, Department of Clinical and Experimental Medicine, University of Pisa, Pisa, Italy, ${ }^{2}$ Department Of Energy and Systems Engineering, University of Pisa, ${ }^{3}$ Department for Integrated Activity Radiology, Vascular and Interventional Radiology, Nuclear Medicine; University of Pisa, Pisa, Italy

Introduction: advanced thyroid cancer (TC) that cannot be treated with radioiodine are orphan of successful therapies.

Aim: to evaluate the clinical benefits of Sorafenib in patients with advanced/aggressive TC. We enrolled 43 patients (19 females, 24 males) with progressive metastatic TC with no therapeutic options and not eligible for clinical trials: 9 de-differentiated papillary TC, 10 follicular/Hurthle TC, 7 poorly differentiated TC, 5 medullary TC and 12 anaplastic TC. This is a still on-going study and at the follow-up is ranging from 15 days to 38 months. To evaluate the efficacy of sorafenib we performed a CT scan after 1 month and every 3 months, biochemical evaluation of disease (thyroglobulin or calcitonin) and a clinical evaluation of the performance status (ECOG).

Results: in 4 cases treatment was discontinued after $<2$ weeks because of the reduced ECOG and these patients were not evaluated for the drug response ( 3 died because of poor clinical conditions and 1 discontinued the drug for severe neurological side effects). $27 / 39$ patients showed a stabilization of the disease (SD) during a treatment period ranging from 1 to $>6$ months, 6/39 had a partial response (PR) and 6/39(15.3\%) a progression of disease (PD). The best responders appeared to be follicular/Hurthle (7/10) and anaplastic (7/12) TC. Unfortunately PD was observed in 6/33(18\%) responders after a mean follow up of 9.0 months; $6 / 33(18 \%)$ patients had severe side effects that caused the discontinuation of treatment; 11/33(33\%) are still successfully continuing the drug ( $9 \mathrm{SD}$ and 2 still PR) after a mean period of 6.5 months (range 2-16 months) while 5/33(15\%) died for deteriorated clinical conditions.

Conclusions: we observed an overall clinical benefit in $33 / 39$ pts $(84.6 \%)$; the escape phenomenon and the severe side effects are the two major limiting factors for the long term disease control operated by the drug.

\section{OP92 \\ DIFFERENTIAL GENE EXPRESSION IN FOLLICULAR THYROID CARCINOMAS SHOWING DIFFERENT DEGREES OF INVASIVENESS}

Ricardo Celestino ${ }^{1}$, Torfinn Nome², Ana Pestana ${ }^{3}$, Eva Sigstad ${ }^{4}$, Ragnhild A. Lothe ${ }^{5}$, Trine Bjøro ${ }^{6}$, Manuel Sobrinho-Simões ${ }^{7}$, Rolf I. Skotheim ${ }^{2}$, Paula Soares ${ }^{8}$

${ }^{1}$ Institute of Molecular Pathology and Immunology of the University of Porto (Ipatimup), Porto, Portugal; School of Allied Health Sciences Estsp, Polytechnic of Porto, Porto, Portugal, ${ }^{2}$ Department of Cancer Prevention, Institute for Cancer Research, Norwegian Radium Hospital, Oslo University Hospital, Oslo; Centre for Cancer Biomedicine, Faculty of Medicine, University of Oslo, Oslo, Norway, ${ }^{3}$ Institute of Molecular Pathology and Immunology of the University of Porto (Ipatimup), Porto Portugal; Institute of Biomedical Sciences of University of Porto, Porto, Portugal, ${ }^{4}$ Department of Pathology, Norwegian Radium Hospital, Oslo University Hospital, Oslo, Norway, ${ }^{5}$ Department of Cancer Prevention, Institute for Cancer Research, Norwegian Radium Hospital, Oslo University Hospital, Oslo; Centre for Cancer Biomedicine, Faculty of Medicine, University of Oslo, Oslo, Norway; Department of Molecular Biosciences, University of Oslo, Oslo, Norway, ${ }^{6}$ Department of Medical Biochemistry, Norwegian Radium Hospital, Oslo University Hospital, Oslo, Norway; Institute of Clinical Medicine, University of Oslo, Oslo, Norway, ${ }^{7}$ Institute of Molecular Pathology and Immunology of the University of Porto (Ipatimup), Porto, Portugal; Medical Faculty, University of Porto, Porto, Portugal; Department of Pathology, Centro Hospitalar de São João, Porto, Portugal, ${ }^{8}$ Institute of Molecular Pathology and Immunology of the University of Porto (Ipatimup), Porto, Portugal; Medical Faculty, University of Porto, Porto, Portugal

Introduction: Follicular thyroid carcinoma (FTC) displays a capsule and is subclassified into minimally ( $\mathrm{mFTC}$ ) and widely invasive FTC (wFTC); the latter can have higher clinical aggressiveness, and carry worse prognosis than mFTC. The molecular mechanisms that differentiate the invasiveness degree of cancer cells in FTCs are poorly understood. Additionally, classification of invasion may be differentially evaluated by individual pathologists in the histological examination of FTC.

We aimed to understand some of the molecular alterations associated to cancer invasion using FTC as an encapsulated tumour model.

Methods: We compared samples from two mFTC and two wFTC using high-throughput paired-end RNA sequencing. A particular attention was done to the identification of novel fusion transcripts and to genes differentially expressed between $\mathrm{mFTC}$ and $\mathrm{wFTC}$.

Results: We identified 6439 candidate fused transcripts in FTCs. By stringent requirements, we selected 29 fusion transcripts for further experimental validation. All were positive by reverse-transcriptase PCR and Sanger sequencing, and in matched normal tissues.

Seventeen candidate genes were differentially expressed between mFTC and wFTC. Validation was done in cancer samples and in matched normal tissue by real-time quantitative PCR, confirming the differential expression both between $\mathrm{mFTC}$ and wFTC, and between FTCs and normal thyroid tissue. Four genes were selected for further experimental studies based in the accuracy of gene expression between cancer samples and normal thyroid tissue samples. Expression of these candidate genes are being validated as potential biomarker of cancer invasion in large series of tumours.

Conclusions: Several novel fusions transcripts were found in FTC, but none of which were cancer specific. Novel differentially expressed genes between wFTC and mFTC are promising as biomarkers. With the ongoing experimental validations in large series of tumours, we expect to find biomarkers that indicate the invasiveness of FTC. 


\section{OP93 \\ SURGICAL STRATEGY IN LOW ADVANCED DIFFERENTIATED THYROID CANCER STAGED T1NOMO - RESULTS OF A PILOT FEASIBILITY STUDY}

Agnieszka Czarniecka ${ }^{1}$, Stanislaw Poltorak ${ }^{1}$, Aleksander Sacher ${ }^{1}$, Adam Maciejewski', Jolanta Krajewska ${ }^{1}$, Michal Jarzab ${ }^{1}$,

Ewa Stobiecka1', Ewa Zembala-Nozynska1, Ewa Chmielik',

Dagmara Rusinek ${ }^{1}$, Monika Kowal',

Malgorzata Oczko-Wojciechowska ${ }^{1}$, Daria Handkiewicz-Junak ${ }^{1}$, Barbara Jarzab ${ }^{1}$

${ }^{1}$ Centre of Oncology M.Sklodowska-Curie Memorial Institute Gliwice Branch

ATA guidelines recommends total thyroidectomy (TT) in all subjects diagnosed preoperatively with DTC larger $>1 \mathrm{~cm}$ and lobectomy for small, low risk unifocal, intrathyroidal carcinomas. Prophylactic central neck dissection (CND) is recommended for more advanced stages (T3 and T4). The possibility of less extensive surgery is now discussed. The question rises whether BRAF mutation assessment is helpful to preoperatively determine the extent of the surgery. Thus, the prospective study has been carried out since 2011. Due to ethical reasons a control group involves patients operated between 2004 and 2006 (retrospective analysis).

The goal: To determine whether such study design is able to answer our question or not.

Material: Prospective group (PG) consisted of 132 DTC patients, staged T1N0M0. 71 with T1a underwent lobectomy whereas in 63, staged T1b, TT with unilateral CND was performed. Retrospective group (RG) involved 210 T1N0M0 DTC subjects, $78 \mathrm{~T} 1 \mathrm{a}$ and $132 \mathrm{~T} 1 \mathrm{~b}$, treated by TT with bilateral CND. The presence of BRAF mutation was assessed in both groups.

Results: An initial clinical stage was confirmed by histopathology in $79 \%$ subjects from PG and 75\% patients from RG (NS). Lymph node metastases were diagnosed less frequently in $\mathrm{PG}$ than in $\mathrm{RG}, 10 \%$ vs $28 \%$ respectively $(\mathrm{p}=0.00)$. The risk of lymph node involvement significantly correlated with tumor diameter and extrathyroideal invasion $(\mathrm{p}=0.0003)$ but not with multifocality. There were $56 \%$ BRAF+ patients in $\mathrm{PG}$ and $48 \% \mathrm{BRAF}+$ in $\mathrm{RG}$ (NS). BRAF mutation was less frequent in T1a patients $(p=0.02)$. There was one relapse (lymph node metastases) in PG, whereas in RG 7 (3.3\%) recurrences were diagnosed. In RG, 5-year disease free survival in T1a and T1b subgroups was respectively: $97 \%$ and $95 \%$.

Conclusion: The prospective study devoted to BRAF-guided surgical strategy in DTC is feasible.

The study was supported by NCN NN 403194340

\section{OP94}

\section{PERCUTANEOUS TREATMENT OF BONE} METASTASES FROM DIFFERENTIATED THYROID CARCINOMAS: RESULTS FROM A COHORT OF 23 PATIENTS

Françoise Bonichon $^{0}$, Cazzato Roberto Luigi ${ }^{1}$, Buy Xavier ${ }^{2}$, Godbert Yann ${ }^{3}$, Henriques de Figueiredo Bénédicte ${ }^{4}$, Pointillart Vincent ${ }^{5}$, Palussiere Jean ${ }^{6}$

${ }^{1}$ Department of Diagnostic Imaging and Interventional Radiology, Università Campus Bio-Medico DI Roma, Rome, Italy, ${ }^{2}$ Department of Interventional Radiology, Institut Bergonié, Bordeaux, France, ${ }^{3}$ Department of Nuclear Medicine, Institut Bergonié, Bordeaux, France, ${ }^{4}$ Department of Radiotherapy, Institut Bergonié, Bordeaux, France, ${ }^{5}$ Spinal Surgery Unit, Department of Orthopaedics, University Hospital of Bordeaux, Bordeaux, France, ${ }^{6}$ Department of Interventional Radiology, Institut Bergonié, Bordeaux, France

Objectives: To present our 12-year experience with interventional radiologic treatments of bone metastases from differentiated thyroid cancer.

Methods: From January 2001 to February 2014, 23 patients (mean age $64.7 \mathrm{yrs}$ ) underwent interventional radiologic procedures on bone metastases from differentiated thyroid cancer ( 7 papillary, 5 follicular, 4 oncocytic, 7 poorly differentiated). Applied interventional treatments were cementoplasty or cryo-/radiofrequency ablations, often combined with surgery (2 lesions) or radiotherapy (29 lesions). End-points were: local efficacy (assessed by com- puted tomography + /- PET-CT and thyroglobulin during follow-up), complications and overall survival after the first interventional radiologic session.

Results: Forty nine bone metastases were treated with cementoplasty (37), cryoablation (8) or radiofrequency ablation (4) in 44 interventional sessions. Lesions locations were into pelvic bones (44\%), spine $(35.6 \%)$, upper or lower limbs (4.4\%), scapula/sternum/ribs (15.6\%). Median delay between initial treatment of the primary thyroid cancer and the first interventional radiologic session was 2.7 years (range $0-22 \mathrm{yrs}$ ). At the time of the procedure, 49\% lesions showed avid I131 uptake. Median hospitalization length was 2 days. Minimal asymptomatic cement leakage occurred in $25 \%$ of the procedures; one case of infection was also noted and treated with antibiotics. Median follow-up was 3.6 years. Local control was obtained in $73.5 \%$ of lesions, sometimes thanks to 2 to 6 repeated sessions. One and 2-year survival was $66 \%$ and $60 \%$, respectively. Long survival was observed in oligometastatic patients.

Conclusions: Percutaneous procedures are safe and effective in the treatment of bone metastatic disease from differentiated thyroid cancer, especially for oligometastatic patients. In slowly progressive disease, multidisciplinary tumor boards should consider interventional procedures as a valid option to treat bone metastases from differentiated thyroid cancer even if they are I131-avid.

\section{OP13 Gene Expression}

\section{OP95 \\ THE TRANSCRIPTION FACTOR NRF2 CONTROLS ANTIOXIDANT RESPONSES AND IMPACTS THYROID-SPECIFIC GENE EXPRESSION AND HANDLING OF EXCESS IODINE}

Panos Ziros ${ }^{1}$, loannis Habeos ${ }^{2}$, Dionysios Chartoumpekis ${ }^{3}$ Michail Mpakakis ${ }^{2}$, Irene Mamali ${ }^{2}$, Stavroula Manolakou ${ }^{1}$, Ioannis Lilis ${ }^{2}$, Nikolaos Papachristou ${ }^{2}$, Venetsana Kyriazopoulou ${ }^{2}$, Thomas Kensler ${ }^{3}$, Dionysios Papachristou ${ }^{2}$, Gerasimos Sykiotis ${ }^{1}$

${ }^{1}$ Lausanne University Hospital, Lausanne, Switzerland, ${ }^{2}$ University of

Patras Medical School, ${ }^{3}$ University of Pittsburgh, USA

Background: Compared to other tissues, the thyroid has increased capacity for defending itself against oxidative stress (OS). Specific antioxidant and detoxification enzymes have been identified that presumably help thyroid cells to maintain their homeostasis by ameliorating oxidative insults. While a minimal oxidative load is a prerequisite for normal thyroid cell function, the gland's antioxidant defense is activated by intra-thyroidal OS occurring during iodine deficiency, goitrogenesis, and high iodine-induced involution. Yet, the precise mechanisms by which thyrocytes sense and respond to OS remain incompletely understood. The transcription factor Nrf2 (Nuclear factor E2-related factor 2) is known to mediate antioxidant transcriptional responses in other tissues, but its role in thyroid physiology has not been addressed.

Objectives: To test whether Nrf2 controls expression of antioxidant and/or thyroid-specific genes and proteins under basal conditions and/or in response to iodine load.

Methods: Wild-type (WT) and Nrf2 knockout (KO) mice were given regular water or a high-iodine water solution for 7 days. Thyroid tissue was removed and analyzed by real-time RT-PCR and Western blotting. The PCCL3 and FRTL5 rat thyroid cell lines were used for in vitro assays.

Results: In rat thyroid cell lines, Nrf2 controlled the basal and/or sulforaphane-stimulated expression of the antioxidant genes NQO1, GPX2 and $T X N R D 1$. In the thyroid of mice, expression of these genes was induced by iodine in an Nrf2-dependent manner. Nrf2 also impacted the basal and/or iodine inducible expression of the thyroid-specific genes NIS, TPO, TG, and TSHR; for NIS and TG, this was further verified at the protein level. While iodine suppressed TG iodination in WT mice, Nrf2 KO mice had dramatically higher iodinated TG levels.

Conclusions: The transcription factor Nrf2 is important for thyroid homeostasis, not only for antioxidant responses to an iodine load, but also potentially for regulation of thyroid hormone synthesis and/or release. 


\section{OP96 \\ THE NUCLEAR COREPRESSOR NCOR IS AN ESSENTIAL MEDIATOR OF THE ANTI- TUMORIGENIC AND ANTI-METASTATIC ACTIONS OF THE THYROID HORMONE RECEPTOR B}

Olaia Martinez-Iglesias ${ }^{1}$, Rosa Martin-Orozco ${ }^{1}$, Elvira Alonso-Merino', Juan Pedro Velasco-Martín ${ }^{2}$, Javier Regadera², Jose Palacios ${ }^{3}$, Pilar González-Peramato ${ }^{4}$, Ana Aranda ${ }^{1}$

${ }^{1}$ Instituto de Investigaciones Biomédicas, ${ }^{2}$ Universidad Autónoma de

Madrid, ${ }^{3}$ Hospital Ramón Y Cajal, ${ }^{4}$ Hospital La Paz

Reduced expression or activity of the thyroid hormone receptors (TRs) are common events in cancer, suggesting that they could act as tumor suppressors. We have previously shown that TRb expression in SK-hep1 hepatocarcinoma cells (SK-TRb cells) reduces tumor growth, invasion, extravasation, and metastasis formation in nude mice. These effects are associated with a decreased expression of prometastatic genes.

We found that the levels of the corepressor $\mathrm{NCoR}$ were increased in SK-TRb cells with respect to the parental cells. NCoR silencing reversed significantly the repressive effect of $\mathrm{TRb}$ on cellular invasion in matrigel assays and on prometastatic gene expression. Promoter activity of various prometastatic genes was also reduced by NCoR over-expression and enhanced after NCoR knock-down. Furthermore, in chromatin immunoprecipitation (ChIP) assays we observed increased recruitment of NCoR and repressive marks to these promoters in SK-TRb cells, demonstrating the important repressive effect of NCoR on prometastatic gene expression.

NCoR expression was higher in tumor xenografts originated by SK-TRb cells than by parental cells and NCoR silencing also obliterated significantly the effect of $\mathrm{TRb}$ expression in vivo, reversing the inhibitory effect of the receptor on tumor growth. The reduced tumor infiltration found in TR?expressing tumors was also abolished in NCoR-depleted cells and there was a tight inverse correlation between NCoR expression and transcripts levels of prometastatic genes. Tumor cell extravasation was also higher in NCoR depleted cells, and the inhibitory effect of TRb on metastasis formation was reversed. These changes were associated with increased expression of prometastatic genes in the metastatic lesions. Finally, NCoR and TRb expression was lower in human hepatocarcinomas tumors than in normal liver.

These results demonstrate the essential role of NCoR in the anti-tumorigenic and anti-metastasic actions of TRb, and suggest the importance of the corepressor as a potential therapeutic target in cancer.

\section{OP97 \\ BEHAVIORAL AND METABOLIC EFFECTS OF 3-IODOTHYROACETIC ACID: ROLE OF HISTAMINE \\ Maria Elena Manni', Alessandro Saba², Annunziatina Laurino', Riccardo Donzelli², Grazia Chiellini2, Riccardo Zucchi², \\ Laura Raimondi ${ }^{1}$ \\ ${ }^{1}$ University of Florence, ${ }^{2}$ University of Pisa, Italy}

Objectives: 3-iodothyroacetic acid (TA1) is the major catabolite of 3 -iodothyronamine in most tissues. The aim of the present work is to determine whether TA1 is physiologically present in rodent brain and whether administration of exogenous TA1 can produce significant functional effects.

Methods: We measured TA1 concentration in mouse brain by HPLC coupled to mass spectrometry and we investigated whether administration of exogenous TA1 modified memory, pain perception and plasma glycemia. Fifteen min after intracerebroventricular (i.c.v.) injection of TA1 $(0.4,1.32$ and $4 \mu \mathrm{gkg}^{-1}$ ) memory acquisition-retention, pain threshold to a hot stimulus and plasma glycemia were evaluated on the light-dark box, on the hot plate $\left(51.5^{\circ} \mathrm{C}\right)$ and by a glucorefractometer respectively. Parallel experiments were performed in mice pre-treated with subcutaneous injections of pyrilamine (10 mgkg ${ }^{-1}$ ) or zolantidine $\left(5 \mathrm{mgkg}^{-1}\right)$, antagonists of histamine $\mathrm{H}_{1}$ and $\mathrm{H}_{2}$ receptors respectively. Furthermore, TA1 (1.32 and $\left.4 \mu \mathrm{gkg}^{-1}\right)$ was i.c.v. injected in histidine decarboxylase null $\left(\mathrm{HDC}^{-} /\right)$mice and in their $\mathrm{HDC}^{+} / /^{+}$littermates.

Results: TA1 was detected in CD1 mouse brain at concentrations on the order of $1 \mathrm{pmol} / \mathrm{g}$ of tissue. Pharmacological administration of TA1 included amnesia (at the dose of $0.4 \mu \mathrm{gkg}^{-1}$ ), stimulation of learning (at 1.32 and 4 $\mu \mathrm{gkg}^{-1}$ ), hyperalgesia (at $0.4,1.32$ and $4 \mu \mathrm{gkg}^{-1}$ ) and hyperglycemia (at 1.32 and $\left.4 \mu \mathrm{gkg}^{-1}\right)$. These responses were inhibited by pyrilamine and zolantidine. In $\mathrm{HDC}^{-/}$mice, TA1 (1.32 and $\left.4 \mu \mathrm{gkg}^{1}\right)$ failed to increase plasma glycemia and to reduce pain threshold.

Conclusions: Exogenous TA1 produces significant behavioral and metabolic effects, possibly through activation of the histaminergic system. It remains to be determined whether similar effects can be produced physiologically by endogenous TA1.

\section{OP98 \\ IDENTIFICATION OF AN INTRA-MOLECULAR DISULFIDE BRIDGE ESSENTIAL FOR THE COVALENT AND FUNCTIONAL INTERACTION BETWEEN DUOX2 AND ITS PARTNER DUOXA2 \\ Carré Aurore ${ }^{1}$, Louzada Ruy², Ameziane El Hassani Rabii ${ }^{3}$, \\ Corinne Dupuy ${ }^{0}$ \\ ${ }^{1}$ Université Paris-Sud 11; Umr 8200 Cnrs; Institut Gustave Roussy, \\ ${ }^{2}$ Laboratório de Fisiologia Endócrina Doris Rosenthal; Universidade \\ Federal Do Rio de Janeiro, ${ }^{3} \mathrm{Ubrm}$, Centre National de L'energie, des \\ Sciences Et des Techniques Nucléaires, Rabat,}

The dual oxidase 2 (DUOX2) protein is a reduced NAD phosphate: $\mathrm{O}_{2}$ oxidoreductase flavoprotein belonging to the NADPH oxidase (NOX/DUOX) family. The discovery of congenital hypothyroidism resulting from inactivating mutations of DUOX2 gene highlighted that DUOX2 is actually the main provider of $\mathrm{H}_{2} \mathrm{O}_{2}$ to the thyroperoxidase. Far from being ubiquitous DUOX2 is not restricted to the thyroid. It is also well-expressed in the respiratory tract epithelium and in the gastrointestinal mucosa where it plays a key role in lactoperoxidase-mediated antimicrobial defense. DUOX2 requires a maturation factor DUOXA2 to exit from the endoplasmic reticulum and to reach the apical plasma membrane. The heterodimerization of DUOX2 and DUOXA2 is a prerequisite for reactive oxygen species production but the nature of their interaction is not known. Using mutagenesis experiments we analyzed the nature of the interaction between DUOX2 and its partner DUOXA2. In our study, we show the identification and the characterization of an intramolecular disulfide bond between the N-terminal domain and an extracellular loop of DUOX2, which has an important structural implication in both export and activity of DUOX2 by promoting covalent interaction between DUOX2 and its partner DUOXA2. In addition, this covalent interaction is critical to DUOXA2 stability indicating that DUOX2 displays a chaperon-like function with respect to its functional partner.

\section{OP99 \\ THYROID HORMONES REDUCE THE TGF $\beta$ - DEPENDENT ACTIONS, ALLEVIATING FIBROTIC RESPONSES \\ Elvira Alonso-Merino ${ }^{1}$, Rosa Martin-Orozco ${ }^{1}$, Lidia Ruíz Llorente ${ }^{1}$, Luis Fernanda Fanjul Rodríguez', Olaia Martinez-Iglesias', Juan Pedro Velasco-Martín ${ }^{2}$, Javier Regadera ${ }^{2}$, Ana Aranda ${ }^{1}$ ${ }^{1}$ Instituto de Investigaciones Biomédicas, ${ }^{2}$ Universidad Autónoma de Madrid, Spain}

Transforming growth factor beta (TGF $\beta$ ), which plays a key role in cancer and fibrotic disorders, mediates its actions mainly through activation of Smad transcription factors, which bind to Smad Binding Elements (SBEs) in target genes. We have previously observed that the thyroid hormone T3 blocks transactivation of SBE-containing reporter plasmids by TGF $\beta$, and represses transcription of endogenous TGF $\beta$ target genes.

We have now analysed the thyroid hormone receptor (TR) isoform as well as the receptor domains responsible for this transcriptional antagonism. In $\mathrm{GH} 4 \mathrm{C} 1$ pituitary cells, the TR $\beta$ specific agonist $\mathrm{GCl}$ is as potent as $\mathrm{T} 3$ to repress SMAD-dependent transactivation. However, expression of TR $\alpha$ in other cell types also mediates repression of TGF $\beta$-dependent transactivation by T3. Using TR $\alpha$ and TR $\beta$ mutants we have observed that the DNA binding domain (DBD) is essential for the repressive effect, whereas the ligand-dependent transcriptional activation domain (AF-2), responsible for coactivators binding, appears to be dispensable. Moreover, we have detected a direct interaction of TR $\alpha$ ?and TR $\beta$ with Smad2/3 and Smad4, which is reversed by T3. Mutations in the AF-2 domain do no alter association with Smads, while the DBD plays an important role in this interaction. 
In chromatin immunoprecipitation (ChIP) assays, we have observed TR recruitment to SBE-containing regions. T3 inhibits this association, as well as TGF $\beta$-dependent recruitment of Smads and coactivators such as $\mathrm{p} 300$.

In agreement with the transcriptional cross-talk between T3 and TGF $\beta$ signaling, hyperthyroidism alleviates the fibrotic response induced by bleomycin in mice skin. In addition, hyperthyroidism decreases Smad2/3 phosphorylation and hepatocyte necrosis in a model of acute carbon tetrachloride administration, reducing liver fibrosis and collagen deposition after chronic administration of this compound.

These results demonstrate that TRs, in a ligand-dependent manner, antagonize TGF $\beta$ responses in cultured cells and appear to play a novel role as suppressors of fibrotic responses in vivo.

\section{OP100 \\ THYROMIMETIC ACTION OF 3,5-T2 IN DIET- INDUCED OBESE MICE}

Julika Lietzow ${ }^{1}$, Wenke Jonas ${ }^{2}$, Priyavathi Dhandapani ${ }^{1}$, Josef Köhrle ${ }^{1}$

${ }^{1}$ Institut Für Experimentelle Endokrinologie, Charité -

Universitätsmedizin Berlin, Germany, ${ }^{2}$ German Institute of Human

Nutrition (Dife), Nuthetal, Germany

Objectives: Administration of 3,5-diiodo-L-thyronine (3,5-T2), an endogenous thyroid hormone $(\mathrm{TH})$ metabolite, is supposed to prevent hyperlipidemia and liver steatosis, without any side effects on the thyroid axis or cardiac activity in rats fed a high-fat-diet. However, little is known about long-term effects of 3,5-T2 in mice. Our aim was to study the potential interference of 3,5-T2 with HPT axis and TH responsive gene expression in diet-inducedobese (DIO) mice.

Methods: Male DIO mice were continuously exposed to high-fat diet. At the age of 20 weeks postpartum the mice received for 14 days a daily application of 3,5-T2 $(0.25$ or $2.5 \mu \mathrm{g} / \mathrm{g} \mathrm{bw}), \mathrm{T} 3(0.03 \mu \mathrm{g} / \mathrm{g} \mathrm{bw})$ or saline. In a further experiment with the same study design DIO mice were treated with saline or the high dose of 3,5-T2 for 28 days.

Results: Chronic administration of 3,5-T2 dose-dependently exerted thyromimetic suppressive effects on the HPT axis, as revealed by alterations in pituitary transcript levels of TH responsive genes and decreased serum concentrations of T4 and T3. In line with these observations, treatment with high dose of 3,5-T2 over 4 weeks resulted in cardiac hypertrophy indicated by increasing heart weight and altered expression of $\mathrm{TH}$ responsive genes. Regarding hepatic gene expression high dose of 3,5-T2 modulated transcript concentrations of genes involved in lipid and energy metabolism similar to T3. Additionally, 3,5-T2 treatment altered gene expression of xenobiotic-metabolizing enzymes in liver.

Conclusions: Treatment of 3,5-T2 in DIO mice mimics T3 action by interfering with the HPT axis and modulating the expression of TH target genes in liver and heart. Contrary to published data in a DIO rat model but in accordance with data on lean rats, long-term treatment with high dose of 3,5-T2 in DIO mice might exert adverse (extra-)hepatic thyromimetic effects.

(Supported by DFG KFO218 TP3 and DFG SPP1629 Thyroid Trans Act)

\section{OP101 \\ SELENIUM INCREASES THYROTROPHIN- INDUCED NIS EXPRESSION THROUGH APE1- DEPENDENT REGULATION OF PAX8 BINDING ACTIVITY \\ Suzana Leoni ${ }^{1}$, Ana Sastre-Perona ${ }^{2}$, Pilar Santisteban ${ }^{1}$, \\ Antonio De la Vieja ${ }^{3}$ \\ ${ }^{1}$ Instituto de Investigaciones Biomédicas (Csic-Uam), ${ }^{2}$ Instituto de Investigaciones Biomédicas (Csic-Uam) - Madrid, ${ }^{3}$ Instituto de Salud Carlos III (Área de Biología Celular Y Desarrollo, Centro Nacional de Microbiología)}

The sodium iodide symporter (NIS) mediates the uptake of $\mathrm{I}^{-}$by the thyroid follicular cell being the first step of hormones biosynthesis. NIS function is stimulated by TSH and requires PAX8 binding within NUE ( $\underline{\text { Nis }}$ upstream enhancer). In this study we investigate the role of selenium on Nis expression through the regulation of Pax8 DNA-binding activity.

Results: Selenium (Se) treatment increased TSH-induced Nis mRNA and protein expressions, as well as Nis activity, in the $\mathrm{PCCl} 3$ thyroid cells. We con- sidered that Pax8 could be involved in this effect, since Se stimulus was only observed for Nis promoter containing NUE. In fact, Se increased Pax8 expression and its DNA-binding activity. Also, in null-Pax8 $\mathrm{PCCl} 3$ cell Nis is not $\mathrm{Se}$ responsive, confirming the crucial role of Pax8 in these responses. Two antioxidant enzymes were considered as putative mediators of this mechanism: TxnRd1 and Ape1. First we inhibited TxnRd1 activity by auranofin and no alteration was observed on Se stimulus. However when Ape1 was inhibited by E3330 both, TSH or TSH/Se stimulus, was no longer observed. Ape1 proved to be essential both for Pax8 binding activity as well as for Nis expression.

Conclusion: Under basal oxidation levels, Nis expression is controlled by Ape1 through a TSH/Se-dependent mechanism. These findings open a new field about the importance of Se in the thyroid physiology.

\section{OP102 \\ 3-IODOTHYROACETIC ACID INDUCES GENERALIZED ITCH AND HYPERSENSITIVITY TO NOXIUS HEAT STIMULI IN MOUSE}

\section{Annunziatina Laurino ${ }^{1}$, Francesco Resta ${ }^{1}$, Riccardo Zucchi ${ }^{2}$,} Laura Raimondi ${ }^{1}$

${ }^{1}$ University of Florence, ${ }^{2}$ University of Pisa, Italy

Objectives: 3-iodothyroacetic (TA1) is an endogenous metabolite putatively produced by 3-iodothyronamine deamination. We have recently suggested that some pharmacological effects of TA1 may be mediated by histamine release. In the present study we investigated if TA1 is able to elicit typical histamine effects, namely itch-induced scratching and thermal hyperalgesia.

Methods: We injected CD1 mice or histidine decarboxylase knock-out mice (HDC-/-), with TA1 $(0.4,1.32$ and $4 \mathrm{mg} / \mathrm{kg}$ ) intraperitoneally (i.p.) or subcutaneously (s.c.). Thermal hyperalgesia was determined after i.p. TA1 administration by the hot plate test, carried out at increasing steps of temperature. Itch was evaluated by measuring the time of occurrence, intensity and duration of scratching after s.c. TA1 injection.

Results: After 5 min from s.c. injection of exogenous TA1, at the doses of 0.4 and $1.32 \mathrm{mg} / \mathrm{kg}, \mathrm{CD} 1$ mice presented itch, with generalized scratching. This effect however was not observed at a higher dose, namely $4 \mathrm{mg} / \mathrm{kg}$. In CD1 mice, i.p. injection of exogenous TA1 reduced pain threshold to noxius heat stimuli. Both itch sensation and hyperalgesia to noxius heat stimuli were absent in $\mathrm{CD} 1$ mice pre-treated with the $\mathrm{H} 1$ histamine receptor antagonist pyrilamine $(10 \mathrm{mg} / \mathrm{kg}$; sc) and in HDC-/- mice.

Conclusions: Our results suggest that TA1 acts as an activator or sensitizer of $\mathrm{H}_{1}$ receptors of the $\mathrm{C}$ fibers of the dorsal root ganglia, which are known to be involved in itch as well as in hypersensitivity to noxius heat stimuli. It remains to be determined whether endogenous TA1 may play a physiological or pathophysiological role in itch and/or pain perception. 


\section{Poster Plan}

\section{European}

\section{Thyroid Journal}

Sunday, 7th September, 12.00-13.00

\begin{tabular}{|l|l|l|}
\hline Session Title & Posters & E-Terminal \\
\hline P01 Graves' Orbitopathy & P01-P12 & 1 \\
\hline P02 Cardiovascular Effects & P13-P17 & 2 \\
\hline P03 Thyroid and Environment 1 & P18-P21, P23-P25 & 3 \\
\hline P04 Thyroid in Reproduction and Development 1 & P26-P36 & 4 \\
\hline P05 Thyroid Function and Development & P37-P44 & 5 \\
\hline P06 Hypothyroidism & P45-P54 & 6 \\
\hline P07 Thyroid Cancer Diagnostics 1 & P55-P62, P64, P65 & 7 \\
\hline P08 Thyroid Cancer Therapeutics 1 & P66-P67, P70-P78 & 8 \\
\hline P09 Medullary Thyroid Cancer & P79-P88 & 9 \\
\hline P010 Goiter and Nodules 1 & P89-P98 & 10 \\
\hline P011 Case Reports 1 & P100-P109 & 11 \\
\hline P012 Imaging and Nuclear Medicine & P110-P115, P117-P121 & 12 \\
\hline
\end{tabular}

Monday, 8th September, 12.00-13.00

\begin{tabular}{|l|l|l|}
\hline Session Title & Posters & E-Terminal \\
\hline P013 Graves' Orbitopathy \& Autoimmunity & P122-P131 & 1 \\
\hline P014 Heart and Thyroid & P132-P139 & 2 \\
\hline P015 Subclinical Thyroid Disease and Hypothyroidism & P140-P150 & 3 \\
\hline P016 Thyroid and Environment 2 & P152-P158+P22 & 4 \\
\hline P017 Thyroid Cancer Diagnostics 2 & P159-P170 & 5 \\
\hline P018 Thyroid Cancer Pathogenesis 1 & P171-P183 & 6 \\
\hline P019 Thyroid Cancer Therapeutics 2 & P184-P195 & 7 \\
\hline P020 Thyroid Hormone Availability and Action & P197-P206 & 8 \\
\hline P021 Thyroid in Reproduction and Development 2 & P207-P218 & 9 \\
\hline P022 Case Reports 2 & P219-P228 & 10 \\
\hline P023 Goiter and Nodules 2 & P229-P238 & 11 \\
\hline
\end{tabular}


Tuesday, 9th September, 12.00-13.00

\begin{tabular}{|l|l|l|}
\hline Session Title & Posters & E-Terminal \\
\hline PO24 Thyroid Autoimmunity & P239-P250 & 1 \\
\hline PO25 Hyperthyroidism & P251-P262 & 2 \\
\hline PO26 Novel Actions of Thyroid Hormones & P263-P268 & 3 \\
\hline PO27 Paediatrics and Pregnancy & P270-P281 & 4 \\
\hline PO28 Thyroid Cancer Diagnostics 3 & P282-P293 & 5 \\
\hline PO29 Thyroid Cancer Diagnostics 4 & P294-P302 & 6 \\
\hline PO30 Thyroid Cancer Pathogenesis 2 & P303-P315 & 7 \\
\hline PO31 Thyroid Hormone and Gene Expression & P316-P322 & 8 \\
\hline PO32 Case Reports 3 & P323-P333 & 9 \\
\hline PO33 Thyroid Cancer Therapeutics 3 & P334-P345 & 10 \\
\hline PO34 Thyroid Cancer Therapeutics 4 & P346-P358 & 11 \\
\hline PO35 Biochemical and Metabolic Parameters & P359-P367 & 12 \\
\hline
\end{tabular}




\section{P01 Graves' Orbitopathy}

\section{P01 \\ DEMOGRAPHIC, CLINICAL, SEROLOGICAL AND QUALITY OF LIFE DATA OF 100 CONSECUTIVE EUTHYROID PATIENTS WITH SEVERE THYROID EYE DISEASE}

Elena Kampmann, Susanne Pitz, Julia Rosbach, George J. Kahaly

Johannes Gutenberg University Medical Center, Mainz, Germany

Objective: Dysthyroidism influences the course of thyroid eye disease (TED). By eliminating this variable we aimed to look for further putative factors contributing to poor quality of life $(\mathrm{QoL})$, clinical severity and activity of TED.

Methods: At an academic joint thyroid-eye clinic, complete ophthalmic, serological and endocrine investigations as well as assessment of healthrelated QoL (EUGOGO questionnaire) were performed in 100 consecutive euthyroid patients with active and severe TED.

Results: Median age was 51 years (range 23-75 years, 72 female, 57 current smokers). Graves' disease (GD), Hashimoto's thyroiditis and a euthyroid TED were present in 94, three and three patients, respectively. Median duration of GD and TED were 16 and 9.5 months, respectively. Diplopia was present in 68 patients with 16 having constant double vision. Chemosis, corneal lesions and asymmetric TED were present in 29,25 and 22 patients, respectively. Median clinical activity (CAS) and severity (CSS) scores were 4 (3-7) and 6.25 (range 1-11), respectively. Upper or lower lid retraction was present in 59 or 42 patients. Median palpebral aperture and proptosis were $11 \mathrm{~mm}(5-17)$ and $21.4 \mathrm{~mm}(12-30)$. Median thyroid binding inhibiting immunoglobulin (TBII) was $8.9 \mathrm{IU} / \mathrm{L}(0.5-174)$. TBII were higher in postmenopausal women $(\mathrm{P}<0.05)$, positively correlated with higher CAS $(\mathrm{P}<0.05)$ and negatively with duration of TED $(\mathrm{P}<0.05)$. Higher CAS was associated with a worse QoL Visual Functioning (VF) score $(\mathrm{P}<0.05)$. Women $(\mathrm{p}=0.01)$, younger patients $(\mathrm{P}<0.05)$ and smokers $(\mathrm{P}<0.05)$ had a lower QoL Appearance score. Older patients scored worse regarding VF $(\mathrm{P}<0.05)$ which also decreased with a higher Gorman diplopia score $(\mathrm{P}<0.001)$. Daily cigarette consumption or pack years had no significant impact on QoL, CAS, CSS, intraocular pressure or TBII.

Conclusions: Subsequent to elimination of dysthyroidism, these innovative data in a large euthyroid TED collective determine further negative impact factors for impaired QoL and disease activity/severity.

\section{P02 \\ PROSPECTIVELY RECORDED SAFETY DATA OF INTRAVENOUS METHYLPREDNISOLONE THERAPY IN GRAVES' ORBITOPATHY}

Michaela Riedl, Elena Kampmann, Elisa Kolbe, Miriam Lehmann, Alexander Pirkl, George J. Kahaly

Johannes Gutenberg University Medical Center, Mainz, Germany

Background: Safety of intravenous (IV) steroid pulses in patients with Graves' orbitopathy (GO) is still controversial while steroid dose and treatment application have not been finalized. Frequency, severity and characterization of eventual steroid-related adverse events (AE) were prospectively analyzed.

Methods: A total of 80 patients (57 or $71 \%$ female, aged $27-75$ years) with active and severe GO were prospectively followed in an academic joint thyroid-eye clinic during a standard schedule of IV methylprednisolone treat- ment (cumulative dose $4.5 \mathrm{~g}, 0.5 \mathrm{~g} / \mathrm{wk}$. for $6 \mathrm{wk}$. then $0.25 \mathrm{~g} / \mathrm{wk} . / 6 \mathrm{wk}$ ). All recorded $\mathrm{AE}$ were coded according to the medical dictionary for regulatory activities (MedDRA), graded if serious and related to drug treatment.

Results: $23 / 80(29 \%)$ patients with GO reported at least one AE and 10 $(12.5 \%)$ reported at least one AE which was related to IV steroid drug treatment. One of $80(1.25 \%)$ patients only stopped IV steroid treatment due to a therapy-related $\mathrm{AE}$ (depression). A total of $34 \mathrm{AE}$ were documented of which 17 were graded as related to IV steroids. Most common reported AE were hot flush and sleeping disorders. All other related AE occurred only once during the observational period and were spread over the known safety profile of IV methylprednisolone. Those were e.g. candida infection, myalgia, metrorrhagia, headache. Several AE (e.g. worsening of visual acuity, dysthyroidism and arrhythmias) were assigned to the underlying disease. Twenty-two $(64.7 \%)$ of all AE were judged as recovered, 10 (29.4\%) were recovering and 2 (5.9\%) $\mathrm{AE}$ in one patient (worsening of psoriasis and lichen ruber planus) were not recovered by the end of the observational period.

Conclusions: IV methylprednisolone pulse therapy is well tolerated. Most $\mathrm{AE}$ are accessory symptoms of the underlying disease and a few AE only were directly related to IV steroids. All related AE fit to the safety profile of IV methylprednisolone.

\section{P03}

\section{STEROID THERAPY IN GRAVES' ORBITOPATHY: COMPARISON OF THE EFFICACY AND SAFETY OF TWO DIFFERENT PROTOCOLS - PARENTERAL VS COMBINED PARENTERAL AND ORAL}

Biljana Beleslin ${ }^{1}$, Jasmina Ciric ${ }^{2}$, Milos Zarkovic ${ }^{2}$, Mirjana Stojkovic ${ }^{3}$, Slavica Savic ${ }^{3}$, Slavica Ciric ${ }^{3}$, Tijana Lalic ${ }^{3}$, Miroslav Knezevic ${ }^{4}$, Bozo Trbojevic ${ }^{5}$

${ }^{1}$ Clinic of Endocrinology, Diabetes and Metabolic Diseases, Clinical Center of Serbia; Medical School University of Belgrade, ${ }^{2}$ Clinic of Endocrinology, Diabetes and Metabolic Diseases, Clinical Center of Serbia; Medical School, University of Belgrade, ${ }^{3} \mathrm{Clinic}$ of Endocrinology, Diabetes and Metabolic Diseases, Clinical Center of Serbia, ${ }^{4}$ Clinic for Ophtalmology, Clinical Center of Serbia; Medical School University of Belgrade, ${ }^{5}$ Clinic of Endocrinology, Diabetes and Metabolic Diseases, Clinical Center of Serbia; Medical School, Univerisity of Belgrade

Objective: The aim of the study was to evaluate efficacy and tolerability of two models of glucocorticoid therapy that has been applied in 132 euthyroid patients with untreated and active moderate to severe Graves' orbitopathy. All patients were treated consecutively with combined intravenous and oral glucocorticoids (Combined GC group) in the initial three years period (66 patients, $49 \pm 10$ years), and with intravenous glucocorticoids (IVGC group) in the second evaluated period ( 66 patients, $50 \pm 11$ years).

Methods: Combined GC therapy included $500 \mathrm{mg}$ of methylprednisolone in $500 \mathrm{ml}$ of saline solution for two alternative days (day 1 and day 3) followed by oral prednisone tapering dose that was repeated each month for the next 5 months. Cumulative dose was $10.2 \mathrm{~g}$. IVGC therapy included infusions of $500 \mathrm{mg}$ of methylprednisolone for the first six weeks, and then infusions of $250 \mathrm{mg}$ for the remaining six weeks. Cumulative dose was $4.5 \mathrm{~g}$. Patients were evaluated at baseline and at the end of therapy (after 6 and 3 months, respectively).

Results: The overall success of the treatment was $67 \%$ in Combined GC group and $52 \%$ in IVGC group $(\mathrm{p}=0.135)$. Improvement in CAS was similar in both goups (Combined GC $83 \%$ vs. IVGC $85 \%, p=0.842$ ). However Combined GC group was more effective in all evaluated ocular measurements (diplopia, proptosis, eyelid aperture, visual acuity) reaching significance only

\section{KARGER \\ (C) 2014 European Thyroid Association \\ Published by S. Karger AG, Basel}


for inferior rectus muscle thickness. In regard to adverse effects of GC therapy, Combined GC therapy induced significantly more mild to moderate adverse events $(75 \%$ vs. $43 \%, \mathrm{P}<0.001)$. After 6 months relapse was observed in no patients treated with Combined GC therapy, and 9/34 (26\%) patients with IVGC therapy $(\mathrm{P}<0.001)$.

Conclusions: Combined GC therapy with high cumulative dose was more efficient with significantly less relapse rate, but with more induced side effects in comparison to IVGC therapy.

\section{P04 \\ PREVAlence Of ACUTE liver damage DURING OR FOLLOWING INTRAVENOUS GLUCOCORTICOID TREATMENT FOR GRAVES' OPHTHALMOPATHY: RESULTS OF AN EPIDEMIOLOGICAL STUDY}

Eleonora Sisti ${ }^{1}$, Eleonora Albano ${ }^{1}$, Marenza Leo ${ }^{1}$, Francesca Menconi ${ }^{1}$, Barbara Mazzi ${ }^{1}$, Francesco Latrofa ${ }^{1}$, Roberto Rocchi ${ }^{1}$, Paolo Vitti ${ }^{1}$, Claudio Marcocci ${ }^{1}$, Michele Marinò ${ }^{1}$

${ }^{1}$ Endocrinology Unit, University of Pisa

Several cases of acute liver damage (ALD), a few of which lethal, have been reported during or following intravenous glucocorticoid (ivGC) treatment for Graves' Ophthalmopathy (GO). These reports have raised doubts on ivGC safety, because of which, in our Institution, several preventive measures have been applied, including an extensive liver evaluation before treatment, a reduction of the ivGC dose, and, in order to prevent autoimmune-rebound hepatitis, the administration of low-dose oral glucocorticoid after completion of ivGC, and, in patients with serum autoantibodies associated with autoimmune hepatitis, also during ivGC. Here we evaluated retrospectively the occurrence of ALD in 500 consecutive patients with GO observed between 2008 and 2012 and who were scheduled to undergo ivGC. Because of the presence of liver steatosis at ultrasound, 24 patients were excluded. Thus, 476 patients ( 113 males and 363 females, age $49 \pm 13.6 \mathrm{yr}$ ) were given ivGC. Liver enzymes were measured every other week up to 3 months after completion of ivGC, and then monthly up to 6 months. DEA, which was defined by the presence of serum ALT values $\geq 300 \mathrm{U} / \mathrm{L}$ and/or of serum AST values $\geq 200 \mathrm{U} / \mathrm{L}$, was observed in five patients, thereby giving an overall morbidity of $1.05 \%$. In all of these five cases liver enzymes returned within the normal range spontaneously, within 2-4 months from the initial observation. No signs or liver failure were detected, both on clinical and biochemical grounds, and patients were asymptomatic. Considering that before 2008 DEA was observed in 14 of approximately 800 patients (morbidity $\sim 1.75 \%$ ) and that in 3 cases it was lethal (mortality $\sim 0.37 \%$ ), we concluded that, because of the new preventive measures we applied, DEA morbidity was slightly reduced and mortality was abolished, and more in general, that if appropriate preventive measures are applied, ivGC is a relatively safe treatment concerning the liver.

\section{P05 \\ LOW DOSE OF RITUXIMAB FOR CORTICOSTEROID-RESISTANT GRAVES' ORBITOPATHY \\ Laurence Du Pasquier Fediaevsky ${ }^{1}$, Sebastien Riviere ${ }^{2}$ \\ Michel Berche ${ }^{1}$, Michel Tazartes ${ }^{1}$, Laurence Leenhardt ${ }^{3}$, \\ Emmanuel Héron ${ }^{1}$ \\ ${ }^{1}$ Centre Hospitalier National D'ophtalmologie des XV XX, ${ }^{2} \mathrm{Hopital}$ \\ Saint-Antoine, ${ }^{3} \mathrm{Groupe}$ Hospitalier Pitié Salpétrière}

There is still limited experience with conventional and low dose Rituximab (RTX) therapy in Graves Orbitopathy (GO).

Objective: To report the outcome of low dose RTX as a third line therapeutic option in a cohort of active (clinical activity score (CAS) $\geq 4$ ), moderate to severe GO.

Methods: From 2011 to 2013, 151 patients were treated with IV methylprednisolone (MP) for active, moderate to severe GO, $39 \%$ of the patients were non responsive or relapsed. Second line treatment was orbital radiotherapy associated with oral steroids $(n=39)$ or oral steroids alone $(n=20)$. Seven patients who remained active and one patient with steroid intolerance were treated by RTX. All of them ( 2 men, 6 women; age range, 29 to 60 years) were euthyroid, three had received orbital radiotherapy 9 months to 5 years before RTX and 5 were dependent on oral steroids.

RTX treatment was given by two IV infusions of $100 \mathrm{mg} 2$ weeks apart.

Results: At the last visit, 6 to 18 months after RTX treatment, mean CAS improved from $4.4 \pm 0.74$ to $1 \pm 1.85(\mathrm{P}<0.01)$ while oral steroids were discontinued for all the patients. Two patients received a third RTX infusion at 2 months and 6 months because of relapse of GO activity. One patient with optic neuropathy failed to improve after RTX. Proptosis, lid signs and diplopia remained unchanged. There was a significant reduction in thyrotropin receptor antibodies $(\mathrm{P}<0.01)$.

RTX infusions were well-tolerated with no significant reaction or adverse event during the follow-up.

Conclusion: In this study, low doses of RTX for steroids and radioresistant active GO was safe and appeared to be associated with resolution of orbital inflammation. Prospective trials are needed to determine the place of RTX in the therapeutic strategy for active GO.

\section{P06 \\ ADJUVANT TREATMENT OF GRAVES DISEASE WITH DICLOFENAC - EFFECTS ON OPHTHALMOPATHY, ANTI-TPO TITERS AND \\ SAFETY}

Mikael Lantz ${ }^{1}$, Jan Calissendorff ${ }^{2}$, Frank Träisk ${ }^{3}$, Leif Tallstedt', Tereza Planck ${ }^{1}$, Ove Törring ${ }^{2}$, Bengt Hallengren ${ }^{1}$, Peter Åsman ${ }^{5}$

${ }^{1}$ Department of Endocrinology, Skåne University Hospital, ${ }^{2}$ Department of Internal Medicine, Division of Endocrinology, Södersjukhuset,

${ }^{3}$ Department of Clinical Neurisciences, St Erik Eye Hospital,

${ }^{4}$ Department of Clinical Neurosciences, St Erik Eye Hospital,

${ }^{5}$ Department of Ophthalmology, Skåne University Hospital

Objective: Due to the knowledge that retrobulbar morphological changes often are present in patients with Graves' disease (GD) already at diagnosis of thyrotoxicosis it may be of importance to interact as early as possible. Cyclooxygenase type 2 (COX-2) is overexpressed in active Graves' ophthalmopathy (GO) and inhibitors of this enzyme may affect the disease. The aim of this trial was to investigate if adjuvant treatment of GD with the COX inhibitor and PPARgamma antagonist diclofenac prevent ophthalmopathy and if laboratory parameters are affected.

Methods: This is a multicenter trial where subjects were randomised to methimazol (block and replace) either with or without diclofenac $50 \mathrm{mg} \mathrm{1 \times 2}$ for 12 months. The primary end-point development of GO after 24 months was evaluated. Smoking habits were registered and the thyroid parameters TSH, fT4, fT3, TRAb and anti-TPO were followed. Safety parameters (kidney, liver and blood) and adverse events were regularly registered.

Results: We have found the adverse event profile acceptable without any severe events related to diclofenac. Both TRAb and anti-TPO titers decreased during treatment with methimazol but the anti-TPO titers were lower in patients treated with diclofenac after 15 months $(p=0.031)$. The TRAb titers were not significantly changed between groups. Smokers had higher titers of TRAb than non-smokers both at diagnosis of GD $(p=0.048)$ and after 15 months $(p=0.042)$. The replacement dose of L-thyroxin and the number of dose changes were higher in the diclofenac treated group after 12 months $(\mathrm{p}=$ $0.037)$. GO developed in $11 \%(n=3)$ of the patients treated with diclofenac and in $21 \%(\mathrm{n}=6)$ of the controls $(\mathrm{p}=>0.05)$.

Conclusions: There was a trend that Diclofenac may reduce development of GO but significance was not reached. Diclofenac reduces anti-TPO titers and seems to be safe to use in GD patients. In addition smokers have increased titers of TRAb. 


\section{P07 \\ PROTECTIVE EFFECTS OF SELENIUM ON CELL VITALITY IN ORBITAL FIBROBLASTS FROM PATIENTS WITH GRAVES' OPHTHALMOPATHY}

Giovanna Rotondo Dottore ${ }^{1}$, Eleonora Sisti', Marenza Leo ${ }^{1}$, Eleonora Albano ${ }^{1}$, Francesca Menconi ${ }^{1}$, Roberto Rocchi', Paolo Vitti', Michele Marinò ${ }^{2}$, Giamberto Casini ${ }^{3}$, Claudio Marcocci ${ }^{1}$

${ }^{1}$ Endocrinology Unit, University of Pisa, ${ }^{2}$ Endocrinology Unit, University Hospital, University of Pisa, ${ }^{3}$ Ophtalmology Unit, University of Pisa

Oxidative stress is one of the main mechanisms involved in the perpetration of the autoimmune reaction responsible for Graves' Ophthalmopathy (GO). In a recent study is was shown that the antioxidant agent selenium has some beneficial effects in patients with mild GO. However, little is known on the actions of selenium in vitro. Here we investigated the effects of selenium in primary cultures of orbital fibroblasts from six patients with GO and five control subjects. To induce oxidative stress, fibroblasts were challenged with $\mathrm{H}_{2} \mathrm{O}_{2}$, which resulted in a mean reduction of cell vitality of $38 \%$ in GO fibroblasts and of $19 \%$ in control fibroblasts. When cells when co-incubated with selenium (selenium-methylcysteine), cell vitality increased by approximately $19 \%$ in both GO and control fibroblasts, an effect that was not observed when cells were co-incubated with methylcysteine, used as a control. Based on these findings, we concluded that oxidative stress reduces cell vitality in orbital fibroblasts, an effect that is less pronounced in control than in GO fibroblasts, which therefore seem to be more sensitive to oxidative stress. Selenium seems to protect from the effects of oxidative stress on cell vitality regardless of the origin of orbital fibroblasts. We considered the possibility that our observations reflected an effect of oxidative stress and of selenium on necrosis and/ or apoptosis, which, however, were not affected. Thus, it is reasonable to postulate that oxidative stress and selenium act mainly on cell cycle, which we are currently investigating. Following $\mathrm{H}_{2} \mathrm{O}_{2}$ challenge, there was a massive release of hyaluronic acid from the cells, which was to some extent prevented by selenium, but not by methylcysteine. Thus, we concluded that in addition to its effect on cell vitality, selenium protects from hyaluronic acid release, one of the major events in determining the pathological changes of GO.

\section{P08 \\ IFN- $\alpha,-\beta$, AND $-\gamma$ MODULATE CXCL8 CHEMOKINE SECRETION IN PRIMARY CULTURES OF THYROCYTES IN GRAVES'

Silvia Martina Ferrari ${ }^{1}$, Poupak Fallahi ${ }^{1}$, Stefano Sellari Franceschini ${ }^{1}$, Alessandro Antonelli ${ }^{\circ}$

${ }^{1}$ University of Pisa

Objective: In chronic inflammation in autoimmune thyroid diseases (AITD), interferons (IFNs) and tumor necrosis factor- $\alpha$ (TNF- $\alpha$ ) cooperate in activating several inflammation-related gene. Much is known about the positive signaling of IFNs to activate gene expression in AITD, while the mechanisms by which IFNs negatively regulate genes remain less studied. While IFNs inhibit (C-X-C motif) ligand 8 (CXCL8) chemokine secretion in several human cell types, their effects on thyroid and orbital cells in Graves' disease were not evaluated. Our aim was to study the interplay between TNF- $\alpha$ and type I or type II IFNs on CXCL8 secretion by these cells.

Methods: CXCL8 was measured in supernatants of primary cultures of thyroid cells, and orbital fibroblasts and preadipocytes basally and after a $24 \mathrm{~h}$ incubation with TNF- $\alpha$.

Results: CXCL8 was detected in all cell types supernatants in basal conditions being significantly increased by TNF- $\alpha$. Twenty-four hours incubation with IFN- $\gamma$ or IFN- $\beta$ or IFN- $\alpha$ dose dependently and significantly inhibited both basal and TNF- $\alpha$-induced CXCL8 secretion. The degree of the inhibitory effect was IFN- $\gamma>$ IFN- $\beta>$ IFN- $\alpha$.

Conclusion: This study demonstrates that type I and type II IFNs downregulate both basal and TNF- $\alpha$-induced CXCL8 secretion by human thyroid and orbital cells in Graves' disease, IFN- $\gamma$ being the most powerful inhibitor.

\section{P09}

CELL DENSITY DEPENDENT PAI-1 EXPRESSION IN ORBITAL FIBROBLASTS

Erika Galgoczi ${ }^{1}$, Florence Jeney ${ }^{1}$, Annamaria Gazdag ${ }^{1}$, Zita Steiber ${ }^{2}$ Bernadett Ujhelyi2, Ferenc Györy ${ }^{3}$, Mónika Katkó ${ }^{1}$, Endre V. Nagy ${ }^{1}$ ${ }^{1}$ Division of Endocrinology, Department of Medicine, University of Debrecen, ${ }^{2}$ Department of Ophthalmology, University of Debrecen,

${ }^{3}$ Department of Surgery, University of Debrecen

Important antiproliferative signals are mediated by cell-cell contacts in fibroblasts. Contact inhibition regulates extracellular matrix (ECM) metabolism of fibroblasts and expression of several proteins, including plasminogen activator inhibitor type 1 (PAI-1). The serine protease inhibitor PAI-1 plays a major role in the turnover of the ECM by inhibition of pericellular proteolysis. Fibroblast proliferation as well as overproduction of the ECM are important factors in the pathogenesis of Graves' orbitopathy (GO), and the role of PAI-1 in this process is still unclear.

Orbital connective tissue explants were obtained from patients undergoing orbital decompression surgery for GO. Control normal orbital tissues were obtained during surgery for non-orbital eye diseases and control dermal connective tissues were obtained during abdominal hernia operations from patients with no history of thyroid diseases. Primary cultures of fibroblasts were established from tissue samples, and cultured at different cell densities. PAI-1 expression was measured by ELISA and RT-PCR.

Regardless of anatomic site of origin, fibroblasts synthesized and released comparable amounts of PAI-1 into the culture medium when normalized for cell number. The protein and mRNA levels of PAI-1 per cell decreased with higher cell density. These observations were similar for all cell lines examined, but it was more pronounced in orbital fibroblast cultures. We found no difference in the cell density dependent PAI-1 expression between normal and $\mathrm{GO}$ orbital fibroblasts.

These in vitro findings indicate that high cell density is a negative regulator of PAI-1 expression in the orbital connective tissue as well. Cell density dependent regulation of PAI-1 expression in the orbit, together with other local factors, might have a regulatory role in the turnover of the ECM typical of the orbit.

This work was supported by the Hungarian Scientific Research Fund (Grant number OTKA K105733).

\section{P10 \\ ROLE OF ORBITAL FIBROBLASTS FOR PATHOLOGICAL VASCULARIZATION IN GRAVES' ORBITOPATHY CAUSED BY INDUCTION OF HYPOXIA-DEPENDENT SIGNALING}

Gina-Eva Görtz' ${ }^{1}$, Mareike Horstmann ${ }^{1}$, Buena Delos Reyes ${ }^{2}$, Klaus-Peter Steuhl', Joachim Fandrey ${ }^{2}$, Anja Eckstein ${ }^{3}$,

Utta Berchner-Pfannschmidt ${ }^{1}$

${ }^{1}$ Department of Ophthalmology, University Hospital Essen, ${ }^{2}$ Institute for Physiology, University Hospital Essen, ${ }^{3}$ Universität Essen; Augenklinik; Abteilung Für Erkrankungen des Vorderen Augenabschnittes, Orthoptik

Purpose: Graves' orbitopathy (GO) is an inflammatory eye disease that affects $25-50 \%$ of patients with Graves' disease. The complex pathophysiology of GO includes accelerated adipogenesis and muscle fibrosis. However, the expansion of the orbital tissue within the space-limited bony orbit may cause tissue hypoxia. Herein, we investigated the vascularization of orbital tissues and hypoxia-dependent signaling in resident orbital fibroblasts (OF).

Methods: We collected retrobulbar fat biopsies from GO patients and control healthy persons. PECAM-1 was used to quantify vessel density in sections of the biopsies. To analyze the potential role of OF for vascularization we isolated primary human OFs from the orbital fat biopsies and analyzed VEGF production using real time RT-PCR and ELISA. To examine whether hypoxia-dependent signaling is responsible for VEGF production, we suppressed the expression of the oxygen-labile $\alpha$-subunit of Hypoxia-inducible factor-1 (HIF-1 $\alpha$ ) by using siRNA approach. HIF-1 $\alpha$ level were determined by real time RT-PCR and Western-blot analysis.

Results: We found an increase in PECAM-1 positive vessels in fat biopsies of GO patients. OF isolated from GO orbital biopsies showed increased VEGF production, which was blocked with HIF-1 $\alpha$ specific siRNA treatment. Consistently, HIF-1 $\alpha$ mRNA and protein were increased in GO-OFs. Finally, 
we found hypoxia-induced stabilization HIF-1 $\alpha$ positively correlated with clinical activity score of GO patients.

Conclusions: Our results indicate that hypoxia-dependent signaling is induced in GO-OF. Our findings suggest that an increase in HIF-1 dependent VEGF production stimulates angiogenesis in orbital fat tissue of GO patients as an adaptive mechanism towards tissue hypoxia.

\section{P11 \\ ALL GRAVES' PATIENTS hAVE ACTIVITY SIGNS IN THE ORBITS IN EARLY STAGE OF GD IRRESPECTIVE OF DEVELOPMENT OR LACK OF GRAVES' ORBITOPATHY DURING A ONE YEAR FOLLOW-UP \\ Eszter Berta ${ }^{1}$, Miklós Bodor², Lajos Szabados ${ }^{3}$, László Galuska³ Erdei Annamaria ${ }^{4}$, Annamária Gazdag ${ }^{5}$, Bernadett Ujhelyi \\ Endre Nagy V. ${ }^{5}$ \\ ${ }^{1}$ Division of Endocrinology, Department of Medicine, University of Debrecen, Debrecen, Hungary; Division of Clinical Pharmacology, Faculty of Pharmacy, University of Debrecen, Debrecen, Hungary, ${ }^{2}$ Department of Medicine, Division of Endocrinology, University of Debrecen, Debrecen, Hungary; Division of Clinical Pharmacology, Faculty of Pharmacy, University of Debrecen, Debrecen, Hungary, ${ }^{3}$ Department of Nuclear Medicine, University of Debrecen, Debrecen, Hungary, ${ }^{4}$ Department of Medicine, Division of Endocrinology, University of Debrecen, Debrecen, Hungary, ${ }^{5}$ Division of Endocrinology, Department of Medicine, University of Debrecen, Debrecen, Hungary, ${ }^{6}$ Department of Ophthalmology, University of Debrecen, Debrecen, Hungary}

Objectives: Graves' orbitopathy (GO) is a common complication of Graves' disease (GD) which is often responding poorly to therapy. GO may develop before, together with or during the course of Graves' disease. In an individual patient, the development of GO cannot be predicted. We assumed that orbital autoimmune activity is predictable using orbital ${ }^{99 \mathrm{~m}} \mathrm{Tc}$-labelled diethylenetriamine pentaacetic acid ( $\left.{ }^{99 \mathrm{~m}} \mathrm{Tc}-\mathrm{DTPA}\right)$ SPECT. We aimed to determine whether any orbital autoimmune activity can be identified in patients who do not develop GO during their follow-up.

Methods: Fifty-four orbits of 27 patients newly diagnosed with GD were entered into the study. Patients with present GO were excluded. None of the patients had received antithyroid drugs or ophthalmic measures before entering the study. An initial ${ }^{99 \mathrm{~m}} \mathrm{Tc}-\mathrm{DTPA}$ SPECT was performed and DTPA uptake as sign of disease activity calculated in each case. SPECT was repeated during follow-up if clinical signs of GO occurred, and a final SPECT was performed at the end of the follow-up period, after one year. Twenty orbits of control patients who underwent DTPA SPECT of the hands for Raynaud's phenomenon served as controls.

Results: During the one year follow-up, 6 out of the 27 patients (22\%) were diagnosed with newly developed GO. The mean initial DTPA uptake of the orbits of GD patients with or without later developing GO was significantly higher than results obtained from the control group $(10.45 \pm 1.72 \mathrm{MBq} /$ $\mathrm{cm}^{3}, 9.18 \pm 1.18 \mathrm{MBq} / \mathrm{cm}^{3}$ and $7.7 \pm 2.44 \mathrm{MBq} / \mathrm{cm}^{3}$, respectively, $\mathrm{P}<0.05$ ). Initial DTPA uptake values of the 6 patients who later developed GO were not different from the rest of the GD group.

Conclusions: All GD patients have mild orbital activity signs with DTPA technique in the early stage of GD, irrespective of development or lack of GO during the course of GD. This uniform ongoing subclinical inflammation may be a reversible early stage of GO.

\section{P12}

TIMELY RADIOIODINE THERAPY IS A SAFE AND ADVANTAGEOUS TREATMENT OF PATIENTS WITH MODERATE-TO-SEVERE GRAVES' ORBITOPATHY

Edvard Pirnat ${ }^{1}$, Simona Gaberšček ${ }^{2}$, Daša Šfiligoj ${ }^{1}$,

Polona Jaki Mekjavic ${ }^{3}$, Katja Zaletel' ${ }^{1}$, Sergej Hojker ${ }^{2}$

${ }^{1}$ University Medical Centre Ljubljana, Department of Nuclear Medicine, 2University Medical Centre Ljubljana, Department of Nuclear Medicine; University of Ljubljana, Faculty of Medicine, ${ }^{3}$ University Medical Centre Ljubljana, Eye Hospital; University of Ljubljana, Faculty of Medicine

Objectives: Radioiodine (RAI) is a safe and effective definitive treatment of Graves' disease (GD) with a risk of worsening of Graves' orbitopathy (GO). In patients with concomitant moderate-to-severe GO, a special caution is usually recommended. Our aim was to retrospectively evaluate the effect of RAI on GO in this group of patients.

Methods: We reviewed records of 724 patients, 569 females and 155 males first diagnosed with GD between 2005 and 2009, and later treated with RAI irrespective of severity or activity of GO. In this study we only included patients with moderate-to-severe active GO (Group 1) and patients without GO before RAI (Group 2) but no patients with mild GO. The course of GO was followed for 12 months after RAI.

Results: Before RAI, we established 37/724 (5.1\%) patients from Group 1 and 553/724 (76.4\%) patients from Group 2. With respect to age, Group 1 and Group 2 did not differ significantly $(47.9 \pm 12.2$ and $46.6 \pm 1.7$, respectively, $\mathrm{p}=0.563$ ). Patients from Group 1 were treated with RAI significantly earlier than patients from Group $2(6.4 \pm 4.6$ and $16.5 \pm 13.9$ months, respectively, $\mathrm{P}<$ $0.001)$, and they received significantly higher first dose of RAI (706.4 \pm 110.9 and $620.4 \pm 105.2 \mathrm{MBq}$, respectively, $\mathrm{P}<0.001)$. Immediately after RAI, all patients from Group 1 received oral or intravenous glucocorticoids for 1 to 3 months, and in Group 2, twelve patients received short-term oral glucocorticoids. Hypothyroidism after RAI was promptly corrected. In Group 1, an amelioration of GO in 35 and no change in 2 patients during the follow-up was established, while in Group 2, an occurrence of GO in 22 patients was noticed (chi-square, $\mathrm{P}<0.001$ ).

Conclusions: In patients with moderate-to-severe GO, timely RAI therapy with concomitant glucocorticoids represents a safe definitive treatment of GD accompanied with the amelioration of GO during the 12-months period after RAI.

\section{PO2 Cardiovascular Effects}

\section{P13 \\ THYROID HORMONE (TH) REDUCED HEART SIRTUIN1 (SIRT1) PROTEIN EXPRESSION, BUT RESVERATROL ADMINISTRATION COULD NOT PREVENT THE TH-INDUCED HEART PHENOTYPE \\ Aline Cordeiro ${ }^{1}$, Lorraine Soares Oliveira ${ }^{1}$, Luana Lopes Souza ${ }^{1}$, Güínever Império ${ }^{1}$, Norma Almeida ${ }^{2}$, \\ Carmen Cabanelas Pazos-Moura ${ }^{1}$ \\ ${ }^{1}$ Federal University of Rio de Janeiro, ${ }^{2}$ Federal Rural University of Rio de Janeiro}

Objectives: (I) To verify whether Sirtuin1 (SIRT1) expression is regulated by thyroid hormone (TH) in the heart and (II) to analyze the effect of possible SIRT1 activation, by resveratrol administration, on ventricular hyperthrophy and gene/protein expression pattern induced by TH.

Methods: (I) Hypothyroid mice (HYPO - propyl-thyouracil $0.15 \%$ chow during 4 weeks), and hyperthyroid ones (HYPER - daily T3 injections $50 \mathrm{mg} / 100 \mathrm{gBW}$ for 14 days). (II) Resveratrol was administrated $(5 \mathrm{mg} / 100$ gBW) by gavage to euthyroid (EU+R) and hyperthyroid (HYPER $+\mathrm{R})$ mice during 14 days, simultaneously to hormone injections, compared to EU and HYPER. Morphometry was analyzed by eosin-and-hematoxylin-stained ventricular histological sections. Protein content was investigated by western blot- 
ting and mRNA and miR expression, by real time PCR. Statistical analysis: One-way ANOVA, post-test Student-Newman-Keuls $\left({ }^{*} \mathrm{P}<0.05, \mathrm{n}=6-8\right)$.

Results: HYPER presented increased ventricle mass and decreased SIRT1 protein content, while HYPO exhibited opposite pattern $(\mathrm{SIRT} 1 \mathrm{HYPO}=1.16 \pm 0.06 *, \quad \mathrm{EU}=1.00 \pm 0.03, \quad \mathrm{HYPER}=0.78 \pm 0.09 *$; mass $: \mathrm{HYPO}=3.86 \pm 0.16^{*}, \mathrm{EU}=4.37 \pm 0.07, \mathrm{HYPER}=5.27 \pm 0.07 * \mathrm{mg} / \mathrm{gBW}$ ), HYPER + R showed increased SIRT1 protein content in relation to HYPER and $\mathrm{EU}\left(\mathrm{EU}=1.00 \pm 0.10, \mathrm{EU}+\mathrm{R}=0.99 \pm 0.09, \mathrm{HYPER}=0.63 \pm 0.10^{*}, \mathrm{HYPER}+\mathrm{R}=\right.$ $1.78 \pm 0.30 *$, while ventricular mass was increased in HYPER and HYPER $+R$ (mass:EU $=62.74 \pm 1.75, \mathrm{EU}+\mathrm{R}=62.78 \pm 0.94, \mathrm{HYPER}=85.45 \pm 1.59^{*}$, HYPER+R: $86.48 \pm 1.56^{*} \mathrm{mg} / \mathrm{cm}$ of tibial length), as well as the hypertrophy marker atrial-natriuretic-factor (ANF) mRNA expression and the miR208a expression, involved in hyperthyroidism-induced cardiac hypertrophy $\left(\mathrm{ANF}: \mathrm{EU}=0.90 \pm 0.86 ; \mathrm{EU}+\mathrm{R}=1.19 \pm 0.18 ; \mathrm{HIPER}=1.53 \pm 0^{*}, 18 ; \mathrm{HIPER}+\mathrm{R}=\right.$ $1.47 \pm 0.25^{*} ; \quad \operatorname{RiR} 208 \mathrm{a}: \mathrm{EU}=0.04 \pm 0.01, \mathrm{EU}+\mathrm{R}=0.04 \pm 0.01$, HYPER $=$ $0.06 \pm 0.005$, HYPER $+\mathrm{R}=0.09 \pm 0.01 *)$. Then, resveratrol could not avoid ventricular hypertrophy development. SIRT1 mRNA levels were unaltered. TH positively-regulated-genes as a-myosin-heavy-chain (MHCa), sarcoplasmic-reticulum-calcium-ATPase (SERCA) and peroxisome-proliferator-activated-receptor-gamma-coactivator-1-alpha (PGCla) mRNA expression were increased in HYPER, while HYPER $+R$ presented intermediary values between EU and HYPER (MHCa:EU $=1.00 \pm 0.06$; $\mathrm{EU}+\mathrm{R}=1.15 \pm 0.15$; HIPER $=$ $1.65 \pm 0.29 *$ HIPER $+\mathrm{R}=1.34 \pm 0.30 ; \quad$ SERCA:EU $=1.00 \pm 0.10 ; \mathrm{EU}+\mathrm{R}=$ $1.98 \pm 0.55 ; \mathrm{HIPER}=5.50 \pm 1.6^{*} ; \mathrm{HIPER}+\mathrm{R}=4.34 \pm 1.6 ; \mathrm{PGC} 1 \mathrm{a}: \mathrm{EU}=1.00 \pm 0.05$, $\mathrm{EU}+\mathrm{R}=2.22 \pm 0.45^{*}, \mathrm{HYPER}=1.94 \pm 0.12 *$, HYPER $+\mathrm{R}=1.37 \pm 0.27$, suggesting SIRT1 may influence TH action. Peroxisome-proliferator-activatedreceptor-alpha (PPARa) protein content was decreased in HYPER and even more in HYPER+R compared to $\mathrm{EU}(\mathrm{EU}=1.00 \pm 0.12, \mathrm{EU}+\mathrm{R}=0.80 \pm 0.12$, HYPER $=0.57 \pm 0.12 *$ HYPER $\left.+\mathrm{R}=0.25 \pm 0.05^{*}\right)$. Reports described PPARa as SIRT1 partner and also a protective factor, by this, its reduction could contribute to resveratrol failure in attenuating ventricular hyperthrophy.

Conclusion: SIRT1 is negatively regulated by TH, however, its protein content increment, and possible activity, induced by resveratrol, was unable to avoid hyperthyroidism-induced ventricle hyperthrophy.

\section{P14 \\ IMPACT OF DRONEDARONE ON THE MYOCARDIAL REMODELING IN EXPERIMENTAL HYPERTHYROID CARDIOMYOPATHY}

Bo Hyun Kim ${ }^{1}$, Kyoung IM Cho ${ }^{2}$, Kang Hee Ahn' ${ }^{1}$, Yoon Jeong Nam ${ }^{1}$, Jong Ho Kim¹, Soo Bin Park', Won Jin Kim¹, Yun Kyung Jeon ${ }^{1}$,

Sang Soo Kim ${ }^{1}$, In Joo Kim ${ }^{1}$

${ }^{1}$ Department of Internal Medicine, School of Medicine, Pusan National University, ${ }^{2}$ Cardiovascular Research Institute, Department of Internal Medicine, Kosin University College of Medicine

Background: This study aimed to evaluate the effects of dronedarone, which blocks multiple cardiac ion channels and $\beta$-adrenoceptors, on the functional and structural manifestations of experimental hyperthyroid cardiomyopathy.

Methods: A rat model of hyperthyroidism was established by daily intraperitoneal injections of L-thyroxine $\left(\mathrm{T}_{4}, 100 \mathrm{mg} / \mathrm{kg}\right.$ per day) for 4 weeks. Twenty-four Sprague-Dawley rats were randomly divided into three groups $\left(\mathrm{n}=8\right.$ each): control group, $\mathrm{T}_{4}$ group $\left(\mathrm{T}_{4}\right.$ alone), and $\mathrm{T}_{4}$ plus dronedarone group ( $\mathrm{T}_{4}$-Dro, $100 \mathrm{mg} / \mathrm{kg}$ dronedarone). All rats underwent echocardiography and cardiac chamber size and strain parameters were measured after 28 days of treatments. Cardiomyocyte diameter and cardiac fibrosis were measured using pathological analysis.

Results: Four-week-thyroxine treatment induced significant tachycardia, reduced systolic function and increased cardiac hypertrophy with fibrosis. However, co-administrated dronedarone decreased heart rate and improved systolic function without improvement of cardiac fibrosis. Specifically, $\mathrm{T}_{4}$-Dro group showed reduced left atrial (LA) dimension and increased peak systolic LA stain.

Conclusions: Dronedarone could significantly attenuate functional remodeling induced by experimental hyperthyroid cardiomyopathy, which is more pronounced in the left atrial structural change.

\section{P15 \\ HYPOTHYROIDISM IS ASSOCIATED WITH RETINAL ARTERIOLAR NARROWING IN THE GENERAL POPULATION}

Till Ittermann ${ }^{1}$, Marcus Dörr ${ }^{2}$, Henry Völzke ${ }^{2}$, Frank Tost ${ }^{2}$, Clemens Jürgens ${ }^{2}$

${ }^{1}$ University Medicine Greifswald; Institut Für Community Medicine Ship/Klinisch-Epidemiologische Forschung, ${ }^{2}$ University Medicine

Greifswald

Objective: Recent studies have shown associations of hypothyroidism with arterial blood pressure, atherosclerosis, and inflammation. Based on these pathways there might also be an association between hypothyroidism and retinal arteriolar narrowing (RAN), a marker of micro-vascular damage from hypertension, atherosclerosis, and inflammation. Against this background the aim of this study was to investigate the putative association between serum thyrotropin (TSH) levels and RAN defined by arterio-venous ratio (AVR) from static vessel analysis.

Material and Methods: We used data from 3189 individuals from the second population-based cohort of the Study of Health in Pomerania (SHIPTREND-0). Thyroid function was defined according to serum TSH and serum diiodothyronine (T2) levels. Low and high serum TSH levels were defined by the cut-offs $0.3 \mathrm{mIU} / \mathrm{L}$ and $3.0 \mathrm{mIU} / \mathrm{L}$. Fundus photography of the central retina was recorded with a non-mydriatic camera and images were evaluated by one experienced reader. An AVR $<0.8$ was defined as decreased. Serum TSH levels, low and high TSH, and serum T2 levels were associated with AVR by linear regression and with AVR $<0.8$ by Poisson regression, both adjusted for age, sex, cigarette smoking, alcohol consumption, and beta blocker intake.

Results: Serum TSH levels were significantly associated with AVR ( $\beta=$ $-0.028 ; 95 \%$ confidence interval $(\mathrm{CI})=-0.049 ;-0.007 ; \mathrm{p}=0.009)$ and with a decreased AVR $<0.8$ (relative risk $=2.05 ; 95 \% \mathrm{CI}=1.13 ; 3.73 ; \mathrm{p}=0.019$ ). Individuals with high TSH had a 1.43 higher risk for a decreased AVR (95\%$\mathrm{CI}=1.04 ; 1.96 ; \mathrm{p}=0.027)$ than individuals with serum TSH levels within the reference range. Serum T2 levels were also associated with a decreased AVR (Relative risk $=0.45 ; 95 \% \mathrm{CI}=0.23-0.87 ; \mathrm{p}=0.017$ ).

Conclusions: Our results substantiate evidence for an association between hypothyroidism and RAN. Potential mechanisms explaining this association are long-term hypertension, atherosclerotic processes, and inflammation.

\section{P16 \\ DEMONSTRATION OF NEW FUNCTIONAL EFFECTS OF T1AM \\ Juliane Dinter ${ }^{1}$, Jessica Müh/haus ${ }^{2}$, Carolin Leonie Piechowski ${ }^{3}$, Maxi Cöster ${ }^{4}$, Gunnar Kleinau ${ }^{3}$, Josef Köhrle ${ }^{5}$, Annette Grüters ${ }^{3}$, Heiko Krude ${ }^{3}$, Torsten Schöneberg ${ }^{4}$, Heike Biebermann ${ }^{3}$ ${ }^{1}$ Charité Universitätsmedizin Berlin Campus Virchow; Institute of Experimental Pediatric Endocrinology, ${ }^{2}$ Charité Universitätsmedizin Campus Virchow-Klinikum; Institute of Experimental Pediatric Endocrinology, ${ }^{3}$ Charité - Universitätsmedizin Berlin Campus Virchow; Institute of Experimental Pediatric Endocrinology, ${ }^{4}$ University of Leipzig; Institute of Biochemistry; Medical Faculty, ${ }^{5}$ Charité - Universitätsmedizin Berlin Campus Virchow; Institute of Experimental Endocrinology}

Injection of T1AM, a thyroid hormone derivative, in rodents results in decreased heart rate and lower body temperature, which is counterintuitive considering the traditional model of thyroid hormone action. Moreover, T1AM has been shown to have cardioprotective effects. These findings make T1AM a potential pharmacological target for treatment options in humans for infarct patients. Since T1AM injection in Taar1-deficient mice produces among no significant changes in body weight and body temperature, and 3-T1 AM injection still leads to above mentioned physiological effects it was speculated that other targets of T1AM might exist.

We took advantage of the label-free EPIC-technology to identify further signaling targets for T1AM. By using this technology we found ADRB1, ADRB2 and ADRA2A as potential T1AM targets after co-stimulation of isoprenaline (ISOP) or norepinephrine (NE) and T1AM. To verify these findings we expressed these receptors in HEK293 cells and measured cAMP accumulation. 
We demonstrate a modulatory effect of T1AM that enhance the ISOP/ NE induced cAMP signalling for ADRB2. In addition, T1AM activates the Gi/O pathway for ADRA2A. For ADRB1 we could not verify a modulating effect of T1AM.

Indeed, T1AM has more targets than TAAR1, namely diverse monoaminergic receptors. However, the previous exhibited negative ionotropic effects of T1AM on heart cannot be confirmed with our cell culture set-up. Our results rather suggest a synergistic effect of T1AM on ADRB2. Possibly, T1AM acts on additional targets or specific receptor-receptor interactions play a role in cardiac T1AM action. In conclusion, this study provides new functional effects of T1AM which have to be taken into consideration prior therapeutical targeting.

Supported by the Deutsche Forschungsgemeinschaft, SPP 1629 "Thyroid Trans Act" BI893/5-1.

\section{P17 \\ T3-INDUCED CARDIAC HYPETROPHY DEPENDS ON FUNCTIONAL THYROID RECEPTOR $\beta$ THAT REGULATES MIR-208A EXPRESSION \\ Güínever Império ${ }^{1}$, Letícia Santiago ${ }^{1}$, Isalira Ramos ${ }^{1}$, \\ Guilherme Pereira ${ }^{1}$, Norma Almeida ${ }^{2}$, Cesar Fuziwara ${ }^{3}$, Edna Kimura ${ }^{3}$ \\ Emerson Olivares ${ }^{2}$, Tania Ortiga ${ }^{1}$ \\ ${ }^{1}$ Federal University of Rio de Janeiro, ${ }^{2}$ Federal Rural University of Rio \\ de Janeiro, ${ }^{3}$ University of São Paulo}

We aimed to investigate if the microRNAs miR-208a and miR-1 were involved in TR $\beta$ regulation of $\mathrm{T}_{3}$-induced cardiac hypertrophy and its correlation with changes in cardiac gene expression and function. Adult wild type (WT) or homozygous (HO) mice for the TR $\beta \Delta 337 \mathrm{~T}$ mutation (does not bind $\mathrm{T}_{3}$ ), underwent hypothyroidism (PTU- $0.15 \%$ in the diet) or hyperthyroidism $\left(\mathrm{T}_{3} 50 \mu \mathrm{g} / 100 \mathrm{~g}\right.$ bw, sc) induction. Echo- and electrocardiogram were performed. Total RNA extraction from hearts was performed by the TRIzol method and mRNA or microRNAs expression were analyzed by qPCR. TR $\beta$ has a fundamental role in adaptive $T_{3}$-induced cardiac hypertrophy, since the hypertrophy expected after $\mathrm{T}_{3}$ treatment was not observed in $\mathrm{HO}$ mice (relative heart weight WT: $119.3 \pm 6.0$ vs. HO: $64.14 \pm 2.21 \mathrm{mg} / \mathrm{cm}, \mathrm{P}<0.001$, and left ventricular mass WT: $200.1 \pm 26.15$ vs. HO: $132.6 \pm 8.78 \mathrm{mg}, \mathrm{P}<0.05)$. The $\mathrm{T}_{3}$-induced cardiac hypertrophy observed in WT mice was considered adaptive, once it produced no impairment in systolic functional parameters, unlike the HO group, which exhibited a contractile deficit, assessed by echocardiogram, with a decrease of the ejection fraction (WT:45.69 $\pm 1.50 \mathrm{vs}$. HO:29.62 $\pm 1.57 \%$, $\mathrm{P}<0.05)$ and on stroke volume (WT:33.84 $\pm 1.80 v s . \mathrm{HO}: 24.65 \pm 1.07 \mu \mathrm{L}, \mathrm{P}<$ 0.05). Also, there was a reduction in $\mathrm{P}-$ Wave duration of $\mathrm{HO}$ mice, by electrocardiogram. Regarding heart gene expression, we identified a group of TR $\beta$ independently regulated genes, as adrenergic receptor (AR)- $\beta 2, \alpha$-myosin heavy chain (MHC) and hyperpolarization-activated cyclic nucleotide-gated channel, that were normally regulated by $T_{3}$ in HO group. However, AR- $\beta 1$, $\beta$-MHC and sarcoplasmic reticulum $\mathrm{Ca}^{++}$-ATPase genes were regulated via TR $\beta$. We also showed that ventricular expression of miR-1 is upregulated by THs independent of the TR $\beta$ pathway. Finally, our results suggested that the miR-208a has an important contribution in TR $\beta$ mediated cardiac hypertrophy, since the increase in its expression observed in WT animals was not verified in HO (WT: $0.0017 \pm 0.00032$ vs. HO: $0.00049 \pm 0.000087$ a.u., $\mathrm{P}<0.05$ ) after $\mathrm{T}_{3}$ treatment.

\section{P03 Thyroid and Environment 1}

\begin{abstract}
P18
PARITY AND 11-YEAR SERUM TSH AND THYROID AUTOANTIBODY CHANGE: A LONGITUDINAL POPULATION-BASED STUDY

Lena Bjergved ${ }^{1}$, Allan Carlé2, Torben Jørgensen ${ }^{3}$,

Hans Perrild ${ }^{4}$, Peter Laurberg ${ }^{5}$, Anne Krejbjerg ${ }^{2}$, Lars Ovesen 6 , Inge Bülow Pedersen ${ }^{2}$, Lone Banke Rasmussen ${ }^{7}$, Nils Knudsen ${ }^{4}$ ${ }^{1}$ Research Centre for Prevention and Health, The Capital Region of Denmark, Glostrup; Department of Endocrinology, Bispebjerg University Hospital, Copenhagen, ${ }^{2}$ Department of Endocrinologi, Aalborg University Hospital, Aalborg, ${ }^{3}$ Research Centre for Prevention and Health, The Capital Region of Denmark; Faculty of Health Sciences, University of Copenhagen; Faculty of Medicine, Aalborg University, Aalborg, ${ }^{4}$ Department of Endocrinology, Bispebjerg University Hospital, Copenhagen, ${ }^{5}$ Department of Endocrinologi, Aalborg University Hospital, Aalborg; Faculty of Medicine, Aalborg University, ${ }^{6}$ Department of Gastroenterology, Slagelse Hospital, Slagelse, ${ }^{7}$ Department of Nutrition, National Food Institute, Technical University of Denmark, Søborg
\end{abstract}

Objectives: A role for female reproductive factors in the etiology of thyroid autoimmunity has been suggested. We investigated the prospective association between parity, abortion, oral contraceptive pill use (OCP), use of hormone replacement therapy (HRT) and 11-year change in serum thyrotropin (TSH) as well as change in thyroid peroxidase autoantibody (TPO-Ab) status.

Methods: A random sample of 4,649 persons aged 18-65 years from a general population participated in the DanThyr C1 study in the period 19971998. We included 1,749 non-pregnant women without current or former treatment for thyroid disease and who participated at the 11-year follow-up examination in 2008-2010. Gynecological exposures were reported in a selfadministered questionnaire at baseline and follow-up. TSH and TPO-Ab were measured at baseline and follow-up. Increased TPO-Ab status during followup was defined as a TPO-Ab below assay cut-off $(<30 \mathrm{kU} / \mathrm{liter})$ at baseline and TPO-Ab $\geq 30 \mathrm{kU} /$ liter at follow-up. Multiple linear regression models were used, adjusted for age, smoking status, and urinary iodine excretion.

Results: We found an inverse association between the number of years on hormone replacement treatment and the risk (odds ratio) of increased TPO-Ab status during follow-up $(0.735(0.558,0.968), \mathrm{P}=0.03)$, but no association with parity, use of OCP, abortion, age at menarche and being pre- or postmenopausal. Also, parity, abortions, use of OCP, HRT use, age at menarche and being pre- or postmenopausal were not significantly associated with 11-year TSH change.

Conclusions: Our results may suggest a protective role for hormone replacement therapy in the etiology of thyroid autoimmunity. The absence of an association between parity and abortion and TSH as well as TPO antibody change in this longitudinal study suggests a minor, if any, role for microchimerism in development of thyroid autoimmunity.

\section{P19 \\ DOES INTRAPARTUM OR NEONATAL USE OF TOPICAL IODINE OR IODINATED CONTRAST MEDIA AFFECT THYROID FUNCTION OF PRETERM INFANTS? A PILOT STUDY}

Fiona Williams ${ }^{1}$, Anita Boelen ${ }^{2}$, Elena Velten ${ }^{3}$, Chris Day ${ }^{4}$, Aung Soe ${ }^{5}$, Sateesh Somisetty ${ }^{6}$

${ }^{1}$ University of Dundee, ${ }^{2}$ Academic Medical Centre, ${ }^{3}$ Ninewells Hospital and Medical School, ${ }^{4}$ Bradford Teaching Hospitals NHS Foundation Trust, ${ }^{5}$ Medway Maritime Hospital, ${ }^{6}$ Luton \& Dunstable Hospital NHS Foundation Trust

Background: The British National Formulary (BNF) states that infants $<32$ weeks gestation should not be exposed to topical iodine for skin disinfection, and recommends its avoidance during pregnancy and breast feeding. The use of iodinated contrast media is not covered by this guidance as it is not a listed drug. Some leaflets recommend monitoring thyroid function after 
administration of contrast media because of the risk of hypothyroidism due to iodine overload, but this is not current clinical practice.

Research Question: Are TSH levels in preterm infants affected by exposure to intrapartum or neonatal topical iodine and/or use of iodinated contrast media?

Methods: A cohort of 173 infants $<32$ weeks gestation was recruited from 5 hospitals in the UK. Maternal and neonatal exposure to iodinated contrast media and topical iodine was recorded; levels of TSH, T4 and TBG were measured from blood spot card on postnatal days 7, 14 and 28.

Results: 125 infants were exposed and 48 infants not exposed to topical iodine or contrast media. The mean gestation for exposed infants was $28 \pm 1.9$ wks and $29 \pm 1.9$ wks for the unexposed group. No infants were treated for hypothyroidism; $5.6 \%$ of the infants exposed to contrast media had raised TSH ( $\geq 6 \mathrm{mU} / \mathrm{L}$ ) blood spot levels (range $1-25 \mathrm{mU} / \mathrm{L}$ ) and $17.6 \%$ of infants exposed to topical iodine during delivery or neonatally had raised TSH levels (range 1-8 mU/L). In the unexposed group, $8.3 \%$ had raised TSH levels (range $1-8 \mathrm{mU} / \mathrm{L}$ )

Conclusions: In the context of this small pilot study some thyroid dysfunction was seen following use of topical iodine and contrast media. A large study is planned which can adjust for gestational age, non-thyroidal illness and quantify iodine exposure. Neonatal thyroid function is not routinely monitored post use of contrast media or following intrapartum exposure; consideration should be given to changing this care pathway.

\section{P20 \\ IS MEASUREMENT OF IODINE CONCENTRATION CONSISTENTLY RELIABLE TO ASSESS IODINE STATUS?}

Simone Johner ${ }^{1}$, Michael Thamm², Roma Schmitz ${ }^{2}$, Thomas Remer ${ }^{1}$

${ }^{1}$ University of Bonn, lel-Nutritional Epidemiology, Donald Study

Centre at the Research Institute of Child Nutrition, Dortmund, ${ }^{2}$ Central

Epidemiology Laboratory, Department of Epidemiology and Health

Reporting, Robert Koch Institute, Berlin

Objectives: Iodine status is one of the most important environmental factors affecting thyroid health. The common way to determine iodine status of a population is to assess median iodine concentration (I-conc) measured in a sufficient number of spot urine samples. Our aim was to compare I-conc with an alternative approach, relying on iodine and creatinine measurements, with regard to the respective interpretational outcome in a representative population.

Methods: Iodine and creatinine concentrations were measured in 7003 spot urine samples from the representative DEGS Study (German Health Interview and Examination Survey for adults, 2008-2011). Total daily iodine excretion was estimated by relating the iodine/creatinine ratio to age- and sexspecific creatinine excretion reference values.

Results: Median iodine concentration was $<65 \mu \mathrm{g} / \mathrm{L}$ in adults indicating mild iodine deficiency in the German population, with more than $70 \%<100$ $\mu \mathrm{g} / \mathrm{L}$. The calculation of total daily iodine excretion derived a median of $>110$ $\mu \mathrm{g} / \mathrm{d}$. When comparing the urinary-based estimated iodine intake $(\mu \mathrm{g} / \mathrm{d})$ to the current dietary reference intakes (DRIs), the proportion of adults with insufficient iodine intake (lying below the average requirement (EAR)) decreased to $30 \%$.

Conclusions: Using the conventional approach for the assessment of iodine status (I-conc) in the current representative nationwide survey suggests a reoccurrence of iodine deficiency in Germany. Assessing daily renal iodine output $(\mu \mathrm{g} / \mathrm{d})$ by the use of additional creatinine measurements and age-\&sexspecific creatinine excretion reference values instead, yields a different picture for German adults: an average (almost acceptable) iodine supply comparable to that recently observed in the representative German survey in children and adolescents. Therefore, our data strongly suggest that hydration status can massively interfere with the epidemiological iodine assessment parameter I-conc, even in large surveys.

This work was financially supported by the German Federal Ministry of Food and Agriculture (BMEL) through BLE, grant number 2813HS013

\section{P21 \\ PREPUBERTAL BISPHENOL A EXPOSURE IN RATS SHOWS A THYROID HORMONE ANTAGONIST ACTION ON HYPOTHALAMUS- PITUITARY-THYROID AXIS}

Marina M.L. Kizys ${ }^{1}$, Kellen C. Oliveira ${ }^{1}$, Renata M. Romano ${ }^{1}$, Gisele Giannocco ${ }^{2}$, Rui Macie ${ }^{\beta}$, Magnus R. Dias-da-Silva ${ }^{1}$,

Marco A. Romano ${ }^{4}$, Maria Chiamolera ${ }^{1}$

${ }^{1}$ Universidade Federal de São Paulo - Unifesp, ${ }^{2}$ Faculdade de Medicina Do Abc - Fmabc, ${ }^{3}$ Universidade Federal de Sao Paulo,

${ }^{4}$ Universidade Estadual Do Centro-Oeste Do Paraná - Unicentro

Bisphenol A (BPA) is one of the major compounds in plastic materials. BPA has been associated with endocrine disruption mostly due to the estrogenic effects on gonadal axis. Alterations in thyroid hormone axis have also been reported; in vitro and in vivo experiments showing that BPA acts as an antagonist to thyroid hormone in thyroid hormone receptors (TRs). The aim of the present study is to analyze BPA action on hypothalamus-pituitary-thyroid axis on gene expression classically regulated by thyroid hormone in vivo. In this study, prepubertal male Wistar rats were orally treated with 5 and 25 $\mathrm{mg} / \mathrm{kg} /$ day BPA from postnatal day 23 (PND23) to PND58 and sacrificed at PND102. Serum concentrations of TSH, T3 and T4 were measured. Total RNA was extracted from hypothalamus, pituitary, liver and heart. Gene expression was performed for the beta isoform of thyrotropin (Tshb)?, type 1 and 3 deiodinase (Dio1 and Dio3), alpha and beta isoforms of TRs (Tra and Trb), TSH release hormone (Trh), and alpha and beta isoforms of myosin heavy chain (a-Mhc and b-Mhc). Animals exposed to BPA presented a drift towards TSH elevation, paradoxically to an increase of total T3 and a reduction of T4, which could be explained by Dio1 gene expression increase observed in liver. In pituitary tissue, Tshb gene expression was elevated and Dio3 gene expression was repressed. In the heart, a-Mhc has reduction of the transcript amount, with no alteration in b-Mhc transcriptional levels. BPA-exposed animal gene expression profiles are similar to what it is expected for hypothyroid ones, which could be explained by the antagonistic action of BPA in TRs showed in former studies. Notwithstanding, BPA estrogenic action could also play a role in the complex regulatory mechanism of gene expression associated to T3.

\section{P22 \\ THE EFFECTS OF IODINE DEFICIENCY ON STOMACH, SALIVARY GLANDS AND MAMMARY GLANDS VASCULARIZATION \\ Jessica Vanderstraeten ${ }^{1}$, Julie Craps ${ }^{1}$, Benoît Lengelé ${ }^{1}$, \\ Marie-Christine Many ${ }^{1}$, Idesbald Colin ${ }^{2}$, Anne-Catherine Gérard ${ }^{2}$ \\ ${ }^{1}$ Université Catholique de Louvain, Morphologie Expérimentale, ${ }^{2} \mathrm{Chr}$ \\ Mons-Hainaut, Clinique Saint-Joseph}

Objectives: Despite the efforts to introduce salt iodization in iodine insufficient countries, iodine deficiency (ID) remains a global problem. In our laboratory, ID impact on microvascular changes in thyroid was extensively studied. We demonstrated that thyrocytes react to ID by secreting VEGF via HIF-1a stabilization, to increase their surrounding blood flow. However, other organs such as stomach, mammary and salivary glands express the sodium/ iodide symporter (NIS) and might also be affected by ID. The objective of this study is to compare the effects of ID on the vascularization of the three aforementioned organs with the effects observed in thyroid.

Methods: Eight week NMRI mice were fed with iodide deficient diet and perchlorate containing water (NIS inhibitor) during zero to ten days, with/ without bevacizumab (VEGF inhibitor). Blood flow was measured with a laser Doppler in mammary and salivary glands. HIF-1a protein levels were measured by western blot in salivary glands. VEGF expression was studied by immunochemistry.

Results: Salivary glands: HIF-1a protein expression was increased at day 1 of the treatment. Blood flow and VEGF protein expression was transiently increased from day 1 to day 4. Mammary glands: a transient increase in blood flow was observed at day 1 . However, there were no differences in VEGF expression between control and ID mice. Treatment with bevacizumab suppressed the increase in blood flow in both glands. Gastric mucosa: VEGF expression was strongly enhanced from day 1 to day 4 .

Conclusion: These data indicate that cells other than thyrocytes can react to ID by inducing transient microvascular changes, probably trying to adapt 
iodide inflow. The pathway seems to be similar since it involves VEGF contribution. As different disorders such as breast atypia, or breast/stomach tumor have been correlated to ID and thyroid enlargement, those results could provide enlightenment to understand this correlation.

\section{P23 \\ THYROID DYSFUNCTION AND ULTRASONOGRAPHY FEATURES IN PATIENTS WITH METASTATIC RENAL CELL CARCINOMA TREATED WITH TYROSINE KINASE INHIBITORS. PRELIMINARY RESULTS FROM A PROSPECTIVE COHORT STUDY \\ Fabiana Pani ${ }^{1}$, Germana Baghino ${ }^{1}$, Francesco Boi ${ }^{1}$, \\ Valentina Moledda ${ }^{1}$, Francesco Atzori², Clelia Madeddu ${ }^{2}$, \\ Maria Teresa Ionta ${ }^{2}$, Stefano Mariotti ${ }^{1}$ \\ ${ }^{1}$ Endocrine Unit, Department of Medical Sciences, University of \\ Cagliari, ${ }^{2}$ Medical Oncology, Department of Medical Sciences, \\ University of Cagliari}

Background: Tyrosine kinase inhibitors (TKI) may induce thyroid dysfunction, but the frequency of this phenomenon and the mechanisms involved remain to be elucidated.

Objective: Prospective evaluation of thyroid function, autoimmunity and morphology during treatment with TKI. To this purpose, 12 consecutive patients with metastatic renal cell carcinoma with comparable tumor staging, normal thyroid function and no evidence of associated thyroid autoimmunity, were studied before and at monthly intervals after beginning TKI (Pazopanib in 1 and Sunitinib in 11 cases) according to standard protocols. In all cases FT3, FT4, TSH and thyroid antibodies (TgAb and TPOAb) were measured together with clinical assessment and thyroid ultrasonography up to six months.

Results: Nine/12 patients $(75 \%)$ became hypothyroid (TSH $8.2 \pm 10.62$, range 114-6.08) within 60 days of therapy, one of them after a short course of transient thyrotoxicosis. High titres of TPOAb (3000 IU/ml and $126 \mathrm{IU} /$ $\mathrm{ml}$ ) became detectable in $2(20 \%)$ patients (all developing hypothyroidism) 4 and 6 months after beginning TKI. Thyroid volume significantly decreased in $11(92 \%)$ patients (from $12.5 \pm 11.6 \mathrm{ml}$ before to $6.2 \pm 4.24$ months after TKI, $\mathrm{P}<0.001$ by paired Student $\mathrm{t}$ test), together with the appearance of mild hypoechogenicity.

Conclusions:

These preliminary data strongly support a direct inhibitory effect of TKI, in keeping with the key role of kinases in thyroid function and growth. Furthermore, the novel appearance of TPOAb in $20 \%$ of the patients suggest that TKI many also trigger/exacerbate thyroid autoimmunity. Larger and longer studies are needed to ascertain the relative role of kinase inhibition and autoimmunity in determining thyroid failure, as well as the reversibility degree of thyroid TKI-induced thyroid dysfunction.

\section{P24 \\ USE OF THYROID MEDICATION AFTER INTRODUCTION OF THE DANISH IODINE FORTIFICATION PROGRAMME IN YEAR 2000}

\section{Betina Thuesen ${ }^{1}$, Charlotte Cerqueira ${ }^{1}$, Nils Knudsen², Lars Ovesen ${ }^{3}$,} Peter Laurberg ${ }^{4}$, Hans Perrild ${ }^{2}$, Lone Banke Rasmussen ${ }^{5}$,

Torben Jørgensen ${ }^{6}$

${ }^{1}$ Research Centre for Prevention and Health, Glostrup,

Denmark, ${ }^{2}$ Bispebjeg Hospital, Department of Endocrinology and

Gastroenterology, Copenhagen, Denmark, ${ }^{3}$ Slagelse Hospital,

Department of Gastroenterology, Slagelse, ${ }^{4}$ Aalborg University

Hospital, Department of Endocrinology, Aalborg, Denmark, ${ }^{5}$ National

Food Institute, Technical University of Denmark, Department of

Nutrition, Søborg, Denmark, ${ }^{6}$ Research Centre for Prevention and

Health, Glostrup, Denmark; University of Copenhagen, Faculty of

Health Sciences, Copenhagen, Denmark; Aalborg University, Aalborg,

Denmark

Objectives: In Denmark, mandatory iodization of salt was initiated in year 2000 because of mild to moderate iodine deficiency. The aim of this study was to evaluate the effect of the increased iodine intake on use of thyroid med- ication - antithyroid medication and thyroid hormone replacement therapy during a time period of 12 years.

Methods: Using the unique personal identification number of all Danes, data on use of thyroid medication during the period 1995-2012 from the Register of Medicinal Product Statistics were linked to information from the Civil Registration register. Use of medication was given as number of users per 100,000 inhabitants. Data were standardized to the age- and sex-composition of the Danish population in year 2000. Based on their residency, the Danish population was divided into two groups with mild (Eastern Denmark) versus moderate (Western Denmark) iodine-deficiency before fortification.

Results: Use of antithyroid medication had increased moderately in both regions from 1995 to 2000 where the mandatory iodization of salt was initiated. After 2000 the prevalence increased until 2003 in Western Denmark (560 users/100,000) and 2004 in Eastern Denmark (340 users/100,000). Since then the prevalence decreased and, in 2011, reached the before-fortification levels in both regions. After further reductions, use of antithyroid medication in 2012 was 432 and 287 users/100,000 Eastern and Western Denmark, respectively. Use of thyroid hormone replacement therapy increased in both regions from about 970 users/100,000 in 1995 to about 1200 users/100,000 in 2000. Since 2000 the prevalence increased steadily and in 2012 the prevalence was 2,108 and 1,923 users/100,000 in Eastern and Western Denmark, respectively.

Conclusions: Use of antithyroid medication increased after initiation of iodine fortification but reached the before-fortification levels after 11 years. Use of thyroid hormone replacement therapy increased steadily - probably partly explained by changes in indications for treatment.

\section{P25 \\ IODINE INTAKE IN COASTAL V INLAND COMMUNITIES - A ROLE FOR SEAWEED? \\ Peter Smyth ${ }^{1}$, Robert Burns ${ }^{1}$, Michael Casey², Karen Mullan ${ }^{3}$, Maria Andersson ${ }^{4}$, Suzanne Dold 4 , Ru Jin Huang ${ }^{5}$, Colin O'Dowd ${ }^{2}$ \\ ${ }^{1}$ Ucd, ${ }^{2} \mathrm{Nui}(\mathrm{G}),{ }^{3}$ Queens University, Belfast, ${ }^{4}$ Eth, Zurich, ${ }^{5} \mathrm{Nui}(\mathrm{G})$}

Conventional wisdom decrees that iodine intake in coastal regions exceeds that in inland areas. This was not borne out by recent studies from Portugal which showed that urinary iodine (UI) concentration was lower in Atlantic island communities than in continental Portugal. We have postulated that iodine intake in coastal communities may be related to the seaweed abundance of the region as seaweed provides the major source of iodine in the marine environment and is a major source of atmospheric $\mathrm{I}_{2}$. This study aimed to establish if living near the sea is a factor in assessment of iodine status by UI. Urine samples were obtained from populations of adult females living in coastal areas, including those residing beside a seaweed hot spot, and inland areas of Ireland. The median UI of $80 \mu \mathrm{g} / \mathrm{L}$ in adults $(\mathrm{N}=132)$ derived from a national population was not significantly different from that of $92 \mu \mathrm{g} / \mathrm{L}$ from subjects living in coastal cities. However both were significantly lower than the median of $136 \mu \mathrm{g} / \mathrm{L}$ obtained in coastal populations $(\mathrm{N}=72)$ living in a coastal area with high seaweed abundance $(\mathrm{P}<0.02)$. Perhaps the greatest difference was the $\%$ of higher $(>150 \mu \mathrm{g} / \mathrm{L})$ values, $(43 \%)$ in the seaweed rich area compared to $16 \%$ in low seaweed coastal and $11.6 \%$ in the national sample. The absence of difference in median UI between coastal and inland communities was also observed in pregnant women In whom identical median UI values of $79 \mathrm{ug} / \mathrm{L}$ was observed in a coastal $(\mathrm{N}=429)$ compared to an inland city $(\mathrm{N}=81)$ in whom higher values were present in $17.9 \%$ and $8.6 \%$ respectively. These findings demonstrate that iodine status, as determined by UI, is not primarily a feature of inland or coastal dwelling but may be influenced by relative seaweed abundance. 


\section{P04 Thyroid in Reproduction and Development 1}

\section{P26 \\ THYROID AUTOIMMUNITY IN WOMEN WITH THYROID DYSFUNCTION DURING THE POSTPARTUM \\ Boyan Nonchev ${ }^{1}$, Antoaneta Argatska², Delyana Davcheva ${ }^{3}$, \\ Maria Orbetzova ${ }^{2}$, Blagovest Pehlivanov ${ }^{4}$, Nikolai Raikov ${ }^{5}$ \\ ${ }^{1}$ Medical University - Plovdiv; Clinic of Endocrinology, ${ }^{2}$ Medical \\ University - Plovdiv, Clinic of Endocrinology, ${ }^{3}$ Medical University - \\ Plovdiv, Central Clinical Laboratory, ${ }^{4}$ Medical University - Plovdiv, \\ Clinic of Obstetrics and Gynecology, ${ }^{5}$ Medical Center Plus}

Background: Autoimmune thyroid disorders (AITD) are frequently observed during the postpartum period. Postpartum thyroiditis is the most common form of AITD but onset of Graves' disease has also been described. Furthermore, thyroid alterations in the first year after delivery are frequently caused by aggravation of pre-existing AITD. However the influence and the dynamics of thyroid autoantibodies (TAAb) need further investigation.

Aim: To reveal the changes in thyroid autoimmunity in postpartal women with thyroid dysfunction

Patients and Methods: A total of 30 females (mean age -30 years; range $-22-38$ years) with postpartum thyroid dysfunction were included in the study. TSH, FT4, FT3, TPOAb, TgAb (ECLIA) and TRAb were measured at the 3rd and 9th month after parturition or miscarriage. In the studied group 14 women $(46.7 \%)$ presented with hypothyroidism and $16(53.3 \%)$ showed hormonal features of thyrotoxicosis at the 3rd month following delivery.

Results: At the 3rd month after delivery positive TAAb were detected in $60 \%(n=18)$ of the studied patients. TPOAb correlated with TSH in the hypothyroid group, and a correlation between TRAb and FT4 was found in the thyrotoxic group. In $55 \%$ of TAAb positive women euthyroid state had been restored by the 9th month and it was associated with significant reduction of $\mathrm{TAAb}$ titers. The remaining $45 \%$ required treatment. $75 \%$ of the TAAb negative patients returned to the euthyroid state at 9 month after delivery.

Conclusions: Thyroid dysfunction during the postpartum is commonly associated with autoimmune alterations. Persistently elevated TAAb is a prerequisite for thyroid dysfunction requiring treatment. Negative TAAb at 3rd month after delivery is strongly associated with spontaneous restoration of euthyroid state.

\section{P27 \\ PROLONGED GESTATIONAL THYROTOXICOSIS IN TWO WOMEN WITH NOVEL TSH RECEPTOR VARIANTS}

Chiho Sugisawa ${ }^{1}$, Kiyomi Abe², Naoko Momotani³, Yukako Inoue ${ }^{3}$, $\mathrm{Kei} \mathrm{Endo}^{4}$, Satoshi Narumi ${ }^{2}$, Matsuo Taniyama ${ }^{4}$

${ }^{1}$ Internal Medicine, Showa University Fujigaoka Hospital, Kanagawa, Japan, ${ }^{2}$ Department of Pediatrics, Keio University School of Medicine, Tokyo, ${ }^{3}$ Tokyo Health Service Association, Tokyo, ${ }^{4}$ Division of

Endocrinology \& Metabolism; Department of Internal Medicine; Showa University Fujigaoka Hospital, Yokohama, Kanagawa

Gestational transient thyrotoxicosis (GTT) is caused by TSH receptor response to HCG. The TSH receptor mutation Lys183Arg was reported in a family with gestational thyrotoxicosis. This mutation was located in the extracellular domain, and the mutational receptor showed hypersensitivity to HCG in vitro. We report prolonged gestational thyrotoxicosis in two Japanese women who harbor TSH receptor variants. In patient 1 , thyrotoxicosis was detected during screening of the thyroid function in pregnant women. TSH receptor antibodies were negative, and she was diagnosed with GTT. Treatment with potassium iodide (KI) was initiated, followed by the addition of methimazole; however, thyrotoxicosis continued until delivery. The thyroid function was normalized after delivery; however, TSH levels remained low. Sequencing of the TSHR gene revealed the Gly336Arg variant, which is located in the extracellular domain. Patient 2 was also diagnosed with GTT after screening. Despite KI treatment, thyrotoxicosis persisted the entire

Poster Presentations pregnancy. After delivery, she experienced postpartum thyroiditis with hypothyroidism, and the thyroid function became normal. Gene analysis revealed the Ile523Phe variant, which is located in the second intracellular loop. The cAMP-producing capacities of the two variant receptors in the presence or absence of HCG were comparable to those of the wild-type receptor, when expressed in HEK293 cells. We speculate that mechanism(s) other than hypersensitivity of the variant TSH receptors to HCG would be responsible for the prolonged thyrotoxicosis in the two cases.

\section{P28 \\ SONOGRAPHIC (US) SCREENING FOR SECOND PRIMARY THYROID CANCER FOLLOWING BREAST CANCER IN THE KOREAN FEMALES \\ Kyoonsoon Jung ${ }^{1}$, Jin Hee Moon ${ }^{1}$, Joo Yeon Cheung ${ }^{1}$, \\ Kwan Seop Lee \\ ${ }^{1}$ Diagnostic Radiology, Hallym University Sacred Heart Hospital}

Objectives: The purpose of this study was to estimate the radiologic features of second primary thyroid cancers incidentally discovered at imaging among patients undergoing breast cancer surgery.

Methods: We performed a retrospective study of data in 727 consecutive patients who underwent screening thyroid US following the diagnosis of breast cancer between January 1, 2007, and March 31, 2014, at a single large academic institution. Thyroid cancer was diagnosed on the basis of pathologic findings after surgery. We compared the cases of pathologically proven thyroid cancer in two groups: during the first six months (group A) and beyond six months after the diagnosis of breast cancer (group B).

Results: Among 727 patients with breast cancer, 40 (5.5\%) had thyroid cancers. Thirty-one of the patients had US-detected incidental thyroid cancers, and an additional 8 had undergone US work-up of incidental thyroid cancers detected at CT (1 of 8) and PET/CT (7 of 8). The risk for developing a thyroid cancer was significantly elevated in group A (65\%). The mean size of thyroid cancers in group A was $10.4 \mathrm{~mm}$ (range, 2-27 mm) at postsurgical pathologic result, compared with a mean size of $4.8 \mathrm{~mm}$ (range, 2-8 $\mathrm{mm}$ ) in group B. Among the 6 thyroid cancers with lymph node metastasis, 5 of them were in group A. Extracapsular invasion of the thyroid cancer was found in 11 patients $(27.5 \%)$ in group A and 4 patients $(10 \%)$ in group B.

Conclusions: Significantly increased risk for thyroid cancer was noted only within six months after the diagnosis of breast cancer. More intensive diagnostic surveillance of breast cancer patients for thyroid cancer is required soon after its diagnosis.

\section{P29 \\ EFFECTIVENESS OF IODINE PROPHYLAXIS IN PREGNANT, LACTATING MOTHERS AND INFANTS IN MILD IODINE DEFICIENCY REGION Natalya Belykh ${ }^{1}$, Marina Mamenko ${ }^{1}$, Nikolay Kovalenko², Nadezhda Minyaylo ${ }^{3}$, Natalya Plugatarenk ${ }^{4}$ \\ ${ }^{1}$ Luhansk State Medical University, ${ }^{2}$ Pervomays Obstetrics Hospital, \\ ${ }^{3}$ Krasnodon Obstetrics Hospital, ${ }^{4}$ Lugansk City Health Department}

Pregnant women and infants are exceptionally vulnerable to iodine deficiency.

Objectives: To evaluate iodine status in pregnant and newborns and effectiveness of antenatal iodine prophylaxis.

Methods: A study was undertaken in 1052 pregnant in third trimester of gestation. Urinary iodine concentration (UIC), breast milk iodine concentration (BMIC), serums TSH, FT4 was determined. Iodine status in newborns evaluated as the results of neonatal screening for congenital hypothyroidism $(n=948)$. A total of 183 pregnant and their newborns were enrolled in the study and assigned to three groups: 46 mothers ( $1^{\text {st }}$ group) were receiving iodine supplementation ( $200 \mu \mathrm{g}$ /day potassium iodide) during pregnancy and lactation, 96 women without it ( $2^{\text {nd }}$ group) and 58 - were intake iodized salt ( $3^{\text {rd }}$ group).

Results: The median UIC in pregnant was $78.2 \mu \mathrm{g} / \mathrm{L}$, the rate of UIC $>150 \mu \mathrm{g} / \mathrm{L}$ was $14.2 \%$. In $1^{\text {st }}$ group the median UIC was $153.5 \mu \mathrm{g} / \mathrm{L} v s$. $67.0 \mu \mathrm{g} / \mathrm{L}$ in $2^{\text {nd }}$ group and $89.4 \mu \mathrm{g} / \mathrm{L}$ in $3^{\text {rd }}$ group $(\mathrm{P}<0.001)$.

Median TSH in pregnant was $1.9 \mathrm{mlU} / \mathrm{L}$. TSH levels in pregnant was highest in $2^{\text {nd }}$ group vs. in $1^{\text {st }}$ and $3^{\text {rd }}$ group $(2.2 \mathrm{mlU} / \mathrm{L} v s .1 .7 \mathrm{mlU} / \mathrm{L}$ and 1.9 $\mathrm{mlU} / \mathrm{L}$ respectively, $\mathrm{P}<0.05), \mathrm{FT} 4$ was lowest $(12.8 \mathrm{pmol} / \mathrm{L}$ vs. $17.1 \mathrm{pmol} / \mathrm{L}$ 
and $13.8 \mathrm{pmol} / \mathrm{L}$ respectively, $\mathrm{P}<0.05$ ). Rate of $\mathrm{TSH}>5.0 \mathrm{mlU} / \mathrm{L}$ was in $20.6 \%$ newborns and it was highest in $2^{\text {nd }}$ group $(24.1 \%$ vs. $13.2 \%$ and $15.2 \%$ in $1^{\text {st }}$ and $3^{\text {rd }}$ groups $)$. Median BMIC was highest in $1^{\text {st }}$ group $(115.3 \mu \mathrm{g} / \mathrm{L} v s$. $56.1 \mu \mathrm{g} / \mathrm{L}$ in $2^{\text {nd }}$ group and $59.2 \mu \mathrm{g} / \mathrm{L}$ in $3^{\text {rd }}$ group, $\left.\mathrm{P}<0.001\right)$. Breast-feeding infants in $1^{\text {st }}$ group have highest the median UIC $(177.1 \mu \mathrm{g} / \mathrm{L} v s .88 .3 \mu \mathrm{g} / \mathrm{L}$ and $81.5 \mu \mathrm{g} / \mathrm{L}$ respectively, $\mathrm{P}<0.05$ ).

Conclusion: Individual iodine supplementation is most effective iodine prophylaxis in pregnant, newborns and lactating mothers.

\section{P30 \\ NEONATAL THYROID-STIMULATING HORMONE SCREENING AS AN INDIRECT METHOD FOR THE ASSESSMENT OF IODINE DEFICIENCY IN CENTRAL SIBERIA}

\section{Irina Osokina ${ }^{1}$, Darya Osokina}

${ }^{1}$ Institute of Medical Studies of the North, Siberian Federal Universty,

${ }^{2}$ Institute of Medical Studies of the North, Russia

Introduction: The World Health Organization (WHO) has proposed to use the results of screening programs for congenital hypothyroidism in neonates as an additional index for the evaluation of iodine status of the population. A frequency of neonatal TSH concentrations above $5 \mathrm{mU} / \mathrm{L}$ below $3 \%$ was proposed as indicating iodine sufficiency. In mild iodine deficiency (MID) the frequency may be $3-19.9 \%$ and the frequencies of $20-39.9 \%$ and above $40 \%$ may be found in moderate and severe iodine deficiency respectively [2-4].

The objective of the present study was to analyze the results of neonatal screening for congenital hypothyroidism to assess iodine status in Central Siberia.

Materials and Methods: We analyzed data of thyroid-stimulating hormone (TSH) from 34980 newborn born in Central Siberia during the 2009: 19585 in Krasnoyarsk territory, 8025 in the Republic of Tyva and 7370 in the Republic of Khakassia

Results: According to the data of the congenital hypothyroidism screening the frequency of TSH $<5 \mathrm{mU} / 1$ was $11.8 \%$ in the Krasnoyarsk territory and corresponded to mild iodine deficiency. The frequency of neonatal TSH concentrations above $5 \mathrm{mU} / \mathrm{L}$ in different regions of the Krasnoyarsk territory fluctuated between 3.5 and $23.7 \%$. The highest values were marked in the Taimyr, in Irbeysky, Suchobuzimsky, Eniseysky, Tuchtetsky, Novoselovsky regions, in towns Zheleznogorsk and Sosnovoborsk (20.9-23.7\%). In the Republic of Khakassia the frequency of TSH $<5 \mathrm{mU} / 1$ was $12.2 \%$. In the Republic of Tyva $-6.6 \%$. It corresponds to mild iodine deficiency.

Conclusion: Our investigations show that in Central Siberia there is mild iodine deficiency demanding continuous adequate iodine prevention.

\section{P31}

\section{IODINE DEFICIENCY AMONG PREGNANT WOMEN IN LATVIA: A NATIONAL CROSS- SECTIONAL SURVEY}

\section{Ilze Konrade ${ }^{1}$, leva Kalere ${ }^{1}$, leva Strele ${ }^{1}$, Anna Jekabsone ${ }^{1}$}

Marina Makrecka ${ }^{2}$, Rudolfs Mezhapuke ${ }^{3}$, Elina Tetere ${ }^{1}$, Vija Veisa ${ }^{1}$

Didzis Gavars ${ }^{4}$, Dace Rezeberga ${ }^{1}$, Valdis Pirags $^{5}$, Kristine Ducena $^{5}$,

Aivars Lejnieks ${ }^{1}$, Maija Dambrova ${ }^{2}$

${ }^{1}$ Riga Stradins University, ${ }^{2}$ Riga Stradins University; Latvian Institute of Organic Synthesis, ${ }^{3}$ Latvian Institute of Organic Synthesis, ${ }^{4} \mathrm{E}$. Gulbis Laboratory, ${ }^{5}$ University of Latvia

Objectives: Low iodine intake during pregnancy may cause thyroid dysfunction, which might result in an inadequate foetal brain development. Although Latvia has been considered iodine replete, newborn TSH screening data suggest some iodine deficiency. In the absence of universal salt iodization programme we conducted a nation-wide of iodine deficiency in pregnant women from all regions of Latvia.

Methods: The study enrolled 829 pregnant women. They were asked to fill a questionnaire on dietary habits concerning iodine intake $(n=749)$. Thyroid function (TSH, FT4) and antibodies (antiTPO-Ab) were measured $(\mathrm{n}=631)$. Urinary iodine was measured with ammonium persulfate method $(\mathrm{n}=716)$.

Results: The median creatinine-standardized urinary iodine concentration (UIC) was 80.7 (IQR = 46.0-130.6 $\mu \mathrm{g} / \mathrm{g} ; 69.0(\mathrm{IQR}=53.6-92.0) \mu \mathrm{g} / \mathrm{l}) \mathrm{dur}-$ ing pregnancy. $81.1 \%$ of pregnant women had UIC below the WHO recommended range of $150-250 \mu \mathrm{g} / \mathrm{g}$. UIC was the lowest during the first trimester of pregnancy $(56.0(\mathrm{IQR}=36.4-100.6) \mu \mathrm{g} / \mathrm{g})$, reaching higher concentrations in the second and third trimester: $87.5(\mathrm{IQR}=46.4-141.7) \mu \mathrm{g} / \mathrm{g}$ and 86.9 $(\mathrm{IQR}=53.8-140.6) \mu \mathrm{g} / \mathrm{g}$, respectively. Women reporting regular iodine supplement, iodized salt or seafood consumption had higher UIC (85.3 vs. 73.6 $\mu \mathrm{g} / \mathrm{g}$, respectively) than women not reporting these iodine sources $(28.9 \%$ of respondents; $\mathrm{p}=0.007)$. The self-reported prevalence of iodized salt consumption was $45.0 \%$, but it did not result in significantly higher UIC (83.2 vs. 79.9 $\mu \mathrm{g} / \mathrm{g}, \mathrm{p}=0.33$ ). Women taking iodine supplements ( $6.4 \%$ of respondents) had non-significantly higher UIC than those without supplementation: 96.2 vs. $79.9 \mu \mathrm{g} / \mathrm{g}$, respectively, $\mathrm{p}=0.30$. AntiTPO-Ab concentration did not correlate significantly with UIC: Spearman's $\rho=-0.012, p=0.78$.

Conclusions: The median UIC indicates iodine deficiency in pregnant women in Latvia. Iodine supplementation (150 $\mu \mathrm{g}$ daily) and regular UIC monitoring should be suggested to overcome iodine deficiency and to reach recommended levels without induction of autoimmune processes.

\section{P32 \\ MATERNAL THYROID FUNCTION AND AUTO-IMMUNITY IN THREE TRIMESTERS OF PREGNANCY AND THEIR OFFSPRINGS' THYROID FUNCTION}

Peimaneh Sarkheil ${ }^{1}$, Ladan Mehran ${ }^{1}$, Sahar Askari ${ }^{1}$,

Zhale Tahmasebinejad ${ }^{1}$, Sormeh Nourbakhsh ${ }^{1}$, Maryam Tohidi $^{2}$, Fereidoun Azizi

${ }^{1}$ Endocrine Research Center, Research Institute for Endocrine

Sciences, Shahid Beheshti University of Medical Sciences, Tehran, I.

R. Iran, ${ }^{2}$ Prevention of Metabolic Disorders Research Center, Research Institute for Endocrine Sciences, Shahid Beheshti University of Medical Sciences, Tehran, I. R. Iran

Objectives: Thyroid dysfunction during pregnancy has significant impacts on thyroid function of infants. We performed this study to evaluate maternal thyroid function abnormalities and autoimmunity during pregnancy and its correlation with thyroid function of offsprings.

Design: Within a cohort study, serum samples of 120 pregnant women were collected in three trimesters and in their 57 cord bloods, 69 neonates, 34, 37, and 36 two, four, and six months infants. Serum TT4, TT3, T3U, TSH, $\mathrm{TPOAb}, \mathrm{TgAb}$ was measured.

Results: Mean age of the pregnant women was $22.0 \pm 4.8$ y (range of 16-38).We found an increasing trend for TT3 (P for trend $<0.0001$ ) and TSH ( $\mathrm{P}$ for trend $=0.01$ ) in three trimesters, but TT4 increased from the first to second trimester and then decreased. Among 120 mothers who followed in three trimesters, 10 mothers $(8 \%)$ had subclinical hyperthyroidism, 18 mothers $(15 \%)$ showed subclinical hypothyroidism. We found one hypothyroid $(0.8 \%)$ and three $(2.5 \%)$ hyperthyroid mothers during pregnancy. There was no correlation between maternal thyroid hormones and auto-antibodies, but correlation has been found between thyroid hormones among three trimesters. A positive correlations between maternal auto-antibodies in all trimesters with cord blood and neonates were found however this correlation was not found after neonatal period and it was stronger for TPOAb than TgAb. Cord blood TSH had good correlation with maternal TSH only in the first trimester $(r=0.29 \mathrm{P}<0.05)$ but not in other trimesters. In addition there was a positive correlation between neonatal TSH and maternal TT4 only in third trimester $(\mathrm{r}=0.25, \mathrm{P}<0.05)$. There was no relationship between TT3 and TT4 of mothers and offspring.

Conclusion: Subclinical hypothyroidism was the most common thyroid dysfunction in pregnancy. The association between maternal auto-antibodies and thyroid hormones of offspring exists mostly in neonatal period and become weaker after one month of age. 


\section{P33}

THYROID HORMONE PROFILES AND UIE DURING PREGNANCY; A LONGITUDINAL STUDY OF A SEGMENT OF INDIAN POPULATION

\section{Nikku Yadav ${ }^{1}$, Atul Kathait ${ }^{1}$, Vineet Sharma ${ }^{1}$, Asha Chandola-Saklani ${ }^{1}$}

${ }^{1}$ Centre for Biosciences and Clinical Research, School of Biosciences,

Apeejay Stya University

Objective: Monitoring of thyroid status in individual healthy young females during pregnancy with a view to understand homeostatic adjustment and establishment of a normative reference range. No such populations based studies are available yet in India.

Methods: The epidemeological observational survey included 300 pregnant women (19-28 year) from 05 villages of Haryana (2013-2014) through Government Primary Health Centers. The inclusion criteria were: normal healthy pregnant women, with no overt thyroid dysfunction and no thyroid supplement. Blood samples were collected in the $10^{\text {th }}, 20^{\text {th }}$ and $30^{\text {th }}$ week of pregnancy for the measurement of TSH, TT4, FT4, TT3 using ELISA UIE was measured spectrophotometrically. Information on dietary intake of iodized salt was obtained through questionnaire.

Results: The overall ranges during pregnancy were- TSH:0.45-4.54 $\mu \mathrm{IU} /$ $\mathrm{ml}$, FT4:8.5-22.91 pmol/1, TT4:9.5-20.02 $\mu \mathrm{g} / \mathrm{dl}$ and TT3:1.5-3.7 $\mathrm{ng} / \mathrm{ml}$. Nonpregnant females TSH: 0.67-3.08 $\mu \mathrm{IU} / \mathrm{ml}$, FT4:10-18.8 pmol/l, TT4:6$12.2 \mu \mathrm{g} / \mathrm{dl}$, TT3:0.5-1.9 ng/ml.

The overall median of UIE was $183 \mu \mathrm{g} / 1,153 \mu \mathrm{g} / 1,162 \mu \mathrm{g} / \mathrm{l}$ and $98 \mu \mathrm{g} / 1$ during first, second, third trimester and control group respectively.

TSH increased from first $(0.50-3.10 \mu \mathrm{IU} / \mathrm{ml})$ to third trimester $(0.62-$ $4.52 \mu \mathrm{IU} / \mathrm{ml}$ ) with an accompanied decline in FT4 from first to third trimester (11.63-22.91 pmol/1, 8.50-19.50 pmol/1, $\mathrm{P}<0.01$ paired t test).TT3 registered increase in second trimester and decrease in third trimester. TT4 increased marginally but not significantly from first to second trimester.UIE decreased significantly from first to third trimester.

Conclusion: The overall increase in hormone levels and UIE in pregnant females compared to controls, reflects the enhanced metabolic demand. The progressive increase in TSH could be interpreted as a tendency towards subclinical hypothyroidism but no individual developed overt symptoms of hypothyroidism.

UIE in pregnant and non-pregnant females are indicative of mild iodine deficiency although subjects used iodised salt.

Distinct inverse correlation between TSH \&FT4 indicates efficient homeostasis in this population and also that these two parameters serve good diagnostic parameters of thyroid status.

Financial assistance from Apeejay Education Society is gratefully acknowledged.

\section{P34}

\section{TSH VALUES IN PREGNANT WOMEN FROM A} POPULATION WITH NORMAL IODINE INTAKE

Mihaela Simescu' ${ }^{1}$, Emilia Nicolaescu' ${ }^{1}$, Claudia Podia Igna ${ }^{2}$

Mihai Varciu ${ }^{3}$, Georgeta Hazi ${ }^{4}$, Cristina Mambet ${ }^{5}$

${ }^{1} \mathrm{Sc}$ Simedis Consult Srl, ${ }^{2}$ Astra Clinic, ${ }^{3}$ Clinical Emergency Hospital

Brasov, ${ }^{4}$ Clinical Emergency Hospital Cluj Napoca, ${ }^{5}$ Synevo

Background: In Romania, following the legislation of universal salt iodization of 2003 iodine intake in population was normalized as shown by general and partial inquires in school children with median urinary iodine concentration (UIC) of $135.2 \mathrm{mg} / \mathrm{l}$. However, data from pregnant women revealed low values of UIC. In this context, it is important to evaluate the thyroid function during pregnancy.

Objective: To determine the thyroid function in pregnant women and to consider the frequency of TSH values above the trimester-specific reference ranges (TSRR)

Methods: We investigated the thyroid function in groups of pregnant women from different regions of the country in 2008 (GRI) and 2013 (GRII). In the first survey, we assessed TSH levels in 210 pregnant women admitted in obstetrics-gynecology clinics before delivery.

For GRII, we measured TSH from 264 pregnant women within all trimesters of pregnancy investigated by gynecologists in a private clinic.

Results: In GRI the median TSH concentration was $2.04 \mathrm{mIU} / 1$ and frequency of TSH values above normality in third trimester was $24.29 \%$ with following distribution $21.43 \%, 40 \%, 32.69 \%$ and $8.33 \%$ for the investigated districts Sibiu, Cluj Napoca, Brasov and Bucharest, respectively.

In GR II, the percentages of TSH values above normality were $22.3 \%$ in the first, $23.38 \%$ in the second and $10.42 \%$ in the third trimester. The corresponding median values were $1.77 \mathrm{mIU} / 1,1.53 \mathrm{mIU} / 1$ and $1.64 \mathrm{mIU} / 1$.

Conclusion: The persistence of high prevalence of elevated TSH values above TSRR in pregnant women imposes urgent, extended and complete national studies as well as programs for early investigation and iodine/LT4 supplementation in pregnancy.

\section{P35 \\ RISK FACTORS FOR DEVELOPMENT OF POSTPARTUM THYROID DYSFUNCTION \\ Antoaneta Argatska ${ }^{1}$, Boyan Nochev' ${ }^{1}$, Delyana Davcheva ${ }^{2}$, Blagovest Pehlivanov ${ }^{3}$, Maria Orbetzova ${ }^{1}$ \\ ${ }^{1}$ Medical University of Plovdiv, Clinic of Endocrinology and Metabolitic Diseases, ${ }^{2}$ Medical University of Plovdiv, Central Clinical Laboratory, \\ ${ }^{3}$ Medical University of Plovdiv, Clinic of Obstetrics and Gynaecology}

Background: Thyroid disorders are frequently observed during the postpartum period. Postpartum thyroiditis (PPT) is the most common cause of thyroid dysfunction after both full-term birth and miscarriage with an estimated mean prevalence of $8.1 \%$. A considerable part of the affected women show clinically evident symptoms of thyroid dysfunction and therefore need appropriate treatment. However, the risk factors for developing thyroid dysfunction during the first year after birth remain a matter of discussion.

Objective: To assess the prevalence of thyroid dysfunction in postpartal women and to evaluate the risk factors for its occurrence.

Patients and Methods: 64 females (mean - 30.5 years; range $-17-38$ years) were included in the study. TSH, FT4, FT3, TPOAb and TgAb (ECLIA) were measured and an US examination of the thyroid gland was performed in the first trimester of pregnancy and 3 months postpartum. The prognostic significance of several patients' characteristics was evaluated. Postpartum thyroid dysfunction was diagnosed in $46.9 \%$ of the studied women.

Results: 3 months following delivery or miscarriage $21.9 \%$ of the patients presented with hypothyroidism (both subclinical and overt) and $25.0 \%$ were thyreotoxic. The other $53.1 \%$ remained euthyroid. No statistically significant differences were detected between the studied groups in terms of age, parity, history of infertility, smoking, family history of thyroid disorders, previously diagnosed thyroid diseases. The only predicting factor of postpartum thyroid dysfunction was TPOAb positivity in the first trimester of pregnancy.

Conclusions: Thyroid dysfunction is commonly detected during the postpartum period. In our series the only predicting factor for its occurrence was the positive titer of TPO antibodies in the early pregnancy. TPOAb positive women could benefit from screening after birth or miscarriage in order to avoid the symptoms of unrecognised thyroid dysfunction and to prevent complications and associated morbidity.

\section{P36}

LEVOTIROXINE TREATMENT IN THYROID ANTIBODY NEGATIVE WOMEN WITH TSH LEVELS BETWEEN 2.5 AND 5.0 IN THE FIRST TRIMESTER OF PREGNANCY

Paloma Iglesias ${ }^{1}$, Amalia Paniagua' ${ }^{1}$, Laura Bartolome ${ }^{1}$, Maria Blanca $^{1}$ Sonsoles Gutierrez

${ }^{1}$ Hospital Rey Juan Carlos

Context: Since August 2012 Guidelines agree that subclinical hypothyroidism in pregnancy requires substitutive treatment.

Objective: The objective of the study was to evaluate the effect of levotiroxine treatment in pregnancy loss and preterm delivery rate in firsttrimester thyroid peroxidase antibody-negative women with TSH values between 2.5 and $5.0 \mathrm{mIU} /$ liter.

Materials and Methods: A retrospective study was conducted at an ambulatory clinics of a community hospital. Twenty-nine thyroid peroxidase antibody-negative women with TSH levels at or below $5.0 \mathrm{mIU} /$ liter were studied from March 2012 to March 2013. Group A ( $=13)$ was treated with LT(4), and group B ( $n=16)$ was not treated. Rates of pregnancy loss and preterm delivery rate were recorded. 
Results: A total of 1 adverse pregnancy outcome were recorded (1 preterm delivery) with no significant difference between the groups. No adverse fetal complications were recorded.

Conclusion: Substitutive treatment with LT(4) in first-trimester thyroid peroxidase antibody-negative women with TSH values between 2.5 and $5.0 \mathrm{mIU} /$ liter did not demonstrate differences pregnancy outcomes.

\section{P05 Thyroid Function and Development}

\section{P37 \\ THE HINGE REGION OF GLYCOPROTEIN HORMONE RECEPTORS IS INFLUENCED BY ITS ADJACENT DOMAINS PARTICULARLY THE TRANSMEMBRANE DOMAIN}

Joerg Schaarschmidt ${ }^{1}$, Sandra Huth ${ }^{1}$, Rene Meier ${ }^{2}$, Ralf Paschke ${ }^{1}$, Holger Jaeschke ${ }^{1}$

${ }^{1}$ University of Leipzig; Department of Internal Medicine, Neurology and Dermatology,; Division of Endocrinology and Nephrology, ${ }^{2}$ University of Leipzig; Institute of Biochemistry

The hinge region (HinR) is a variable structure of glycoprotein hormone receptors (GPHRs) and connects the hormone binding leucine-rich repeat (LRR) domain with the transmembrane domain (TMD). The central question how extracellular ligand binding triggers the conformational changes in the TMD necessary for $G$ protein activation remains unanswered mainly due to missing information about the interplay between the HinR and the TMD. To gain further insights into the interplay between LRR domain, HinR and TMD we generated chimeras between the thyroid stimulating hormone receptor (TSHR) and the follicle stimulating hormone receptor (FSHR) and explored their functionality. The results show that the HinR of GPHRs is highly influenced by its adjacent domains. While the FSHR TMD is incompatible with the TSHR HinR regarding folding and cell surface expression, no incompatibility between the TSHR TMD and the FSHR HinR is observed. Furthermore, modifying the charge distributions at the C-terminal part of the LRR domain of GPHRs affects ligand binding and signaling even though the changes are not within the binding interface. The chimeras also show that the binding and signaling properties of bovine TSH are more sensitive to changes in the HinR than human TSH, whereas those of the FSH analog TR4401 and human FSH are almost unaffected by the same changes. In conclusion the HinRs of GPHRs do not only share similar characteristics but also behave as receptor specific structural and functional entities.

\section{P38 \\ IN VIVO REGULATION OF DUOX1/DUOXA1 EXPRESSION IN THE THYROID OF A NOVEL TRANSGENIC MOUSE OVEREXPRESSING INTERLEUKIN-4}

Eskalli Zineb ${ }^{1}$, Françoise Miot ${ }^{1}$, De Deken Xavier ${ }^{1}$

${ }^{1}$ Université Libre de Bruxelles

NADPH oxidases DUOX1 and DUOX2 constitute the $\mathrm{H}_{2} \mathrm{O}_{2}$-generating system required for thyroid hormone synthesis. DUOX apical cell surface targeting, site of their catalytic activity is regulated by the maturation factors, DUOXA1 and DUOXA2, which share the same bidirectional promoter with their respective DUOX partner. However, the molecular mechanisms regulating their expression are still unclear. $\mathrm{H}_{2} \mathrm{O}_{2}$ production in thyroid is tightly regulated, but under oxidative stress conditions excessive $\mathrm{H}_{2} \mathrm{O}_{2}$ could promote tumorigenesis by altering the DNA integrity.

In primo-cultured mouse thyrocytes, the Th2 cytokines, IL-4 and IL-13, positively modulate the $\mathrm{H}_{2} \mathrm{O}_{2}$-generating system through an up-regulation of both DUOX1/DUOXA1 and DUOX2/DUOXA2 genes. As these cytokines induce $D U O X$ and $D U O X A$ gene expression, we decided to generate a transgenic mouse model, TG-mIL4-GFP, in which murine IL4 expression is under the control of the thyroid specific thyroglobulin (TG) promoter. We are cur- rently characterizing the TG-mIL4-GFP mouse. We first confirmed the presence of a high expression of the mIL4 transgene at mRNA and protein levels in the thyroid tissue of transgenic lines compared to wild-type mice. Our first results showed an up-regulation of DUOX1/DUOXA1, but not DUOX2/ $D U O X A 2$, gene expression in the thyroid of the transgenic lines. We plan to measure the level of $\mathrm{H}_{2} \mathrm{O}_{2}$ production, characterize the thyroid phenotype from a histological point of view and estimate the oxidative stress and differentiation status of the thyroid gland. We suggest that DUOX/DUOXA overexpression could trigger a local high level of $\mathrm{H}_{2} \mathrm{O}_{2}$ production and hypothetically promotes an oxidative stress in the mouse thyroid TG-mIL4-GFP. This transgenic mouse model could be a useful model to better understand the involvement of an oxidative stress in thyroid inflammations or thyroid cancers.

\section{P39}

\section{A NEW CANDIDATE PROTEIN FOR THE FUNCTION OF THE APICAL THYROCYTE IODIDE CHANNEL}

Jacques Dumont $^{1}$, Twyffels Laure ${ }^{2}$, Van Sande Jacqueline ${ }^{2}$

${ }^{1}$ University Brussels, ${ }^{2}$ Ulb

The iodide uptake in the thyroid follicular lumen requires its first active transport by the $\mathrm{Na}+/ \mathrm{I}-$ symporter NIS, at the basal side followed by its efflux from the cell at the luminal side. The latter step is generally believed to be mediated by pendrin.

We have identified a new candidate thyroid apical iodide channel (TAIC). We show that the TAIC is expressed in human and rat thyroid.

To determine the properties of TAIC and pendrin we have expressed both pendrin and TAIC in HEK293T cells and compared their properties to those of the existing channel in PCCl3 and FRTL5 rat thyroid cell lines. The properties of TAIC but not those of pendrin nicely fit those of the channel functional in the cell lines. We therefore conclude that TAIC is a functional iodide channel in rat thyroid. It is not excluded that in different species several channels, including pendrin, may carry this function.

\section{P40 \\ ROLE OF FOXE1 IN THE REGULATION OF EMT GENES IN THYROID EPITHELIAL CELLS}

\section{Lara P. Fernandez ${ }^{1}$, Pilar Santisteban 1}

${ }^{1}$ Instituto de Investigaciones Biomédicas (Csic-Uam)

FoxE1 is a pioneer transcription factor that is essential during thyroid development and also for the maintenance of the thyroid differentiated phenotype. Moreover, FOXE1 has emerged as the best validated thyroid cancer susceptibility gene. It has been hypothesized that FOXE1 can induce a motile advantage in malignant thyroid cells. Recently, we have reported cadherin 1 $(C d h 1)$, a gene extensively implicated in epithelial to mesenchymal transition (EMT), as new FoxE1 target gene in differentiated thyroid cells. However FoxE1 role in regulating thyroid cell proliferation, migration and invasion and/or EMT has not yet been analysed in detail.

The aim of this work is the characterization of new FoxE1 target genes involved in EMT in thyroid differentiated follicular cells.

For this purpose, we have selected from our previous study, a group of 7 candidate EMT genes whose expression seems to be deregulated in the absence of FoxE1. We perform an in silico search for putative FoxE1 binding sites followed by an in vivo analysis of FoxE1 binding to their promoter regions. Experimental validation is done by RT-PCR, Western-blot and Chromatin Immunoprecipitation (ChIP) assays.

After FoxE1 silencing, we confirm deregulation of 6 out the 7 EMT genes selected. Among them, Zebl expression is greatly decreased in the absence of FoxE1. Moreover, we find a highly conserved FoxE1 binding domain in the promoter of human, mouse and rat Zeb1. ChIP analysis is performed in order to study Zeb1 direct regulation by FoxE1.

In search of FoxE1 potential downstream EMT mediators, together with $C d h 1$, we identified Zeb1 as FoxE1 target gene. To our knowledge, this is the first evidence regarding the implication of Zeb1 in executing the transcriptional programme triggered by FoxE1, in thyroid epithelial cells. 


\section{P41 \\ EMBRYONIC BASEMENT MEMBRANES GUIDE POSITIONING OF C CELL PRECURSORS AND MATURE C CELLS IN THE DEVELOPING MOUSE THYROID}

Ellen Johansson ${ }^{1}$, Mikael Nilsson $^{1}$

${ }^{1}$ Sahlgrenska Cancer Center, University of Gothenburg, Sweden

Thyroid follicular cells and parafollicular C cells typically share the same basement membrane (BM). BM underlying epithelia constitute a barrier that separates the epithelium from stromal cells. This raises the question how $\mathrm{C}$ cells get this position during organogenesis. From studies on avian development it is assumed that neural crest cells invade the ultimobranchial body (UB) and later differentiate into C cells. Here we investigate the prerequisites for migration and thyroid entry of $\mathrm{C}$ cell precursors related to BM enveloping primordial tissues.

Mouse embryos comprising all developmental stages from delamination from foregut endoderm to functional differentiation of the orthotopic gland (E10.5-E17.5) were collected. Frozen sections were immunostained for laminin, a major BM constituent, E-cadherin to distinguish epithelia from mesenchyme, Nkx2-1 to identify progenitor cells of both midline thyroid and UB, Pax8 to distinguish between follicular and UB epithelial cells, and calcitonin to localize mature $\mathrm{C}$ cells.

Laminin formed a continuous BM that enveloped respectively the thyroid bud and UB, demarcating distinct borders to adjacent mesenchyme. Mesenchymal cells did not penetrate the BM at any time during UB development. At E13.0 fusion of thyroid primordia started with laminin disappearing from the UB surface and overgrowth of Pax8+ cells. A new BM surrounding both Pax8+ and Pax- epithelial cells was established. The first $C$ cells appeared at E14.5 located in the UB remnant that was all invested by Pax8+ cells. Spreading of C cells occurred within cords of Pax $8+$ parenchyma enveloped by BM.

Both thyroid anlagen are clearly separated from surrounding tissue by laminin+ BMs during primordial growth. The BM temporarily dissolves to enable close contact between cells of the two anlagen during fusion of primordia. This explains the final positioning of thyroid $\mathrm{C}$ cells. Lack of invading neural crest cells supports previous notions of an endodermal origin of mammalian $\mathrm{C}$ cells.

\section{P42 \\ STUDY OF PENDRIN EFFECT IN CELL PROLIFERATION}

\section{Joana Rodrigues ${ }^{1}$, Maria del Carmen Suarez ${ }^{1}$,}

Angela Rodriguez García-Rendueles ${ }^{1}$, Sihara Perez ${ }^{1}$,

Laura Fugazzola ${ }^{2}$, José Cameselle-Teijeiro ${ }^{3}$, Clara Alvarez Villamarín ${ }^{1}$

${ }^{1}$ Center for Research in Molecular Medicine and Chronic Diseases

(Cimus-Idis), University of Santiago de Compostela, ${ }^{2}$ Department

of Clinical Sciences and Community Health, University of Milan;

Endocrine Unit, Fondazione Policlinico IRCCS, Milan, Italy,

${ }^{3}$ Department of Pathology, Clinical University Hospital of Santiago de

Compostela, Sergas, University of Santiago de Compostela

The Pendred's Syndrome is a rare disease leading to congenital deafness and less than $75 \%$ has goiter whose mechanism is not well known. Our group established a humanized culture medium, the $\mathrm{h} 7 \mathrm{H}$ medium, where concentrations of seven groups of additives were adapted to the physiological range found in human serum (Bravo et al, JCEM 2013). We had established a bank of human primary thyroid cultures (BANTTIC) from surgical thyroid pieces from the patients of Clinical University Hospital of Santiago de Compostela (CHUS).

The aim of this work was to study the pendrin effect in normal thyroid cells proliferation. Due to pendrin is an iodine simporter we want to study cell proliferation with different iodine concentrations.

Our group obtained two stable cell cultures infecting shRNA lentivirus in normal thyroid cells: T-NT2-SH-sc (scramble) and T-NT2-SH-PDS. The interested cells were selected by puromycin. We validated pendrin absence in T-NT2-SH-PDS by western blot and the mRNA expression by taqman. The range of iodine concentration used was between $1-100 \mathrm{ug} / \mathrm{L}$. We measure proliferation by MTT assay.

Poster Presentations
The pendrin expression was reduced to the half in T-NT2-SH-PDS. We observed that T-NT2-SH-sc cell proliferation was higher when comparing with T-NT-SH-PDS (1,7 times more).

With different iodine concentrations we observed a significant decrease, around $50 \%$, in T-NT2-SH-sc and no difference in the absence of pendrin (T-NT-SH-PDS) during several weeks. Iodine has an effect in the proliferation of normal thyroid cells, which does not exist in cells with low PDS.

This could allow us understand the genetic thyroid disease and thyroid cancer.

\section{P43 \\ A GLUCOSE-REGULATED PROTEIN MODULATES THYROTROPIN RECEPTOR SIGNALING}

Jana Fischer ${ }^{1}$, Anne Müller ${ }^{1}$, Heiko Krude ${ }^{1}$, Annette Grüters ${ }^{1}$,

Gunnar Kleinau ${ }^{1}$, Heike Biebermann ${ }^{1}$

${ }^{1}$ Charité Universitätsklinikum, Institut Für Experimentelle Pädiatrische

Endokrinologie

The thyrotropin receptor (TSHR) belongs to the family of glycoprotein hormone receptors, a subfamily of G-protein coupled receptors (GPCRs) and is involved in thyroid hormone synthesis and secretion as well as in the expression of thyroglobulin or the $\mathrm{Na} / \mathrm{I}$ - symporter. Common diseases such as non-autoimmune hyperthyroidism or TSH resistance are associated with the TSHR. Since several years it is known that the TSHR is able to form homodimers which has an influence on TSH binding shown as negative cooperativity. Besides homo- and hetero-oligomerization of GPCRs interactions with other cytosolic and membrane bound proteins indicate that GPCRs function in a protein network that modulate GPCRs action in its cellular context. Thereby interacting proteins can play an important role in fine-tuning the function of GPCRs.

For the identification of new TSHR interaction partners we screened a human thyroid cDNA library by applying a bimolecular fluorescence complementation. This assay is based on the complementation of two Yellow Fluorescent Protein (YFP)-halves that were fused to human TSHR cDNA and a human thyroid cDNA library. In a case of an interaction of the TSHR with a protein from the cDNA library the two halves reconstitute and form a full functioning YFP. Positive interaction pairs were selected by FACS (fluorescence-activated cell sorting) analysis.

In the first screen we identified one interaction partner, a glucose-regulated protein that is a chaperone highly expressed in the thyroid gland. This chaperon increases IP3 signaling via Gq of TSHR after TSH stimulation whereas cAMP levels remain unchanged. Our results indicate a novel pathway-selective influence of glucose-regulated proteins in the thyroid gland.

\section{P44 \\ STRUCTURAL AND FUNCTIONAL CHARACTERIZATION OF A NOVEL THYROID- SPECIFIC LONG NON-CODING RNA}

Barbara D'Andrea ${ }^{1,3}$, Angela Pascarella ${ }^{1}$, Miriane De Oliveira',

Sara Credendino', Elena Amendola', Pina Marotta², Paolo Sordino ${ }^{3}$,

Rosa Maria Sepe ${ }^{3}$, Marzia Scarfón, Roberto Di Lauro',

Gabriella De Vita ${ }^{1}$

${ }^{1}$ Dipartimento di Medicina Molecolare e Biotecnologie Mediche, Universita' Federico II, Napoli, Italy, ${ }^{2}$ Istituto di Ricerche Genetiche G.Salvatore, Biogem s.c.ar.I, Ariano Irpino, Avellino, Italy, ${ }^{3}$ Stazione Zoologica Anton Dohrn Napoli Italy

To search for genes involved in thyroid morphogenesis we performed an unbiased search for transcripts enriched in the early mouse thyroid primordium (E10.5). At the top of the list we found a novel gene for a long noncoding RNA that we named Thybe1 for Thyroid bud-enriched 1. Thybe 1 shows strong and highly specific expression in mouse thyroid both during development and in the adult. Its genomic locus shows a partially overlapping head-to-head arrangement with that of the protein encoding gene Klhl14, also included in our list of thyroid-enriched genes. Klhl14 is a poorly characterized gene, whose expression in thyroid has not been previously highlighted. To shed light on the function of this overlapping gene pair, we analyzed their expression in a panel of adult mouse tissues. Interestingly, these genes resulted invariably co-expressed in several tissues, thus suggesting either a common regulation or a reciprocal regulation. Thybe1 and Klhl14 gain- and loss-of- 
function experiments in FRTL-5 cells, however, did not reveal mutual effects on expression levels of each gene, while oncogene-induced de-differentiation is paralleled by downregulation of both genes. To investigate the in vivo function of Thybe1, we generated a Thybel floxed allele in order to obtain both constitutive and conditional KO mouse lines. Phenotype of both mouse lines is currently under investigation and will provide definitive results on the role of such gene in the whole organism and more specifically in the thyroid gland. As this gene pair together with its genomic organization is also well conserved in the zebrafish genome, we performed morpholino-based Thybe1 knockdown in this model. Preliminary data indicate that inhibition of Thybe 1 expression elicits a dramatic phenotype in zebrafish, although further experiments are required to confirm these data and to check if thyroid phenotype is either morphologically and/or molecularly altered in morphants.

\section{P06 Hypothyroidism}

\section{P45 \\ QUALITY OF LIFE AND EMOTIONAL STATUS OF PATIENTS AFTER RADICAL TREATMENT \\ Alexander Dreval', Timur Britvin ${ }^{2}$, Olga Nechaeva ${ }^{3}$, \\ Tamara Mamedova ${ }^{0}$, Tatyana Shestakova ${ }^{3}$, Irina Komerdous ${ }^{3}$, Irina Chikh ${ }^{3}$ \\ ${ }^{1}$ Moscow Regional Research Clinical Institute N.A. Vladimirskiy, \\ ${ }^{2}$ Moscow Regional Research Clinical Institute N.A.Vladimirsky, \\ ${ }^{3}$ Moscow Regional Clinical Research Institute}

Materials and Methods: we observed 71 patients at the age from 25 to 80 y o (60 women and 11 man) who were treated for Grave's disease by surgical treatment (34 patients) and by radioactive iodine (37 patients). Evaluation of quality of life was assessed with SF-36, anxiety and depression was carried out using the test of Spielberg-Hanin and Beck Depression scale.

Results: Median TSH level was $2.5 \mathrm{mkME} / \mathrm{ml}$ and median dose of thyroxine was $100 \mathrm{mcg}$ in both group.

Median of quality of life was measured by 8 scales SF-36 that ranged from 54 points (vital activity) to 75 (pain) in surgical group and from 50 points (life activity) to 73 (pain) in RAI group. The level of depression was 14 and 12 points, which corresponds to mild depression, situational anxiety was 41 and 38 points, personal anxiety 42 and 37 points, which corresponds to a moderate level of anxiety in both groups. Statistically significant difference was noted on a scale of mental health $(\mathrm{P}<0.05)$, which was higher in radioiodine group, and the level of depression $(p=0.006)$, anxiety-like personality $(p=0.004)$ and situational anxiety $(\mathrm{p}=0.05)$ wich were higher in surgical group.

Conclusions: Quality of life and psyhoemotional status of patients were at high level in both groups, but no significant difference between the groups was noted. In the group of patients who received radioiodine better performance of mental and emotional status was achieved.

\section{P46}

\section{EFFECT OF TEMPERATURE ON THE STABILITY OF TSH ON DRIED BLOOD SPOT COLLECTED ON FILTER PAPER}

\section{Patrícia Magalhães ${ }^{1}$, Carlos Miranda ${ }^{1}$, Fernando Villar ${ }^{1}$}

Ludmilla Cardoso ${ }^{1}$, Andre Schmidt ${ }^{1}$, Roberta Rodrigues ${ }^{1}$, Lea Macie/ ${ }^{1}$

${ }^{1}$ Ribeirao Preto Medical School

Introduction: In Brazil, neonatal screening was based on TSH determination on dried blood spot on filter paper.

Objectives: To evaluate the influence of drying and storage temperature (T) of samples on the stability of TSH.

Material and Methods: Blood samples with heparin were collected from 29 volunteers and $50 \mu 1$ were dispensed on filter paper Schleicher \& Schuell 2992: 10 samples with normal TSH $(\mathrm{x} \pm \mathrm{SD}=1.1 \pm 0.5 \mathrm{mU} / \mathrm{L}), 9$ samples from decompensated hypothyroid patients $(\mathrm{x} \pm \mathrm{SD}=52.4 \pm 44.1 \mathrm{mU} / \mathrm{L})$ and 10 from patients with subclinical hypothyroidism $(\mathrm{x} \pm \mathrm{SD}=6.9 \pm 2.4 \mathrm{mU} / \mathrm{L})$. Half of the samples were dried at $22^{\circ} \mathrm{C}$ and the other half at $35^{\circ} \mathrm{C}$ for 3 hours. Then, the samples were stored at $22^{\circ} \mathrm{C},-4^{\circ} \mathrm{C}$ or $-20^{\circ} \mathrm{C}$. TSH measurements are obtained by
AutoDELFIA technology at 0 (D0), 7 (D7), 30 (D30), 60 (D60), 180 (D180) and 360 (D360) days of storage.

Results: The drying T didn't interfere with the measurement of TSH on D0. The samples that were dried at $35^{\circ} \mathrm{C}$ and stored at $22^{\circ} \mathrm{C}$ were stable until D30. The samples dried and stored at $22^{\circ} \mathrm{C}$ remained stable until D60. Samples stored at $22^{\circ} \mathrm{C}$ lost stability from D30, whereas samples stored at $-4^{\circ} \mathrm{C}$ and $-20^{\circ} \mathrm{C}$ remained stable until D180. On D360 there was significant difference in levels of TSH from baseline regardless of storage $\mathrm{T}\left(22^{\circ} \mathrm{C},-4^{\circ} \mathrm{C}\right.$ or $\left.-20^{\circ} \mathrm{C}\right)$.

Conclusions: The high drying $\mathrm{T}$ of blood on filter paper interfere with the stability of the TSH. Storage in freezer is not advantageous compared to storage in the refrigerator. Ideally, the samples should be stored in refrigerator, which does not occur in routine newborn screening laboratories. At the end of 1 year, if the confirmation of the initial result is required, the reduction of TSH concentrations should take into account.

\section{P47 \\ PATIENTS WITH ATROPHIC GASTRITIS WHO RECEIVED L-T4 IN TABLET FORMULATION AFTER SWITCHING TO AN ORAL LIQUID FORMULATION: REVERSIBLE NORMALISATION OF SERUM TSH LEVELS}

Ilaria Ruffilli ${ }^{1}$, Silvia Martina Ferrari ${ }^{1}$, Andrea Di Domenicantonio ${ }^{1}$, Alda Corrado ${ }^{1}$, Poupak Fallahi ${ }^{1}$, Alessandro Antonelli ${ }^{0}$

${ }^{1}$ University of Pisa

Objective: Absorption of oral L-thyroxine (L-T4) therapy is influenced by several factors, including the age of the patient, adherence to therapy, dietary habits, absorption kinetics, malabsorption, and interference of other drugs. A potential concern in patients with autoimmune atrophic gastritis is the drug malabsorption.

Methods: In this work, we show reversible normalisation of serum thyroid-stimulating hormone (TSH) levels in three patients with autoimmune atrophic gastritis, previously treated with L-thyroxine in tablet formulation, switched to the therapy with L-T4 in oral liquid formulation.

Results: Serum TSH levels are normalised by the passage from tablets to oral liquid formulation, while the switch back to tablets caused thyrotropin levels to worsen. This fact leads us to believe that absorption of thyroxine is greater with oral liquid formulations in these patients.

Conclusion: In conclusion, we report three patients with autoimmune gastritis, in whom oral liquid L-thyroxine induced a reversible normalisation of thyrotropin levels. The obtained results suggest that the L-T4 oral liquid formulation could circumvent the $\mathrm{pH}$ alteration resulting from autoimmune atrophic gastritis.

\section{P48 \\ HYPOTHYROIDISM, CENTRAL OBESITY AND METABOLIC SYNDROME: CORRELATIONS BETWEEN THYROID STIMULATING HORMONE, ADIPOCYTOKINES AND INDICES OF INSULIN RESISTANCE \\ Bojan Lozanov', Radka Argirova', Lachezar Lozanov², \\ Vesselina Koleva', Mira Vankova', Desislava Gorcheva ${ }^{1}$, \\ Radoslav Petrov', Boyka Kostova ${ }^{1}$, Mircho Vukov' \\ ${ }^{1}$ Tokuda Hospital, ${ }^{2}$ Tokuda Hospital Sofia; Endocrinology Deprtment}

The objective of the study was to investigate the correlations between serum TSH, leptin, the parameters of insulin resistance (IR) and some indices of systemic inflammation in patients with metabolic syndrome (MetS), central obesity and hypothyroidism (Hashimoto's thyroiditis).

Material and Methods: 41 patients (29 f, $12 \mathrm{~m}$ ) with MetS and central obesity with BMI $>35 \mathrm{~kg} / \mathrm{m}^{2}$ were assessed and divided into 2 groups: gr.A/ with hypothyroidism (Hypot.) $\mathrm{n}=20$ pts at m.age $42 \pm 13 \mathrm{y}$; gr. B/ MetS without Hypot $\mathrm{n}=21 \mathrm{pts}, \mathrm{m}$.age $38.2 \pm 12 \mathrm{y}$, and gr.C/ age-matched healthy controls $\mathrm{n}=20$ (BMI $\left.21 \pm 3 \mathrm{~kg} / \mathrm{m}^{2}\right)$. Laboratory parameters included: TSH $(0.35$ - $4.94 \mathrm{mU} / \mathrm{l}$ ), FT4 (9-19 pmol/1), leptin (ELISA, ref.r. 4.5 - $20.8 \mathrm{ng} / \mathrm{ml}$ ), interleukin-6 (IL-6, ECLIA, ref.r. 0-7 ng/ml), TNF-alpha, CRP, OGTT: (0-60-120 min) glucose + insulin (CMIA), HOMA-IR, HDL cholesterol, and triglycerides (TG). Statistical analyses were performed by SPSS for Windows 13. 
Results: The hypothyroid patients (gr.A) compared to controls (gr.C) showed a 4-fold higher leptin mean value $(40.25 \pm 15 \mathrm{vs} 11.7 \pm 8.4 \mathrm{ng} / \mathrm{ml})$ corresponding to the significantly higher BMI $\left(39.5 \mathrm{vs} 21.1 \mathrm{~kg} / \mathrm{m}^{2}\right)$ and HOMA-IR (3.6 vs 1.4). These parameters did not differ significantly between gr.A and gr.B (euthyroid patients with MetS only). The mean value of TSH in gr.A $(10.14 \pm 5.57 \mathrm{mU} / \mathrm{l})$ was up to 6 times higher than TSH in gr.B and in the controls $(1.9 \pm 05$ and $1.7 \pm 1.0 \mathrm{mU} / 1$, respectively). Positive correlations were found between leptin and TSH $(r=0.54, \mathrm{P}=0.02)$, CRP and BMI $(\mathrm{r}=0.54$, $\mathrm{P}=0.044)$, CRP and TSH $(\mathrm{r}=0.668, \mathrm{P}=0.02)$. Most of the studied patients in gr.A and gr.B were positive for CRP or IL-6.

Conclusion: The data obtained demonstrate that hypothyroidism and metabolic syndrome are characterized by a variety of similar disturbances of metabolic regulation. These include insulin resistance, chronic inflammation of adipose tissue, resulting in production of adipocytokines like leptin, which seems to be concordant with thyroid hormone deficiency.

\section{P49 \\ COMPARISON BETWEEN DIRECT IMMUNOASSAY AND TANDEM MASS SPECTROMETRY IN FREE T4 MEASUREMENT FOR THE EVALUATION OF PATIENTS WITH CENTRAL HYPOTHYROIDISM \\ Ruth Percik', Offie Soldin ${ }^{2}$, Aharon Lubetzky ${ }^{3}$, Jacob llany ${ }^{1}$, \\ David Olchovsky' \\ ${ }^{1}$ Institute of Endocrinology, Sheba Medical Center, ${ }^{2}$ Georgetown \\ University Medical Center, ${ }^{3}$ Coagulation Institute, Sheba Medical \\ Center, ${ }^{4}$ Medical Ward A, Sheba Medical Center}

Objectives: Thyroid hormone replacement therapy for central hypothyroidism is based on free serum T4 measurements, with a therapeutic target of Ft4 levels above the 50th percentile of normal range. Liquid chromatographytandem mass spectrometry (LC-MS/MS) is a novel technique which enables quantification of the analyzed hormone especially in condition with instability in binding protein levels. Our objective was to compare laboratory FT4 measurements based on direct immunoassay with LC-MS/MS in a prospective cohort of patients with central hypothyroidism.

Methods: Subjects attended 4 office visits during a year of follow-up. In each visit we assessed hypothyroidism using a validated clinical score and laboratory measurements including FT4, FT3 and TSH, both in the chemiluminescent immunoassay and in LC-MS/MS.

Results: Among 12 subjects, FT4 measurements in the two techniques correlated significantly $(\mathrm{R}=0.79$, $\mathrm{P}$ value $=0.0001$; with significant correlation between FT4 and TSH levels in both techniques $(\mathrm{R}=-0.604 \mathrm{P}$ value $=0.0001$; $\mathrm{R}=-0.354, \mathrm{P}$ value $=0.05$ respectively). We found a significant correlation between FT4 and clinical scores in both techniques $(\mathrm{R}=-0.372$, $\mathrm{P}$ Value $=0.03$ and $\mathrm{R}=0.336$, $\mathrm{P}$ value $=0.042$ respectively). On R.O.C. analysis there are sta-

Table 1. Characteristics of 12 participants with hypopituitarism (for Abstract P49)

\begin{tabular}{ll}
\hline Number of men / women & 12 \\
\hline Age - years (mean; range) & $54(29-74)$ \\
Primary Diagnosis & 3 \\
Microadenoma & 3 \\
Non-functioning macroadenoma & 2 \\
Craniopharyngioma & 2 \\
Apoplexy & 1 \\
Head Trauma & 1 \\
Histiocytosis X & 8 \\
Pituitary surgery & 4 \\
Sellar irradiation & $127 \pm 38$ \\
Thyroxin dosage (mqg/d, mean \pm SEM) & $\mathrm{N}$ \\
Concurrent Hormonal replacement & 10 \\
Glucocorticoid & 6 \\
Testosterone & 2 \\
GH & 1 \\
LH & 1 \\
FSH & 1 \\
DHEA & 3 \\
Desmopressin & \\
\hline
\end{tabular}

tistically insignificant differences between the two R.O.C. curves of the clinical score as a predictor of FT4 levels in the two techniques.

Conclusions: Chemiluminescent immunoassay and LC-MS/MS are comparably accurate in measuring FT4 in central hypothyroidism despite possible hormone binding protein instability. In addition, the clinical score is a reliable predictor for FT4 measurements in the two laboratory techniques.

\section{P50 \\ UNDIAGNOSED SUBCLINICAL AND CLINICAL HYPOTHYROIDISM IN TYPE 1 DIABETES PATIENTS \\ Salome Glonti \\ Unimed Ajarabatumi Refferal Hospital; Theraphy; Endocrinology, \\ Batumi, Georgia}

Objectives: Hypothyroidism(HT) is often under-diagnosed due to the lack of symtoms and increases the risk of lipid disturbances of diabetes mellitus (DM). In consideration of high prevalence of thyroid pathology of Adjara (iodine deficiency district of Georgia), we have examined it in random selection of type $1 \mathrm{DM}$ patients, with an early unexamined thyroid function.

Materials and Methods: We examined 123 type 1 DM patients over 18 (middle age $37 \pm 1.2$ ) who had no connection with thyroid gland(TG) deseases. All patients were examined TSH, free T4, Anti - TPO, HBA1C and thyroid ultrasound(TU).

Results: Subclinical hypothyroidism(SH) was found in 31 from 123 type 1 DM patients $(25.2 \%)(\mathrm{TSH}-5,2 \pm 0.9 \mathrm{mU} / \mathrm{ml})$. In 10 from $123(8.1 \%)$ was found HT (TSH - $10.2 \pm 0.8 \mathrm{mU} / \mathrm{ml})$. In $67-(54.5 \%)$ had increased degree of Anti-TPO, within all 31 with HT, and 10 with SH. 26 type 1 DM patients with Anti-TPO had no disorder of TG function. Elevated HbA1c was observed in $14.8 \%$ of HT patients versus $12.1 \%$ in euthyroid patients $(\mathrm{P}>0.05)$. According to the TU research data, patients with $\mathrm{SH}$ had diffuse or nodular goiter $(64.3 \%)$. In the group of HT-hypoplasia or atrophy of TG (61.7\%).

Conclusion: High prevalence of undiagnosed HT of type1 DM patients in Adjara: SH diagnosed to $25.2 \%$, HT -to $8.1 \%$. Physicians should anticipate the possibility of HT in patients with type $1 \mathrm{DM}$ by examination of the TG and serum TSH levels at the onset of DM and yearly thereafter. While HT was significantly associated with type $1 \mathrm{DM}$, poor diabetes control did not seem to predispose to HT and was not associated with severety of the disease.

\section{P51 \\ PATIENT CHARACTERISTICS IN SUBCLINICAL HYPOTHYREOSIS}

\section{Esa Soppi}

Eira Hospital, Helsinki, Finland

Introduction: In Finland, close to 290,000 (about $5.3 \%$ of the population) patients used levothyroxine in 2003 , based on the prescription statistics of the Social Insurance Institution. Approximately 200,000 patients were not entitled to special reimbursement, meaning that these patients have subclinical hypothyreosis. The number of levothyroxine users is increasing rapidly: from 2012 to 2013 about 20,000 new patients were prescribed levothyroxine. This trend is apparently a result of an increased awareness of the high prevalence of thyroid diseases. The diagnosis of subclinical hypothyreosis is difficult and requires exclusion of many conditions with similar symptoms, e.g., iron and D-vitamin deficiency, subclinical diabetes and sleep deprivation. After exclusion of other diseases test treatment with levothyroxine is often needed to establish the diagnosis. I describe a group of patients with subclinical hypothyreosis who responded favorably to levothyroxine treatment.

Patients and Results: 214 patients with subclinical hypothyreosis from office based practice were treated with levothyroxine. Patients were divided into two groups based on their initial TSH-values (Table). No clinical or laboratory characteristics can be used to separate these patients from each other. The higher the TSH-value was, the more common it was to identify TPOantibodies. Similarly, the latter group needed a higher average maintenance dose of thyroxine. A careful history together with sufficiently long treatment time with levothyroxine with a favorable outcome are essential to establish the diagnosis of subclinical hypothyreosis. 


\section{P52}

THE FREQUENCY OF HYPOTHYROIDISM IN SUBJECTS WITH METABOLIC SYNDROME, DIABETES, ARTERIAL HYPERTENSION, HYPERCHOLESTEROLEMIA, VITAMIN D DEFICIENCY

Anna-Maria Borissova ${ }^{1}$, Alexander Shinkov ${ }^{1}$, Jordan Vlahov ${ }^{1}$ Lilia Dakovska $^{1}$, Lidia Kassabova ${ }^{2}$, Dobrin Svinarov ${ }^{2}$

${ }^{1}$ Medical University of Sofia, University Hospital of Endocrinology,

${ }^{2}$ Medical University of Sofia, University Hospital "alexandrovska"

Hypothyroidism is a well-known cardiovascular risk factor. The aim of the study was to determine the prevalence of hypothyroidism in subjects with other disorders associated with increased cardiovascular risk.

Material: We studied 1966 subjects, 20-80 years, 1049 females and 917 males. Body weight, height, waist circumstance, arterial blood pressure, plasma glucose, cholesterol, HDL-ch, TSH, FT4 and 25(OH)D were measured. The subjects were divided into two groups: 1. with hypothyroidism subclinical (SHT) and overt (OHT); 2. Without hypothyroidism.

Results: Diabetes was found in $9.6 \%$, Arterial Hypertension - in $39.2 \%$, Hypercholesterolemia - in $68.2 \%$, vitamin D deficiency in $-21.3 \%$ of subjects. SHT was found in $8.8 \%$ and OHT in $2.2 \%$ of the subjects or the total hypothyroidism was $10 \%$. The prevalence of hypothyroidism in subjects with diabetes, arterial hypertension, hypercholesterolemia, vitamin D deficiency is presented in the table.

Conclusion: Hypothyroidism was significantly more prevalent in subjects with other disorders associated with high cardiovascular risk, presumably contributing to CV morbidity and therefore it should be promptly detected and corrected.

\section{P53}

\section{PERIOPERATIVE MANAGEMENTS PITfalls OF} PATIENTS WITH HYPOTHYROIDISM

Richard Parkhimovich ${ }^{1}$, Irina Chikh ${ }^{1}$, Tamara Mamedova ${ }^{0}$

${ }^{1}$ Moscow Regional Clinical Research Institute

Two patients with hypothyroidism complicated perioperative period.

1) Patient B., 46 years old male was admitted due to severe form of snoring and sleep apnoe, increasing tiredness during 1.5 years. Laser uvulopalatoplastic was performed to minimize snoring. Within a day asphyxia developed, intubation followed, but respiration volume remain 150-200 ml, mechanical ventilation was started . Echocardiography without changes, sinus tachycardia. Abdomen volume increased due to bloating and bowel atony; mesenterial thrombosis was suspected. Hb $90 \mathrm{~g} / \mathrm{l}$, leucocyte formula - normal. High level of serum kreatinkinase and LDH (3112 and $879 \mathrm{U} / 1)$ and hard edema witnessed about possibility of hypothyroidism and hypothyroid myopathy). The TTH level occured $72 \mathrm{mU} \backslash \mathrm{ml}$, and T3-0.5 nmol $\backslash 1$ (1-2.8); but this information was too late. On the next day the patient died due to hypoxia (respiratory muscules myopathy plus the start of pneumonia); in addition, increasing hepato-renal insufficiency first of all as a result of "myxedema ileus". Autopsia: autoimmune thyreoiditis, pneumonia, distrophia and fragmentation myocardiocytes.

2) Patient M., 67 years old female was operated on brain(benign cyst). Before and after operation - nausea, vomiting, anorexia and as the result .hypoproteinemia (serum albumine $=22 \mathrm{~g} \backslash 1$ )-. Abdominal troubles increased: extreme bloating, bowel gas and atony, obstipation . Multispiral RCT: bowel with uneven dilatation, elongation of sigmoidea, uneven wide of bowel wall; lymphoma or inflammation and surgery were considered. These time the information was got, that patient had hypothyroidism and the last two months did not receive thyroxine. The fresh data: $\mathrm{TSH}=29 \mathrm{mU} \backslash \mathrm{ml}$, and $\mathrm{fT} 4=1.5 \mathrm{pmol} \backslash 1$ (11-23) - confirmed hypothyroidism presence. Abdominal troubles were considered to be "myxedema ileus" or Ogilvie syndrome, thyroxine dosis during 3 days became $150 \mathrm{mcg}$ with good In conclusion: do not miss hypothyroidism and remember about "myxedema ileus" and hypothyroid myopathy

\section{P54 \\ REGULATION OF NR4A IN WHITE ADIPOSE TISSUE BY DIFFERENT PHYSIOLOGICAL STATES AS HYPOTHYROIDISM}

Sonia Pérez ${ }^{0}$, Miguel López ${ }^{1}$, Rubén Nogueiras ${ }^{1}$, Sulay Tovar $^{1}$

${ }^{1}$ Cimus, Spain

Objectives: Nur77, Nurr1 and Nor-1 (NR4A1, NR4A2 and NR4A3, respectively) are member of the NR4A subfamily of the orphan nuclear receptors. They are implicated in numerous biological processes (DNA repair, arteriosclerosis, cell apoptosis, carcinogenesis and metabolism).

The aim of this work was to elucidate the regulation of NR4A1, NR4A2 and NR4A3 in white adipose tissue (WAT) under different physiological and pathophysiological settings involved in energy balance such as hormonal deficiency, fasting, postnatal development, gender, and pregnancy.

Methods: Sprawe-Dawley rats are separated in different groups with several conditions: Several pituitary factors; Different nutritional status; Different states of development $(25,45,60$ and 90 days); Pregnant rats of different days; Ovariectomized and Gonadectomized rats; etc. We analyze the expression of mRNA of NR4A1, NR4A2 and NR4A3 in gonadal adipose tissue.

Results: Our results shows as the hormonal deficiency in thyroid produced a decreased of Nur77, Nurr1 and Nor1 expression whereas GH-deficiency only affects expression of Nur77. The food status changes NR4A expression levels and the pregnancy a special metabolic produced the increased expression. In addition, the age and sexual status affect the Nur77, Nurr1 and Nor1 expression, increased along of the development indicate the regulation of NR4A1, NR4A2 and NR4A3 by different metabolic status.

Conclusions: Our data indicate that NR4A subfamily could be exerting an action in the metabolism of the rat acting as a mediator in physiological stages.

Table 1. Two patient populations with subclinical hypothyreosis dichotomized by TSH-levels (for Abstract P51)

\begin{tabular}{|c|c|c|c|c|c|c|c|c|}
\hline $\begin{array}{l}\text { Group } \\
\text { TSH-level } \\
(\mathrm{mU} / 1)\end{array}$ & $\mathrm{N}$ & $\begin{array}{l}\text { Thyroid diseases } \\
\text { in close relatives } \\
(\%)\end{array}$ & $\begin{array}{l}\text { Males } \\
(\%)\end{array}$ & $\begin{array}{l}\text { Mean age } \\
\text { (yrs) }\end{array}$ & $\begin{array}{l}\text { Mean } \\
\text { TSH } \\
(\mathrm{mU} / 1)\end{array}$ & $\begin{array}{l}\text { Mean freeT4 } \\
(\mathrm{pmol} / \mathrm{l})\end{array}$ & $\begin{array}{l}\text { TPOAb positive } \\
(\%)\end{array}$ & $\begin{array}{l}\text { Mean } \\
\text { thyroxine dose } \\
(\mathrm{mg})\end{array}$ \\
\hline $2.1-4.9$ & 121 & 48 & 17 & 41 & 4.0 & $\begin{array}{l}9-18 \\
(\text { mean 13) }\end{array}$ & 40 & 0.117 \\
\hline $5.0-9.9$ & 93 & 48 & 10 & 43 & 6.7 & $\begin{array}{l}9-18 \\
(\text { mean 13) }\end{array}$ & 57 & 0.142 \\
\hline
\end{tabular}

Table 1. (for Abstract P52)

\begin{tabular}{|c|c|c|c|c|c|c|c|}
\hline \multicolumn{2}{|c|}{ Diabetes mellitus } & \multicolumn{2}{|c|}{ Arterial Hypertension } & \multicolumn{2}{|l|}{ Cholesterol } & \multicolumn{2}{|l|}{ Vitamin D } \\
\hline Yes & NO & $>140 / 90 \mathrm{mmHg}$ & $<140 / 90 \mathrm{mmHg}$ & $>5.2 \mathrm{mmol} / 1$ & $<5.2 \mathrm{mmol} / 1$ & $<25 \mathrm{nmol} / 1$ & $>25 \mathrm{nmol} / 1$ \\
\hline $30 *$ & $186^{*}$ & 94 & 122 & $163 * *$ & $53 * *$ & $58 *$ & $158 *$ \\
\hline $16.5 \%$ & $10.4 \%$ & $12.3 \%$ & $10.2 \%$ & $12.2 \%$ & $8.4 \%$ & $14.8 \%$ & $10 \%$ \\
\hline
\end{tabular}

\footnotetext{
$* \mathrm{P}<0.05 ; * * \mathrm{P}<0.01$
} 


\section{P55 \\ CORE NEEDLE BIOPSY IS MORE USEFUL FOR MANAGEMENT DECISION THAN REPEAT FINE- NEEDLE ASPIRATION CYTOLOGY IN BOTH SUBCATEGORIES OF AUS/FLUS THYROID NODULES}

Donggyu Na' ${ }^{1}$, Hye Sook Min², Hunkyung Lee ${ }^{3}$, Hyo Bin Seo ,

Ji-Hoon Kim ${ }^{2}$, Yeokyu Youn ${ }^{2}$

${ }^{1}$ Human Medical Imaging \& Intervention Center, ${ }^{2}$ Seoul National University Hospital, ${ }^{3}$ Ewha Clinical Laboratory, ${ }^{4}$ Healthcare Research Institute, Seoul National University Hospital Healthcare System Gangnam Center

Objectives: The purpose of this study was to determine whether core needle biopsy (CNB) is more useful than repeat fine-needle aspiration cytology (rFNAC) for the management decision of AUS/FLUS nodules in each subcategory of AUS/FLUS.

Methods: This study included 214 thyroid nodules from 207 consecutive patients who previously had AUS/FLUS results and underwent both rFNAC and CNB after the initial FNAC. The AUS/FLUS category was subcategorized into AUS (nuclear atypia) and FLUS (architectural atypia). We compared the inconclusive results, the rate of malignancy diagnosis (category 5 or 6), and sensitivity for surgically diagnosed malignant tumors between rFNAC and CNB in each subcategory of AUS/FLUS nodules.

Results: In each subcategory of AUS ( $n=141)$ and FLUS $(n=73)$, CNB showed a lower rate of inconclusive results (category 1 and 3 ) than rFNAC (AUS, $26.8 \%$ vs. $57 \%, \mathrm{P}<0.001$ and FLUS, $21.9 \%$ vs. $49.3 \%, \mathrm{P}=0.001$ ). $\mathrm{CNB}$ showed a higher rate of category 2 and 4 results than rFNAC in both AUS and FLUS nodules ( $\mathrm{P}=0.01$ and $\mathrm{P} \leq 0.013$, respectively). The rate of malignancy diagnosis of CNB was higher than that of rFNAC in AUS nodules $(\mathrm{P}=0.001)$ and malignancy diagnosis was found only by CNB in FLUS nodules. The sensitivity of CNB for surgically diagnosed malignant tumors $(\mathrm{n}=$ $35)$ was higher than that of rFNAC in overall ( $62.9 \%$ vs. $22.9 \%, \mathrm{P}=0.001)$, AUS (70.4\% vs. $29.6 \%, \mathrm{P}=0.001)$, and FLUS nodules $(37.5 \%$ vs. $0 \%)$.

Conclusions: CNB showed a lower rate of inconclusive results, a higher rate of malignancy diagnosis, and higher sensitivity for malignant tumors in both subcategories of AUS/FLUS nodules. CNB will be more useful for management decision than rFNAC in both subcategories of AUS/FLUS nodules.

\section{P56 \\ MRI AND ULTRASONOGRAPHY DETECTION OF CERVICAL LYMPH NODE METASTASES IN DIFFERENTIATED THYROID CARCINOMA BEFORE REOPERATION}

\section{Zeming Liu}

Department of Breast and Thyroid Surgery, Union Hospital, Tongji Medical College, Huazhong University of Science and Technology, WuHan, Hubei, China

Objective: The purpose of this study was to compare the diagnostic capabilities of magnetic resonance imaging (MRI) and ultrasonography (US) for cervical lymph nodal metastases in differentiated thyroid carcinoma (DTC) before reoperation.

Material and Method: From June 2011 to May 2013, preoperative MRI and ultrasound data were collected from differentiated thyroid cancer patients who underwent a reoperation. The following characteristics were assessed: the sensitivity, specificity, positive predictive value, negative predictive value and accuracy of MRI and US. The MRI and ultrasound findings were correlated with the histological diagnosis after reoperation.

Results: One hundred and thirty-eight cases were included in the analysis. $88.4 \%(122 / 138)$ of which had evidence of residual thyroid cancer tissue or metastatic nodal involvement at final histology. Lymph nodal metastases were confirmed in the central compartment in $76.42 \%(81 / 106)$ of patients and in lateral compartment in $73.28 \%(85 / 116)$ of patients. The sensitivity, specificity and accuracy of MRI VS US for detecting central compartment metastases was $75 \%$ VS $41.67 \%(\mathrm{P}=0.04), 90.91 \%$ VS $100 \%(\mathrm{P}=1)$ and $80 \%$ VS $60 \%$ $(\mathrm{P}=0.618)$, respectively; For detecting lateral compartment metastases was $83.33 \%$ VS $77.78 \%(P=1), 25 \%$ VS $50 \%(P=0.606)$ and $65.38 \%$ VS $69.23 \%$ $(\mathrm{P}=1)$, respectively. There was statistically significant difference between the sensitivity of MRI and ultrasound for diagnose of central compartment metastases. The MRI features with the greatest correlation with positive lymph nodal metastases were fusion and enhancing lesions. The ultrasound features with the greatest correlation with positive lymph nodal metastases were hypoechoic and microcalcifications.

Conclusion: MRI is more sensitive than ultrasonography in detecting central compartment metastases in papillary thyroid carcinoma. There is no significant difference in diagnosis of lateral neck node metastases between MRI and US.

\section{P57 \\ FOLLICULAR THYROID NEOPLASMS ON FNA REQUIRING SURGERY AND THE FINAL PATHOLOGY: IS FOLLICULAR THYROID CANCER THE REAL THREAT?}

Ron Pery 1 , Gil Har-Zahav¹, Esther Shabtai², Mordechai Guttman',

Moshe Shabtai ${ }^{3}$

${ }^{1}$ Dept. of Surgery, Sheba Medical Center; Tel Aviv University,

${ }^{2}$ Statistics, Communication Disorders; Tel Aviv University, ${ }^{3}$ Endocrine

Surgery, Dept. of Surgery; Sheba Medical Center, Sackler School of

Medicine; Tel Aviv University

Background: The presence of indeterminate cytology such as follicular neoplasm is usually an indication for surgery due to the inability of FNA to discriminate between benign and malignant follicular tumors. Subsequent to a clinical impression that follicular cancers are very infrequent, this study aimed at evaluating the actual pathology in thyroid specimens removed surgically for follicular lesion. (The study was approved by the local IRB).

Patients and Methods: The relevant demographic, clinical, and pathological data of all consecutive patients undergoing partial or total thyroidectomy in the last 3 years were entered into a data base. All patients with pre-operative diagnosis of follicular neoplasm Bethesda III-IV were included. The final permanent pathology was noted and correlated to age, gender and extent of operation.

Results: Of 195 thyroidectomies, 46 were performed for follicular neoplasms. Mean age was 54.6 (34-80) and 40 were females. Operations performed were hemithyroidectomy+Isthmectomy $(\mathrm{HI})(\mathrm{n}=36)$ and total thyroidectomy (TT) $(\mathrm{n}=10)$. Overall there were $10(21.7 \%)$ malignant and $36(78.3 \%)$ benign pathological results. One follicular carcinoma $(2.2 \%)$ was observed while 8 were follicular variant of papillary cancer and 1 was medullary thyroid cancer. The most frequent benign lesions were follicular adenoma $(\mathrm{n}=18,39 \%)$, nodular hyperplasia $(\mathrm{n}=9,19.6 \%)$ and colloid nodules and other benign nodules $(\mathrm{n}=9,19.5 \%)$.

Conclusions: These results concur with the notion that $20 \%$ of follicular lesion will be malignant but differ largely from the concept that these malignancies will be follicular thyroid cancer. Further and larger studies are required to validate these conclusions and target the cases requiring surgery more precisely.

\section{P58 \\ DESCRIPTIVE STUDY OF OVER 1,800 PATIENTS WITH DIFFERENTIATED THYROID CARCINOMA TREATED IN THE PERIOD 1998-2012 IN 11 HOSPITALS IN CATALONIA}

Zafon Carles ${ }^{1}$, Manel Puig-Domingo ${ }^{2}$, Dídac Mauricio ${ }^{2}$

${ }^{1}$ Hospital Vall D'hebron; on Behalf of the Consortium for the Study of Thyroid Cancer (Cecat), ${ }^{2}$ Hospital Germans Trias I Pujol; on Behalf of the Consortium for the Study of Thyroid Cancer (Cecat)

Introduction: Collaboration between institutions in order to combine both patient care and research strategies is mandatory in those diseases with a relatively low incidence. Recently, the consortium for the study of thyroid cancer (CECaT), which includes 18 centers, has been created in Catalonia (Spain).

Objective: The first initiative of the group was to carry out a descriptive analysis about the characteristics of patients with differentiated thyroid carcinoma (DTC) treated over a period of 15 years (1998-2012).

Material and Methods: Patients from eleven centers of the CECaT were included. Several clinical and histological variables were analyzed. Data are expressed as proportions, percentages and means (standard deviation). 
Results: The cohort included 1,855 patients, 1,470 (79.2\%) women, with a mean age of 47.7 (15.7) years. Prior to surgery, the fine needle aspiration biopsy revealed the diagnosis of malignancy / suspected malignancy in $63 \%$ of the cases. A total of 1,650 (89\%) were papillary carcinomas. The average tumor size was $21.5(16) \mathrm{mm}, 30 \%$ were smaller than $1 \mathrm{~cm}$ and $31 \%$ were multifocal. In 353/896 (39\%) patients regional lymph nodes were involved. After surgery, 890/1,056 (84\%) subjects were treated with I131 with a mean dose of 117 (35) $\mathrm{mCi}$. Over $24 \%$ had some form of persistence and / or recurrence of the disease. A total of 72/1,246 distant metastases (5.7\%) were recorded. After a follow-up period of 5.5 (3.7) years, out of 1,355 patients with available information, $79 \%$ were free of disease, $17 \%$ had no tumor persistence and $4 \%$ were deceased.

Conclusions: This is one of the largest series of DTC described in Europe. The patient characteristics analyzed are similar to those reported in other parts of the world.

\section{P59 \\ WHY IS MORE PAPILLARY THYROID CANCER (PTC) AND LESS FOLLICULAR THYROID CANCER (FTC) DIAGNOSED IN ONE REGION COMPARED WITH NEIGHBOURING REGIONS IN ENGLAND? \\ Robert Goldspring ${ }^{1}$, Georgina Gerrard ${ }^{2}$, Sarah Lawton ${ }^{2}$ \\ Lavanya Mariappan ${ }^{2}$, Brinda Sethugavalar ${ }^{2}$, Preetha Chengot ${ }^{1}$ \\ ${ }^{1}$ St James University Hospital, Leeds, UK, ${ }^{2}$ St James Institute of \\ Oncology, Leeds, UK}

Objectives: Five year survival of thyroid cancer was found to be higher in one region of England compared with its 2 neighbours. The Northern and Yorkshire Cancer Registry audited the histological diagnoses of all thyroid cancer patients in these three regions looking for a reason to explain the difference in survival.

Methods: The region is sub divided into three areas, X, Y and Z. For each area, the number of patients with each histological type of thyroid cancer was found between January 2009 and December 2010 via the regional cancer registry. The overall thyroid cancer survival rate was calculated for each region as well as the number of thyroidectomies per surgeon, oncological practice and MDT review rate.

Results: 576 patients with differentiated thyroid cancer (DTC) were diagnosed in the three areas combined. The results were similar in regions $\mathrm{Y}$ and $\mathrm{Z}$ and for brevity were grouped together as $\mathrm{Y} / \mathrm{Z}$. PTC was found in $67 \%$ (235/348) of DTC patients in Y/Z but was $77 \%(177 / 228)$ in X area. FTC was diagnosed in $24 \%(83 / 348)$ in $\mathrm{Y} / \mathrm{Z}$ compared with only $14 \%(33 / 228)$ in $\mathrm{X}$ $(\mathrm{P}<0.05)$. Hurthle Cell thyroid cancer was found in $9 \%$ of patients in all 3 regions. The incidence of anaplastic thyroid cancer was the same in each area. Five year survival was significantly greater for X compared with the other two regions. Surgical site specialisation was similar throughout the region. The oncologists in all three areas followed national guidelines. All histology in the three regions were reviewed at regular MDT meetings.

Conclusion:

We are unsure as to why more patients are histologically diagnosed to have PTC than FTC in one region compared with the other two areas. Possibly more small incidental PTC were found and we will review and present the staging data.

\section{P60}

INTRA- AND INTER OBSERVATORY VARIATION OF THYROID SHEAR WAVE ELASTOGRAPHY A VARIABILITY STUDY

Kristine Rubeck ${ }^{1}$, Steen Bonnema ${ }^{2}$, Marie Louise Jespersen ${ }^{3}$, Peer Christiansen ${ }^{4}$, Viveque Nielsen ${ }^{1}$

${ }^{1}$ Department of Otorhinolaryngology and Head \& Neck Surgery, Aarhus University Hospital, ${ }^{2}$ Department of Endocrinology, Odense University

Hospital, ${ }^{3}$ Department of Pathology, Aarhus University Hosipal,

${ }^{4}$ Surgical Department P, Section of Breast and Endocrine Surgery,

Aarhus University Hospital

Objectives: The main goal when diagnosing thyroid nodules is to differentiate the few malignant from the overwhelming background of benign lesion. Currently available pre-operative tests are unable to rule out malignancy in a subset of patients, thus making diagnostic surgery necessary. Thyroid nodules constitute a diagnostic challenge subject to continuous research worldwide, and novel biochemical, genetic, radiological, and cytological tools have been suggested in an attempt to limit diagnostic thyroid surgery.

The aim of the study is to evaluate the reproducibility of ultrasonographic Shear Wave Elastography (SWE) as a novel tool for pre-operative evaluation of thyroid nodules. Hereby, we hope to contribute to a more restrictive approach when selecting patients for diagnostic thyroid surgery.

Methods: SWE assesses tissue elasticity in a quantitative manner, depicting findings as a definite variable (Elasticity Index, EI) guided by a colorcoded map.

The inter- and intraobserver and day-to-day variation of thyroid SWE will be evaluated prospectively by inclusion of 50 patients with thyroid nodules. All SWE examinations will be performed by two blinded and independent operators from January to September, 2014.

Results: Inclusion is ongoing and results will be presented at the European Thyroid Association $38^{\text {th }}$ Annual Meeting.

Conclusions: Studies have proposed a potential role of thyroid SWE in the differentiation between malignant and benign nodules, as a higher EI has been described in malignant lesions. The method is time-efficient and easily performed, allowing it to be implemented clinically. However, as the diagnostic accuracy of SWE is not clear, more studies are needed.

\section{P61 \\ CLINICAL UTILITY AND COST EFFECTIVENESS OF GALECTIN-3 THYROTEST IN THE SELECTION OF CYTOLOGICALLY INDETERMINATE THYROID NODULES (THY3) FOR SURGERY. ANALYSIS ON 336 NODULES WITH EXTENDED FOLLOW-UP}

Salvatore Sciacchitano ${ }^{1}$, Leila Baghernajad Salehi ${ }^{2}$, Luca Lavra $^{2}$, Fiorenza Magi ${ }^{2}$, Alessandra Ulivieri ${ }^{2}$, Stefano Amendola ${ }^{3}$, Tommaso Porcelli ${ }^{3}$, Giampaolo De Francesco ${ }^{4}$, Carlo Bellotti ${ }^{5}$, Armando Bartolazzi ${ }^{6}$

${ }^{1}$ Dept. of Clinical and Molecular Medicine, Sapienza University of Rome, Italy; Laboratory of Bio-Medical Research, "niccolò Cusano" University of Rome, Italy, ${ }^{2}$ Laboratory of Bio-Medical Research, "niccolò Cusano" University of Rome, Italy, ${ }^{3}$ Dept. of Clinical and Molecular Medicine, Sapienza University of Rome, Italy, ${ }^{4}$ Breast Unit, Sant'andrea University Hospital, Rome, Italy, ${ }^{5}$ Chair of Surgery, Sapienza University of Rome, Rome, Italy, ${ }^{6}$ Pathology Unit, Sant'andrea University Hospital, Rome, Italy

Galectin-3 Thyrotest has been proposed as a pre-surgical diagnostic test in cytologically indeterminate thyroid nodules (Thy3), but its clinical impact and cost effectiveness still need to be evaluated. This study was conducted to analyze the performance and costs of the routine application of this test to all Thy 3 thyroid nodules and to compare these results to that reported applying the conventional approach indicating surgery. A total of 336 Thy 3 thyroid nodules were subjected to Galectin-3 Thyrotest. Surgery was performed in all Galectin-3 positive cases $(n=93)$ and in 75 of them a malignant lesion was confirmed at final histology. Galectin-3 negative cases $(n=243)$ were monitored by US for a mean period of $25.3 \pm 19.4$ months (6-132 months). Among these patients, surgery was performed in 86 cases because of marked 
increase in nodule volume, oncocytic features at cytology or patient's choice and a malignancy was detected in 6 of them. All monitored nodules showed a volume that was reduced in $28 \%$, unchanged in $52 \%$ or slightly increased in $20 \%$ of cases. Our combined approach of Galectin-3 Thyrotest followed by US monitoring allowed us: (i) to identify malignancy in 81 cases, equal to $24.1 \%$ of our total Thy 3 nodules, comparable to the prevalence of thyroid cancer in Thy3 lesions reported in the literature $(27.8 \%$; 95\% CI $23-33 \%)$; (ii) to reduce thyroidectomies, compared to conventional approach $(53.3 \%$ vs $100 \%$ ); (iii) to increase the percentage of malignancy in thyroidectomized patients $(45.3 \%$ vs $27.8 \%)$. Our test is cheaper (100 Euro/test), compared to the two new genetic ones, namely the miRInform, Asuragen (2,200 USD/test) and the Gene Expression Classifier, Veracyte (3,200 USD/test). In conclusion, our results indicate that selection of Thy 3 thyroid nodules based on Galectin-3 Thyrotest and subsequent US follow-up is an effective and cheap strategy and should be recommended in the clinical management of such patients.

\section{P62 \\ THYROID LESIONS IN PATIENTS WITH GERMLINE MUTATION OF PTEN GENE (PTEN- HAMARTOMA TUMOR SYNDROME) \\ José Cameselle-Teijeiro ${ }^{1}$, José-Manuel Cabezas-Agrícola ${ }^{2}$ \\ Rosa Reyes-Santías ${ }^{3}$, Natividad Alfonsín-Barreiro ${ }^{4}$, Ihab Abdulkader ${ }^{3}$, \\ Felipe Casanueva-Freijo ${ }^{2}$, Ana Vega-Gliemmo \\ ${ }^{1}$ Department of Pathology, Clinical University Hospital of Santiago \\ de Compostela, Sergas, University of Santiago de Compostela, \\ ${ }^{2}$ Department of Endocrinology, Clinical University Hospital, Santiago \\ de Compostela, Spain, ${ }^{3}$ Department of Pathology, Clinical University \\ Hospital, Santiago de Compostela, Spain, ${ }^{4}$ Department of Pathology, \\ Clinical University Hospital, Vigo, Spain, ${ }^{5}$ Medicina Xenómica, \\ Fundación Pública Galega de Medicina Xenómica, Clinical University \\ Hospital, Santiago de Compostela, Spain}

Objectives: Phosphatase and tensin homolog deleted on chromosome ten (PTEN)-hamartoma tumor syndrome (PHTS) is an autosomal dominant disorder caused by germline inactivating mutations of the PTEN tumor suppressor gene. PHTS includes Cowden syndrome (CS), Bannayan-Riley-Ruvalcaba (BRRS), and Proteus-like syndromes. Affected individuals develop both benign and malignant neoplasms in a variety of tissues, including thyroid. We report the thyroid pathology in a series of patients with PHTS

Methods: This study investigates the thyroid pathology and germline PTEN mutations in a series of 9 patients with PHTS.

Results: Nine patients with PHTS (CS $[n=7]$; BRRS $[n=2])$, ( 8 female and 1 male -BRRS) had a mean age at thyroidectomy of 23.2 years (range 9-35), 26.8 years (18-35) for CS and 10.5 (9 and 12) for BRRS. Germline PTEN mutations were located at exons: 1, 2, 5 (3 patients), 6, 7, and 8 (2 patients). Thyroid pathological findings were: multiple adenomatous nodules in all $(100 \%)$ patients, lymphocytic thyroiditis in $8(88.8 \%)$, follicular adenomas (with 2 adenolipomas) in $8(88.8 \%$ ), papillary carcinoma (PTC) (with 4 microcarcinomas [mPTC]) in $5(55.5 \%)$, follicular carcinoma (FC) in $2(22.2 \%)$, C-cell hyperplasia in $1(11.1 \%)$, intrathyroidal thymic tissue in $1(11.1 \%)$, parathyroid gland within thyroid in $1(11.1 \%)$, and adipose tissue another $(11.1 \%)$. No correlation was found between specific PTEN germline mutations and pathological findings: PTC and/or mPTC was detected in patients with PTEN mutation at exons 5, 6 and 8, while FC at exons 2 and 6 .

Conclusions: No correlation was found between specific PTEN germline mutations and pathological findings; the histological findings, however, of multiple adenomatous nodules and/or adenomas with thyroiditis, particularly in children and young adults, should alert to the possibility of PHTS. This work was supported by Grant PI12/00749-FEDER from Instituto de Salud Carlos III, Ministry of Economy and Competitiveness, Madrid, Spain.

\section{P63}

Poster has been withdrawn

\section{P64}

THE THYMIC HYPERPLASIA (TH) AND BIOCHEMICAL PERSISTENCE OF DISEASE IN PATIENTS WITH DIFFERENTIATED THYROID CARCINOMA (DTC)

Carla Gambale ${ }^{1}$, Antonio Matrone ${ }^{2}$, Eleonora Molinaro ${ }^{1}$, Laura Agate ${ }^{1}$, Valeria Bottici', Agnese Biagini', David Viola', Salvatore Mazzeo ${ }^{3}$,

Francesca Bianchi', Federica Brozzi ${ }^{1}$, Paolo Vitti ${ }^{1}$, Rossella Elisei ${ }^{1}$

${ }^{1}$ Department of Clinical and Experimental Medicine-Unity of

Endocrinology- University of Pisa, ${ }^{2}$ University of Pisa, Endocrine Unit Department of Clinical and Experimental Medicine, Pisa, Italy, ${ }^{3}$ Unity of Radiology-University of Pisa - Pisa

Introduction: Thyroglobulin ( $\mathrm{Tg}$ ) is a sensitive marker of disease in patients with differentiated thyroid carcinoma (DTC). However it is not unusual to find patients in follow-up with low but detectable values of Tg and negative imaging for metastatic lesions.

Methods: We analyzed features of 21 patients treated with total thyroidectomy and 131I for DTC, who had TH, detected by CT scan or MRI.

Results: 21 PTC patients (15 women, 6 men), with a mean age at diagnosis of 30.1 years (16-49) and with a CT scan or MRI showing the persistence of thymus were followed for a median period of 4 years (1-16 years). Whole body scan (WBS) after 131I ablative dose showed a mediastinal uptake compatible with TH in 6/21(28.6\%): $2 / 6$ continued to have mediastinal uptake at the last WBS, in 2/6 the last WBS was negative and in 2/6 only one WBS was performed because they were cured after this. At variance, 15/21 had no mediastinal uptake at the 1st WBS but $9 / 15(60 \%)$ showed a mediastinal uptake at last WBS. 2 out of 15 patients with no mediastinal uptake showed lung and/or neck lymphnode uptake and were retreated with 131I: 19/21(90.5\%) patients performed several treatments with 131I. Nevertheless, 18/21(85.7\%) patients continued to have detectable values of basal $\mathrm{Tg}(0.75 \pm 1.13 \mathrm{ng} / \mathrm{ml})$ at the last clinical and biochemical control.

Conclusions: 1) In young patients with DTC and low but detectable values of Tg, without any radiological finding of metastases, TH may be considered even if no mediastinal uptake is observed at WBS; 2 ) in the presence of TH, confirmed by CT scan or MRI, and negative imaging for metastatic lesions, low detectable levels of Tg are not indicative of a persistent disease and no further 131I activities should be administered.

\section{P65 \\ PROGNOSis VAlUe of STAGe PT3' Clinical AND PATHOLOGIC CHARACTERISTICS IN WELL DIFFERENTIATED THYROID CANCER}

Carolina Moreno ${ }^{1}$, Miguel Melo', Cristina Ribeiro², Luísa Ruas², Joana Saraiva ${ }^{1}$, Daniela Guelho ${ }^{2}$, Luís Cardoso², Nuno Vicente ${ }^{2}$, Gracinda Costa ${ }^{2}$, Francisco Carrilho ${ }^{2}$

${ }^{1}$ Coimbra Hospital and University Centre; Faculty of Medicine of the University of Coimbra, ${ }^{2}$ Coimbra Hospital and University Centre

Introduction: The TNM staging system for well differentiated thyroid cancer(WDTC) considers tumours over $4 \mathrm{~cm}$ diameter limited to the thyroid or tumours with minimal extra-thyroid extension to be classified as pT3. This stage can be quite heterogeneous, as different clinical and pathologic characteristics seem to have distinct prognosis value.

Aims: To determine prognostic factors amongst clinical, laboratorial, histologic and radiologic characteristics of WDTC pT3 patients followed in our department.

Material and Methods: Transverse observational study of 129 patients with WDTC pT3 treated in our institution between January/2006December/2011. The following parameters were analysed: age, gender, personal and family history, histologic type, size, number of foci, presence of capsule, extra-thyroid extension, ${ }^{131} \mathrm{I}$ activity administered, post- ${ }^{131} \mathrm{I}$ wholebody scintigraphy, using SPSS $21.0^{\circledR}$. Patients were considered disease-free if there was no uptake outside the thyroid area in the post-dose scintigraphy, normal cervical ultrasound and stimulated thyroglobulin $<2 \mathrm{ng} / \mathrm{mL} 9-15$ months after initial treatment.

Results: Patients were followed for $48.2 \pm 16$ months. At the end, $72.1 \%(n=93)$ patients were disease-free - group $A$, and $27.9 \%(n=36)$ had residual disease or relapse - group B. The size of primary tumour was significantly inferior in group A compared with group $\mathrm{B}(21.5 \pm 12.9 \mathrm{~cm}$ Vs 
$30.6 \pm 20.8 \mathrm{~cm} ; \mathrm{p}=0.004)$, as the vascular invasion $(36.4 \% \mathrm{Vs} 63.6 \% ; \mathrm{P}<$ $0.001)$ and tumoral extension to adipose and fibromuscular tissue $(38.5 \% \mathrm{Vs}$ $61.5 \% ; \mathrm{P}<0.001)$. In the post- ${ }^{131} \mathrm{I}$ whole-body scintigraphy, the latero-cervical uptake was significantly less in group $\mathrm{A}(19 \% \mathrm{Vs} 81 \% ; \mathrm{P}<0.001)$. Mediastinic and distant metastasis were found only in group $\mathrm{B}(100 \% \mathrm{Vs} 0 \% ; \mathrm{P}<0.001)$. Thyroglobulin 3 months after surgery was significantly inferior in group $\mathrm{A}(0.3 \pm 0.3 \mathrm{Vs} 15 \pm 42.9 ; \mathrm{p}=0.001)$. By logistic regression were identified as the most important independent factors predictive of cure: absence of latero-cervical uptake in post- ${ }^{131}$ I whole-body scintigraphy $(\mathrm{OR}=0.048 ; \mathrm{P}<0.001)$ and tumour extension to adipose and fibromuscular tissues $(\mathrm{OR}=0.255 ; \mathrm{p}=0.021)$.

Conclusions: In our cohort several clinical, pathological and radiological factors were associated with good prognosis(disease-free). In pT3 tumours, absence of node involvement and considerable extra-thyroid extension were the most relevant factors predictive of cure.

\section{P08 Thyroid Cancer Therapeutics 1}

\section{P66 \\ EFFECT OF PHOTODYNAMIC THERAPY BY INTRATUMORAL PHOTOFRIN INJECTION IN ORTHOTOPIC MOUSE MODEL OF HUMAN ANAPLASTIC THYROID CARCINOMA \\ Pil Sang Chung ${ }^{1}$, Jin-Cheol Ahn ${ }^{2}$ \\ ${ }^{1}$ Department of Otolaryngology-Head and Neck Surgery, Dankook University Hospital; Beckman Laser Institute Korea, Dankook \\ University, ${ }^{2}$ Biomedical Engineering, Dankook University}

Objective: Anaplastic thyroid cancer is one of the most aggressive solid tumors. Multimodal treatments do not meaningfully improve survival of anaplastic thyroid cancer. Consequently, new effective therapeutic modalities are needed. This study investigated the effectiveness of PDT by intratumoral photofrin injection in orthotopic mouse model of human anaplastic thyroid carcinoma.

Material \& Method: FRO cells were injected into the thyroid glands of athymic female nude mice. Fourteen days post-implantation, mice were divided randomly into a PDT group and control group. The survival time and body weight of nude mice were examined. Tissue was collected for subsequent histological assay after autopsy of mice.

Result: There was no PDT related mortality. Histological examination revealed that PDT induced severe necrosis and cytotoxicity of neoplastic cells. Animals in the PDT group in a repeat experiment survived significantly longer than animals in the other groups.

Conclusion: PDT using photofrin is effective in delaying the tumor growth in orthotopic mouse model of ATC. This result suggest that intratumoral PDT may be applied to the palliative treatment of ATC.

\section{P67}

\section{BRAF: A POTENT INDICATOR FOR}

DETERMINING THE EXTENT OF SURGERY IN

PAPILLARY THYROID CANCER

Bong Kyun Kim${ }^{1}$, Jee Soo Kim 1 , Jung-Han Kim ${ }^{1}$, Jun-Ho Choe ${ }^{1}$, Seo Ki Kim ${ }^{1}$, Hyun Chul Lee ${ }^{1}$, Jun Ho Lee ${ }^{1}$, Jae Hoon Jang ${ }^{2}$

${ }^{1}$ Samsung Medical Center, Sungkyunkwan University School of

Medicine, ${ }^{2}$ Division of Breast and Endocrine Surgery, Department of

Surgery,

Background: Recent studies have shown that BRAF mutation is a promising diagnostic and prognostic marker for papillary thyroid cancer(PTC). However, other studies have raised questions about the diagnostic and prognostic value of BRAF mutation.

Objective: The aim of our study was to investigate whether BRAF mutation analysis may assist determining the extent of surgery in PTC patients.

Study design. Case-control study.

Patients and Methods: From January 2008 to December 2012, data was collected retrospectively on 3111 PTC patients who underwent thyroidectomy with cervical lymph node dissection. From this group, we examined the relationships between BRAF mutation and the various clinicopathologic factors from the results of fine needle aspiration(FNA), ultrasonography(US), and histopathology reports. BRAF mutation was analyzed by allele specific polymerase chain reaction(AS-PCR), direct sequencing and real time PCR(RTPCR) from FNA specimens or surgical specimens.

Results: The prevalence of BRAF mutation in PTC patients was 2530 of 3111 cases $(81.3 \%)$, and multivariate analysis showed that BRAF mutation was independently associated with male PTC(OR: $1.538,95 \%$ CI 1.199-1.976, P < $.001)$, classic variant of PTC(OR: 3.096, 95\% CI 1.972-4.878, P<.001), both thyroid lobe involvement(OR: $1.653,95 \%$ CI $1.266 \sim 2.160, \mathrm{P}<.001)$, extrathyroidal extension(OR: $1.617,95 \%$ CI 1.328-1.970, $\mathrm{P}<.001$ ), central lymph node(CLN) metastasis(OR: 1.416, 95\% CI 1.146-1.748, P = .004). However, there were no associations between BRAF mutation and other factors as follows: age, tumor size, multicentricity in single lobe, lymphovascular invasion.

Conclusions: This study clearly demonstrates that BRAF mutation is strongly associated with the both thyroid lobe involvement, extrathyroidal extension, and CLN metastasis. In addition to conventional indications, BRAF mutation analysis can be used as an indicator for determining the extent of surgery in PTC.

P68

Poster has been withdrawn

P69

Poster has been withdrawn 


\section{P70 \\ COMPARISON BETWEEN ENDOSCOPIC THYROIDECTOMY AND CONVENTIONAL OPEN THYROIDECTOMY}

Sang Seol Jung ${ }^{1}$, Sohee Lee ${ }^{1}$, Byung Joo Song ${ }^{1}$, Ja Seong Bae ${ }^{1}$

${ }^{1}$ General Surgery, Catholic University of Korea

Background: Recently, endoscopic thyroidectomy has been applied to thyroid surgery during the last 10 years. It has been used only to a limited patient due to some technical and anatomical limitation.The aim of this study was to compare the complication of endoscopy and conventional open thyroidectomy.

Method: One thousand seven patients underwent open lobectomy and isthmectomy(Open group), and 223 patients underwent endoscopic lobectomy and isthmectomy via the axilla (Endo group) between January 2011 and August 2013. These patients were analyzed for the clinicopathologic characteristics and postoperative complication.

Results: A total of 1230 patients underwent lobectomy and isthmectomy. Endoscopic lobectomy and isthmectomy via axilla approach(Endo group) was performed in 223 patients $(18.1 \%)$ and conventional open lobectomy and isthmectomy(Open group) was performed in 1007 patients(81.9\%). Compared with the Open group, the Endo group patients presented younger age (mean age 36.25 vs. $46.15, \mathrm{p}=0.000$ ). The operative time in the Endo group was longer compared to the Open group $(85.75$ vs. $82.61, p=0.000)$. There was no statistical difference between the Endo group and the Open group in relation to postoperative hospital stay ( 2.91 vs. $2.7, \mathrm{p}=0.089$ ), central lymph node dissection(CND)(206 vs. $937, \mathrm{p}=0.628)$, tumor size( 0.60 vs. $0.83, \mathrm{p}=$ 0.250 ), histology(benign vs. malignancy, 6 vs. 217,24 vs. $980, p=0.856$ ), and number of retrieved lymph node( 4.95 vs. $6.10, p=0.416)$. In the malignant lesion, the Open group had higher stage $(\mathrm{p}=0.011)$.Postoperative complication rate was $2.88 \%$ and $6.73 \%$, respectively (Open group vs. Endo group, $\mathrm{p}=$ $0.005)$. There were no differences in bleeding, transient, permanent hypocalcemia, and permanent RLN injury. Transient RLN injury occurred in $2.38 \%$ and $6.28 \%$ of patients, respectively $(\mathrm{p}=0.002)$.

Conclusion: Compared to conventional open thyroidectomy, endoscopic thyroidectomy shows insignificant postoperative complication, as well as good cosmetic results. Endoscopic thyroidectomy can be safe, so it can be extended to more patients.

\section{P71 \\ COMPLEMENTARY RADIOIODINE (RAI) TREATMENT IS BENEFICIAL IN LOW RISK DIFFERENTIATED THYROID CANCER (DTC)}

Jolanta Krajewska ${ }^{1}$, Michal Jarzab ${ }^{1}$, Agnieszka Czarniecka ${ }^{1}$,

Jozef Roskosz' ${ }^{1}$ Daria Handkiewicz-Junak ${ }^{1}$, Aleksandra Kukulska ${ }^{1}$,

Elzbieta Gubala ${ }^{1}$, Ewa Paliczka-Cieslik ${ }^{1}$, Barbara Jarzab ${ }^{1}$

${ }^{1}$ M.Sklodowska-Curie Memorial Cancer Center and Institute of

Oncology, Gliwice Branch

Complementary RAI treatment after total thyroidectomy constitutes the main strategy in postoperative management of DTC patients. However, its routine administration in low risk subjects remains controversial due to the lack of unequivocal evidences of its effectiveness.

The aim of the study was a retrospective evaluation of long-term outcomes of combined DTC treatment to assess the impact of adjuvant RAI therapy in the low risk group.

Primary hypothesis was: "if adjuvant RAI treatment was unnecessary, a delay in RAI administration would have no impact on long-term outcomes".

Material and Methods: The study group consisted of 510 DTC patients, staged pT1b-T4N0-N1M0, treated with total thyroidectomy followed by RAI therapy. On the basis of initial stage and postoperative stimulated serum $\mathrm{Tg}$ level 272 patients were classified as a low risk group (T1-T3, N0Nx and Tg $<10 \mathrm{ng} / \mathrm{ml}$ ), 90 as a high risk (stimulated $\mathrm{Tg}>30 \mathrm{ng} / \mathrm{ml}$ ), whereas 148 remaining constituted an intermediate risk group. Median follow-up was 12.1 years (range 1.5-15.2)

Results: To properly assess the efficacy of adjuvant RAI therapy all groups were divided depending on time of RAI administration. Next, subjects treated with RAI up 9 months after cancer diagnosis were compared to subgroups treated later: within 9-24 and $>24$ months. In the low risk group, patients treated with RAI up to nine months, showed no recurrences, whereas among those, treated 9-24 months and $>24$ months after diagnosis, the risk of recurrence were $5.5 \%$ and $7.1 \%$, respectively $(\mathrm{p}=0.035)$. There were no significant time depending differences in intermediate and high risk groups.

Conclusion: Complementary RAI therapy, administered within a short time period after the operation significantly reduces the risk of cancer recurrence in the low risk group. We believe that the exclusion of low risk patients from RAI therapy, suggested by the ATA and European guidelines, is not justified.

\section{P72}

OUTCOME AND SURVIVAL IN DIFFERENTIATED THYROID CANCER WITH DISTANT METASTASES AT DIAGNOSIS: LONG FOLLOW-UP OF 60

\section{PATIENTS}

Barbara Vlassopoulou ${ }^{1}$, Sofia Tsirona', Haris Vassilopoulos ${ }^{1}$,

George loannidis ${ }^{1}$, Efi Botoula ${ }^{1}$, Panagiotis Trivizas ${ }^{1}$,

Stylianos Tsagarakis ${ }^{1}$

${ }^{1}$ Evangelismos Hospital; Department of Endocrinology, Diabetes and Metabolism

Background \& Aim: Differentiated thyroid cancer (DTC) presenting with distant metastases is uncommon. The aim of this study was to evaluate the outcome \& survival of patients with DTC presenting with distant metastases at diagnosis.

Methods: A retrospective study of 60 patients with DTC with distant metastases treated at our department from 1966 to 2009 (minimum FU: 5 years).

Results: The mean follow-up of patients was 9.1 years. Histology identified 45 papillary, 13 follicular and two Hürthle-cell cancers. Sites of distant metastases comprised 20 lung (33.3\%), 14 bone (23.3\%), nine lung \& bone $(15 \%)$ and 17 multiple sites $(28.3 \%)$. All patients received radioiodine (RAI) ablation and subsequent RAI therapy (mean cumulative dose $610 \mathrm{mCi}$ ). External beam radiotherapy was given to 13 patients. During the follow-up period a total of 31 deaths have occurred; 27 due to thyroid cancer and 4 to unrelated causes. The overall survival rates at 5 and 10 years were $65 \%$ and $58.3 \%$ respectively, with cause-specific survival rates of $68.3 \%$ and $63.3 \%$ respectively. At the time of last follow-up 29 patients were alive, 20 with persistent disease and nine were disease free. In order to determine time-related trends in outcome, the study was divided into two different eras: before 1990 (group A) and between 1991-2009 (group B); the numbers of patients treated during each of these periods were 23 and 37 respectively. Cancer-related mortality occurred in 13 patients from group A \& 14 from group B $(p=0.19)$. A statistically significant difference was not found when comparing the 5- and 10 -year mortality rates of groups A \& B ( $p=0.39 \& p=0.42$ respectively).

Conclusion: Patients with DTC presenting with distant metastases have a worse outcome, approximately half of them die of disease, and only a limited number survive free of disease. The high mortality rates remained unchanged with time.

\section{P73}

HEMITHYROIDECTOMY VERSUS TOTAL THYROIDECTOMY FOR UNILATERAL PAPILLARY THYROID MICROCARCINOMA: A COMPARATIVE STUDY

Kang Dae Lee ${ }^{1}$, Hyoung Shin Lee ${ }^{1}$, Jun Woong Song ${ }^{2}$,

Sung Won Kim ${ }^{2}$, Jong Chul Hong ${ }^{3}$, Taejung Park ${ }^{2}$

${ }^{1}$ Department of Otolaryngology-Head and Neck Surgery, Kosin

University College of Medicine, ${ }^{2}$ Kosin University College of Medicine,

${ }^{3}$ Dong-A University College of Medicine

Purpose: The optimal surgical extent of thyroidectomy for unilateral papillary thyroid microcarcinoma (PTMC) is controversial. In this study, we compared the oncologic outcomes between patients who underwent hemithyroidectomy (HT) versus total thyroidectomy (TT) for a unilateral PTMC.

Methods: A retrospective study was conducted with 160 patients who underwent thyroidectomy with central lymph node dissection (CLND) for unilateral PTMC. The patients were divided into two groups as group A (HT and CLND, $n=41)$ and group B (TT and CLND, $n=119)$. The clinicopathologic 
factors were compared between the two groups and correlation of the factors to recurrence was analyzed.

Results: Total thyroidectomy (group B) had independent correlation to tumor size larger than $5 \mathrm{~mm}(\mathrm{p}=0.008, \mathrm{OR}=3.224)$ and radioiodine ablation therapy $(\mathrm{p}=0.008, \mathrm{OR}=4.290)$. Recurrence occurred in 3 cases $(7.3 \%)$ of group A and 5 cases $(4.2 \%)$ of group B with no significant difference $(\mathrm{p}=$ 0.423 ) during the median follow-up period of 75 months (range 36 to 176 months). Lateral lymph node metastasis at initial presentation $(\mathrm{p}=0.014$, $\mathrm{OR}=7.744)$ was the only independent correlated factor to recurrence in patients of two groups

Conclusion: Hemithyroidectomy versus TT when conducted with CLND for patient with unilateral PTMC showed no significant difference regarding tumor recurrence in this study.

\section{P74 \\ THE SUCCESSFUL RATE FOR RADIOACTIVE IODINE REMNANT ABLATION IN PATHOLOGICAL N1B PAPILLARY THYROID CARCINOMA PATIENTS ACCORDING TO RISK GROUP \\ Tae Yong Kim 1 , Min Ji Jeon ${ }^{2}$, Won Gu Kim 1 , Eun Kyung Jang ${ }^{2}$ Dong Eun Song ${ }^{2}$, Jin-Sook Ryu', Won Bae Kim 1 , Young Kee Shong ${ }^{1}$ ${ }^{1}$ Asan Medical Center; University of Ulsan College of Medicine, ${ }^{2}$ Asan Medical Center}

Lateral cervical lymph node (CLN) involvement of papillary thyroid carcinoma (PTC), pathological N1b, is regarded as a significant risk factor for differentiated thyroid carcinoma. However, some of N1b PTC patients showed favorable prognosis and should be considered different from those with poor prognosis.

To identify the high risk pathological N1b PTC patients, we analyzed various characteristics of lateral CLN status as prognostic factors.

This retrospective cohort study included 389 classical PTC patients with pathological N1b disease who underwent initial thyroid surgery and remnant ablation in our hospital. All patients underwent fixed dose of 5.6 GBq I-131. Patients with primary tumor sized more than $4 \mathrm{~cm}$, extrathyroidal gross invasion, maximal CLN sized larger than $3 \mathrm{~cm}$, metastatic CLNs number over 20 , or bilateral lateral CLN involvement were classified into high risk N1b group. The others were classified into intermediate risk group

Among 389 patients, 74 (19\%) were classified into high risk group and $315(81 \%)$ were classified into intermediate risk group. The successful ablation rate was only $16 \%$ in high risk group but, was $59 \%$ in intermediate risk group $(\mathrm{P}<0.001)$. These trends were also significant in the following subgroup analyses according to age and sex. Eligible patients were followed up with median 5.1 years. Distant metastases, including both synchronous and metachronous, occurred as significantly higher in high risk group than intermediated group ( $18 \%$ vs. $5 \%, \mathrm{P}<0.001)$. The recurrence rate was also significantly different according to the risk stratification ( $43 \%$ in high risk, $18 \%$ in intermediate risk, $\mathrm{P}<0.001$ ).

Subclassification of N1b PTC patients showed significant different clinical outcome. This finding suggests that the dose of RAI ablation should be tailored according to the risk stratification.

\section{P75 \\ COMPARISON OF POSTOPERATIVE VOICE OUTCOME BETWEEN THYROIDECTOMY USING HARMONIC SCALPEL AND LIGASURE}

Kijeong Lee ${ }^{1}$, Soon-Youn Kwon'², Jeong-Soo Woo ${ }^{2}$, Seung-Kuk Baek', Jae-Gu Jo ${ }^{2}$, Min Woo Park', Kwang Jin Jung ${ }^{1}$, Si-Youn Song ${ }^{3}$, Kwang-Yoon Jung ${ }^{1}$

${ }^{1}$ Department of Otolaryngology-Head and Neck Surgery, Korea University College of Medicine; Thyroid Center, Korea University Medical Center, ${ }^{2}$ Department of Otolaryngology-Head and Neck Surgery, Korea University College of Medicine, ${ }^{3}$ Yeungnam University College of Medicine

Introduction: Voice symptoms are frequently reported after thyroidectomy even in absence of vocal fold paralysis. Both ultrasonic coagulation (Harmonic Scalpel) (HS) and bipolar coagulation (Ligasure) (LS) are new energy devices commonly used in conventional thyroidectomy. The objective of this study was to comparing the voice outcome of open total thyroidectomy between HS and LS.

Method \& Materials: Prospective non-randomized study of 98 patients who underwent open total thyroidectomy from April 2012 to December 2012 was conducted. Of these, patients with insufficient voice data, vocal fold paralysis, gross extrathyroidal extension (pT4), concomitant lateral neck dissection were excluded. The acoustic analysis (jitter, shimmer, NHR, F0), electroglottography (CQ), voice range profile, auditory perceptual evaluation (GRBAS), videolaryngostroboscopy, voice handicap index-30 (VHI-30) were obtained preoperatively and 1 week, 1 month, 3 month, 6 month after thyroidectomy.

Results: Total 85 patients (HS: 55, LS: 30 ) were enrolled in this study. Mean age was 51.3 year and female was 67 patients (78\%). Demographics, tumor stage and operating time were not significantly different between the HS and LS groups. The parameters of acoustic analysis, electroglottography, auditory perceptual evaluation and videolaryngostroboscopy did not significantly change after thyroidectomy and were not different between the HS and LS groups.

Conclusion: In this study, there was no significantly difference in the postoperative voice outcome between the two devices.

\section{P76 \\ EFFECT AND TOXICITY OF SMALL MOLECULE TYROSINE KINASE INHIBITORS IN PATIENTS WITH THYROID CARCINOMA: SYSTEMATIC REVIEW AND META-ANALYSIS \\ Esther Klein Hesselink ${ }^{1}$, Danielle Steenvoorden², Ellen Kapiteijn², Eleonora Corssmit ${ }^{2}$, Anouk van der Horst-Schrivers ${ }^{1}$, Joop Lefrandt ${ }^{1}$, Thera Links ${ }^{1}$, Olaf Dekkers ${ }^{2}$ \\ ${ }^{1}$ University Medical Center Groningen, ${ }^{2}$ Leiden University Medical Center}

Purpose: Different tyrosine kinase inhibitors (TKIs) have been studied in the past years in patients with thyroid carcinoma (TC). However, the effect and toxicity of various TKIs in TC patients have not been directly compared, while this is of great importance for clinical decision-making. The aim of the present systematic review and meta-analysis was to summarize effect and toxicity of treatment with small-molecule TKIs in patients with TC.

Methods: PubMed, Embase, Web of Science, Cochrane, Academic Search Premier and CINAHL were systematically searched for publications on TKIs in patients with TC until January 2014. Primary endpoint was objective response (complete and partial responses); secondary endpoints were clinical benefit (complete response, partial response, and stable disease of at least 24 weeks), percentage TKI dose reduction and/or TKI discontinuation because of adverse events, hand-foot syndrome, diarrhea and nausea/vomiting. Meta-analysis was performed using an exact likelihood approach. Pooled percentages and $95 \%$ confidence intervals (CIs) were reported.

Results: A total of 1,535 publications were identified, of which 24 (reporting on 1,274 patients) were included in the meta-analysis. Pooled percentages for objective response ranged from $0 \%$ for gefitinib to $35 \%$ for pazopanib, and were $20(95 \%$ CI $14-26) \%, 32(27-37) \%, 27(22-32) \%$ and $8(5-13) \%$ for the best studied TKIs sorafenib, vandetanib, cabozantinib and motesanib, respectively. Pooled percentages for clinical benefit ranged from $50-78 \%$ for these TKIs. All TKIs were associated with considerable toxicity.

Conclusion: The currently studied TKIs have a modest effect and considerable toxicity. Therefore, careful selection of TC patients for whom the benefits of treatment outweigh toxicity is warranted.

\section{P77 \\ PREDICTORS OF HYPERTHYROIDISM IN PATIENTS TREATED WITH LEVOTHYROXINE SUPPRESSIVE DOSES FOR WELL- DIFFERENTIATED THYROID CANCER \\ Tatiana Mityukova $^{1}$ \\ ${ }^{1}$ The Institute of Physiology of the National Academy of Sciences; \\ Belarusian Medical Academy for Postgraduate Education}

Recent data indicate that levothyroxine (L-T4) dose for well-differentiated thyroid cancer (WDTC) treatment requires an optimization. The purpose of 
our study was to search for possible predictors of hyperthyroidism in young WDTC patients.

Young adults (N-114; 90 females; mean age 28.42 \pm 0.29 years) who are under standard L-T4 suppressive therapy for WDTC have been investigated. The control group included 73 healthy age-and sex-matched subjects. In all the participants, the height, weight and body mass index (BMI, $\mathrm{kg} / \mathrm{m}^{2}$ ) were measured and thyroid function tests (Elisa-Kits DRG, USA).

It has been demonstrated that the patients under standard suppressive therapy (median daily L-T4 dose $2.6 \mu \mathrm{g} / \mathrm{kg}$ ) had higher TT4 and FT4 levels than in the control (12.6 vs. $10.9 \mu \mathrm{g} / \mathrm{dl}$ and 1.8 vs. $1.6 \mathrm{ng} / \mathrm{dl}$, respectively; $\mathrm{P}<0.001)$. About half of the patients, had moderately suppressed TSH $(0.1-0.5 \mathrm{mIU} / \mathrm{l})$; the other part had stronger suppression $(\mathrm{TSH}<0.1 \mathrm{mIU} / 1)$. In both subgroups, the proportions of elevated free thyroid hormones' fractions were similar (FT4 $18-19 \%$, FT3 8-12\%). In thyroid cancer patients with weight excess and obesity $\left(\mathrm{BMI}>25 \mathrm{~kg} / \mathrm{m}^{2}\right)$, TSH suppression $<0.5 \mathrm{mIU} / 1$ was achieved with lower $\mathrm{L}-\mathrm{T} 4$ daily doses than in those with normal BMI $(2.2$ vs. $2.8 \mu \mathrm{g} / \mathrm{kg} ; \mathrm{P}<0.05)$. Every second female patient $(51.5 \%)$ and $29 \%$ of the men had elevated T4 levels. In men, FT3 raise was found out more frequently compared to women $(20.8 \%$ vs. $7.8 \%)$. In WDTC men with normal weight, a combination of elevated FT3 levels and decreased FT4/FT3 ratio less than 5 dominated; such findings may indicate the hyperthyroidism risk under treatment with standard suppressive L-T4 doses.

The need for L-T4 in WTDC patients depends on BMI and should be individualized. An elevated FT3 level and decreased FT4/FT3 ratio (less than 5) may indicate hyperthyroidism in such patients.

\section{P78 \\ THE MANAGEMENT OF RADIOIODINE RESISTANT DIFFERENTIATED THYROID CANCER - A LONGITUDINAL 10-YEARS FOLLOW-UP}

Ruxandra Dobrescu ${ }^{1}$, Andrei Goldstein ${ }^{2}$, Anca Simioniuc $^{3}$, Dumitru loachim ${ }^{2}$, Corin Badiu ${ }^{4}$

${ }^{1}$ National Institute of Endocrinology, ${ }^{2}$ National Institute of Endocrinology "Cl Parhon", ${ }^{3}$ National Institute of Endocrinology "CI Parhon", ${ }^{4}$ National Institute of Endocrinology; "Carol Davila" University of Medicine \& Pharnacy

Differentiated thyroid carcinoma (DTC), is generally characterized by excellent long-term prognosis, and, with total thyroidectomy \pm radioiodine therapy is curable in most cases. ${ }^{131} \mathrm{I}$ resistance is complicated by the morbidity of cumulative RAI doses greater than $600 \mathrm{mCi}$, and the lack of effectiveness of chemotherapy.

Objective: We conducted a retrospective study on patients with ${ }^{131}$ I resistant DTC between 2002-2012.

Patients and Methods: From a total of 5024 cases admitted with DTC, 37 met our criteria (29 women and 8 men), aged between $38.56 \pm 13.48$ years; 5/37(13.51\%) had previous FNAB suggestive for malignancy, while $32 / 37(86.49 \%$ ) were discovered post-thyroidectomy. TNM classification was as follows: $21 / 37(56.75 \%)$ stage T4a with lesions of $3.61 \pm 2.51 \mathrm{~cm}$ and $3 / 37$ patients $(8.10 \%)$ had FTC, the rest $34 / 37(91.90 \%)$ had PTC, in which the majority $26 / 37(70.27 \%)$ were sclerosis variant; $5 / 26(19.23 \%)$ presented multifocal PTC.

Results: Initially, 27/37(72.97\%) underwent total thyroidectomy, and $10 / 37(27.03 \%)$ partial thyroidectomy. Completion thyroidectomy was done in $14 / 37(37.83 \%)$. During the follow-up stage, $18 / 37(48.64 \%)$ were identified with metastases. After thyroidectomy, patients receive between 5-12 times. The initial dose was $82.81 \pm 37.58 \mathrm{mCi}^{131}$; the average total dose of $\mathrm{I}^{131}$ administered was $852.16 \pm 249.59 \mathrm{mCi}$. At the end of the follow-up $93.25 \pm 41.39$ months, stimulated thyroglobulin levels were $106.06 \pm 49.86 \mathrm{ng} / \mathrm{ml}$, recommended level $(<2 \mathrm{ng} / \mathrm{ml})$ being found only on $5 / 37(13.51 \%)$ patients. The last ultrasound revealed solid mass in the thyroid bed in 13/37(35.13\%). During the follow-up 9/37 patients had negative whole body scintigraphy (WBS) despite elevated levels of thyroglobulin $(42.31 \pm 54.23 \mathrm{ng} / \mathrm{ml}) ; 6 / 9$ patients with negative WBS received additional doses of $437.5 \pm 167.92 \mathrm{mCi} \mathrm{I}^{131}$; thyroglobulin levels at follow-up were $30.5 \pm 40.8 \mathrm{ng} / \mathrm{ml}$.

Conclusions: Radioiodine resistant DTC has a poor prognosis, a small group of patients reaching normal levels of thyroglobulin. In perspective we need to characterize the exact cytogenetic subset of DTC in order to develop targeted therapies.

\section{P09 Medullary Thyroid Cancer}

\section{P79 \\ MEN2A SPANISH NATIONAL DATABASE: CLINICAL PROFILE OF MEDULLARY THYROID CARCINOMA CAUSED BY GERMLINE RET CYS634TYR MUTATION}

Paloma Portillo ${ }^{1}$, Nuria Valdes Gallego ${ }^{2}$, Elena Navarro ${ }^{3}$, Lluís Forga ${ }^{4}$,

Anna Casterás ${ }^{5}$, Jordi Mesa ${ }^{5}$, Javier Tébar ${ }^{1}$

${ }^{1}$ Hospital Virgen de la Arrixaca, ${ }^{2}$ Hospital Central de Asturias;

Endocrinologia Y Nutricion, ${ }^{3}$ Hospital Virgen del Rocío, ${ }^{4}$ Complejo

Hospitalario de Navarra, ${ }^{5}$ Hospital Vall D’hebrón

Introduction: genotype-phenotype correlation has been described, and there are codons-specific guidelines, but information about single nucleotide and amino acid substitution genotype-specific clinical risk profiles is scarce, and it could be useful for tailoring more precise clinical care.

Objective: to study the medullary thyroid carcinoma (MTC) clinical profile of a large number of MEN2A patients with the specific Cys634Tyr (C634Y) RET mutation which is the most common RET mutation in Spanish genetic carriers.

Patients and Methods: data from the Spanish MEN national online database was analysed, focusing on patients with C634Y mutation.

Results: our series comprises 176 genetic carriers from 48 unrelated families. 173 patients have thyroid disease: C-cell hyperplasia was diagnosed in 25 patients at a mean age of $8.4 \pm 6.3(0.5-28.6)$ years and 148 subjects had MTC at a mean age of $32.9 \pm 32.8(4-75)$ years. Age-related penetrance for MTC was $46 \%$ by age 30 and $85 \%$ by age 50 . Lymph node metastases were observed in 26 patients at a mean age of $37.8 \pm 15.8(16.5-75.1)$ years and 5 patients had also distant metastases aged 34-63.70. 92 (65.7\%) out of 140 patients were biochemically free of disease after thyroidectomy at the last follow up, mean time of follow up 6.9?6.3 (1-32) years. All patients younger than fourteen years at the time of thyroidectomy were cured, and 7 (30.4\%) out of 23 patients with lymph nodes metastases at the time of surgery were biochemically cured. The estimated cumulative frequency of being biochemically free of disease after thyroidectomy was $68 \%$ at 5 years and $64 \%$ at 10 years.

Conclusion: These data provide RET C634Y mutation specific MTC neoplastic risk profile in a large number of patients. This information might facilitate risk assessment, genetic counselling, and surveillance of C634Y genetic carriers.

\section{P80 \\ COMPARISON OF PENTAGASTRIN TEST \\ AND CALCIUM STIMULATION TEST FOR PREOPERATIVE DIAGNOSIS OF MEDULLARY THYROID CARCINOMA}

Philipp Ubl', Alois Gessl', Christian Scheuba ${ }^{1}$, Bruno Niederle ${ }^{1}$,

Marcus Hacker ${ }^{1}$, Shuren L.I. ${ }^{1}$

${ }^{1}$ Medical University of Vienna

Aim: the aim of this study was to compare pentagastrin test (PGT) with calcium stimulation test (CST) for preoperative diagnosis values of medullary thyroid cancer (MTC) in patients with thyroid nodule.

Patients and Methods: Patients with nodular thyroid disease $(n=526)$ were included in the study. 29 patients had thyroidectomy after both pentagastrin test and calcium stimulation test. The calcitonin was examined in serum samples obtained before and 2, 3, as well as 5 minutes after iv stimulation using pentagastrin $(0.5 \mu \mathrm{g} / \mathrm{kg}$ body weight $)$ or calcium gluconate $(2.5 \mathrm{mg} / \mathrm{kg})$.

Results: Among 29 patients having thyroidectomy, 19 patients had MTC, 7 had $\mathrm{C}$-cell hyperplasia $(\mathrm{CCH})$ and 3 had neither MTC nor $\mathrm{CCH}$ (negative histological results). Under PGT the maximal values of stimulated CT were 97 to $178842 \mathrm{ng} / \mathrm{L}, 19$ to $522 \mathrm{ng} / \mathrm{L}$ and 30 to $94 \mathrm{ng} / \mathrm{L}$ for MTC, CCH and the negative histological results, respectively. The ranges of maximal stimulated CT values after CST for MTC, CCH and the negative histological results were 372 to $209186 \mathrm{ng} / \mathrm{L}, 161$ to $1163 \mathrm{ng} / \mathrm{L}$ and 200 to $714 \mathrm{ng} / \mathrm{L}$, respectively.

Conclusion: PGT and CST are both sensitive and specific test for preoperative evaluation of medullary thyroid cancer. A significant direct correlation 
is found between PGT and CST. However, CST has a more wide range of reference values as compared with PGT.

\section{P81 \\ UTILITY OF 18F-DIHYDROXYPHENILALANINE PET SCAN IN PATIENTS WITH RECURRENT MEDULLARY THYROID CARCINOMA}

Ana Romero Lluch ${ }^{1}$, Ignacio Cuenca Cuenca ${ }^{2}$,

Raquel Guerrero Vázquez¹, Antonio Jesús Martínez Ortega', Ignacio Jiménez Varo ${ }^{1}$, Beatriz González Aguilera ${ }^{1}$,

Noelia Gros Herguido ${ }^{1}$, Elena Navarro González ${ }^{1}$

${ }^{1}$ Endocrinology, Virgen del Rocio University Hospital, ${ }^{2}$ Nuclear

Medicine, Virgen del Rocio University Hospital

The aim of this study was to evaluate the potential value of 18-dihydroxiphenylalanine PET (18F-DOPA) as a localization method in patients with recurrent medullary thyroid cancer (MTC) and to compare the usefulness of this technique over 18F-FDG PET scan.

Methods: We prospectively examined, between november 2011-2012, 17 patients with MTC diagnosis and suspicion of recurrence either by imaging techniques $(n=2)$, or by elevated calcitonin levels $(n=15)$. Each patient underwent a 18F-FDG PET scan first and 15 days later a 18F-DOPA PET. Sex, age, type of MTC, number and location of the lesions were studied. The correlation between calcitonin, CEA levels and calcitonin doubling time and the positivity of PET was analyzed.

Results: Note that quantitative variables are defined as mean \pm SD.

$\mathrm{n}=17(71 \%$ women $)$; age $48.1 \pm 12.8$ years. 10 patients had sporadic MTC and 7 patients had MEN 2A. Abnormal uptakes were detected with $18 \mathrm{~F}$-DOPA in 10 patients and only in 8 patients with $18 \mathrm{~F}-\mathrm{FDG}$ (sensitivity of $60 \%$ vs $46.6 \%$ ). 24 total lesions were detected with 18F-DOPA versus 13 lesions with 18F-FDG. None of our patients showed additional lesions with 18F-FDG in comparison with 18F-DOPA. 7 patients had negative results with both scans. Calcitonin levels were significantly higher in patients $(\mathrm{n}=10)$ with positive PET $(524.09 \pm 406.51 \mathrm{pg} / \mathrm{ml}$ vs $95.48 \pm 72.68 \mathrm{pg} / \mathrm{ml} ; \mathrm{p}=0.02)$. In all patients with calcitonin levels $>260 \mathrm{pg} / \mathrm{ml}, 18$-FDOPA PET was able to demonstrate persistent disease. In patients with positive PET, there were no differences in calcitonin levels between 18F-DOPA and 18F-FDG $(524.09 \pm 406.51 \mathrm{pg} / \mathrm{ml} \mathrm{vs}$ $481.50 \pm 415.58 \mathrm{pg} / \mathrm{ml} ; \mathrm{p}=0.82$ ). No correlation between calcitonin doubling time and PET positivity was found. Histological confirmation was obtained in 7 patients.

Conclusions: 18F-DOPA PET seems to be superior to 18F-FDG PET in detecting lesions in patients with recurrent MTC. This technique is especially useful when other morphologic imaging techniques are negatives and calcitonin levels are $>260 \mathrm{pg} / \mathrm{ml}$.

\section{P82 \\ MANAGEMENT STRATEGY IN A RET PROTO- ONCOGENE MUTATION CARRIER IN A SETTING OF CONGENITAL HYPOTHYROIDISM}

Alptekin Gursoy ${ }^{1}$, Seyfettin Ilgan ${ }^{1}$, Erdinc Aygenc ${ }^{1}$, Banu Bilezikci ${ }^{1}$

${ }^{1}$ Ankara Guven Hospital Thyroid Center

We hereby describe follow-up and management of 21-year-old female patient with congenital hypothyroidism who was carrying germline ret protooncogene of Val804Met in exon 14. As a routine procedure, in a mutant gene carrier, prophylactic thyroidectomy is a rule before the development of medullary thyroid carcinoma (MTC). However, this patient has "entirely unique" situation in which she had congenital hypothyroidism and taking thyroid hormone replacement therapy since birth. We have searched for possible thyroid tissue with thyroid scintigraphy. There was functional uptake in the base of tongue which was compatible with lingual thyroid. Her calcitonin level was less than $2 \mathrm{ng} / \mathrm{mL}$ (below the lover limit of detection). Considering the ontogeny of parafollicular cells (C-cells), we suggested the possible presence of parafollicular in lingual thyroid gland. We considered surgery to remove lingual thyroid gland to prevent future development of MTC. Histopathology revealed neither MTC nor C-cell hyperplasia. Scanty amount of C-cells were present on calcitonin immunostaining.

\section{P83 \\ VEGF, VEGFR3 AND PDGFRB PROTEIN EXPRESSION IS INFLUENCED BY RAS MUTATIONS IN MEDULLARY THYROID CARCINOMA}

Lucía Inglada Pérez ${ }^{1}$, Veronika Mancikova², Maria Curras-Freixes ${ }^{2}$, Aguirre A. De Cubas ${ }^{2}$, Álvaro Gómez², Rocío Letón ${ }^{2}$,

Iñaki Comino-Mendez ${ }^{2}$, María Apellaniz-Ruiz², Lara Sánchez²,

Alberto Cascón ${ }^{1}$, Julia Sastre-Marcos ${ }^{3}$, Cristina Rodríguez-Antona ${ }^{1}$,

Mercedes Robledo ${ }^{1}$

${ }^{1}$ Hereditary Endocrine Cancer Group, Spanish National Cancer

Research Centre (Cnio); Isciii Center for Biomedical Research on Rare Diseases (Ciberer), ${ }^{2}$ Hereditary Endocrine Cancer Group, Spanish

National Cancer Research Centre (Cnio), ${ }^{3}$ Servicio de Endocrinología

Y Nutrición, Complejo Hospitalario de Toledo

Background: Tyrosine kinase inhibitors (TKIs) have achieved remarkable clinical results in medullary thyroid carcinoma (MTC) patients. However, the considerable variability in patient response to treatment with TKIs remains largely unexplained. There is evidence that it could be due, at least in part, to alterations in genes associated with the disease via their effect on the expression of TKI targets. The objective of this study was to evaluate the influence of $R A S$ mutations on the expression levels in MTC tumors of eight key TKI target proteins.

Methods: We assessed by immunohistochemistry the expression of EGFR, KIT, MET, PDGFRB, VEGF, VEGFR1, VEGFR2 and VEGFR3 in a series of 84 primary MTC tumors that had previously been molecularly characterized, including 14 RAS-positive, $18 R E T^{\mathrm{M} 918 \mathrm{~T}}$-positive and $24 R E T^{\mathrm{C} 634}$ positive tumors, as well as 15 wild type (WT) tumors with no mutations in the RET or $R A S$ genes.

Results: In contrast to RET-positive tumors, $R A S$-positive tumors did t express neither PDGFRB nor MET (p-value $=0.0060$ and 0.047 , respectively). Similarly, fewer $R A S$-positive than RET-related tumors expressed VEGFR3 (p-value $=0.00062$ ). Finally, WT tumors expressed VEGF more often than both $R A S$ - and $R E T$-positive tumors ( $\mathrm{p}$-value $=0.0082$ and 0.011 , respectively).

Conclusions: This is the first study identifying that the expression of TKI targets differs according to the presence of $R A S$ mutations in MTC. This information could potentially be used to select the most beneficial TKI treatment for these patients.

This work was supported by Fondo de Investigaciones Sanitarias-FIS project PI11/01359 (to MR) and the project from the Spanish Ministry of Science and Innovation SAF2012-35779 (to CR-A). LI-P is supported by CIBERER. VM, AAdeC and MA-R are predoctoral fellows of the "la Caixa"। CNIO international PhD programme.

\section{P84 \\ CALCITONIN STIMULATION TEST-EXPERIENCE OF ONE CENTRE}

Elwira Przybylik-Mazurek ${ }^{1}$, Anna Kurzynska ${ }^{1}$,

Alicja Hubalewska-Dydejczyk ${ }^{1}$

1Jagiellonian University Medical College; Department of Endocrinology

Objectives: Medullary thyroid carcinoma (MTC) is the third most common of all thyroid cancers. Calcitonin $(\mathrm{Ct})$ is a significant marker for the diagnosis and follow-up of MTC. We would like report our own experience with calcitonin stimulation test (CtST).

Methods: Fifty five females and thirty one males with MTC followed up in Department of Endocrinology of University Hospital in Krakow in years 1982-2014 were retrospectively analysed. The mean age at diagnosis was 49 for females and 50 for males. Twenty six cases of MTC were hereditary (FMTC). Patients were monitored by routine measurement of basal Ct levels, neck ultrasound and additional imaging tests (CT, scintigraphy). The CtST was performed in 37 patients ( $\mathrm{Ct}$ level was measured at baseline and 1.2,5.10.15 min after intravenous pentagastrin or calcium gluconate).

Results: All patients with pentagastrin stimulated test, but only few with calcium stimulation test demonstrated mild or serious side effects. Among 2/18 patients with normal basal $\mathrm{Ct}<10 \mathrm{ng} / \mathrm{l}$ more than tenfold increase of calcitonin level was observed, 10/11 patients with basal Ct between 10.1-100 ng/1 had significant increase of calcitonin level: in 7 cases eight to tenfold and in those cases the residual disease or recurrence was confirmed in imaging; in 3 
patients with renal insufficiency three to ninefold elevation was observed). In 8 patients with elevated basal Ct level $>100.1 \mathrm{ng} / \mathrm{l}$ (all of them with residual disease or recurrence confirmed in imaging) also increased $\mathrm{Ct}$ level during test was detected. More often positive CtST was observed in patients with FMTC.

Conclusions: Calcium stimulation calcitonin test is very useful for the diagnosis and monitoring patients with MTC, especially those with basal $\mathrm{Ct}$ level between 10.1-100 ng/l. Calcium stimulation calcitonin test is better tolerated and has less side-effects comparing to pentagastrin test. Calcitonin stimulation test seems to be more useful for monitoring patients with FMTC.

\section{P85 \\ FAMILIAL MEDULLARY THYROID CARCINOMA PRESENTING AS CUSHING SYNDROME: ANALYSIS OF RET GENE}

Jessica Ares Blanco ${ }^{1}$, Lorena Suárez ${ }^{1}$, María-Galiana Rodríguez', Lucía Díaz', Daniel Caravia', Joaquín Pertierra' ${ }^{1}$, Edelmiro Menéndez ${ }^{1}$

${ }^{1}$ Hospital Universitario Central de Asturias

Medullary thyroid cancer (MTC) accounts for approximately 5\% of all thyroid cancers and occurs either sporadically ( $75 \%$ of cases) or in a hereditary pattern $(25 \%)$. Familial form can either be isolated or belong to multiple endocrine neoplasia type 2 (MEN 2) syndrome. MTC can produce ACTH in up to 40 per cent of cases by immunohistochemistry, however Cushing Syndrome (CS) is rare $(0.6 \%)$.

We describe the case of a family with MEN 2A whose index case presented with CS. The 72 year-old man initially consulted for worsening hypertension, fatigue, depression, lower extremity edema and muscle weakness. Further evaluation diagnosed ectopic ACTH production from medullary thyroid carcinoma.

The specific features of this case are the clinical presentation as CS and the coexistence of a genetic mutation and an alteration in the sequence of RET (REarranged in Transfection) proto-oncogene. The confirmed one is a mutation in exon 10 (618 codon), which consists in the change of aminoacyd p.C618Y at the protein. Genetic testing also reveals another alteration in the sequence of codon 844 of exon 14, changing the aminoacyd p.R844Q. His daughter inherited the confirmed mutation and underwent total thyroidectomy; his son and sister inherited the alteration in RET gene with uncertain meaning. Calcitonine and metanephrines are at normal ranges in the last two, so we decided not to do any surgery yet and closely monitor both of them.

No mutation (genetically studied)

Uncertained meaning mutation (codon 844 of exon 14 which confies aminoacid change $\mathrm{p} . \mathrm{R} 844 \mathrm{Q})$

No mutation (genetically studied)
Uncertained meaning mutation (codon 844 of exon 14 which
Confies aminoacid change p.R844Q)
RET gene mutation (mutation in exon 10, at codón 618 of RET
gene, which changes aminoacid p.C618Y at the protein)
Not studied
Coexistence of mutations in exon 10 at codón 618 and in codon
844 of exon 14 of the RET gene

Fig. 1. (for Abstract P85).
RET gene mutation (mutation in exon 10, at codón 618 of RET gene, which changes aminoacid p.C618Y at the protein).

Not studied

Coexistence of mutations in exon 10 at codón 618 and in codon 844 of exon 14 of the RET gene

\section{P86 \\ USING NEXT GENERATION SEQUENCING METHOD IN RET MUTATION-NEGATIVE FAMILIES WITH HEREDITARY MEDULLARY THYROID CARCINOMA \\ Eliska Vaclavikova ${ }^{1}$, Sarka Dvorakova ${ }^{1}$, Vlasta Sykorova ${ }^{1}$, Josef Vcelak', Tereza Halkova ${ }^{1}$, Petr Vlcek ${ }^{2}$, Bela Bendlova ${ }^{1}$ ${ }^{1}$ Institute of Endocrinology, Dept. of Molecular Endocrinology, ${ }^{2} 2 \mathrm{nd}$ \\ Faculty of Medicine, Charles University, Department of Nuclear \\ Medicine and Endocrinology}

Objectives: Germline mutations in the RET proto-oncogene are the only one major cause of familial cases of medullary thyroid carcinoma (MTC). Nevertheless, very small number of families left unresolved without the causing inherited mutation. New techniques of next generation sequencing give hope in finding the new candidate genes that could be involved in the pathogenesis of these MTC families.

Methods: Our cohort consists of 470 families with MTC (470 index patients and 380 at-risk-relatives). The germline $R E T$ mutation was detected in 58 families $(12.3 \%)$. No mutation in all RET exons was found in six families with clinically supposed hereditary form of MTC (4 FMTC and 2 MEN2A phenotype). Six index patients of these families were analyzed using Illumina panel sequencing 94 genes associated with cancer. After filtering common single nucleotide polymorphisms, the data were focused on novel or rare non-synonymous variants in coding sequences and tested in silico (SIFT, PolyPhen) for possible damaging effect.

Results: In each patient, the analysis has discovered several promising variants in genes involved in key processes for cancer development such as repair genes. Considering SIFT and PolyPhen evaluation, we have identified very rare variants in genes $R H B D F 2, X P A, M E T$ and $C H E K 2$. The variants detected in $A P C$ and $E Z H 2$ were previously investigated in association with other cancers. Three patients have a pathogenic polymorphism in HNF1A.

Conclusions: The search for the new candidate genes in families with hereditary MTC is important. Several detected alterations are promising, however, it is necessary to verify them by other techniques, and primarily, to verify them in other affected family members. It can represent genetic modifying factors or crucial genes in each family.

Supported by the grant projects IGA MH CZ NT13901-4 and MH CZ DRO 00023761.

\section{P87 \\ MEN2A SPANISH NATIONAL DATABASE: RET CYS634ARG MUTATION IS ASSOCIATED WITH HIGHER RISK OF CLINICAL AGGRESSIVENESS OF MEDULLARY THYROID CARCINOMA THAN RET C634Y MUTATION}

Nuria Valdes Gallego ${ }^{1}$, Elena Navarro ${ }^{2}$, Paloma Porillo ${ }^{3}$, Javier Tébar ${ }^{3}$ Jordi Mesa ${ }^{4}$, Anna Casterás ${ }^{4}$, Lluís Forga ${ }^{5}$

${ }^{1}$ Hospital Central de Asturias; Endocrinologia Y Nutricion, ${ }^{2}$ Hospital Virgen del Rocío, ${ }^{3} \mathrm{Hospital}$ Virgen de la Arrixaca, ${ }^{4} \mathrm{Hospital}$ Vall

D’hebrón, ${ }^{5}$ Complejo Hospitalario de Navarra

Introduction: genotype-phenotype correlation has been described between specific codon mutation and age of onset, penetrance and clinical aggressiveness of medullary thyroid carcinoma (MTC) in Multiple Endocrine Neoplasia type 2A (MEN 2A). But data are scarce about whether there could be any difference between clinical profiles based on the type of nucleotide and amino acid substitution at the same codon.

Objective: to study clinical differences between genetic carriers of Cys634Arg (C634R) mutation and individuals carrying Cys634Tyr (C634Y) mutation.

Patients and methods: we collected data included in the Spanish online national database from MEN 2A patients carrying C634Y and C634R muta- 
tion. The study was a retrospective multicentric one, and the median time of follow up was $6.5 \pm 7.9(1-32)$ years.

Results: 173 patients from 49 unrelated families were C634Y mutation carriers, 26 patients from 8 different families had C634R mutation. We found higher age related penetrance of MTC in C634R mutation carriers than in $\mathrm{C} 634 \mathrm{Y}$ mutated individuals $(\mathrm{P}=0.0001)$. Kaplan-Meier estimate of cumulative lymph node and distant metastases rates showed that these events occurred earlier in individuals harbouring C634R mutation $(\mathrm{P}=0.001) .92$ $(65.7 \%)$ out of 140 patients with C634Y mutation were biochemically free of disease after thyroidectomy at the last follow up, in contrast to only $6(30 \%)$ out of 20 carriers of $C 634 \mathrm{R}$ mutation $(\mathrm{P}=0.001)$. The estimated cumulative frequency of being biochemically free of disease was lower in C634R than in $\mathrm{C} 634 \mathrm{Y}$ genetic carriers $(\mathrm{P}=0.00)$. Multivariate adjusted Cox regression analysis showed C634R mutation to be an independent factor for persistent/ recurrent disease (hazard ratio, $3.17 ; 95 \%$ confidence interval: $1.66-6.03 ; \mathrm{P}=$ $0.001)$

Conclusion: our study suggests that $\mathrm{C} 634 \mathrm{R}$ mutation is associated with higher risk of clinical aggressiveness of MTC than C634Y mutation. These results could be useful in tailoring clinical care.

\section{P88 \\ MUTATIONS IN THE RET PROTO-ONCOGENE IN A COHORT OF 36 PATIENTS WITH SPORADIC MEDULLARY THYROID CARCINOMA \\ Saskia Ting ${ }^{1}$, Vera Tiedje ${ }^{2}$, Thomas Herold ${ }^{3}$, Karl Worm ${ }^{1}$, \\ Dagmar Führer-Sakel ${ }^{2}$, Kurt Werner Schmid ${ }^{1}$ \\ ${ }^{1}$ Institute of Pathology; University Hospital Essen, ${ }^{2}$ Department of \\ Endocrinology and Metabolism; University Hospital Essen, ${ }^{3}$ Institute of Pathology; University Hospital Essen; German Cancer Consortium \\ (Dktk) Heidelberg}

Objectives: Mutations in the RET proto-oncogene are driver mutations in familial medullary thyroid carcinoma (MTC). In sporadic cases somatic RET mutations have been reported in up to $30-50 \%$ (ETA guidelines, Eur thyroid $\mathrm{J}, 2012 ; 1: 5-14)$. The role of these mutations and clinical course of disease as well as the role in response to TKI e.g. vandetanib therapy is not fully understood. The aim of our study was to analyse the role of RET mutations in patients with sporadic MTC.

Methods: Paraffin embedded primary tumor tissue was available from 36 patients with sporadic MTC treated at the Department of Endocrinology and Metabolism (University Hospital Essen). Sanger sequencing of exons 10, 11, $13,14,15,16$ of the RET proto-oncogene was performed.

Results: Nineteen (53\%) patients were diagnosed with metastatic and $17(47 \%)$ with local disease. In 21/36 (58\%) patients RET mutations were detected. The Met918Thr mutation in exon 16 was more frequent in patients with metastatic disease (10 versus 4 patients). Eleven patients diagnosed with metastatic disease were treated with vandetanib. All patients that responded to TKI treatment (RECIST) $(6 / 10 ; 60 \%)$ had a somatic RET mutation, whereas only $1 / 4$ patients that did not respond to the vandetanib therapy, had a mutation in the RET proto-oncogene. Furthermore we detected new RET mutations in tumor tissue that have not been reported yet.

Conclusion: In a small study we detected a high frequency of mutations in the RET proto-oncogene in a cohort of sporadic MTC patients. As previously suggested the RET Met918Thr mutation in exon 16 may be a potential biomarker for aggressive disease. Furthermore in our study response to vandetanib therapy was more frequently observed in patients with somatic RET mutations. In addition, the biological impact of the new detected RET mutations is unclear.

\section{P010 Goiter and Nodules 1}

\section{P89 \\ DOES THE EFFICACY OF FINE-NEEDLE ASPIRATION OF THYROID NODULES VARY WITH SIZE AND TI-RADS SCORE?}

Claude Bigorgne $^{1}$, Gilles Russ ${ }^{2}$, Bénédicte Royer ${ }^{3}$, Agnès Rouxel ${ }^{1}$ Marie Bienvenu ${ }^{4}$

${ }^{1}$ Centre de Pathologie Et D'imagerie; La Pitié-Salpêtrière Hospital, ${ }^{2}$ Centre de Pathologie Et D'imagerie; La Pitié-Salpêtrière Hospital; Pierre and Marie Curie University, ${ }^{3}$ Centre de Pathologie Et D'imagerie, ${ }^{4}$ Centre de Pathologie Et D'imagerie; Cochin Hospital

Objectives: to determine if the efficacy of ultrasound-guided fine-needle aspiration (US-FNA) of thyroid nodules varies with the size of the nodules and their TI-RADS (thyroid imaging-reporting and data system) score.

Methods: prospective study on 7144 nodules referred for US-FNA in 18 months. All nodules were scored with ultrasound (US) according to the TI-RADS score and cytological results were analyzed according to the Bethesda's classification and divided in two groups: non-diagnostic (group A) and sufficient for diagnostic (group B). Nodules were divided in 5 classes by size (i.e $\leq 0 \mathrm{~mm},>10$ and $\leq 20 \mathrm{~mm},>20$ and $\leq 30 \mathrm{~mm},>30 \mathrm{~mm}$ and $\leq 40 \mathrm{~mm}$ and $>40 \mathrm{~mm}$ ). TI-RADS scores ranged from 1 to 5 (normal to very probably malignant, respectively). Size distributions and $\%$ of each class size were compared between groups A and B using Mann-Whitney's test and chi-square's test, respectively. Absolute risk of obtaining a non-diagnostic smear was calculated for each class of size. Finally, TI-RADS scores were also compared between groups $\mathrm{A}$ and $\mathrm{B}$ using the chi-square test.

Results: There were 250 non-diagnostic smears (3.5\%). Size distribution was significantly different between groups A and B. Non-diagnostic results were more frequent in subcentimeter nodules $(p=0.05)$ and did not vary significantly among other size classes. However, the risk of obtaining a nondiagnostic result in subcentimeter nodules was only slightly increased (4.3\%) compared to supracentimetric nodules $(3.3 \%$ to $3.6 \%)$ and remains very low. Regarding TI-RADS score, there was no difference between the two groups. In particular, highly suspect nodules (TI-RADS scores 4B and 5) do not bear a higher risk to provide a non-diagnostic result in subcentimeter nodules.

Conclusions: risk of obtaining a cytologically non-diagnostic result of US-FNA is only slightly increased in subcentimeter nodules and does not vary with TI-RADS score. Small suspect nodules can undergo US-FNA, if judged useful, with a high probability of achievement.

\section{P90 \\ GREY-SCALE ANALYSIS MAY IMPROVE THE ULTRASONOGRAPHIC EVALUATION OF THYROID NODULES}

\section{Giorgio Grani ${ }^{1}$, Mimma D'Alessandri ${ }^{2}$, Giovanni Carbotta ${ }^{2}$,}

Angela Nesca ${ }^{2}$, Marianna Del Sordo ${ }^{2}$, Martina Vitale ${ }^{2}$,

Flavia Pigliacelli ${ }^{2}$, Angela Fumarola ${ }^{2}$

${ }^{1}$ Sapienza University of Rome; Dept. of Experimental Medicine,

${ }^{2}$ Sapienza University of Rome

Background: Subjective analysis of ultrasonography (US) images is the first-line method to assess thyroid nodules, but it is limited by interobserver variability and experience. The inter-observer agreement seems to be relatively good; however, a merely slight agreement was reported for echogenicity and echotexture. It is possible to perform a quantitative evaluation of echo-pattern and echogenicity, thus obtaining an objective estimate of the degree of hypoechogenicity and homogeneity, associated with risk of thyroid malignancy.

Methods: From January 2010 to October 2012, 908 nodules of 839 consecutive patients underwent US-guided FNA. In a single US image, three regions of interest (ROIs) were drawn: the first encompassing the nodule; the second including the adjacent thyroid parenchyma; and the third, the strap muscle. Histogram analysis was performed, obtaining the median, mean and $\mathrm{SD}$ of the pixels comprising each ROI. Echogenicity was expressed as a ratio: the nodule/parenchyma and parenchyma/muscle median grey ratios were cal- 
culated. The heterogeneity index was calculated as the coefficient of variation of grey histogram for each of the three ROIs.

Results: Nodule/parenchyma median grey ratio was significantly lower (i.e. more hypoechoic) in nodules found to be suspicious for cancer, according to the cytology report $(\mathrm{p}=0.006)$ and in confirmed malignant nodules $(\mathrm{p}=0.02)$. A nodule/parenchyma median grey ratio $<0.46$ (cut-off point determined by ROC analysis) has a sensitivity of $53.6 \%$ and specificity of $71.1 \%$ in predicting malignancy (OR 2.84; $\mathrm{p}=0.01)$. It can also be used as a continuous measure of hypoechogenicity and risk of malignancy (OR 0.20; $p=0.02$ ).

Discussion: We developed a method to perform a quantitative measurement of thyroid echogenicity, in a real clinical context, without the need for fixed operating conditions. Evaluation of nodule echogenicity according to the nodule/parenchyma median grey ratio allows for an objective stratification of nodule structure and risk of malignancy.

\section{P91 \\ INITIAL EXPERIENCE WITH CONTINUOUS INTRAOPERATIVE NEURAL MONITORING DURING THYROIDECTOMY}

\section{Marcin Barczynski ${ }^{1}$, Malgorzata Stopa ${ }^{1}$, Krzysztof Pragacz $^{2}$,}

Aleksandra Papier ${ }^{1}$, Aleksander Konturek ${ }^{1}$, Wojciech Nowak

${ }^{1}$ Third Department of General Surgery, Jagiellonian University Medical College, ${ }^{2}$ Department of General Surgery, Public Health Care Medical Center

Background: Continuous intraoperative neural monitoring (c-IONM) with automatic periodic stimulation (APS) via vagal nerve has a potential to improve recurrent laryngeal nerve (RLN) protection when compared to intermittent intraoperative neural monitoring (i-IONM). The aim of this study was to evaluate a novel c-IONM system in comparison to i-IONM technique during thyroidectomy.

Methods: A prospective cohort study of consecutive patients undergoing total thyroidectomy with i-IONM and c-IONM between 02/2012 and 12/2012 was undertaken. c-IONM was performed with APS Electrode. Clinical, pathological and follow-up data, signal stability, and accuracy of loss of signal (LOS) in prognostication of RLN injury during thyroidectomy were analyzed.

Results: A series of 100 total thyroidectomies equal to 200 RLNs at risk (NAR) were analyzed. Signal stability of c-IONM was good in all operations. Unilateral LOS appeared in 5 NAR. RLN injury was segmental in 2 NAR and global in 3 NAR. Transient unilateral RLN paresis was found in 4 NAR. Positive versus negative predictive value of c-IONM was $80 \%$ versus $99 \%$. None of the patients had permanent RLN palsy.

Conclusions: c-IONM with APS electrode provides good signal stability. Negative predictive value of c-IONM is excellent while factors affecting positive predictive value of the method have yet to be defined.

\section{P92 \\ LONG-LASTING THYROID NODULES SHRINKAGE AFTER RADIOFREQUENCY ABLATION AT 1 YEAR FOLLOW-UP ON A PROSPECTIVE STUDY \\ Maurilio Deandrea ${ }^{1}$, Francesca Garino ${ }^{1}$, Alberto Mormile ${ }^{1}$, \\ Federico Ragazzoni', Paolo Piero Limone ${ }^{1}$ \\ ${ }^{1}$ Department of Endocrinology, Diabetes and Metabolism, Ao Mauriziano}

Objectives: Percutaneous radiofrequency thermal ablation (RFA) was reported as an effective tool for the management of thyroid nodules (TNs) but long term follow-up on standardized populations are lacking at present time. The aim of this study was to prospectively evaluate the volume reduction of benign nonfunctioning medium sized thyroid nodules after a single session of RFA with a "moving-shot technique".

Methods: 30 patients with medium sized $(10-20 \mathrm{ml})$ non-functioning thyroid nodules were enrolled. In all patients, a repeated fine-needle aspiration cytology was performed to exclude a thyroid malignancy. All patients were clinically, biochemically, and morphologically evaluated at baseline and after 1.6 and 12 months.

Results: Volume reduction of the nodules was significant starting at the first month of follow-up (median volume at 1 month $12.1 \mathrm{ml}$ vs 15.4 at base- line, $\mathrm{P}<0.001$ ), but the most significant reduction was recorded at 6 months (median volume al 6 months $6.25 \mathrm{ml}, \mathrm{P}<0.001$ vs baseline, $\mathrm{P}<0.05$ vs 1 month). After 1 year follow-up nodules appeared further slightly reduced of volume (median $5.45 \mathrm{ml}$ ), while not significantly vs 6 months (p 0.29 ). The overall volume reduction at 1 year follow-up was $68.4 \%$ (IQR 55.4-83.1) vs baseline $(\mathrm{P}<0.001)$. RFA was safe and well tolerated in all patients without any significant side effect.

Conclusions: This trial shows good efficacy of RFA on benign nonfunctioning solid thyroid nodules in term of volume reduction; data on a longer follow-up are needed to confirm longterm efficy. The study shows that RFA may represent a valid therapeutic approach in patients with TNs not receiving conventional treatments.

\section{P93 \\ THYROID VOLUMES OF TEHRANIAN SCHOOLCHILDREN WHO HAVE LIVED THEIR ENTIRE LIVES IN AN IODINE SUFFICIENT AREA \\ Hossein Delshad ${ }^{1}$, Atieh Amouzegar ${ }^{1}$, Fereidoun Azizi ${ }^{1}$ \\ ${ }^{1}$ Endocrine Research Center, Research Institute for Endocrine \\ Sciences, Shahid Beheshti University of Medical Sciences, Tehran, I. \\ R. Iran}

Background and Purpose: There is a lack of consensus of opinions on universal reference values for thyroid volume and it is recognized that for local populations it is necessary to use and establish their specific reference ranges especially for the purpose of goiter surveys. Our objective was to describe thyroid volume measured by ultrasonography in Tehranian schoolchildren who had been living in iodine sufficient status during their whole life period.

Methods: This cross-sectional study was performed fifteen years after implementing the universal salt iodization in Iran, on 464 Tehranian schoolchildren, aged 7-15 yr. Data were collected on age, sex, weight, height and urinary iodine. Thyroid size was ascertained by palpation and ultrasonography by an expert endocrinologist.

Results: The prevalence of goiter was $4.6 \%$ by palpation. All goitrous subjects had grade 1 goiter. Median urinary iodine was $140 \mu \mathrm{g} / \mathrm{L}$. Thyroid volumes determined by ultrasonography were comparable in boys and girls of all ages. The best predictors of thyroid volume were age, weight, and body surface area. The median thyroid volumes of Tehranian schoolchildren in this study were lower in all age groups compared with our previous 2001 study and updated provisional WHO/ICCIDD reference values.

Conclusions: Tehranian schoolchildren currently living in an iodine sufficient area, where they have spent their entire lives, have smaller thyroid glands than recommended international references. Using population standards rather than universal ones in assessing thyroid volume should be considered.

\section{P94 \\ SERUM TSH LEVELS AND MALIGNANCY RISK IN PATIENTS WITH THYROID NODULES}

Mira Siderova ${ }^{1}$, Kiril Hristozov', Ivan Krasnaliev², Maria Petrova', Yana Bocheva ${ }^{3}$

${ }^{1}$ Clinic of Endocrinology, University Hospital "St. Marina", ${ }^{2}$ Department of Pathology, University Hospital "St. Marina”, ${ }^{3}$ Clinical Laboratory, University Hospital "St.Matina"

Objectives: Recent studies have reported that higher levels of TSH are associated with an increased incidence of thyroid malignancy in patients with nodular thyroid disease. However, different conditions may affect TSH concentration. The aim of our study was to correlate serum TSH levels in patients with thyroid nodules with cytological and histological results.

Materials and Methods: 1483 consecutive patients (1339 women and 144 men) with nodular thyroid disease were evaluated by ultrasound-guided fine needle aspiration biopsy (FNAB) with cytological results classified as nondiagnostic, benign, follicular lesion, suspicious or malignant. Serum levels of TSH, $\mathrm{FT}_{4}, \mathrm{FT}_{3}, \mathrm{TPO}$ and $\mathrm{Tg}$-antibodies were measured at presentation by chemiluminescent immunoassay. 380 of the patients were submitted to surgery and final histology proved 83 carcinomas and 307 benign thyroid nodules.

Results: The overall sensitivity and specificity of FNAB in predicting malignancy were $90.32 \%$ and $81.11 \%$, respectively. Mean TSH in cytologically benign nodules was $1.703 \pm 2.569 \mathrm{mU} / 1$, in follicular lesions $2.393 \pm 4.119$ $\mathrm{mU} / 1$, in suspicious nodules $2.326 \pm 2.317 \mathrm{mU} / 1$ and in malignant on cytology 
nodules $3.045 \pm 2.781 \mathrm{mU} / \mathrm{l}$. Although in the reference range TSH levels in malignant cytologies were significantly higher that those in benign cases $(\mathrm{P}<$ 0.0001). This relationship was confirmed among operated cases where mean preoperative TSH was significantly higher in histologically malignant than benign nodules (TSH 3,387 vs. 1,613 mU/1; P $<0.0001$ ). In order to eliminate the factors influencing TSH concentration, we excluded all patients with thyroid autonomy, Hashimoto's thyroiditis (positive antibodies, US appearance, lymphocytic infiltration on histology) and those on levothyroxin or thyrostatic treatment. Among the rest of the patients, mean TSH value remained significantly lower in benign than malignant cases $(1,370$ vs. $2,805 \mathrm{mU} / \mathrm{l} ; \mathrm{P}<$ 0.0001).

Conclusion: Our results suggest a direct relationship between serum TSH and risk of malignancy in thyroid nodules both in cytological and histological series after excluding the influence of different thyroid diseases and medication.

\section{P95 \\ HEMITHYROIDECTOMY FOR BENIGN EUTHYROID GOITER AFFECTS MITOCHONDRIAL-RELATED GENE EXPRESSION \\ Tina Toft Kristensen ${ }^{1}$, Anne-Dorthe Feldthusen ${ }^{2}$, \\ Palle Lyngsie Pedersen ${ }^{2}$, Jacob Larsen ${ }^{2}$, Søren Jelstrup ${ }^{1}$, Jan Kvetny ${ }^{2}$ ${ }^{1}$ Department of Otorhinolaryngology and Maxillofacial Surgery, Køge University Hospital, Region Zealand, ${ }^{2}$ Mitochondrial Research Unit, \\ Næstved Hospital, Region Zealand}

Introduction: Thyroid hormones are known to regulate mitochondrial function. Previously in the same cohort we have demonstrated decreased thyroid hormone levels one year after hemithyroidectomy with a concomitant increase in the mitochondrial membrane potential, supposedly leading to increased formation of reactive oxygen species (ROS). The genomic cellular response to decreased serum $\mathrm{T}_{4}$ concentration after hemithyroidectomy has not previously been studied.

Patients and methods: Twenty-eight patients were examined before and $1,3,6$, and 12 months after hemithyroidectomy for benign euthyroid goiter. TSH and thyroid hormones were measured, body weight was measured to the nearest $0.5 \mathrm{~kg}$, and whole blood gene expressions of selected mitochondrialrelated and thyroid hormone-regulated genes were examined by RT-qPCR, including peroxisome proliferator-activated receptor gamma coactivator1B (PGC1B), mitochondrial transcription factor A (TFAM), nuclear respiratory factor 2 (NRF2) and the ROS-scavenging enzyme mitochondrial superoxide dismutase 2 (SOD2), which might also regulate central metabolic pathways in the mitochondrion.

Results: One year after hemithyroidectomy, $\triangle \mathrm{SOD} 2$ and $\triangle \mathrm{NRF} 2$ gene expressions correlated inversely with $\Delta \mathrm{fT}_{4}(\mathrm{r}=-0.42, \mathrm{p}=0.036),(\mathrm{r}=-0.43$, $p=0.033)$, respectively. Half of the patients gained weight $(n=13)$ in the first postoperative year after hemithyroidectomy with a median weight gain of 3.4 (IQR: 1.6-6.2) kg. In this patient group SOD2 gene expression was decreased one year after hemithyroidectomy compared with preoperative levels (median 2.27 a.u. (IQR:1.83-3.57) vs. 1.53 a.u. $(1.12-1.95), \mathrm{p}=0.02$ ), whereas SOD2 gene expression was unchanged in patients without postoperative weight gain.

Conclusion: A decrease in serum $T_{4}$ concentration within the reference range induced by hemithyroidectomy for benign euthyroid goiter affects the expression of the mitochondrial-related genes NRF2 and SOD2, possibly explained by the existence of individual optimal levels of serum concentrations of thyroid hormones. Decreased SOD2 gene expression might compromise the antioxidant capacity, and in selected patients possibly lead to weight gain.

\section{P96 \\ DISAPPEARANCE OF THYROID NODULES IS COMMON AFTER THE DANISH IODIZATION PROGRAM - AN ELEVEN-YEAR DANTHYR FOLLOW-UP STUDY}

Anne Krejbjerg ${ }^{1}$, Lena Bjergved ${ }^{2}$, Inge Bülow Pedersen ${ }^{3}$, Nils Knudsen ${ }^{4}$, Torben Jørgensen ${ }^{5}$, Hans Perrild ${ }^{4}$, Lars Ovesen ${ }^{6}$, Lone Banke Rasmussen ${ }^{7}$, Peter Laurberg ${ }^{1}$

${ }^{1}$ Department of Endocrinology, Aalborg University Hospital, Aalborg, Denmark; Department of Clinical Medicin, Aalborg University, Aalborg, Denmark, ${ }^{2}$ Research Centre for Prevention and Health, The Capital Region of Denmark, Glostrup, Denmark; Department of Endocrinology, Bispebjerg University Hospital, Copenhagen, Denmark, ${ }^{3}$ Department of Endocrinology, Aalborg University Hospital, Aalborg, Denmark, ${ }^{4}$ Department of Endocrinology, Bispebjerg University Hospital, Copenhagen, Denmark, ${ }^{5}$ Research Centre for Prevention and Health, The Capital Region of Denmark, Glostrup, Denmark; Faculty of Medicin, Aalborg University, Aalborg, Denmark; Faculty of Health Sciences, Copenhagen, Denmark, ${ }^{6}$ Department of Internal Medicine, Slagelse Hospital, Slagelse, Denmark, ${ }^{7}$ Department of Nutrition, National Food Institute, Technical University of Denmark, Søborg, Denmark

Objective:To clarify how iodine fortification (IF) influenced existing thyroid nodules in two regions with different baseline iodine intake (Copenhagen, mild iodine deficiency (ID) and Aalborg, moderate ID).

Methods: In a longitudinal population-based study (DanThyr) we examined 2,465 adults before (1997) and after (2008) the Danish mandatory IF of salt (2000). Ultrasonography was performed by the same sonographers using the same equipment, after controlling performances. Participants treated for thyroid disease were excluded from analyses.

Results: During the 11-year follow-up period, the prevalence of multinodularity had increased $(10.1-14.4 \%, \mathrm{P}<0.001)$, especially in Aalborg $(9.2$ $-15.5 \%, \mathrm{P}<0.001$ ), whereas no change in prevalence was observed for solitary nodules $(6.1-5.3 \%, \mathrm{p}=0.21)$. However, when we took aging into account, and considered participants around 40 years of age at follow-up and participants around 40 years at baseline as two independent cohorts, and compared the prevalences of nodules; both solitary $(\mathrm{p}=0.004)$ and multiple nodules $(\mathrm{P}<$ 0.001 ) were more frequent at baseline than at follow-up.

Disappearance of thyroid nodules during the 11 years were common, with an overall normalization rate of 21.2 (CI 17.9-24.9) per 1000 person years. Solitary nodules had a significantly higher normalization rate than multiple nodules (normalization rate ratio 0.47 (CI $0.32-0.67$ ) and there was a regional difference (Aalborg vs. Copenhagen) between normalization rates of multiple nodules (normalization rate ratio 0.29 (CI $0.12-0.64)$ ), but not solitary nodules (normalization rate ratio 0.81 (CI $0.53-0.1 .21)$ ).

In a multivariate logistic regression model, region (proxy for iodine intake) was a predictor for disappearance of multinodular structure (OR 0.26 (CI $0.10-0.71), \mathrm{p}=0.01$ with Copenhagen as reference).

Conclusions: Normalization of the thyroid gland with disappearance of thyroid nodules is common after an iodization program. Thus, thyroid nodularity is not always an irreversible abnormality. 


\section{P97 \\ UTILITY OF INTRA PROCEDURAL CONTRAST ENHANCED ULTRASOUND EVALUATION IN THE PERCUTANEOUS LASER ABLATION OF LARGE BENIGN THYROID NODULES. FIRST EXPERIENCE IN SPAIN}

Mattia Squarcia ${ }^{1}$, Mireia Mora ${ }^{2}$, Irene Halperin ${ }^{2}$, Enrique Carrera ${ }^{3}$, Joan Berenguer 4 , Ricard Valero ${ }^{3}$, Gloria Aranda ${ }^{2}$,

Felicia Alexandra Hanzu ${ }^{2}$

${ }^{1}$ Department of Radiology Hospital Clinic Barcelona; Laboratory of Endocrine Diseases Idibaps, ${ }^{2}$ Department of Endocrinology and Nutrition Hospital Clinic Barcelona; Laboratory of Endocrine Diseases Idibaps, ${ }^{3}$ Department of Anestesiology Hospital Clinic Barcelona,

${ }^{4}$ Department of Radiology Hospital Clinic Barcelona

Objective:Percutaneous laser ablation (PLA) is an efficient therapy of benign symptomatic thyroid nodules not eligible for surgery. Nevertheless, there are still no correlation studies between the initial ablation area $(A b A r)$ and the final volume reduction (DV). We evaluate the utility of intraprocedural contrast enhanced ultrasound (CEUS) with sonovue in the evaluation of the $A b A r$ and in the prediction of the final nodules DV.

Subjects: $N=4 ; 3 \mathrm{f} / 1 \mathrm{~m}$, age $48-83$, with large symptomatic solid thyroid nodules, V: 60-90 ml, with benign fine needle cytology's were included after informed consent.

Methods and Results: Subjects were submitted, to a unique ultrasound guided PLA session using a continuous-wave laser device of $1064 \mathrm{~nm}$ (MULTIDIODE PL3D ${ }^{\text {TM }}$ INTERmedic, Spain). One 240 um optic fiber was inserted in the nodule through a $21 \mathrm{G}$ needle. We estimated the output power $(3$ W) and the total energy (E) delivery on the basis of the published data's. $50 \%$ of the total $\mathrm{E}$ was administrated initially and the rest after the direct quantification of the $A b A r$. After 5 min to permit the withdrawal of the hiperecogenicity formed during the procedure we evaluated the $A b A r$ with CEUS. We administrated a $2^{\text {nd }} \mathrm{E}$ amount based on the observed $A b A r$ and the initial estimated E. Final total delivered $\mathrm{E}$ was variable: in 2 patients significantly lower $(900 \mathrm{vs}$ $1200 \mathrm{~J}$, respectively $1250 \mathrm{vs} 1600 \mathrm{~J}$ ) and in one higher (1500 vs $1400 \mathrm{~J})$ than the initially calculated $\mathrm{E}$, due to the variation in the $A b A r$ probably as function of histological type and vascularization. DV was of $45-49 \%$ at 1 month. No secondary effects were recorded. 3; 6 months follow-up and increasing of the $\mathrm{N}$ are ongoing.

Conclusion: CEUS is a low cost procedure that permits the intraprocedural delimitation of the treated area and the safe single session ablation with a unique fiber of large nodules decreasing the total cost of PLA.

\section{P98 \\ QUALITY OF LIFE IN PATIENTS WITH BENIGN NON-TOXIC GOITRE AS COMPARED TO THE GENERAL POPULATION: BASELINE AND 6 MONTHS POST-TREATMENT \\ Per Cramon ${ }^{1}$, Åse Krogh Rasmussen ${ }^{1}$, Jakob Bue Bjorner ${ }^{2}$, \\ Steen Joop Bonnema ${ }^{3}$, Daniel Mark Frendl ${ }^{4}$, Mogens Groenvold ${ }^{5}$, Laszlo Hegedüs ${ }^{3}$, Ulla Feldt-Rasmussen ${ }^{1}$, Torquil Watt ${ }^{1}$ \\ ${ }^{1}$ Copenhagen University Hospital Rigshospitalet, ${ }^{2}$ Qualitymetric, \\ ${ }^{3}$ Odense University Hospital, ${ }^{4}$ University of Massachusetts Medical \\ School, ${ }^{5}$ University of Copenhagen}

Objectives: To evaluate thyroid-related quality of life in patients with non-toxic goitre, as compared to the general population, before and 6 months after treatment.

Methods: Quality of life was assessed with the 85 item ThyPRO survey which is scored as 13 scales (range: 0-100). Data included baseline and 6 month post-treatment assessments from 123 patients with goitre who underwent radioiodine therapy (35\%), hemithyroidectomy $(50 \%)$, total thyroidectomy $(11 \%)$, and cyst aspiration with ethanol instillation (3\%). At baseline and 6 months after treatment, $90 \%$ and $85 \%$ of the patients were euthyroid, respectively. The remaining patients had subclinical thyroid dysfunction. Normative data were collected from 754 Danish individuals for the 9 ThyPRO scales that do not make attributions to thyroid disease. ThyPRO score differences between patients and the general population were analysed with multivariate linear regression, adjusting for: age, gender, comorbidity and educational sta- tus. Changes in patient scores with treatment were analysed with the paired t-test. Magnitudes of score differences were evaluated as effect-sizes $(0.2-0.5$ indicating small, 0.5-0.8 moderate, and 0.8+ large effects).

Results: Patients had worse scores than the general population at baseline on all 9 comparable ThyPRO scales, with large effects on the Goitre Symptoms and Anxiety scales. Patients' ThyPRO scores improved 6 months after treatment on Goitre Symptoms (large effect), Anxiety (moderate effect), and Hyperthyroid Symptoms, Eye Symptoms, Tiredness, Emotional Susceptibility and Impaired Daily Life (small effects). However, the post-treatment scores remained worse than the general population on five scales, with large effects on Goitre Symptoms and small effects on Hypothyroid Symptoms, Tiredness, Depressivity, and Emotional Susceptibility scales.

Conclusions: Patients had greatest impairment on the Goitre Symptoms and Anxiety scales, as compared to the general population, and the largest post-treatment improvements were also observed on these two scales. However, a quality of life deficit persisted 6 months after treatment.

\section{P99}

Poster has been withdrawn

\section{P011 Case Reports 1}

\section{P100 \\ CASE REPORT: SUPERIOR VENA CAVA SYNDROME SECONDARY TO INTRATHORACIC GOITER \\ Bruno Francisco García-Bray ${ }^{1}$, Nieves Cruz Felipe-Pérez ${ }^{1}$, \\ Ricardo Darias-Garzón ${ }^{1}$, Benigno Rivero-Melián ${ }^{1}$, \\ José Gregorio Oliva-García ${ }^{1}$, Estefania González-Melo ${ }^{1}$, \\ Alejandra Mora-Mendoza1, Beatriz Gómez-Alvarez¹, Ignacio Llorente-Gómez de Segura ${ }^{1}$ \\ ${ }^{1}$ Hospital Universitario Nuestra Señora de la Candelaria}

Introduction: We introduce a case involving the appearance of superior vena cava syndrome secondary to intrathoracic goiter. We discuss the diagnosis, prognosis and treatment applied to this pathology.

Case Report: 67 year old woman who presents respiratory distress, cough and weight loss. After physical examination and a chest radiography showing a mass that occupied the upper and middle right lung lobes, a neoplasm is suspected and at first, palliative radiotherapy is indicated. Chest and abdomen CT are done before treatment and an intrathoracic goiter is diagnosed. Following this finding, surgical treatment is performed.

Conclusions: When a superior vena cava syndrome is diagnosed, additional tests should be performed to discover the real ethiology of the syndrome, which in this case corresponds to a giant intrathoracic goiter. Thyroid function tests and imaging techniques such as ultrasound or CT are indicated in these cases. The treatment of choice of the intrathoracic goiter is surgical removal, especially in cases where compression of adjacent structures is present. 
P101

A CASE OF SECOND DEGREE AV BLOCK AND SEVERELY IMPAIRED CONTRACTILITY IN CARDIAC MYXEDEMA

Apostolos Chatzitomaris ${ }^{1}$, Michael Scheeler ${ }^{2}$, Michael Gotzmann ${ }^{2}$, Janice Schildroth ${ }^{2}$, Kathy Miriam Knyhala ${ }^{2}$, Roland Koeditz²,

Harald H. Klein ${ }^{2}$, Johannes W Dietrich ${ }^{3}$

${ }^{1}$ Bergmannsheil University Hospitals, ${ }^{2}$ Bergmannsheil University

Hospitals Bochum, ${ }^{3}$ Bergmannsheil University Hospitals; Medical

Hospital I; Dept. of Endocrinology \& Diabetes

The heart is a major target organ for thyroid hormone action. Cardiac function may significantly change in patients suffering from hypothyroidism. Severe overt hypothyroidism can result in diastolic hypertension, lowered cardiac output, impaired left ventricular contractility, diastolic relaxation, pericardial effusion and bradycardia. However the function of the atrial pacemaker is usually normal and the degree of slowing of the heart rate is often modest. Here we report a case of a 20 year old male Caucasian with severe overt hypothyroidism. He presented with syncope due to second degree atrioventricular block type Mobitz 2 and heart failure with reduced ejection fraction $(38 \%)$. The diagnosis of hypothyroidism was made on the basis of markedly reduced levels of plasma free total thyroxine $(\mathrm{fT} 4,<3.9 \mathrm{pmol} / \mathrm{L})$ and plasma free triiodothyronine (fT3, $2.4 \mathrm{pmol} / \mathrm{L}$ ) associated with massive rise in plasma thyrotropin (TSH, $108 \mathrm{mU} / \mathrm{L}$ ). The cause of the overt hypothyroidism was hypothyroid Graves' disease. Magnetic resonance imaging of the heart demonstrated decreased cardiac contractility and pericardial effusion, suggesting peri-myocarditis. Plasma levels for BNP and troponin I were low, however. Additionally, testing for cardiotropic viruses in a blood sample delivered normal results, which made a possible infectious cause unlikely. After achieving euthyroidism the second degree the atrioventricular block type Mobitz 2 reverted to normal sinus rhythm. Additionally, the left ventricular ejection fraction returned to normal and the pericardial effusion that was demonstrated in MRI dissolved. Concluding, we describe a case of cardiac myxedema that affected both electrophysiology and contractility, manifestations that were reversible in response to treatment with levothyroxine.

\section{P102}

\section{THREE CLINICAL CASES OF ECTOPIC THYROID (ABERRANT GOITER) WITH DIFFERENT THYROID FUNCTION AT DIAGNOSIS}

\section{Timur Britvin ${ }^{1}$, Alexander Dreval ${ }^{2}$, Tatiana Shestakova ${ }^{1}$,}

Irina Komerdus

${ }^{1}$ Moscow Regional Research Clinical Institute N.A. Vladimirsky,

${ }^{2}$ Moscow Regional Research Clinical Institute N.A. Vladimirskiy

Background: We present three cases of aberrant goiter with different thyroid function, diagnosed in our clinic for past year.

Case 1: Woman, 51 y. o., thyrotoxicosis for 8 years. Several relapses of thyrotoxicosis were controlled by antithyroid medication (ATM). She had signs of endocrine ophthalmopathy, inactive. On ATM her TSH- 0.01 $\mathrm{mU} / 1$, fT4-16.6 pmol/L (11-23). On US thyroid volume was $68.1 \mathrm{ml}, 2$ nodules in lobes, $2.5 \mathrm{~cm}$ and $1.9 \mathrm{~cm}$. On May 2013 thyroidectomy performed. Postoperative course was complicated by bleeding and hypoparathyroidism. The levothyroxine, calcium and vitamin $\mathrm{D}$ were prescribed. Two months later patient complained of palpitations, weakness, insomnia. Subclinical hyperthyroidism was revealed, TG $-115 \mathrm{U} / \mathrm{ml}$. On CT-the mass of anterior mediastinum, $9 \times 9 \times 7 \mathrm{~cm}$. On September 2013 the mass has been removed surgically. Histological exam: diffuse parenchymal colloid goiter with signs of functional activity of the follicular epithelium.

Case 2: Woman 62 y.o. had thyroidectomy on 2009 - papillary thyroid carcinoma. Chest radiography revealed widening of the superior mediastinal shadow. On $100 \mathrm{mcg}$ of L-T4 her TSH was $0.02 \mathrm{mU} / 1$, f T4 $13.2 \mathrm{pmol} / \mathrm{L}$. On CT the multinodullar inhomogeneus mass with muicrocalcification was revealed in the superior mediastinum, $8.8 \times 14 \times 15 \mathrm{~cm}$. It was surgically removed. Histologically - micro and macrofollicullar colloid goiter.

Case 3. Woman, 34 y.o., for 2 past years suffered from hyperhidrosis, weakness, mood lability. Hormonal evaluation did not reveal any abnormalities. On US and CT two nodular masses were found between carotid and jugular vein and the 2 -nd one located partly behind the sternum $(2 \times 3.5$ and $3.4 \times 4.4 \mathrm{~cm}$ ). Thyroid scintigraphy showed low uptake of radionuclide by the thyroid and fixation of the radionuclide by the mass located behind the sternum. Both masses were surgically removed. Histological exam is in process.

Conclusion: Ectopic thyroid (aberrant goiter) is a rare condition and it may be undiagnosed by clinicians.

\section{P103 \\ PITFALLS IN ULTRASOUND GUIDED FINE NEEDLE ASPIRATION}

Cláudia Freitas ${ }^{1}$, André Couto-Carvalho ${ }^{1}$, Ana Rita Caldas ${ }^{1}$

Ana Maia Silva ${ }^{1}$, Rita Sampaio ${ }^{1}$, Ana Duarte ${ }^{1}$, Fátima Borges ${ }^{1}$

${ }^{1}$ Centro Hospitalar Do Porto - Hospital Santo António

The criteria used to perform a fine needle aspiration (FNA) in thyroid nodules are increasingly based on ultrasound (US) features. Recently, a new classification system was proposed, supporting that nodules characteristically benign in US (TIRADS 2) don't need to perform FNA.

Authors present two cases of TIRADS 2 nodules, which, in fact, were malignant.

The first is a case of a 36 years old woman with hypothyroidism due to Hashimoto Thyroiditis. She appeared in our outpatient clinic with a very large goiter with an US revealing a hyperechogenic, well shaped nodule with $26 \mathrm{~mm}$ in the left lobe and micronodules with the same features in the right one, like the ones described as "white-knights". She had already performed a FNA and the result was follicular neoplasm. She was submitted to a total thyroidectomy and the histological exam revealed a follicular variant of the papillary carcinoma, $2 \mathrm{~cm}$ in size, on the left lobe, and multifocal micro foci on the other side.

The second case is of a 66 years old woman with known thyroid nodules since two years ago, sent to our outpatient clinic to perform FNA last January. The larger nodule had $18 \mathrm{~mm}$ in size and had a complete eggshell calcification. We performed a FNA, and the result was suspected of malignancy. She was submitted to a total thyroidectomy. The histological exam confirmed the existence of a $2 \mathrm{~cm}$ size classical variant of papillary carcinoma and detected one lymph node metastasis.

These two cases illustrate that, despite the well recognised value of the US benign features, there are exceptions. These are good examples of TIRADS 2 nodules, which could have not been submitted to FNA and were already malignant. Authors suggest that the size should be considered when selecting thyroid nodules to FNA, regardless the US features.

\section{P104}

\section{CLINICAL, PATHOLOGiCAL AND MOLECULAR} ANALYSIS OF TSH. OMA PATIENT

Esther Díaz Rodríguez ${ }^{1}$, Sihara Perez ${ }^{2}, E^{2}$ a Fernandez ${ }^{3}$,

Ramón Serramito ${ }^{4}$, Alfredo García ${ }^{4}$, Ignacio Bernabeu ${ }^{4}$,

Clara Alvarez Villamarín ${ }^{2}$

${ }^{1}$ Molecular Medicine and Chronic Diseases (Cimus), University of Santiago de Compostela; Universidad de Santiago de Compostela, ${ }^{2}$ Molecular Medicine and Chronic Diseases (Cimus), University of Santiago de Compostela, ${ }^{3}$ Endocrinology Division. Chus-Sergas. Medicine Department, ${ }^{4}$ Endocrinology Division Chus sergas. Medicine Department

Thyrotropin-secreting pituitary adenomas are rare tumors, and prevalence is about one case per million. Normal or elevated thyrotropin levels in hyperthyroid patients are characteristic of TSH-secreting pituitary adenoma, these tumors are uncommon although number of reported cases are tripled in the last decade. Subjects with TSH-omas are often misdiagnosed as having Graves disease, and misdiagnosis may leads to enlargement of tumor size.

Methodology: To develop a clinical, pathological and molecular data analysis of TSH-omas, a total RNA extraction, qRT-PCR were made from human samples. A tumor from one patient was analyzed comparing to human pituitary gland (Poly A+ RNA) and acromegaly samples. 26 genes were evaluated, pituitary hormones: GH, PRL, POMC, $\beta$ FSH, $\beta \mathrm{LH}, \beta \mathrm{TSH}, \alpha$ Subunit; Somatostatin receptors: SSTR1 SSTR2 SSTR3 SSTR5; Dopamine receptors: DR1, DR2, DR4, DR5; Hormone receptors: GHRH-R, GnRH-R, CRH-R1, AVPR1b, GHS-R1a; Selected markers: Ki-67, PTTG-1.

Results: A 31 year old woman was admitted in the CHUS, diagnosed with hypogonadism and_central hyperthyroidism, clinical test presented high levels of FT4 $(2,490 \mathrm{ng} / \mathrm{L})$ and TSH was nonsuppressed $(4,11 \mu \mathrm{U} / \mathrm{ml})$, also 
image test showed a macroadenoma pituitary tumor. After administration of Octreotide, during 3 months, levels of FT4 decreased until $1 \mathrm{ng} / \mathrm{L}$ and TSH levels were reduced too, but treatment failed to reduce tumor sized. As a pure tumor population the mainly pituitary hormones detected, by qRT-PCR, were $\beta T S H$ and $\alpha \mathrm{GSU}$. An increase in Somatostatin receptors and Dopamine receptors was observed as well as GHS-R overexpresión: 26 times more than the control and 5 more comparing to acromegaly tumors.

Conclusions: Initial analysis indicates a close parallelism of the molecular profile of the adenoma with previously reported data and the clinical phenotype of the patients. Further studies will be needed to understand how this information could help to predict the hormonal response to therapy in order to improve the management of patients.

\section{P105 \\ AN UNUSUAL ENCOUNTER OF TWO COMMON ENDOCRINE DISORDERS AFTER AN EPISODE OF DRESS \\ Chee Kian Chew \\ Department of Endocrinology, Tan Tock Seng Hospital, Singapore}

A 52 years old gentleman, with chronic sinusitis, was admitted, for generalized pruritic skin rash, loss of appetite, fever and lethargy one week after taking bactrim for severe sinusitis. He had leucocytosis with eosinophilia and deranged liver function tests with transaminitis. He was diagnosed to have Drug Reaction with Eosinophilia and Systemic Symptoms (DRESS) secondary to bactrim in view of the development of cutaneous eruption with hepatitis and eosinophilia after taking bactrim. He was started on oral prednisolone and the DRESS resolved.

He was readmitted two weeks later for epigastric pain and vomiting. Biochemical tests were consistent with diabetic ketoacidosis: plasma glucose $32.2 \mathrm{mmol} / \mathrm{L}$ (4.0-7.8), bicarbonate $2.9 \mathrm{mmol} / \mathrm{L}$ (19-31), $\mathrm{pH} 7.105$ (7.35-7.45), beta-hydroxybutyrate $4.4 \mathrm{mmol} / \mathrm{L}(0-0.6)$. His thyroid function was normal: free T4 $19 \mathrm{pmol} / \mathrm{L}$ (8-21), TSH $0.41 \mathrm{mIU} / \mathrm{L}(0.34-5.60)$. He was treated and discharged with insulin for his newly diagnosed diabetes mellitus. The positive GAD autoantibody of $6.7 \mathrm{U} / \mathrm{ml}(0-0.8)$ confirmed the diagnosis of autoimmune type 1 diabetes mellitus.

Three months after the onset of DRESS, he complained of weight loss but he denied palpitation, heat intolerance, diarrhoea, lethargy or giddiness. On examination, there were no goiter, thyroid eye signs or postural hypotension. Thyroid function test confirmed thyrotoxicosis: free T4 $34 \mathrm{pmol} / \mathrm{L}(8-21)$, TSH $0.02 \mathrm{mIU} / \mathrm{L}$ (0.34-5.60). Interestingly, although TRAb was normal, thyroid stimulating immunoglobulin (TSI) was elevated to $300 \%(50-179)$, confirming the diagnosis of Grave's disease. His 8 am serum cortisol was normal. He was started on carbimazole.

DRESS is a rare and potentially fatal drug reaction. It is known to be associated with multiple autoimmune sequalae including autoimmune endocrine disorder such as autoimmune thyroid disease and type 1 diabetes mellitus. It is characterized by sequential reactivation of various herpesviruses causing activation and clonal expansion of autoreactive lymphocytes resulting in autoimmune diseases. Patient with DRESS should be followed-up life-long for the development of these autoimmune diseases.

\section{P106 \\ DIAGNOSTIC AND EVOLUTIVE PECULIARITIES IN A CASE SERIES OF MALIGNANT STRUMA OVARII \\ Ruxandra Dobrescu ${ }^{1}$, Diana Coles ${ }^{1}$, Andrei Goldstein ${ }^{1}$, Dumitru loachim ${ }^{1}$, Mihai Magurean ${ }^{2}$, Iuliana Ceausu' ${ }^{3}$, Corin Badiu ${ }^{4}$ \\ 1"c.l. Parhon" National Institute of Endocrinology, ${ }^{2}$ "Colentina" University Hospital, Dpt Orthopedics, "3"I. Cantacuzino" University Hospital, Dpt. Gynecology; "C. Davila” University of Medicine and Pharmacy, ${ }^{4}$ National Institute of Endocrinology; "Carol Davila" University of Medicine \& Pharnacy}

Background: Struma ovarii accounts for $5 \%$ of all ovarian teratomas, being a rare cause of thyrotoxicosis, malignancy being dictated by histological appearance and the presence of metastases. Graves' disease associated with follicular thyroid carcinoma is particularly rarely reported in the literature.

Case Reports: We present 2 cases of women with metastatic malignant struma ovarii.
The first case aged 48, admitted for persistent thyrotoxicosis 2 years after total thyroidectomy for hyperthyroidism by Graves disease along with a $6 \mathrm{~cm}$ benign thyroid nodule and with a history of total hysterectomy and left ovariectomy 22 years ago (for uterine perforation). Restarting thyamazole established euthyroid status. Abdominal ultrasonography revealed a $6 \mathrm{~cm}$ complex cystic mass on the right ovary, while CT scan revealed multiple pulmonary and vertebral metastases that caused paraparesis. Spinal tumor pathology confirmed metastases of follicular thyroid carcinoma (FTC). ${ }^{131} \mathrm{I}$ whole body scan (WBS) showed functioning ectopic thyroid tissue in pulmonary areas, right pelvis, axial skeleton, pelvic bones, skull but also intense iodine uptake in thyroid region. This finding imposed a histopathologic review revealing well differentiated FTC, stage pT3NxM1 and the rest of the gland was consistent with Graves' disease. Right ovariectomy confirmed malignant struma ovarii with microfoci of FTC; she underwent a further resection of $3 \mathrm{~cm}$ skull metastasis and afterwards received ${ }^{131} \mathrm{I}, 3$ times at 3 months interval.

The second case, aged 30, was operated for an ovarian teratoma suggesting malignant struma. After 3 months, thyroidectomy was performed showing normal thyroid. WBS 1 month after revealed lung metastases, therefore she was submitted to ${ }^{131} \mathrm{I}$ therapy.

Conclusion: Persistence of thyrotoxicosis could be consequence of malignant struma ovarii and/or functioning metastases and impact upon treatment of thyroid malignancy. Completion thyroidectomy is required when ${ }^{131} \mathrm{I}$ becomes part of the treatment, even when thyroid is apparently normal.

\section{P107 \\ SUNITINIB-INDUCED TRANSIENT THYROTOXICOSIS IN A PATIENT WITH RENAL CELl CARCinOMA \\ Raluca Trifanescu ${ }^{1}$, Oana-Gabriela Trifanescü, Mara Carsote ${ }^{3}$ \\ Catalina Poiana ${ }^{4}$ \\ 1"Carol Davila" University of Medicine and Pharmacy; "C.I. Parhon" Institute of Endocrinology, 2“Al. Trestioreanu” Institute of Oncology; "Carol Davila" University of Medicine and Pharmacy, ${ }^{3 " C a r o l ~ D a v i l a " ~}$ University OD Medicine and Pharmacy; "C.I. Parhon" Institute of Endocrinology, "“Carol Davila" Univeristy of Medicine and Pharmacy; "C.I. Parhon" Institute of Endocrinology}

Introduction: Thyroid dysfunction is a frequent side effect of tyrosine kinase inhibitors. Up to $60 \%$ of patients treated with sunitinib developed thyroid dysfunction, especially hypohyroidism, after a median treatment duration of 16 weeks. Thyrotoxicosis was seldom reported.

Case Report: A 39 years old Caucasian woman, resident in an iodine sufficient area, was treated with sunitinib for renal cell carcinoma. Thyroid function tests (TSH, FT4, FT3, measured by chemiluminescence) and antithyroid antibodies (TPO Abs, TRAb) were normal during first six months of treatment. Thereafter, the patient developed tachycardia, tremor of extremities, anxiety and arterial hypertension. Biochemical data confirmed thyrotoxicosis $(\mathrm{TSH}=0.03 \mathrm{mIU} / \mathrm{L}, \mathrm{FT} 4=37.6 \mathrm{pmol} / \mathrm{L}, \mathrm{FT} 3=14.72 \mathrm{pmol} / \mathrm{L}) ; \mathrm{TPO}$ antibodies $(10 \mathrm{IU} / \mathrm{mL})$ and TRAb $(0.686 \mathrm{UI} / \mathrm{l})$ were negative; radioiodine uptake was decreased both at 2 hours $(4 \%)$ and 24 hours $(1 \%)$ and serum thyroglobulin was markedly increased $(300 \mathrm{ng} / \mathrm{mL})$, revealing destructive thyrotoxicosis. Ultrasound measured thyroid volume was normal, with normal vascularization at Doppler examination. A short term glucocorticoid therapy was administered with rapid normalization of thyroid hormones within 3 weeks $(\mathrm{TSH}=0.29$ $\mathrm{mIU} / \mathrm{L}, \mathrm{FT} 4=11.1 \mathrm{pmol} / \mathrm{L}$ ); sunitinib treatment was continued; one month later, the patient developed subclinical hypothyroidism $(\mathrm{TSH}=7.21 \mathrm{mIU} / \mathrm{l}$, FT4 $=15.35 \mathrm{pmol} / \mathrm{L}, \mathrm{FT} 3=4.6 \mathrm{pmol} / \mathrm{L}$ ), followed by another episode of thyrotoxicosis $(\mathrm{TSH}=0.1 \mathrm{mIU} / \mathrm{L})$.

Conclusion: close moitoring of thyroid function is recommended in sunitinib treated patients, irrespective of treatment duration. Recurrent episodes of destructive thyrotoxicosis may occur, followed by hypothyroidism. 


\section{P108}

TWO PORTUGUESE FAMILIES OF FAMILIAL PAPILLARY THYROID CARCINOMA

\section{Eva Lau ${ }^{1}$, Ana Isabel Oliveira' ${ }^{1}$, Davide Carvalho'}

${ }^{1}$ Endocrinology, Diabetes and Metabolism Department, Centro

Hospitalar São João, Faculty of Medicine, Porto University

Introduction: Familial papillary thyroid carcinoma (FPTC) is defined by the presence of 2 or more first-degree relatives with non-medullary thyroid carcinoma (NMTC), in the absence of another familial syndrome. It is estimated a familial origin in $3.5 \%-6.2 \%$ of patients with NMTC.

Case Report: Family 1: Index case - Female, 52 years old, who underwent subtotal thyroidectomy in 1982 by pappillary thyroid cancer (PTC). The tumor was $5.5 \times 3 \mathrm{~cm}$ diameter and frank lymphatic invasion, although without capsule invasion. During that year thyroidectomy was totalized, along with lymph node dissection for metastatic PTC. Three therapeutic radioiodine I-131 were performed $(70 \mathrm{mCi}, 60 \mathrm{mCI}$ and $100 \mathrm{mCi}$ in 1982,1983 and 1985 , respectively). Local recurrence excision, with vascular invasion and resection margin with cancer (2009) and new therapy with I-131 (100mCi - 2010) was performed. Currently, despite no recurrent thyroid masses or lymphadenopathy in ultrasonographic study and no abnormal uptake in FDG-PET, thyroglobulin keeps up increased $(42.1 \mathrm{ng} / \mathrm{mL})$ with negative anti-thyroglobulin antibodies. Family history revealed a niece with PTC of $10 \mathrm{~mm}$. Of 6 brothers, 2 of them have CPT history, diagnosed at 62 and 61 years, both underwent surgery and therapy with I-131. Family 2: Index case - female, 20 years old, followed in Endocrinology outpatient clinic for a $10 \mathrm{~mm}$ nodule with calcifications. Fine needle biopsy revealed a PTC. Total thyroidectomy was perfomed, confirming a multicentric PTC with a metastatic lymph node, but without capsule invasion. Subsquently, she was submitted to I-131 therapy $(100 \mathrm{mCi})$. Family history revealed more 3 familiar with CPT operated. Family screening, detected 3 familiar with benign thyroid nodules, already submitted to surgery.

Conclusion: FPTC appears to confer increased risk of multifocal disease, local invasion and lymphatic metastasis. As there is no genetic test to identify those at risk, screening of at-risk families is essential in the earlier recognition and treatment of these tumors.

\section{P109 \\ IN QUEST OF ORAL THYROXINE RESISTANCE - THREE CASE REPORTS WITH ASSUMED INTESTINAL MALABSORPTION OF LEVOTHYROXINE}

Bruhnke Gabriele ${ }^{1}$, Richard Voigtländer ${ }^{1}$, Jaeger Andrea ${ }^{1}$,

Lars Moeller ${ }^{2}$, Ulrich Schweizer ${ }^{3}$, Dagmar Führer ${ }^{1}$

${ }^{1}$ Department of Endocrinology and Metabolism and Division of

Laboratory Research, University of Duisburg-Essen, Essen, Germany,

${ }^{2}$ University Duisburg-Essen, Department of Endocrinology and

Metabolism, ${ }^{3}$ Rheinische Friedrich-Wilhelms-Universität Bonn, Institut

Für Biochemie und Molekularbiologie

Oral substitution of thyroid hormone is the most common hormone replacement world-wide, but some patients fail to reach euthyroidism despite an elevated levothyroxine (LT4) dosage. If other causes are excluded, oral thyroxine resistance may be assumed which proposes a deficient intestinal uptake of LT4. We present three patients with abnormally high LT4 requirements. In case 1 , an 18 year old hypothyroid female with Hashimoto's thyroiditis claimed a daily intake of $600 \mu \mathrm{g}$ LT4. During thyroxine resorption testing, complete uptake of the administered LT4 dose was confirmed. Furthermore, test evaluation uncovered, that our patient must have taken about $600 \mu \mathrm{g}$ LT4 by herself at the start of testing. When asked about this, she admitted noncompliance in the past. Currently, she receives a LT4-replacement adapted to body weight. In case 2 , a 72 year old male patient had undergone surgical removal of benign goiter in 2012. He presented with a TSH of $2.3 \mathrm{mU} / 1$ under $400 \mu \mathrm{g}$ LT4. Type B gastritis was diagnosed by gastroscopy and antibiotic treatment was initiated. Thyroid metabolism will be re-examined after gastric disease is cured. In case 3, a 57 year old female patient presented with 24 hours subcutaneous administration of LT4 filled in a morphine pump. This route of application was chosen because oral supplementation had failed after thyroid removal because of papillary cancer in 2001. Resorption testing revealed a four hours delayed uptake of ingested LT4. Moreover, abnormal aminoaciduria was diagnosed and could reflect a deficient transporter molecule local- ized in kidney and gut. Intestinal tissue is now analyzed for abnormalities in thyroid hormone/amino acid transporters. In summary, the etiology behind thyroxine malabsorption is broad, and non-compliance as well as objectively abnormal kinetics of thyroxine uptake has to be taken into consideration. This project is funded by DFG SPP 1629 FU356/8-1.

\section{P012 Imaging and Nuclear Medicine}

\section{P110 \\ THE THYROID-RELATED QUALITY OF LIFE MEASURE THYPRO HAS GOOD RESPONSIVENESS AND ABILITY TO DETECT RELEVANT TREATMENT EFFECTS}

Torquil Watt ${ }^{1}$, Per Cramon ${ }^{2}$, Laszlo Hegedüs ${ }^{3}$, Jakob Bue Bjorner ${ }^{4}$ Steen Joop Bonnema ${ }^{3}$, Åse Krogh Rasmussen ${ }^{1}$,

Ulla Feldt-Rasmussen ${ }^{1}$, Mogens Groenvold ${ }^{4}$

${ }^{1}$ Department of Endocrinology, Copenhagen University Hospital

Rigshospitalet, Denmark, ${ }^{2}$ Copenhagen University Hospital

Rigshospitalet, ${ }^{3}$ Department of Endocrinology and Metabolism, Odense

University Hospital, Denmark, ${ }^{4}$ University of Copenhagen, Denmark

Background and Purpose: Patient-reported outcomes have become important endpoints in comparative effectiveness research and in patient-centered health care. Valid patient-reported outcome measures detect and respond to clinically relevant changes. The purpose of this study was to evaluate responsiveness of the thyroid-related quality of life (QoL) instrument ThyPRO in patients undergoing relevant clinical treatments for benign thyroid diseases, and to compare it with responsiveness of the generic SF-36 Health Survey V2.

Methods: A sample of 435 patients undergoing treatment completed ThyPRO and SF-36 Health Survey at baseline and 6 months after treatment initiation. Responsiveness was evaluated in three thyroid patient-groups: Patients with hyper- $(n=66)$ and hypothyroidism $(n=84)$ rendered euthyroid, and patients with a clinically detectable non-toxic goiter treated with surgery or radioactive iodine and remaining euthyroid $(n=62)$. Changes in QoL were evaluated in terms of effect size and compared to the changes predicted by clinical experts. The responsiveness of equivalent scales from ThyPRO and SF-36 Health Survey V2 were compared with the relative validity index.

Results: The ThyPRO demonstrated good responsiveness across the whole range of QoL aspects in patients with hyper- and hypothyroidism. Responsiveness to treatment of non-toxic goiter was also demonstrated for physical and mental symptoms and overall QoL, but not for impact on social life or cosmetic complaints, in contrast to clinicians' predictions. For all comparable scales except one, the ThyPRO was more responsive to treatment than the SF-36 Health Survey.

Conclusions: The ThyPRO was responsive to treatment across the range of benign thyroid diseases. We suggest implementing this measurement instrument as a patient-reported outcome in clinical studies and in clinical management

\section{P111}

\section{A PRACTICAL SCORING MODEL FOR DETECTING MALIGNANT THYROID NODULES BY USING ULTRASONOGRAPHIC FINDINGS} Shirzad Nasiri" ${ }^{1}$, Nassim Sodagari ${ }^{1}$

${ }^{1}$ Tehran University of Medical Sciences

Background and Aims: About $10 \%$ of thyroid nodule FNAs (Fine Needle Aspiration) are reported indeterminate. About $20 \%$ of these nodules are malignant. We decided to evaluate the ultrasonographic features of malignant thyroid nodules, in order to find a predictive scoring model for thyroid nodules and consequently reduce the unnecessary thyroid surgeries.

Materials and Methods: 114 patients with thyroid nodule who were candidate for thyroid surgery between November 2011 and December 2012 were gathered. All the patients were assessed by ultrasonography of thyroid before 
surgery. By the gold standard of permanent pathology, and by using the logistic regression analysis, a predictive scoring model was suggested.

Results: according to scoring model, if a patient with thyroid nodule and ultrasonography was referred to us, in case of female, 1.1 score, the nodule size $\leq 36 \mathrm{~mm}, 0.9$ score, the oval shape of nodule, 0.9 score, hypoechogenicity of nodule, 1.8 score, concurrent lymphadenopathy, 1.1 score, microcalcification of nodule, 1.1 score and a thick or incomplete halo around nodule, 1.5 score was given to patient. If the sum of scores are more than 4.7 , with sensitivity of $90.9 \%$, specificity of $76.1 \%$, positive predictive value of $71.4 \%$, negative predictive value of $92.7 \%$, positive likelihood ratio of 3.81 and negative likelihood ratio of 0.12 , this patient has malignant thyroid nodule and should undergo thyroid surgery.

Conclusions: By higher rate of sensitivity in comparison with specificity in our suggestive model, the missing of thyroid cancer will be low. This can be a helpful and complementary tool along with FNA for early and more accurate diagnosis of malignant thyroid nodule.

\section{P112 \\ RADIOFREQUENCY ABLATION IS A THYROID. FUNCTION-PRESERVING TREATMENT FOR PATIENTS WITH BILATERAL BENIGN THYROID NODULES}

Min Ji Hong ${ }^{1}$, Jung Hwan Baek', Young Jun Choi', Jeong Hyun Lee ${ }^{1}$, Hyun Kyung Lim², Young Kee Shong ${ }^{3}$, Suck Joon Hong ${ }^{4}$

${ }^{1}$ Department of Radiology and Research Institute of Radiology, University of Ulsan College of Medicine, Asan Medical Center,

${ }^{2}$ Department of Radiology, Soonchunhyang University Hospital, ${ }^{3}$ Asan Medical Center; University of Ulsan College of Medicine, ${ }^{4}$ Department of Surgery, University of Ulsan College of Medicine, Asan Medical Center

Background and Purpose: Our objective was to evaluate the efficacy and safety, especially the preservation of thyroid function, of RF ablation for treating bilateral thyroid nodules.

Materials and Methods: Between January 2007 and October 2012, the bilateral thyroid nodules of 18 patients ( 16 females, 2 males; mean age, 49.9 years; range $27-81$ years) were included in this study, as determined by the following criteria: (1) the presence of bilateral thyroid nodules; (2) patients with pressure symptoms or cosmetic problems; (3) cytological confirmation of benignancy; and (4) refusal of surgery.

Results: The mean initial nodule size was $4.1 \pm 1.9 \mathrm{~cm}$, although it had significantly decreased by the time of the last follow-up $(P<.001,2.5 \pm 1.4 \mathrm{~cm})$. The initial nodule volume was $24.4 \pm 32.2 \mathrm{~mL}$ and was decreased at the last follow-up $(6.3 \pm 19.0 \mathrm{~mL}, P<.001)$, with a mean volume reduction of $75.9 \pm 19.0 \%$. The symptom $(P<.001)$ and cosmetic score $(P<.001)$ were decreased. Serum hormone levels did not differ significantly prior to treatment and at the last follow-up $(P>0.05)$.

Conclusion: RF ablation improves cosmetic and symptomatic problems as well as preserves thyroid function in patients with bilateral thyroid nodules.

\section{P113 \\ ROLE OF F-18 FLUORODEOXYGLUCOSE PET/ \\ CT IN THE EVALUATION OF NON-IODINE- AVID METASTASES/DISEASE RECURRENCE IN THYROID CARCINOMA}

Syed Zama Ali ${ }^{\circ}$, Anabalagan Kannivelu ${ }^{1}$

${ }^{1}$ Khoo Teck Puat Hospital, Singapore

Purpose: To evaluate the role of F-18 Fluorodeoxyglucose (FDG) PET/ $\mathrm{CT}$ in the investigation of suspected non-iodine-avid residual metastases/disease recurrence in cases of thyroid carcinomas with previous total thyroidectomy and Radioactive Iodine (RAI) treatment.

Material and Methods: In this retrospective study, we evaluated 35 patients of known thyroid carcinomas with F-18 FDG PET/CT done (between April 2012 to March 2014) for suspected metastases/recurrence due to rising serum levels of thyroglobulin ( $\mathrm{Tg}$ ) or thyroglobulin antibodies ( $\mathrm{TgAb})$. All patients had previous negative low dose $(30 \mathrm{mCi})$ or high dose $(100-150$ $\mathrm{mCi} 131$ Iodine Whole-Body scintigraphy within 0 to 3 months of the PET/ CT study.

Results: Out of 35 patients, 32 patients were of papillary thyroid carcinoma (PTC) and 3 patients of follicular thyroid carcinoma (FTC). F-18 FDG PET/CT detected foci of increased F-18 FDG uptake suspicious of metastases/ disease recurrence in 29 patients. In twelve patients (all PTC) increased F-18 FDG uptake was noted in the cervical lymph nodes with SUVmax between 3.0 to 21.7. In eight patients (7 PTC, 1FTC) lung nodules were noted (size $=0.5$ to $2.1 \mathrm{~cm}$ ) with SUVmax range from 1.5 to 21.4. Four patients (all PTC) had foci of F-18 FDG uptake in thyroid bed with SUVmax from 2.7 to 5.1 and four patients (2 PTC, 2 FTC) had uptake in mediastinal nodes with SUVmax from 4.5 to 20.6. The sensitivity and specificity of F-18 FDG PET/CT in detection of non-iodine-avid metastases was estimated as $96.7 \%$ and $80 \%$, respectively. The sensitivity, specificity, positive predictive value and negative predictive value of elevated stimulated thyroglobulin level $(>2 \mathrm{ng} / \mathrm{ml})$ for presence of metastatic/recurrent lesions were $93.3 \%, 40 \%, 90.3$ and $50 \%$ respectively.

Conclusion: F-18 FDG PET/CT is useful in the evaluation of patients with suspected recurrence or residual metastases in cases of thyroid carcinomas with negative 131 Iodine-Whole body scintigraphy and rising $\mathrm{Tg} / \mathrm{TgAb}$.

\section{P114 \\ PULSED DOPPLER EVALUATION OF THYROID BLOOD FLOW IN DIFFUSE THYROID DISORDERS}

Mariela Bachvarova ${ }^{1}$, Kiril Hristozov², Branimir Kanazirev ${ }^{3}$

${ }^{1}$ Varna Medical University, ${ }^{2}$ Clinic of Endocrinology, University Hospital "St. Marina "Varna, ${ }^{3}$ Varna Medical University; St Mariana University Hospital

Objective:To evaluate blood flow velocities in the inferior thyroid artery according to underlying thyroid disease and hormone status.

Materials and Methods: 65 patients were studied with pulsed Doppler. Patients were divided twofold: into 3 groups according to their hormonal status-hyperthyroid(H), subclinical hyperthyroid(SH) and euthyroid(EU) and into five groups according to their underlying clinical nosology: G1-euthyroid with Graves' disease (21 patients), G2-hyperthyroid with Graves' disease

Table 1. Indices of Pulsed Doppler velocities in all investigated groups (for Abstract P114)

\begin{tabular}{lllll}
\hline Groups & PSV & MnV & PI & RI \\
\hline G1 & $57.7 \pm 34.7 * * *$ & $41.8 \pm 25^{* * *}$ & $0.716 \pm 0.16^{*}$ & $0.513 \pm 0.07 * *$ \\
G2 & $83.5 \pm 24.4^{* * *}$ & $58.5 \pm 18.2 * * *$ & $0.793 \pm 0.22^{*}$ & $0.540 \pm 0.08^{* *}$ \\
G3 & $76.6 \pm 10.1 * * *$ & $58.6 \pm 8 * * *$ & $0.552 \pm 0.02^{*}$ & $0.422 \pm 0.01 * *$ \\
G4 & $26.6 \pm 11.2^{* * *}$ & $18.3 \pm 8.2 * * *$ & $0.838 \pm 0.12^{*}$ & $0.570 \pm 0.06^{* *}$ \\
G6 & $8.8 \pm 1.8^{* * *}$ & $5.7 \pm 0.5^{* * *}$ & $0.910 \pm 0.24^{*}$ & $0.590 \pm 0.09 * *$ \\
Control & $17.7 \pm 5.5^{* * *}$ & $12.2 \pm 4 * * *$ & $0.867 \pm 0.22^{*}$ & $0.583 \pm 0.08^{* *}$ \\
H & $65.6 \pm 34.6 * * *$ & $45.7 \pm 24.6^{* * *}$ & $0.820 \pm 0.22$ & $0.553 \pm 0.90$ \\
SH & $54.0 \pm 34.9^{* * *}$ & $40.1 \pm 27.7 * * *$ & $0.713 \pm 0.17$ & $0.514 \pm 0.95$ \\
EU & $28.2 \pm 26^{* * *}$ & $19.7 \pm 18.3 * * *$ & $0.835 \pm 0.20$ & $0.566 \pm 0.83$ \\
\hline
\end{tabular}

$\mathrm{P}<0.001 * * *, \mathrm{P}<0.01 * *, \mathrm{P}<0.05^{*}$ 
(21), G4-autoimmune Hashimoto thyroiditis (12), G5-bacterial and subacute thyroiditis (7), G5-atrophic thyroiditis (4) and 29 age-matched controls. PSV, MnV, PI, RI of the inferior thyroid artery were measured and compared between groups. Thyroid status- FT3, FT4, TSH, anti-TPO, TRAK were determined in all.

Results: Significant differences between $\mathrm{H}, \mathrm{SH}$ and $\mathrm{EU}$ were found in PSV and MnV. Significant differences between groups according to underlying pathology of all measured indices- PSV, MnV, PI, RI were also found (table 1).

Conclusions: Pulsed Doppler of inferior thyroid artery provides additional diagnostic information concerning thyroid pathology and hormonal state of patients.

\section{P115 \\ ROLE OF 131-I SPECT-CT IN THE RISK STRATIFICATION OF DIFFERENTIATED THYROID CANCER}

Shirly Margarita Nieves Maldonado ${ }^{1}$, Patricia Fierro Alanis ${ }^{1}$ Virginia Pubul Nuñez', Ines Dominguez Parado',

Sonia Argibay Vazquez"1, José Manuel Cabezas Agrícola1, Alejandro Bejarano Garcia', Jesus Lopez Urdaneta ${ }^{1}$,

Julia Cortez Hernández ${ }^{1}$, Álvaro Ruibal More/ ${ }^{1}$

${ }^{1}$ Centro Hospitalario Santiago de Compostela

Pourppose: To assess the added contribution of the 131 -I SPECT -CT compared to the whole body scan (WBS), in the risk stratification in differentiated thyroid cancer.

Materials and Methods: Retrospective and observational study, in which 38 patients (28 women, 10 men) with the histopathological diagnosis of differentiated thyroid cancer (29 papillary and 9 follicular) were included. These patients underwent a WBS and a SPECT-CT in our nuclear medicine department (post ablative dose/ post- therapeutic dose), between the years 2008 and 2013. The interpretation of the images (planar WBS and SPECT-CT) was performed independently by two observers initially blinded for clinical information; in cases of discrepancy between the observers the images were evaluated by a third specialist under the same conditions. The radiotracer deposits observed in both study types were assessed according to their location and were interpreted as pathological or physiological, evaluating the changes in TNM (7th Edition) and the ATA risk classification.

Results: Changes in the risk stratification were observed in $50 \%$ of patients $(n=19 / 38)$ when comparing the findings of the WBS and SPECT-CT; $89 \%(n=17 / 19)$ of these changes where at the expense of nodal component (N). From the group of 19 patients without changes on tumor stage or the risk stratification, the SPECT-CT image provided greater accuracy in the location of distant metastases in a $32 \%(n=6 / 19)$.

Conclusion: The SPEC-CT images provide relevant information which allows a more precise location of nodal and distant metastatic involvement, thereby improving risk stratification of differentiated thyroid cancer patients.

\section{P117 \\ 99MTC-METHOXYISOBUTYLISONITRILE (MIBI) SCINTIGRAPHY IS AN USEFUL AND COST- EFFECTIVE TOOL FOR ASSESSING THE RISK OF MALIGNANCY IN THYROID NODULES WITH INDETERMINATE FINE NEEDLE CYTOLOGY (FNAC) \\ Alfredo Campenni', Luca Giovanella², Salvatore Giovinazzo ${ }^{3}$ Rosaria Certo ${ }^{3}$, Marina Raffaella Galletti ${ }^{3}$, Massimo Siracusa ${ }^{4}$, Teresa Manuela Vicchio ${ }^{3}$, Mariapaola Cucinotta ${ }^{4}$, Francesco Trimarchi3, Sergio Baldari ${ }^{4}$, Rosaria Maddalena Rugger ${ }^{3}$ \\ ${ }^{1}$ Nuclear Medicine Unit, University of Messina; Aou P Policlinico G. Matino Messina, ${ }^{2}$ Nuclear Medicine AND Pet/Ctcentre Oncology Institute OF Southern Switzerland-Bellinzona, Switzerland, \\ ${ }^{3}$ Endocrinology Unit \&, ${ }^{4}$ Nuclear Medicine Unit University OF Messina}

Objectives: To assess the performance of sestaMIBI scintigraphy in the diagnostic evaluation of thyroid nodules with non-diagnostic (Thyr 1) or inconclusive (Thyr3) FNAC.

Material and Methods: This prospective study was conducted on 30 patients $(F=25, M=5$; mean age $47.4 \pm 11.8$ years $)$ with cold thyroid nodules at ${ }^{99 \mathrm{~m}} \mathrm{Tc}$-pertechnetate scintigraphy, greater than $1.5 \mathrm{~cm}$ in diameter (mean size: $28.4 \mathrm{~mm}$; range 15-45). The patients had undergone FNAC, with indeterminate results: Thyr1, $\mathrm{n}=5$, and Thyr3, $\mathrm{n}=25$. MIBI uptake in thyroid nodules was evaluated both qualitatively (compared with that in contralateral thyroid lobe) and quantitatively, by using region of interest (ROI). All patients underwent total-thyroidectomy.

Results: All the cold nodules were MIBI-positive, with different intensity of MIBI uptake at qualitative analysis: low ( $n=11$ patients), moderate $(n=13$ patients) and high ( $\mathrm{n}=6$ patients). By quantitative analysis, the patients were arbitrarily subdivided in three groups: $\mathrm{A}(\mathrm{n}=11)$ with a wash-out index (woi) $\geq-40 \%$; Group B, $(n=13)$ : woi between -20 and $-40 \%$; Group C $(n=6)$ : woi $\leq-20 \%$. We assumed that a woi $\leq-20 \%$ was suspicious for malignancy, while a woi $\geq-40 \%$ was predictive of a benign lesion. Compared to hystopathologic findings, all patients of the group A were negative for thyroid cancer (sensitivity and negative predictive value $100 \%$ ). All patients affected by benign adenomas except one were included in Group B (sensitivity of 93\%). Finally, five out of six patients of the Group $\mathrm{C}$ had a differentiated thyroid cancer (all papillary thyroid carcinoma) (specificity and positive predictive value $83.3 \%$ ). The only false positive was a patient affected by oxyphil adenoma with a borderline value of woi (-19\%)

Conclusions: We suggest the use of MIBI-scan (by using quantitative analysis) in the work-up of cold nodule with indeterminate cytology to assess the risk of malignancy and lower the rate of unnecessary thyroidectomies.

\section{P116}

Poster has been withdrawn 


\section{P118 \\ EFFECTIVENESS OF AN INDIVIDUALIZED \\ RADIOIODINE ACTIVITY CALCULATION \\ METHOD IN PATIENTS WITH \\ HYPERTHYROIDISM}

Daniel González Duarte ${ }^{1}$, Yolanda Santaella Guardiola²,

Lourdes García García-Doncel', Milagros Cardoso Rodiguez ${ }^{3}$,

Jesús Melgar Pérez $z^{4}$, Manuel Aguilar Diosdado

${ }^{1}$ Ugc Endocrinology and Nutrition, Cádiz. Hospital Punta Europa de

Algeciras, ${ }^{2}$ ucg Radiology. Nuclear Medicine Department. Hospital

Punta Europa de Algeciras, ${ }^{3}$ Ugc Radiology. Nuclear Medicine

Department. Hospital Punta Europa de Algeciras, ${ }^{4}$ Radiation Physics

Department. Hospital Punta Europa de Algeciras, ${ }^{5}$ Ugc Endocrinology

and Nutrition, Cádiz. Hospital Puerta del Mar de Cádiz

There is still controversy regarding the best method to determine the radioiodine-131(I-131) activity necessary for an optimal treatment of hyperthyroidism

Purpose: The aim of our study was to compare the clinical outcomes of patients with hyperthyroidism using an individualized radioiodine dose method versus fixed doses and to analyze possible predictor factors related to euthyroidism.

Methods: 143 hyperthyroid patients treated with an individualized I-131 dose (ID) and 102 with a fixed dose (FD) were followed prospectively during a year in Hospital Punta Europa, Algeciras. Clinical outcome was determined after one year of the I-131 treatment. Both groups were compared through bivariate statistical analysis. Chi-square test was used for qualitative variables and Student's t-test for quantitative variables. Multivariate analysis was performed to identify potential independent factors associated with the cure of hyperthyroidism.

Results: 106 patients treated with ID (74\%) were euthyroid at the end of the year compared to only 37 in the FD group $(26 \%), \mathrm{P}<0.0005$. Regarding hypothyroidism, 33 patients in the ID group $(23 \%)$ were hypothyroid versus 45 in the FD group (44\%), P $<0.001$. Median I-131 doses used were 8 and $14.5 \mathrm{mCi}$ in patients with ID and $\mathrm{FD}$, respectively $(\mathrm{P}<0.0005)$. Using an individualized dose protocol was independently and positively associated with euthyroidism(odds ratio[OR] 5.18, 95\% Confidence interval[CI] 2.84-9.44, $\mathrm{P}<0.0005)$. Euthyroidism was also more common in multinodular goiter/ toxic adenoma than Graves' disease in the multivariate analysis (OR 4.94; $95 \%$ CI $1.12-5.88 \%, \mathrm{P}<0.026$ ).

Conclusions: Treatment of hyperthyroidism with an individualized I-131 algorithm instead of the commonly used fixed dose protocol clearly improves clinical outcomes after a year in patients with hyperthyroidism. Improvement in clinical outcomes can be achieved using much lower I-131 doses and therefore optimizing the treatment with less radiation risk.

\section{P119 \\ SALIVARY GLAND SIDE EFFECTS AFTER 131-IODINE ADMINISTRATION FOR THYROID REMNANT ABLATION: IS THERE ANY RELATIONSHIP WITH THE METHOD OF PATIENT'S PREPARATION (RHTSH/ LT4 WITHDRAWAL) OR THE ADMINISTERED IODINE ACTIVITY?

loannis lakovou ${ }^{1}$, Euanthia Giannoula', Iordanis Konstantinidis',
Savvas Frangos ${ }^{3}$
${ }^{1}$ Nuclear Medicine Dpt Aristotle University Papageorgiou Hsp,
${ }^{2}$ Otorhinolaryngology Dpt Aristotle University Papageorgiou Hsp,
${ }^{3}$ Department of Nuclear Medicine Bank of Cyprus Oncology Centre

Aim: The aim of this study was to determine the incidence of salivary glands pathology (SGP) after 131-I administration for thyroid remnant ablation in cases of not high risk differentiated thyroid cancer and to investigate any relationship of this phenomenon with the method of patient's preparation (rhTSH vs T4 withdrawal) as well as the administered iodine activity.

Materials \& Methods: A total of 124 patients were equally divided into 4 groups depending on the ablation method used: group A (rhTSH \& $3700 \mathrm{MBq}$ ), group B (rhTSH \& $2590 \mathrm{MBq})$, group C (T4 withdrawal \& $3700 \mathrm{MBq})$ and group D (T4 withdrawal \& $2590 \mathrm{MBq}$ ). The result of the ablation was deter- mined with a whole-body scan performed at the end of the 12th month postablation and a calculation of stimulated thyroglobulin. Summated Xerostomia Inventory (the Dutch version) was used to determine the incidence of salivary gland side effects at the end of the 1st and 12th month after the ablation.

Results: Successful ablation rate $(86 \%$ in total) was the same, with no statistical difference among groups. SGP was reported by $31 \%$ of all patients within the 1 st month, but only by $12 \%$ at the end of the 1 st year. The incidence was significantly higher in unsuccessfully ablated patients at the end of the 1 st year, whereas, it was significantly lower in the groups that used rhTSH as a method of preparation $(\mathrm{P}<0.05)$. There was no relationship between dose administered and SGP appearance.

Conclusion: The use of rhTSH in the patient's preparation for ablation in cases of DTC with no distant metastases reduces the incidence of SGP, interestingly showing no dependence on the dose of 131-I administered.

\section{P120 PREDICTIVE FACTORS OF RADIOACTIVE IODINE THERAPY FAILURE IN GRAVES' DISEASE}

Joana Nunes ${ }^{1}$, Elisabete Rodrigues ${ }^{2}$, Ana Oliveira $^{3}$, Tiago Vieira ${ }^{3}$ Victor Alves ${ }^{3}$, Berta Perez ${ }^{3}$, Ricardo Correia ${ }^{4}$, Davide Carvalho², Jorge Pereira ${ }^{3}$

${ }^{1}$ Endocrinology Chsj, ${ }^{2}$ Department of Endocrinology, Diabetes and Metabolism, Centro Hospitalar São João, /Faculty of Medicine University of Porto, Porto, Portugal, ${ }^{3}$ Department of Nuclear Medicine, Centro Hospitalar São João, Porto, Portugal, ${ }^{4}$ Department of Radiology, Centro Hospitalar São João, Porto, Portugal / Faculty of Medicine University of Porto, Porto, Portugal

Aims: To study some of the factors that may interfere with the success rate of radioactive iodine therapy (RAI) in Graves' disease.

Methods: Retrospective analysis of all patients submitted to RAI after thionamides failure, in the last 5 years in our institution. Gender, age at diagnosis, disease duration, ophthalmopathy, thyroid size, antithyroid drugs, TSH, FT4, TRABs, ${ }^{131}$ I uptake prior to therapy and radiodine doses were evaluated. Successful treatment was defined as euthyroidism/hypothyroidism 12 months after RAI, without thionamides. Prior to RAI, 30 patients were receiving methimazole and 29 propylthiouracil (all withdrawn medication for 5-7 days). Eighty-four patients were submitted to RAI but 10 were lost for follow-up and were excluded. Univariate and multivariate analyses were performed using SPSS $®\left(\mathrm{v} 21.0\right.$ Windows $\left.{ }^{\circledR}\right)$.

Results: Nineteen patients became euthyroid (25.7\%), 33 hypothyroid $(44.6 \%)$ and 22 remained hyperthyroid. In the univariate analysis, higher TRABs $(p=0.02)$, higher ${ }^{131}$ I uptake at 2 hours $(p=0.000), 4$ hours $(p=0.001)$ and 24 hours $(\mathrm{p}=0.015)$ and treatment with propylthiouracil $(\mathrm{p}=0.024)$ were associated with RAI failure. There was no association with gender, age at diagnosis, disease duration, ophthalmopathy, thyroid size, TSH and FT4. Multiple logistic regression (adjusted for thyroid size) showed higher failure rate with higher TRAbs $(30.3 \pm 15.07 \mathrm{U} / \mathrm{L}$ vs $18.7 \pm 14.02 \mathrm{U} / \mathrm{L}, \mathrm{p}=0.013, \mathrm{OR}=1.052)$, higher ${ }^{131} \mathrm{I}$ uptake at 2 hours $(73.3 \pm 22.03 \%$ vs $49.5 \pm 22.77 \%, \mathrm{p}=0.006 \mathrm{OR}=$ $1.047)$ and at 4 hours $(82.4 \pm 17.92 \%$ vs $62.4 \pm 23.36 \%, \mathrm{p}=0.048 \mathrm{OR}=1.036)$ and lower radiodine doses $(7.2 \pm 1.97 \mathrm{mCi}$ vs $8.5 \pm 2.95 \mathrm{mCi}, \mathrm{p}=0.041, \mathrm{OR}=$ 0.712). RAI failures was predicted by TRAbs $>33.7 \mathrm{U} / \mathrm{L}(\mathrm{AUC}=72.0 \%)$ with specificity $=80.0 \%$ and sensitivity $=63.6 \%$, by ${ }^{131} \mathrm{I}$ uptake at 2 hours $>71.6 \%$ $\left(\mathrm{AUC}=78.0 \%\right.$ ) with specificity $=80.0 \%$ and sensitivity $=61.9 \%$ and by ${ }^{131} \mathrm{I}$ uptake at 4 hours $>84.8 \%(\mathrm{AUC}=74.8 \%)$ with specificity $=80.0 \%$ and sensitivity $=55.0 \%$.

Conclusion: Higher TRABs, lower radiodine doses and higher ${ }^{131}$ I uptake were associated with RAI failure. Elevated iodine plasma clearance in Graves' disease and fast cellular turnover, with the consequent reduction of iodine retention in thyroid gland, may explain this fact. 


\section{P121}

PREDICTION OF POSITIVE SERUM

THYROGLOBULIN ANTIBODY BY 18F-FDG PET/

CT IN THE RESIDUAL THYROID GLAND AFTER UNILATERAL THYROID LOBECTOMY

Jeong Won Lee ${ }^{1}$, Arthur Cho ${ }^{1}$, Mijin Yun ${ }^{1}$, Jong Doo Lee

${ }^{1}$ Department of Nuclear Medicine, Yonsei University College of

Medicine

Purpose: In this study, we investigated the clinical role of ${ }^{18} \mathrm{~F}$-fluorodeoxyglucose $\left({ }^{18} \mathrm{~F}\right.$-FDG) positron emission tomography/computed tomography (PET/CT) in predicting the positive serum thyroglobulin antibody $(\mathrm{Tg}-\mathrm{Ab})$ using ${ }^{18} \mathrm{~F}$-FDG uptake in the residual thyroid lobe in differentiated thyroid cancer (DTC) patients who underwent unilateral thyroid lobectomy.

Methods: A total of 113 DTC patients who underwent ${ }^{18} \mathrm{~F}-\mathrm{FDG}$ PET/CT scan and serum $\mathrm{Tg}-\mathrm{Ab}$ level measurement after unilateral thyroid lobectomy were enrolled. Serum Tg-Ab levels were periodically checked during followup after PET/CT (mean follow-up duration, 18.0 7.8 months). The maximum and mean standardized uptake value (SUVmax and SUVmean), thyroid-toliver uptake ratio (TLR), and Hounsfield unit (HU) of the residual thyroid lobe were measured.

Results: Of the 113 patients, 59 patients $(52.2 \%)$ had thyroiditis on the resected thyroid lobe. The number of patients with positive Tg-Ab was 65 $(57.5 \%)$ at the time of PET/CT and $57(50.4 \%)$ at the last follow-up. The patients with thyroiditis on the resected thyroid lobe showed greater SUVmax, SUVmean, and TLR, but lower HU values than those without thyroiditis $(\mathrm{P}<$ $0.05)$. Further, there were significant differences in SUVmax, SUVmean, TLR, and HU between the patients with positive $\mathrm{Tg}-\mathrm{Ab}$ and negative $\mathrm{Tg}-\mathrm{Ab}$ at the time of PET/CT $(\mathrm{P}<0.05)$, and serum Tg-Ab levels significantly correlated with TLR in patients with positive $\mathrm{Tg}-\mathrm{Ab}(\mathrm{r}=0.332, \mathrm{p}=0.007)$. The positivity of serum $\mathrm{Tg}-\mathrm{Ab}$ at the last follow-up could be predicted by SUVmax $(83.8 \%)$, SUVmean $(81.6 \%)$, and TLR $(88.4 \%)$ with high positive predictive value.

Conclusion: Using ${ }^{18} \mathrm{~F}$-FDG uptake in the residual thyroid lobe, positive serum $\mathrm{Tg}-\mathrm{Ab}$ can be predicted with a high positive predictive value. ${ }^{18} \mathrm{~F}-\mathrm{FDG}$ $\mathrm{PET} / \mathrm{CT}$ could provide effective information for the prediction of the positivity of serum $\mathrm{Tg}-\mathrm{Ab}$ after unilateral thyroid lobectomy in DTC patients.

\section{P013 Graves' Orbitopathy \& Autoimmunity}

\section{P122 \\ SERUM LEVELS OF THE IMMUNE REGULATORY MOLECULE GALECTIN-9 ARE INCREASED IN PATIENTS WITH GRAVES' DISEASE HYPERTHYROIDISM \\ Ana Serrano ${ }^{1}$, Ana Maria Ramos Levi', \\ Miguel A. Sampedro-Núñez', Ivan Sánchez ${ }^{1}$, Alicia Vicuña ${ }^{1}$, \\ Ana Rodriguez-Muñoz ${ }^{1}$, Rebeca Martinez ${ }^{1}$, Hortensia de la Fuente ${ }^{1}$, Roberto Gonzalez-Amaro ${ }^{2}$, Monica Marazuela ${ }^{1}$ \\ ${ }^{1}$ Hospital Universitario Princesa. Instituto de Investigación Princesa, \\ ${ }^{2}$ Universidad San Luis Potosi}

Background: Patients with autoimmune thyroid disease (AITD) exhibit aberrant immune-regulatory mechanisms. Galectins (Gal) are a family of glycan-binding proteins which may be involved in immune regulation. However, the association with concurrent biochemical thyroid status remains unknown. In this study we evaluated serum Gal-1 and Gal-9 in AITD patients.

Materials and Methods: Peripheral blood samples from 22 patients with Graves' disease (GD) (11 with untreated hyperthyroidism, 6 euthyroid with treatment and 5 with iatrogenic hypothyroidism), 21 Hashimoto's thyroiditis (HT) (16 hypothyroid and 5 euthyroid with treatment), 12 non-GD hyperthyroid patients (NG) and 24 healthy controls were studied. Serum thyroid hormone and antibody levels were measured on the same day as serum Gal-1 and Gal-9. FT4 was quantified by RIA, TSH, Tg-Ab and TPO-Ab by immunora- diometric assays, and TSHR-Ab, Gal-1 and Gal-9 by ELISA. Patients were grouped according to clinical diagnosis and thyroidal status.

Results: Serum Gal-1 (ng/mL) and Gal-9 $(\mathrm{pg} / \mathrm{mL})$ were lower in controls (1.425 and 7.699, respectively), compared to GD $(4.614,9.188)$, HT (3.566, 8.770) and NG $(3.688,9.375)(\mathrm{P}<0.05)$. However, there were no significant differences in Gal levels between these three groups. When considering biochemical status in AITD patients, Gal-9, but not Gal-1, was higher in hyperthyroid cases; in fact, Gal-9 correlated positively with FT4 and negatively with TSH $(r=0.383, p=0.010$ and $r=-0.365, p=0.009$, respectively). Also, higher levels of Gal-9 were observed in hyperthyroid GD compared to NG (10.113 vs $8.885, p=0.034)$, even though FT4 levels were not significantly different between both groups. No significant association was found with thyroid antibodies or GO. Hyperthyroid GD patients who became euthyroid with treatment showed a reduction of Gal levels.

Conclusions: Galectins may be involved in the severity and pathogenesis of autoimmune hyperthyroidism, and could be potentially used as a diagnostic and therapeutic marker.

\section{P123 \\ HASHIMOTO'S THYROIDITIS ASSOCIATED WITH TYPE 1 DIABETES PRESENTS WITH GENDER SPECIFIC CHARACTERISTICS AND MAY DEVELOP BEFORE, WITH OR AFTER THE ONSET OF HYPERGLYCEMIA}

Adele Latina ${ }^{1}$, Giulia Pezzino ${ }^{1}$, Maria Luisa Arpi ${ }^{1}$, Laura Sciacca ${ }^{1}$, Sebastiano Squatrito ${ }^{1}$, Damiano Gullo ${ }^{1}$

${ }^{1}$ Endocrine Unit, Garibaldi-Nesima Hospital, University of Catania Medical School

Background: Hashimoto's thyroiditis (HT) can be associated with type 1 diabetes mellitus (T1D). Most studies on the association of both diseases have been focused on the predisposing genetic background. Less is known on the timing of thyroiditis development. In a cohort of T1D patients of different age groups we evaluated the clinical and epidemiological characteristics of concurrent HT

Patients and Methods: In 115 T1D caucasic patients ( $\mathrm{F}=81, \mathrm{M}=34$; mean age $18.8 \mathrm{yrs}$, median $16 \mathrm{yrs}$ ) defined according to ADA criteria, in which HT was diagnosed on the basis of antithyroid antibodies, thyroid function and clinical evolution of HT was retrospectively assessed.

Results: HT was diagnosed before T1D in $12.3 \%$ of females and in $8.8 \%$ of males (mean 6.3 and 3.7 yrs, respectively). Contemporary diagnosis occurred in $22.2 \%$ of females and $44.1 \%$ of males and in $30.8 \%$ of them hypothyroidism was diagnosed, with no gender differences. HT followed T1D diagnosis in $65.4 \%$ in females and $47.1 \%$ in males. In 76 euthyroid cases with mean follow-up period of 6 years we found that most patients maintained normal thyroid function; however, $24.5 \%$ of females and $13.0 \%$ of males developed hypothyroidism.

Conclusions: In our series of patients at the time of T1D diagnosis about one-tenth of cases presented with previous diagnosis of HT, a high number of patients HT was diagnosed concomitantly (F 22\%, M 44\%) and a third of them were diagnosed as hypothyroid, with no gender differences. Positivation of the antithyroid antibodies after T1D diagnosis was more frequent in females than in males. At follow-up most patients remained euthyroid but females were more prone than males to develop hypothyroidism. As a consequence, a serum TSH level should be obtained at regular intervals in patients with T1D and euthyroid HT.

\section{P124 \\ INTERNAL OPHTHALMOPLEGIA ASSOCIATED WITH GRAVES' HYPERTHYROIDISM}

\section{Ken Okamura' ${ }^{1}$, Hiroko Ibayashi², Kaori Sato', Megumi Fujikawa',}

Sachiko Bandai', Hiroshi Shibasaki ${ }^{3}$, Takanari Kitazono ${ }^{1}$

${ }^{1}$ Dept of Medicine and Clinical Science; Graduate School of Medicine; Kyushu University, ${ }^{2}$ lbayashi Clinic, ${ }^{3}$ Dept Neurology; Kyoto University Graduate School of Medicine

Introduction: Graves' disease is commonly associated with external ophthalmoplegia, but it may also be associated with internal ophthalmoplegia. 
Case: A 28-year-old Japanese female complained of difficulty in seeing near, palpitation, tremor and weight loss. Struma was palpable with elevated serum $\mathrm{fT}_{4}$, suppressed TSH level and positive TSH receptor antibody and antinuclear antibody. She was diagnosed as Graves' hyperthyroidism and treated with thionamide. She also complained of intermittent ocular pain and diplopia. Ophthalmologically, bilateral blepharoptosis and upward gaze palsy were observed. Bell phenomenon and doll's head eye phenomenon were preserved. Ocular fundi were normal. Edrophonium test and acetylcholine receptor antibody were negative. Slight exophthalmos (Rt $12 \mathrm{~mm}$, Lt $8 \mathrm{~mm}$ ) was noted, and brain MRI revealed slightly swollen bilateral inferior rectus muscle. Initially, pupils were isocoric with slightly slow response to light. When ocular pain was severe, visual acuity decreased from 1.5 to 0.15 (Rt) and from 1.5 to 0.4 (Lt). There was also marked accommodation disturbance with the near point being $20 \mathrm{~cm}$ (Rt \& $\mathrm{Lt}$ ), and the far point being $25 \mathrm{~cm}$ (Rt) and $30 \mathrm{~cm}(\mathrm{Lt})$, suggesting 'tonic ciliary muscle'. Steroid pulse therapy was ineffective, but pain was relieved after retrobulbar steroid injection. When the right eye was painful, the pupil was dilated and almost unresponsive to pen light but slightly responsive to outdoor sunlight. The pupil was unresponsive to $2 \%$ pilocarpine eye drop. Neurological examination revealed no other abnormalities. She has suffered from these symptoms for 5 years, but the attacks of fluctuating and alternating painful internal ophthalmoplegia improved gradually, although blepharoptosis and upward gaze palsy persisted.

Conclusion: Graves' disease in this patient was associated with internal ophthalmoplegia involving the ciliary muscles and pupillary sphincters in addition to blepharoptosis and upward gaze palsy.

\section{P125 \\ THYROGLOBULIN AUTOANTIBODIES SWITCH TO IGG1 AND IGG3 SUBCLASSES AFTER 131I TREATMENT FOR GRAVES' HYPERTHYROIDISM: AUTOANTIBODIES SUBCLASSES ARE RELATED TO THE ACTIVITY OF AUTOIMMUNE THYROID DISEASE}

Debora Ricci' ${ }^{1}$, Michele Marinò ${ }^{1}$, Lucia Montanelli' ${ }^{1}$, Paolo Piaggi ${ }^{1}$, Barbara Mazzi', Francesca Bianchi', Federica Brozzi', Pierina Santini', Emilio Fiore ${ }^{1}$, Massimo Tonacchera ${ }^{1}$, Paolo Vitti' ${ }^{1}$, Francesco Latrofa ${ }^{1}$

${ }^{1}$ Endocrinology Unit, University Hospital, University of Pisa

Thyroglobulin autoantibodies ( $\mathrm{TgAb}$ ) are considered non-pathogenic and their subclass distribution is debated. Radioidine $\left({ }^{131} \mathrm{I}\right)$ treatment for Graves' disease (GD) is one of the events inducing a rise in TgAb levels. Sera with positive TgAb (AIA-Pack, Tosoh Bioscience, Japan) were collected from 25 GD patients (18 females and 7 males), age $41.5(30.8-56.8) \mathrm{yr}$, at the time of ${ }^{131}$ I treatment and three and six months thereafter. To prevent the exacerbation of Graves' ophthalmopathy, all patients received oral prednisone. Total TgAb, $\mathrm{TgAb}$ light chains and $\mathrm{TgAb}$ subclasses were measured by ELISA at each time point. Data were analysed by the non-parametric Friedman test and, when the differences were significant, values at baseline were compared with those after three and six months in the post-hoc analysis.

Total $\mathrm{TgAb}$ IgG assessed by ELISA rose significantly $(\mathrm{p}=0.024): 1: 100$ $(1: 100-1: 330)$ before ${ }^{131} \mathrm{I}$ treatment, $1: 330(1: 100-1: 660)$ at three months $(\mathrm{p}=$ $0.10)$ and $1: 330(1: 330-1: 1000)$ at six months $(\mathrm{p}=0.048)$. TgAb $\mathrm{k}$ chains did not change $(\mathrm{p}=0.052)$, while $\mathrm{TgAb} 1$ chains increased significantly $(\mathrm{p}=$ $0.001)$, being $0(0-1: 100)$ before ${ }^{131} \mathrm{I}$ treatment, $0(0-1: 330)$ at three months $(\mathrm{p}=0.03)$ and $1: 100(0-1: 330)$ at six months $(\mathrm{p}=0.002)$. A significant rise in $\mathrm{IgG1}$ and $\mathrm{IgG} 3$ levels was observed after ${ }^{131} \mathrm{I}(\mathrm{p}=0.008$ and 0.006 , respectively), while IgG2 and IgG4 levels did not change. The increase in IgG1 levels was persistent: $0(0-1: 100)$ before ${ }^{131} \mathrm{I}$ treatment, $1: 100(0-1: 100)$ at three months $(\mathrm{p}=0.028)$ and $1: 100(0-1: 100)$ at six months $(\mathrm{p}=0.024)$. At variance, the increase in TgAb IgG3 was transient: $0(0-1: 100)$ before ${ }^{131} \mathrm{I}$ treatment, $1: 100(0-1: 330)$ at three months $(\mathrm{p}=0.028)$ and $0(0-1: 100)$ at six months $(\mathrm{p}=0.72)$.

In conclusion, ${ }^{131} \mathrm{I}$ treatment for GD increases the levels of the complement-activating $\operatorname{IgG1}$ and $\mathrm{IgG} 3$ subclasses. In autoimmune thyroid disease the rise in $\mathrm{TgAb}$ levels is associated with the switch in subclass distribution.

\section{P126 \\ POSSIBLE ROLE OF PHYSIOLOGICAL AND PATHOLOGICAL THYROTROPIN RECEPTOR (TSHR) ACTIVATION IN SKIN AND ITS DISEASES}

Fiona Grennan-Jones ${ }^{1}$, Ibtisam Elmansori ${ }^{2}$, Mohd Shazli Draman ${ }^{1}$, Lei Zhang ${ }^{1}$, Fiona Ruge ${ }^{3}$, Tammy Easter ${ }^{2}$, Aled Rees ${ }^{1}$, Aziz Elgadi', Claude Marcus ${ }^{4}$, Marian Ludgate ${ }^{1}$, Rebecca Porter ${ }^{2}$

${ }^{1}$ Institute of Molecular \& Experimental Medicine; School of Medicine; Cardiff University, ${ }^{2}$ Department of Dermatology \& Wound Healing; School of Medicine; Cardiff University, ${ }^{3}$ Cardiff-Peking Cancer Institute; School of Medicine; Cardiff University, ${ }^{4}$ Department of Clinical Science, Intervention \& Technology; Karolinska Institutet; Karolinska University

Hospital Huddinge

Objectives: Several skin diseases, including pretibial myxedema, alopecia areata, and vitiligo, are associated with hyper- and hypo-thyroidism. Apart from variations in thyroid hormone, increased signalling through the TSH receptor (TSHR), by thyroid stimulating antibodies or elevated TSH will affect any tissue expressing a functional TSHR. We aimed to determine whether functional TSHR is expressed in the skin and clarify its location. We also investigated whether physiological activation of extra-thyroidal TSHR might be via locally produced thyrostimulin.

Methods: RT-PCR and QPCR using primers specific for full-length TSHR, the major TSHR isoform (exons 1-8, TSHRV) or the subunits of thyrostimulin on mouse and human tissues and cell lines, including mouse skins obtained at different growth (anagen) and resting (telogen) stages of the hair cycle. Immunohistochemistry with TSHR antibodies (for ectodomain and membrane-spanning region) applied to frozen human thyroid and skin. Skin histology of adipose-specific TSHR KO mice was examined.

Results: Full-length but not variant TSHR transcripts and both thyrostimulin subunits were detected in whole human skin. TSHR immunoreactivity was detected in isolated fibroblast-like cells in the dermis, especially around sebaceous and sweat glands using the ectodomain antibody. TSHR and TSHRV transcripts were demonstrated in whole mouse skin, were at the limit of detection in telogen but increased during anagen. Expression of TSHR and TSHRV also increased during 3T3L1 adipogenesis. Skin morphology of adipose-specific TSHR KO mice appeared normal.

Conclusions: Our results indicate that TSHR and Thyrostimulin subunits are co-expressed in few tissues including whole human skin. The up-regulation in TSHR transcripts observed during the hair cycle may be due to expansion of the subcutaneous adipose layer required to accommodate anagen hair follicles. Further work is needed to investigate effects of thyrostimulin on skin but our results provide some rationale for the frequent association of skin/hair disorders with thyroid dysfunction involving TSHR activation.

\section{P127 \\ INACTIVATION OF ACTIVE MODERATE-SEVERE GRAVES' ORBITOPATHY (GO) AFTER SMOKING CESSATION: A CASE REPORT}

Danila Covelli', Guia Vannucchi², Irene Campi ${ }^{2}$, Nicola Currò ${ }^{3}$,

Mario Salvi

${ }^{1} 1$ graves' Orbitopathy Unit, Endocrinology, Fondazione Ca'

Granda Irccs, University of Milan, Italy, ${ }^{2} 1$ graves' Orbitopathy Unit,

Endocrinology, Fondazione Ca' Granda Irccs, University of Milan, Italy,

${ }^{3}$ 2ophthalmology, Fondazione Ca' Granda Irccs, University of Milan, Italy

GO is a debilitating complication occurring in approximately half of Graves'disease (GD) patients. Typically it presents with an initial phase of progressive exacerbation followed by spontaneous regression in two thirds of cases and by deterioration in $15 \%$ of patients. Preventive measures, such as smoking cessation and prompt achievement of euthyroidism, may prevent progression of GO to more severe forms. We report on a patient whose GO became inactive as a direct consequence of having quit smoking.

Case Report: A 42 year-old woman was referred to our centre in 2003 for worsening of GO. At that time she was an active cigarette smoker (20/ day) since she was thirteen. She was diagnosed of GD with GO in 2008 and treated with methimazole until 2011 when she underwent I-131 treatment with oral steroids prophylaxis. Thereafter she has been euthyroid on L-T4 therapy. 
In 2012 she developed active GO, initially treated with low dose oral steroids elsewhere (prednisone $50 \mathrm{mg}$ for 10 days, tapered down in 1 month) without clinical improvement. When we saw her in 2013 she has active moderatesevere GO with clinical activity score (CAS) of 5/7 (gaze evoked orbital pain, severe lid edema, conjunctival and lid redness in both eyes; chemosis in left eye) and NOSPSCES score 2c3b4a5060. Local treatment and smoking cessation were recommended, while the patient was screened for immunosuppressive treatment. Six weeks later she returned with significant reduction activity and severity signs (CAS 2/10; NOSPECS2a3b4a5060) after having stopped smoking, as suggested.

Definitive GO inactivation was assessed three months later, without further therapy (CAS 1/10; NOSPECS2a3a4a5060).

In this patient a significant improvement of inflammatory eye signs and symptoms was observed a few weeks after smoking cessation with subsequent progressive GO inactivation. Thus, the possibility that quitting smoking by itself may ameliorate active GO should not be underestimated.

\section{P128 \\ DEMOGRAPHIC CHARACTERISTICS OF THE PATIENTS WITH GRAVES' ORBITOPATHY IN SERBIA}

Mirjana Stojkovic ${ }^{1}$, Biljana Beleslin², Mihailo Dragicevic ${ }^{1}$, Slavica Savic ${ }^{1}$, Tijana Lalic ${ }^{1}$, Milos Zarkovic ${ }^{2}$, Jasmina Ciric ${ }^{2}$

${ }^{1}$ Clinic of Endocrinology, Diabetes and Metabolic Diseases, Clinical

Center of Serbia, ${ }^{2} \mathrm{Clinic}$ of Endocrinology, Diabetes and Metabolic

Diseases, Clinical Center of Serbia; Medical School University of

Belgrade

Aim: The aim of this study was to examine demographic characteristics of the patients with Graves' orbitopathy (GO).

Material and Methods: The study group included 88 patients with GO (67 female, 21 male) seen for the first time in our Clinic during the year of 2010. The last Serbian census was taken 2009. and collected data (age and sex structure, employment and qualification) were available in public at the begining of 2011. Data were reported by Statistical Office of the Republic of Serbia.

Results: GO was distributed by age in an expected manner (mean 50.5 years; range $16-76), 69.31 \%$ of patients were $40-59$ years old. The mean value of GO duration was 9.77 months (range $1-48$ ). In 51 patients GO was active and in 37 patients inactive. There were 23 patients with mild and 65 patients with moderately severe GO. Distribution of education levels in severe vs mild GO was as follows: primary school 7.9 vs $4.3 \%$; secondary school 74.6 vs $87 \%$; higher education 20.6 vs $8.7 \%$. Distribution of higher education was similar to those in general population: primary school $20.76 \%$, secondary school $48.93 \%$, higher education $16.24 \%$. There was no signifficant correlation between the education level and severity of the disease. The most frequent occupations in GO patients were: housewife $20.45 \%$, retiree $25 \%$, employee $20.45 \%$, toiler $9.09 \%$, nurse $4.45 \%$, unemployed $3.4 \%$. The majority of patients were married $(86 \%)$ and had children $(84 \%)$, but marital status was not correlated with the severity of GO. About $60 \%$ of patients were smokers, and they tend to have a more severe form of GO. According to the Ministry of Health report $33.4 \%$ of Serbian are smokers.

Conclusion: There was no significant correlation of the GO severity and education level, occupation and marrital status in the group of Serbian population.

\section{P129 \\ COMPARISON OF HOLTER ANALYSIS DATA BETWEEN HYPERTHYROID PATIENTS WITH GRAVES' DISEASE VS. TOXIC NODULAR GOITER}

Elif Turan ${ }^{1}$, Ilknur Can ${ }^{2}$, Yasar Turan ${ }^{3}$, Mehmet Uyar ${ }^{4}$, Mehtap Cakir ${ }^{1}$ ${ }^{1}$ Necmettin Erbakan University, Meram School of Medicine, Division of Endocrinology and Metabolism, ${ }^{2} \mathrm{Necmettin}$ Erbakan University, Meram School of Medicine, Department of Cardiology, ${ }^{3}$ Konya Meram Research Hospital, Department of Cardiology, ${ }^{4} \mathrm{Necmettin}$ Erbakan University, Meram School of Medicine, Department of Public Health

Objective: Most common causes of hyperthyroidism are Graves' disease and toxic nodular goiter. Previous studies have demonstrated the relationship between hyperthyroidism and increased risk of cardiac arrhythmias. The aim of our study was to demonstrate if mechanism of hyperthyroidism thus autoimmunity has an impact on the type of cardiac arrythmias accompanying hyperthyroidism.

Methods: 16 patients with Graves' disease and 20 patients with toxic nodular goiter with overt hyperthyroidism were included in our study. Age, sex, thyroid hormone levels, thyroid autoantibody positivity, thyroid ultrasonography and scintigraphy results were recorded. 24-hour Holter ECG monitoring was performed in all patients.

Results: The results of the study are presented in Table 1. In 24-hour Holter ECG recordings; there were no significant differences in terms of maximum and minimum heart rates, number of premature ventricular and supraventricular contractions and supraventricular tachycardia rates. Nonsustained ventricular tachycardia was noted only in the Graves' disease group ( $18 \%$ vs. $0 \%, p=0.043$ ), while atrial fibrillation was noted only in the toxic nodular group ( $24 \%$ vs. $0 \%, \mathrm{p}=0.016)$.

Conclusions: Significantly higher atrial fibrillation rate noted in the toxic nodular goiter group may be due to older age of this group. On the other hand, significantly higher rate of nonsustained ventricular tachycardia noted in the Graves' disease group is a novel finding of our study and may be due to either significantly higher serum free T3 levels or autoimmune etiology.

\section{P130 \\ GRAVES' OPHTHALMOPATHY WITH OVERT AUTOIMMUNE HYPOTHYROIDISM - 4 CASE REPORTS}

Horea loan Ursu' ${ }^{1}$, Mariana Purice ${ }^{2}$, Dan Hortopan ${ }^{2}$, Anda Dumitrascu², Daniela Alexandrescu², Monica Livia Gheorghiu ${ }^{1}$

1"C.I.Parhon" National Institute of Endocrinology, Bucharest; "C. Davila" University of Medicine and Pharmacy, Bucharest, 2"C.I.Parhon"

National Institute of Endocrinology, Bucharest

In a group of 166 patients with Hashimoto thyroiditis with overt hypothyroidism, Graves' ophthalmopathy was diagnosed in 4 patients $(2.4 \%)$. Age range was 36 - 56 yrs, mean age was 46.2 yrs and sex ratio (female to male) was 3:1. Risk factors for the occurrence of hypothyroid Graves' ophthalmopathy are thyroid dysfunction (hypothyroidism), age (older patients), cigarette smoking and increased thyrotropin receptor antibodies (TRAb). Before the onset of Graves' ophthalmopathy, overt hypothyroidism was untreated or discontinuously treated in all cases. One of the female patients was in the $6^{\text {th }}$ decade. Current smokers were 3 patients and a patient was nonsmoker. TRAb

Table 1. (for Abstract P129)

\begin{tabular}{llll}
\hline & Graves disease group & Toxic nodular goiter group & $\mathrm{P}$ value \\
\hline Age $($ mean $\pm \mathrm{SD})$ & $48.9 \pm 8.6$ & $62.9 \pm 11.5$ & $\mathrm{P}=0.001$ \\
Free T3 $($ mean $\pm \mathrm{SD})$ & $7.87 \pm 3.90$ & $5.21 \pm 1.53$ & $\mathrm{P}=0.033$ \\
Free T4 $($ mean $\pm \mathrm{SD})$ & $2.12 \pm 1.00$ & $1.65 \pm 0.68$ & $\mathrm{P}=0.263$ \\
$\mathrm{TSH}($ mean $\pm \mathrm{SD})$ & $0.040 \pm 0.017$ & $0.035 \pm 0.032$ & $\mathrm{P}=0.124$ \\
Atrial fibrillation $(\%)$ & $\% 0(\mathrm{n}=0 / 16)$ & $\% 30(\mathrm{n}=6 / 20)$ & $\mathrm{P}=0.016$ \\
$\begin{array}{l}\text { Nonsustained ventricular } \\
\text { tachycardia }(\%)\end{array}$ & $\% 18(\mathrm{n}=3 / 16)$ & $\% 0(\mathrm{n}=0 / 20)$ & $\mathrm{P}=0.043$ \\
\hline
\end{tabular}


were evaluated in 3 patients, being negative in one case $(<1 \mathrm{IU} / \mathrm{mL})$, slightly increased in the second $(1.67 \mathrm{IU} / \mathrm{mL})$ and moderately increased in the third patient $(5.5 \mathrm{IU} / \mathrm{mL}$; positive $>1.5)$. Orbital $\mathrm{CT}$ scan revealed both muscle enlargement and an increased volume of orbital fat in 3 patients and only an increased volume of orbital fat in a patient with mild Graves' ophthalmopathy. In 3 out of the 4 patients an asymmetric eye involvement was found: proptosis side difference of $2 \mathrm{~mm}$ in 2 patients and of $3 \mathrm{~mm}$ in one case. Mild Graves' ophthalmopathy was revealed in 2 patients (only levothyroxine therapy and smoking cessation being recommended) and moderate to severe Graves ophthalmopathy was found in 2 patients, being associated with permanent diplopia (treated with intravenous glucocorticoids in both cases, plus orbital radiotherapy in one case). Diplopia was refractory to treatment in one case and remitted in the other case. Two of the four patients developed severe autoimmune comorbidities (nephrotic syndrome and systemic sclerosis) a few years after the onset of Graves ophthalmopathy.

Conclusion: Graves' ophthalmopathy with overt autoimmune hypothyroidism is rare, but with variable severity. In our patients TRAb titers were only slightly increased and the proptosis asymmetry was frequent.

\section{P131 \\ INDIVIDUALS WITH LOW MANNOSE-BINDING LECTIN LEVELS MAY BE PROTECTED AGAINST GRAVES' DISEASE \\ Annamaria Gazdag ${ }^{1}$, Monika Katko', Domonkos M. Nagy ${ }^{1}$, Annamaria Erdei', Maria Papp ${ }^{2}$, Miklos Bodor ${ }^{1}$, Eszter Berta ${ }^{1}$, Bernadett Ujhelyi ${ }^{3}$, Endre V. Nagy ${ }^{1}$ \\ ${ }^{1}$ Division of Endocrinology, Department of Medicine, University of Debrecen, ${ }^{2}$ Division of Gastroenterology, Department of Medicine, University of Debrecen, ${ }^{3}$ Department of Ophthalmology, University of Debrecen}

Introduction: Mannose-binding lectin (MBL) is an important member of the lectin pathway of the complement system, and is part of the innate immune system. The connection between MBL levels and certain autoimmune diseases is well established. Thyroid hormones increase MBL synthesis in the hepatocytes. The aim of this study was to analyse MBL levels in patients with Graves' disease (GD) with and without Graves' orbitopathy (GO).

Methods: Serum MBL levels of 130 GD patients were measured by ELISA and compared to that of 116 age and gender matched healthy controls. TSH, fT4, fT3 and TSH-receptor antibody (TRAb) levels were also determined. Results were categorized in three groups: low $(<100 \mathrm{ng} / \mathrm{ml})$, intermediate $(100$ to $1000 \mathrm{ng} / \mathrm{ml})$ and high $(>1000 \mathrm{ng} / \mathrm{ml})$.

Results: 67 patients of the GD group suffered from active or inactive EOP. There weren't significant differences in the serum levels of MBL between the euthyreoid GD patients' and healthy control groups $(849$; 394-1571 vs. 827; 243-1753 ng $/ \mathrm{ml}$, respectively). Patients with and without endocrine orbitopathy $(996 ; 517-1834$ vs. $826 ; 422-1632 \mathrm{ng} / \mathrm{ml}$, ns) were also not different in this respect. MBL and fT3 levels showed significant correlation in all patients' groups. When patients were grouped according to their MBL levels, there were significantly less patients with low MBL level in the GD group $((2 / 64$; $3.1 \%)$ than in the healthy controls' group $(16 / 116 ; 13.8 \%, \mathrm{p}=0.035)$.

Conclusion: There is a strong correlation between MBL and fT3 levels. MBL deficiency was rarely found in GD patients, which may suggest a protecting role against thyroid autoimmunity.

This work was supported by the Hungarian Scientific Research Fund (Grant number OTKA K105733).

\section{P014 Heart and Thyroid}

\section{P132 \\ THE IMPACT OF SUBCLINICAL THYROID DYSFUNCTION ON CORONARY VASOSPASM IN PATIENTS WITHOUT ASSOCIATED CARDIOVASCULAR RISK FACTORS}

\section{Kyoung Im Cho ${ }^{1}$, Bo Hyun $\mathrm{Kim}^{2}$}

${ }^{1}$ Cardiovascular Research Institute, Department of Internal Medicine, Kosin University College of Medicine, ${ }^{2}$ Department of Internal

Medicine, School of Medicine, Pusan National University

Background and Objectives: Subclinical hypothyroidism is associated with endothelial dysfunction and impaired coronary flow reserve. However, the effect of subclinical thyroid dysfunction or thyroid autoimmunity on variant angina has not been cleared yet.

Materials and Methods: Among 414 consecutive patients, who underwent coronary angiography with ergonovine provocation test (EPT) without associated cardiovascular risk factors, 177 with positive EPT (EPT (+)) and 237 with negative EPT (EPT(-)) were studied. Subclinical hypothyroidism was defined as a thyroid-stimulating hormone (TSH) level $>4.0 \mathrm{mIU} / \mathrm{l}$ and a normal free thyroxine level $(0.89-1.76 \mathrm{ng} / \mathrm{dl})$; and subclinical hyperthyroidism was defined as a TSH level $<0.45 \mathrm{mIU} / 1$ and a normal free thyroxine level. We excluded the patients with the presence of fixed organic stenosis. The relationship between coronary artery spasm and the presence of subclinical thyroid dysfunction as well as serum thyroidperoxidase autoantibody (TPO Ab) was evaluated.

Results: The proportion of subclinical hypothyroidism in patients with EPT (+) was significantly higher than in the patients with EPT(-) $(17 \%$ vs. $10 \%, \mathrm{p}=0.023)$. However, there was no significant difference in the propotion of subclinical hyperthyroidism between the groups. Moreover, patients with EPT $(+)$ showed more positive TPO Ab $(31 \%$ vs. $13 \%, \mathrm{P}<0.0001)$ than those with EPT (-). There was a positive correlation between EPS $(+)$ and TPO positive $(r=0.226, P<0.001)$, subclinical hypothyroidism $(r=0.112, p=$ $0.033)$, and body mass index $(\mathrm{r}=0.123, \mathrm{p}=0.018)$. Multiple linear regression analysis revealed that the only significant predictors of EPT $(+)$ were body mass index(standardized $\beta$ coefficient $=0.108, p=0.024$ ) and the presence of TPO Ab (standardized $\beta$ coefficient $=0.205, \mathrm{P}<0.001$ ) after adjustment for traditional risk factors.

Conclusion: Subclinical hypothyroidism, not a subclinical hyperthyroidism, is associated with coronary spasm as established by ergonovine test. Presence of TPO Ab is significantly associated with increased coronary vasospam in patients without cardiovascular risk factors. 


\section{P133 \\ NONINVASIVE ECHOCARDIOGRAPHIC EVALUATION OF LEFT VENTRICULAR FUNCTION IN PATIENTS WITH HYPER- AND HYPOTHYROIDISM \\ Maria Dimova ${ }^{1}$, Branimir Kanazirev ${ }^{1}$ \\ ${ }^{1}$ Varna Medical University; St Mariana University Hospital}

Purpose: To evaluate thyroid hormone impact on cardiovascular system (CVS) we compared left ventricular function, systolic time intervals and systemic vascular resistance in patients with thyroid dysfunction and in healthy controls.

Material and Methods: We investigated 103 subjects: 39 patients with hyperthyroidism, 24 patients with hypothyroidism and 40 controls by echocardiography and systolic time intervals (STI). Heart rate (HR), pre-ejection period (PEP), corrected PEPc, ejection time (ET) and its corrected value (ETc) and $\mathrm{PEP} / \mathrm{ET}$ ratio were studied. End-diastolic, end-systolic dimensions (EDD, ESD), shortening fraction (SF), mean velocity of circumferential fiber shortening (MVCFS), left ventricular mass index (LVMi) and systemic vascular resistance (SVR) were examined.

Results: STI in hyperthyroidism were significant different: HR was increased, ET, ETc, PEP, PEPc were shorter and PEP/ET was decreased. CI was greater, MVCFS was increased and SF was higher, SVR was decreased and LVMi was greater. STI in hypothyroid patients were significant changed: PEP and PEP/ET were increased. CI was lower and SVR was higher, MVCFS was decreased and LVMi was greater, SF and ESD were changed too. Significant correlations were seen when plotting thyroxin hormone levels against MVCFS and also between values of MVCFS and SVR.

Conclusion: The mechanism of hyper-dynamic high-output state of the CVS in hyperthyroidism is characterized by a significant increase in CI secondary to increased HR, unchanged stroke volume at the expense of significantly reduced SVR achieved by high speed ejection with shortened STI and shorter and quicker diastolic filling. The mechanisms of hypo-dynamic lowoutput state of the CVS in hypothyroidism are characterized by a significant reduction in $\mathrm{CI}$, unchanged $\mathrm{HR}$ at the expense of reduced stroke volume and EF with a significant increase in STI and SVR.

\section{P134}

\section{MASKED HYPERTENSION IN A COHORT OF} NEWLY DIAGNOSED HYPOTHYROID PATIENTS

Daniela Gallo ${ }^{1}$, Eliana Piantanida ${ }^{1}$, Nadia Pariani $^{1}$, Giovanni Veronesi ${ }^{1}$,

Adriana Lai ${ }^{1}$, Lorenza Sassi ${ }^{1}$, Maria Laura Tanda ${ }^{1}$, Marco Ferrario',

Luigi Bartalena

${ }^{1}$ University of Insubria, Varese, Italy

Objectives: Masked hypertension $(\mathrm{MH})$ is characterized by the association of normal clinical blood pressure (BP) with high ambulatory or home BP. MH is associated with target-organ damage and increased cardiovascular morbidity and mortality, similar to true hypertension (TH). Few data are available about its prevalence in hypothyroidism. Aim of our study was to evaluate the prevalence of MH in clinical (c-HYPO) and subclinical (s-HYPO) hypothyroid patients.

Methods: Sixty-four consecutive patients were enrolled, 38 with newly diagnosed s-HYPO (43.7 $\pm 12.4 \mathrm{yr}, \mathrm{F} 94.7 \%)$ and 26 with c-HYPO $(47.6 \pm 12.6$ yr, F 84.6\%), without known hypertension, cardiac diseases or diabetes; 50 euthyroid controls (EU) matched for gender and age were included. Each patient underwent clinical BP measurement, 24-h ambulatory BP monitoring, and FT3, FT4, and TSH measurement. Following the 2013 ESH/ESC Guidelines, $\mathrm{MH}$ was diagnosed with clinical $\mathrm{BP}<140 / 90 \mathrm{mmHg}$ and 24-h BP $\geq 130$ (systolic) and/or 80 (diastolic) $\mathrm{mmHg}$.

Results: At clinical measurement, diastolic BP values were significantly higher in c-HYPO $(83.3 \pm 10.0 \mathrm{mmHg})$ and in $\mathrm{s}-\mathrm{HYPO}(80.4 \pm 8.8 \mathrm{mmHg})$ than in $\mathrm{EU}(75.3 \pm 8.5 \mathrm{mmHg}, \mathrm{p}=0.001)$. The results of 24-h ambulatory BP monitoring showed a significant higher prevalence of MH both in s-HYPO (26.3\%) and c-HYPO $(15.4 \%)$ than in EU $(10 \%, \mathrm{p}=0.05)$; moreover, TH had significantly higher prevalence in c-HYPO (11.5\%) and s-HYPO $(10.5 \%)$ than in $\mathrm{EU}(8 \%, \mathrm{p}=0.03)$. The odds ratio $(\mathrm{OR})$ for hypertension vs normotension confirmed a significantly increased risk to have MH in HYPO vs EU (3.29, 1.08-10.08; $\mathrm{p}=0.02$ ). Considering s-HYPO and c-HYPO distinctly, OR for
MH was significantly higher in s-HYPO $(4.00,1.21-13.28)$ than in c-HYPO (2.29, 0.54-9.73) compared with EU.

Conclusions: According to our data, $\mathrm{MH}$ seems to be more frequent in hypothyroid patients than in euthyroid subjects.

\section{P135}

\section{SERUM CONCENTRATION OF CYTOKINES (IL- 6, IL-8, IL-10 AND TNF-ALPHA) IN PATIENTS SUFFERING FROM GRAVES'S DISEASE AND HEART FAILURE}

Arina Frolova ${ }^{0}$, Tatiana Rodionova

${ }^{1}$ Saratov State Medical University N.A. V.I. Razumovsky

Objectives: to study concentration of IL-6, IL-8, IL-10, TNF-alpha in blood serum of patients with Graves's disease (GD) complicated by heart failure (HF).

Methods: 32 patients (18 to 50 years old) with GD and having HF were studied. Concentrations of IL-6, IL-8 and IL-10 and TNF-alpha were studied by enzyme-linked immunosorbent assay. Patients suffered from the I class HF (NYHA Classification) were excluded from the group under study as well. The comparison group consisted of 30 people who did not suffer from thyroid function abnormality and were comparable in age and sex.

Results: 32 patients under study (18 women, 14 men). The age median is 41.0 [28.0; 50.0]. Class II HF was found in 21 patients $(65.6 \%)$, class III - in 9 patients $(28.15 \%)$, class IV - in 2 patients $(6.25 \%)$. TSH receptor autoantibodies (TRAbs) level median in the group under study was $15.7[8.2 ; 36.7]$ $\mathrm{u} / 1$ which is significantly higher than the same index in the control group $\mathrm{Me}=0.4[0.01 ; 1.1] \mathrm{u} / 1(\mathrm{P}>0.05)$. Cytokine serum concentration median in patients with GD complicated by HF was: IL-6 - $14.3[8.6 ; 18.1] \mathrm{pcg} / \mathrm{ml}$, IL-8 - $138.9[98.2 ; 150.3] \mathrm{pcg} / \mathrm{ml}$ and IL-10- $49.2[29.8 ; 77.8] \mathrm{pcg} / \mathrm{ml}$, TNF-alpha $7.2[3.3 ; 25.6] \mathrm{pcg} / \mathrm{ml}$. The mentioned indices in the control group were as follows: IL-6 - $4.4[2.1 ; 6.6] \mathrm{pcg} / \mathrm{ml}, \mathrm{IL}-8$ - $9.2[4.7 ; 9.5] \mathrm{pcg} / \mathrm{ml}$ and IL-10- 9.2 $[3.7 ; 12.5] \mathrm{pcg} / \mathrm{ml}$, TNF-alpha $-4.7[2.0 ; 6.1] \mathrm{pcg} / \mathrm{ml}$. Cytokine serum concentration median of IL-6, IL-8, IL-10 and TNF-alpha was higher in patients with GD complicated by HF $(\mathrm{P}>0.05)$.

Conclusion: TRAbs level in patients with GD having II-IV class of HF as a complication is significantly higher than that of the controlled group. Also, cytokine concentration in such patients increases alongside with proinflammatory (IL-6, IL-8 and TNF-alpha) and anti-inflammatory (IL-10) fractions.

\section{P136 \\ B1-ADRENORECEPTOR GENE POLYMORPHISM GLY389ARG AND CARDIOVASCULAR PARAMETERS IN PATIENTS WITH THYREOTOXICOSIS}

\section{Alina Babenko $^{0}$, Elena Grineva1, Dariya Savitskaya",}

Vladislav Solncev ${ }^{1}$, Anna Kostareva ${ }^{1}$

${ }^{1}$ Federal State Budget Institution "Federal Medical Research Center Named after V.A. Almazov"

Heart $\beta_{1}$-adrenoreceptors $(\beta 1-\mathrm{AR})$ performs a crucial role in mediating the cardiostimulant effects of norepinephrine and Gly389Arg polymorphism $\beta 1-\mathrm{AR}$ can influence on the cardiovascular parameters. Now the significance of Gly389Arg polymorphism in the human heart in patients with thyrotoxicosis is not known. We've studied the Gly389Arg polymorphisms of $\beta 1-\mathrm{AR}$ gene in relation to the Echo-cardiography parameters in 165 normotensive patients with thyrotoxicosis (TT) without any CVD before and during the thyreostatic treatment. There was no difference in parameters that may affect the development of cardiovascular complications (age, level of thyroid hormones (TH)) between the groups. In first study the EchoCG parameters had no significant difference in groups with different genotype. In second study after a year of thyreostatic therapy no significant difference in clinical parameters and $\mathrm{TH}$ was revealed. In EchoCG parameters there was multidirectional dynamics of LVMI in carriers of various genotypes. The difference between two assessments for genotype GG was $-35.5(-60 ;-11)$, for genotype GC $--1(-11$; $10.6)$ and for genotype $\mathrm{CC}-0(-16 ; 4)(\mathrm{p}=0.03)$. Left ventricular hypertrophy $(\mathrm{LVH})$ was detected in $11 \%$ of people in group 1 (GG), in group 2 (CG) - in $26.5 \%$ and in group $3(\mathrm{CC})-$ in $22.6 \%$. The frequency of various types of LV geometry has been analyzed. It was noted that in the carriers of GG geno- 
type concentric $\mathrm{LVH}(\mathrm{CH})$ is not met. After one year in group of patients with GG genotype LVM normalized. In other groups percent of patients with LVH and distribution of various types of geometry has changed slightly. Nobody of GG genotype carriers had atrial fibrillation (AF), heterozygous patients had $\mathrm{AF}$ in $19 \%$, homozygous CC carriers $-9.7 \%$. Thereby, we detected tendency for lower frequency of $\mathrm{LVH}$ and no presence of $\mathrm{CH}$ and $\mathrm{AF}$ in GG genotype carriers.

\section{P137 \\ GRAVES' DISEASE AND CARDIOVASCULAR RISK FACTORS \\ Celestino Neves ${ }^{1}$, César Esteves ${ }^{1}$, Helena Greenfield ${ }^{1}$, Miguel Pereira', Oksana Sokhatska², Carmo Palmares ${ }^{2}$ \\ Davide Carvalho', Luís Delgado², José Luís Medina ${ }^{1}$ \\ ${ }^{1}$ Endocrinology Service, São João Hospital, Faculty of Medicine, University of Porto, Portugal, ${ }^{2}$ Immunology Department, São João Hospital, Faculty of Medicine, University of Porto, Portugal}

Background: Graves' disease (GD) has important health implications including increased risk of metabolic abnormalities and cardiovascular disorders.

Objectives: To evaluate the interrelationships between thyroid function in GD and cardiovascular risk factors.

Methods: We evaluated 104 subjects (94\% female) with GD, 49 with hyperthyroidism $[\mathrm{TSH}=0.01(0.00-0.13) \mathrm{UI} / \mathrm{mL}]$ and 55 with euthyroidism $[\mathrm{TSH}=1.34(0.75-1.78) \mathrm{UI} / \mathrm{mL}]$

We recorded the levels of TSH, FT3, FT4, TRAb, anti-TPO and antiTg, total cholesterol (total-C), LDL-Cholesterol (LDL-C), HDL-Cholesterol (HDL-C), triglycerides (TG), apolipoprotein B (apoB), apolipoprotein A1 (apoA1), lipoprotein(a) [Lp(a)], CRP, homocysteine, folate and vitamin B12. We determined the HOMA-IR, HISI (Hepatic Insulin Sensitivity Index) and WBISI (Whole-Body Insulin Sensitivity Index). Statistical analysis was performed using the Mann-Whitney test, the logistic regression, and the Spearman's correlation test. Results were expressed as median and percentiles (25-75). A two-tailed $\mathrm{P}<0.05$ was considered statistically significant.

Results: No differences were found between the groups with respect to age [44(32-54) vs 41(33-53)years] and BMI [24.75(22.45-28.92) vs $24.82(21.48-28.58) \mathrm{kg} / \mathrm{m}^{2}$.]. Significantly higher levels of TRAb were found in the hyperthyroid patients. Levels of folate [5.1(3.6-6.5) vs 6.9(5.1-9.4) $\mathrm{ng} / \mathrm{mL} ; \mathrm{P}<0.001]$ and WBISI [4.39(2.49-6.15) vs 5.50(4.08-7.79); $\mathrm{p}=$ $0.015]$ were significantly lower in the hyperthyroid group. Levels of CRP were significantly higher in the hyperthyroid group [0.265(0.090-0.760) vs $0.190(0.080-0.335) \mathrm{mg} / \mathrm{dL} ; \mathrm{p}=0.076]$. TSH levels were positively correlated with folate $(\mathrm{r}=0.240, \mathrm{p}=0.021)$, HISI $(\mathrm{r}=0.217, \mathrm{p}=0.046)$ and WBISI $(\mathrm{r}=0.356, \mathrm{p}=0.001)$ and negatively correlated with TRAb $(\mathrm{r}=-0.461, \mathrm{P}<$ $0.001)$ and HOMA-IR $(r=-0.218, p=0.045)$. The levels of FT3 and FT4 were positively correlated with HOMA-IR $(r=0.284, p=0.008$ and $r=0.261, p=$ 0.016 respectively) and negatively correlated with HISI $(\mathrm{r}=-0.283, \mathrm{p}=0.009$ and $\mathrm{r}=-0.261, \mathrm{p}=0.016$ respectively) and WBISI $(\mathrm{r}=-0.233, \mathrm{p}=0.032$ and $r=-0.260, p=0.016$ respectively). Negative correlations were found between FT4 levels and HDL-C $(r=-0.198, p=0.046)$.

Conclusions: The interrelationships between thyroid function, TRAb, insulin resistance, folate and CRP indicate an increased cardiovascular risk in hyperthyroidism due to GD.

\section{P138 \\ THE OCCURRENCE RATE FOR CARDIOVASCULAR DISEASES IN THE CASES OF TYPE 2 DIABETES MELLITUS AND THYROID DEFICIENCY \\ Armine Khroyan}

Yerevan State Medical University, Endocrinology, Armenia

Objectives of the current investigation is to establish in which group cardiovascular diseases are more frequent: in the first group, consisting of patients with type 2 diabetes mellitus, taking L-thyroxine, or in the second patients, who do not take it

Methods: The investigations have been carried out in "Muratsan" University Hospital 2009-2014. 180 patients with type 2 diabetesmellitus and thyroid hypofunction have been taken under investigation, being divided
Table 1. (for Abstract P138)

\begin{tabular}{|c|c|c|}
\hline & I group & II group \\
\hline Glucose & $\begin{array}{l}80 \% \text { - up to } 7 \mathrm{mmol} / \mathrm{l} \\
20 \% \text { - above } 7 \mathrm{mmol} / \mathrm{l}\end{array}$ & $\begin{array}{l}64 \% \text { - up to } 7 \mathrm{mmol} / \mathrm{l} \\
36 \% \text { - above } 7 \mathrm{mmol} / 1\end{array}$ \\
\hline $\mathrm{UbA}_{1} \mathrm{C}$ & $\begin{array}{l}95 \% \text { - up to } 6.5 \% \\
5 \% \text { - above } 6.5 \%\end{array}$ & $\begin{array}{l}72 \%-\text { up to } 6.5 \% \\
28 \%-\text { above } 6.5 \%\end{array}$ \\
\hline ECG & $\begin{array}{l}82 \%-\text { norm } \\
18 \% \text { - left ventricle hypertrophy }\end{array}$ & $\begin{array}{l}70 \%-\text { norm } \\
30 \%-1 \text { vh }\end{array}$ \\
\hline Echocardiography & $\begin{array}{l}91 \%-\text { norm } \\
9 \%-\text { FE below }\end{array}$ & $\begin{array}{l}83 \%-\text { norm } \\
17 \% \text { FE below }\end{array}$ \\
\hline Lipid profile & $\begin{array}{l}75 \% \text { - norm } \\
25 \% \text { - dyslipidemia }\end{array}$ & $\begin{array}{l}63 \% \text { - norm } \\
37 \% \text { - dyslipidemia }\end{array}$ \\
\hline
\end{tabular}

into 2 groups. The first group consisted of 84 patients, having primary hypothyroidism with TSH above $6 \mathrm{mlU} / \mathrm{ml}$ and FT4 below $11 \mathrm{pg} / \mathrm{ml}$, now take L-thyroxine. The second group consisted of 96 patients with subclinical hypothyroidism, whose TSH was above $5 \mathrm{mlU} / \mathrm{ml}$ and $\mathrm{FT}_{4}$ was $12-20 \mathrm{pg} / \mathrm{ml}$.

Results: We can state that cardiovascular diseases are more frequent among the patients with subclinicalhypothyroidism and diabetesmellitus.

In case of any thyroid hypofunction, it is required to prescribe L-thyroxine or iodinesupplement

The timely prescription of L-thyroxine prevents insulinresistance.

Prescription of L-thyroxine in combination with anti-diabetic medication not only leads to a better compensation of diabetes mellitus, but also prevent further complications -particularly cardiovascular complications, i.e. myocardial infarction.

\section{P139 \\ AUTOIMMUNE THYROIDITIS AND CARDIOVASCULAR RISK FACTORS \\ Helena Greenfield ${ }^{1}$, Celestino Neves ${ }^{1}$, César Esteves ${ }^{1}$, Miguel Pereira ${ }^{1}$, Oksana Sokhatska ${ }^{2}$, Carmo Palmares ${ }^{2}$, \\ Davide Carvalho ${ }^{1}$, Luís Delgado², José Luís Medina ${ }^{1}$ \\ ${ }^{1}$ Endocrinology Service, São João Hospital, Faculty of Medicine, University of Porto, Portugal, ${ }^{2}$ mmmunology Department, São João Hospital, Faculty of Medicine, University of Porto, Portugal}

Objectives: To evaluate the relationship between thyroid function and cardiovascular risk factors in patients with autoimmune thyroiditis.

Methods: We analysed thyroid function, antithyroid antibodies, insulin resistance markers comprising the HOMA-IR, HISI (Hepatic Insulin Sensitivity Index), WBISI (Whole-Body Insulin Sensitivity Index) and the levels of total cholesterol, HDL, LDL-cholesterol, triglycerides (TG), apolipoprotein B (ApoB), ApoA1, lipoprotein(a) (Lp[a]), homocysteine, CRP (C-reactive protein), folic acid and vitamin B12 levels, in 255 patients with autoimmune thyroiditis. We defined two groups of patients; in group $1(\mathrm{n}=$ 186) we included patients with values of TSH between 0.35 and $2.50 \mu \mathrm{UI} / \mathrm{ml}$, FT4 between 0.70 and $1.48 \mathrm{ng} / \mathrm{dl}$, and FT3 between 1.71 and $3.71 \mathrm{pg} / \mathrm{ml}$. In group $2(\mathrm{n}=69)$ we included patients with TSH values higher than $2.50 \mu \mathrm{UI} /$ $\mathrm{ml}$, FT4 between 0.70 and $1.48 \mathrm{ng} / \mathrm{dl}$, and FT3 between 1.71 and $3.71 \mathrm{pg} / \mathrm{ml}$. For the statistical analysis we used the Mann-Whitney test, logistic regression and Spearman correlations. The results were adjusted for age and body mass index and are expressed in median \pm percentile 25 and 75 . A bilateral value of $\mathrm{P}<0.05$ was considered statistically significant.

Results: $94 \%$ of individuals were female. Median age was significantly higher in group 1 [49(36-60) vs 42(30-58)years, $p=0.025]$. The individuals with higher total cholesterol $(\mathrm{OR}=1.008 ; \mathrm{p}=0.03), \mathrm{CRP}(\mathrm{OR}=1.684 ; \mathrm{p}=$ $0.04)$ or anti-thyroglobuline antibodies $(\mathrm{OR}=1.002 ; \mathrm{p}=0.02)$ had an increased risk of having a TSH level higher than $2.50 \mu \mathrm{UI} / \mathrm{ml}$. In the whole group of patients there was a positive correlation between TSH and CRP $(r=0.132 ; \mathrm{p}=$ $0.043)$ and HOMA-IR $(r=0.158 ; p=0.029)$. TSH levels were negatively correlated with HISI $(r=-0.158 ; p=0.029)$ and WBISI $(r=-0.164 ; p=0.024)$. In the group with TSH higher than $2.50 \mu \mathrm{UI} / \mathrm{ml}$ there was a negative correlation between FT3 and homocysteine $(r=-0.357 ; \mathrm{p}=0.011)$ and between FT4 and anti-TPO antibodies $(\mathrm{r}=-0.281 ; \mathrm{p}=0.020)$. 
Conclusions: This study suggests that high TSH levels within the reference range may be associated with cardiovascular risk factors in patients with autoimmune thyroiditis.

\section{P015 Subclinical Thyroid Disease and Hypothyroidism}

\section{P140 \\ THE ASSOCIATION OF MATERNAL AND CHILD'S THYROID FUNCTION ON CHILD'S INTELLECT AND SCHOLASTIC PERFORMANCE \\ Fanni Päkkilä̈ ${ }^{1}$ Tuija Männistö ${ }^{2}$, Anneli Pouta ${ }^{3}$, Anna-Liisa Hartikainen ${ }^{4}$, Aimo Ruokonen², Heljä-Marja Surceß ${ }^{3}$, BSc Aini Bloigu ${ }^{3}$, \\ Marja Vääräsmäki ${ }^{4}$, Marjo-Riitta Järvelin ${ }^{5}$, Irma Moilanen ${ }^{6}$, \\ Eila Suvanto ${ }^{4}$ \\ ${ }^{1}$ Department of Obstetrics and Gynecology, University of Oulu and Oulu University Hospital; Institute of Health Sciences, University of Oulu; Department of Children, Young People and Families, National Institute for Health and Welfare, ${ }^{2}$ Department of Clinical Chemistry, University of Oulu and Oulu University Hospital, ${ }^{3}$ Department of Children, Young People and Families, National Institute for Health and Welfare, ${ }^{4}$ Department of Obstetrics and Gynecology, University of Oulu and Oulu University Hospital, ${ }^{5}$ Institute of Health Sciences, University of Oulu; Department of Children, Young People and Families, National Institute for Health and Welfare; Department of Epidemiology and Biostatistics, Mrc Health Protection Agency (Hpa) Centre for Environment and Health, School of Public Health, Imperial College London, ${ }^{6}$ Clinic of Child Psychiatry, University of Oulu and Oulu University Hospital}

Objectives: To study the effect of maternal thyroid dysfunction on child's neuropsychological development.

Methods: The Northern Finland Birth Cohort 1986 included all expected births within a year ( $\mathrm{N}=9362$ women, $\mathrm{N}=9479$ children) from the Northern Finland region. Maternal serum samples obtained at early pregnancy $(\mathrm{N}=$ $5791)$ and serum samples of children obtained at age $16(\mathrm{~N}=5904)$ were analyzed for thyrotropin (TSH), free thyroxine (fT4) and thyroid peroxidase antibodies (TPO-Abs). Child's school performance was evaluated by their main teacher at the age of 8 years and adolescents filled in self-evaluations at age 16. Data on possible intellectual deficiency (ID) were collected from multiple sources for all children. Logistic regression estimated child's odds of ID and poor school performance associated with exposure to maternal thyroid dysfunction and odds of poor school performance associated with child's own thyroid function at age 16. Results are presented as odds ratios (ORs) with $95 \%$ confidence intervals $(95 \% \mathrm{CI})$. Analyses were adjusted for maternal/family covariates and stratified by child's gender due to interaction.

Results: Odds of ever having repeated a class in school was higher among children exposed to maternal subclinical hypothyroidism (OR $2.14,95 \% \mathrm{CI}$ 1.01-4.53) or hypothyroxinemia (OR 3.49, 95\% CI 1.06-11.48). Children of hyperthyroid mothers had increased odds of poor performance in mathematics (OR 1.56, 95\% CI 1.03-2.38). Maternal thyroid dysfunction did not increase child's odds of having ID. At age 16, girls who were hyperthyroid had higher odds of having difficulties in Finnish (OR 2.82, 95\%CI 1.42-5.61) and boys with hypothyroxinemia at age 16 had higher odds of having difficulties in Finnish or mathematics (OR 2.13, 95\%CI 1.26-3.62)

Conclusions: Maternal thyroid dysfunction during early pregnancy had some effect on the overall scholastic performance of the child. Additionally, child's own thyroid dysfunction affected their self-evaluated school performance.

\section{P141 \\ ELDERLY PATIENTS WITH OVERT HYPOTHYROIDISM EXPRESS ONLY FEW SYMPTOMS, AND THYROID FUNCTIONS TEST SHOULD BE PERFORMED ON WIDE INDICATIONS. A POPULATION-BASED STUDY \\ Allan Carlé 1 , Inge Bülow Pedersen ${ }^{1}$, Nils Knudsen², Hans Perrild², Lars Ovesen ${ }^{3}$, Lone Banke Rasmussen ${ }^{4}$, Torben Jørgensen ${ }^{5}$, Peter Laurberg 6 \\ ${ }^{1}$ Department of Endocrinology, Aalborg University Hospital, Aalborg, Denmark, ${ }^{2}$ Endocrine Unit, Medical Clinic I, Bispebjerg Hospital, Copenhagen, Denmark, ${ }^{3}$ Department of Internal Medicine, Slagelse Hospital, Slagelse, Denmark, ${ }^{4}$ Department of Nutrition, National Food Institute, Technical University of Denmark, Søborg, Denmark, ${ }^{5}$ Research Centre for Disease Prevention and Health, Copenhagen County, Denmark, ${ }^{6}$ Aalborg University Hospital}

Background: Thyroid function testing is normally performed when patients present symptoms suspicious for thyroid failure. We studied the predictive power of hypothyroidism in a population of old and young hypothyroid patients and their matched controls.

Methods: Patients newly diagnosed with overt autoimmune hypothyroidism ( $\mathrm{n}=140$, medium TSH of $54.5 \mathrm{mU} / \mathrm{L}$, total T4 of $37.5 \mathrm{nmol} / \mathrm{L})$ and controls free of thyroid disease $(\mathrm{n}=560$, medium TSH of $1.24 \mathrm{mU} / \mathrm{L})$ recruited from the same population answered questionnaires regarding symptoms suggestive of hypothyroidism. Among 33 symptoms investigated, we identified 13 hypothyroidism-associated complaints $(\mathrm{P}<0.0015)$. The association between age at diagnosis and symptom presence was investigated. Diagnostic odds ratios (DORs) were calculated as measures for the association between participant status (case vs. control) and presence of symptoms (yes vs. no).

Results: Younger hypothyroid patients reported a higher prevalence of tiredness $(<60 / \geq 60$ years, $86 / 65 \%)$, dry skin $(71 / 40 \%)$, anterior neck pain (20/5\%), mood lability (56/20\%), difficulty swallowing (36/13\%), constipation $(45 / 23 \%)$, globulus $(42 / 20 \%)$, and restlessness $(36 / 25 \%)$; marginally higher prevalence of hair loss (34/20\%) and palpitations (39/23\%); but no difference in wheezing, shortness of breath, and dizziness. All 13 hypothyroidism-associated complaints were more often reported by young patients $(<60$ years) compared to their age- and sex-matched controls $(\mathrm{P}<0.001)$. Odds ratios $(95 \% \mathrm{CI})$ for being a hypothyroid case in the presence of each symptom spanned from $2.40(1.40-4.12)$ for wheezing to $8.17(4.40-15.2)$ for tiredness. In contrast, the elderly patients only reported tiredness (OR: 3.62 (1.77-7.43)) and wheezing (OR: 3.33 (1.47-7.51)) more often than elderly controls.

Conclusions: The presence of hypothyroidism-associated symptoms are suggestive for hypothyroidism in young subjects, but elderly patients with overt hypothyroidism have nearly the same complaints as old euthyroid people. Thus, thyroid function testing should be performed on wide indications in the old.

\section{P142 \\ COMPARATIVE EFFECTIVENESS OF REPLACEMENT MONOTHERAPY WITH L-THYROXINE AND COMBINATION WITH L-THYROXINE AND TRIIODOTHYRONINE IN WOMEN WITH POSTOPERATIVE AND AUTOIMMUNE HYPOTHYROIDISM}

$\underline{\text { Valentin Fadeyev }}^{1}$, Meruert Madiyarova ${ }^{1}$, Tatyana Morgunova ${ }^{1}$

${ }^{1}$ I.M.Sechenov First Moscow State Medical University, Department of Endocrinology

Objective: According to the literature, some patients with hypothyroidism receiving adequate L-thyroxine replacement still have persistent symptoms of hypothyroidism or dyslipidemia. The aim was to compare effectiveness of L-T4 monotherapy and combination L-T4+T3 in such patients with postoperative hypothyroidism (PO) and autoimmune hypothyroidism (AIH).

Patients and Methods: 40 women (18-45 y.o.) with hypothyroidism on adequate L-T4 and persistent symptoms (score $\geq 10$, TSQ) or dyslipidemia were included. 24 - AIH, 16 - postoperative (for Graves' disease). Patients received L-T4, then L-T4+T3 ( 25 mcg of L-T4 were replaced with $12.5 \mathrm{~T} 3$ ). Evaluation at baseline and after 3 months of L-T4+T3 included: quality of life 
(SF36), Hospital Anxiety and Depression Scale, TSQ, Inventory of memory and attention, TSH, TC, LDL.

Results: TSH levels were not different between L-T4 and after transition to L-T4+T3. There was significant reduction of TC and $\mathrm{LDL}$ in $\mathrm{AIH}$ and $\mathrm{PO}$ groups during L-T4+T3 $(P<0.02)$. The dynamics of TC was more substantial in PO hypothyroidism; and the decrease of TC, LDL was more pronounced at initially higher values $(P<0.02)$. QOL improved on L-T4+T3 in AIH (social functioning, mental health); no difference in $\mathrm{PO}$ hypothyroidism. Reduction in TSQ, improvement of cognitive functions were in both groups on combined treatment; in AIH these changes were accompanied by reduction in severity of depression $(P<0.02)$. Of 40 patients $35 \%$ preferred L-T4+T3 treatment: in this group the severity of symptoms initially was higher ( $\geq 17$ points) vs. no preference. Treatment with L-T4+T3 also led to more substantial TSQ decrease, QoL improvement $(P<0.05)$.

Conclusions: In young women with compensated hypothyroidism and persistent symptoms and/or dyslipidemia treatment with L-T4+T3 led to improvement in lipid profile; most substantial improvement was in PO hypothyroidism. The most significant improvement in psychoemotional features and QoL was observed in patients with AIH or TSQ score $\geq 17$.

\section{P143 \\ CARDIOVASCULAR DYSFUNCTION IN PATIENTS WITH AUTOIMMUNE THYROIDITIS AND SUBCLINICAL HYPOTHYROIDISM: CHARACTER OF DISORDERS AND RELATIONSHIP TO TSH VALUES \\ Leonid Strongin ${ }^{1}$, Tatiana Nekrasova ${ }^{1}$ \\ ${ }^{1}$ Nizhny Novgorod State Medical Academy}

Objectives: Recent studies established that the influence of subclinical hypothyroidism $(\mathrm{SCH})$ on cardiovascular risk factors might be proportional to the degree of TSH elevation. However, clinical cut-off level of TSH associated with increased risk remains unclear. The aim of the study was to evaluate the association between TSH values and cardiovascular dysfunction in patients with autoimmune thyroiditis (AT) and $\mathrm{SCH}$.

Methods: We investigated thyroid status, diastolic, endothelial function and relationship between these parameters in 186 women with AT; 65 among them were euthyroid and 121 had SCH. Diastolic function was estimated by tissue Doppler imaging; arterial function was evaluated by ultrasound assessment of flow-mediated endothelium-dependent and independent dilation.

Results: In the ROC analysis, a level of TSH $\geq 4.6 \mathrm{mu} / \mathrm{L}$ predicted diastolic dysfunction with $70.8[48.9 ; 87.3] \%$ sensitivity and $69.1[55.2 ; 80.8] \%$ specificity (AUC: $0.678[0.563 ; 0.778], p=0.036$ ). TSH value of $\geq 5.25 \mathrm{mu} / \mathrm{L}$ predicted endothelial dysfunction and associated metabolic abnormalities (AUC $>0.6, \mathrm{P}>0.05$ for most disorders).

Conclusion: TSH "cutoff "in respect of its relationship to most cardiovascular abnormalities occurred within the range of $4.6-5.25 \mathrm{mU} / \mathrm{L}$. Actually, the level of TSH over $5.25 \mathrm{mu} / \mathrm{L}$ might be considered a predictive marker for increased risk of $\mathrm{SCH}$ associated disorders.

\section{P144 \\ LIOTHYRONINE (L-T3) THERAPY FOR HYPOTHYROIDISM INCREASED THREE-FOLD IN DENMARK DURING 2013}

Luba Freja Michaelsson ${ }^{1}$, Bjarke Medici ${ }^{2}$, Christian Selmer ${ }^{2}$,

Michael Roeder ${ }^{3}$, Jens Faber ${ }^{2}$, Hans Perrild4', Nils Knudsen ${ }^{5}$, Blrte Nygaard ${ }^{2}$

${ }^{1}$ Herlev Hospital, ${ }^{2}$ Department of Endocrinology, Herlev Hospital, ${ }^{3}$ Danish Medicines Agency, ${ }^{4}$ Department of Endocrinology, Bispebjerg University Hospital, Copenhagen, Denmark, ${ }^{5}$ Department of

Endocrinology, Bispebejrg University Hospital, Copenhagen, Denmark

Hypothyroidism is a common disease in Denmark and 118000 patients corresponding to $2 \%$ of the population are treated with thyroid hormone. Standard therapy is levothyroxine (L-T4), and Guidelines suggest that only specialists should initiate a combination therapy with liothyronine (L-T3) due to risk of overtreatment. In May 2013 a popular-science book was published, and a demand for therapy with L-T3 and desiccated thyroid was seen, inducing a threefold increase in the sale of L-T3 and desiccated thyroid during 2013 to
Table 1. Symptoms on L-T4 therapy (for Abstract P144)

\begin{tabular}{lll}
\hline & $\begin{array}{l}\text { Primary } \\
\text { symptom (\%) }\end{array}$ & $\begin{array}{l}\text { Other } \\
\text { symptoms (\%) }\end{array}$ \\
\hline Being tired & 26 & 80 \\
Low energy & 16 & 82 \\
Cognitive problems & 10 & 77 \\
Muscular skeleton symptoms & 11 & 72 \\
Weight & 9 & 72 \\
Depression & 3 & 37 \\
Constipation & 2 & 41 \\
\hline
\end{tabular}

an estimated 750 patients. One third of the sale was desiccated thyroid and two third was L-T3.

Aim: To investigate 1) Who were given this therapy, 2) How the therapy was monitored, 3) How the patient experienced the effect of the therapy, and 4) If the medication was prescribed by a specialist or a general practitioner (GP).

Method: Internet-based survey through the Thyroid Patient Society.

Results: 260 Patients finished the survey (95\% women, $80 \%$ between 30 and 60 years old, $58 \%$ had an intermediate or long education).

The medication was prescribed by the GP in $48 \%$, by specialist in $47 \%$ or acquired without prescription on the internet by $5 \%$. The dose was adjusted by the doctor related to thyroid function parameters in $50 \%$ and related to symptoms in $20 \%$. In $30 \%$ the dose was adjusted by the patients themselves correlated to symptoms.

Conclusion: This survey demonstrates that public, nonprofessional focus on treatment of hypothyroidism resulted in decision about treatment as well as control during treatment that did not follow current guidelines.

\section{P145 \\ HYPOTHYROIDISM IS A PREDICTOR OF DISABILITY PENSION AND LOSS OF LABOUR MARKET INCOME. A DANISH REGISTER-BASED STUDY}

Marianne Thvilum $^{1}$, Frans Brandt ${ }^{1}$, Thomas H. Brix ${ }^{1}$, Laszlo Hegedüs ${ }^{1}$

${ }^{1}$ Odense University Hospital

Background: During the last decades hypothyroidism has repeatedly been associated with an increased burden of somatic as well as psychiatric morbidity. Whether hypothyroidism has any socio-economic consequences such as early retirement or income discrepancies is at present unclarified.

Aim: To examine, and compare with an age and sex matched control group, the risk of receiving disability pension (before the age of 60) and effect on labour market income in patients diagnosed with hypothyroidism.

Methods: Observational register-based cohort study. By record-linkage between different Danish health registers, 1745 hypothyroid singletons diagnosed before the age of 60 were each matched with 4 non-hypothyroid controls and followed for a mean of 5 years (range 1-31). Additionally, we included 277 same sex twin pairs discordant for hypothyroidism. The risk of disability pension was evaluated by the Cox regression analysis. Changes in labour market income progress over 5 years were evaluated using a difference in difference model.

Results: With a hazard ratio (HR) of 2.24 (95\% confidence interval (CI): 1.73-2.89), individuals diagnosed with hypothyroidism had a significantly increased risk of disability pension. This remained significant when adjusting for educational level and comorbidity (HR 1.89; CI: 1.42-2.51). In an analysis of labour market income, two years before compared with two years after the diagnosis of hypothyroidism, the hypothyroid individuals had on average a $1605 €$ poorer increase than their euthyroid controls $(\mathrm{P}<0.001)$. Essentially similar results were found in the twin population, suggesting that confounding due to genetic or environmental factors is negligible.

Conclusion: A diagnosis of hypothyroidism in young adults is associated with an $89 \%$ increased risk of disability pension compared to non-hypothyroid controls. Socio-economic consequences are also seen even among hypothyroid individuals staying on the labour market following the diagnosis of hypothyroidism, reflected by a decreased progress in labour market income. 


\section{P146 \\ ORAL SOLUTION (OS) LEVOTHYROXINE (L-T4) PERMITS TO REACH TARGET TSH LEVELS IN PATIENTS WHO TAKE TWO OR MORE DRUGS KNOWN TO IMPAIR THE INTESTINAL ABSORPTION OF L-T4}

Roberto Vita $^{1}$, Giovanna Saraceno ${ }^{1}$, Francesco Trimarchi',

Salvatore Benvenga ${ }^{1}$

${ }^{1}$ Endocrinology, Department of Clinical \& Experimental Medicine,

University of Messina

Objectives: To challenge the better absorption profile of a liquid formulation (OS) of L-T4 over tablet L-T4 by verifying whether OS would correct the tablet L-T4 malabsorption caused by the co-ingestion of two or more interfering drugs.

Methods: Thus far we have enrolled 11 patients who took tablet L-T4 either for replacement (REP group, $\mathrm{n}=5$ ) or for TSH suppression (SUP group, $\mathrm{n}=6$ ) and had serum TSH above target because they were also taking at least two of: proton-pump inhibitors $(n=9)$, calcium carbonate $(n=6)$, ferrous sulfate $(\mathrm{n}=5)$, sevelamer $(\mathrm{n}=4)$, magnesium/aluminum hydroxide $(\mathrm{n}=$ $1)$, sodium alginate $(n=1)$. We switched the tablet with the OS (Tirosint ${ }^{\circledR}$ soluzione orale, IBSA Italia s.r.1.), while maintaining the same daily dose, and checked serum TSH (mU/L) at least twice, eight weeks apart. Data are $\mathrm{m} \pm \mathrm{SD}$. Statistics is based on Wilcoxon test and Fisher's exact test.

Results: In the REP group, serum TSH was lower under the OS compared to the tablet $(2.7 \pm 1.1$ vs. $5.8 \pm 3.2, \mathrm{P}=0.004)$. The rate of TSH values $\leq 4.12$ or $\leq 2.5$ was $9 / 9(100 \%)$ or $4 / 9(44.4 \%)$ under the $\mathrm{OS}$, but $5 / 12(41.7 \%, \mathrm{P}=0.007)$ or $0 / 12(0 \%, \mathrm{P}=0.02)$ under the tablet. Target serum TSH levels $(\leq 2.5)$ were achieved already at the first or second determination in $4 / 5$ patients $(80 \%)$ under the OS, but in none under the tablet $(\mathrm{P}=0.05)$. In the SUP group, TSH was also lower under the OS $(0.5 \pm 0.6$ vs. $3.2 \pm 2.6, \mathrm{P}<0.0001)$. The rate of TSH values $\leq 0.10$ was $11 / 24(45.8 \%)$ under the OS, but $0 / 14$ under the tablet $(\mathrm{P}=0.02)$. Target serum TSH levels $(\leq 0.1)$ were achieved already at the first or second determination in $4 / 6$ patients $(66.7 \%)$ under the OS, but in none under the tablet $(\mathrm{P}=0.06)$.

Conclusions: In patients taking $\geq 2$ drugs that interfere with L-T4 intestinal absorption, the OS ensures an absorption of L-T4 far better than the tablet.

\section{P147 \\ CHARACTERISATION OF PATIENTS NOT SATISFIED WITH THE OUTCOME OF L-T4 MONOTHERAPY FOR HYPOTHYROIDISM \\ Bjarke Medici ${ }^{1}$, Jens Faber ${ }^{1}$, Blrte Nygaard ${ }^{1}$ \\ ${ }^{1}$ Department of Endocrinology, Herlev Hospital}

Symptoms of hypothyroidism persist despite biochemically euthyroidism in some L-T4-treated hypothyroid patients. ETA Guidelines (1) suggest that L-T4/L-T3 combination therapy could be considered for these patients.

Aim: to characterize these patients and the effect of combination therapy.

Method: All patients referred to one specialized clinic to obtain T4/T3 combination therapy from September 2012 to December 2013 were included. Medical history and symptoms were recorded and blood samples including a panel of routine tests were taken. Patients eligible for combination therapy were followed up after 3 months according to Guidelines with evaluation of effect.

Results: One-hundred and sixteen patients were referred (110 females), median BMI 27.6 (range 18.3-44.6) (73\% having BMI $>25)$ median age 52 (range 22-86). S-TSH levels were in median $1.14 \mathrm{mU} / \mathrm{L}$ (range 0.01-56.10), within normal range $(0.35-4.0 \mathrm{mU} / \mathrm{L})$ in $58 \%,<0.35 \mathrm{mU} / \mathrm{L}$ in $27 \%$, and $>4$ $\mathrm{mU} / \mathrm{L}$ in $15 \%$. 65 patients were dysregulated or we suspected another explanation of the symptoms; heart failure, iron deficiency, alcoholism, depression, menopause, vitamin D deficiency. The 5 most common symptoms were fatigue $(69 \%)$, cognitive problems (47\%), weight (44\%), unspecified illness $(37 \%)$ and muscular-skeleton complaints (34\%). Fifty-one patients started T4/ T3combination therapy. So far 41 have been followed up after 3 months, of which $20 \%$ experienced no improvement of symptoms, whereas $80 \%$ felt better with improvement on fatigue $(36 \%)$, cognitive problems $(36 \%)$, need of sleep (36\%), weight (34\%) and muscular-skeleton complaints (24\%), and thus wanted to continue the treatment.
Conclusion: $44 \%$ referred to the clinic to obtain T4/T3 combination therapy, fullfilled the criteria for this treatment (ETA Guidelines), and $80 \%$ of these experienced an improvement during follow up. Thus using the Guidelines we identified a subgroup of patients in whom $80 \%$ seemed to profit from T4/T3 combination therapy.

(1)Wiersinga et al 2012

\section{P148 \\ PREDICTIVE RISK MODEL OF SEVERE CORONARY ATHEROSCLEROSIS IN HEART ISCHEMIC DISEASE PATIENTS WITH SUBCLINICAL HYPOTHYROIDISM}

Anna Volkova ${ }^{0}$, Svetlana Dora ${ }^{1}$, Olga Berkovich ${ }^{1}$, Elena Grineva1, Olga Dygun

${ }^{1}$ Saint-Petersburg Medical University Named Acad. I.P. Pavlov

In clinical trials of recent years there were shown that subclinical hypothyroidism $(\mathrm{SCH})$ can participate in the formation of coronary atherosclerosis. Mild thyroid failure can mediate atherogenic dyslipidemia, hyperhomocysteinemia, insulin resistance and so on. As a rule $\mathrm{SCH}$ is the result of autoimmune thyroiditis wich to some extent depends from iodine supplementation of the region. There is elevation of iodine supplementation in Saint Petersburg due to iodine profilactice and autoimmune hypothyroidism can be revealed more frequent.

Objective: The aim of our study was to evaluate the results of coronarography in heart ischemic disease (HID) patients in accordance with thyroid gland function.

Materials and Methods: 870 of HID patients participated in our study. For all patients results of coronarography were performed. To evaluate thyroid function TSH level was measured. We also considered sex, age of our patients, smoking history, presence of hypertension, history of cardiovascular events, genetic predisposition.

Results: In accordance with the results of coronarography among our patients $16.1 \%$ did not have significant stenosis, $43.6 \%$ had $1-2$ of damaged vessels and $40.3 \%$ of patients had severe coronary atherosclerosis.As severe coronary atherosclerosis, we considered damage of 3 vessels and trunk of left coronary artery (LCA). As for TSH level in $12.1 \%$ patients $\mathrm{SCH}$ was revealed. In patients with $\mathrm{SCH} 57.4 \%$ had severe coronary atherosclerosis, and in euthyroid patients multivessel damage was in $38.1 \%$ of cases $(p=0.01)$.In the other hand, in patients with severe coronary atherosclerosis $\mathrm{SCH}$ was revealed in $17.4 \%$ of cases, when in patients without significance stenosis $\mathrm{SCH}$ was revealed in $8.3 \%$ of cases $(\mathrm{p}=0.01)$.

We calculated predictive risk of severe coronary atherosclerosis in HID patients by classification tree model. In our model SCH (THS $\geq 4.0 \mathrm{mUI} / \mathrm{l}$ ) had $\mathrm{OR}=2.31$ and very high predictive risk after infarction and age of our patients.

\section{P149 \\ SCREENING FOR HYPOTHYROIDISM IN PATIENTS WITH TYPE 1 DIABETES MELLITUS: HOW AND WHEN?}

Ludmilla Cardoso ${ }^{1}$, Marisa Tucci ${ }^{1}$, Patrícia Magalhães ${ }^{1}$, Sonir Antonini ${ }^{1}$,

Lea Maciel ${ }^{1}$

${ }^{1}$ Ribeirao Preto Medical School

The prevalence of hypothyroidism (hypo) in patients with type 1 diabetes (DM1) is higher than in the general population and there is no consensus how often screening should be performed.

Objectives: To assess the prevalence and the time interval until detection of hypo in DM1. To verify if there is a basal TSH value that discriminates individuals at higher risk for developing hypo. To evaluate the role of measurement of antibody anti-peroxidase (anti-TPO) in screening.

Patients and Methods: 232 patients with DM1 aged 20.0 \pm 5.0 years; [med $=19.9(5.2-32.5)], 50.9 \%$ male and $49.1 \%$ female, were followed for $10.3 \pm 3.5$ years $[10.2(0.4-22.3)]$. Serum anti-TPO and TSH were performed at intervals of $0.4 \pm 0.5$ and $0.9 \pm 1.2$ times per year of follow up, respectively. The hypo was defined by 2 or more serum TSH $>4.0 \mu \mathrm{IU} / \mathrm{mL}$.

Results: The age at diagnosis of DM1 was $7.6 \pm 4.2$ years $[\mathrm{med}=7.0(0.6-$ 19)]. $14.2 \%$ of them presented hypo at $12.4 \pm 5.4$ years old [10.5(6.1-26.7)] (69.7\% female). $71 \%$ developed the hypo until 7.3 years after the diagnosis of 
DM1. $63.6 \%$ of patients with hypo were anti-TPO+, of which $47.6 \%$ had the anti-TPO elevation at 1.7 years (med) before the TSH rise; in these cases, the hypo emerged after 6.7 (med) years of diagnosis of DM1. In the other patients, hypo emerged after 4.1 years (med). The basal serum $\mathrm{TSH} \geq 1.8 \mu \mathrm{IU} / \mathrm{mL}$ was able to predict hypo with sensitivity of $78 \%$ and specificity of $62 \%$.

Conclusions: The high prevalence of hypo justifies the screening. Our data suggest that screening should be performed with serum TSH, and should be more frequent in the first 7.3 years of the onset of DM1. Female patients have higher risk of developing the thyroid disease than male patients. Basal $\mathrm{TSH}$ values $\geq 1.8 \mu \mathrm{IU} / \mathrm{mL}$ indicate greater likelihood of developing hypo. The determination of anti-TPO has not contributed to increased surveillance of the disease.

\section{P150 \\ PATIENTS WITH SUBCLINICAL HYPOTHYROIDISM TREATED WITH L-T4 IN TABLET FORMULATION SHOW REVERSIBLE NORMALISATION OF SERUM TSH LEVELS AFTER SWITCHING TO AN ORAL LIQUID FORMULATION \\ Poupak Fallahi ${ }^{1}$, Silvia Martina Ferrari ${ }^{1}$, Alda Corrado ${ }^{1}$, \\ Andrea Di Domenicantonio ${ }^{1}$, Ilaria Ruffilli ${ }^{1}$, Alessandro Antonelli ${ }^{0}$ \\ ${ }^{1}$ University of Pisa}

Objective: One of the crucial step in the management of substitutive therapy in patients with autoimmune thyroiditis and subclinical hypothyrodism is L-Thyroxine (L-T4) absorption.

Methods In this study, we report that 15 patients with autoimmune thyroiditis and subclinical hypothyrodism (in absence of other causes of L-T4 malasorption) who received L-T4 in tablet formulation after switching to the same dosage of an oral liquid formulation, have the reversible normalisation of serum thyroid-stimulating hormone (TSH) levels.

Results The change from tablets to liquid oral formulation normalised serum TSH levels, while the switch back to tablet formulation caused thyrotropin levels to worsen in 8 among the 15 considered patients. These results lead us to believe that absorption of thyroxine was greater with oral liquid formulations in these patients.

Conclusion Our results suggest that the L-T4 oral liquid formulation could circumvent some problems related to the use of high L-T4 doses in the substitutional treatment of hypothyroidism.

\section{P016 Thyroid and Environment 2}

P151

Poster has been withdrawn

\section{P152}

THE PREVALENCE OF IODINE DEFICIENCY IN POPULATION OF REPRODUCTIVE AGE BY EXAMPLE OF STUDENTS AND DOCTORS OF SAINT-PETERSBURG

Daria Soboleva ${ }^{1}$, Svetlana Dora ${ }^{1}$, Anna Volkova $^{1}$

${ }^{1}$ The First Acad. I.P. Pavlov State Medical University, St. Petersburg

Objectives: The purpose of the study is to assess the iodine status in population of reproductive age.

Methods and Results: In 2013 we surveyed 111 medical students and doctors (87 women and 24 men), from 19 up to 24 years old. All the participants were examined by the endocrinologist, the thyroid palpation was performed, once daily urine was taken in order to determine urinary iodine concentration by reaction of Sandell-Kolthoff and to calculate median urinary iodine concentration. Depending on the level of the median urinary iodine concentration the participants were divided into 4 groups: the median urinary iodine concentration of more than $100 \mathrm{mg} / 1$ (group 1 - iodine sufficiency), the median urinary iodine concentration $99-50 \mathrm{mg} / 1$ (group 2 - mild iodine deficiency), the median urinary iodine concentration 49-20 mg/l (group 3 moderate iodine deficiency), the median urinary iodine concentration less than $20 \mathrm{mg} / 1$ (group 4 - severe iodine deficiency). This work was supported by the grant from the competition of scientific projects of young scientists of The First academician I.P. Pavlov State Medical University of Saint-Petersburg. The median urinary iodine concentration was $97.3 \mathrm{mg} / 1(12.4-395.0 \mathrm{mg} /$ 1). Only $46.8 \%$ of participants had iodine sufficiency, $40.5 \%$ - mild iodine deficiency, 9\% - moderate iodine deficiency, and 3.6\% - severe iodine deficiency. Goiter by palpation was found in $9 \%$ of participants. Our findings are significantly worse results of the Saint-Petersburg's study of schoolchildren was performed in 2010 (the median urinary iodine concentration was 148.0 $\mathrm{mg} / 1$, goiter by palpation was found in $1.6 \%$ of schoolchildren).

Conclusions: Most of the examined of reproductive age have mild iodine deficiency. The voluntary model of using iodized salt as prevention of iodine deficiency conducted in the Russian Federation is not effective enough even among people with medical education.

\section{P153 \\ ADJUVANT MITOTANE TREATMENT IN PATIENTS WITH ADRENOCORTICAL CANCER CAUSES CENTRAL HYPOTHYROIDISM Marco Russo ${ }^{1}$, Claudia Scollo ${ }^{1}$, Gabriella Pellegriti ${ }^{1}$, \\ Pasqualino Malandrino ${ }^{1}$, Andrea Caff', Maria Luisa Arpi ${ }^{1}$, Gisella Parrinello ${ }^{1}$, Sebastiano Squatrito ${ }^{1}$, Damiano Gullo ${ }^{2}$ \\ ${ }^{1}$ Endocrine Unit, Garibaldi-Nesima Hospital, University of Catania Medical School, Catania, Italy, ${ }^{2}$ Endocrine Unit, Garibaldi-Nesima Hospital, University of Catania Medical School}

Introduction: Mitotane, a steroidogenesis inhibitor with adrenolytic properties used to treat adrenal cortical cancer (ACC), can affect thyroid function. A reduction of FT4 levels with normal FT3 and TSH has been described in these patients. Effects of mitotane on thyroxine-binding globulin and deiodinase activity have been hypothesized. Recently, using an in vitro murine model, mitotane has been shown to inhibit the secretory capacity of thyrotropic cells.

Objective:To investigate the pathogenesis of thyroid abnormalities in mitotane-treated AAC patients evaluating the thyrotropic axis activity.

Patients and Methods: Five female AAC patients treated with mitotane as an adjuvant therapy (age $50.0 \pm 13.5 \mathrm{yrs} ; \mathrm{m} \pm \mathrm{SD}$ ) underwent a standard TRH test $(200 \mu \mathrm{g}$ i.v). Mitotane dosage and plasma concentration were $1.9 \pm 0.8 \mathrm{~g}$ / day and $12.7 \pm 2.0 \mathrm{mg} / \mathrm{L}$, respectively.

Results: Basal TSH was $1.63 \pm 0.42 \mathrm{mU} / \mathrm{L}$ (n.v. 0.4-4.0), FT4 $8.55 \pm 0.87$ $\mathrm{pmol} / \mathrm{L}$ (9.0-20.6), FT3 3.84 $\pm 0.44 \mathrm{pmol} / \mathrm{L}$ (2.9-6.0). After TRH administration peak TSH was reached at 30' (5.7 $\pm 0.7 \mathrm{mU} / \mathrm{L}$, min-max 4.9-6.8). $\Delta \mathrm{TSH}$, absolute TSH response (peak TSH minus basal TSH) was $4.1 \pm 0.8 \mathrm{mU} / \mathrm{L}$ (range 3.5-5.3). Fold TSH response (peak/basal TSH) was $3.7 \pm 1.0 \mathrm{mU} / \mathrm{L}$ (range 2.6-4.7). PRL secretion was normal. Therefore, mitotane-treated patients showed low FT4, normal FT3 and TSH and impaired TSH response to TRH, characteristic of central hypothyroidism.

Conclusions: This is the first description of an insufficient TSH production in ACC patients on adjuvant mitotane therapy, suggesting central hypothyroidism. This finding thus confirms in vitro studies and may have 
a therapeutic implication for treatment with thyroid hormones as suggested from current guidelines for this specific condition.

\section{P154 \\ BENEFICIAL EFFECT OF AN INCREASE IN IODINE SUPPLY ON THE TYPE AND SEVERITY OF IODINE-INDUCED THYROID DISORDERS}

Simona Gaberšček ${ }^{1}$, Vid Bajuk ${ }^{2}$, Katja Zaleteß ${ }^{\beta}$, Edvard Pirnat ${ }^{3}$,

Sergej Hojker ${ }^{1}$

${ }^{1}$ University Medical Centre Ljubljana, Department of Nuclear Medicine; University of Ljubljana, Faculty of Medicine, ${ }^{2}$ University of Ljubljana,

Faculty of Medicine, ${ }^{3}$ University Medical Centre Ljubljana, Department of Nuclear Medicine

Objectives: Data on the association between iodine supply and the incidence of iodine-induced thyroid disorders are very scarce. Therefore, our aim was to establish the occurrence of iodine-induced hyperthyroidism (II-Hyper) and iodine-induced hypothyroidism (II-Hypo) before and after an increase in salt iodization. In 1999, Slovenia increased mandatory iodization of kitchen salt from previous $10 \mathrm{mg}$ of potassium iodide to $25 \mathrm{mg}$ per $\mathrm{kg}$.

Methods: We reviewed records of all patients diagnosed with II-Hyper or II-Hypo between 1998 and 2009 at the Thyroid Department of the University Medical Centre Ljubljana which has a stable catchment area of 1 million inhabitants for more than 20 years. Diagnosis based on medical history of iodine excess, laboratory results, thyroid ultrasound, and if appropriate, on thyroid scintigraphy with Technetium-99m pertechnetate.

Results: Out of all patients first examined at our department between 1998 and 2009, relatively less patients presented with II-Hypo in the period between 1998 and 1999 than in the period between 2000 and 2009 (5 out of $6745,0.07 \%$ and 157 out of $40818,0.38 \%$, respectively, $\mathrm{P}<0.001)$. In the period 1998-1999, the ratio between the number of patients with II-Hyper and II-Hypo was significantly higher than in the period 2000-2009 (85/5, 17:1 and $157 / 441,2.8: 1$, respectively, $\mathrm{P}<0.001)$. In patients presented with II-Hyper, the level of $\mathrm{fT}_{4}$ did not differ significantly between 1998-1999 and 2000-2009 $(35.4 \pm 18.6$ and $31.8 \pm 17.3 \mathrm{pmol} / \mathrm{L}$, respectively, $\mathrm{p}=0.085)$. However, the level of $\mathrm{fT}_{3}$ was significantly higher in the period 1998-1999 than in the period 2000-2009 (8.7 \pm 5.2 and 7.3 $\pm 4.2 \mathrm{pmol} / \mathrm{L}$, respectively, $\mathrm{p}=0.005)$.

Conclusions: After an increase in mandatory salt iodization, iodineinduced thyroid dysfunction more frequently presents as hypothyroidism than before the increase. Additionally, following an increase in iodine supply, patients with iodine-induced hyperthyroidism are less severely hyperthyroid.

\section{P155 \\ PHARMACOKINETICS OF SELENOMETHIONINE IN PATIENTS WITH HYPOTHYROIDISM AND HYPERTHYROIDISM: A SINGLE-DOSE STUDY \\ Leonidas Duntas ${ }^{1}$, Theodoros Karakonstantakis ${ }^{2}$, Emilia Mantzou', loannis Papassotiriou ${ }^{2}$ \\ ${ }^{1}$ Unit of Endocrinology, Diabetes and Metabolism, Evgenidion Hospital, University of Athens, ${ }^{2}$ Department OF Clinical Biochemistry, "aghia Sophia" Children's Hospital}

Objective:To study the pharmacokinetics of selenomethionine (SeMet) in patients with various metabolic states, as insufficient data are available in humans.

Methods: A single-dose of L-selemethionine $400 \mu \mathrm{g}$ in bioavailable soft gel capsule form was administered in 3 groups of patients: Moderate hypothyroidism (TSH: 6-10 mU/L; Gr1) due to autoimmune thyroiditis, hyperthyroidism (TSH: < $0.1 \mathrm{mU} / \mathrm{L}$; Gr2) due to Graves' disease (GD) (4/6 with acute mild/moderate endocrine ophthalmopathy, EO) and 6 euthyroid controls (Gr3). Blood samples were taken at 0, 2, 4, 8, 12 and $24 \mathrm{~h}$. Various pharmacokinetic parameters were calculated. Selenium is a natural dietary constituent, thus wide variations in serum baseline concentrations are usually observed.

Results: Baseline concentrations were widely variable among patients and controls: Gr1: $68+17 \mu \mathrm{g} / 1$ (nr: 40-140 $\mu \mathrm{g} / \mathrm{l}), \mathrm{Gr} 2: 64+22 \mu \mathrm{g} / \mathrm{l}$ and $\mathrm{Gr} 3$ : $73+17 \mu \mathrm{g} / \mathrm{l}$. These baseline values were taken into consideration in the pharmacokinetics analysis. Se was steadily increased with a maximum $\left(C_{\max }\right)$ between 4 and $8 \mathrm{~h}$ and there was no statistically significant difference among the groups. The concentrations plateaued at $8 \mathrm{~h}-12 \mathrm{~h}$ and declined at $24 \mathrm{~h}$. No signs of toxicity were observed, except a transitory change in sense of taste $(n=4)$. It is of interest that patients with the lowest basal levels exhibited the highest $\mathrm{C}_{\max }$ and the shortest time peak ( $\mathrm{T}_{\text {peak }}$ ), while 3/4 EO patients reported eye pressure relief 8-12 h following administration of SeMet.

Conclusions: SeMet in the form studied is sufficiently bioavailable and well absorbed, while its absorption is not influenced by the metabolic state. On the other hand, its bioavailability is likely to be dependent on the basal serum concentrations, which may reflect increased demand or nutrient depletion. Effectiveness of Se administration probably requires an increasing $\mathrm{Se}$ concentration.

\section{P156 \\ DI-ETHYLHEXYL-PHTHALATE (DEHP) IS METABOLISED BY HUMAN THYROID CELLS BUT DOES NOT SEEM TO INFLUENCE THYROGLOBULIN PRODUCTION}

Juliana Frohnert Hansen"1, Marianne Moller Brorson"1, Marie-Louise Hartoft-Nielsen ${ }^{1}$, Malene Boas², Katharina Main², Hanna Frederiksen ${ }^{2}$, Jacob Hofman-Bang ${ }^{1}$, Åse Krogh Rasmussen ${ }^{1}$, Ulla Feldt-Rasmussen $^{1}$

${ }^{1}$ Department of Medical Endocrinology, Rigshospitalet, Copenhagen University Hospital, ${ }^{2}$ Department of Growth and Reproduction,

Rigshospitalet, Copenhagen University Hospital

Objectives: Phthalates are suspected to influence thyroid function in epidemiologic and experimental animal studies. The aim of the study was to investigate the ability of primary human thyroid cell cultures to metabolise phthalates and a possible concentration-dependent response of phthalates on thyroglobulin (Tg) production.

Methods: Human thyrocytes obtained from thyroidectomies were cultured to confluent monolayers and exposed to DEHP in concentrations $10^{-9}$ to $10^{-4} \mathrm{M}$, with thyroid stimulating hormone (TSH) (1 IU/1) for 24,48 or $72 \mathrm{~h}$. DEHP metabolites and Tg were quantified in cell supernatants (liquid chromatography-tandem mass spectrometry and ELISA, respectively), and mRNA by RT-qPCR. Statistical analyses were performed using two-way anova (SAS-institute).

Results: Cell cultures metabolised DEHP $\left(10^{-7}-10^{-4} \mathrm{M}\right)$ to its primary metabolite mono-ethylhexyl-phthalate (MEHP). Time studies indicated this to occur gradually, with most DEHP metabolised during the first $24 \mathrm{~h}$. However, the conversion to MEHP varied: relatively less was metabolised with increasing DEHP $\left(10^{-9}\right.$ to $\left.10^{-4} \mathrm{M}\right)$ added (measured after $72 \mathrm{~h}$ DEHP exposure).

Though results indicated a concentration dependent inhibition of Tg-secretion of the cells after $48 \mathrm{~h}$ DEHP exposure $\left(10^{-9}-10^{-5} \mathrm{M}\right)(\mathrm{p}=0.04$, $\mathrm{n}=$ three culture in single determination), Tg-mRNA expression of the same cultures was not affected by DEHP $(p=0.37)$. No influence on Tg release nor Tg-mRNA could be detected at any of the other studied time points $(24 \mathrm{~h} \mathrm{Tg} /$ Tg-mRNA: $\mathrm{p}=0.37 / 0.10 ; 72 \mathrm{~h} \mathrm{Tg} / \mathrm{Tg}$-mRNA: $\mathrm{p}=0.48 / 0.18, \mathrm{n}=$ three cultures in single determination).

Conclusion: DEHP was internalised and metabolised by primary human thyroid cell cultures, a rapid process mainly occurring during the first $24 \mathrm{~h}$ of exposure. The relative conversion, however, decreased inversely proportional to the DEHP concentration added, indicating an active saturable process in the thyroid cells. A possible inhibition of the Tg-secretion of the cell cultures could not be confirmed at the level of gene expression. Tg production from human thyrocytes therefore seems unaffected by DEHP exposure. 


\section{P157 \\ RADIATION-INDUCED MOLECULAR AND CELLULAR MODULATIONS IN THYROID CELLS UNDER IODINE DEFICIENCY}

Hanane Derradjii', Marie Hoyas ${ }^{1}$, Jessica Vanderstraeten ${ }^{2}$, Jasmine Buset ${ }^{1}$, Arlette Michaux ${ }^{1}$, Kevin Tabury ${ }^{1}$, Sarah Baatout ${ }^{1}$, Ides Colin ${ }^{3}$, Anne-Catherine Gérard ${ }^{3}$

${ }^{1}$ Radiobiology Unit, Laboratory of Molecular \& Cellular Biology, Belgian Nuclear Research Centre, Sck•cen, Mol, Belgium, ${ }^{2}$ Pôle de Morphologie, Institut de Recherche Expérimentale Et Clinique, Université Catholique de Louvain-la-Neuve, Brussels, Belgium, ${ }^{3}$ Service D'endocrino-Diabétologie, Centre Hospitalier Régional (Chr) Mons-Hainaut, Belgium

Objectives: Thyroid cancer is one of the malignancies induced by radiation exposure as shown by epidemiological studies performed after the Chernobyl accident and on patients undergoing external radiation therapy Besides radiation, iodine deficiency (ID) is a risk factor for thyroid toxicity and remains nowadays a problem in many geographical areas. Therefore, this work is focused on the study of the molecular and cellular modulations induced by low doses of radiation and ID on thyroid cells

Methods: FRTL-5 cells were grown at least 7 days in Coon's medium ( $5 \%$ calf serum $/ 6$ hormone mixture) supplemented or not with iodine. Six hours before irradiation ( $0.5 \mathrm{~Gy}$ and $3 \mathrm{~Gy}$ of X-rays), the cells were iodine deprived by medium replacement. DNA damage was checked using DNA ladder by gel electrophoresis, apoptosis was estimated using flow cytometry, and ERK1/2, NFk-Bp52 and Bad protein expressions were measured by western blot.

Results: No DNA damage was observed in the irradiated and/or iodine deprived FRTL-5 cells as measured by gel electrophoresis. Likewise, no SubG1 peak was observed in the irradiated and/or iodine deficient cells using flow cytometry. ERK2 expression was increased in the 3Gy irradiated iodine sufficient cells and in both 0.5 and 3 Gy irradiated iodine deprived cells. NFk-Bp52 was highly increased by both treatments and ID reinforced radiation effects. No difference was found for Bad expression.

Conclusions: Our results indicate that FRTL-5 cells show a radioresistant profile. Furthermore, anti-apoptosis, cell survival and proliferation mechanisms are turned on as evidenced by the absence of apoptosis and the expression of the ERK2 and NFk-Bp52, two key molecules of proliferation and survival pathways which were increased upon irradiation and both treatments, respectively. Noteworthy, radiation effects were reinforced by ID. All these data point to resistant, cell survival, proliferation and anti-apoptosis profiles, all of them prerequisite for precancerous transformation.

\section{P158 \\ ETHNIC DIFFERENCE IN URINARY CREATININE EXCRETION INFLUENCE EVALUATION OF IODINE NUTRITION IN GREENLAND INUIT AND CAUCASIANS \\ Stig Andersen ${ }^{1}$, Marie Dehnfeld ${ }^{2}$, Lone B. Rasmussen ${ }^{3}$ \\ Peter Laurberg ${ }^{4}$ \\ ${ }^{1}$ Aalborg University Hospital, ${ }^{2}$ Arctic Health Research Centre, ${ }^{3}$ National \\ Food Institute, ${ }^{4}$ Department of Endocrinology}

Background: Human contamination and iodine excretion is commonly assessed by the analysis of urine. A 24 hour urine sample is ideal but it is inconvenient, inaccurate and unreliable. Creatinine adjustment is widely used for estimation of 24 hour urinary excretion from spot urine samples.

Methods: We collected 104 consecutive spot- and 24 hour urine samples from Greenland Inuit and Caucasians and measured creatinine using the Jaffe method, iodine using the ceri-arsen method, and para-amino benzoic acid (PABA) by the HPLC method for estimation of completeness of sampling.

Results: Participants were recruited from the capital city $(n=36)$, a major town $(n=48)$ and a settlement $(n=20)$. They were 30 through 69 years with 78 Inuit and 26 non-Inuit. Inuit were smaller than Caucasians (height, 163 vs $177 \mathrm{~cm}, \mathrm{P}<0.001$; weight, $71 \mathrm{vs} 84 \mathrm{~kg}, \mathrm{P}<0.001)$. Urinary creatinine excretion was lower in Inuit than in Caucasians (men, 1344/1807 mg/24 h; women $894 / 1259 \mathrm{mg} / 24 \mathrm{~h} ; \mathrm{p}=0.002$ and 0.02$)$. Creatinine excretion was influenced by age $(\mathrm{P}<0.001)$, gender $(\mathrm{P}<0.001)$, weight $(\mathrm{P}<0.001)$ and ethnicity $(\mathrm{p}=$ 0.047 ) while unaffected by traditional Inuit diet (ns) in multivariate linear regression model. Data suggest a similar influence of age on creatinine excre- tion in Inuit and in Caucasians. Iodine excretion was higher in Inuit than in Caucasians ( 75 vs $57 \mu \mathrm{g} / 24 \mathrm{~h}$ ) and decreased with lower intake of Inuit foods: $>60 \% / 40-60 \% /<40 \% 95 / 74 / 69 \mu \mathrm{g} / 24 \mathrm{~h}$

Conclusion: Ethnicity influences creatinine excretion and it is important to consider ethnicity when estimating $24 \mathrm{~h}$ urinary iodine excretion from spot urine samples.

P017 Thyroid Cancer Diagnostics 2

\section{P159 \\ COMPARISONS OF LIQUID-BASED CYTOLOGY AND CONVENTIONAL CYTOLOGY ON THYROID NODULES: PROSPECTIVE STUDY WITH IMAGING-CYTOLOGIC CORRELATIONS ACCORDING TO BETHESDA SYSTEM}

Soo Jin Kim ${ }^{1}$, Sung Hee Park', Semin Chong ${ }^{1}$, Han Suk Ryu ${ }^{2}$

${ }^{1}$ Department of Radiology, Chung-Ang University Hospital,

${ }^{2}$ Department of Pathology, Seoul National University Hospital

Background: Liquid-based cytology (LC) is new developed cell preparation method for overcoming limitations of conventional cytology (CC).

Objective: We compared sonographic (US) features predicting nondiagnostic result and diagnostic performance between $\mathrm{LC}$ and $\mathrm{CC}$ on thyroid nodule.

Materials and Methods: From September to December 2011, this prospective study included 753 nodules in consecutive 601 patients underwent US-guided FNAB prepared into LC and CC simultaneously. A pathologist reviewed each method (CC, LC and combination) with a month interval. We evaluated US differences between $\mathrm{LC}$ and $\mathrm{CC}$ in predicting nondiagnostic result (NDR). Of 753 nodules, 526 nodules, proven histologic result by operation or unchanged for two years, were included for assessing diagnostic performance of three methods. Receiver operator characteristic (ROC) curves were calculated for each preparation methods.

Results: NDR was higher in inexperienced performers, $<1 \mathrm{~cm}$, solid, and calcified nodules $(P<0.05)$ in all methods. Mixed composition was more prevent in NDR using CC than LC $(P=0.004)$. Nondiagnostic rate was $26.9 \%$, $18.2 \%$ and $15.8 \%$ in CC, LC and combination, respectively. Sensitivity ( $92.3 \%$ vs $91.5 \%$ vs $92.3 \%$ ), specificity ( $60.2 \%$ vs $66.4 \%$ vs $70.1 \%$ ), positive predictive value $(46.1 \%$ vs $50.1 \%$ vs $53.2 \%)$ and negative predictive value $(95.5 \%$ vs $95.5 \%$ vs $96.1 \%)$ were similar. Area under curve (AUC) was 0.762 , 0.790 and 0.821 , respectively.

Conclusion: LC is more effective in reducing repeat FNA with better diagnostic performance than CC. LC may be preferred in a mixed thyroid nodule for reducing NDR than CC.

\section{P160 \\ THE CLINICAL SIGNIFICANCE OF TUMOR SUPPRESSOR GENE MRNA AND PROTEIN EXPRESSION, METHYLATION CHANGES IN NODULAR THYROID DISEASE}

\section{Wei Feng ${ }^{1}$, Wang Zhaoxia' ${ }^{1}$, Wu Yun ${ }^{1}$, Li Yongchao', Wang Jing ${ }^{1}$}

${ }^{1}$ Department of Endocrinology, the First Affiliated Hospital of Baotou

Medical College, Inner Mongolia University of Science and Technology, Baotou China

Objective:The tumor suppressor gene methylation lead to gene silencing, it plays an important role in thyroid tumor occurrence and development.This study examined the tumor suppressor gene (PTEN, DAPK) DNA methylation, mRNA and protein expression status in the patients with nodular thyroid disease. Discuss its clinical significance.

Method: Select 140 cases of nodular thyroid disease diagnosed (Including 66 cases of nodular goiter, 50 cases of thyroid adenoma, 24 cases of thyroid cancer)and 60 cases of normal control group in September 2009 - December 2013, Mining fasting peripheral venous blood collected, by the MSPPCR to detect PTEN, DAPK Methylation, by RT-PCR to detect mRNA expression. 
By Western Blotting to detect the protein expression in thyroid nodules tissue. Using SPSS 17.0 statistical software.

The result 1) In the high methylation status of DAPK, PTEN gene in the peripheral blood and thyroid nodule tissue of thyroid cancer, And to compare the methylation rate of thyroid cancer $>$ adenoma $>$ nodular goiter.

2) DAPK, PTEN mRNA and protein expression in thyroid cancer and adenoma reduce or even missing, And was negatively correlated with two genes methylation.

3) DAPK, PTEN gene promoter methylation and mRNA and protein expression was realated with lymph node metastasis in thyroid cancer patients. No relationship between Nodule size, calcification and ECT.

Conclusion:

1, PTEN, DAPK genes promoter methylation and the low mRNA, protein expression or missing are common in molecular biological events in the thyroid tumors.Closely related to the occurrence and development of thyroid tumors.

2, For the benign and malignant thyroid nodules early identification of molecular diagnosis is based.

\section{P161 \\ THE USE OF THE BETHESDA SYSTEM FOR REPORTING THYROID CYTOPATHOLOGY IN KOREA: A NATION-WIDE MULTICENTER SURVEY FROM THE KOREAN SOCIETY OF ENDOCRINE PATHOLOGIST}

Hyo Jin Park ${ }^{1}$, Hye Sook Min ${ }^{2}$, Hyeong Ju Kwon ${ }^{3}$, Chan Kwon Jung ${ }^{4}$, Hyunju Yoo ${ }^{5}$, Yoo Duk Choi ${ }^{6}$, Mi Ja Lee ${ }^{7}$, Jeong Ja Kwak ${ }^{8}$,

Soon Won Hong ${ }^{9}$, Mi Kyung Shin ${ }^{10}$

${ }^{1}$ Department of Pathology Seoul National University Bundang Hospital, Seongnam, Korea, ${ }^{2}$ Department of Pathology Seoul National University Hospital, Seoul, Korea, ${ }^{3}$ Severance Hospital, Yonsei University College of Medicine, Seoul, Korea, ${ }^{4}$ Department of Hospital Pathology College of Medicine The Catholic University of Korea, ${ }^{5}$ Department of Pathology, Thyroid Center, Daerim St. Mary's Hospital, Seoul, Korea, ${ }^{6}$ Department of Pathology, Chonnam National University Medical School, Gwangju, Korea, ${ }^{7}$ Department of Pathology, College of Medicine, Chosun University, Gwangju, Korea, ${ }^{8}$ Department of Pathology, Soon Chun Hyang University Hospital, Bucheon, Korea, ${ }^{9}$ Thyroid Cancer Center, Department of Pathology, Gangnam Severance Hospital, Yonsei University College of Medicine, Seoul, Korea, ${ }^{10}$ Department of Pathology Hallym University Medical Center Kangnam Sacred Heart Hospital, Seoul, Korea

Background: The Bethesda system for reporting thyroid cytopathology (TBSRTC) has standardized reporting of thyroid cytology specimens. The objective of the curreht study was to evaluate the nationwide usage of TBSRTC and assess the malignancy rates in each categories of TBSRTC in Korea.

Methods: Questionnaire surveys were used for data collection about the FNA of thyroid nodules in each institute at 2012. The incidences and follow up malignancy rates of each category diagnosed from January to December of 2011 in each institute were also collected and analyzed.

Results: Sixty out of 74 institutes answering the surveys have reported the results of thyroid FNA by TBSRTC. The average of malignancy rates upon resected cases of 14 institutes were as follow: nondiagnostic, $45.6 \%$; benign, $16.5 \%$; atypical of undetermined significance (AUS), $68.8 \%$; suspicious for follicular neoplasm (SFN), 30.1\%; suspicious for malignancy (SM), 97.6\%; malignancy, $99.7 \%$.

Conclusions: More than $80 \%$ of Korean institutes are using TBSRTC at 2012. The malignancy rates other than SFM and malignancy categories were higher than those of reports by other country. Therefore, the guidelines for treating patients with thyroid nodules in Korea would be better revisited based on the malignancy rates of this study.

\section{P162 \\ FALSE-NEGATIVE BRAFV600E MUTATION IN FINE-NEEDLE ASPIRATION CYTOLOGY OF PAPILLARY THYROID CARCINOMA}

Se Hyun Paek ${ }^{1}$, Byung Seup Kim¹, Kyungho Kang ${ }^{1}$, Soo Jin Kim¹, Hee Sung Kim ${ }^{1}$, Sung Jun Park ${ }^{1}$

${ }^{1}$ Chung-Ang University Hospital, Chung-Ang University College of Medicine

Introduction: The BRAF V600E mutation is highly specific to papillary thyroid carcinoma (PTC). A test for this mutation can increase the diagnostic accuracy of fine-needle aspiration cytology (FNAC). But false-negative rate of BRAF V600E on FNAC has been reported considerably. In this study, we investigated the risk factors associated with false-negative results of BRAF V600E mutation on FNAC compared to corresponding formalin-fixed, paraffin embedded (FFPE) surgical samples.

Methods: BRAF V600E mutation results of total 223 nodules confirmed as PTC in permanent biopsy from December 2011 to June 2013 were reviewed retrospectively. The results of BRAF V600E mutation between preoperative FNAC and postoperative FFPE section were investigated using sensitivity, specificity, positive predictive value and negative predictive value. And risk factors associated with false-negative results of BRAF V600E mutation on FNAC were investigated.

Results: Of total 223 PTC nodules, 152 nodules $(68.2 \%)$ were BRAF V600E mutation positive on FNAC and 185 nodules $(83.0 \%)$ were BRAF V600E mutation positive on FFPE. Sensitivity, specificity, positive predictive value and negative predictive value of the results of BRAF V600E mutation on FNAC were $80.5 \%, 92.1 \%, 98.0 \%$ and $49.3 \%$ respectively. 36 nodules (16.1\%) were BRAF V600E mutation negative on FNAC but positive on FFPE. Risk factors for this false negative results of BRAF V600E mutation on FNAC were virtually acellular specimen, AUS, and suspicious for PTC on FNAC in multivariate analysis.

Conclusions: BRAF V600E mutation analysis may be a useful adjunct technique for confirming the diagnosis of malignancy. However, the falsenegative rate of BRAF mutation test of FNA on thyroid nodule is increased in virtually acellular specimen, AUS, and suspicious for malignancy. Surgical treatment plan according to the BRAF mutation result on FNA should be made carefully in these cases.

\section{P163 \\ PAPILLARY THYROID CANCER IN PATIENTS WITH PRIMARY HYPERPARATHYROIDISM: OUR EXPERIENCE}

Julia Bernal' ${ }^{1}$, Eduardo Ferrero Herrero ${ }^{1}$, Blanca Cristobal',

Lorena Brandáriz', Danae Gil-Díez¹, Tamara Fernández',

Virginia García ${ }^{1}$, María García-Conde ${ }^{1}$, Javier García Borda ${ }^{1}$,

Manuel Lomas'

${ }^{1} \mathrm{H} .12$ de Octubre, Spain

Introduction: Primary hyperparathyroidism (PHPT) is an increasingly prevalent disease affecting all age groups, and the incidence of synchronous thyroid pathology ranged $17.8 \%$ to $84.3 \%$. In large series, rates of coexistent differentiated thyroid cancers were reported $2.7 \%$ to $9.5 \%$. Thyroid carcinoma associated may cause difficulties in the diagnosis, localization and therapy of PHPT.

Materials and Methods: We retrospectively examined 103 consecutive patients who underwent parathyroid surgery.

Results: This group was composed of 82 women and 21 men. The mean age was 60.17 years (range: 26-84). All of the patients had a diagnosis of PHPT preoperatively and underwent radiological assessment which included ultrasound and Tc99-sestamibi gammagraphy to help localise involved parathyroid tissue. Coexistent thyroid pathologies were investigated by physical examination, ultrasonography or CT. 10 patients $(9.7 \%)$ had coexistent papillary thyroid cancer, with female to male ratio of $7: 3$. The mean age was 60 years (range: 39-81). The histopathologic exam revealed 6 carcinomas T1a $(60 \%), 1 \mathrm{~T} 1 \mathrm{~b}(10 \%), 1 \mathrm{~T} 2(10 \%), 1 \mathrm{~T} 3(10 \%)$ and $1 \mathrm{~T} 4 \mathrm{a}(10 \%) .2$ patients had cervical lymph node metastasis $(20 \%)$, and 4 microcarcinoma $(40 \%)$. The carcinoma was unifocal in 6 patients $(60 \%)$ and unilateral in $8(80 \%)$. The average size of the tumor was $13.1 \mathrm{~mm}$ (range: 2-30). Total thyroidectomy was performed in the majority of cases $(\mathrm{n}=8,80 \%)$, with 2 patients undergo- 
ing thyroid lobectomy (20\%). In only 1 case was need to perform laterocervical dissection.

Conclusions: Coexistent papillary cancers may be very frequent especially in endemic goitre areas, and this cause difficulties in the diagnosis and management of PHPT. Pre and intraoperative thyroid examination should be performed in PHPT to avoid overlooking important thyroid pathologies, and similarly patients suspected or known to have thyroid carcinoma, should received adequate screening for PHPT, because a delay in operating would increase morbidity associated with a second neck exploration.

\section{P164 \\ PREDICTING THE LIKELIHOOD OF THYROID MALIGNANCY BY USING BRAF MUTATION ANALYSIS: COMPARISON OF BRAF DETECTION METHODS \\ Seo Ki Kim ${ }^{1}$, Jee Soo Kim¹, Jun Ho Lee', Jung-Han Kim", \\ Jun-Ho Choe', Bong Kyun Kim ${ }^{1}$, Jae Hoon Jang ${ }^{2}$, Hyun Chul Lee ${ }^{1}$ \\ ${ }^{1}$ Samsung Medical Center, Sungkyunkwan University School of \\ Medicine, ${ }^{2}$ Division of Breast and Endocrine Surgery, Department of \\ Surgery,}

Background. Owing to either emotional or insurance issues, many patients with various thyroid disease want to know their confirmative diagnosis as soon as possible. Although some studies have shown that BRAF mutation analysis enhanced the diagnostic value of fine needle aspiration(FNA) and ultrasonography(US), there have been no tools for predicting the likelihood of thyroid malignancy before thyroidectomy.

Objectives. The goal of this study was to provide information about the likelihood of thyroid malignancy for each patient, and the comparison of BRAF detection methods were conducted to assess false detections.

Study design. Case-control study

Patients and Methods: From January 2008 to December 2012, we retrospectively reviewed 3297 patients who underwent thyroidectomy for various thyroid disease. In this study, an individually applicable prediction model was designed by combining the results of FNA, US and BRAF mutation. BRAF mutation was analyzed by allele specific polymerase chain reaction(AS-PCR), direct sequencing and real time PCR(RT-PCR).

Results: Thyroid nodules, which showed indeterminate US, atypia of undetermined significance(AUS) FNA, had different potential for PTC according to the presence of BRAF mutation(negative: $29.2 \%$ vs positive: $87.5 \%$, respectively). Other thyroid nodules which showed benign FNA/US and negative BRAF mutation had considerable potential for PTC(18.6\%). These false detections could be explained by the relatively low sensitivity of BRAF mutation $(81.3 \%)$ which appeared in our study. The sensitivity and specificity of RT-PCR( $86.2 \%$ and $94.7 \%$, respectively) were superior to those of other two methods. The sensitivity of AS-PCR was significantly higher than that of direct sequencing $(77.1 \%$ vs $73.2 \%, \mathrm{P}<.001$, respectively), and the specificity of AS-PCR was lower than that of direct sequencing, but not significantly $(91.1 \%$ vs $94.1 \%, \mathrm{P}=.267$, respectively $)$.

Conclusion. Although, our study design was biased to thyroidectomy cases, the study enables both clinicians and patients to assess the likelihood of thyroid malignancy.

\section{P165 \\ FDG PET/CT THYROID INCIDENTALOMA: DO ULTRASOUND CRITERIA OF SUSPICION OF MALIGNANCY MODIFY OUR DECISION TO PERFORM FINE NEEDLE ASPIRATION CYTOLOGY (FNAC)?}

Anne Dierick-Gallet ${ }^{1}$, Isabelle Borget ${ }^{1}$, Sophie Bidault ${ }^{1}$,

Charlotte Lepoutre ${ }^{1}$, Philippe Vielh ${ }^{1}$, Abir Al Ghuzlan',

Amandine Berdelou', Elizabeth Girard ${ }^{1}$, Desiree Deandreis ${ }^{1}$,

Marie Terroir', Jean Lumbroso', Haitam Mirghani', Eric Baudin ${ }^{1}$,

Martin Schlumberger ${ }^{1}$, Sophie Leboulleux

${ }^{1}$ Gustave Roussy, Université Paris-Sud, Villejuif, France

Background: Fine Needle Aspiration Cytology (FNAC) is recommended in all thyroid incidentalomas with ${ }^{18} \mathrm{~F}$-fluorodeoxyglucose (FDG) uptake because of a risk of malignancy of $35 \%$, regardless of ultrasound characteris- tics. The aim of this study was to determine the usefulness of diagnostic index of ultrasound (US) criteria of suspicion of malignancy using Thyroid Imaging Reporting And Data System (TIRADS) score in this population.

Material and Methods: This is a retrospective monocentric study of 38 consecutive patients with thyroid incidentaloma found on FDG PET/CT between 2011 and 2013. Forty nodules (mean size: 23.4x14.8x17.4 mm, mean SUV max: 6.78) form the basis of this report. The gold standard was histopathology $(n=12)$, or cytology $(n=28)$. Among the 40 nodules, 10 were malignant (thyroid cancer in 6 cases, thyroid metastases in 4 cases)

Results: TIRADS score of the thyroid nodules was 3 in 8 cases $(20 \%), 4 \mathrm{~A}$ in 20 cases $(50 \%)$, 4B in 8 cases $(20 \%)$, and 5 in 4 cases $(10 \%)$. The sensitivity (Se) and specificity (Sp) of microlobulated or irregular margins was $80 \%$ and $87 \%$, respectively. For microcalcifications Se was $30 \%$ and Sp $100 \%$. For marked hypoechogenicity Se was $40 \%$ and Sp was $87 \%$ and for taller than wide shape Se was $40 \%$ and $\mathrm{Sp} 100 \%$. The positive predictive value of TIRADS score was $0 \%$ for score $3,10 \%$ for score $4 \mathrm{~A}, 50 \%$ for score $4 \mathrm{~B}$ and $100 \%$ for score 5 .

Conclusion: These preliminary results show that TIRADS score has to be taken into account to select among FDG avid thyroid incidentaloma which nodules need to be explore by FNAC to detect thyroid carcinoma or intrathyroidal metastases. Data collection is ongoing to increase our statistical power

\section{P166 \\ INCREMENTAL VALUE OF A COMPLEMENTARY NECK ACQUISITION DURING 18F-FDG-PET/ CT IN PATIENTS WITH DIFFERENTIATED OR POORLY-DIFFERENTIATED THYROID CANCER}

Renaud Ciappuccini ${ }^{1}$, Nicolas Aide ${ }^{1}$, Jean-Pierre Rame ${ }^{1}$,

Natacha Heutte ${ }^{1}$, Jean-Jacques Michels ${ }^{1}$, Elske Quak',

Stephane Bardet ${ }^{2}$

${ }^{1}$ Centre Baclesse, ${ }^{2}$ Centre Francois Baclesse

Objectives: 18-fluorodeoxyglucose-positron-emission-tomography with computed tomography $\left({ }^{18} \mathrm{~F}\right.$-FDG-PET/CT) can evidence persistent/recurrent disease (PRD) in patients with differentiated or poorly-differentiated thyroid cancer, especially for iodine-refractory lesions. The aim of the study was to compare the performances for neck PRD detection of the routinely performed whole-body acquisition (WBA) and of a complementary neck acquisition (CNA) performed during the same PET/CT study.

Methods: Twenty-six consecutive patients with a PET/CT study (Biograph 6, Siemens) combining WBA and CNA who subsequently underwent neck surgery, were retrospectively reviewed. ${ }^{18} \mathrm{~F}-\mathrm{FDG}$ uptake in cervical lymph-nodes or in the thyroid bed was blindly scored by two independent readers using a 5 -point scale ( 1 , definitely benign; 2 , probably benign; 3 , indeterminate; 4 , probably malignant; and 5 , definitely malignant), both on WBA and CNA. Surgical and pathological data were used as gold standard.

Results: Of 26 patients, 25 had histology proven neck nodal $(n=23)$ or local disease $(\mathrm{n}=2)$. Fifteen patients $(60 \%)$ displayed FDG-avid lesions on either WBA or CNA. In these 15 patients, the number of true-positive FDG foci per patient was higher on CNA than on WBA (2.2 vs. $1.3, \mathrm{P}<0.01)$. Of 27 malignant lymph-nodes removed, $21(78 \%)$ were detected on CNA versus $12(44 \%)$ on WBA $(\mathrm{P}<0.01)$. Three false-negative WBA studies $(3 / 15=20 \%)$ were upstaged as true-positive on CNA. The median size of the largest nodal metastasis was $13.5 \mathrm{~mm}$ for lesions visible both on WBA and CNA, $6.3 \mathrm{~mm}$ for the lesions detected by CNA but not by WBA, and $5 \mathrm{~mm}$ for those not visible on PET/CT $(\mathrm{P}<0.001)$.

Conclusions: CNA improves the detection of neck PRD and should be routinely added to the commonly performed WBA PET/CT study. 
P167

PEDIATRIC DIFFERENTIATED THYROID CARCINOMA IN CENTRAL HOSPITAL

OF SANTIAGO DE COMPOSTELA - A RETROSPECTIVE STUDY

Eduarda Resende ${ }^{1}$, Virginia Pubul Nuñez ${ }^{2}$, Marta Nascimento ${ }^{3}$,

Paloma Cabanas Rodriguez ${ }^{4}$, Lidia Castro Feijoo ${ }^{4}$,

Gemma Novoa Gomez ${ }^{5}$, Rebeca Saborido Fiaño ${ }^{4}$,

Jesús Barreiro Conde ${ }^{4}$

${ }^{1}$ Department of Endocrinology - Central Hospital of Funchal,

${ }^{2}$ Department of Nuclear Medicine - Chus, ${ }^{3}$ Department of Pediatrics

- Pedro Hispano Hospital, ${ }^{4}$ Department of Pediatric Endocrinology -

Chus, ${ }^{5}$ Department of Pediatrics - Ourense Hospital

Introduction: The prevalence of palpable thyroid nodules in children under 21 years is around 0.8 to $1.8 \%$, considerably inferior to the prevalence in adult population; however, the risk of malignancy is higher. The aim of this study is to revise the cases of differentiated thyroid carcinoma (DTC) in people under 21 years and to analyse the patient's characteristics.

Methods: Retrospective study identifying the cases of DTC diagnosed in people under 21 years, between 1992 and 2013, in Santiago de Compostela.

Results: We found 19 cases of DTC in people under 21 years: 17 were girls and only 2 were boys. Diagnosis under the age of 10 years was found in just one patient, and $67 \%$ of the cases were diagnosed between 15 and 19 years. One patient had history of cervical irradiation and none have positive familiar history. Two patients had history of previous use of growth hormone (GH). The histology was papillary carcinoma in the vast majority of the patients (18 cases), and $44 \%$ of these had cervical lymph node metastasis at diagnosis. All the patients were submitted to a total thyroidectomy and radioiodine ablation, with a median activity of $3385 \mathrm{MBq}$; in 7 cases it was needed more than one treatment with radioiodine. A surgical re-intervention was necessary in 6 cases. The median time of follow-up was 13.5 years, and 10 patients are free of disease, 7 have persistent disease and in 2 patients there is loss of follow-up.

Discussion/Conclusion: In the two patients with history of previous $\mathrm{GH}$ use the immunohistochemical staining of the tumors showed positivity for GH receptors, which is interesting.

The majority of the patients were diagnosed in the adolescence, and a considerable percentage of these had cervical lymph node metastasis at diagnosis - as described in the literature about DTC in children.

\section{P168 \\ DEVELOPMENT OF ANTI-THYROGLOBULIN ANTIBODIES (ANTI-TG) AFTER IODINE-131 ABLATION IN PATIENTS WITH WELL DIFFERENTIATED THYROID CANCER WDTC AND THEIR INTERFERENCE WITH THE MEASUREMENT OF THYROGLOBULIN (TG) \\ Savvas Frangos ${ }^{1}$, Nicolaos Eftychiou ${ }^{1}$, Anna Vanezi ${ }^{1}$, Maria Petrou ${ }^{1}$, Evanthia Giannoula'2, Ioannis lakovou ${ }^{2}$ \\ ${ }^{1}$ Department of Nuclear Medicine; Bank of Cyprus Oncology Centre, \\ ${ }^{2}$ Nuclear Medicine Department; Aristotle University, Papageorgiou Hospital}

Objectives: Anti-TG are commonly identified in patients with well differentiated thyroid cancer. When present, they interfere with the measurement of TG. Concentration of $<22 \mathrm{IU} / \mathrm{mL}$ is unlikely to cause clinically significant TG assay interference. Scope of the study is to discover if TG could be positive in the presence of Anti-TG and to observe the development of Anti-TG in patients with positive antibodies. We considered positive the patients with Anti-TG $>23 \mathrm{IU} / \mathrm{ml}$

Material and Method: 198 patients with WDTC stage T1aN0M0 to T2N1bM0 underwent I-131 ablation therapy between Jan2011 and Oct2013. 76 patients (38.4\%) [66F/10M, age 20-66] had Anti-TG $>23 \mathrm{IU} / \mathrm{ml}$ on therapy day (T0).For all the patients Anti-TG and TG were estimated at T0 and about two months later (T1) .For 71 patients in 6-12 months later (T2) and for 51 on the day of WB scan about one year after therapy.

Results: The level of Anti-TG at T0 was $23-1723 \mathrm{IU} / \mathrm{ml}$. TG was detectable in 46 patients. (24 pts $0.2-2 \mathrm{ng} / \mathrm{ml}, 15 \mathrm{pts} 2.2-8 \mathrm{ng} / \mathrm{ml}$ and $7 \mathrm{pts} 10.1-$ $84 \mathrm{ng} / \mathrm{ml}$ ) At T1 12 pts showed increased levels of Anti-TG (7.9-872.8 mIU/l) and 64 decreased (1.4-948.8) 10 pts became negative. 9 pts show detectable TG (0.2-5.4 ng/ml) all with positive Anti-TG. At T2 4 pts showed increased levels (7.9-872.8 IU/ml) 64 pts showed decreased levels (1.4-948.8) 27 pts became negative, $5 \mathrm{pts}$ showed detectable TG $(0.2-4 \mathrm{ng} / \mathrm{ml}) 2$ of them with positive Anti-TG. 12 out of $51 \mathrm{WB}$ scans were positive. 4 with negative Anti-TG ( 3 with detectable TG) and 8 with positive Anti-TG (2 with detectable TG). Out of 39 negative WB 23 pts have negative Anti-TG (6 showed detectable TG.) 16 pts have positive Anti-TG (7 detectable TG)

Conclusions: $38.4 \%$ of our patients have positive Anti-TG which is more than the literature. TG was detectable in 46 out of 76 patients. Most of the patients showed decreased Anti-TG. In positive WB scan half the patients have positive Anti-TG.

\section{P169 \\ PROGNOSTIC VALUE OF USING THE \% CHANGE IN INITIAL TGAB CONCENTRATION TO MONITOR TGAB-POSITIVE PAPILLARY THYROID CANCER (PTC) PATIENTS FOR PERSISTENT/RECURRENT DISEASE}

Shireen Fatemi ${ }^{1}$, Jonathan LoPresti ${ }^{2}$, Ivana Petrovic ${ }^{2}$, Carole Spencer ${ }^{3}$ ${ }^{1}$ Southern California Permanente Medical Group, ${ }^{2}$ Usc Endocrine Laboratories, ${ }^{3}$ Usc Endocrine Laboratory; University of Southern California

Background: $\sim 25$ percent of PTC patients have Tg autoantibodies(TgAb) causing Tg interference during post-operative PTC monitoring. Because changes in $\mathrm{Tg}$ antigen prompt changes in $\mathrm{TgAb}$ concentrations, $\mathrm{TgAb}$ can act as a surrogate tumor-marker. There is currently limited information regarding interpreting the magnitude and temporal pattern of serum $\mathrm{TgAb}$ changes.

Study Design: Selected patients had TgAb detected (Kronus/RSR) within 3 months of thyroidectomy (TTx) for PTC and anatomic imaging that established disease-status at the end of $>3$ years follow-up monitoring. $\mathrm{TgAb}$ was calculated as percent initial (1-3 month postoperative) value (corrected for assay functional sensitivity).

Study Patients: 27 patients (mean age at TTx $47.5 \pm 11.0(\mathrm{sd})$ years) were judged disease-free(DF) $5.5 \pm 3.3$ years after TTx, and 27 patients (age $40.0 \pm 14.7$ years) had disease(DZ) detected $4.4 \pm 3.2$, range 0.9 to 11.7 years after TTx.

Results: Initial $\mathrm{TgAb}$ concentrations for $\mathrm{DF}$ versus $\mathrm{DZ}$ groups were comparable (median 11.1 versus $12.1 \mathrm{kIU} / \mathrm{L}$, respectively, $\mathrm{P}>0.90$ ). One-year after TTx, median DF $\% \mathrm{TgAb}$ was 28 , range $3-86 \%$, and by two-years 15 , range $0-64 \%$. At the end of $7.3 \pm 3.4$ years follow-up, DF $\% \mathrm{TgAb}$ was $<10 \%$ in $89 \%$ with $12 / 27(44 \%$ ) becoming TgAb-negative. In contrast, the DZ group at one-year had median $\% \mathrm{TgAb}$ of 60 , range $6-1177 \%$ and at the time recurrence was detected had median $\% \mathrm{TgAb}$ of 137 , range $6-1779 \%$. The appearance of $\mathrm{TgAb}$ was the first sign of recurrence in one patient.

Conclusions:

1. A progressive decline in $\operatorname{TgAb}$ following TTx suggests the absence of disease.

2. In DF patients $\% \mathrm{TgAb}$ typically falls to $<10 \%$ initial value after 3 years follow-up.

3. Disappearance of $\operatorname{TgAb}$ is more likely when initial post-operative $\operatorname{TgAb}$ is low.

4. Patients with persistent/recurrent disease either displayed rising $\operatorname{TgAb}$, a de novo $\mathrm{TgAb}$ appearance, or had stable $\% \mathrm{TgAb}$ that remained above $10 \%$ after 3 years follow-up. 
P170

\section{ESTABLISHMENT OF HUMAN RELEVANT} CULTURE FROM FNABS

Angela Rodriguez García-Rendueles ${ }^{1}$, Maria del Carmen Suarez ${ }^{1}$, Valentina Cirello ${ }^{2}$, Joana S. Rodrigues ${ }^{1}$ Ana Senra ${ }^{1}$, Sihara Perez ${ }^{1}$, Javier Rodríguez-García ${ }^{3}$, Laura Fugazzola², Clara Alvarez Villamarín ${ }^{1}$ ${ }^{1}$ Center for Research in Molecular Medicine and Chronic Diseases (Cimus), University of Santiago de Compostela, ${ }^{3}$ Department of Clinical Sciences and Community Health, University of Milan, Milan, Italy; Endocrine Unit, Fondazione Policlinico Irccs, Milan, Italy; ${ }^{3} \mathrm{Clinical}$ Biochemistry, University of Santiago de Compostela (USC) and Clinical University Hospital of Santiago de Compostela

Pendred's Syndrome is a rare disease where goiter is associated to congenital deafness. The biological cause for the goiter is largely unknown. Our group developed a special culture medium, the $\mathrm{h} 7 \mathrm{H}$ medium (Bravo et al, JCEM 2013) where concentration of every component is adjusted to the expected physiological levels in human serum. This medium allow us obtaining primary cultures of human thyrocytes forming FLS (follicle-like-structures) that maintain thyroid phenotype derived from surgical thyroid pieces of patients. Some Pendred's Syndrome patients can not be operated so it would be necessary to develop a personalized culture to study the specific alterations for each patient and have better knowledge of goiter biological mechanisms in this disease.

The aim of this work is develop a cellular model from FNAB (fine-needle aspiration biopsy) from Pendred's Syndrome patients.

Two diagnostic FNAB's in h7H medium were sent from Italy to Spain. This patient has a homozigotic mutation (T721M). One of them was washed out the blood from cells and the other one not. In our laboratory, the first one was seeded in two plates (T-PS5a,b) and the last one, in one plate (T-PS5c). Thyroid phenotypic markers were performed by inmunohistochemistry and we measure secreted tyroglobulin in culture medium.

In T-PS5a,b the cells attached to the plate and grew faster than T-PS5c. Despite of this, after one month we could obtain a clonal cell culture whose cells formed FLS. The T-PS5 a,b cells did not secret TG to the medium and were only positive for calcitonin. The T-PS5c culture secreted TG and was positive for TTF1, TG, CK7 and total CK, but negative for calcitonin.

We could achieve a cell culture model of FNAB from Pendred's Syndrome patients that would allow study cellular mechanisms implicated in this kind of goiter and international collaboration with other centers.

\section{P018 Thyroid Cancer Pathogenesis 1}

\section{P171 \\ TALL CELL VARIANT OF PAPILLARY THYROID CARCINOMA: A CLINICOPATHOLOGICAL STUDY WITH BRAFV600E MUTATIONAL ANALYSIS \\ Rocío Villar-Taibo ${ }^{1}$, Diego Peteiro-González², \\ José-Manuel Cabezas-Agrícola ${ }^{3}$, Clara Ruiz-Ponte ${ }^{4}$, José Cameselle- Teijeiro $^{5}$ \\ ${ }^{1}$ Endocrinology Department. University Hospital of León, \\ ${ }^{2}$ Endocrinology Section. Sierrallana Hospital, Torrelavega (Cantabria), \\ ${ }^{3}$ Department of Endocrinology, Clinical University Hospital, 15706 \\ Santiago de Compostela, Spain, ${ }^{4}$ Fundación Pública Galega de \\ Medicina Xenómica, Ciberer, Santiago de Compostela, ${ }^{5}$ Department \\ of Pathology, Clinical University Hospital of Santiago de Compostela, \\ Sergas, University of Santiago de Compostela}

Objectives: Tall cell variant (TCV) of papillary thyroid carcinoma (PTC) is characterized by tall columnar cells whose height is at least three times their width. TCV usually presents at older ages, has a larger size, and shows more extrathyroidal extension and metastases than classical PTC. We compare TCV with both classical and follicular variants to determine if, regardless of age and size, TCV is more aggressive than its classical and follicular counterparts.

Methods: Sixteen $(3.66 \%)$ patients with TCV were identified in a series of 437 PTC patients from the Clinical University Hospital, Santiago de
Compostela, Spain (1990-2010). We compared their clinicopathologic features and $B R A F^{\mathrm{V} 600 \mathrm{E}}$ mutational status with 34 cases of classical and follicular variants of PTC matched in size and age. The TCV series included 11 women and 5 men aged 15-74 years (median, 57 years). Fifteen (93.8\%) patients underwent total/near total thyroidectomy, 1 case lobectomy, and $5(31.3 \%)$ lymph node dissection.

Results: In the tall cell variant series, tumor size ranged from 5- $45 \mathrm{~mm}$ (median $19 \mathrm{~mm}$ ). Extrathyroidal extension appeared in $9(56.3 \%)$ cases and lymph node metastases in $9(56.3 \%)$. Eight patients $(50 \%)$ harbored multifocal papillary carcinomas, with lymphovascular invasion in $6(37.5 \%)$ and distant metastases in $1(6.2 \%)$. Ten patients $(62.5 \%)$ presented at stage III/ IV. $B R A F^{\mathrm{V} 600 \mathrm{E}}$ mutation was detected in $12(80 \%)$ tumors. The control group showed less extrathyroidal extension and lymph node metastases: $5(14.7 \%)$ $(\mathrm{p}=0.007)$ and $9(26.5 \%)$ cases $(\mathrm{p}=0.04)$, respectively; $9(26.5 \%)$ tumors were multifocal, with lymphovascular invasion in $10(29.4 \%)$, no distant metastases, and $7(20.5 \%)$ patients presenting at stage III/IV $(\mathrm{p}=0.04)$. BRAF mutation was higher in the TCV ( $80 \%$ vs. $28 \%$; p = 0.004).

Conclusions: The TCV of PTC is usually associated with BRAF mutation and behaves more aggressively than its classical and follicular counterparts regardless of size and age.

\section{P172 \\ WARTHIN-LIKE VARIANT OF PAPILLARY THYROID CARCINOMA: A COMPARISON WITH CLASSIC TYPE IN THE PATIENTS WITH COEXISTING HASHIMOTO'S THYROIDITIS}

Chan Kwon Jung ${ }^{1}$, Min-kyung Yeo ${ }^{2}$, Ja Seong Bae ${ }^{3}$, Sohee $L^{2} e^{3}$, Min Hee Kim ${ }^{4}$, Dong Jun Lim ${ }^{4}$

${ }^{1}$ College of Medicine, The Catholic University of Korea; Department of Hospital Pathology, ${ }^{2}$ Chungnam National University, ${ }^{3}$ Department of Surgery, Catholic University of Korea College of Medicine, Seoul St. Mary's Hospital Seoul, Republic of Korea, ${ }^{4}$ College of Medicine, The Catholic University of Korea

Objectives: The Warthin-like variant of papillary thyroid (WLPTC) is a rare subtype of papillary thyroid carcinoma (PTC) resembling Warthin tumor of salivary glands. It is characterized by oncocytic cells with typical PTC nuclear features and extensive lymphoplasmacytic infiltration in the stalks of papillary structures. Due to its rarity and relatively indolent clinical behavior, the clinicopathologic and molecular features of WLPTC are still unclear. We aimed to investigate demographic and clinical characteristics of patients with WLPTC comparing them with those of classic PTC.

Methods: Out of 2.139 patients who underwent surgical treatment for PTC at Seoul St. Mary hospital from 2012 to 2013, 40 patients with WLPTC were identified and compared to 200 consecutive patients with classic PTC. BRAF mutation was tested with pyrosequencing.

Results: There were no significant differences in mean age, predilection for women, tumor location, multifocality, extrathyroidal extension, and lymph node metastasis between WLPTC and classic PTC. But, WLPTCs were more commonly associated with Hashimoto's thyroiditis than classic PTCs $(93 \%$ vs. $36 \%, \mathrm{P}<0.001)$ and showed significantly lower rate of BRAF mutation when compared to classic PTCs (65\% vs. $84 \%, \mathrm{P}=0.007)$. In patients with classic PTC, the frequency of BRAF mutations was negatively correlated with coexisting Hashimoto's thyroiditis. When we compared WLPTCs and classic PTCS in the patients with coexisting Hashimoto's thyroiditis, there were no significant differences in clinicopathologic characteristics and BRAF mutation rate between the two groups.

Conclusions: Patients with WLPTC and coexisting Hashimoto's thyroiditis had similar demographic, clinical, pathologic and molecular characteristics to those with classic PTC and Hashimotos' thyroiditis. 


\section{P173}

HLA-G 3'UNTRANSLATED REGION POLYMORPHISMS IN THYROID TUMORS: POTENTIAL DIAGNOSTIC AND PROGNOSTIC MARKERS?

Lea Maciel', Nathalie Figueiredo-Feitosa ${ }^{1}$, Patrícia Magalhães ${ }^{1}$,

Daiani Alves', Gustavo Palomino ${ }^{1}$, Celso Mendes-Junior',

Eduardo Donadi ${ }^{1}$

${ }^{1}$ Ribeirao Preto Medical School

Human leukocyte antigen G (HLA-G) is a nonclassical major histocompatibility complex molecule that plays a role in immune response against tumor cells.

Objectives: To evaluate the polymorphic sites of 3'untranslated region (3'UTR) of HLA-G in colloid goiter (CG), follicular adenoma (FA), papillary (PTC) and follicular thyroid carcinomas (FTC) and its association with clinical outcome.

Methods: DNA was obtained from 21 patients with CG, 20 with FA, 22 with FTC, 72 with PTC and 156 healthy controls. Polymorphic sites from 3'UTR region (14bpIN/DEL $+3003 \mathrm{C} / \mathrm{T}+3010 \mathrm{C} / \mathrm{G}+3027 \mathrm{~A} / \mathrm{C}+3035 \mathrm{C} / \mathrm{T}$ $+3142 \mathrm{C} / \mathrm{G}+3187 \mathrm{~A} / \mathrm{G}+3196 \mathrm{C} / \mathrm{G})$ were characterized by sequencing. Fisher's exact test was used to compare allelic and genotypic frequencies.

Results: PTC had higher frequency of 14bpDEL $(p=0.03),+3010 \mathrm{G}(p=$ $0.034),+3010 \mathrm{CG}(p=0.044),+3142 \mathrm{G}(p=0.03)$ and $+3142 \mathrm{CG}(p=0.04)$ than CG. PTC had higher prevalence of $+3035 \mathrm{C}(p=0.05)$ and $+3187 \mathrm{GG}$ $(p=0.032)$ and FTC higher frequency of $14 \operatorname{bpDEL} / \mathrm{INS}(p=0.02)$ than controls. CG had higher frequency of polymorphisms associated with lower HLA-G production than PTC: $14 \mathrm{pbINS} / \mathrm{INS}(p=0.05),+3010 \mathrm{CC}(p=0.004)$, $+3142 \mathrm{GG}(p=0.004)$ and $+3142 \mathrm{G}(p=0.03)$. There were no differences in frequencies of $+3003 \mathrm{C} / \mathrm{T},+3027 \mathrm{~A} / \mathrm{C}$ and $+3196 \mathrm{C} / \mathrm{G}$. The UTR-5 haplotype was more prevalent in CG $(p=0.04)$ and controls $(p=0.03)$ than in PTC. The ICCCCGAG haplotype occurred only in PTC. The +3003TT was more frequent in PTC patients older than 45 years $(p=0.04)$. Male patients had a higher prevalence of $+3196 \mathrm{GG}(p=0.05)$. Tumor multicentricity was associated with UTR-2 $(p=0.05)$. The joint analysis of PTC and FTC showed that tumor size $<2 \mathrm{~cm}$ was associated with $14 \mathrm{bpINS} / \mathrm{INS}(p=0.03)$ and multicentricity with $+3035 \mathrm{CC}(p=0.03)$ and $+3196 \mathrm{GG}(p=0.03)$.

Conclusion: The polymorphic sites of 3'UTR region of HLA-G associated with greater magnitude of HLA-G production (14pb DEL, 14pb DEL/ INS, +3010G, +3010CG, +3035C, +3035CC, +3142CG, +3187GG) were associated with thyroid tumors and some factors of worse prognosis, confirming the negative role of HLA-G in thyroid carcinogenesis.

FAPESP\#10/51649-9, CNPq\#457231/2013-0

\section{P174 \\ NO SIGNIFICANT DIFFERENCES IN THE LONG TERM OUTCOME BETWEEN FAMILIAL (FDTC) AND SPORADIC (SDTC) CASES OF FOLLICULAR CELL DERIVED THYROID CARCINOMAS (DTC)} Katerina Saltiki ${ }^{1}$, Michael Apostolakis ${ }^{1}$, Fani Kanouta ${ }^{2}$, George Papadakis², Theodora Pappa', Anastasia Athanasiadou', Gianna Rentziou', Eleni Anastasiou', Anastasios Pappas², Maria Alevizaki ${ }^{1}$

${ }^{1}$ Endocrine Unit, Dept Medical Therapeutics, Alexandra Hospital, Athens University School of Medicine, Athens, Greece, ${ }^{2}$ Dept of Endocrinology, Metaxa Hospital, Athens, Greece

Objectives: $5-10 \%$ of DTCs have familial disease. Aim of the study was to assess differences in clinical features and prognosis between fDTC and sDTC.

Methods: 995 DTC patients $(22.7 \%$ men), were followed-up for $1-44$ years (mean $6.4 \pm 7.8$, median 3.3 years). 80 patients $(8.0 \%$ ) had fDTC. These were pooled with a further 29 patients with fDTC. Extent of disease at diagnosis and long term outcome during follow-up were recorded.

Results: No difference in mean age at diagnosis was found between the two groups (fDTC: $43.92 \pm 14.2$, vs sDTC: $42.48 \pm 15.1$ yrs). Familial disease prevalence was slightly higher in women than in men $(\mathrm{p}=0.06)$. The frequency of thyroid remnant ablation was similar (fDTC: $71.2 \%$ vs $75.1 \%$ ). No differences in histological type, microcarcinoma prevalence, tumour size, multifocality, capsular and/or tissue invasion or autoimmunity were found.
fDTC had less frequently lymph node invasion $(17.1 \%$ vs $27.3 \%, p=0.025)$ however, in this group, lymph node dissection had been less frequently performed $(8.2 \%$ vs $15.7 \%, p=0.048)$. Of those who underwent thyroid remnant ablation with $\mathrm{I}^{131}$ no differences in thyroglobulin levels during the post-iodine assessment (basal, stimulated) were found. fDTC had more frequently disease persistence compared to sDTC during the post-iodine assessment $(42.2 \% \mathrm{vs}$ $23.9 \%, p=0.002)$. However, during follow-up and after further therapeutic interventions (surgery and/or $\mathrm{I}^{131}$ ) fDTC did not differ significantly in the outcome compared to sDTC. The 10-year probability of lack of disease progression did not differ between groups $\left(98.9 \%\right.$ vs $97.1 \%, x^{2}=0.69, p=0.4 \log$ Rank).

Conclusions: Although fDTC present during the $1^{\text {st }}$ post-iodine assessment more frequently disease persistence, they have similar 10-year probability of lack of disease progression as sDTC. Women may have increased awareness for thyroid testing when they have positive family history for DTC.

\section{P175 \\ ROLE OF MIRNA IN CONTROL OF THYROID TUMORIGENESIS}

Jacques Dumont ${ }^{1}$, Pita Jaime ${ }^{2}$, Van Sande Jacqueline ${ }^{2}$,

Maenhaut Carine ${ }^{2}$

${ }^{1}$ University Brussels ${ }^{2}$ Ulb

miRNAs exert a multidirectional control on gene expression: each miRNA has multiple target mRNAs and each mRNA is the target of several miRNAs. It is therefore surprising that the literature abounds in articles reporting a unidirectional interpretation of miRNA expression in tumors: one modulated miRNA, one target, one effect. In this work we compare miRNA and mRNA expressions in human thyroid cells in vitro submitted to various treatments and in various thyroid tumors and tumor cell lines. We show that if miRNA difference in expression may account for the chronic effect of the tumorigenic process in human tumors in vivo (autonomous adenomas, thyroid papillary and anaplastic carcinoma) or thyroid cancer cell lines in vitro, they do not account for the shorter term $(24,48 \mathrm{~h})$ in vitro regulations.

These findings will be analyzed within the framework of our concepts on miRNA roles.

\section{P176 \\ EVIDENCE OF MACROPHAGES INFILTRATION IN POORLY DIFFERENTIATED (PDTC) AND ANAPLASTIC THYROID CARCINOMA (ATC): IMPACT ON MOLECULAR ANALYSIS}

Alessia Tacito ${ }^{1}$, Clara Ugolini ${ }^{2}$, Cristina Romei ${ }^{1}$, Raffaele Ciampi ${ }^{1}$,

Francesca Casella1, Liborio Torregrossa², Fulvio Basolo²,

Rossella Elisei ${ }^{1}$

${ }^{1}$ Department of Clinical and Experimental Medicine, University of Pisa, ${ }^{2}$ Department of Surgical Pathology, Medicine, Molecular and Critical Area, Univerisy of Pisa

Tumor-associated macrophages (TAM) are associated with a more advanced stage of disease and poorer prognosis in PDTC and ATC. Their presence has been considered a limit for detection of oncogene mutations.

Aim of this work was to evaluate the presence of TAM in PDTC and ATC in relation to the presence of somatic mutations.

Among 37 available cases (15 ATC, 20 PDTC and 2 ATC/PDTC) the search of TAM was performed up to now on 20 samples (11 ATC, 9 PDTC).

The analysis of TAM was performed with immunohistochemistry on paraffin-embedded tissues using an anti-CD68 antibody; somatic mutations in BRAF, RAS, b-catenin, PIK3CA, TP53, Axin1, PTEN, APC and ALK were studied by direct sequencing.

All samples were positive for CD68. In particular the ratio TAM/ATC cells was $1: 1$ in 7 cases, $1: 2$ and $1: 3$ in 2 case respectively and 1:50 in 2 cases; the ratio TAM/PDTC cells was 1:50 in 1 case, 1:100 in 4 cases and 1:200 in 4 cases. In PDTC the ratio TAM/PDTC cells was always lower than 1:1, moreover the prevalence of TAM cells was significantly higher $(\mathrm{p}=0.04)$ among ATC cells than PDTC cells.

Somatic mutations (R273H and R267W of p53, Q97R of PTEN, $\mathrm{V} 600 \mathrm{E}$ of BRAF) were present in 4/11 ATC and in 4/9 PDTC (BRAF V600E in 2 cases, p53 H1047R and AKT Q17K). Such mutations have been found even in cases in which the ratio TAM/tumor cells was $1: 1$. 
Our data demonstrate that: a) TAM are present in all cases but the ration TAM/ tumoral cells was never $>$ than $1: 1 ; b$ ) TAM are significantly more frequent in ATC than in PDTC; c) somatic mutations are present in $36 \%$ of ATC and in $44.4 \%$ of PDTC, d) despite the presence of TAM, sequence analysis is able to identify somatic mutations.

\section{P177 \\ LDL CHOLESTEROL REGULATE THE EXPRESSION OF LDL-R IN FOLLICULAR THYROID CELLS}

Eugènia Mato ${ }^{1}$, Aleida Pujol ${ }^{2}$, Joan Carles Escola ${ }^{3}$, Cintia Gonzalez, Olga Bell ${ }^{5}$, David Santos ${ }^{6}$, Antonio Moral Duarte ${ }^{7}$, Jose Ignacio Pérez ${ }^{7}$, Francisco Blanco-Vaca ${ }^{8}$, Alberto de Leiva ${ }^{9}$

${ }^{1}$ Networking Research Center on Bioengineering, Biomaterials and Nanomedicine (Ciber-Bbn), Eduab-Hsp Hospital Santa Creu I Sant Pau, Autonomous University, Barcelona, ${ }^{2}$ Institut de Recerca de L'hospital de la Santa Creu I Sant Pau, Institut D'investigacions Biomèdiques (lib) Santa Creu I Sant Pau, Institut de Recerca de L'hospital de la Santa Creu I Sant Pau, Institut D'investigacions Biomèdiques (lib) Santa Creu I Sant Pau, Ciber de Diabetes Y Enfermedades Metabólicas Asociadas. Ciberdem. Barcelona, Spain, ${ }^{4}$ Networking Research Center on Bioengineering, Biomaterials and Nanomedicine (Ciber-Bbn), Hospital Santa Creu I Sant Pau, Autonomous University, Barcelona, Endocrinology Department, Hospital Sant Pau, Autonomous University, Barcelona, Spain, ${ }^{5}$ Networking Research Center on Bioengineering, Biomaterials and Nanomedicine (Ciber-Bbn), Hospital Santa Creu I Sant Pau, Autonomous University, Barcelona, ${ }^{6}$ Institut de Recerca de L'hospital de la Santa Creu I Sant Pau, Institut D'investigacions Biomèdiques (lib) Sant Pau and, Ciber de Diabetes Y Enfermedades Metabólicas Asociadas. Ciberdem, Barcelona, ${ }^{7}$ General Surgery, Hospital de la Santa Creu I Sant Pau, Barcelona, ${ }^{8}$ Institut de Recerca de L'hospital de la Santa Creu I Sant Pau, Institut D'investigacions Biomèdiques (lib) Sant Pau And; Ciber de Diabetes Y Enfermedades Metabólicas Asociadas. Ciberdem. Barcelona, ${ }^{9}$ Networking Research Center on Bioengineering, Biomaterials and Nanomedicine (Ciber-Bbn), Hospital Santa Creu I Sant Pau, Autonomous University, Barcelona, Endocrinology Department, Hospital Sant Pau, Autonomous University, Barcelona, Spain; Dept. of Endocrinology \& Nutrition

Introduction: It is known that cancer cells require cholesterol to maintain a high level of proliferation. Low and high density lipoproteins (LDL and HDL) are considered the major suppliers of cholesterol to cancer cells. However, the relationship between these lipoproteins and epithelial thyroid neoplasm remains unknown.

Aim: 1. Characterization of lipoprotein profile in patients with adenomes and epithelial thyroid carcinomas. 2. Investigate the effect of human LDL and HDL lipoproteins in two thyroid cell lines.

Methods: Serum from patients affected with Adenomas $(\mathrm{n}=7)$, WDPTC $(\mathrm{n}=10)$, WDFTC $(\mathrm{n}=8)$, PDTC $(\mathrm{n}=3)$ were analyzed to determine their lipoprotein profile. Furthermore, Nthy-ori (thyroid cells) and Cal-62 (anaplastic thyroid cells) were treated during $72 \mathrm{~h}$ with $5 \%$ of human lipoprotein-depleted serum (LPDS), LDL (100 $\mu \mathrm{M}$ of apoB) and HDL $(100 \mu \mathrm{M}$ of apoA-I). Gene expression of ATP-binding cassette (ABC) $A B C A 1, A B C G 1$, apolipoprotein A1, LDL receptor (LDLR), Scavenger receptor type B-I (SCARB1) genes was measured by qRT-PCR. Proliferation study was measured by MTT assay. Statistical analyses were preformed by GraphPad Prism and DataAssist softwares.

Results: Patients with PDTC had lower mean serum apolipoprotein (apoB) levels $(0.58 \pm 0.02 \mathrm{~g} / \mathrm{L})$ than adenomas $(0.84 \pm 0.04 \mathrm{~g} / \mathrm{L})$ or WDTC $(0.82 \pm 0.04$ $\mathrm{g} / \mathrm{L})$. No significant changes were found in the mean serum level of other lipoproteins and apolipoproteins. LDL promoted: 1) Proliferation in Nthyori and CAL-62 (13.7\%, 15.8\%; respectively), 2) up-regulation of $A B C A 1$ (3.98-fold-change, 2.14-fold-change; respectively) and $A B C G 1$ (5.16-foldchange, 4.97-fold-change; respectively) genes, 3) down-regulation of $L D L R$ gene (0.29-fold-change, 0.51 -fold-change; respectively). HDL treatment does not affect cellular proliferation but show down-regulation in $A B C A 1$ ( 0.8 -foldchange, 0.16 -fold-change respectively) and $A B C G 1$ ( 0.34 fold-change, 0.16 fold-change respectively) genes.

Summary: Preliminary findings suggest a possible role for apoB as a biomarker in patients with aggressive thyroid tumours. Moreover, LDL was able

Poster Presentations to promote proliferation in thyroid cells which was associated with changes in lipoprotein-related genes expression.

\section{P178 \\ SERUM TSH, FREQUENCY AND SIZE OF PAPILLARY THYROID CARCINOMA IN PATIENTS WITH NODULAR THYROID DISEASE}

Maria Annateresa Provenzale ${ }^{1}$, Liborio Torregrossa², Letizia Pieruzzi',

Eleonora Molinaro', Emilio Fiore ${ }^{1}$, Fulvio Basolo ${ }^{2}$, Paolo Vitti ${ }^{1}$

${ }^{1}$ Department of Clinical and Experimental Medicine - Pisa,

${ }^{2}$ Department of Surgical Pathology - Pisa

A relationship between serum TSH and risk of papillary thyroid carcinoma (PTC) has been shown in patients with nodular thyroid disease. However, the association between TSH level and PTC size has not yet been clarified.

Aim of the study was to analyze serum TSH in patients with PTC greater than $1 \mathrm{~cm}$ (macroPTC) and smaller than $1 \mathrm{~cm}$ (microPTC), the latter subdivided into microPTC detected before surgery at ultrasound examination (clin-mPTC) and microPTC clinically occult, incidentally found at histology (inc-mPTC). The study included 590 patients submitted to thyroid surgery for the size of goiter $(n=157)$ or for nodules with an indeterminate $(n=297)$ or suspicious/indicative of PTC $(n=136)$ cytology.

On histology 276 patients had a diagnosis of benign nodular thyroid disease (BNTD), 182 of macroPTC, 132 of microPTC, subdivided in 70 clin$\mathrm{mPTC}$ and 62 inc-mPTC. Inc-mPTC (median value $4 \mathrm{~mm}$, interquartile range, IR $2-6.5 \mathrm{~mm})$ were significantly smaller $(\mathrm{P}<0.0001)$ with respect to clin-mPTC $(8 \mathrm{~mm}$, IR 6-10 mm). TSH values were significantly lower $(\mathrm{P}<$ $0.0001)$ in inc-mPTC $(0.53 \mu \mathrm{U} / \mathrm{ml}$, IR $0.37-0.90 \mu \mathrm{U} / \mathrm{ml})$ compared to clin$\mathrm{mPTC}(0.94 \mu \mathrm{U} / \mathrm{ml}$, IR $0.57-1.33 \mu \mathrm{U} / \mathrm{ml})$. TSH was not statistically different between inc-mPTC and BNTD $(0.65 \mu \mathrm{U} / \mathrm{ml}$, RI 0-33-1.07 $\mu \mathrm{U} / \mathrm{ml})$ and between clin-mPTC and macroPTC $(0.84 \mu \mathrm{U} / \mathrm{ml}$, IR $0.55-1.29 \mu \mathrm{U} / \mathrm{ml})$. In both macroPTC and clin-mPTC, TSH was significanlty higher compared to $\operatorname{BNTD}(\mathrm{P}<0.0001$ and $\mathrm{p}=0.04$, respectively).

In summary, inc-mPTC are significantly smaller and have TSH values significantly lower compared to clin-mPTC and not different compared to BNTD. In clin-mPTC, TSH values are not different compared to macroPTC, but significantly higher compared to BNTD.

In conclusion, clinically occult microPTC are more frequently detected in patients with lower TSH values (patients with nodular goiter and thyroid autonomy), while higher TSH values are more often associated with clinically detectable PTC.

\section{P179 \\ TUMORIGENESIS OF PAPILLARY THYROID CANCER IS NOT BRAF-DEPENDENT IN PATIENTS WITH ACROMEGALY}

Ho-Cheol Kang ${ }^{1}$, Hee Kyung Kim ${ }^{1}$, Jee Hee Yoon ${ }^{1}$, Soo Jeong Kim ${ }^{1}$

${ }^{1}$ Department of Internal Medicine, Chonnam National University Medical School

Purpose: Acromegaly is associated with an increased risk of malignant tumors. Several studies have reported a high frequency of PTC in patients with acromegaly. The aim of this study was to determine the prevalence and predictors of thyroid cancer in patients with acromegaly and to investigate the frequency of the BRAFV600E mutation in PTC patients with and without acromegaly.

Materials and Methods: 60 patients with acromegaly (33 females and 27 males) were referred to Chonnam National University Hwasun Hospital between April 2004 and April 2013. Thyroid US and US-guided FNA were performed on nodules with suspicious US features. We selected 16 patients with non-acromegalic PTC as a control group. The BRAFV600E mutation was analyzed in paraffin-embedded surgical specimens of PTC by real-time polymerase chain reaction.

Results: The prevalence of thyroid nodules was $75.0 \%$ (45/60), and thyroid cancer was found in $15(25.0 \%)$ patients. No differences in age, sex, initial growth hormone (GH) and IGF-1 percentage of the upper limit of normal values or treatment modalities were observed between patients with and without PTC. Acromegaly was active in 12 of 15 patients at the time of PTC diagnosis; uncontrolled acromegaly had a significantly higher frequency in the PTC group $(60 \%)$ than in the non-PTC group $(28.9 \%)(\mathrm{p}=0.030)$. The 
BRAFV600E mutation was present in only $9.1 \%$ (1/11) of PTC patients with acromegaly, although $62.5 \%(10 / 16)$ of control patients with PTC had the mutation $(\mathrm{p}=0.007)$.

Conclusion: The prevalence of PTC in acromegalic patients was high $(25 \%)$. Therefore, we suggest that acromegalic patients should be routinely screened for thyroid cancer. An uncontrolled hyperactive GH-IGF-1 axis may play a dominant role in the development of PTC rather than the BRAFV600E mutation in patients with acromegaly

\section{P180 \\ THE IMPACT OF CLAUDIN-1 ON THYROID CANCER AGGRESSIVENESS}

Denise Zwanziger ${ }^{1}$, Stefanie Rehn ${ }^{1}$, Julia Badiong ${ }^{1}$, Andrea Jaeger ${ }^{1}$, Dagmar Führer'

${ }^{1}$ University Hospital Essen

Objective:In epithelial cells the tight junction protein claudin- 1 is located in the cell membrane to maintain the paracellular barrier-integrity. In human tumors, claudin-1 is often observed in subcellular compartments and thereby seems to be involved in cell proliferation and differentiation. In addition, a high claudin-1 expression seems to correlate with human tumor aggressiveness. In follicular thyroid carcinoma, the second most common thyroid cancer, a low expression of claudin- 1 has been observed by immunohistochemistry. However, a potential relation between claudin-1 and tumor aggressiveness as well as other pathogenic features of thyroid carcinoma has not yet been investigated.

Methods \& Results: Here we investigated claudin-1 in two follicular thyroid carcinoma cell lines, FTC-133 (derived from a primary tumor) and FTC-238 (derived from a lung metastasis). On immunofluorescence, both cell lines reveal claudin-1 localized in the cell nucleus. By Western blot analysis an increased claudin-1 expression in FTC-238 as compared to FTC-133 cells was observed. Elevating the pathogenic character of FTC-133 by transient transfection of RasV12 increases cell migration and claudin-1 expression as compared to empty-vector transfected cells. Conversely, down-regulation of claudin-1 in FTC-238 by claudin-1 siRNA leads to a decreased cell migration as compared to control siRNA transfected cells. Interestingly, claudin-1 siRNA transfection decreases levels of activated protein kinase C (PKC).

Conclusion: Claudin-1 expression seems to increase with the aggressiveness and a more pathogenic character of follicular thyroid carcinoma in cell culture. Moreover, claudin-1 expression seems to correlate with activated PKC expression. These preliminary results could be a hint of a functional role of claudin-1 in follicular thyroid carcinoma.

\section{P181 \\ RET GERMLINE HETEROZYGOUS MUTATION ALA883THR IS NOT ASSOCIATED WITH MEDULLARY THYROID CARCINOMA \\ Laura Valerio', Cristina Romei', Alessia Tacito ${ }^{1}$, Raffaele Ciampi', Valeria Bottici ${ }^{1}$, Rossella Elise ${ }^{1}$ \\ ${ }^{1}$ Endocrine Unit, Department of Clinical and Experimental Medicine, University of Pisa, Pisa, Italy}

Introduction: In the last 20 years we collected genetic data of 140 families with hereditary form of medullary thyroid carcinoma (MTC). Ala883Thr is present in only $1 / 140(0.7 \%)$ family and MTC affected members are homozygous for the mutation. We found Ala883Thr in one other family with 2 members, both heterozygous, but only one of them was affected by MTC.

Aim: To verify the relationship between the heterozygous Ala883Thr mutation and the MTC in this other family.

Methods: The parents of the index case were investigated for Ala883Thr and the mother was positive. She was $62 \mathrm{yr}$ old and showed a nodular goiter but was negative both for basal and pentagastrin stimulated calcitonin. The father was negative both for RET mutation and clinical/biochemical examination. He was positive for the polymorphism Gly691Ser. RET gene mutation screening was performed in the patient's tumor tissue by sequencing analysis but no somatic mutations were found in any RET exon.. The analysis of several RET polymorphisms showed the presence of heterozygous exon 11 polymorphism (Gly691Ser) in our patient.

Conclusions: We confirm that RET Ala883Thr heterozygous mutation is not sufficient to induce MTC as demonstrated by the absence of the disease in the mother of our patient. The MTC of our patient can be due to the simultaneous presence of Ala883Thr and Gly691Ser since this latter has been demonstrated to confer a predisposition to the development of the disease. Functional studies to demonstrated this hypothesis are ongoing.

\section{P182 \\ EXPRESSION OF LAMININ-332 IN PAPILLARY THYROID CARCINOMA WITH OR WITHOUT LYMPH NODE METASTASIS}

Kyoung Ho Oh${ }^{1}$, Won-gue Han ${ }^{1}$, Jun Yoo ${ }^{1}$, Soon Young Kwon ${ }^{1}$

${ }^{1}$ Korea University Ansan Hospital

Background and Objectives: Laminin-332, a major component of the extracellular matrix, is an important autocrine factor produced by cancer cells to promote tumorigenesis. We aimed to investigate the relationship between laminin-332 expression in papillary thyroid carcinoma and lymph node metastasis

Materials \& Methods: Forty patients with papillary thyroid carcinoma (7 men and 33 women) were enrolled in the study. Twenty patients presented with lymph node metastasis, whereas the other 20 did not. Laminin-332 expression was determined by immunohistochemical staining with antibodies against the laminin- $5 \gamma 2$ chain in all 40 patients. The correlation between laminin-332 expression and clinical factors such as tumor size, multiplicity, and lymph node metastasis were investigated.

Results: Laminin-332 was expressed specifically within tumor tissue compared to normal thyroid tissue. The frequency of laminin- $5 \gamma 2$ chain expression showed significant correlation with cervical lymph node metastasis $(p=0.003)$. As the tumor size increased, laminin-332 showed a tendency for higher expression, although the correlation was not statistically significant $(p=0.082)$. No relationship between the presence of multiple tumors and the expression of laminin-332 was identified.

Conclusion: Laminin-332 expression may be helpful in the diagnosis of papillary thyroid carcinoma, and our results suggest that high levels of laminin-332 expression may be a useful marker for predicting lymph node metastasis of papillary thyroid carcinoma.

\section{P183 \\ SECOND PRIMARY MALIGNANCY IN PATIENTS WITH THYROID CANCER}

Stylianos Mandanas ${ }^{1}$, Paschalia Iliadou ${ }^{1}$, Pavlos Papakotoulas ${ }^{1}$, Evanthia Giannoula ${ }^{1}$, Vasiliki Chatzipavlidou ${ }^{1}$,

Kalliopi Pazaitou-Panayiotou ${ }^{1}$

${ }^{1}$ Theagenio Cancer Hospital

Objectives: This study aimed to evaluate the association of a second primary malignancy in patients with thyroid carcinoma (TC).

Methods: The medical records of patients with two different primary malignancies (TC and any other primary malignancy, identified with histological examination) were retrospectively studied. Patients were divided into three groups: Group A included patients who first presented thyroid cancer; group B included patients who presented other types of cancer before thyroid cancer; group $\mathrm{C}$ included patients who were diagnosed and treated for both thyroid and other primary cancer, simultaneously. Data analysis was performed with the statistical package SPSS (version 17.0; SPSS Inc., Chicago, IL).

Results: 126 patients, 35 males (28\%), with mean age $( \pm$ SD) $50 \pm 12$ years at the diagnosis of first cancer were recruited. The histological subtypes of TC were: $80 \%$ papillary, $9 \%$ follicular, $4 \%$ medullary, $3 \%$ Hurthle cell, $1.5 \%$ poorly differentiated, $1.5 \%$ mixed and $1 \%$ anaplastic. 30 patients of group $\mathrm{A}$ $(56.67 \%)$ underwent radioactive iodine (RAI) ablation. Most second primary malignancies derived from breast $(37 \%)$, while less common primary sites were skin (8\%), larynx (7\%), colon (5.5\%), endometrium (5\%), prostate (4\%) and other rare sites. Group A included 53 patients (42\%), group B 60 patients $(48 \%)$ and group $\mathrm{C}$ included 13 patients $(10 \%)$. The mean age of onset of TC was significantly different $(\mathrm{p}=0.002)$ among three groups (group A: $50 \pm 12$ years, group B: $58 \pm 11$ years, group C: $53 \pm 15$ years).

Conclusions: The approximately same proportion of second primary malignancy in patients of groups A and B indicates that the appearance of a second primary malignancy is independent from the treatment applied for thyroid cancer. Large prospective studies are needed to verify our results. 


\section{P019 Thyroid Cancer \\ Therapeutics 2}

\section{P184 \\ THE IMPACT OF THE NUMBER OF HARVESTED CENTRAL LYMPH NODES ON THE LYMPH NODE RATIO AND POSTOPERATIVE ATHYROGLOBULINEMIA}

\section{Eun-Jae Chung ${ }^{1}$, Young-Soo Rho ${ }^{2}$}

${ }^{1}$ Ilsong Memorial Institute of Head and Neck Cancer, Hallym University, College of Medicine, ${ }^{2}$ Ewha Womans University, College of Medicine

Objects The purpose of this study was to analyze the impact of lymph node harvest on the lymph node ratio (LNR) and postoperative athyroglobulinemia.

Methods We prospectively enrolled 118 patients who underwent a total thyroidectomy and bilateral central lymph node neck dissection (CND). The inclusion criteria were as follows: (1) patients diagnosed preoperatively with PTMC (papillary thyroid microcarcinoma) by ultrasound-guided fine-needle aspiration biopsy (2) no evidence of central or lateral neck nodal metastasis after the preoperative clinical and imaging evaluation, and (3) patients who underwent a total thyroidectomy with a bilateral CND. Patients who had extracapsular spread or lymphovascular invasion in the final pathologic result (including minimal ECS) were excluded to eliminate other sources of bias.

Results The mean number of retrieved_lymph nodes in the central compartments was 6.5. There was a significant association between the number of retrieved nodes and the LNR. The high node volume group $(\geq 6.5)$ had a significantly higher rate of postoperative athyroglobulinemia ( $38.6 \%$ vs. $56.3 \%$ ). The multivariate analysis confirmed the number of retrieved lymph nodes in the central compartments was a risk factor for high LNR $(p=0.006$, odds ratio 3.848). The rates of vocal fold palsy and hypoparathyroidism did not differ according to the number of retrieved lymph nodes.

Conclusions The more lymph nodes that are retrieved during CND, the better the chance of clearing metastatic nodes in level VI.

Table 1. Multivariate analysis of the association between the lymph node ratio (LNR) and clinicopathologic variables (for Abstract P184)

\begin{tabular}{llll}
\hline Clinicopathologic variables & \multicolumn{3}{l}{ Lymph node ratio (LNR) } \\
\cline { 2 - 4 } & p-value & Odd ratio & $95 \%$ CI \\
\hline $\begin{array}{l}\text { Number of retrieved nodes } \\
\leq 6.5\end{array}$ & $0.006^{*}$ & 3.848 & $1.467-10.091$ \\
$>6.5$ & & & \\
\hline
\end{tabular}

\section{P185 \\ REOPERATIVE NECK DISSECTION IN DIFFERENTIATED THYROID CANCER WITH RECURRENT/PERSISTENT DISEASE: EFFICACY OF FIRST REOPERATION}

Livia Lamartina', Dana Hartl', Amandine Berdelou ${ }^{1}$, Abir Al Ghuzlan ${ }^{1}$, Borget Isabelle ${ }^{1}$, Desiree Deandreis ${ }^{1}$, Eric Baudin ${ }^{1}$, Haitam Mirghani', Martin Schlumberger ${ }^{2}$, Sophie Leboulleux ${ }^{1}$

${ }^{1}$ Gustave Roussy, Université Paris-Sud, Villejuif, France, ${ }^{2}$ Department of Nuclear Medicine and Endocrine Oncology, Gustave Roussy and Université Paris-Sud, Villejuif, France

Background: Persistent/recurrent disease in the neck occurs in up to $30 \%$ of patients with differentiated thyroid cancer (DTC).

Objective:To assess efficacy, safety, long-term outcome and prognostic factors of first neck reoperation (FR) in DTC patients without distant metastases treated in a tertiary centre.

Methods: Retrospective study of 157 consecutive DTC patients $(64 \%$ females, median age 35 years, 96\% papillary DTC) with FR between 1995-2013 for recurrent/persistent disease after initial treatment (surgery+radioiodine) with a follow-up of $\geq 6$ months. DTC was pT3 in $42 \%$ and pT4 in $10 \%$, pN1 in $74 \%(64 \% \mathrm{pN} 1 \mathrm{~b})$ and $25 \%$ had an aggressive histotype. The indication for surgery was determined by the tumor board. Following FR, disease status was either: complete remission (CR) (thyroglobulin $(\mathrm{Tg}) £ 1 \mathrm{ng} / \mathrm{mL}$ with normal imaging), biochemical incomplete response (BIR) $(\mathrm{Tg}>1 \mathrm{ng} / \mathrm{mL}$ or detectable anti-Tg antibodies with normal imaging), or persistent disease (PD) (abnormal imaging).

Results: The median number of metastatic lymph nodes (N1) removed on FR was 4 (mean size of the largest N1: $14 \mathrm{~mm}$ ). Extracapsular spread was found in 88 patients, thyroid bed relapse in 4 . One patient died of a complication (hematoma). Following FR, 19 patients had PD (12\%), 33 had BIR (21\%) and $104(67 \%)$ had CR among which 31 later relapsed. At final assessment, after a median follow-up of 5 years, $96(62 \%)$ patients had CR, of which 14 after further therapies. Nine patients initially with BIR had spontaneous CR secondarily. Rates of permanent hypoparathyroidism, laryngeal nerve palsy and other complications were $2 \%, 3.8 \%$ and $6 \%$. Age, aggressive pathology, bilateral and/or contralateral relapse, $>10 \mathrm{~N} 1$ at FR and N1 size $>10 \mathrm{~mm}$ were significantly associated with $\mathrm{BIR}+\mathrm{PD}$ at final assessment. Only male sex was an independent risk factor (p 0.001; OR 3.4 95\% CI 1.6-7.1). DTC.

Conclusion: In experienced hands FR is effective for persistent/recurrent

\section{P186 \\ THYROID PAPILLARY CARCINOMAS: COMPARISON BETWEEN THE CLASSICAL AND THE FOLLICULAR VARIANT AS TO CLINICAL PATHOLOGICAL CHARACTERISTICS AND FOLLOW-UP \\ Flávia Ramos ${ }^{1}$, Jose Vicente Tagliarini ${ }^{1}$, Emanuel Castilho ${ }^{1}$, Mariangela Marques ${ }^{1}$, Yoshio Kiy ${ }^{1}$, Carlos Roberto Padovani ${ }^{2}$, Celia Regina Nogueira ${ }^{1}$, Gláucia Mazeto ${ }^{1}$ \\ ${ }^{1}$ Botucatu Medical School - Sao Paulo State University - Unesp. \\ ${ }^{2}$ Institute of Bioscience - Sao Paulo State University - Unesp}

Objective: To compare patients with the classical (CPC) and follicular variant (FVPC) papillary carcinoma regarding clinical pathological characteristics, as well as postoperative evolution.

Methods: A retrospective chart review of patients was performed, comparing 100 cases of CPC and 34 cases of FVPC, as to its initial presentation (tumor characteristics, stage and postoperative follow-up exams), treatment [neck lymph nodes dissection; radioactive iodine $\left({ }^{131} \mathrm{I}\right)$ first $\left(1^{\text {st }}\right)$ dose, total number of doses and total cumulative dose] and status at the follow-up (time of disease free survival; disease-specific mortality; presence of persistence, recurrence, persistence/recurrence of the neoplasm and of active disease in the last evaluation). Parameters related to worst prognosis, defined as permanence and/or recurrence of the neoplasm or presence of active disease in the last evaluation, were also analyzed.

Results: Compared to FVPC group, CPC presented higher percentage $(\mathrm{P}<0.05)$ of cases with capsular invasion $(40 \%$ versus $23.1 \%)$, classification of the Latin-American Thyroid Society (LATS) as very low risk $(23 \%$ versus $6.1 \%)$, persistence (28\% versus $11.8 \%)$ and persistence/recurrence (33\% versus $17.6 \%)$ of the neoplasm. FVPC presented higher percentage of low risk cases $(69.7 \%$ versus $43 \%$; $\mathrm{P}<0.05)$, according to the staging proposed by the LATS, than CPC group. The remaining parameters did not differ between groups. The tumor variant had an influence on the persistence of the disease, with CPC presenting a higher risk than FVPC [OR: 4.009; $p=0.038$; CI $(95 \%): 1.0083 ; 14.843]$. The LATS staging was a predictor of active disease at the last evaluation [OR: $0.208 ; p=0.021 ; \mathrm{CI}(95 \%): 0.055 ; 0.793]$ : the high risk cases presented $79 \%$, the low risk, $45 \%$, and the very low risk, $15 \%$ of chances to exhibit it.

Conclusions: In this study, it has been observed that patients with CPC presented higher rates of capsular invasion and persistence/recurrence than the ones with FVPC. 
P187

PREDICTIVE FACTORS OF CONTRALATERAL CENTRAL LYMPH NODE METASTASIS IN UNILATERAL PAPILLARY THYROID CARCINOMA

Jun Ho Kim ${ }^{1}$, Yun Beom Ryu ${ }^{1}$, Lee Su Kim ${ }^{1}$

${ }^{1}$ Division of Breast and Endocrine Surgery, Hallym University Sacred

Heart Hospital, Hallym University College of Medicine

Introduction: The role of prophylactic bilateral central neck dissection (CND) in clinically node negative papillary thyroid carcinoma (PTC) patients is controversial. We aimed to investigate the incidence and the risk factors of contralateral central lymph node (LN) metastasis in patients with unilateral PTC.

Methods: A total of 107 patients who underwent total thyroidectomy and prophylactic bilateral CND for unilateral PTC were analyzed.

Results: Occult central neck LN metastasis was present in $23.4 \%$ $(25 / 107)$. Of these patients, contralateral central LN metastasis was present in $48 \%(12 / 25)$. The rate of contralateral central LN metastasis was significantly higher in cases of carcinoma with a maximal diameter of greater than $1 \mathrm{~cm}$, multifocality, thyroiditis or histologically proven metastasis to ipsilateral central LN $(P<0.05)$. Multivariate analysis showed that the presence of ipsilateral central LN metastasis was the only independent predictor for the presence of contralateral central LN metastasis (odds ratio $7.055,95 \%$ confidence interval 1.663 to $29.928 ; p=0.008$ ).

Conclusion: Ipsilateral central LN metastasis is a potential independent predictor of synchronous contralateral central LN metastasis in unilateral PTC. These finding may guide the necessity and extent of prophylactic bilateral or unilateral CND.

\section{P188 \\ ONE-STEP NUCLEIC ACID AMPLIFICATION (OSNA) ASSAY TO EVALUATE LYMPH NODE STATUS IN PATIENTS WITH PAPILLARY THYROID CARCINOMA}

Gabriel Obiols ${ }^{1}$, Zafon Carles², Iglesias Carmela ${ }^{1}$, Gonzalez Oscar ${ }^{1}$

Garcia-Burillo Amparo', Temprana Jordi', Serres Xavier ${ }^{1}$

${ }^{1}$ Hospital Vall D'hebron, ${ }^{2}$ Hospital Vall D'hebron; on Behalf of the

Consortium for the Study of Thyroid Cancer (Cecat)

Introduction: Routine prophylactic lymph node (LN) dissection is a matter of debate in papillary thyroid cancer (PTC). Accordingly, authors advocate finding diagnostic strategies to select those patients who may benefit from LN resection. OSNA (One-Step Nucleic Acid Amplification) is a molecular biological technique that allows the intraoperative evaluation of LN status in several human cancers. OSNA assay measures the amount of CK19 mRNA (copy numbers, $\mathrm{CN}$ ) which is directly related with the size of metastatic foci. The utility of OSNA in PTC has not been assessed.

Objective: To describe the results of OSNA analysis in a series of patients with PTC that underwent total thyroidectomy and LN dissection.

Material and Methods: Twelve consecutive patients with PTC were included. Selective sentinel LN biopsy (SSLNB) using a radioisotope method was used for identify LN. Sentinel LN status was evaluated by OSNA. Those SSLNB OSNA-positive cases underwent LN dissection of the affected compartment. All LNs were evaluated with imprint cytology and analysed by OSNA. According to OSNA results, LNs were classified as negatives, micrometastases and macrometastases.

Results: A total of $154 \mathrm{LN}$ were removed (nineteen were sentinel). Sixty percent LN were in the central compartment, and $32 \%$ in the lateral compartment. OSNA was positive in 57 (37\%) LN (25 micrometastases and 32 macrometastases). OSNA CN were significantly related with the weight of the LN $(\mathrm{p}=0.01)$. CN was higher in sentinel $\mathrm{LN}$ than in non sentinel LN $(p=0.01)$. There were no $\mathrm{CN}$ differences between both compartments. OSNA and imprint cytology analyses were concordant in $140(91 \%)$ cases, whereas 11 LNs were positive for OSNA but negative for cytology, and the contrary occurred in $3 \mathrm{LNs}$.

Conclusions: OSNA could be a useful technique to detect LN metastases in PTC.
P189

AGE IS THE ONLY RISK FACTOR RELATED TO DISEASE DEATH IN PAPILLARY THYROID CANCER PATIENTS WITH DISTANT METASTASIS AT THE TIME OF INITIAL SURGERY

Kenichi Matsuzu' ${ }^{1}$ Kiminori Sugino' ${ }^{1}$, Mitsuji Nagahama ${ }^{1}$, Wataru Kitagawa', Hiroshi Shibuya', Keiko Ohkuwa'1, Takashi Uruno', Akifumi Suzuki', Junko Akaishi', Chie Masaki', Fumi Saito ${ }^{1}$, Yuna Ogimi', Michikazu Kawano', Nobuyasu Suganuma ${ }^{2}$, Yasushi Rino ${ }^{3}$, Munetaka Masuda ${ }^{3}$, Koichi Ito

${ }^{1}$ Ito Hospital, ${ }^{2}$ Yokohama City University; Department of Surgery,

${ }^{3}$ Yokohama City University

Introduction: In general, prognosis of papillary thyroid cancer (PTC) is excellent, however small part of them will die of this disease. The aimof the present study were to investigate the long-term clinical outcome of PTC patients presenting with distant metastasis and to elucidate the risk factor for disease death.

Patients and Methods: Among 5700 PTC patients who underwent an initial surgery at our hospital between 1986 and 2004, 74 patients (1.3\%) who had distant metastasis at the time of initial surgery were adopted as the subjects. Patients' characteristics and clinical outcomes were collected retrospectively from original medical records. The median follow-up period was 8.9 years. Possible risk factors of cause specific survival (CSS) were analyzed by univariate and multivariate analyses using the Cox proportional hazard model. Analyzed factors were; age, sex, tumor size, extrathyroidal invasion, clinical lymph node metastasis, site of distant metastasis, and radioiodine avidity.

Results: There were 12 males and 62 females with a median age of 58 year-old (9-82 year-old). Median tumor size was $30 \mathrm{~mm}$. Twenty patients were died of this disease. The 5-, 10-, 15-, 20-year CSS rates were 85.3, 72.0, 62.5 and $62.5 \%$. Older age than 45 year-old at the time of initial surgery was the only significant factor for disease death (HR: 12.8, $\mathrm{p}=0.0003$ ). Other studied factors, including radioiodine avidity, were not associated with CSS.

Conclusion: Age is the only significant risk factor for disease death in PTC patients presenting with distant metastasis, and patients younger than 45 year-old can expect long survival.

\section{P190 \\ PILOTING A COMPUTERISED FOLLOW UP SYSTEM FOR THYROID CANCER PATIENTS WHO HAVE AN EXCELLENT RESPONSE TO INITIAL TREATMENT SUCH THAT THEIR SERUM THYROTROPHIN CAN BE IN THE LOW - NORMAL RANGE}

Georgina Gerrard ${ }^{1}$, Katie Spencer ${ }^{1}$, Lisa Owen ${ }^{1}$, Brinda Sethugavalar ${ }^{1}$, Lavanya Mariappan ${ }^{1}$, Robert D. Murray ${ }^{2}$

${ }^{1}$ St James Institute of Oncology, Leeds, UK, ${ }^{2}$ St James University

Hospital, Leeds, UK

Objectives: The 2014 British Thyroid Association Guidelines state that the need for TSH suppression should be based on dynamic risk stratification about a year after thyroidectomy and radioiodine (RRA). Patients with an excellent response to treatment i.e. stimulated thyroglobulin (sTg) $<1$ and a satisfactory neck ultrasound, should aim for the TSH in the low - normal range. Such patients are currently followed in outpatients and we plan to retrospectively risk stratify them, reduce their dose of levothyroxine and follow up via a computer system.

Methods: All patients in our region who have been given RRA for thyroid cancer since 2002 are to be contacted. They will be risk stratified based on a sTg and scans. They will be offered a consultation to discuss the risks and benefits of TSH suppression and supervise levothyroxine dose reduction. Post menopausal women will have a FRAX score and osteoporosis treatment if required.

Results: 1440 patients have been identified to be contacted. These patients were seen in endocrinology, oncology or nurse led clinics. sTg and scan results are available from electronic records but risk stratification can be difficult in the presence of $\mathrm{Tg}$ antibodies. The majority of patients are in the excellent response category. It can be challenging explaining that their levothyroxine dose can be reduced as they have often been warned that a high TSH can cause 
their cancer to return. Explanation of the risks of long term TSH suppression is necessary.

Conclusion: At the end of the project, patients who have had an excellent response to treatment over the last 12 years will have a target TSH in the low normal range. These patients could have life long follow up via a computerised recall system avoiding hospital clinic attendance. This is being piloted in our region and results will be presented.

\section{P191 \\ ONCOLOGIC OUTCOMES OF PAPILLARY THYROID CARCINOMA: A 16-YEAR SINGLE SURGEON EXPERIENCE}

Hyo Sang Park', Kang Dae Lee ${ }^{2}$, Hyoung Shin Lee ${ }^{2}$, Sung Won Kim ${ }^{3}$, Jun Woong Song ${ }^{3}$, Gi Yun $\mathrm{Nam}^{3}$, Jong Chul Hong ${ }^{4}$

${ }^{1}$ Sharing and Happiness Hospital, ${ }^{2}$ Department of Otolaryngology-

Head and Neck Surgery, Kosin University College of Medicine, ${ }^{3}$ Kosin

University College of Medicine, ${ }^{4}$ Dong-A University College of Medicine

Objective: We sought to evaluate the oncologic outcomes of patients with papillary thyroid carcinoma (PTC) who were initially treated at department of Otolaryngology Head and Neck Surgery at Kosin university Gospel hospital and to analyze the clinicopathologic factors related to recurrence and death due to PTC.

Methods: A retrospective chart review of 681 patients who initially underwent surgery at our center from 1998 to 2009 was performed. KaplanMeier curves and Cox proportional hazards model were used to identify risk factors for recurrence and death. Risk factors included age, sex, TNM status, extrathyroidal extension, local invasion, multifocality, bilaterality, lymph node metastasis and radioactive iodine treatment.

Results: Mean age of the patients was $46.6 \pm 12.9$ years (range 13-80 years) including $302(44.3 \%)$ patients $<45$ years and $379(55.7 \%)$ patients $\geq$ 45 years of age and $128(18.8 \%)$ male and $553(81.2 \%)$ female. Median follow up was 62 months (range 1-176 months). The 10-year disease free survival, disease specific survival and overall survival was $83.4 \%, 98.9 \%$ and $95.7 \%$, respectively. Male, T stage, $\mathrm{N}$ stage, lymph node metastasis, multifocality, bilaterality and local invasion were correlated to recurrence on univariate analysis while male $(\mathrm{OR}=2.230)$ and lymph node metastasis $(\mathrm{OR}=4.602)$ were independently related to recurrence. Male, age $\geq 45$ years, $\mathrm{T}$ stage, $\mathrm{N}$ stage, extrathyroidal extension, lymph node metastasis and local invasion were correlated to death due to PTC on univariate analysis while local invasion $(\mathrm{OR}=$ 26.907) was the only correlated factor on multivariate analysis.

Conclusion: Male and lymph node metastasis at initial surgery should be considered as high risk factors for recurrence, while patients with local invasion have high risk for death due to PTC.

\section{P192 \\ PATTERNS AND PREDICTIVE FACTORS OF CONTRALATERAL CENTRAL NODAL METASTASIS IN THYROID PAPILLARY CARCINOMA: PROSPECTIVE STUDY OF BILATERAL CENTRAL LYMPH NODE DISSECTION}

\section{Young-Soo Rho' ${ }^{1}$ Eun-Jae Chung ${ }^{2}$}

${ }^{1}$ Ewha Womans University, College of Medicine, ${ }^{2}$ Ilsong Memorial Institute of Head and Neck Cancer, Hallym University, College of Medicine

Objectives: The objective of this study was to determine the incidence and the risk factors for occult contralateral central neck lymph node metastasis.

Methods: A prospective study was conducted with data obtained from 121 PTC patients with clinically node-negative contralateral central neck between 2012 and 2013 at the Hallym University, College of Medicine. Total thyroidectomy and bilateral CCND was routinely performed in addition to thyroid surgery in all cases. The central neck compartment specimens were divided into dephian, ipsi/ contralateral paratracheal, and pretracheal department.

Results: Sixty-five patients (53.7\%) had central lymph node metastasis, and 32 patients $(26.4 \%)$ had lateral neck compartment lymph node metastasis. On univariate analysis, Age $<45(P<0.001)$, mean tumor size $\geq 1 \mathrm{~cm}(p=$ $0.007)$, extrathyroidal extension $(P<0.001)$, pretracheal nodal metastasis $(P<$
0.001), ipsilateral paratracheal nodal metastasis $(P<0.001)$, lateral neck nodal metastasis $(P<0.001)$, delphian nodal metastasis $(P<0.001)$ and number of retrieved lymph nodes in the contralateral paratracheal node $(p=0.026)$ were significantly associated with contralateral central lymph node metastasis. Age $<45$ ( $p=0.009$, odds ratio 0.084) and lateral neck compartment nodal metastasis $(p=0.011$, odds ratio 11.795$)$ were significantly correlated with contralateral central lymph node metastasis with multivariate analysis.

Conclusions Contralateral central lymph node metastases were significantly associated with Age $<45$ and lateral neck compartment node metastasis.

\section{P193 \\ THYROID CANCER OUTCOME IN CHILDREN AND YOUNG ADULTS}

Marinella Tzanela ${ }^{1}$, Christina Kanaka-Gantenbein ${ }^{2}$, Olga Karapanou ${ }^{3}$, Phoebe Rontogianni4, Aikaterini Dacou-Voutetakis ${ }^{2}$,

Stylianos Tsagarakis ${ }^{1}$

${ }^{1}$ Department of Endocrinology, Evangelismos Hospital, ${ }^{2} 3$ division of Endocrinology, Metabolism and Diabetes, 1st Department of Pediatrics, University of Athens, Aghia Sophia Children's Hospital, ${ }^{3} 1$ department of Endocrinology, Evangelismos Hospital, ${ }^{4}$ Department of Nuclear Medicine, Evangelismos Hospital

Thyroid cancer is relatively rare in children and young adults. Despite an increasing incidence of $1.1 \%$ annually, data on its outcome is scarce. In the present study we analyzed the presentation and outcome of such patients over the last 20 years. We retrospectively studied 97 patients, aged $15.8 \pm 0.31$ years (range 5-20 years, 21 males and 76 females). The majority of patients (78.3\%) underwent near total thyroidectomy; in $18.5 \%$ of patients lymph node dissection was also performed. Papillary thyroid carcinoma was found in $95.9 \%$ of patients, with aggressive histology (tall cell, insular, columnar cell carcinoma) in $14.4 \%$ of the patients. At diagnosis, $45.3 \%$ patients had lymph node metastases and $7.2 \%$ pulmonary metastases. Mean time of follow-up after diagnosis was $45.5 \pm 0.9$ months. At their latest follow-up $76.6 \%$ of patients were in remission (defined as undetectable $\mathrm{Tg}$ in the absence of antiTg, no evidence of neck disease by ultrasound and negative ${ }^{123} \mathrm{I}$ scan), while $23.3 \%$ had persistent disease. Patients with persistent disease had a higher prevalence of lymph node metastases at presentation $(87.5 \%$ vs $39.1 \%$ of patients on remission, $\mathrm{p}=0.006)$ and received a higher total therapeutic radioiodine dose compared to patients in remission $(104.64 \pm 8.6 \mathrm{mCi}$ vs $79.02 \pm 4.6 \mathrm{mCi}, \mathrm{p}=0.011)$. All patients with pulmonary metastases had papillary cancer (6 multifocal and 1 with a single foci of insular subtype) and 5 patients had additional neck lymph node involvement . They received a total ${ }^{131} \mathrm{I}$ dose of $379.9 \pm 46.3 \mathrm{mCi}$ in 2 to 4 doses. After $37.7 \pm 8.1$ months of follow up 3 patients were in remission while 4 had persistent but stable disease.

These findings demonstrate that lymph node metastases is the most important morbidity factor in children and young adult patients with thyroid carcinoma. Appropriate management with surgery and radioiodine, even in the presence of advanced disease, results in disease remission in the majority of patients.

\section{P194 \\ FOLLOW UP OF LOW RISK THYROID CANCER PATIENTS BY SPECIALIST NURSE PHONE CONSULTATIONS RATHER THAN VIA CLINIC VISITS}

Emma Dugdale ${ }^{1}$, Georgina Gerrard ${ }^{1}$, Lynn Priestley' ${ }^{1}$ Lavanya Mariappan ${ }^{1}$, Ee Siang Choong

${ }^{1}$ St James Institute of Oncology, Leeds, UK

Objectives: Low risk thyroid cancer patients have an excellent prognosis. Life long follow up is recommended to monitor thyroid function tests, treatment toxicity and to detect early recurrence. In 2009, to help the problem of limited capacity in clinics, nurse led telephone follow up was established. This study was to assess this service and to plan the future now that the phone clinic is full.

Methods: The patients eligible for the nurse led service had DTC treated with thyroidectomy and radioiodine and a stimulated thyroglobulin $(\mathrm{sTg})<1$ (at the nine month follow up scan), no Tg antibodies and an undetectable suppressed Tg. Patients on calcium supplementation were followed by endocri- 
nologists. Patients were given an appointment for the phone consultation and had blood tests prior to the appointment and the specialist nurse discussed the test results and symptoms over the phone. 50 patients using the service were obtained and a patient satisfaction survey was sent out.

Results: $40 / 50$ patients replied and $100 \%$ were very satisfied with the service. It avoided time off work, child care issues and travel into the city clinic. However, within 4 years the phone clinic was at capacity and no funding was available to expand it. The TSH target is in the low - normal range for patients with an excellent response to initial treatment. These patients could have annual thyroid function tests and Tg in the community via a computerised recall system and a patient questionnaire.

Conclusion: Life long follow up of thyroid cancer patients can be challenging because of increase in the numbers of patients requiring follow up and clinics become full. The specialist nurse phone clinic was a popular, effective and efficient mode of follow up but was at capacity after 4 years. Alternative methods of follow up which will be discussed.

\section{P195 \\ INTRAOPERATIVE RLN MONITORING (IONM): NORMATIVE RANGE ASSOCIATED WITH NORMAL POSTOPERATIVE VOCAL CORD FUNCTION: A PRELIMINARY STUDY \\ Young-Soo Rho ${ }^{1}$, Eun-Jae Chung ${ }^{2}$ \\ ${ }^{1}$ Ewha Womans University, College of Medicine, ${ }^{2}$ Ilsong Memorial Institute of Head and Neck Cancer, Hallym University, College of Medicine}

Objectives The objective of this study was to determine normative quantitative intraoperative EMG parameters associated with postoperative normal cord function.

Methods A prospective study was conducted with data obtained from 50 PTC patients (91 cases) with thyroid surgery by a single surgeon between 2012 and 2013 at the Hallym University, College of Medicine. Total thyroidectomy $(n=41)$ and hemithyroidectomy $(n=9)$ were performed. IONM quantitative parameters included threshold stimulation current, evoked amplitude during surgery at $1-\mathrm{mA}$ stimulation, normal evoked amplitude $(\mu \mathrm{V})$ at end of surgery, mean with the 5 th percentile and 95 th percentile. Postoperative laryngoscopy was performed until postoperative 6 months duration.

Results Mean threshold stimulation current was $0.289 \mathrm{~mA}(0.2-0.4 \mathrm{~mA})$. Mean amplitude at threshold stimulation was $130.6 \mu \mathrm{V}(63.8-237.9)$. Mean initial evoked amplitude were 315.5 (Rt, 160.6-619.6) and 369.5 (Lt, 109.8854.2). Mean evoked amplitude at the end of surgery for cricoaryenoid joint was $429.5 \mu \mathrm{V}(134.1-1038.3)$, and for $4 \mathrm{~cm}$ point from cricoaryenoid joint was $433.4 \mu \mathrm{V}(105.1-1017.5)$. IONM result was consistant with postoperative vocal cord function in $96 \%$ (48 among 50 patients).

Conclusions Final prognostic EMG parameters for IONM were correlate with normal vocal cord function. Thyroid surgeon can move to the second side of surgery during planned bilateral thyroid surgery after identifying the neuropraxic segment under IONM.

\section{P196}

Poster has been withdrawn

\section{P020 Thyroid Hormone Availability and Action}

\section{P197}

\section{ANALYZING THE ENERGY METABOLISM IN MCT8-DEFICIENT NEURONS}

Franziska Mever ${ }^{1}$, Eva K. Wirth', Ulrich Schweizer ${ }^{2}$

${ }^{1}$ Institut Für Experimentelle Endokrinologie, Charite Universitätsmedizin Berlin, ${ }^{2}$ Institut Für Biochemie und Molekularbiologie, Rheinische

Friedrich-Wilhelms-Universität Bonn

Thyroid hormones $(\mathrm{TH})$ influence the energy metabolism throughout the whole body. They act on heat production, skeletal muscle development, increase oxygen consumption and ATP production. For their local action, TH needs to enter the cell via specific transmembrane transporters. Among them, the monocarboxylate transporter 8 (Mct8) has been identified as the most specific one. Mutations in MCT8 lead to a severe form of psychomotor retardation in combination with low thyroxine (T4), high triiodothyronine (T3) and normal to elevated TSH levels in humans, the Allan-Herndon-Dudley syndrome. Expression of Mct8 in mouse brain, including neurons, suggested the syndrome to be caused by neuronal T3-deficiency. So far, nobody has analyzed the energy metabolism in Mct8-deficiency. By using the Extracellular Flux Analyzer (Seahorse Bioscience, Massachusetts, US) and a defined serum-free self-made growth supplement, we were able to measure the oxygen consumption rate (OCR) under different TH conditions in primary cultures of $M c t 8$ deficient neurons compared to wild type littermates. A five day treatment with or without T3 $(3 \mathrm{nM}, 10 \mathrm{nM})$ lead in both, WT and Mct8-deficient neurons, to an increase in OCR, whereas Mct8-deficient cells have a lower basal and maximal OCR. ATP production was not different in both cultures in the presence of T3. When we treat both cultures with or without T4 $(3 \mathrm{nM}, 10 \mathrm{nM})$, we showed that Mct8-deficient neurons cannot deal very well with both T4 concentrations in comparison to WT neurons concerning OCR as well as ATP production. That there is still an uptake of T3 indicates the presence of another TH transporter. One possible transporter is the L-type amino acid transporter 2 (Lat2). By the use of a specific Lat inhibitor we will test our hypothesis.

\section{P198}

\section{3-IODOTHYRONAMINE RESCUES $\beta$-AMYLOID DEPENDENT LONG TERM POTENTIATION IMPAIRMENT IN THE ENTORHINAL CORTEX}

Chiara Criscuolo ${ }^{1}$, Martina Sabatini ${ }^{2}$, Riccardo Donzelli ${ }^{2}$,

Alessandro Saba ${ }^{2}$, Riccardo Zucchi ${ }^{2}$, Nicola Origlia ${ }^{1}$

${ }^{1} \mathrm{Cnr}$ Neuroscience Institute, ${ }^{2}$ University of Pisa

Objectives: Beta-amyloid (Abeta) is the principal component of senile plaques, which represent a prominent feature of Alzheimer's disease (AD). However, loss of neuronal function and cognitive impairment precedes Abeta plaque formation. We have previously reported that soluble Abeta oligomers impair synaptic transmission and plasticity in the entorhinal cortex, an area crucially involved in cognitive functions and early affected in AD. Recently 3-iodothyronamine (T1AM), a novel endogenous chemical messenger, was shown to stimulate memory acquisition in mouse. Our aim was to investigate whether T1AM can exert a protective effect versus Abeta-induced synaptic plasticity impairment.

Methods: Synaptic function was studied recording extracellular field potentials evoked in cortical layers II-III of entorhinal cortex slices; long term potentiation (LTP) was elicited by high frequency stimulation. T1AM concentration was measured by HPLC coupled to mass spectrometry.

Results: We first evaluated the effects of T1AM perfusion, and identified a concentration $(5 \mu \mathrm{M})$ which does not interfere with either basal synaptic transmission or LTP induction and maintenance. As previously reported, Abeta oligomeric peptide at the concentration of $200 \mathrm{nM}$ was capable of inhibiting LTP without affecting basic synaptic transmission. However perfusion with T1AM before high frequency stimulation delivery was able to rescue LTP in Abeta treated slices. Assaying endogenous T1 AM concentration in the entorhinal cortex yielded values on the order of $6 \mathrm{pmol} / \mathrm{g}$.

Conclusions: Our results highlight the neuroprotective properties of T1AM, an endogenous thyroid hormone relative which can be detected in 
brain, and particularly in the entorhinal cortex. A better understanding of the mechanisms underlying the effect of T1AM against Abeta-induced neuronal dysfunction might allow the development of new pharmacological strategies to delay disease progression in $\mathrm{AD}$ patients.

\section{P199 \\ THYROID HORMONE TRANSPORTER LAT2: ANALYSIS OF SUBSTRATE CHARACTERISTICS BY VARIATION OF THYROID HORMONE LIKE SCAFFOLDS \\ Katrin Hinz ${ }^{1}$, Katja Meyer ${ }^{1}$, Michael Lisurek ${ }^{1}$, Josef Köhrle ${ }^{2}$, Anita Kinne ${ }^{1}$, Gerd Krause ${ }^{1}$ \\ ${ }^{1}$ Leibniz-Institut Für Molekulare Pharmakologie (Fmp), ${ }^{2}$ Institut Für \\ Experimentelle Endokrinologie, Charite-Univ. Medizin Berlin}

Thyroid hormones (TH) are transported across cell membranes by different transmembrane transporter proteins. We are studying transport mechanisms of the L-type amino acid transporter Lat2 in TH transport. Recently we investigated structure-function relationships of the Lat2 and identified it as a transporter for $3.3^{\prime}-\mathrm{T} 2$. Homology model-driven mutations lead to the localization of sensitive residues at Lat2 that are involved in its transport mechanism.

For a better understanding of the structure-function relationships we are now investigating the counterpart and study the transport of various substrates by oocytes from Xenopus laevis.

Using our own FMP in-house prepared database of commercially available screening compounds ( 9.1 million unique compounds from 39 vendors) a substructure search on various thyroid hormone-like scaffolds was performed. Out of the resulting sub-library, the most promising derivatives were manually chosen and ordered from the respective company.

Virtual screening at our Lat2 model of the resulting sub-library of potential substrates supported the selection of suitable compounds which we purchased. Moreover, it augmented the structure-function analysis for substrate interactions.

Uptake studies by Lat2 indicated that substrates even with bulky substituents at the first aromatic ring are transported. Enlargements at the second ring are transported when containing hydrophobic substituents.

The analysis of allowed conformational states revealed remarkable differences for the studied compounds. Transported substrates are more flexible and can form a straight but slim shape, which cannot be adopted by rigid and bulkier compounds such as T4 that are not transported.

Taken together we report substrate properties for Lat2: (i) An amino acid like moiety is needed likely as initial recognition site at Lat2 (comparable to other transporters like in AdiC), (ii) attached by bulky hydrophobic fragments and further (hydrophobic) enlargements. (iii) Conformational flexibility of the substrate must allow the formation of a straight but slim shape of the molecule.

\section{P200}

T3 REGULATED GENES IN BROWN ADIPOCYTES

Raquel Martinez de Mena ${ }^{1}$, Maria Jesus Obregon ${ }^{2}$

${ }^{1}$ Instituto de Investigaciones Biomédicas Uam-Csic, ${ }^{2}$ Inst.

Investigaciones Biomedicas, Csic

T3 has pleiotropic actions regulating many processes in different tissues. In brown adipose tissue $\mathrm{T} 3$ regulates thermogenesis, lipid metabolism and mitochondrial function. T3 actions include increases in UCP1, Dio2 and on many genes regulating lipid, carbohydrate and mitochondrial metabolism.

Aim: To explore the actions of $\mathrm{T} 3$ in brown adipocytes using genomic techniques.

Materials and Methods: Brown adipocytes in primary culture were grown with or without insulin and serum (24-48 h). T3 was added for $24 \mathrm{~h}$ and NE for $8 \mathrm{~h}$ to some groups. RNA was isolated and Agilent microarrays performed. Genomic results were analyzed and only significant differences were considered. Some genes were validated by quantitative PCR using Taqman probes.

Results: A large number of genes were regulated by T3 in brown adipocytes, belonging to carbohydrate metabolism, glycogen synthesis, ion transport, cell differentiation and communication, immunity, signal transduction and mRNA transcription and transcription factors. Among those up-regulated we found structural genes as keratin23 (Krt23, x27); lipid-related genes as adiponutrin (x7), Chrebp (x4), S14 (Thrsp), ME1, lipoamide (Pdhb); several SLCs as Glut4 (Scl2a4), tramdorin1 (Scl36a2), OATO3A1 (Slco3a1), Scl24a3 and mitochondrial transporters, known T3-regulated genes as Atp1b2 (x6), parvalbumin (x5), Fgf21, UCP1, UCP3, Adora2A, Sult1a1, alpha1-microglobulin/bikunin (Ambp) and others less studied as T3 targets as chondroitinsulfate-proteoglycan3 (Cspg3, x4), Hemopexin (hpx), Ret, Cd38 or Amphiregulin (Areg, S14). Less genes were downregulated as Pepkc1, K-voltage channel as Kcnh2 and tomoregulin (Tmeff1)

The results also show high synergy between T3 and NE for genes as UCP1 (x286), Dio2 (x25), Hsd17b2 (x10). Certain actions of T3 were enhanced in the absence of insulin (HydroxymethylglutarylCoA Synthase 2 (hmgcs2, x90), but others were inhibited because those genes require insulin (Me1, Tshr, Acaca, Scd1, Scl2).

Conclusions: Genomic techniques (microarrays) of brown adipocytes revealed potent regulation of gene expression by $\mathrm{T} 3$ and its synergies with $\mathrm{NE}$ and insulin.

\section{P201 \\ THE CHICKEN EMBRYO AS A MODEL FOR MCT8 DEFICIENCY DURING CEREBELLAR DEVELOPMENT}

Joke Delbaere $^{1}$, Stijn L.J. Van Herck ${ }^{1}$, Nele Bourgeois ${ }^{1}$,

Veerle M. Darras

${ }^{1}$ Laboratory of Comparative Endocrinology, Ku Leuven, Belgium

Objectives: Thyroid hormone (TH) deficiency has detrimental effects on brain development, among them an abnormal organization of the cerebellum. Biological TH activity is in part determined by the action of transporters facilitating the cellular uptake and efflux of iodothyronines. One of the key factors for TH entry into neuronal cells is the monocarboxylate transporter 8 (MCT8). We investigated the cell-specific expression profile and the impact of knockdown of MCT8 during cerebellar development using the chicken embryo as a model.

Methods: MCT8 mRNA distribution was studied in chicken cerebellum from embryonic day 6 to 18 (E6-E18). Different cell populations were identified by a combination of in situ hybridization and immunohistochemistry using cell-specific markers. Based on the expression data we set up an in vivo knockdown strategy to silence early MCT8 expression by electroporation of a chicken-specific miRNA-based RNAi construct in the cerebellar anlage at E3.

Results: MCT8 mRNA was already abundant in the cerebellum at E6. At this stage, we demonstrated co-localization with the Retinoic acid receptorrelated orphan receptor alpha (ROR $\alpha$ ), a marker controlling early Purkinje cell (PC) dendritic differentiation. At later stages, MCT8 expression was colocalized with the calcium-binding protein calbindin-D28k in PCs. Monitoring transfection efficiency after electroporation showed that expression of the MCT8 RNAi construct was predominantly localized in PCs and deep cerebellar nuclei, implicating that knockdown is highly efficient in both cell types. Analysis of knockdown embryos showed a reduction of ROR $\alpha$ expression in the transfected region. Embryos are further analyzed with respect to alterations in the degree of PC dendritic branching and synaptic contacts of climbing fibers with $\mathrm{PC}$ dendrites.

Conclusions: Our data show that MCT8 is already expressed in PC precursors during early cerebellar development. Detailed analysis of the neurological phenotype following in vivo knockdown will further unravel the role of MCT8 in PC development. 


\section{P202}

EVALUATION OF TRIAC ACTIVITY IN MCT8 KO MICE

Soledad Barez-Lopez ${ }^{1}$, Maria Jesus Obregon ${ }^{2}$,

Raquel Martinez de Mena ${ }^{1}$, Ainhoa Iglesias ${ }^{3}$, Jose Carlos Moreno ${ }^{4}$,

Juan Bernal ${ }^{5}$, Ana Guadaño-Ferraz ${ }^{1}$, Beatriz Morte ${ }^{6}$

${ }^{1}$ Instituto de Investigaciones Biomédicas Uam-Csic, ${ }^{2}$ Inst.

Investigaciones Biomedicas, Csic, ${ }^{3}$ Thyroid Molecular Lab. Institute for

Medical and Molecular Genetics (Ingemm). La Paz University Hospital,

${ }^{4}$ Molecular Thyroid Laboratory, Institute for Medical and Molecular

Genetics (Ingemm), La Paz University Hospital, Madrid, Spain,

${ }^{5}$ Instituto de Investigaciones Biomédicas Csic; Center for Biomedical

Research on Rare Diseases Ciberer; Center for Biomedical Research

on Rare Diseases, ${ }^{6}$ Instituto de Investigaciones Biomedicas Csic-Uam;

Center for Biomedical Research on Rare Diseases

The monocarboxylate transporter 8 (MCT8) is a thyroid hormone (TH)specific cell membrane transporter that is essential for T3 transport to the brain. MCT8 mutations lead to severe neurological impairments and abnormal serum TH levels. To date, therapeutic options for patients with MCT8 mutations are limited. The acetic acid derivative of T3 TRIAC has potential therapeutic value. Here we have analysed the in vivo effects of TRIAC on wild type (WT) and Mct8 knockout (KO) mice after administration of $30 \mathrm{ng} / \mathrm{g}$ bw/day in the drinking water from P21 to P30. TRIAC, T4 and T3 were measured by specific RIAs. Serum TRIAC reached the same concentration in WT and Mct8KO after treatment. TRIAC treatment greatly decreased serum T4 in the WT and the Mct8KO, and decreased T3 to normal levels in the Mct8KO. Serum T3 did not change in the WT after treatment. Liver D1 activity and mRNA were increased after TRIAC treatment in both genotypes. To assess the effect of TRIAC on the brain we measured D2 activity and the expression of the TH-regulated genes $\mathrm{Hr}, \mathrm{Cbr} 2$, Itih3, and Flywch2 in the cerebral cortex. D2 activity was elevated in the Mct8KO, and did not change after treatment, in correspondence with the low circulating T4. There were no effects of TRIAC on $\mathrm{Hr}, \mathrm{Cbr} 2$, and Itih3 expression, but Flywch 2 was reduced in the Mct8KO. The results indicate that TRIAC treatment induced a state of hypothyroidism partially compensated by its thyromimetic actions on peripheral tissues.

\section{P203 \\ MCT 8 AND LAT 2 EXPRESSION IN DIFFERENTIALLY FUNCTIONING BENIGN AND MALIGNANT THYROID TUMORS}

Dagmar Führer-Sakel', Julia Badziong1, Vera Tiedje', Saskia Ting ${ }^{2}$, Sien-Yi Sheu ${ }^{2}$, Lars C Moeller ${ }^{1}$, Jonas Weber ${ }^{3}$, Kurt Werner Schmid ${ }^{2}$, Klaudia Brix ${ }^{3}$, Denise Zwanziger ${ }^{1}$

${ }^{1}$ Department of Endocrinology and Metabolism; University of DuisburgEssen, ${ }^{2}$ Institute of Pathology; University of Duisburg-Essen, ${ }^{3}$ School

of Engineering and Science; Jacobs University Bremen

Objectives: Monocarboxylate transporter 8 (MCT8) is a specific thyroid hormone $(\mathrm{TH})$ transporter contrary to the L-type-amino acid transporter 2 (LAT2) which also transports other substrates. Both are located in several organs, including the thyroid. We hypothesized, that the expression of MCT8 and LAT2 expression is altered in benign and malignant thyroid tissue showing distinct biological properties.

Methods: MCT8 and LAT2 expression were evaluated by immunohistochemistry in 200 thyroid tumor samples of follicular adenoma (FA), follicular thyroid carcinoma (FTC), papillary thyroid carcinoma (PTC), poorly differentiated thyroid carcinoma (PDTC) and anaplastic thyroid carcinoma (ATC). Tumor staining intensity was graded by calculating the 'hybrid' $(\mathrm{H})$ score. Additionally, mRNA expression was determined by qRT-PCR of thyroid cancer and normal thyroid tissue. Moreover, protein expression of MCT8 and LAT2 were investigated by western blot analysis of the human benign nthy-ori cell line and different human thyroid cancer cell lines (B-CPAP, BHT-101,
C-643, FTC-133, -236, -238, K1, TPC-1) and the rat thyroid cell line PCCL3 cultured with and without TSH.

Results: The median H-Score for MCT8 and LAT2 differed between the entities (Table 1).

Strong upregulation of MCT8 and LAT2 mRNA was found in toxic adenoma (TA) compared to folicular adenoma. MCT8 and LAT2 protein expression was increased in PCCL3 cells cultered with TSH versus PCCL3 cells cultured without TSH.

Conclusion: Upregulation of MCT8 and LAT2 in TA is consistent with involvement of TH transporters in increased TH release during thyrotoxicosis. Partially altered TH transporter expression was observed in thyroid carcinomas, whereby LAT2 expression may be linked with other LAT2 transporter properties involving e.g. amino acid transport rather than TH efflux.

\section{P204 \\ LINKS BETWEEN HYPOTHALAMIC THYROID HORMONE SIGNALLING AND BAT THERMOGENESIS IN DIET-INDUCED OBESITY RESISTANT WSB/EIJ MICE}

Marie-Stéphanie Clerget-Froidevaux ${ }^{1}$, Stéphanie Decherf', Jérémy Terrien ${ }^{1}$, Bolaji Seffou ${ }^{1}$, Isabelle Seugnet ${ }^{1}$, James Bowers ${ }^{1}$, Barbara Demeneix ${ }^{1}$

${ }^{1} \mathrm{Mnhn} / \mathrm{Cnrs}$ Umr7221

The wild-type derived WSB/EiJ mouse strain is resistant to diet-induced obesity (DIO). Although euthyroid, this strain exhibits lower than average serum tT4 concentrations (Yuan et al., Aging Cell 8, 277-287 2009). Our initial studies confirmed the reported DIO-resistance and low tT4 of WSB/EiJ versus $\mathrm{C} 57 \mathrm{BL} / 6 \mathrm{~J}$ mice as well as their striking metabolic plasticity.

We tested the hypothesis that these WSB/EiJ phenotypic characteristics, notably metabolic plasticity, longevity and low tT4 levels, are inter-dependent. Studies were conducted in 3-month old male C57BL/6J and WSB/EiJ mice on different diets: control ( $10 \% \mathrm{Kcal}$ from fat), short-term or long-term high fat diet (HFD) (3 day or 8 week $45 \% \mathrm{Kcal}$ from fat, respectively).

We hypothesized that the DIO-resistance observed in WSB/EiJ mice could be influenced by a difference in central TH availability. This hypothesis derives from first, our observations on increased expression of deiodinase 2 (Dio2) mRNA levels in the hypothalamus of HFD WSB/EiJ mice and second, published data linking central T3 levels to energy expenditure (Lopez et al., Nat Med 16, 1001-1008 2010). Thus, in WSB/EiJ, HFD treatment could increase hypothalamic T3, driving increased BAT thermogenic capacity. Using Western-Blot, we compared UCP1 and PGC1 $\alpha$ expression in BAT from all groups studied. We also recorded BAT heat production using an infra-red thermal camera. We are currently investigating the implications of central $\mathrm{TH}$ availability in this thermogenic activity by ICV injections of rT3 to inhibit central D2 activity and measuring the downstream consequences on BAT thermogenic activity. We are also analysing hypothalamic and peripheral inflammation in all groups studied (HFD being known to induce inflammatory responses), hypothesising that another component of WSB/EiJ DIO-resistance could be a differential inflammatory response.

Our results provide mechanistic data to explain how WSB/EiJ mice maintain energetic balance under HFD, and the implication of thyroid signalling in this highly controlled metabolic plasticity.

Table 1. (for Abstract P203)

\begin{tabular}{llllll}
\hline Median H-Score & FA & FTC & PTC & PDTC & ATC \\
\hline MCT8 & 54.17 & 31.79 & 33.29 & 46.13 & 3.33 \\
LAT2 & 77.02 & 73.33 & 106.62 & 87.32 & 40.13 \\
\hline
\end{tabular}




\section{P205}

\section{A STUDY OF FETAL BRAIN STRUCTURE FOLLOWING SEVERE IODINE DEFICIENCY FETAL THYROIDECTOMY AND MATERNAL THYROIDECTOMY IN THE SHEEP}

Zorka Milicevic $^{1}$

${ }^{1}$ Department of Molecular Biology and Endocrinology, Institute of

Nuclear Sciences "Vinca" University of Belgrade, Belgrade, Serbia

A comparison has been made of the effect of a severely iodine deficient diet $(<10 \mu \mathrm{gI} / \mathrm{Kg})$ on sheep prenatal brain structure with that of successful fetal thyroidectomy at 50-60 days and at 98 days; and maternal thyroidectomy before pregnancy. Measurements of brain weight, DNA and protein content have also been carried out. These histological examinations include the cell density of various regions of the brain.

Developing brains are small with closely packed nerve cells when mitotic production of nerve cells comes to an end, and the subsequent large growth of brain volume is mainly due to elaboration of axonal-dendritic synaptic connections. Consequently, number of nerve cells in the tissue volume decreases, and thus declining neuronal density can be used as a measure of brain maturation. In brains fixed shortly before birth (140-150 days gestation), we have identified structural targets that are sensitive to thyroid or iodine deficiency.

In areas CA1 and CA4 of the hippocampus increased density of pyramidal cells $(+32 \%$ and $+19 \%)$ was found after development on the iodine deficient (I.D.) diet, while fetal thyroidectomy (FTX) at 56-98d gestation caused $24 \%$ or $12 \%$ increase in density, but maternal thyroidectomy (MTX) before conception had no detectable effect. In the cerebellum the connective molecular area was decreased by $22 \%$ after I.D. and $6 \%$ after FTX but was unaffected by MTX. The density of Purkinje cells was increased by $50 \%$ after I.D. and $13 \%$ after FTX but was unaffected by MTX. In contrast, the cerebellar granule cells, which are among the last neurons to be formed, were decreased in density by $15 \%$ after MTX.

Thus some sensitive targets in the structure of the cerebrum and cerebellum have been identified, and iodine deficiency has been found to cause more retardation of development than fetal thyroidectomy which in turn is more damaging than maternal thyroidectomy.

\section{P206 \\ EXPRESSION PROFILES OF THYROID HORMONE TRANSPORTERS MCT10 AND LAT2 WITHIN THE DIFFERENT INTESTINAL SEGMENTS OF YOUNG AND AGED WILD-TYPE MICE \\ Richard Voigtländer ${ }^{1}$, Petzsch Kristina ${ }^{1}$, Brix Klaudia ${ }^{2}$, \\ Schmidt Mathias ${ }^{1}$, Jaeger Andrea ${ }^{1}$, Rehders Maren ${ }^{2}$, \\ Zwanziger Denise ${ }^{1}$, Lars Moeller ${ }^{3}$, Ulrich Schweizer ${ }^{4}$, Dagmar Führer ${ }^{1}$ ${ }^{1}$ Department of Endocrinology and Metabolism and Division of Laboratory Research, University of Duisburg-Essen, Essen, Germany, ${ }^{2}$ Department of Cell Biology, Jacobs University Bremen, Bremen, Germany, ${ }^{3}$ University Duisburg-Essen, Department of Endocrinology and Metabolism, ${ }^{4}$ Rheinische Friedrich-Wilhelms-Universität Bonn, Institut Für Biochemie und Molekularbiologie}

Localized in the basolateral membrane of human small intestines, thyroid hormone transporters MCT10 and LAT2 are the proposed molecules involved in visceral thyroid hormone metabolism. To our best knowledge, the age-dependent expression patterns of these transporters have not been evaluated. Yet, alterations in transcription may be anticipated, because thyroid metabolism in general changes with age. We therefore analyzed the expression profiles of primary thyroid hormone transporter Mct8 and secondary thyroid hormone transporters Mct10, Lat1 \& 2, Ntcp and Oatps within the intestines of young and aged C57BL/6-mice by quantitative PCR. Young mice (age $=4$ months) and old mice (age $=18$ months) were sacrificed and RNA was isolated from the different intestinal segments. Highest expression levels were shown for candidate molecules Mct10 and Lat2, confirming their assumed role in small intestine. Other thyroid transporters could be neglected due to low transcription in intestinal tissues. We now examined Mct10- and Lat2-expression of old compared to young mice

\begin{tabular}{|l|c|c|}
\hline & Mct10 & Lat2 \\
\hline $\begin{array}{l}\text { Duodenum } \\
n=8 \text { (old) } n=5 \text { (young) }\end{array}$ & $\Phi /(\uparrow)$ & $\emptyset$ \\
\hline $\begin{array}{l}\text { Jejunum } \\
n=6 \text { (old) } n=4 \text { (young) }\end{array}$ & $\bigotimes /(\downarrow)$ & $\emptyset /(\downarrow)$ \\
\hline $\begin{array}{l}\text { Ileum } \\
n=8 \text { (old) } n=5 \text { (young) }\end{array}$ & $\downarrow$ & $\Phi /(\downarrow)$ \\
\hline $\begin{array}{l}\text { Colon } \\
n=8 \text { (old) } n=4 \text { (young) }\end{array}$ & $\bigotimes$ & $\varnothing /(\downarrow)$ \\
\hline
\end{tabular}

Age-dependent expresion pattern of Mct10 and Lat2 in the respective tissues. Old C57BL/6-mice (mean age $=18$ months) were compared to yound (mean age $=4$ months). $\downarrow$ : distinct down-regulation, $\Phi$ : no distinct difference, $(\uparrow)$ : latent up-regulation, $(\downarrow)$ : latent down-regulation.

Fig. 1. (for Abstract P206).

(Fig. 1). A distinct age-dependent down-regulation of Mct10 was observed in ileum. In the remaining intestinal parts transcription of both molecules seems to be unaffected from age. A latent up-regulation may be estimated for Mct10 in duodenum and jejunum and a latent down-regulation for Lat2 in jejunum, ileum and colon. In summary, our results have to be classified as preliminary and further analysis based on larger mouse groups and additional immunohistochemistry will be necessary. This project is funded by DFG SPP 1629 FU356/8-1.

\section{P021 Thyroid in Reproduction and Development 2}

\section{P207 \\ THYROID DYSFUNCTION AND THYROID AUTOIMMUNITY IN PREGNANT WOMEN}

\section{Hernando Vargas-Uricoechea}

Universidad del Cauca, Internal Medicine Department, Popayan-

Cauca, Colombia

Background: Thyroid Dysfunction (TD) and Thyroid Autoimmunity (TA) are prevalent among women of reproductive age and are associated with adverse pregnancy outcomes, the frequency of TD in pregnancy is of the $2-3 \%$, and is mainly caused by TA. The diagnosis is determinant because of the high risk of complications (postpartum hemorrhage, abruptio placentae, gestational hypertension in the mother and disordered brain development and/ or intrauterine growth retardation in the fetus).

Objective: Determine the frequency of TA in 121 pregnant women.

Materials and Methods: 121 pregnant women were screened during the third trimester of pregnancy, exclusion criteria were: antecedents of replacement with thyroxine or previous thyroid disease. TSH, FT4, FT3, Thyroid Peroxidase Antibodies (TPO-Ab) and Thyroglobulin Antibodies (Tg-Ab) testing was performed. The chemiluminescence assays for detection of TPO-Ab and Tg-Ab implemented on the IMMULITE 2000 system, a numerical result greater than or equal to $35 \mathrm{UI} / \mathrm{mL}$ indicates an elevated TPO-Ab level in 
Table 1. (for Abstract P208)

\begin{tabular}{|c|c|c|c|c|c|}
\hline & & $1^{\circ} \mathrm{T}$ & $2^{\mathrm{a}} \mathrm{T}$ & $3^{\circ} \mathrm{T}$ & $\begin{array}{l}\text { POSTPARTUM } \\
\text { (6/12 months) }\end{array}$ \\
\hline AbTPO (-) & TSH/FT4 & $\begin{array}{l}3.13 \pm 0.78 / \\
1.18 \pm 0.14\end{array}$ & $\begin{array}{l}3.19 \pm 1.4 \\
/ 1 \pm 0.10\end{array}$ & $\begin{array}{l}3.19 \pm 1.4 / \\
0.79 \pm 0.25\end{array}$ & $\begin{array}{l}2.22 \pm 1.24 / \\
1.18 \pm 1.93\end{array}$ \\
\hline \multirow[t]{2}{*}{ AbTPO (+) } & TSH/FT4 & $\begin{array}{l}2.52 \pm 1.05 / \\
1.19 \pm 0.12\end{array}$ & $\begin{array}{l}2.29 \pm 1.13 / \\
1.07 \pm 0.11\end{array}$ & $\begin{array}{l}2.52 \pm 1.05 / \\
0.97 \pm 0.93\end{array}$ & $\begin{array}{l}12.17 \pm 31.8 / \\
1.06 \pm 0.34\end{array}$ \\
\hline & $\%$ Ab ТРО & $100 \%$ & $76.5 \%$ & $52.9 \%$ & $95 / 100 \%$ \\
\hline
\end{tabular}

serum. Whereas that a numerical result greater than or equal to $40 \mathrm{IU} / \mathrm{mL}$ indicates an elevated $\mathrm{Tg}-\mathrm{Ab}$ level in serum.

Results: In this poblation, TPO-Ab and Tg-Ab were positive in $16 \%$ and $18 \%$ of women, respectively. 12 women $(10 \%)$ met criteria for subclinical hypothyroidism, wich $12 / 12(100 \%)$ had elevated TPO-Ab level and $11 / 12$ $(92 \%)$ had elevated Tg-Ab level. None of de women met criteria for subclinical hyperthyroidism.

Conclusion: The prevalence of TA and TD in pregnant women can be higher than normally described, for this reason, it is important to identify early and understand thyroid status in pregnant women in order to reduce potential maternal and fetal complications.

\section{P208 \\ PROSPECTIVE STUDY: IS THERE A RELATIONSHIP BETWEEN AUTOIMMUNITY AND THYROID ULTRASOUND IN PREGNANT WOMEN?}

Lorena Suarez Gutierrez ${ }^{1}$, Nuria Valdés ${ }^{1}$, Jessica Ares ${ }^{1}$,

María Galiana Rodríguez Caballero ${ }^{1}$, Lucía Díaz Naya ${ }^{1}$, Noelia Abello ${ }^{1}$, Edelmiro Menéndez Torre ${ }^{1}$

${ }^{1}$ Hospital Universitario Central de Asturias

Introduction: The function of the prognosis and diagnostic of thyroid ultrasound in pregnant women with positive thyroid autoimmunity is still uncertain. The aim of this study is to describe the development of the thyroid function and autoimmunity throughout pregnancy in a group with positive antibodies $(\mathrm{Ab})$ and its relationship with the characteristics of the thyroid ultrasound.

Methods: We carried out a descriptive and prospective cohort study comprised by 17 patients with positive $\mathrm{Ab}$ and 13 patients with negative $\mathrm{Ab}$, both showing thyroid normofunction. We measured TSH, free T4 and antiperoxidase $\mathrm{Ab}$ (TPO) at each pregnancy trimester (T) and also at 6 and 12 months postpartum. Besides, we made a thyroid ultrasound (US) at the end of the first pregnancy trimester and also at 6 months postpartum.

Results: We found no significant differences $\left(1^{\circ} \mathrm{T} \mathrm{P}<0.056,2^{\circ} \mathrm{T} \mathrm{P}<0.18\right.$ y $\left.3^{\circ} \mathrm{T} \mathrm{P}<0.81\right)$ on the levels of TSH and free $\mathrm{T} 4\left(1^{\circ} \mathrm{T} \mathrm{P}<0.56,2^{\circ} \mathrm{T} \mathrm{P}<0.82\right.$ y $\left.3^{\circ} \mathrm{T} \mathrm{P}<0.059\right)$ between the two groups.

Surprisingly, the rate of earlier abortions in this group was $52.9 \%$ versus $15.38 \%$ in the negative $\mathrm{Ab}$ group.

Conclusions:

1) We did not find hypothyroidism in the studied groups. There were no significant differences in the levels of TSH nor free T4, probably due to the reduced sample size and the low correlation between ultrasound and positive $\mathrm{Ab}$ TPO results.

2) In agreement with previous literature, this study shows a higher abortion risk in the positive $\mathrm{Ab}$ group.

\section{P209 \\ IODINE STATUS OF PREGNANT WOMEN FROM ISTANBUL}

Engin Oral', Abdullah Serdar Açıkgöz'1, Begüm Aydoğan², Berna Imge Aydoğan ${ }^{3}$, Hasniye Çelik Acıoğlư , Gökçe Anık İlhan ${ }^{4}$, Banu Dane ${ }^{5}$, Ayşegül Öze/5, Hakan Erene/2, Bülent Tandoğan', Erbil Çakar ${ }^{6}$, Herman Iş̧̧i ${ }^{7}$, Başak Kayan ${ }^{7}$, Halil Aslan ${ }^{8}$, Ali Ekiz ${ }^{8}$, Seda Sancak ${ }^{9}$, Ayhan Çelik ${ }^{9}$, Tevfik Yoldemir ${ }^{10}$, Özgür Uzun ${ }^{10}$, Murat Faik Erdoğan ${ }^{3}$

${ }^{1}$ İstanbul University Cerrahpaşa School of Medicine, Department

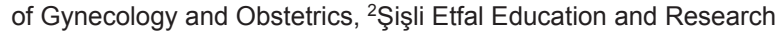
Hospital, Department of Gynecology and Obstetrics, ${ }^{3}$ Ankara University School of Medicine, Department of Endocrinology and Metabolism Diseases, ${ }^{4}$ Kartal Lütfi Kırdar Education and Research Hospital, Department of Gynecology and Obstetrics, ${ }^{5}$ Bezmi Alem University, Department of Gynecology and Obstetrics, ${ }^{6}$ Zeynep Kamil Obstetrics and Pediatrics Hospital, Department of Gynecology and Obstetrics, ${ }^{7}$ Florance Nightingale Bilim University, Department of Gynecology and Obstetrics, ${ }^{8}$ Kanuni Sultan Süleyman Education and Research Hospital, Department of Gynecology and Obstetrics, ${ }^{9} \mathrm{Fatih}$ Sultan Mehmet Education and Research Hospital, Department of Gynecology and Obstetrics, ${ }^{10}$ Marmara University Pendik Education and Research Hospital, Department of Gynecology and Obstetrics

Background: Iodine deficiency (ID), especially when it is severe, may have considerable consequences other than endemic goiter. ID in prenatal period and early childhood, affects cerebral development unfavorably.

Aim: The goal of this study was to evaluate the iodine status of pregnant women in İstanbul, where, median urinary iodine concentration(UIC) of School Age Children was $164 \mu \mathrm{g} / \mathrm{L}$ in 2007.

Method: In this multicenter study, 3700 pregnant women from eight different gynecology and obstetrics centers from the İstanbul City were recruited. Age, gestational weeks, smoking habbits, consumption of iodized salt, dietary salt restriction, history of stillbirth, abortus and congenital malformations were questioned. Spot urine samples were analyzed for(UIC) in Ankara University Iodine Laboratory. 2287 of 3700 samples had been completed at the moment.

Results: The mean age of subjects was 29.5 5.5. 621 women were in first trimester, 717 were in second trimester and 895 were in third trimester of pregnancy. The median UIC was $80 \mu \mathrm{g} / \mathrm{L}(1-324), 75 \mu \mathrm{g} / \mathrm{L}(1-600)$ and 70 $\mu \mathrm{g} / \mathrm{L}(1-750)$ in 3 trimesters of pregnancy, respectively. In the whole group UIC $<50 \mu \mathrm{g} / \mathrm{L}$ was observed in $35.4 \%$ (n:809), UIC $<150 \mu \mathrm{g} / \mathrm{L}$ was observed in $92.7 \%$ (n:2120) of pregnant women. Associations between the other study parameters will be given in the whole group .

Conclusion: Although the median UIC among SAC had been reported to be sufficient in İstanbul, in 2007, this study, showed that more than $90 \%$ of the pregnant women is facing with some degree of iodine defficiency during pregnancy. Results of the whole group and need for suplementation will be discussed in details. 


\section{P210 \\ IODINE NUTRITIONAL STATUS IN PREGNANT AND LACTATING WOMEN IN NORTH-EAST HUNGARY}

Mónika Katkó ${ }^{1}$, Laszlo Samson ${ }^{1}$, Zsuzsa Varga ${ }^{1}$, Miklos Bodor $^{1}$, Ildiko Hircsu' ${ }^{1}$, Andrea A. Gazso ${ }^{1}$, Domonkos M. Nagy ${ }^{1}$, Erika Galgoczi', Annamaria Gazdag ${ }^{1}$, Annamaria Erdei ${ }^{1}$, Endre V. Nagy ${ }^{1}$

${ }^{1}$ Division of Endocrinology, Department of Medicine, University of Debrecen

Mild iodine deficiency, which is present in North-East Hungary, often worsens during pregnancy and lactation. Pregnant and lactating women living in this area are supposed to be given iodine supplementation. However, not all pregnancy multivitamins contain iodine, and the household use of iodized salt is optional.

The aim of this study was to investigate factors affecting maternal iodine nutrition among pregnant and lactating women living in a mildly iodine deficient area in North-East Hungary. Intake of supplementary iodine, age, educational status and smoking habits were assessed by a questionnaire. As a biomarker of iodine status, spot urine iodine concentration corrected for creatinine and iodine concentration of breast milk were measured by inductively coupled plasma mass spectrometry (ICP-MS). Maternal smoking was verified by cotininuria.

During pregnancy and lactation, median urinary iodine excretion was 56.3 and $58 \mu \mathrm{g} / \mathrm{g}$ creatinine, respectively. $30 \%$ of pregnant women and nearly $50 \%$ of them following delivery did not take any form of iodine supplementation. In this study, the use of iodized salt alone was not enough to achieve adequate iodine intake in these life stages. Smoking and non-smoking mothers had similar levels of urinary iodine. However, breast milk of smoking mothers contained reduced iodine levels indicating the effect of thiocyanate. Smoking was found to be common in young, low educated women; the use of iodine containing supplements was also rare among them.

These results draw attention to the urgent need to review the Hungarian health policies on iodine deficiency control. Antenatal health promotion strategies should focus on the use of iodine supplementation, especially among less educated women in their early twenties.

The work is supported by the TÁMOP-4.2.2.A-11/1/KONV-2012-0031 project. The project is co-financed by the European Union and the European Social Fund.

\section{P211 \\ LOW-NORMAL LEVELS OF T4 FREE HORMONE AS A CAUSE OF GIRLS OVARIAN DISFUNCTION}

Maria Badalyan $^{0}$, Viktoria Hakobyan ${ }^{1}$, Gayane Bayburdian ${ }^{1}$

${ }^{1}$ Yerevan State Medical University

The study included 18 girls aged 14-18 with normosthenic constitution and with complaints of ovarian dysfunction during the last 6 months -2 years.

In $80 \%$ of cases (14 girls) there were noticed cognitive disfunctions (bad memory, attention, low mental activity) and also austenic symptoms (intense, tiredness, total fatigue, feeling of alarm, disfunction of sleep and/or daytime sleepiness).

Ultrasound examination of the thyroid gland revealed diffuse changes (10 girls $-55.6 \%$ )

Definition of the functional status of the thyroid gland: TSH $-2.96 \pm 1 \mathrm{uIU}$ $/ \mathrm{ml}$;

$\mathrm{T}_{4}$ free $-0.9 \pm 0.2 \mathrm{ng} / \mathrm{dl}$, which corresponds to low normal values of this hormone.

15 girls (83.3\%) receiving Levothyroxine (Euthyrox) at a dose of 50-75 $\mathrm{mg}$ had a recovery of menstrual cycles during 4-6 months.

It is necessary to take notice of the level of $\mathrm{T}_{4}$ free while searching for the reason of menstrual dysfunction at normal level of TSH.

\section{P212}

THE IODINE EXCRETION RATE AND ITS EFFECTS ON THYROID FUNCTION AND MORPHOLOGY DURING PREGNANCY IN AN IODINE SUFFICIENT REGION

Seda Sancak ${ }^{0}$, Mümtaz Ozarar ${ }^{1}$, Mehmet Sargın², Ömer Kan³, Gülgün Aslan ${ }^{4}$, Hasan Aydın ${ }^{5}$, Ali Özdemir ${ }^{3}$, Hakkı Muammer Karakaş ${ }^{1}$ ${ }^{1}$ Fatih Sultan Mehmet Training and Educational Hospital, Department of Radiology, Istanbul, Turkey, ${ }^{2}$ Kartal Lutfi Kirdar Education and Research Hospital, Diabetes Department, Istanbul, Turkey, ${ }^{3}$ Fatih Sultan Mehmet Training and Educational Hospital, Departman of Internal Medicine, Istanbul, Turkey, ${ }^{4}$ Kartal Lutfi Kirdar Education and Research Hospital, Departman of Internal Medicine, Istanbul, Turkey,

${ }^{5}$ Yeditepe University Medical Faculty, Department of Endocrinology and Metabolism, Istanbul, Turkey

Background and Aims: It is well known that if increased demand for iodine during pregnancy cannot be met, it may result in thyroid dysfunction and changes in the morphology of the thyroid gland. The aim of this study was to assess the adequacy of iodine status during pregnancy and its effects on thyroid function and morphology in Istanbul City which is accepted as an iodine sufficient region.

Materials and Methods: Otherwise healthy pregnant women with singleton pregnancies $(n=292$, mean age $29 \pm 5$ years $)$ in their first trimester were included into the study.

Thyroid volume by ultrasound examination, thyroid function tests (TSH, free T4, and free T3) and thyroid autoantibodies (thyroid peroxidase antibody and thyroglobulin antibody) and urinary iodine excretion were measured at first and third trimester of pregnancy. The pregnant women were divided into three categories: euthyroid $(n=207)$, hypothyroid $(n=40)$ and hyperthyroid $(\mathrm{n}=45)$.

Results: Considering all pregnant women, urine iodine excretion rate was found to be lower than the recommended (mean $108.8 \pm 66.7 \mu \mathrm{g} / \mathrm{L}$ ). Twentyone percent of women had severe iodine deficiency and only $49.6 \%$ were adequate. There was no correlation between iodine excretion rate and thyroid function test and thyroid volume throughout the pregnancy. Demographic characteristics were comparable between groups. Urinary iodine excretion rate was similar in each group. It did not differ between first and third trimester in each group. Thyroid autoantibody prevalence was similar among groups. There was no correlation between thyroid function tests, urinary iodine excretion (108.8 \pm 66.7 and $92.5 \pm 55.9$ respectively), thyroid autoimmunity and the thyroid volume in any of the groups.

Conclusion: Iodine deficiency is still a problem of pregnant women in Turkey even in iodine sufficient regions. However, this does not affect the thyroid function or thyroid volume. The effect on children is not known yet.

\section{P213}

\section{PREVALENCE AND PERINATAL OUTCOME} OF EARLY ISOLATED MATERNAL HYPOTHYROXINEMIA

Raluca Maria Furnica ${ }^{1}$, Damien Gruson ${ }^{2}$, Chantal Daumerie ${ }^{3}$

${ }^{1}$ Department of Endocrinologie, Cliniques Universitaires Saint-Luc, Université Catholique de Louvain, Brussels, ${ }^{2}$ Department of Laboratory Medicinecliniques Universitaires Saint-Luc, Université Catholique de Louvain, Brussels, ${ }^{3}$ Cliniques Universitaires Saint-Luc; Université Catholique de Louvain

Objectives: Isolated hypothyroxinemia $(\mathrm{IH})$ is defined as a thyroxine level below the $5^{\text {th }}$ percentile of the pregnancy related reference range and a normal TSH. The prevalence and the consequences of IH remain unknown. Perinatal outcome and effect on neurocognitive development are subject of controversies.

The aims of our study were to evaluate the prevalence of IH in the first trimester of pregnancy and the impact of maternal IH on the perinatal outcome.

Methods: The study population consisted of 742 consecutive singleton pregnant women followed in our tertiary center between July 2012 and July 2013. IH was defined for values of FT4 below the 5 th percentile $(\leq 0.7 \mathrm{ng} / \mathrm{dl}$, DxI Beckman Coulter) (n: 103). Women with overt thyroid dysfunction (n: 20) or other disease influencing thyroid hormone levels were excluded (n: 7). 
Table 1. Reference ranges for TSH, fT3, fT4 in subsequent trimesters (for Abstract P215)

\begin{tabular}{llll}
\hline & 1st trimester $(\mathrm{n}=175)$ & 2nd trimester $(\mathrm{n}=175)$ & 3rd trimester $(\mathrm{n}=175)$ \\
\hline TSH, mIU/1 & $0.009(0.008-0.010)$ & $0.050(0.014-0.110)$ & $0.110(0.034-0.195)$ \\
& $3.177(2.963-3.830)$ & $3.442(3.051-3.770)$ & $3.530(2.944-3.943)$ \\
\hline fT3, pmol/1 & $3.63(3.43-3.71)$ & $3.29(3.16-3.41)$ & $3.10(2.92-3.26)$ \\
& $6.55(6.23-6.84)$ & $5.45(5.20-5.64)$ & $5.37(5.04-5.55)$ \\
\hline fT4, pmol/1 & $11.99(11.52-12.15)$ & $10.46(10.34-10.67)$ & $8.96(8.63-9.62)$ \\
& $21.89(20.84-23.92)$ & $16.67(16.03-17.20)$ & $17.23(16.20-18.32)$ \\
\hline
\end{tabular}

The selected IH women (n: 76) were matched with singleton pregnant women without hypothyroxinemia (FT4 $\geq 0.8 \mathrm{ng} / \mathrm{dl}$ ).

Maternal characteristics, infant variables and perinatal obstetrical complications, fetal growth restriction and neonatal morbidity were compared.

Results: FT4 values below the 5 th percentile were found in 76 women $(11 \%)$. The mean age at the time of pregnancy was 32 years $( \pm 4.7)$ and the mean weight was $66 \mathrm{~kg}( \pm 12.8)$. Thyroid parameters were evaluated between 11 and 13 weeks of gestation.

The hypothyroxinemia group had a higher rate of gestational diabetes $(10 \%)$ compared with the control group $(4.5 \%)$. Ten infants $(13 \%)$ were preterm in $\mathrm{IH}$ versus $2.2 \%$ of the control group. No other significant difference were observed

Conclusions:

In our population, the prevalence of IH was $11 \%$.

Isolated hypothyroxinemia was associated with a higher gestational diabetes and neonatal hypotrophy.

\section{P214}

\section{IODINE NUTRITION AND THYROID FUNCTION IN PREGNANT WOMEN OF OVIEDO}

Edelmiro Menendez ${ }^{1}$, Antonio Rabal Artal ${ }^{1}$, Lorena Suarez Gutierrez ${ }^{1}$, María Galiana Rodríguez Caballero ${ }^{1}$, Jessica Ares Blanco ${ }^{1}$,

Lucía Díaz Naya ${ }^{1}$

${ }^{1}$ Hospital Universitario Central de Asturias

Background and Objective:In Asturias (Norhern región of Spain), where iodine deficiency was eradicated in school children by the year 2000 , iodine deficiency persisted in pregnant women, who were recommended to use of iodine supplementation. The aim of this study was to determine the iodine nutrition of pregnant women in our area and whether or not iodine supplements are needed.

Material and Methods: Throughout May and June 2013 we studied the iodine nutrition and thyroid function during the first trimester of pregnancy in 173 women in the health area of Oviedo.

Results: The median urinary iodine was $197 \mathrm{~g} / \mathrm{L}$. Iodinated supplements were used by $47 \%$ of women, which had a yoduria median higher than those not taking iodinated supplements (247 vs. $138 \mathrm{~g} / \mathrm{L} ; \mathrm{P}<.001)$, and also a higher TSH (2.30 vs $1.94 \mathrm{mU} / \mathrm{L})$ although not significantly different. Yoduria was also higher in women who took more than 2 servings of dairy products (median: $230 \mathrm{~g} / \mathrm{L}$ ) than those who took less (median: $191 \mathrm{~g} / \mathrm{L}$ ). Within the group of women who were not taking iodine supplements, those regularly using iodized salt in the kitchen $(47 \%)$ had a median urinary iodine concentration of $190 \mathrm{~g} / \mathrm{L}$ indicating iodine sufficiency.

Conclusions: Iodinated supplements seem unnecessary nowadays in pregnant women of Oviedo who regularly take iodized salt and our recommendation in these cases should be to continue the use of iodized salt in the recommended amounts during pregnancy and consume at least two daily servings of milk or dairy products

\section{P215}

REFERENTIAL VALUES OF TSH AND FREE THYROID HORMONES IN HEALTHY PREGNANT WOMEN - A MULTICENTER STUDY IN POLAND

Marta Kostecka-Matyja ${ }^{1}$, Ewa Bar-Andziak ${ }^{2}$, Tomasz Bednarczuk ${ }^{2}$, Monika Buziak-Bereza'1, Paulina Dumnicka ${ }^{3}$, Anna Fedorowicz ${ }^{1}$, Maria Górska ${ }^{4}$, Małgorzata Krasnodębska², Beata Niedźwiedzka², Dorota Pach ${ }^{1}$, Marek Ruchała ${ }^{5}$, Katarzyna Siewko ${ }^{4}$, Bogdan Solnica ${ }^{6}$, Jerzy Sowiński ${ }^{5}$, Małgorzata Szelachowska ${ }^{4}$,

Małgorzata Trofimiuk-Müldner ${ }^{1}$, Katarzyna Wachowiak-Ochmańska ${ }^{5}$ Alicja Hubalewska-Dydejczyk ${ }^{1}$

${ }^{1}$ Chair and Department of Endocrinology, Jagiellonian University, Collegium Medicum, Krakow, Poland, ${ }^{2}$ Department of Internal Medicine and Endocrinology, Medical University, Warsaw, Poland, ${ }^{3}$ Department of Medical Diagnostics, Jagiellonian University, Collegium Medicum, Krakow, Poland, ${ }^{4}$ Department of Endocrinology, Diabetology and Internal Medicine, Medical University, Bialystok, Poland, ${ }^{5}$ Department of Endocrinology, Metabolism and Internal Medicine, University of Medical Sciences, Poznan, Poland, ${ }^{6}$ Department of Diagnostics, Chair of Clinical Biochemistry, Jagiellonian University, Collegium Medicum, Krakow, Poland

Objectives: For a few years the endocrinologists' community has been discussing the best-care of pregnant women with an existing thyroid dysfunction, and the proper assessment of their thyroid function.

"Guidelines for the Diagnosis and Management of Thyroid Disease During Pregnancy and Postpartum" published in 2012, recommend setting the referential values for Thyroid Hormones $(\mathrm{TH})$ by a local laboratory. Considering a current difference within geophysical, socioeconomic conditions, and iodine prophylaxis in every population, it's advisable to assess referential values of $\mathrm{TH}$ specific to a region of residence.

The objective was to assess the referential values of TSH, fT3, fT4 in respective trimesters of pregnancy for Polish women.

Methods and Results: Our study included pregnant women from four centers in Poland, (175 patients). The age range was $27-47$ years. Healthy pregnant women had the following qualifications: a negative thyroid disease, a normal thyroid peroxidase antibodies range, a valid thyroid ultrasound image, a proper iodine prophylaxis, and a normal TSH in newborns. TSH, fT3, fT4, and anti-TPO were performed in each trimester.

The reference intervals were evaluated by percentile, as recommended by the International Federation of Clinical Chemistry, using the bootstrap method.

Conclusions: Reference ranges for pregnant women when compared to the general population showed a lower concentration of TSH in every trimester of pregnancy and lower fT 4 in $2^{\text {nd }}$ and $3^{\text {rd }}$ trimester.

Presenting referential values will help in better care of pregnant women. It will prevent misdiagnosis and inadequate treatment. 


\section{P216 \\ IODINE STATUS, THYROID VOLUME AND NODULARITY DURING PREGNANCY}

Aynur Aktaş', Ünsal Aydın'1, Berna Imge Aydoğan², Kudret Erkenekli Gönül Koç ${ }^{1}$, Zehra Berberoğlu', Yalçın Aral', Murat Faik Erdoğan ${ }^{2}$

${ }^{1}$ Ankara Education and Research Hospital, Department of

Endocrinology and Metabolism Diseases, ${ }^{2}$ Ankara University School

of Medicine, Department of Endocrinology and Metabolism Diseases,

${ }^{3}$ Ankara Zekai Tahir Burak Obstetrics Hospital, Department of

Gynecology and Obstetrics

Background: Thyroid gland undergoes functional and anatomical changes during pregnancy. iodine has long been recognized an important micronutrient for maternal thyroid as well as fetal growth and development.

Aim: The goal of this study was to asses the iodine status thyroid function, volume, nodularity of pregnant women in Ankara, which is reported to be a borderline iodine sufficient environement in 2007.

Method: A total of 315 pregnant women participated in this longitudinal study. Subjects with a history of thyroid dysfunction were excluded. Pregnant women were studied trough first, second and third trimester for thyroid function tests, thyroid volume, nodule formation and growth, and urinary iodine concentrations(UIC), without any intervention.

Result: The mean age of subjects was $28(17-45)$. The percentage of pregnant women with UIC below $150 \mathrm{mcg} / \mathrm{L}$ were $77 \%, 83 \%, 93 \%$ for women in first, second, third trimester of pregnancy, respectively. Analysis for other study parameters are stil going on.

Conclusion: Although the median UIC among SAC had been reported to be borderline sufficient in Ankara, in 2007, this study, showed that about $77 \%$ of the pregnant women were iodine defficient at the first trimestr. UIC significantly fall throughout the pregnancy without intervention and $93 \%$ of the pregnant women, from Ankara is facing with some degree of iodine defficiency in the last trimestr of pregnancy. Results on thyroid volume and nodularity and need for suplementation will be discussed in details.

\section{P217 \\ THYROID VOLUME AND CALCITONIN LEVELS DURING PREGNANCY}

Michela Perrino $^{1}$, Danila Covelli ${ }^{1}$, Beatrice Vigo ${ }^{1}$, Laura Fugazzola ${ }^{1}$, Guia Vannucchi ${ }^{1}$

${ }^{1}$ Endocrine Unit, Fondazione Ca' Granda Irccs, University of Milan, Italy

The increase of thyroid volume (TV) during pregnancy has been reported by some Authors. Moreover, calcitonin (CT) is known to promote the outgrowth of trophoblast cells and it is predicted to increase during pregnancy. We enrolled 151 women (mean age 32.3 years, range 21.2-45.9) before the 12 week of pregnancy and evaluated TV and CT levels during pregnancy. TPOAb were evaluated only at baseline, while serum CT and TSH were measured at each trimester. In addition, the thyroid volume was assessed by ultrasound at each trimester by the same expert, and defined according to the ellipsoid formula. A familial history for thyroid diseases was present in $66 \%$ patients, while $25 \%$ of women had a previous diagnosis of nodular goiter or hypothyroidism. Interestingly, at the time of enrollment, 59/151 (39\%) women were on iodine supplementation, and 70/151 (46.3\%) had adequate iodine salt intake. Any significant TV variation was observed during pregnancy: mean TV was $8.9 \pm 2.5 \mathrm{ml}$ at baseline, and $8.8 \pm 2.6 \mathrm{ml}$ and $9.4 \pm 2.7 \mathrm{ml}$ in the second and third trimester, respectively $(\mathrm{P}=0.15)$. In patients with nodular goiter at baseline, no variations in the number/volume of nodules were observed. In one patient without thyroid lesions at baseline, the appearance of a $6 \mathrm{~mm}$ solid nodule was recorded during the second trimester. In nine women, L-T4 replacement therapy was introduced during pregnancy due to the detection of TSH levels higher than the normal values for pregnancy. Interestingly, mean $\pm \mathrm{SD}$ serum CT levels were $2.1 \pm 0.8 \mathrm{ng} / \mathrm{dl}$ at baseline and remained stable at the second $(2.1 \pm 0.7)$ and third trimester $(2.0 \pm 0.6)(P=0.14)$. Serum $C T$ levels did not correlate with neither TV $(\mathrm{P}=0.59)$ nor BMI $(\mathrm{P}=0.54)$.

In conclusion, we demonstrated that TV remains stable during pregnancy, at least in women with a sufficient iodine supplementation. Moreover, no variation of serum CT levels were observed during pregnancy.

\section{P218 \\ IN A MILDLY IODINE DEFICIENT PREGNANT POPULATION SERUM TG CONCENTRATION IS A WEAK INDICATOR OF IODINE STATUS}

Eftychia Koukkou ${ }^{1}$, Ioannis llias ${ }^{1}$, Irene Mamali², Georgios Adonakis ${ }^{3}$, Stavros Kravaritis ${ }^{3}$, Kostas Markou ${ }^{0}$

${ }^{1}$ Endocrine Department, "Elena Venizelou" Hospital, Athens, ${ }^{2}$ Division of Endocrinology, University of Patras Medical School, Patras, ${ }^{3}$ Obst

and Gyn Clinic, University of Patras Medical Scho

Objectives: Iodine status of a population is traditionally evaluated mainly by Urinary Iodine Excretion (UIE). Serum Tg is a suitable marker of the recent iodine status in a population. hCG stimulates thyroid hormone secretion by binding to the TSH receptor. The aim of our study is to estimate the reliability of $\mathrm{Tg}$, as a marker of iodine intake, in pregnancy.

Subjects and Methods: 299 pregnant women (mean age \pm SD: $30.52 \pm 4.83$ years $)$ in the first $(n=91)$, second $(n=83)$ and third $(n=125)$ trimester were studied. In every trimester Urine Iodine Excretion (UIE), plasma TSH, Tg, fT4, anti-TPO and hCG levels were measured. Normality of the measured parameters was assessed with Kolmogorov-Smirnov test. Statistics were done with correlation matrix implementing Spearman's rank correlation, the Kruskal-Wallis test and with Quade's nonparametric analysis of covariance; statistical significance was set at $\mathrm{P}<0.05$.

Results: Tg was weakly negatively correlated to UIE ( $\rho:-0.128, p: 0.028)$, fT4 was negatively correlated to TSH and the trimester of pregnancy ( $\rho$ : -0.160 , p: $0.006 \& \rho:-0.570, p: 0.001$, respectively) and positively to hCG ( $\rho$ : +0.179 , p: 0.002). The latter fell significantly as pregnancies advanced (first, second and third trimester medians: 35925, $11500 \& 13570 \mathrm{mIU} / \mathrm{mL}$, respectively, p: 0.0001), while UIE did not show any appreciable variation over time (first, second and third trimester medians: $124.3,132.0 \& 125.7 \mu \mathrm{g} / \mathrm{L}$, respectively, ns). When covarying the effect of UIE, Tg levels were fairly stable from the first to second trimester (medians: $14.4 \& 13.4 \mathrm{ng} / \mathrm{mL}$, respectively, p: 0.176 ) and increased significantly in the third trimester (median: $18.4 \mathrm{ng} / \mathrm{mL}$, p: 0.025).

Conclusion: In contrast to what happens in the general population, it seems that in pregnancy serum $\mathrm{Tg}$ concentration is a weak indicator of iodine status, at least in a mildly iodine deficient population. This is probably due to $\mathrm{hCG}$ interference in thyroid function.

\section{P022 Case Reports 2}

\section{P219 \\ HYALINIZING TRABECULAR TUMOR OF THE THYROID: A CASE REPORT \\ Sorina Martin ${ }^{1}$, Minodora Betivoiu' ${ }^{2}$, Florin Andrei ${ }^{3}$, Cosmin Giulea ${ }^{4}$ Simona Fica ${ }^{5}$ \\ ${ }^{1}$ Elias Hospital, Endocrinology Department; Carol Davila University of Medicine and Pharmacology, Endocrinology Department, ${ }^{2}$ Elias Emergency Hospital, Endocrinology Department, ${ }^{3}$ Elias Hospital, Pathology Department, ${ }^{4}$ Elias Hospital, Surgery Department, ${ }^{5}$ Elias Emergency Hospital, Endocrinology Department; Carol Davila University of Medicine and Pharmacology, Endocrinology Department}

Introduction: Hyalinizing trabecular tumor (HTT) is a very rare and controversial thyroid tumor that was first described by Carney and colleagues in 1987. This tumor has morphological and architectural similarities with paraganglioma, medullary thyroid carcinoma and the nuclear features and RET/ PTC1 rearrangements of papillary thyroid carcinoma, which makes its diagnosis challenging.

Case Report: We report the case of a 50-year-old woman, nonsmoker, with no family history of thyroid disorders, known with a $10 \mathrm{~mm}$ right thyroid lobe nodule for 15 years, slightly growing, with repeatedly normal thyroid function. She presented in November 2013 with complaints of cervical discomfort, anxiety and tremor. Thyroid ultrasound revealed a $16 / 10 \mathrm{~mm}$ hypoechoic right thyroid lobe nodule with irregular borders, microcalcifications and high internal blood flow on Doppler imaging, no pathological limph nodes. TSH, FT4, TPOAb, anti-thyroglobulin antibodies and calcitonin were 
in normal ranges. Fine needle aspiration was recommended and refused by the patient, so thyroidectomy was decided. Pathology report raised the suspicion of HTT or medullary thyroid carcinoma (MTC). IHC: TTF1 diffuse positive in tumor cells, Calcitonin negative, CROMO negative, Ki 67 characteristic intense cytoplasmic tumor reaction, diagnostic for HTT. After surgery substitutive treatment with L-thyroxine was started. The patient is followed with physical examination, TSH, FT4, serum thyroglobulin, anti-thyroglobulin antibodies and neck US.

Conclusions: HTT can pose diagnostic difficulties in the differentiation from MTC and experienced pathologists should be aware of its presence and its similarities with other thyroid tumors types. Even if HTT is considered a benign neoplasm, a rigorous diagnostic and regular monitoring of patients are necessary to avoid the very rare cases of malignant behavior with local invasion and metastases or a tumor recurrence.

\section{P220 \\ ATYPICAL PRESENTATION OF UNCONTROLLED GRAVES' DISEASE - POSTERIOR REVERSIBLE ENCEPHALOPATHY SYNDROME}

\section{Aileen Grace Salalima - Dela Paz ${ }^{1}$, Christy Yao}

${ }^{1}$ The Medical City - Section of Endocrinology and Metabolism

Introduction: The clinical spectrum of Posterior Reversible Encephalopathy Syndrome (PRES) is rarely reported in the presence of autoimmune thyroid disease. We report a case of uncontrolled Graves' disease in thyroid storm, with radiologic findings and neurologic manifestations consistent with PRES.

Clinical Case: A 33-year-old Filipino woman, with Graves' disease for 7 years, presented with sudden complete loss of vision and thyroid storm (Burch and Wartofsky score - 80). Cranial MRI showed multiple cortical/subcortical foci of T2/FLAIR prolongation in the occipital lobes and parasagittal regions of parietal and frontal lobes which were bilateral and symmetric in distribution. Baseline thyroid function tests showed a TSH of $0(0.35-4.94 \mathrm{uIU} / \mathrm{mL})$, elevated FT3 and FT4 at $6.11(1.71-3.71 \mathrm{pg} / \mathrm{mL})$ and $2.29(0.70-1.48 \mathrm{ng} /$ dL) respectively. She was given Propanolol and Propylthiouracil, which was later shifted to Methimazole.

Spontaneous improvement in vision was noted a few hours after onset of symptoms. On the $5^{\text {th }}$ hospital day, she had sudden dyspnea and went into cardiopulmonary arrest. The patient was resuscitated after nine minutes and underwent therapeutic hypothermia. Intravenous antibiotics for possible aspiration pneumonia and vasopressors were given. The patient developed acute renal failure with multiple electrolyte imbalance thus renal replacement therapy was started. She had multiple arrhythmias with findings of diastolic failure secondary to hyperthyroidism and ventricular diastolic dysfunction.

The patient's sensorium gradually improved and she was discharged with complete recovery of vision and no neurologic deficits after more than one month of hospitalization. Repeat cranial MRI done outpatient showed resolution of previous neuroimaging findings. Autoimmune work-up (ANA) was negative. She underwent RAI therapy 3 months after admission and is presently clinically and biochemically euthyroid.

Conclusion: PRES can lead to significant morbidity and mortality. Early determination of its underlying etiology is necessary for opportune management.

\section{P221}

\section{THYROID CANCER IN PATIENTS WITH HYPERTHYROIDISM}

Martin Taylor ${ }^{1}$, Anthony Skene ${ }^{1}$, Joan McCutcheon 1

${ }^{1}$ Royal Bournemouth Hospital

For many years it was established dogma that patients that presenting with a toxic multinodular goitre are unlikely to have a thyroid cancer; although this is true (as thyroid cancer is uncommon), it is now recognised that toxic multinodular goitres not infrequently contain foci of papillary thyroid carcinoma.

We review the evidence that patients with hyperthyroidism are at increased risk of thyroid cancer and whether this increased risk merits further investigate prior to definitive treatment form hyperthyroidism whether it be surgical treatment or I131 treatment.
We present three patients with hyperthyroidism due to differing aetiological cause who came to surgery and were found to have occult thyroid carcinomas.

A 44 year old women presented with a toxic nodule confirmed on technetium scan. She was offered lobectomy or radioiodine treatment. Due to childcare issues she opted for surgery and was found to have an unsuspected $8 \mathrm{~mm}$ papillary carcinoma of the thyroid adjacent to the toxic nodule, with a separate $2 \mathrm{~mm}$ focus of papillary carcinoma in lymphoid tissue.

The second patient, a 58 year old woman with a history of Graves Disease who had severe thyroid eye disease. Because of concerns about the use of radio-iodine in the presence of thyroid eye disease she proceeded to near total thyroidectomy. The patient was found to have a $9.5 \mathrm{~mm}$ minimally-invasive follicular carcinoma of the thyroid.

The third patient, a 63 year old woman had her primary treatment modalities at another Hospital. She presented with a large goitre and was found to have a hot nodule. The patient underwent a thyroidectomy and was found to have an incidental $0.9 \mathrm{~mm}$ papillary carcinoma of the thyroid. She was treated with ablative radio-iodine.

We review Risk Stratification and Prognosis for patients with Differentiated Thyroid Carcinoma when diagnosed in the presence of concurrent hyperthyroidism.

\section{P222 \\ REVERSIBLE THYROTOXICOSIS DUE TO DESTRUCTIVE THYROIDITIS AFTER PERCUTANEOUS ETHANOL INJECTION THERAPY (PEIT) IN AN INOPERABLE PATIENT WITH TOXIC ADENOMA AND UNSTABLE ANGINA PECTORIS}

Johannes W. Dietrich ${ }^{1}$, Apostolos Chatzitomaris ${ }^{2}$, Michael Scheeler ${ }^{2}$ Andreas Muegge ${ }^{2}$, Harald H. Klein ${ }^{3}$, Assem Aweimer ${ }^{2}$

${ }^{1}$ Bergmannsheil University Hospitals; Medical Hospital I; Dept. of

Endocrinology \& Diabetes, ${ }^{2}$ Bergmannsheil University Hospitals,

${ }^{3}$ Bergmannsheil University Hospitals Bochum

It is known for nearly 200 years that thyrotoxicosis may affect cardiovascular function. Often, cardiovascular symptoms are the most characteristic and disabling manifestations of hyperthyroidism. Toxic adenoma is a common cause for thyrotoxicosis in iodine-deficient or previously iodine-deficient regions. Treatment of toxic adenoma with radioiodine may take several months to be effective and can lead to short-term worsening of cardiovascular function. Percutaneous ethanol injection therapy (PEIT) is an alternative therapeutic approach. The sclerosing properties of ethanol have been recognized for many years, and the mechanism of action is coagulative necrosis and concomitant small vessel thrombosis. Markers of efficacy comprise thyroid function, scintigraphy, and ultrasonography including changes in nodular volume. Here we present the case of a 65-year-old in-patient who suffered from coronary heart disease with unstable angina and thyrotoxicosis due to toxic adenoma. Since the risk of thyroid surgery was expected to be high and the time-scale of radioiodine ablation would be too long to be effective we conducted PEIT in 3 sessions, in each of these $5 \mathrm{ml}$ ethanol $95 \%$ was injected in the toxic adenoma under ultrasonographic guidance. The presented side effect was neck pain. PEIT resulted in a transient rise of fT3 and fT4 suggesting destructing thyroiditis, however without worsening of clinical symptoms. Within 5 days hormone levels returned to normal values. Additionally, a normalized ${ }^{99 \mathrm{~m}} \mathrm{Tc}-$ uptake was demonstrated in a second scintigraphy. Concluding, PEIT is an alternative approach in the management of toxic adenomas and should be preserved for patients who cannot or will not undergo standard therapy. However, it may be associated with destructive thyroiditis, resulting in transient rise of circulating thyroid hormones. 


\section{P223 \\ MASSIVE THYROID METHASTASIS FROM LONGSTANDING BREAST CARCINOMA}

Mario Cappagli ${ }^{0}$, Prospero Magistrelli ${ }^{1}$, Paolo Dessanti ${ }^{2}$ Antonella Montepagani ${ }^{3}$, Michele Moroni ${ }^{4}$, Pierfrancesco Bonfanti ${ }^{5}$

${ }^{1}$ Surgery Department S.Andrea Hospital La Spezia, ${ }^{2}$ Pathology

S.Andrea Hospital La Spezia, ${ }^{3}$ Endocrinology S.Andrea Hospital La

Spezia, ${ }^{4}$ Pathology S.Andrea Hospital la Spezia, ${ }^{5}$ Surgery S.Andrea

Hospital La Spezia

Case History: A 48 yrs old female arrived at Endocrinology Department for a lasting tachycardia. She was operated for breast cancer, first time 31 years old and again, for relapse, 40 years. Cancer follow-up was negative still one year ago. Clinical examination showed: stable weight $(70 \mathrm{Kg})$; sweaty skin; normal cardiovascular and respiratory apparatus; blood pressure: 140/90; two firm nodules, one for each thyroid lobe: thyroid US showed an enlarged gland, many hypoechoic micronodular areas and hypoechoic nodules of $41 \mathrm{~mm}$ on the right lobe and $21 \mathrm{~mm}$ on the left one without calcifications; no pathological lymphnode was appreciated. TSH, free T4, free T3 and Calcitonin were normal; AbTpo (108 UI/ml) and AbTg $(71 \mathrm{UI} / \mathrm{ml})$ moderately elevated. ${ }^{99 \mathrm{~m}} \mathrm{TC}$ scan showed some cold areas, two of them corresponding to the hypoechoic nodules. The first clinical diagnosis was: non toxic autoimmune multinodular goiter. A FNAB of both nodules was performed with this cytological result: "epithelial cells, isolated or groups with enlarged hyperchromic nucleus, poor cytoplasm, suggesting carcinoma". Himmunochemistry: TTF1 negative and ER positive.

Conclusion: methastatic thyroid infiltration from breast cancer (BC). The patient was submitted to thyroidectomy and cervical lymphnode dissection of central compartment. Hystology confirms: "diffuse methastatic thyroid infiltration from $\mathrm{BC}$ ".

Comment: Thyroid methastasis from breast cancer are rare but they can appear also ten years later from diagnosis of BC. It is possible that thyroid high blood flow facilitate neoplastic cell implantation. In this patient cytology and immunochemistry were able to get an accurate diagnosis. The peculiarity of this case report is the long time from breast cancer diagnosis and the massive neoplastic infiltration which substituted almost completely the normal thyroid tissue.

\section{P224 \\ IMMUNOGLOBULIN G4-RELATED ORBITOPATHY MIMICKING GRAVES' ORBITOPATHY}

Erdei Annamaria ${ }^{1}$, Steiber Zita ${ }^{2}$, Ujhelyi Bernadett ${ }^{2}$, Szanto Antonia ${ }^{3}$, Miklós Bodor ${ }^{4}$, Eszter Berta ${ }^{5}$, Annamaria Gazdag ${ }^{6}$, Katko Monika ${ }^{1}$, Nagy Endre ${ }^{1}$

${ }^{1}$ Department of Medicine, Division of Endocrinology, University of Debrecen, Debrecen, Hungary, ${ }^{2}$ Department of Ophthalmology, University of Debrecen, Debrecen, Hungary, ${ }^{3}$ Department of Medicine, Division of Immunology, University of Debrecen, Debrecen, Hungary, ${ }^{4}$ Department of Medicine, Division of Endocrinology, University of Debrecen, Debrecen, Hungary; Division of Clinical Pharmacology, Faculty of Pharmacy, University of Debrecen, Debrecen, Hungary, ${ }^{5}$ Division of Endocrinology, Department of Medicine, University of Debrecen, Debrecen, Hungary; Division of Clinical Pharmacology, Faculty of Pharmacy, University of Debrecen, Debrecen, Hungary, ${ }^{6}$ Division of Endocrinology, Department of Medicine, University of Debrecen

Introduction: Immunoglobulin G4 (IgG4)-related disease is a recently described entity characterized by lymphoplasmacytic infiltration and tissue fibrosis. It typically affects the lacrimal glands, orbits, salivary glands, lungs, pancreas, biliary ducts and retroperitoneal tissue.

Case Report: A 25-year-old woman has been referred to the endocrine clinic with suspected Graves' orbitopathy. Bronchial asthma and recurrent skin rashes of unknown aetiology beginning 10 years earlier were present in her medical history. She was treated for dacroadenitis in 2009. She had eyelid swelling during her pregnancy in 2012, which had been considered the consequence of pregnancy. Her symptoms worsened after delivery; eyelid redness and retrobulbar pain evolved. The degree of exophthalmos measured by Hertel exophthalmometry was $24 / 105 \mathrm{~mm}$ in the right side and 21/105 $\mathrm{mm}$ in the left side. Orbital MR showed eyelid oedema and swollen superior and lateral rectus muscles in both orbits. Thyroid hormone levels were in the normal range and aTPO, aTG and TRAb were all negative. In addition, the patient complained about a palpable "nodule" in the left side of her face, which was diagnosed as an unusual swollen limb of the parotid gland. Based on her medical history, laboratory and imaging results, the possibility of IgG4-related orbitopathy has been raised. On oral methylprednisolone, rapid improvement in the status of the eyes was observed. Elevated IgG4 level reconfirmed the diagnosis.

Conclusions: IgG4-realetd orbitopathy may easily mistaken for endocrine orbitopathy. All patients with suspected Graves' orbitopathy in whom thyroid autoantibodies are not present have to be evaluated towards IgG4-related orbitopathy.

\section{P225 \\ INTERFERON-ALPHA-INDUCED GRAVES DISEASE IN A PATIENT WITH CHRONIC hePATITIS C AND PRE-EXISTING AUTOIMMUNE THYROIDITIS}

Pedro Rodrigues ${ }^{1}$, Joana Menezes ${ }^{1}$, Cláudia Nogueira ${ }^{1}$,

Davide Carvalho

${ }^{1}$ Endocrinology, Diabetes and Metabolism Department, Centro

Hospitalar São João; Faculty of Medicine, Porto University

Introduction: Interferon-induced thyroiditis is a major clinical problem for patients receiving interferon-alpha (INF- $\alpha$ ) treatment for chronic hepatitis $\mathrm{C}$ virus $(\mathrm{HCV})$ infection. INF- $\alpha$-treated patients are at increased risk for developing thyrotoxicosis, especially those with pre-existing thyroid autoimmunity. Thyrotoxicosis in this setting can be due to either painless thyroiditis or Graves' disease.

Case Report: A 53-year-old male patient with chronic HCV infection, genotype $3 \mathrm{a}$, was treated with pegylated INF- $\alpha$ and ribavirin for 6 months. Prior to INF- $\alpha$ therapy, laboratory tests showed normal thyroid function, negative antithyroglobulin antibodies (TgAbs) $1.4 \mathrm{UI} / \mathrm{mL}(<4.11)$ and positive antithyroid peroxidase antibodies (TPOAbs) $14.4 \mathrm{UI} / \mathrm{mL}(<5.61)$. Thyroid ultrasound revealed a discrete inhomogeneous pattern and no nodules. During treatment he maintained normal thyroid function but higher TPOAbs titer 192.5 UI/mL. Eight months after cessation of INF- $\alpha$ therapy he developed clinical features of thyrotoxicosis with weight loss, increased appetite, palpitations, nervousness and fatigability. On physical examination he presented a symmetrical goiter and fine distal tremor. No eye involvement. Laboratory assays revealed TSH $0.001 \mu \mathrm{UI} / \mathrm{mL}(0.35-4.94)$, free $\mathrm{T}_{4} 2.09 \mathrm{ng} / \mathrm{dL}(0.70-$ $1.48)$ and free $T_{3} 16.83 \mathrm{pg} / \mathrm{mL}(1.71-3.71)$. Diagnosis of Graves' disease was confirmed by the presence of TSH receptor antibodies (TRAbs) $21.8 \mathrm{U} / \mathrm{L}$ $(0-1.8)$ in addition to a diffuse homogeneous high radioiodine uptake at ${ }^{131} \mathrm{I}$ thyroid scintigraphy. HCV infection was not cured. Since he had persistently elevated liver transaminases, more than 3 times the upper limit of normal, it was decided to perform ${ }^{131} \mathrm{I}$ therapy. Three months after radioiodine treatment ( $7 \mathrm{mCi}$ ) he presents TSH $0.31 \mu \mathrm{UI} / \mathrm{mL}$, free $\mathrm{T}_{4} 0.70 \mathrm{ng} / \mathrm{dL}$ and free $\mathrm{T}_{3} 3.65 \mathrm{pg} /$ $\mathrm{mL}$.

Conclusions: It is postulated that INF- $\alpha$ induces thyroiditis by immune stimulatory effects and by direct effects on the thyroid. Regular screening and monitoring of thyroid dysfunction during and after INF- $\alpha$ therapy is essential in order to obtain early detection and appropriate treatment.

\section{P226 \\ AGGRESSIVE TREATMENT OF A 51 YEAR OLD PATIENT WITH ANAPLASTIC THYROID CANCER WITH OVER ONE YEAR SURVIVAL}

Lars C Moeller ${ }^{1}$, Vera Tiedje ${ }^{1}$, Saskia Ting ${ }^{2}$, Kurt Werner Schmid ${ }^{2}$,

Dagmar Führer-Sakel ${ }^{1}$

${ }^{1}$ Department of Endocrinology and Metabolism; University of

Duisburg-Essen, ${ }^{2}$ Institute of Pathology; University of Duisburg-Essen

Anaplastic thyroid cancer (ATC) is a very rare and aggressive disease with a median survival of 5-6 months. The American Thyroid Association proposes a combined radiochemotherapy.

We present a 51 year old male patient diagnosed with ATC in the beginning of December 2012. No metastases were detected in the initial staging. After R1 resection of the tumor, the patient received radiation therapy (cumulative dose $60.4 \mathrm{~Gy}$ ). He was referred to our centre in March 2013. On staging the patient 
showed pulmonary metastases. Molecular pathological analysis of primary tumor tissue showed absence of BRAF, KRAS, ALK, EGFR and PI3KCa mutations. After three cycles of chemotherapy with Carboplatin (AUC2) and Paclitaxel $\left(50 \mathrm{mg} / \mathrm{m}^{2}\right)$ re-staging showed stable pulmonary metastases, but new hepatic lesions. We then treated the patient with the vascular disrupting agent Fosbretabulin in combination with a higher dosage of carboplatin (AUC6) and paclitaxel $\left(200 \mathrm{mg} / \mathrm{m}^{2}\right)$, according to the protocol by Sosa et al. which has recently been evaluated in 80 ATC patients and has been shown to increase progression free survival. Unfortunately, the patient had progressive disease after three treatment cycles. Thus, we offered the patient a third line treatment with Sorafenib. Under this treatment the patient showed partial response and stable disease during 4 months. In the staging in December 2013 progressive disease was diagnosed. We offered a forth line treatment with vandetanib. The state of the patient worsened quickly in January and February 2014 and the patient died in the beginning of march.

This case demonstrates that the treatment of ATC is a challenge due to its very aggressive nature and the lack of an established therapy. Therefore, we propose a uniform treatment protocol for Germany in order to harmonize and prospectively evaluate treatment of ATC using a molecular based approach.

\section{P227}

\section{A NOVEL NUCLEAR IMAGING METHODS IN MEDULlaRY THYROID CANCER: A CASE REPORT}

Hrvojka Tomic Brzac ${ }^{1}$, Sanja Kusacic Kuna ${ }^{1}$

Gordana Horvatic Herceg ${ }^{1}$, Drazen Huic ${ }^{1}$

${ }^{1}$ Clinical Department of Nuclear Medicine and Radiation Protection,

Clinical Hospital Centre Zagreb

Introduction: Patients with residual thyroid medullary cancer disease postoperatively can be easily identified biochemically (by elevated calcitonine level) and then could be selected for further evaluation. Unfortunately, radiographic detection of distant metastatic disease is unlikely when the $\mathrm{Ct}$ level is $<250 \mathrm{pg}=\mathrm{mL}$, and no single imaging test provides optimal whole-body imaging. Fluorodeoxyglucose positron emission tomography (FDG PET) is less sensitive to identify metastases of medullary thyroid cancer. The aim of report is to present a novel nuclear imaging methods in detecting of unknown foci of distant metastases in patient with medullary thyroid cancer.

Case Report: A 51-year old women referred for ultrasound examination because of diarrhea of unknown origin. An ultrasound of the neck showed node in the upper pole of left thyroid lobe, and suspitious nodes in the neck. Ultrasound guided fine needle-aspiration biopsy with immunocitochemistry revealed medullary thyroid carcinoma with metastatic lymph nodes in neck region and patient underwent total thyreoidectomy with a lateral neck dissection. Patohistologic finding confirmed medullary thyroid cancer. Three years later patient underwent left lateral dissection because of metastatic disease on contralateral side of the neck. During next few years, an elevated calcitonin level persist, but in spite of extensive examination a precise localisation of metastatic site could not found. Finally, patient underwent to SPECT/ CT scintigraphy with Tc-99m-tektrotyde that showed suspected accumulation of activity in sacrum. At that time, positron emission tomography with 18 F-DOPA was performed and showed multiple foci of pathologic accumulation (in three other places except sacral region). In conclusion, a novel imaging methods in nuclear medicine could be ussefull in cases of persistent elevation of calcitonin or CEA level in patients suspected for metastatic medullary thyroid carcinoma.

\section{P228}

\section{ALOPECIA AREATA IN A PATIENT WITH AUTOIMMUNE THYROID DYSFUNCTION} Joana Oliveira ${ }^{1}$, Pedro Rodrigues $^{1}$, Paula Freitas ${ }^{2}$, Davide Carvalho² ${ }^{1}$ Endocrinology, Diabetes and Metabolism Department, Centro Hospitalar São João; Faculty of Medicine, Porto University, ${ }^{2}$ Endocrinology, Diabetes and Metabolism Department, Centro Hospitalar São João; Faculty of Medicine, Porto University - Porto

Introduction: Alopecia areata(AA) is a common form of localized, nonscarring hair loss. AA is frequently associated with autoimmune thyroid disorders, which occur in 8 to $11 \%$ of patients. The presence of antithyroglobulin antibodies(TgAbs) and antithyroid peroxidase antibodies(TPOAbs) seems to be associated with the disease. However, the association with TSH receptor antibodies(TRAbs) is less frequently reported.

Case Report: A 34-year-old woman was referred to an endocrinology appointment due to subclinical hyperthyroidism - TSH $0.05 \mu \mathrm{UI} / \mathrm{mL}(0.25-5)$, FT4 $1.14 \mathrm{ng} / \mathrm{dL}(0.89-1.76)$ and positive antithyroid autoantibodies - TgAbs 96.8 U/mL $(<18)$, TPOAbs $213.9 \mathrm{U} / \mathrm{mL}(<8)$. She complained of weight loss, thin hair and irritability and denied tachycardia, insomnia, diarrhoea or ocular symptoms. There was no history of recent exposure to iodinated contrast or other iodinated products. Physical examination revealed a goiter but no palpable nodules, murmur or thrill. She had normal heart rate $(84 \mathrm{bpm})$, no tremor or eye involvement. Seven weeks later, she developed intense fatigue, distal tremor and palpitations. Laboraty tests showed clinical hyperthyroidism - TSH $0.001 \mu \mathrm{UI} / \mathrm{mL}(0.25-5)$, FT4 $2.85 \mathrm{ng} / \mathrm{dL}(0.89-1.76)$, FT3 $14.26 \mathrm{pg} / \mathrm{mL}(1.71-$ 3.71). Thyroid ultrasound showed normal volume and a heterogeneous structure with small solid nodules, the largest one in the left lobe $(9 \mathrm{~mm})$. Thyroid scintigraphy revealed a high diffuse radioiodine uptake and TRAbs were positive $-8.3 \mathrm{U} / \mathrm{L}(0-1.8)$. Initially she started propranolol with improvement of symptoms and after confirmation of hyperthyroidism methimazole was initiated. About one month later the patient presented significant hair loss, with extensive areas of diffuse alopecia. She was referred to a dermatology appointment, performed scalp biopsy that confirmed the diagnosis of AA and started oral corticosteroids.

Conclusions: Graves' disease and Hashimoto thyroiditis are autoimmune thyroid disorders that can be associated with AA. AA is caused by an abnormal T-cell response against hair follicle self-antigens, similar to T-cell mediated autoimmune thyroid diseases. AA should always be considered in patients with Graves' disease or Hashimoto thyroiditis as a possible associated autoimmune disorder.

\section{P023 Goiter and Nodules 2}

\section{P229 \\ MINIMALLY INVASIVE OPEN APPROACH WITH HARMONIC SCALPEL FOR THE TREATMENT OF SOLITARY THYROID NODULES IN THE CHILDHOOD}

Rumen Nenkov' ${ }^{1}$, Radoslav Radev

${ }^{1}$ Medical University, Varna

The minimally invasive techniques become more and more acknowledged in the field of thyroid surgery in recent years. Their role however, in the surgical treatment of thyroid nodular disease in children is not still well established.

Aim: To present our results with the surgical treatment of solitary thyroid nodules using harmonic scalpel through a minimally invasive open approach in childhood.

Materials and Methods: For the period from 01 of January to 31 of December 2013, 862 patients with thyroid nodules have been operated on in our clinic, using minimally invasive open approach with harmonic scalpel. 84 of these patients were children ( 65 girls and 19 boys, aged from 5 to 18 years). The thyroid resections were performed through a central collar incision, 2.5 to $3.0 \mathrm{~cm}$ in length. A harmonic scalpel (Ethicon Endo-Surgery Care) has been used in all cases. The operative incision length, extent of surgery, use of hemostatic instruments, duration of operative time, incidence of complications, length of hospital stay and quality of cosmetic results were examined.

Results: The size of removed thyroid nodules varied between 2.0 and $3.5 \mathrm{~cm}$. In 21 patients a near total resection of one thyroid lobe was performed and in 63 patients - lobectomy. Hemo static instruments and sutures were not used. The procedure was completed without need of draining in most cases. Intraoperative and postoperative complications were not observed. The operative time varied between 26 and 38 minutes. The postoperative hospital stay did not exceed 24 hours. The cosmetic results were considered as perfect and satisfactory in all patients and their relatives.

Conclusions: Our results demonstrate that minimally invasive open approach using harmonic scalpel is a feasible, effective technique with very good cosmetic results in the surgical treatment of solitary thyroid nodules in childhood. 


\section{P230 \\ THE INDIVIDUALLY TAILORED THYROXINE \\ DOSE AS A TOOL TO UNVEIL OCCULT \\ AUTONOMOUS THYROID FUNCTIONING AREAS \\ Maria Giulia Santaguida ${ }^{1}$, Nunzia Bruscaํㅜ, Camilla Virili' \\ Susanna Carlotta Del Duca ${ }^{1}$, Ilenia Gatto ${ }^{1}$, Miriam Cellini', \\ Lucilla Gargano $^{2}$, Marco Centanni ${ }^{1}$ \\ 1"sapienza" University of Rome Dept of Medico-Surgical Sciences and \\ Biotechnologies Latina, ${ }^{2}$ Endocrinology Unit AusI Latina, Latina}

Autonomous functioning thyroid areas (AFTA), frequently associated to heart arrhythmias, are often occult and may be associated to TSH values in the low-normal range. Despite the administration of an individually tailored dose (ITD) of thyroxine $\left(\mathrm{T}_{4}\right)$ in the treatment of multinodular goitre (MNG), a serum TSH lower than expected may be obtained as a consequence of the presence of AFTA. This study was aimed at characterizing the existence of hidden AFTA in patients hyperresponding to treatment with an ITD of thyroxine. In a cohort of about 3000 consecutively examined outpatients, we assessed 94 patients ( $84 \mathrm{~F}$ and $10 \mathrm{M}$; median age $=56$ years) with $\mathrm{MNG}$ in treatment with ITD, who showed an unexpected lower serum TSH as compared to the one observed in a reference group $(\mathrm{n}=123 ; 109 \mathrm{~F}$ and $14 \mathrm{M}$; median age $=54$ years $)$ in whom iodine and drug interferences, as well as AFTA, were positively excluded. Patients in the study group showed significantly lower median TSH ( $0.052 \mathrm{vs}$ $0.20 \mathrm{mU} / 1, \mathrm{P}<0.0001)$, despite a significantly lower dose administered (1.19 vs $1.49 \mu \mathrm{g} / \mathrm{Kg} /$ day, $\mathrm{P}<0.0001$ ). When separately analyzed according to age, these differences were confirmed both in adult patients $<60$ years (median TSH:0.06 vs $0.2 \mathrm{mU} / 1, \mathrm{P}<0.0001$; median $\mathrm{T}_{4}$ dose: 1.29 vs $1.56 \mu \mathrm{g} / \mathrm{Kg} /$ day, $\mathrm{P}<0.0001$ ) and in older patients $>60$ years (median TSH:0.05 vs $0.22 \mathrm{mU} / 1$, $\mathrm{P}<0.0025$; median $\mathrm{T}_{4}$ dose: 0.94 vs $1.33 \mu \mathrm{g} / \mathrm{Kg} /$ day, $\mathrm{P}<0.0005$ ). Interestingly, when treatment was withdrawn, $86 / 94$ patients $(91.5 \%)$ had serum TSH in the normal range and the median TSH of the whole study group was $1.09 \mathrm{mU} / 1$. The presence of autonomous functioning areas has been detected, through thyroid scintiscan and radioactive iodine uptake test (RAIU- $4^{\text {th }}$ and $24^{\text {th }}$ hour), in 65 out of 94 patients $(69 \%)$. RAIU at $4^{\text {th }}$ hour $(17$ vs $13 \%, \mathrm{P}<0.0199)$ and $24^{\text {th }}$ hour $(33.5$ vs $28 \%, \mathrm{P}<0.001)$ were both significantly higher in patients with autonomous functioning areas. These results show that the ITD of $\mathrm{T}_{4}$ represents a novel tool to detect hidden autonomous functioning thyroid areas.

\section{P231 \\ INTRATHYROIDAL ECTOPIC THYMIC TISSUE MIMICKING A THYROID NODULE IN A 4-MONTH-OLD BABY}

\section{Gülay Karagüzel ${ }^{0}$, Aysenur Ökten ${ }^{1}$}

${ }^{1}$ Karadeniz Technical University, School of Medicine; Department of

Pediatric Endocrinology; Trabzon, Turkey

Ectopic intrathyroidal thymus tissue that may be present as a thyroid nodule is rarely reported in childhood. We present a case of a 4-month-old boy who referred to our clinic with a solitary thyroid nodule diagnosed by ultrasound (US) as 'incidentalomas'. His thyroid function tests, complete blood count and biochemistry were normal. Thyroid US was repeated and showed a hypoechoic nodule $(6 \times 3 \mathrm{~mm})$ with multiple hyperechoic areas in the left lobe. It was reported as an intrathyroidal ectopic thymic tissue. Fine-needle aspiration biopsy (FNAB) was not done because it is an invasive diagnostic procedure. During his one year follow-up, nodular enlargement was not seen.

Ultrasonographic findings are tipic for thymic tissue as a hypoechoic pattern with multiple regular linear and punctate bright echogenicities representing connective septa and blood vessels within the septa. Thymus has a unique appearance at US and ectopic thymic tissue can be diagnosed and followed-up safely by US. Intrathyroidal thymic inclusions should be considered in the differential diagnosis of thyroid nodules in children and adolescents. If the nodule is stable or regressing during the follow-up, FNAB is an invasive and expensive method.

\section{P232 \\ ULTRASOUND-GUIDED HIGH-INTENSITY FOCUSED ULTRASOUND ABLATION OF NON- FUNCTIONING BENIGN THYROID NODULES - THREE MONTHS OF FOLLOW-UP}

Roussanka Kovatcheva ${ }^{1}$, Jordan Vlahov², Julian Stoinov ${ }^{3}$, Katja Zalete/ ${ }^{4}$

${ }^{1}$ University Hospital of Endocrinology, Department of Thyroid and Metabolic Bone Disorders, Sofia Medical University, ${ }^{2}$ University Hospital of Endocrinology, Department of Thyroid and Metabolic Bone Disorders, ${ }^{3}$ University Hospital of Endocrinology, Department of Endocrine Surgery; Sofia Medical University, ${ }^{4}$ University Medical Centre Ljubljana, Department of Nuclear Medicine

Objectives: To assess the short-term efficacy and safety of ultrasound (US)-guided high-intensity focused ultrasound (HIFU) for the treatment of non-functioning benign thyroid nodules.

Materials and Methods: HIFU treatment was performed under US-guidance and conscious sedation in 20 euthyroid patients (mean age, 44.5 years) with benign solitary or dominant thyroid nodule. Thyroid volume, structure, and vascularisation were assessed at baseline, 1 and 3 months after treatment. Adverse events associated with HIFU were evaluated.

Results: Starting from $4.96 \mathrm{~mL} \pm 2.79$ (standard deviation), the mean nodule volume decreased to $3.58 \mathrm{~mL} \pm 1.99$ at 1 -month follow-up $(P=0.080)$ reaching $3.05 \mathrm{~mL} \pm 1.96$ by the $3^{\text {rd }}$ month $(P=0.017)$. At 3 -month follow-up, the mean volume reduction was $38.5 \% \pm 21.6(P<0.001)(>50 \%, \mathrm{n}=6 ; 30 \%$ $50 \%, \mathrm{n}=7 ;<30 \%, \mathrm{n}=7)$. Isoechoic nodules showed greater reduction at 1 month as compared to the hypoechoic nodules $(31.6 \pm 18.1$ vs $16.4 \pm 8.6, P=$ $0.053)$. Nodules with markedly increased blood flow showed smaller volume reduction at 3 months if compared with less vascularised nodules $(10.9 \% \pm$ 14.5 vs $41.5 \% \pm 20.3, P=0.054$ ). Minor transient complications (subcutaneous edema, mild skin redness) were observed in 2 patients.

Conclusion: US-guided HIFU ablation is an effective and safe procedure for treatment of benign solid thyroid nodules. Initial US pattern and vascularisation seem to influence effective ablation.

\section{P233}

\section{SURGERY IN INTRATHORACIC GOITER}

$Y_{\text {Vvana Anavy Martínez Mateo }}{ }^{0}$, Francisco Juan de Santos Iglesias ${ }^{1}$, Jose Manuel Gonzalez Gonzalez ${ }^{1}$, Guillermo Jesus Vaquero Gajate ${ }^{1}$, Leyre Rodrigo Hernanz ${ }^{1}$

${ }^{1}$ Hospital San Pedro de Alcantara

Introduction: Intrathoracic goiter is a dificult disease to define because there isn't an universal classification about how much volumen of thyroid gland must stay intrathoracic, neither how far it must downfall. That means there is a great difference between its incidence in the diverses trials $(0.2 \%-$ $50 \%$ ) If we use the Kaltic definition, intrathoracic goiter are $1-4 \%$ of goiters. Comprehensive sympthoms and/or cervical mass are the most frequent clinical manifestations, however between 30\%-40\% are asympthomatics. Intrathoracic goiter treatment is surgery after imaging studies, computarized tomography (CT) or Magnetic Resonance (MR) The 95\% of these thyroidectomies could be make by cervical incision.

Case Report: We present a 77 years old woman who had a cervical mass from the thyroid gland that downfall intrathoracic, it's checked a $5 \mathrm{~cm}$ nodule from left thyroid lobule and it's no possible to touch the lower poles. The patient has dyspnea and dysphagia for solids. The functional analyses show an hyperthiroidism. The cervical ultrasound shows a great thyroid gland with a lot of nodules. The cervical CT scan check a great mutinodular thyroid that downfalls behind the upper cava vein and supraaortics artheries, closed to aortic arch. The upper poles are closed to the mandibular angle. This intrathoracic goiter was treated by surgery. It was made a thyroidectomy by cervical incision without complication, the upper and lower vascular elements were easily identified and the great thyroid gland was excised through the cervical incision.

Conclusions: The $95 \%$ of intrathoracic goiters can be excised by cervical incisión, if these is as wide to excised the thyroid gland through it, without complications neither recurrences, exceptions are throids advanced cancer, thyroids closed to carina and thyroids located in posterior mediastinum. 


\section{P234 \\ EFFECTS OF GH REPLACEMENT THERAPY ON THYROID VOLUME AND NODULES DEVELOPMENT IN GROWTH HORMONE DEFICIENT ADULTS}

Lorenzo Curtò ${ }^{1}$, Salvatore Giovinazzo ${ }^{1}$, Angela Alibrandi ${ }^{2}$,

Francesco Trimarchi ${ }^{1}$, Rosaria Ruggeri ${ }^{0}$

${ }^{1}$ Endocrinology Unit, University of Messina, ${ }^{2}$ Department of Statistical Sciences

Objectives: Our aim was to define the effects of GH replacement therapy (GHRT) on thyroid volume (TV) and nodular growth in patients with GH deficit (GHD).

Patients and Methods: We retrospectively evaluated 96 subjects $(47 \mathrm{M}$, $49 \mathrm{~F}$, aged 16-78 years) with childhood onset $(\mathrm{CO}, \mathrm{n}=28)$ and adulthood onset (AO, $n=68$ ) GHD. Sixty-seven had also a central hypothyroidism $(\mathrm{CH})$. The median disease's duration was 7 years (range 1-34), and replacement treatment's duration 5 years (range $1-30$; mean GH dose $=4.30 \mu \mathrm{g} /$ daily in men and $5.65 \mu \mathrm{g} /$ daily in women).

Results: Pre-treatment TV, as determined by ultrasounds, was smaller in GHD patients than healthy subjects from the same geographic area $(\mathrm{P}<.030)$. $\mathrm{TV}$ was positively correlated with body weight $(\mathrm{Rho}=0.21 ; \mathrm{P}=.049)$, serum IGF-I $(\mathrm{Rho}=0.35 ; \mathrm{P} .001)$ and TSH $(\mathrm{Rho}=0.48 ; \mathrm{P} .001)$. During GHRT, TV significantly increased $(\mathrm{P}=.016$ for the entire group; $\mathrm{P}=.014$ in GHD patients without $\mathrm{CH}$ ), the main predictor for TV change being serum TSH $(\beta=1.009$; $\mathrm{CI}=.034$ and 1.984; $\mathrm{P}=.043$ ). Before starting GHRT, 17 patients harbored thyroid nodules. During GHRT, the nodules' size slightly increased in 7 of them, and appearance of new thyroid nodules was observed in 9. Among the 79 patients without thyroid lesions before GHRT, 17 developed one or more thyroid nodules. The prevalence of $\mathrm{CH}$ was not different in GHD patients with or without thyroid nodules $(23 / 34$ vs $44 / 62 ; \chi 2=0.01 ; \mathrm{P}=.915$; or $11 / 17 \mathrm{vs}$ $44 / 62$ excluding patients with pre-therapy nodular goitre; $\chi 2=0.04 ; \mathrm{P}=.841$ ). In the multivariate analysis, the main predictor for nodule development was serum IGF-1 $(\mathrm{OR}=1.856 ; \mathrm{P}=.03)$

Conclusions: GHRT is associated with TV increase in GHD patients without CH. $27 \%$ of our GHD patients developed thyroid nodules during GHRT, irrespective of TSH deficit, mainly depending on IGF-1 values.

\section{P235 \\ INTRATHYROID LOCATION OF PARATHYROID GLAND: A SIGNIFICANT CAUSE OF INADVERTENT PARATHYROIDECTOMY DURING THYROID SURGERY?}

Nick Michalopoulos ${ }^{1}$, Efterpi Margaritidou' ${ }^{2}$, Theodossis Papavramidis' ${ }^{1}$, Ioannis Kanellos ${ }^{1}$, Kalliopi Pazaitou-Panayiotou ${ }^{2}$

${ }^{1}$ Ahepa Hospital, ${ }^{2}$ Theagenio Cancer Hospital

Objectives: Total thyroidectomy (TT) is a safe and effective operation with virtually zero mortality and minimal morbidity rates in experienced centers of endocrine surgery. The most frequent complication after TT is hypoparathyroidism. Surgical trauma, devascularization of parathyroid glands and inadvertent parathyroidectomy have been identified as the main causes resulting in postoperative hypocalcaemia. The objective of this study is to investigate the incidence of intrathyroid parathyroid glands (ITPGs) in a large cohort of 4500 patients who underwent TT and to clarify the characteristics of ITPGs.

Methods: This is a retrospective study of two specialized centers in thyroid diseases in Greece. The medical records of patients with a history of TT for thyroid malignant or benign disease were analyzed. The presence of parathyroid tissue in the histological specimen was searched. Exclusion criteria included patients who underwent lobectomy or near total thyroidectomy, intentional parathyroidectomyfor concurrent parathyroid diseases, patients with previous parathyroidectomy and patients with inadequate data. Patients were divided in two groups based on the finding of parathyroid tissue in thyroid specimen: those with intrathyroid parathyroid gland (ITPG) and those with extrathyroid parathyroid gland (ETPG).

Results: 605 patients were excluded from the study and a data base was created with the remaining 3895 TTs. Inadvertentparathyroidectomy (IP) was presented in 424 cases $(10.89 \%)$. Intrathyroid parathyroid glands were presented in 68 cases $(1.75 \%)$. Pathological characteristics were similar in ITPGs and ETPGs concerning the size, the localization and the weight. Cystic degeneration was presented in ITPGs $(\mathrm{p}=0.01)$.

Conclusions: The presence of ITPGs in our cohort is $1.75 \%$. IP does not increase the risk for clinical hypocalcaemia. Surgeons should inform patients for the probability of the intrathyroid presence of parathyroid glands.

\section{P236 \\ RESULTS OF RADIOIODINE TREATMENT IN PATIENTS WITH GOITER MULTINODULAR AND TOXIC ADENOMA}

Concepción Muñoz Jiménez ${ }^{0}, M^{a}$ Rosa Alhambra Expósito ${ }^{1}$, Rodrigo Bahamondes Opazo ${ }^{1}, M^{a}$ Ángeles Gálvez Moreno', Inmaculada Prior Sánchez ${ }^{1}$, Aura Herrera Martínez ${ }^{1}$

${ }^{1}$ Servicio Andaluz de Salud

Background: In adult population, multinodular goiter (MNG) and toxic adenoma are common causes of hyperthyroidism, with significant cardiac and arrhythmogenic consequences that can destabilize or trigger different diseases. Radioactive iodine induces a high response rate and a decrease in goiter size safely.

Objective:To analyze the thyroid function 6 months and one year after a fixed dose of $15 \mathrm{mCi}$ radioiodine administrated to patients with BMN or toxic adenoma.

Material and Methods: Descriptive study. 138 patients treated with radioiodine for toxic nodule or BMN between 2010 and 2012 were included. We analyzed the history and thyroid function at 6 months and one year after receiving radioiodine. Data were analyzed with SPSS 15.0.

Results: 138 patients were included $(82.6 \%$ women, mean age $61.74 \pm 14.56$ years). $64.5 \%$ of patients were diagnosed of MNG and $35.5 \%$ of toxic adenoma. 6 months after radioiodine, in the MNG group: $62 \%$ were euthyroid, $11.4 \%$ hyperthyroid and $24.6 \%$ hypothyroid. In toxic adenoma group: $61.4 \%$ were euthyroid, $6.8 \%$ hyperthyroid and $31.8 \%$ hypothyroid. One year after radioiodine, in the MNG group: $46.9 \%$ were euthyroid, $6.3 \%$ hyperthyroid and $46.9 \%$ hypothyroid; in toxic adenoma group: $49 \%$ were euthyroid, $5.8 \%$ hyperthyroid and $45.8 \%$ hypothyroid. No statistically significant differences between groups there were.

Conclusions: The fixed-dose radioiodine $15 \mathrm{mCi}$ in MNG and toxic adenoma has a high percentage of remission and euthyroid at 6 months and one year later. Given the simplicity of the technique, the absence of complications and the prevalence of elderly population with multiple comorbidities, radioiodine should be considered as first-line treatment of MNG and toxic adenoma. In our series, there were not different in thyroid function 6 months and one year after radioiodine treatment.

\section{P237 \\ ROLE OF US-GUIDED THIN CORE NEEDLE BIOPSY FOR CYTOLOGICALLY INDETERMINATE THYROID NODULES. A CONTROLLED PROSPECTIVE TRIAL}

Enrico Papini', Giancarlo Bizzarri², Irene Misischi ${ }^{1}$, Palma Specchia ${ }^{3}$, Antonio Bianchini', Rinaldo Guglielmi', Filomena Graziano',

Luca Turrini ${ }^{5}$, Silvia Taccogna ${ }^{5}$

${ }^{1}$ Department of Endocrinology, Regina Apostolorum Hospital, Albano Laziale, Rome, Italy, ${ }^{2}$ Department of Diagnostic of Imaging, Regina Apostolorum Hospital, Albano Laziale, Rome, ${ }^{3}$ Sapienza University of Rome, Rome, Italy, ${ }^{4}$ Department of Diagnostic of Imaging, Regina Apostolorum Hospital, Albano Laziale, Rome, Italy, ${ }^{5}$ Section of

Pathology, Ospedale Regina Apostolorum, Albano Laziale Rome, Italy

Background: Fine needle aspiration biopsy represents the gold standard for the preoperatory management of thyroid nodules. However, thyroid nodules which are indeterminate at cytological evaluation are frequently submitted to diagnostic surgery due to their uncertain malignant potential.

Aim of the Study: To assess in a single center controlled series of indeterminate thyroid nodules the predictive role for malignancy of thin core needle biopsy.

Materials and Methods: Fifty consecutive patients with indeterminate thyroid cytology were enrolled in the study. After local anesthesia, a 21-G cutting needle was inserted under US-guidance into the nodule through a 
trans-isthmic approach. The samples were fixed in formalin and were blindly evaluated by two experienced pathologists. The cytomorphology of the target lesion, its capsule and the relationship with surrounding parenchyma were assessed. A panel of three histochemical markers (HBME-1, Galectin-3 and CK-19) was performed.

Results: Forty-eight out of fifty samples were adequate for diagnosis. Thirty-three nodules were classified as "benign" (hyperplastic nodules), 15 nodules were classified as "follicular neoplasm", and 1 case as "malignant". Morphologically benign nodules with negative immunohistochemical panel had close US and clinical follow-up up to 12 months, while the 16 nodules classified as follicular neoplasm or malignant lesion underwent surgery. Ten $(66 \%)$ of these cases resulted to be papillary or follicular thyroid carcinomas, while $6(34 \%)$ were follicular adenomas or hyperplastic nodules. None of the benign and immunohistochemically negative nodules showed suspicious clinical or US changes at follow-up.

Conclusions: US-guided thin core needle biopsy was rapid, safe and welltolerated. Its use, combined with an immunohistochemical panel, identified as follicular neoplasm or malignant $33 \%$ of cytologically indeterminate lesions and $66 \%$ of them resulted to be malignant. None of the indeterminate nodules classified as benign showed suspicious changes at clinical and US follow-up.

\section{P238}

\section{SMALL NODULAR LESIONS OF THE THYROID GLAND IN CHILDREN: CONSIDER AN ECTOPIC THYMUS}

Natallia Akulevich ${ }^{1}$, Yulia Makarava², Julia Boiko², Irina Khmara²

${ }^{1}$ State Center for Medical Rehabilitation, Minsk, Belarus; The Institute of Physiology of the National Academy of Sciences of Belarus, ${ }^{2}$ State Center for Medical Rehabilitation, Minsk, Belarus

The use of ultrasound (US) leads to detection of small $(<1 \mathrm{~cm})$ thyroid nodules even in young children. About $80 \%$ of cases are benign; however, they require differential diagnosis with thyroid cancer. Another challenging condition is the ectopy of thymus to the thyroid.

We analyzed evolution of ectopic intrathyroid tymus in children.

Patients were 26 children ( 14 girls) aged $7.2 \pm 3.5$ years, with no history of head/neck irradiation. The time of follow-up was $1.6 \pm 0.6$ years. Patients were referred for different reasons: parents caution due to positive family history for thyroid diseases $-27 \%$; other endocrine problems in the child $-38.5 \%$; in $34.5 \%$ of cases non palpable thyroid nodules were detected by US at health check. An ectopic thymic tissue had typical US characteristics such as singular or multiple hypoechoic foci with hyperechoic inclusions, avascular, of regular or irregular shape, from 2 to $9 \mathrm{~mm}$ in maximal size. The vast majority of children had no goiter. Other sonographic features found were thyroiditis - in 3, thyroid microcysts - in 4, cycts of the neck - in 2 children. Mean TSH level was $3.2 \pm 1.5 \mathrm{mIU} / \mathrm{l}$. An elevated TPO-Ab and TSH $>4 \mathrm{mIU} / 1$ levels were $38.5 \%$ each. Till the end of observation, $24 / 26(92.3 \%)$ of patients had no growth of thyroid lesions; in 3/24 children decrease in size was observed. In 2 children, the nodule had slightly grown in puberty. In $3 / 26$ children FNB was performed including a girl with growing nodule whose mother had thyroid cancer. In all cases, cytology described the lymphoid cells of different stage of maturity. No surgery was done and all children are under follow-up.

Differential diagnosis of small thyroid nodules in young children should consider an ectopic intrathyroid tymus, follow-up in mandatory, and surgery in not always necessary.

\section{P024 Thyroid Autoimmunity}

P239

\section{PREVALENCE OF AUTOIMMUNE THYROID DISORDER IN PEDIATRICS TYPE 1 DIABETES MELLITUS IN MEERUT, INDIA}

Manish Gutch ${ }^{1}$, Sukriti Kumar ${ }^{2}$, Sanjay Saran ${ }^{3}$, Keshav Kumar Gupta ${ }^{1}$ ${ }^{1}$ Department OF Endocrinology, LIrm Medical College, Meerut, India, ${ }^{2}$ Department OF Radiodaignosis, Sgpgi, Lucknow, India, ${ }^{3}$ Department OF Endocrinology, LIrm Medical College, Garh Road, Meerut, India

Background: Various autoimmune disorder are associated with pediatrics type 1 diabetes mellitus, but are usually ill-defined and not usually suspected until the disease become advance, and the prevalence of these autoimmune conditions are usually not very well defined in developing part of world.

Aim: To find out the prevalence of various autoimmune disorders associated with pediatrics type 1 diabetes mellitus.

Materials and Method: Total of one hundred and sixty four patients was screened ( 90 males and 74 females) during the study period of one year, patients were evaluated for the clinical signs, biochemical investigations and family history of autoimmune disorders in tertiary care health center in western U.P

Results: Autoimmune thyroiditis was found to be most commonly associated with type 1 diabetes, followed by the celiac diseases, and graves diseases, other less common were pernicious anemia, juvenile rheumatoid arthritis and vitiligo.

Conculsion: Autoimmune hypothyroidism was found to be significantly associated with type 1 diabetes, timely identification of these disorder are of paramount important for better glycemic control and to reduced the morbidity and mortality associated with the conditions.

Table 1. Autoimmune disorder associated with type 1 diabetes mellitus (for Abstract P239)

\begin{tabular}{lll}
\hline Parameters & $\begin{array}{l}\text { Males } \\
(90 \text { males })\end{array}$ & $\begin{array}{l}\text { Females } \\
(74 \text { females })\end{array}$ \\
\hline Autoimmune hypothyroidism & 07 & 21 \\
Celiac diseases & 03 & 02 \\
Graves diseases & 01 & 02 \\
Pernicious anemia & 01 & 01 \\
Juvenile rheumatoid arthritis & 01 & 00 \\
Vitiligo & 03 & 00 \\
Alopecia & 00 & 00 \\
Immune thrombocytopenic purpura & 00 & 00 \\
Myasthenia gravis & 00 & 00 \\
\hline
\end{tabular}

\section{P240 \\ GLYCOSYLATION OF PURIFIED ANTI- THYROGLOBULIN IGG FROM HASHIMOTO'S PATIENTS AND HEALTHY BLOOD DONORS}

Yang Zhang ${ }^{1}$, Shanshan Yuan ${ }^{1}$, Lanlan Zhao ${ }^{1}$, Ying Gao ${ }^{1}$, Xiaohui Guo ${ }^{1}$

${ }^{1}$ Department of Endocrinology, Peking University First Hospital, Beijing, China

Objective:Anti-thyroglobulin ( $\mathrm{TgAb}) \operatorname{IgG}$ has been found in serum of patients with Hashimoto's thyroiditis and healthy blood donors. Alterations of glycosylation of IgG have been found in many autoimmune diseases. The aim of our study was to investigate the differences of glycosylation of TgAb IgG between Hashimoto's patients and healthy blood donors.

Methods: $\mathrm{TgAb}$ IgG was purified from sera of Hashimoto's patients (HT, $\mathrm{n}=32$ ) and healthy blood donors (control, $\mathrm{n}=15$ ) by affinity chromatography. HT patients were further divided into two subgroups: patients with moderate levels (mHT, $n=15$ ) and with high levels of TgAb (hHT, $n=17$ ). Matrixassisted laser desorption/ionization quadrupole ion trap time-of-flight mass 
spectrometry (MALDI-QIT-TOF-MS) was performed to identify glycosylation profiles of purified $\mathrm{TgAb}$ IgG. Letin microassay was used to compare the different abundance of glycans of TgAb between Hashimoto's patients and controls.

Results: There was no difference of glycosylation profiles of purified $\mathrm{TgAb} \operatorname{IgG}$ between Hashimoto's patients and healthy blood donors by MALDI-QIT-TOF-MS. The lectin microassay found that there was more mannose, terminal sialic acid, core fucose and Galb1-4GlcNAcb1-2Man glycans on $\mathrm{TgAb} \operatorname{IgG}$ from HT group than that from control group (all $P<0.001$ ), and on $\mathrm{TgAb}$ IgG from hHT group than that from mHT group (all $P<0.05$ ).

Conlusions: Glycosylation levels of $\mathrm{TgAb} \operatorname{IgG}$ in Hashimoto's patients were greater than that of natural $\mathrm{TgAb} \mathrm{IgG}$ in healthy subjects. It might provide new clues for exploring the role of $\mathrm{TgAb}$ in pathogenesis of $\mathrm{HT}$.

\section{P241 \\ IMPACT OF IL-10 AND IL-12B SINGLE NUCLEOTIDE POLYMORPHISMS ON CIRCULATING CYTOKINE LEVEL IN DEVELOPMENT OF HASHIMOTO'S THYROIDITIS} Julieta Gerenova ${ }^{1}$, Irena Manolova ${ }^{2}$, Spaska Stanilova ${ }^{3}$

${ }^{1}$ Department of Endocrinology, University Hospital; Medical Faculty, Trakia University - Stara Zagora, ${ }^{2}$ Department of Health Care,

Medical Faculty, Trakia University, ${ }^{3}$ Department of Molecular Biology, Immunology and Medical Genetics, Medical Faculty, Trakia University

This study was designed to investigate the correlation of immunoregulatory circulating cytokines Interleukin (IL) -10 and IL-12p40 with their genotypes in Hashimoto's thyroiditis (HT) development.

Circulating level of IL-10 and IL-12p40 in serum of HT patients $(n=124)$ and healthy controls $(n=72)$ was determined by ELISA. Genotyping for the 3'UTRA/C IL12B polymorphism was performed using RFLP-PCR and genotyping for $-1082 \mathrm{~A} / \mathrm{G}$ by ARMS-PCR assay. HT patients were divided according to the thyroid function in euthyroid and hypothyroid stages.

We examined the significant increase in serum level of IL-10 for group of hypothyroid stage compared to both control and euthyroid (1.98 vs 0.74 and 0.48 respectively; $\mathrm{P}<0.05$ ). Moreover, quantity of circulating IL-10 decreased after treatment with levothyroxine. When patients was stratified by $-1082 \mathrm{~A} / \mathrm{G}$ SNP genotype we established the statistically significant enhanced level of circulating IL-10 in hypothyroid patients with GG genotype compared to AA and AG genotypes: 3.45 vs 0.59 and 0.24 respectively; $p=0.048$. We also detected significant differences between circulating IL-10 quantity in hypo and euthyroid patients with GG genotypes: 3.45 vs $0.96 ; p=0.028$. In contrast genotypes AA and AG did not reveal differences in circulating IL-10 among patients with different stages.

The quantity of IL-12p40 in serum was enhanced in both eu- and hypothyroid stages compared to healthy controls $(82.3$ and 83.4 vs $62.2 ; p=0.025)$ and unsignificantly diminished after levothyroxine treatment (72.3). Regarding the 3'UTRA/C IL12B polymorphism it was shown the significantly higher level for AA genotype in HT (58.97 vs 78.9; $\mathrm{p}=0.019)$ in comparison with AA healthy individuals.

In conclusion we showed that IL-12p40 and IL-10 serum level in patients with HT demonstrated significant differences depending of genotype and HT stages. The result provides evidence that enhanced serum level of IL-10 in combination with enhanced IL-12p40 is associated with hypothyroid stages in HT development.

\section{P242 \\ VESTIBULAR DISORDERS IN EUTHYROID PATIENTS WITH HASHIMOTO'S THYROIDITIS: RESULTS FROM A MULTICENTER STUDY}

Sara Tognini ${ }^{0}$, Giuseppe Chiarella ${ }^{1}$, Andrea Nacci ${ }^{2}$, Roberta Sieli ${ }^{3}$, Giuseppe Pasqualetti ${ }^{3}$, Giuseppe Costante ${ }^{4}$, Bruno Fattori ${ }^{2}$,

Diego Russo ${ }^{4}$, Fabio Monzani ${ }^{3}$

${ }^{1} 1$ department of Experimental and Clinical Medicine, Audiology and Phoniatrics Unit, University of Catanzaro Magna Graecia, Italy, ${ }^{2}$ Audiology Phoniatric Unit, Department of Neuroscience, University of Pisa, Italy, ${ }^{3}$ department of Clinical and Experimental Medicine, University of Pisa, Italy, ${ }^{4}$ Department of Health Sciences, University of Catanzaro Magna Graecia, Italy

Objectives: a relationship between vestibular disorders and Hashimoto's thyroiditis (HT) independently from thyroid function has been postulated. To shed more light on the actual relationship between these disorders, we examined vestibular function in euthyroid HT patients as compared to either agematched patients with benign multinodular goitre (MNG) or healthy subjects without any thyroid disorder.

Methods: forty-seven patients with HT ( $89.4 \% \mathrm{~F}$; aged $48.3 \pm 12.7$ years), 21 with MNG $(57.1 \% \mathrm{~F} ; 54.1 \pm 9.8$ years) and 30 healthy volunteers $(56.7 \% \mathrm{~F}$; $50.7 \pm 13.9$ years) were enrolled. Inclusion criteria were the presence of normal thyroid function tests and no clinical history of vestibular dysfunction. Each subject was submitted to complete vestibular evaluation [Caloric Test, Vestibular-evoked myogenic potentials (VEMPs), Head Shaking Test (HST)].

Results: $52.2 \%$ of HT patients showed an alteration of VEMPs and $44.7 \%$ of Caloric test $(\mathrm{P}<0.0001$ for both). None of the MNG patients showed any vestibular alteration, while one healthy control an altered Caloric test. According to a serum TPOAb cut-off level, defined as three times the upper limit of the normal range, HT patients with high-titre showed a significantly higher prevalence of alterations of both VEMPs and HST than those with lowtitre $\left(65.4 \%\right.$ Vs $33.3 \%, \mathrm{p}=0.03 ; 42.8 \% V_{S} 7.1 \%, \mathrm{p}=0.01$, respectively). By logistic regression analysis, the absence of thyroid autoimmunity significantly reduced the risk of vestibular alterations: HR 0.25 (95\%CI: $0.003-0.19, \mathrm{p}=$ $0.0004)$ for caloric test; HR 0.07 (95\%CI: $0.02-0.425, \mathrm{P}<0.0001)$ for VEMPs and HR 0.22 (95\%CI: $0.06-0.7, p=0.01)$ for HST.

Conclusion: the present study demonstrates the existence of a strong association between thyroid autoimmunity and subclinical vestibular damage, regardless of thyroid function. Specifically, a significant association between vestibular alterations and the degree of circulating TPOAb titre was observed. This finding suggests a possible role of thyroid autoimmunity per se in the development of vestibular disease

\section{P243 \\ ASSOCIATION OF COMMON POLYMORPHISMS OF THE RAGE (RECEPTOR FOR ADVANCED GLYCATION END PRODUCTS) GENE WITH AUTOIMMUNE PARAMETERS IN WOMEN WITH HASHIMOTO'S THYROIDITIS}

Maria Giannakou ${ }^{1}$, Katerina Saltiki ${ }^{1}$, Eleni Loukari ${ }^{1}$, Aimilia Mantzou ${ }^{1}$ George Philippou ${ }^{1}$, Konstantinos Terzidis ${ }^{1}$, Charalampos Stavrianos ${ }^{1}$, Maria Alevizaki

1Dept of Endocrinology and Metabolism,"Evgenidion" Hospital, Athens University School of Medicine, Athens, Greece

Objectives: Oxidative stress may influence the development of autoimmune thyroiditis (AITD). Dietary oxidative stress markers, such as advanced glycation end products (AGEs) intensify the immune response and the process of inflammation. Polymorphisms of their pro-inflammatory receptor RAGE have been associated with the development and the severity of autoimmune diseases, such as type 1 diabetes and multiple sclerosis. Associations of $R A G E$ gene polymorphisms in euthyroid patients with autoimmune thyroiditis have not been studied.

Methods: 300 consecutive euthyroid women were examined (range 20-65 years): 200 women had thyroid antibodies $(\mathrm{ThAb}(+))$ of whom 100 were without and 100 with T4 replacement. 100 women with $\mathrm{ThAb}(-)$ and (-) AITD family history served as controls. The $R A G E$ polymorphisms $-374 \mathrm{~T}>\mathrm{A}$ $M f e 1$ and $-429 \mathrm{~T}>\mathrm{C}$ Alu 1 were studied in peripheral blood. 
Results: Overall the frequency of the $-429 \mathrm{~T}>\mathrm{C} A l u 1$ was: non-carriers (TT) $87.3 \%$ and carriers (CC/CT) $12.7 \%$. The frequency of the $-374 \mathrm{~T}>\mathrm{A} M f e 1$ was: non- carriers (TT) $27.6 \%$ and carriers (TA/AA) $72.4 \%$. No association between the polymorphisms and the ThAb positivity was found. Alu1 carriers $(\mathrm{CC} / \mathrm{CT})$, compared to non-carriers, had more frequently antiTg-ThAb ( $56 \%$ vs $35.4 \%, \mathrm{p}=0.049$ ) as well as higher anti-TPO and antiTg ThAb titles $(\mathrm{p}=0.034$ and $\mathrm{p}=0.055$, respectively). Furthermore, hypothyroidism was more common amongst $A l u 1$ carriers compared to non-carriers $(47.4 \%$ vs $30.9 \%, \mathrm{p}=0.003)$. Also Alu 1 carriers had more frequently another autoimmune disease when compared to non-carriers $(24.3 \%$ vs $11.4 \%, \mathrm{p}=0.038)$. As far as $M f e 1$ polymorphism is concerned, carriers suffered less frequently from another autoimmune disease when compared to non-carriers $(10.2 \% \mathrm{vs}$ $20.3 \%, p=0.031$.

Conclusions: The $R A G E$ polymorphism $-429 \mathrm{~T}>\mathrm{C} A l u 1$, which has been associated with increased transcriptional activity of $R A G E$, may predispose to ThAb positivity and to further autoimmune diseases. This finding may indirectly support the possibility of an association between oxidative stress and thyroid autoimmunity.

\section{P244 \\ EVALUATION OF THE INCIDENCE OF THYROID DISORDERS IN PATIENTS WITH SYSTEMIC LUPUS ERYTHEMATOSUS}

Andrea Di Domenicantonio ${ }^{1}$, Silvia Martina Ferrari ${ }^{1}$, Poupak Fallahi ${ }^{1}$, Ilaria Ruffilli ${ }^{1}$, Marta Mosca ${ }^{1}$, Alessandro Antonelli

${ }^{1}$ University of Pisa

Objective: No study has evaluated the incidence of new cases of thyroid disorders in systemic lupus erythematosus (SLE) patients. In this study, we show the incidence of new cases of clinical and subclinical thyroid dysfunctions in women with SLE.

Methods: Transversal study: We have evaluated the thyroid disorders in 211 women with SLE, in comparison with 211 gender- and age-matched controls.

Longitudinal study: We have excluded SLE patients with thyroid dysfunctions $(n=47)$ at the initial evaluation, then the appearance of new cases of thyroid disorders was observed in 164 SLE and in 164 matched controls, with similar iodine intake (median follow-up 85 months in SLE, vs 91 in controls).

Results: The results of Transversal study were: mean TSH value, the titre and prevalence of thyroid peroxidase antibodies (AbTPO), the percentage of a thyroid hypoechoic pattern and a small thyroid volume were significantly $(\mathrm{P}<$ 0.05 ) higher in women with SLE than in controls. In the Longitudinal study, we have shown a high incidence of new cases of hypothyroidism, thyroid dysfunctions, AbTPO positivity, and appearance of a hypoechoic thyroid pattern in $\operatorname{SLE}(17,23,12,15 / 1000$ female SLE per year; respectively) in comparison with the control group, despite a significantly longer observational period in the control group.

The appearance of hypothyroidism in female SLE was related to a border line high initial TSH, AbTPO positivity, a hypoechoic pattern, and a small thyroid volume (data obtained by a logistic regression analysis).

Conclusion: In this study, we first show a high incidence of new cases of hypothyroidism, thyroid dysfunctions, AbTPO positivity, and appearance of a hypoechoic thyroid pattern in female with SLE in comparison with the control group. High risk female SLE patients [high (even if in the normal range) TSH, positive AbTPO, hypoechoic and small thyroid] should have periodically thyroid follow-up.

\section{P245 \\ INVESTIGATING THE SODIUM IODIDE SYMPORTER (NIS) AS A POSSIBLE CANDIDATE SHARED ANTIGEN IN THYROID AUTOIMMUNITY AND BREAST CANCER}

Ilaria Muller ${ }^{1}$, Fiona Grennan-Jones ${ }^{1}$, Lei Zhang ${ }^{1}$, Claudio Giani², Colin Dayan ${ }^{1}$, Marian Ludgate ${ }^{1}$

${ }^{1}$ Institute of Molecular \& Experimental Medicine; School of Medicine; Cardiff University, ${ }^{2}$ Department of Endocrinology; School of Medicine; University of Pisa

Introduction: women with breast cancer $(\mathrm{BC})$ have a higher prevalence of serum thyroid autoantibodies $(\mathrm{Ab})$ and women with $\mathrm{Ab}$ to thyroid peroxidase (TPO) have improved BC prognosis.

Objectives: we hypothesized an immune response to an antigen shared by thyroid and BC cells, since they both express the sodium iodide symporter (NIS) and have a peroxidase activity. NIS is a possible candidate but previous studies have already excluded Ab to NIS (NISAb) which block activity. We aimed to investigate neutral NISAb, i.e. those which bind without affecting function.

Methods: 42 human sera (all women) were collected: 12 affected by BC, 11 with thyroid autoimmunity (TA) having positive serum Ab to TPO and/ or thyroglobulin, 10 with both $\mathrm{BC}$ and TA, 9 with non autoimmune thyroid diseases.

We generated $\mathrm{CHO}$ cells stably expressing human NIS; following antibiotic selection, individual clones were generated by limiting dilution. PCR using NIS primers identified clones for future study (CHO-NIS). NIS mRNA was expressed in CHO-NIS and absent in $\mathrm{CHO}$ cells transfected with empty pcDNA3 (CHO-Empty).

Cell-surface expression in NIS clones was demonstrated by flow cytometry using a human NIS mouse monoclonal antibody followed by an antimouse-IgG AlexaFluor488-conjugate. The fluorescence intensity was $10^{1}$ with CHO-Empty and increased to $10^{3}$ in 'low' but $10^{4}$ in 'high' CHO-NIS clones. Controls without first antibody were at $10^{1}$ in all clones.

Results: CHO-NIS and CHO-Empty were incubated with heat-inactivated human sera followed by anti-human-IgG AlexaFluor488-conjugate. In CHONIS 'low' none of the 42 sera displayed a significant increase in fluorescence intensity compared with CHO-Empty (Kolmogorov-Smirnov Test). However, in CHO-NIS 'high' a substantial proportion of the sera had increased fluorescence intensity. Controls lacking first antibody were consistently at $10^{1}$.

Conclusions: Our results are consistent with low titre neutral NISAb in some patients with $\mathrm{BC}$ and/or TA but further studies are required to confirm specificity.

\section{P246 \\ SKIN SCORES OF PATIENTS WITH SYSTEMIC SCLEROSIS (SSC) CORRELATE WITH CIRCULATING THYROTROPIN (TSH) LEVELS}

Rosaria Ruggeri ${ }^{0}$, Gianluca Bagnato ${ }^{1}$, William Neal Roberts ${ }^{2}$,

Rosaria Certo ${ }^{3}$, Francesco Trimarchi ${ }^{3}$, Gianfilippo Bagnato ${ }^{1}$

${ }^{1}$ Rheumatology Unit, University of Messina, ${ }^{2}$ Rheumatology, Virginia

Commonwealth University, Richmond, USA, ${ }^{3}$ Endocrinology Unit,

University of Messina

Objectives: SSc is a connective tissue disease, characterized by cutaneous and multi-organ fibrosis, and vascular abnormalities. The association of SSc with Hashimoto's thyroiditis (HT) and hypothyroidism has been reported in a wide range of variability (2-25\%). Skin thickening is a characteristic feature of SSc and resemble myxedematous skin. Our aim was to correlate the degree of skin involvement in SSc patients with serum TSH, since it is well known that TSH receptors are widely expressed in human tissues, including the skin.

Materials and Methods: We enrolled $43 \mathrm{SSc}$ patients (18 with diffuse SSc, 25 with localized SSc), all female, with a mean age $50 \pm 13$ years and a mean disease duration $7.9 \pm 6$ years. In all patients, we measured TSH, free thyroxine (FT4), anti-thyroperoxidase and anti-thyroglobulin antibodies, all by chemiluminescence immunoassay. Subjects under L-thyroxine therapy were excluded. Skin thickness was evaluated using modified Rodnan total skin scores (TSS): skin thickening was assessed by palpation in 17 areas of the body, using a scale of 0 (normal skin) to 3 (severely thickened; skin unmovable on pinching). 
Table 1. (for Abstract P248)

\begin{tabular}{|c|c|c|c|c|}
\hline & \multicolumn{2}{|c|}{ Data before treatment } & \multicolumn{2}{|c|}{ Data after treatment } \\
\hline & TSH (мкME/мл) & Св.Т4 (пмоль/л) & TSH (мкME/мл) & Св.Т4 (пмоль/л) \\
\hline $\mathrm{BVI}+$ & 1.4 & 18.16 & 1.66 & 13.51 \\
\hline BVI - & 2.8 & 16.2 & 2.55 & 12.6 \\
\hline
\end{tabular}

Results: TSSs for the entire group of SSc patient averaged 9.40 \pm 5.05 , while the mean TSH was $2.05 \pm 1.14 \mathrm{mIU} / \mathrm{L}$. TSH levels were significantly higher in dcSSc than in $1 \mathrm{SSc}$ patients $(3.14 \pm 2.39$ vs $1.63 \pm 0.93 \mathrm{mUI} / \mathrm{L} ; \mathrm{P}<$ $0.02)$. Spearman's rank correlation between TSS and TSH was statistically significant, either considering SSc patients as a whole $(\mathrm{r}=0.75, \mathrm{P}<0.0001)$ or subdividing them into dSSc $(\mathrm{r}=0.63 ; \mathrm{P}<0.0001)$ and $1 \mathrm{SSc}(\mathrm{r}=0.81 ; \mathrm{P}<$ $0.0001)$. Anti-thyroid antibodies were found in $14 / 43 \mathrm{SSc}$ patients $(30 \%, 8$ $\mathrm{Ab}-\mathrm{Tg}+\mathrm{ve}$ e $5 \mathrm{Ab}-\mathrm{TPO}+\mathrm{ive}$ ), but poorly correlated with skin scores.

Conclusions: Serum TSH is higher in SSc patients with more severe disease and significantly correlate with the TSS. TSH could play a role in the development of cutaneous fibrotic changes in SSc patients.

\section{P247}

\section{TPO EXPRESSED IN THE THYROID AND IN THE BREAST TISSUE SHARE SIMILAR ANTIGENIC PROPERTIES}

Katarzyna Arczewska ${ }^{1}$, Marlena Godlewska ${ }^{1}$, Magdalena Rudzińska ${ }^{1}$, Karolina Hanusek ${ }^{1}$, Wanda Krasuska ${ }^{1}$, Anna Łyczkowska', Mirosław Kiedrowski², Jean Ruf ${ }^{3}$, Barbara Czarnocka

${ }^{1}$ Department of Biochemistry and Molecular Biology; Centre of Postgraduate Medical Education, ${ }^{2}$ Department of Pathology; Maria Skłodowska-Curie Memorial Cancer Center and Institute of Oncology, ${ }^{3}$ Aix-Marseille Université (Amu) and Institut de Recherche Biomédicale des Armées (Irba); Umr Md2, Faculté de Médecine Nord, Marseille, France; Institut National de la Santé Et de la Recherche Médicale (Inserm), France

The association between breast cancer $(\mathrm{BC})$ and thyroid autoimmunity was postulated for a long time. Particularly, the protective function of TPO autoantibodies $(\mathrm{aAb})$ present in sera of patients with $\mathrm{BC}$ was evidenced. TPO $\mathrm{aAb}$ recognize conformational epitopes limited to two immunodominant regions (IDRs), discovered using TPO-specific mAbs, and confirmed using Fabs. Recently, presence of TPO in BC tissue was demonstrated.

Objectives: In current study we characterized immunogenicity of TPO in breast tissues and breast cancer cell lines to confirm that antigenic structure of TPO expressed in breast tissue is the same as the one expressed in thyroid. Moreover, expression of TPO in human normal $(\mathrm{BN})$ and malignant (BC) breast tissue samples was assayed to determine the association between its expression level and extent of disease.

Methods: TPO expression was evaluated in $\mathrm{BN}$ and $\mathrm{BC}$ tissue samples, as well as paraffin-embedded sections from 55 patients and several breast cancer cell lines by Q-RT-PCR and immunological methods, IHC, IF and Western blotting, using a panel of anti-human TPO mAbs.

Results: IHC, IF and Western blotting analyses confirmed that TPO mAbs \#47, A4, \#15, \#64, \#18 and ab76935 (Abcam) recognize TPO expressed in BN and $\mathrm{BC}$ tissues and breast cancer cell lines.

In Q-RT-PCR analysis TPO mRNA was detected in both breast cancer and normal tissues, with the downregulation of expression in breast tumour $(0.0047 \pm 0.0003$ vs $0.0010 \pm 0.0002)$. Finally we observed that TPO mRNA expression is associated with clinical stage of breast cancer showing the lowest level in G3 tumours.

Conclusions: We confirm that TPO is expressed in breast tissue and shows the same antigenic structure as the one present in thyroid. Moreover, TPO expression is associated with clinical stage of breast cancer what may constitute a hint in understanding the protective role of thyroid autoantibodies in breast tumour.

\section{P248 \\ TSH LEVEL AND EFFICIENCY OF TREATMENT OF HCV \\ Olga Nechaeva ${ }^{1}$, Alexander Dreval $^{2}$, Tamara Mamedova $^{0}$, Tatyana Shestakova ${ }^{1}$, Irina Komerdous ${ }^{1}$, Irina Chikh ${ }^{1}$ \\ ${ }^{1}$ Moscow Regional Clinical Research Institute, ${ }^{2}$ Moscow Regional \\ Research Clinical Institute N.A. Vladimirskiy}

It was a pilot project of investigation of affecting TSH level on result of treatment of patients with chronic hepatitis C.

The retrospective group included 48 patients with hepatitis $\mathrm{C}$ genotype 3 A, who took the antiviral therapy course during 24 weeks with pegilated interferon and ribavirin, число $(40 \%)$ of them women, число $(60 \%)$ men, mean age (средний между группами). There were no patients with known thyroid dysfunction in this group. 32 (66) \% patients had a rapid virological response (RVR), 16 people (33.3\%) did not reach this criterion.

The groups were matched by sex. Patients with RVR were significantly younger ( $32.3 \pm 7.2 \mathrm{y} \mathrm{o}$ ) than patients without RVR $(40.63 \pm 8.66 \mathrm{y} \mathrm{o})$. In the primary analysis, before treatment there were no statistical differences in the levels of free T4 and TSH. But after 24 weeks of treatment course there was a significant difference between the groups, the lower the value of TSH before treatment (less than $2.5 \mathrm{mU} / \mathrm{ml}$ ), the more marked presence of rapid virologic response .

Conclusion: The lower level of TSH (less than $2.5 \mathrm{mU} / \mathrm{ml}$ ) can affect the possibility of rapid virologic response, suggesting that it may improve the effectiveness of antiviral therapy. We need more studiers to investigate a possibility of medical treatment of patients with hepatitis $\mathrm{C}$ without thyroid dysfunction with levothyroxin to support TSH level less than $2.5 \mathrm{mU} / \mathrm{ml}$ before antiviral therapy.

\section{P249}

\section{THE CLINICAL ROLE OF SERUM} CONCENTRATIONS OF SELECTED CYTOKINES: IL-1 $\beta$, TNF- $\alpha$ AND IL-6 IN DIAGNOSIS OF AUTOIMMUNE THYROID DISEASE (AITD) IN

\section{CHILDREN}

Hanna Mikos ${ }^{1}$, Marcin Mikos², Barbara Rabska-Pietrzak ${ }^{1}$

Marek Niedziela ${ }^{1}$

${ }^{1}$ Department of Pediatric Endocrinology and Rheumatology, Poznan University of Medical Sciences, Poland, 'Department of Pneumology, Allergology and Clinical Immunology, Poznan University of Medical

Sciences, Poland

Chronic autoimmune thyroiditis cAIT leads to hypothyroidism due to T cell-mediated cytotoxicity in most cases. By contrast, Graves' disease (GD) with thyrotropin receptor stimulatory autoantibodies cause hyperthyroidism. Cytokines play a crucial role in modulating immune response in both disorders. The aim of study was to evaluate the concentrations of cytokines: IL- $1 \beta$, TNF- $\alpha$ and IL- 6 in these two opposite clinical and hormonal thyroid diseases. The study group consisted of 64 children, 44 newly diagnosed, untreated children with cAIT ( $\mathrm{n}=22$; with hypothyroidism) and GD $(\mathrm{n}=22$; hyperthyroidism), and the control group of 20 healthy children. Cytokine concentrations were evaluated using the ELISA technique. The studied groups of children did not differ significantly in concentrations of IL-6 ( $p=0.48)$ and TNF- $\alpha(\mathrm{p}=0.067)$. In children with hypothyroidism we found significantly higher concentrations of IL-1 $\beta$ (median $2.16 \mathrm{pg} / \mathrm{mL}$, IQR 0.87 ) compared to hyperthyroidism (median $1.39 \mathrm{pg} / \mathrm{L}, \mathrm{IQR} 1.27)(\mathrm{P}<0.01)$ and the 
control group (median $1.88 \mathrm{pg} / \mathrm{mL}$, IQR 1.04) $(\mathrm{P}<0.05)$. The results of ROC curve analysis demonstrated the usefulness of IL-1 $\beta(\mathrm{AUC}=0.77, \mathrm{p}=0.003)$ and TNF- $\alpha(\mathrm{AUC}=0.691, \mathrm{p}=0.034)$ as diagnostic parameters in cAIT which enable discrimination of children with autoimmune thyroid disease from healthy individuals. Concentrations of these markers are increased in autoimmune hypothyroidism. We found no significant sex differences in the tested parameters. In conclusion, IL- $1 \beta$ and TNF- $\alpha$ may be considered as markers of hypothyroidism, and could efficiently discriminate between healthy and autoimmune hypothyroid children. Significantly higher concentrations of IL-1 $\beta$ in children with hypothyroidism may be used to distinguish children with cAIT from GD patients.

\section{P250 \\ ANALYSIS OF RS3761549G/A POLYMORPHISM IN FOXP3 GENE IN CHILDREN AND ADOLESCENTS WITH AUTOIMMUNE THYROID DISEASES}

Artur Bossowski ${ }^{1}$, Anna Bossowska ${ }^{2}$, Hanna Borysewicz-Sańczyk ${ }^{3}$, Joanna Goscik ${ }^{4}$, Natalia Wawrusiewicz-Kurylonek ${ }^{5}$, Adam Krętowski ${ }^{5}$ ${ }^{1}$ Medical University in Białystok, Poland, ${ }^{2}$ Dep. of Cardiology. Ministry Hospital in Bialystok, ${ }^{3}$ Dep. of Pediatric Endocrinology, Diabetology With Cardiology Division. Medical University. Bialystok, ${ }^{4} 4$ centre for Experimental Medicine, Medical University of Bialystok, ${ }^{5}$ Department of Endocrinology and Diabetes With Internal Medicine, Medical Univ

Forkhead box P3 (Foxp3) is an important regulatory factor for the development and function of T regulatory cells (Tregs). Moreover it has been established that deficiency of the Foxp3 gene in Treg cells suppresses their regulatory function leading to the development of autoimmune thyroid diseases (AITDs). The aim of our study was to estimate the association of three polymorphism of FOXP3 gene with the predisposition to GD and HT in children.

The study was performed in the group of 145 patients with GD (mean age, 16.5 \pm 2 ), 87 patients with HT (mean age, 15.2 \pm 2.2 ) and 161 healthy volunteers (mean age, 16.3 \pm 3 ). DNA was extracted from the peripheral blood leukocytes using a classical salting out method. The three SNPs rs3761549 (-2383C/T), rs3761548 (-3279G/T) and rs3761547 (-3499T/C) in the FOXP3 gene were genotyped by TaqMan SNP genotyping assay using the real-time PCR method.

In our study, rs $3761549 \mathrm{G} / \mathrm{A}$ genotype was more frequent in females with GD in comparison to healthy female subjects $(15 \%$ vs. $7 \%, \mathrm{p}=0.033)$ with $\mathrm{OR}=2.15$ and $95 \%$ confidence interval for OR: 1.07-4.63. We also observed rs $3761547 \mathrm{~T} / \mathrm{C}$ to be more frequent in females with GD in comparison to control females and this difference was close to being statistically important $(13 \%$ vs. $7 \%, p=0.066)$ with $\mathrm{OR}=1.99$ and $95 \%$ confidence interval for OR: $0.96 ; 4.48$. There were no significant differences in males in the analyzed SNPs and in females with rs3761548 SNP.

In conclusion, these results may suggest that rs3761549G/A polymorphism in Foxp3 gene could contribute to GD development in females

\section{P025 Hyperthyroidism}

\section{P251 \\ CLINICAL AND MORPHOLOGICAL CRITERIA OF RECURRENT HYPERTHYROIDISM IN SURGICALLY TREATED PATIENTS WITH GRAVES' DISEASE \\ Svetlana Dora $^{0}$, Anna Volkova ${ }^{1}$, Madina Gudieva ${ }^{1}$ \\ ${ }^{1}$ Saint-Petersburg Medical University Named Acad. I.P. Pavlov}

Currently there is absence of some criteria wich can make prognosis of Graves' disease duration and outcomes after surgery. Search of such criteria is very important for clinical practice and science.

Methods: We included 122 patients with Graves' disease, wich was diagnosed from 1970 up to 2010 . Patients were undergone surgery due to absence of remission. Levels of TSH, fr.T4, fr.T3, TSAb were measured by the immunoferment method. During surgery histological slices of thyroid gland samples were taken. 1200 histological samples were performed for morphological analysis. We evaluated several morphological parameters: height of thyrocytes (HT), follicular volume (FV), epithelium-colloidal index (ECI)

Results: The results of surgery treatment we evaluated one year later. In $50.8 \%$ of patients there was euthyroidism, $25.4 \%$-hypothyroidism, recurrence of thyrotoxicosis was in $23.8 \%$ of cases. Frequency of Graves' ophtalmopathy was significantly higher in group of patients with postoperative thyrotoxicosis (48\%); in group of patients with postoperative hypothyroidism frequency of Graves' ophtalmopathy was in $24 \%$ of cases $(p=0.02)$. Volume of thyroid gland before surgery treatment was significantly larger in group of patients with postoperative thyrotoxicosis (PT) $\left(52.96 \pm 3.74 \mathrm{ml}^{3}\right)$ than in postoperative hypothyroidism $(\mathrm{PH})$ group $\left(41.79 \pm 3.39 \mathrm{ml}^{3} ; \mathrm{p}=0.02\right)$. The levels of TSAb before surgery treatment was time higher in group of PT patients, than in $\mathrm{PH}$ patients $(38.5 \pm 9.03 \mathrm{mU} / 1$ vs. $5.69 \pm 1.03 \mathrm{mU} / 1 ; \mathrm{p}=0.001)$. HT to patients with PT was significantly higher than in group of patients with PH $(5.94 \pm 0.41 \mu \mathrm{m}$ vs. $4.88 \pm 0.25 \mu \mathrm{m} ; \mathrm{p}=0.013)$. FV in patients with PT was significantly less than in group of patients with $\mathrm{PH}(7500 \pm 1200 \mu \mathrm{m}$ vs. $12790 \pm 2100 \mu \mathrm{m} ; \mathrm{p}=$ $0.01)$. ECI was significantly higher in PT patients than in patients with $\mathrm{PH}$ $(1.07 \pm 0.009$ vs. $0.73 \pm 0.08 ; \mathrm{p}=0.001)$.

Conclusion: Clinical and morphological criteria in patients with recurrence of thyrotoxicosis after surgery treatment have showed significantly higher functional activity of thyroid gland initially.

\section{P252 \\ HYPERTHYROID OR NOT: A CASE OF T3 PREDOMINANT GRAVES' DISEASE}

Barbara Lucchini ${ }^{0}$, Stefan Bilz ${ }^{1}$

${ }^{1}$ Kantonsspital San Gallen

Introduction: A dysproportionate increase of T3 when compared to T4 serum concentrations is well established in hyperthyroid Graves' disease (GD). Up to two thirds of circulating T3 may originate from the thyroid both through enhanced thyroidal deiodination of T4, mainly by type 1 iodothyronine deiodinase (D1), and direct formation of T3-iodotyrosine in severely hyperthyroid patients. In a subset of patients, also referred to as T3 predominant GD, T3 concentrations may remain elevated even after T4 has returned to the normal range following antithyroid drug therapy due to an additional increase in thyroidal iodothyronine deiodinase type 2 (D2) activity and even more enhanced thyroidal $\mathrm{T} 3$ formation.

Clinical Case: A 50 year old woman was referred after GD had been treated with carbimazole (CMZ, 5-30 mg q.d.) for more than one year without achieving euthyroidism, as indicated by continuously suppressed TSH levels, despite free T4 (fT4) values that were within or even below the normal range. She appeared hyperthyroid with signs of ophtalmopathy and a WHO grade III goiter. On $10 \mathrm{mg} \mathrm{CMZ} \mathrm{q.d.} \mathrm{TSH} \mathrm{remained} \mathrm{suppressed}(0.059 \mathrm{mU} / \mathrm{l})$ with discordant results for free T4 and T3 concentrations (fT4 $4.1 \mathrm{pmol} / 1,6.8$ - 18; fT3 $13.1 \mathrm{pmol} / 1 ; 3.2-6.0$ ). A diagnosis of T3 predominant GD was made. Therapy with potassium iodide resulted in rapid lowering of fT3 into the normal range and a total thyroidectomy was performed.

Discussion and Conclusion: T3 predominant GD needs to be considered if TSH fails to return to the euthyroid range after fT4 concentrations have been adequately lowered with antithyroid drug therapy. Propylthiouracil (PTU), a specific inhibitor of D1 is preferred over CMZ in these patients. However, since even PTU frequently fails to restore euthyroidism, thyroidectomy is recommended early in the course of this specific subtype of GD.

\section{P253}

\section{Clinical OUTCOMES OF GRAVES' DISEASE USING A TARGETED 131-I ABSORBED DOSE OF 60 GY TO THE THYROID}

Stephen L Hyer ${ }^{1}$, Brenda Pratt ${ }^{2}$, Matthew Gray ${ }^{2}$, Kate Newbold ${ }^{3}$, Glenn Flux ${ }^{2}$, Clive Harmer ${ }^{2}$

${ }^{1}$ St. Helier Hospital; Dept. of Endocrinology \& Diabetes, ${ }^{2}$ Royal Marsden Hospital, ${ }^{3}$ The Royal Marsden Hospital National Health Service Trust, London, England, UK

Background: The optimal ${ }^{131}$ I activity to successfully treat Graves' disease remains controversial. With the advent of accurate dosimetry and imaging, it is possible to minimize risks to the patient by avoiding unnecessarily large activities. 
Objective: To assess the long term clinical outcome of patients with Graves' disease treated with a target absorbed ${ }^{131} \mathrm{I}$ dose of $60 \mathrm{~Gy}$.

Method: Following dosimetric study, 283 patients (median age 46 yrs) were treated with ${ }^{131} \mathrm{I}$ aiming to achieve a dose of $60 \mathrm{~Gy}$ to the thyroid. The administered radioactivity was calculated using the MIRD formula taking into account the thyroid volume, maximal ${ }^{131}$ I uptake and effective half-life. Patients with thyrocardiac disease (heart failure or atrial fibrillation) were excluded. Clinical outcomes were analyzed at 6 months, 1, 3, 5 and 10 years with a median follow-up of 37.5 months.

Results: The required radioactivity to achieve 60 Gy was highly variable ranging from $17.4-1377 \mathrm{MBq}$ (mean $106 \mathrm{MBq}$; median $78 \mathrm{MBq}$ ). At 6 month follow-up, 158 (56\%) remained hyperthyroid, defined as patients still requiring antithyroid medication. By 12 months, the number still hyperthyroid had fallen to $65(23 \%)$ patients. $38 \%$ (108 patients) required a second ${ }^{131} \mathrm{I}$ administration with a mean of 16.4 months after initial ${ }^{131}$ I therapy. Of the 175 patients treated with a single $60 \mathrm{~Gy}$ dose, $38 \%$ were euthyroid on no specific medication at 3 years, $32 \%$ at 5 years and $26 \%$ at 10 years. Patients with persistent hyperthyroidism had significantly larger thyroid volumes.

Conclusions: A targeted radioiodine absorbed dose of 60 Gy to the thyroid can delay the long term onset of hypothyroidism in about a quarter of patients with Graves' disease. This approach is best suited for younger patients with smaller thyroid volumes where urgent control of hyperthyroidism is not required. In this group of patients, it may well be the treatment of choice.

\section{P254}

\section{NATURE AND PREVALENCE OF PREGNANCY ASSOCIATED THYROTOXICOSIS}

Akane Ide $^{1}$, Nobuyuki Amino ${ }^{1}$, Takumi Kudo ${ }^{1}$, Shino Kang ${ }^{1}$,

Waka Yoshioka' ${ }^{1}$, Mako Fujiwara ${ }^{1}$, Eijun Nishihara ${ }^{1}$, Mitsuru Ito ${ }^{1}$,

Hirotoshi Nakamura ${ }^{1}$, Akira Miyauchi ${ }^{1}$

${ }^{1}$ Kuma Hospital

Objective: Pregnancy influences markedly the clinical course of thyroid disease. In early pregnancy, hCG can lead to gestational thyrotoxicosis (GT), and possibly induce the onset of Graves' thyrotoxicosis. However there are no reports on prevalence of 'pregnancy induced Graves' disease' (PrGr). After delivery, postpartum destructive thyrotoxicosis (PPT) and Graves' thyrotoxicosis (PPGr) are also frequently observed. We studied the prevalence of these types of thyrotoxicosis in our outpatient clinic of Kuma Hospital.

Methods: We performed a retrospective review of 1.197 consecutive nontreated patients with thyrotoxicosis from January 2013 to December 2013. Diagnosis of these four different thyrotoxicosis was made by measurement of FT4, FT3, TSH, TSH receptor antibody and thyroid blood flow.

Results: Among patients with thyrotoxicosis, Graves' disease, painless thyroiditis, subacute thyroiditis and Plummer' disease were found in $70.7 \%$, $17.3 \%, 7.1 \%$ and $2.8 \%$, respectively. Of these, $1,011(84.5 \%)$ were female and $186(15.5 \%)$ were male patients. GT, PrGr, PPT and PPGr were found in 24 (6.1\%), $4(1.0 \%), 15(3.8 \%)$, and 31(7.9\%) patients, respectively, within 392 female patients of child-bearing age (20-39 yo). PPGr was found in 31 of 107 (28.9\%) parous women aged 20-39 with Graves' disease.

Conclusion: A clinically significant number of women develop Graves' disease in the postpartum period. The prevalence of pregnancy induced Graves' disease was much less than that of postpartum Graves' disease and gestational thyrotoxicosis. These results may explain partly the female preponderance of thyrotoxicosis.

\section{P255}

\section{THE VALUE OF COLOUR FLOW DOPPLER SONOGRAPHY IN EVALUATION OF HYPERTHYROIDISM INDUCED BY HUMAN CHORIONIC GONADOTROPIN}

\section{Katja Zaletel I', Simona Gaberšček ${ }^{2}$, Edvard Pirnat ${ }^{1}$, Sergej Hojker ${ }^{2}$}

${ }^{1}$ University Medical Centre Ljubljana, Department of Nuclear Medicine,

${ }^{2}$ University Medical Centre Ljubljana, Department of Nuclear Medicine;

University of Ljubljana, Faculty of Medicine

Introduction: Symptomatic hyperthyroidism, induced by human chorionic gonadotropin (hCG), rarely occurs as initial presentation of placental or germ cell tumours. In such cases, routine clinical methods do not enable the thyroid specialist to obtain adequate information to suspect hCG-secreting tumor.

Case Report: We report 3 cases, directed to the thyroid department for symptomatic hyperthyroidism in the period between January 2008 and March 2013. Patient 1 was 31-years old female 3 weeks after delivery with TSH $0.024 \mathrm{mU} / \mathrm{L}, \mathrm{fT}_{4} 87.8 \mathrm{pmol} / \mathrm{L}$ and $\mathrm{fT}_{3} 30.8 \mathrm{pmol} / \mathrm{L}$. Patient 2 was 45 -years old male with TSH $0.008 \mathrm{mU} / \mathrm{L}, \mathrm{fT}_{4} 24.3 \mathrm{pmol} / \mathrm{L}$ and $\mathrm{fT}_{3} 7.67 \mathrm{pmol} / \mathrm{L}$. Patient 3 , a 36-years old female, was 11 months pregnant and 5 months after previous abortion, presenting with TSH $0.008 \mathrm{mU} / \mathrm{L}, \mathrm{fT}_{4} 25.0 \mathrm{pmol} / \mathrm{L}$ and $\mathrm{fT}_{3} 8.51$ $\mathrm{pmol} / \mathrm{L}$. All three cases were negative for TPO, Tg and TSH-receptor antibodies. On ultrasound, thyroid volume in patients 1,2 and 3 was $30,6.9$ and $5 \mathrm{~mL}$, respectively, and in all, structure was isoechoic and homogenous. To elucidate the cause of hyperthyroidism, colour flow Doppler (CFD) was performed. Increased thyroid vascularity and peak systolic velocity (PSV) were shown: Doppler pattern 3 with PSV $26.1 \mathrm{~cm} / \mathrm{s}$ in patient 1 , pattern 2 with PSV 12.3 $\mathrm{cm} / \mathrm{s}$ in patient 2 and pattern 2 with PSV $9.1 \mathrm{~cm} / \mathrm{s}$ in patient 3 . As CFD indicated thyroid stimulation in patients without apparent thyroid disease, $\mathrm{hCG}$ was measured. In patient 1 with $\mathrm{hCG}>1.000 .000 \mathrm{U} / \mathrm{L}$ metastatic choriocarcinoma was diagnosed, in patient 2 with hCG $525.647 \mathrm{U} / \mathrm{L}$ metastatic seminoma was confirmed, while in patient 3 with $\mathrm{hCG}>20.000 \mathrm{U} / \mathrm{L}$ molar pregnancy was established.

Conclusion: When hyperthyroidism cannot be explained by apparent thyroid disease, CFD is a useful tool in the diagnostic procedure. If thyroid vascularity is increased, hCG should be measured as a possible cause of thyroid stimulation.

\section{P256 \\ HYPERTHYROIDISM IS ASSOCIATED WITH SEVERE WORK DISABILITY. A DANISH REGISTER-BASED STUDY}

Frans Brandt ${ }^{1}$, Marianne Thvilum ${ }^{1}$, Laszlo Hegedüs ${ }^{1}$,

Thomas Heiberg Brix ${ }^{1}$

${ }^{1}$ Odense University Hospital

Background: Hyperthyroidism, independent of aetiology, is associated with increased morbidity and mortality. This ought intuitively lead to socioeconomic consequences, such as early retirement or loss of income.

Aim: To examine the degree of work disability, as measured by the risk of receiving disability pension (before the age of 60) and lower labour market income in patients diagnosed with hyperthyroidism.

Methods: Observational register- and population-based cohort study. By record-linkage between different Danish health registers, 1942 hyperthyroid singletons diagnosed before the age of 60 were each matched with 4 non-hyperthyroid controls and followed for a mean of 9 years (range 1-20). Additionally, we included 584 same sex twin pairs discordant for hyperthyroidism. The risk of disability pension was evaluated by the Cox regression analysis. Changes in labour market income progress over 5 years were evaluated using a difference in difference model.

Results: Hyperthyroid individuals more often received disability pension; hazard ratio (HR) 1.88, (95\% confidence interval (CI): 1.57-2.24). Subdividing according to the cause of hyperthyroidism yield essentially similar results; Graves' disease (GD) (HR 1.51, 95\% CI: 0.87-2.63) and toxic nodular goitre (TNG) (HR 2.10, 95\% CI: 1.02-4.36). In an analysis of labour market income, two years before compared with two years after the thyroid diagnosis, hyperthyroid individuals had increased on average $1189 €$ less than their euthyroid controls $(\mathrm{P}<0.001)$. This difference in income was even more pronounced in GD (2539€) but attenuated in individuals with TNG (132 $€)$. Essentially similar results were found in the population of monozygotic twins, suggesting that confounding due to genetic or environmental factors is negligible.

Conclusion: A diagnosis of hyperthyroidism is associated with severe work disability as reflected by a nearly $90 \%$ increased risk of receiving disability pension and loss of labour market income. 
SURGICAL OUTCOMES OF SUBTOTAL, NEARTOTAL, AND TOTAL THYROIDECTOMY FOR GRAVES' DISEASE

Hiroyuki Iwasaki ${ }^{1}$, Michiko Fukahori', Saito Shirayama ${ }^{1}$, Naoki Gotoh $^{2}$, Shinsuke Hatori ${ }^{3}$, Nobuyasu Suganuma ${ }^{4}$

${ }^{1}$ Department of Surgery, Nomura Hospital, ${ }^{2}$ Department of Surgery, luhw Atami Hospital; Department of Surgeru, Nomura Hospital, ${ }^{3}$ Department of Surgery, Ashigarakami Hospital, ${ }^{4}$ Department of Surgery, Yokohama City University

Introductions: Surgical treatment for Graves' disease has been verified for over 100 years. In recent years, to avoid relapse and to remedy severe exophthalmos, total thyroidectomy has been accepted in several countries. We studied proper surgical treatment for patients with various degrees of Graves' disease.

Methods: Surgical outcomes were analysed in the following three groups. 66 patients underwent subtotal thyroidectomy for normalizing thyroid function without taking medicine, 40 patients underwent near-total thyroidectomy to avoid relapse, and 56 patients underwent total thyroidectomy for remedying severe exophthalmos or giant goiter. Their estimated remnant thyroids were $3-5 \mathrm{~g}$ in the subtotal group and $1-3 \mathrm{~g}$ in the near-total group, respectively. Postoperative thyroid function and TRAb were followed up for over 5 years.

Results: In the subtotal group, 40 patients $(60.6 \%)$ remained normal thyroid function, 18 patients $(27.3 \%)$, subclinical hypothyroid and 8 patients $(12.1 \%)$ relapsed into hyperthyroid. In the near-total group, 16 patients $(40.0 \%)$ remained normal thyroid function, $15(37.5 \%)$, subclinical hypothyroid, $7(17.5 \%)$, permanent hypothyroid, and two $(5.0 \%)$ relapsed into hyperthyroid. Surgical complications (i.e. temporary hypoparathyroid) were recognized in 5 patients (4.7\%) out of 106 subtotal and near-total group, and 25 patients (44.6\%) in total thyroidectomy group.

Conclusions: Most patients successfully underwent the surgery and felt happy being free from their large goiters or taking tablets everyday. As far as normalizing the thyroid functions by subtotal or near-total thyroidectomy, it is strictly impossible to get perfect results in spite of our efforts verifying remnant thyroids for many years. Ultimately, total thyroidectomy is acceptable surgical procedure for complete recovery from the disease even though the patients have to take thyroid hormone for the rest of their life.

\section{P258 \\ THE HIGH INCIDENCE OF PAINLESS THYROIDITIS AS A CAUSE OF THYROTOXICOSIS IN KOREA}

Sang Jin Kim ${ }^{0}$, Yeo Joo Kim

${ }^{1}$ Department of Internal Medicine, Subdivision of Endocrinology and

Metabolism, Soonchunhyang University, Cheonan, Korea

Background: The most common cause of thyrotoxicosis is Graves' disease (GD), followed by painless thyroiditis (PT). In Korea, the treatment of choice for GD is antithyroid drugs (ATDs). Most cases of PT spontaneously improve under observation. Therefore, a differential diagnosis is very important. The prevalence of PT varies among countries, ranging from $0 \%$ to $20 \%$. In Korea, there is no official report for the prevalence of PT.

Methods: Ninety-nine thyrotoxic patients, excluding pregnant or lactating women, were routinely examined with a $99 \mathrm{~m}$ technetium thyroid scan. We assessed the clinical characteristics, serum levels of Free T4, thyroid stimulating hormone (TSH), thyroid peroxidase antibody (TPOAb), thyroglobulin antibody (TGAb), and thyrotropin-binding inhibitory immunoglobulin (TBII), as well as findings of $99 \mathrm{~m}$ technetium thyroid scans.

Results: Of the 99 thyrotoxic patients, 69 were diagnosed with GD and 30 were diagnosed with PT. All GD patients, who were diagnosed on the basis of the scan findings, showed improved and normalized thyroid hormone levels following ATD therapy. All PT patients improved spontaneously without ATDs.

Fourteen (46.7\%) PT patients and 44 (64.7\%) GD patients were positive for TPOAb, whereas $20(66.7 \%)$ PT patients and $43(63.2 \%)$ GD patients were positive for TGAb. TBII positivity was observed in only $70.1 \%$ of GD patients. Some PT patients had the highest level of FT4 level.

Conclusions: PT accounted for very high proportion of thyrotoxicosis in this study. Parameters such as age, sex, goiter size or nature, as well as lev- els of FT4, TPOAb or TGAb, and TBII cannot differentiate between GD and PT. Therefore, routine thyroid scans should be carried out for all thyrotoxic patients, except pregnant or lactating women.

\section{P259 \\ FACTORS INFLUENCING PROLONGED TREATMENT OF GRAVES' DISEASE}

Jasmina Ciric ${ }^{1}$, Branka Lazic ${ }^{2}$, Milos Zarkovic ${ }^{1}$, Biljana Beleslin ${ }^{1}$, Mirjana Stojkovic², Slavica Savic ${ }^{2}$, Tijana Lalic ${ }^{2}$

${ }^{1}$ Clinic of Endocrinology, Diabetes and Metabolic Diseases, Clinical Center of Serbia; Medical School University of Belgrade, ${ }^{2}$ Clinic of Endocrinology, Diabetes and Metabolic Diseases, Clinical Center of Serbia

Introduction: Graves' disease (GD) is treated by drugs $12-18$ months or longer, by surgery or by radioiodine. Recurrence is seen in $50 \%$ of cases.

Aim: The aim of the study was to examine the influence of different parameters recognized as possible risk factors for longer duration of GD.

Material and Methods: In the group of 59 patients with GD treated longer than 12 months we analyzed the influence of goitre size, orbitopathy (GO), thyroid antibodies, smoking and other risk factors for the achievement of remission.

Results: The range of age was $32-81$ years, $68 \%$ were female. Family history was positive in $20 \%$ and concomitant autoimmune diseases in $1.7 \%$. There were $64.4 \%$ of smokers; among them $79 \%$ were women. GO appeared before GD in $8.5 \%$ of patients; at the same time in 20.3 , and during GD in $71.2 \%$. The mean value of GO duration after the diagnosis of GD was $12.8 \pm 9.1$ months (active GO -36, moderately severe -43 patients). Mean value of GD duration was $20 \pm 9.5$ months (11-50). Low level of TSH was measured for $1-50$ months (mean 15.8 \pm 10.5 ). Mean value of TRAb was $12.5 \pm 18.5$, and persistence time $19.1 \pm 11.6$ months. Prolonged treatment by thionamides was the choice in 46 patients. Eight women had large goiter, 4 were treated by surgery after 12 months. Three patients were treated by radioiodine because of unstable disease. Six patients had prolonged low dose block-and-replace therapy becouse of high level of TRAb and active GO. A significant positive correlation of GD duration and TRAb level $(\mathrm{P}<0.05)$, GO duration $(\mathrm{P}<0.01)$, GO onset $(\mathrm{P}<0.05)$ and goitre size $(\mathrm{P}<0.05)$ was found.

Conclusion: We found that factors influencing the duration of GD and a definite choice of treatment were the level and persistence of TRAb, large goitre, prolonged duration of GO, and later onset of GO.

\section{P260 \\ SELF-REPORTED WORK ABILITY VARIES ACCORDING TO TYPE OF THYROID DISEASE}

Mette Andersen Nexø $\emptyset^{1}$, Torquil Watt ${ }^{2}$, Steen Joop Bonnema ${ }^{3}$,

Laszlo Hegedüs ${ }^{3}$, Åse Krogh Rasmussen², Ulla Feldt-Rasmussen², Jakob Bue Bjørner ${ }^{1}$

${ }^{1}$ The National Research Center for the Working Environment, ${ }^{2}$ Copenhagen University Hospital (Rigshospitalet), ${ }^{3}$ Odense University Hospital

Objective:Thyroid diseases impact quality of life (QOL). The ability to work is an important aspect of QOL, yet few studies have examined work ability among patients with thyroid diseases. This study examined work ability in a wide range of thyroid diseases.

Methods: Employed patients $(\mathrm{n}=507)$ with non-toxic goitre, toxic nodular goitre, Graves' disease, Graves' orbitopathy, Hashimoto's hypothyroidism, or other thyroid diseases were recruited from two Danish hospitals (Odense and Copenhagen University hospital). Of these 203 patients were newly diagnosed ( $<1$ year). Using linear regression, the thyroid diagnostic subgroups were compared on the role physical and role emotional subscales from the SF-36 and on a thyroid specific work disability scale. Longitudinal analyses examined whether an item from the thyroid specific work disability scale predicted early retirement. Analyses were adjusted for age, gender, job type, and years with disease.

Results: Compared to patients with non-toxic goitre, patients with Graves' disease had worse function on the role physical (-4.0, 95\% CI: $-6.3 /-1.7)$, role emotional (-2.8; 95\% CI: $-5.2 /-0.4)$, and thyroid specific scales $(-11.4 ; 95 \%$ CI: -15.6/-7.2). Patients with Hashimoto's hypothyroidism had worse function on the role emotional $(-3.4,95 \%$ CI:-5.7/-1.2) and thyroid specific $(-4.2 ; 95 \%$ 
CI: $-8.2 /-0.2)$ scales. The low work ability was most pronounced within the first year of diagnosis. Thyroid specific work disability predicted labour market exclusion within the next 4 years [OR $=5.0,95 \% \mathrm{CI}: 2.7-9.1]$.

Conclusions: Self-reported work role limitations and thyroid specific work disability is most pronounced in Graves' disease and Hashimoto's hypothyroidism; in particular within the first year of diagnosis. Self-assessed work disability is a risk factor for permanent exclusion from the labour market.

\section{P261 \\ TREATMENT OF HYPERTHYROIDISM IN SWEDEN \\ Kristina Byström $^{1}$, Gabriel Sjölin ${ }^{2}$, Mirna Abraham-Nordling ${ }^{3}$, Jan Calissendorff', Helena Filipsson ${ }^{5}$, Bengt Hallengren ${ }^{6}$, \\ Mats Holmberg ${ }^{4}$, Selwan Khamisi ${ }^{7}$, Mikael Lantz ${ }^{8}$, Ove Törring ${ }^{9}$, Göran Wallin ${ }^{0}$ \\ ${ }^{1}$ End Klin Örebro, ${ }^{2}$ Dept Surg Örebro, ${ }^{3}$ Dept Surgery Danderyd, ${ }^{4}$ Dept End Karolinska, ${ }^{5}$ Dept End Sahlgrenska Academy, ${ }^{6}$ Dept End Skåne University Hospital, ${ }^{7}$ Dept End Uppsala, ${ }^{8}$ Dept End Skånes University Hospital, ${ }^{9}$ Dept End Södersjukhuset}

Introduction: The incidence of hyperthyroidism was found to be 27.1 / 100000 inhabitants (children included) / year in a prospective study 2003 2005 in Sweden, including approximately $40 \%$ of the entire Swedish population of 9 million inhabitants. Sweden is considered iodine sufficient. Living Patients have been followed up by a Questionnaire and we have studied treatment, cure, complications and quality of life.

Methods: 2915 Patients of all ages, who were diagnosed with overt hyperthyroidism in the years 2003-2005, were prospectively registered in a multicenter study (cities; Malmö, Karlskrona, Göteborg, Örebro, Eskilstuna / Katrineholm, Stockholm and Uppsala) organized by the "Swedish Thyroid Study Group" (Vetenskapsrådets Planeringsgrupp för Tyreoideasjukdomar). For each patient - the diagnosis (Graves' disease (GD), toxic multinodular goiter (TMG) and solitary toxic adenoma (STA)), smoking, initial treatment, occurrence of Thyroid Associated Ophthalmopathy (TAO) and demographic data were registered. The participating clinicians were specialists in medicine, endocrinology, oncology, nuclear medicine or surgery and all shared interest in thyroid diseases. 2451 living patients have been asked to participate in a follow up study. 1442 (58\%) patients are included in this study.

Results: All patients euthyroid today.

$24 \%$ of the patients have had medical treatment, without radioiodine or Surgery

$47 \%$ have been treated with radioiodine

$25 \%$ of the patients have been operated

$4 \%$ have been treated with both radioiodine and surgery

Conclusion: $24 \%$ of the patients in this material are cured with medical treatment. $47 \%$ have been treated with radioiodine. $25 \%$ of the patients have been operated and $4 \%$ have had a combination of all treatment options. There are geographical differences both according incidence and treatment between the different hospitals. Further evaluation of this patient material is ongoing

\section{P262 \\ CLINICAL AND IMMUNOLOGIC ASSESSMENT OF EFFICIENCY AND SAFETY OF VARIOUS SCHEMES APPLICATION FOR CURING GRAVES'S DISEASE}

Tatiana Rodionova ${ }^{1}$, Arina Frolova ${ }^{0}$

${ }^{1}$ Saratov State Medical University N.A. V.I. Razumovsky

Objectives: to assess symptoms regress and levels of cytokines in patients with Graves's disease when using different treatment regimens.

Methods: were studied 68 patients (18 to 50 years old) with Graves's disease. Drug-induced euthyroidism, complications induced by thyrostatic drugs, interleukines levels (IL-6, IL-8 and IL-10) were assessed.

Results: were studied 47 women and 21 men. Their average age is 36 [28.0; 42.0]. Beginning daily dose of Thiamazole was $30 \mathrm{mg}$. The euthyroidism was reached in 8-10 weeks. Then patients were divided into 2 equal groups randomly. The Thiamazole dose for the first group was gradually reduced to the supporting treatment dose, the second group patients received combined treatment: $10-15 \mathrm{mg}$ of Thiamazole and $25-50 \mathrm{mcg}$ of Levothyroxine a day.
No agranulocytosis in patients was detected at the time of assessing the safety of the both treatment regimens. In the beginning cytocines plasma levels were: IL-6 - 12.7 [7.8; 16.4] pcg/ml, IL-8 - 127.4 [99.2; 135.8] pcg/ml and IL-10- 48.1 [32.7; 51.4$] \mathrm{pcg} / \mathrm{ml}$. In 3.5 months after division into subgroups, the cytokine level in all the patients decreased, but there were no significant differences between the groups. In 1 group: IL-6 - 7.1 [6.8; 8.5] pcg/ml), IL-8 - $64.2[59.6 ; 72.3] \mathrm{pcg} / \mathrm{ml}, \mathrm{IL}-10$ - 10.8 [9.2; 11.7] pcg/ml. In 2 group: IL-6 - $6.9[6.2 ; 8.1] \mathrm{pcg} / \mathrm{ml}(\mathrm{P}>0.05), \mathrm{IL}-8-62.9$ [57.7; 71.3] pcg/ml $(\mathrm{P}>0.05)$, IL-10 - $9.9[9.0 ; 11.4] \mathrm{pcg} / \mathrm{ml}(\mathrm{P}>0.05)$.

Conclusion: The treatment methods used to cure Graves's disease (titration and block and replace) are comparable in efficiency and safety. As there were no accurate differences in cytokine concentration in 6 months of treatment in two groups under study, the higher dose of Thiamazole prescribed alongside with Euthyrox does not have any significant influence on the autoimmune process activity.

\section{P026 Novel Actions of Thyroid Hormones}

\section{P263 \\ 3-IODOTHYRONAMINE AS A POTENTIAL ANTI- TUMOR COMPOUND}

Sandra Ghelardoni ${ }^{1}$, Leonardo Lorenzini ${ }^{1}$, Claudio Ghezzani ${ }^{1}$,

Vittoria Carnicelli ${ }^{1}$, Grazia Chiellini ${ }^{1}$, Alessandra Salvetti ${ }^{1}$,

Riccardo Zucchi ${ }^{1}$

${ }^{1}$ University of Pisa

Objectives: 3-iodothyronamine (T1AM) is an endogenous messenger chemically related to thyroid hormone, which produces significant effects on energy metabolism and modulates mammalian sirtuin expression. In particular, subchronic T1AM administration was reported to increase the expression of mammalian sirtuin 6 (SIRT6), which has a pro-apoptotic role. Therefore in the present study we evaluated the effects of T1AM on viability and proliferation of cancer and non cancer cell lines.

Methods: Four cell lines (HepG2, liver carcinoma; H9c2, cardiomyoblasts; Caco-2, colon carcinoma; and WI-38, lung fibroblasts) were cultured for $24 / 48 \mathrm{~h}$ in the presence or absence of different concentrations of exogenous T1AM (0.1-1-10 mM). Cell viability was determined by crystal violet staining and transmission electron microscopy (TEM) analysis was performed. To evaluate changes in SIRT 6 gene expression, cells were incubated with the same concentrations of T1AM, and gene expression was assessed by real-time PCR.

Results: In HepG2 cells, T1AM significantly reduced cell viability $(\mathrm{P}<$ 0.05 with $10 \mathrm{mM}$ T1AM) while cell proliferation increased in H9c2 and WI-38 cells $(\mathrm{P}<0.05$ with $1-10 \mathrm{mM}$ T1AM). After exposure to $10 \mathrm{mM}$ T1AM, SIRT6 gene expression increased in HepG2 $(\mathrm{P}<0.05)$ while in H9c2 cells it was reduced $(\mathrm{P}<0.05)$. No changes were detected in Caco-1 and WI-38 cell lines. TEM analysis revealed morphological changes in mitochondria and endoplasmic reticulum upon $48 \mathrm{~h}$ of treatment with T1AM $(1 \mathrm{mM})$ in either H9c2 or HepG2 cell lines. Nuclear alterations were observed only in HepG2 cells.

Conclusion: Our results suggest that T1AM may induce overexpression of SIRT6 and selectively reduce the proliferation of liver cancer cells. 


\section{P264}

NEW SIGNALING PATHWAY FOR TRACE AMINE ASSOCIATED RECEPTORS: TAAR8 SHOWS BASAL GI SIGNALING

Jessica Mühlhaus ${ }^{1}$, Juliane Dinter ${ }^{2}$, Daniela Nürnberg ${ }^{2}$, Maren Rehders ${ }^{3}$, Georg Homuth ${ }^{4}$, Chun-Xia Y ${ }^{5}$,

Carolin Leonie Piechowski², Gunnar Kleinau ${ }^{2}$, Heiko Krude ${ }^{2}$, Annette Grüters ${ }^{2}$, Josef Köhrle ${ }^{2}$, Klaudia Brix ${ }^{3}$, Matthias Tschöp ${ }^{5}$, Heike Biebermann ${ }^{2}$

${ }^{1}$ Charité Universitätsmedizin Campus Virchow-Klinikum; Institute of Experimental Pediatric Endocrinology, ${ }^{2}$ Charité Universitätsmedizin Campus Virchow-Klinikum, ${ }^{3}$ Jacobs University Bremen Ggmbh,

${ }^{4}$ Universitätsmedizin Greifswald, ${ }^{5} \mathrm{Helmholtz} \mathrm{Zentrum} \mathrm{München} \mathrm{-}$

German Research Center for Environmental Health

Objective:Trace amine associated receptors (TAAR) are a family of G-protein-coupled receptors evolutionarily conserved throughout diverse vertebrates. TAAR, as characterized so far, show high ligand promiscuity. Though, most of them are still orphan receptors. The thyroid hormone derivative 3-iodothyronamine (3T1AM) is a known agonist for TAAR1, activating Gs signaling pathway in vitro. Previous studies reported that application of 3T1AM in vivo in both, wild type and TAAR1 knockout mice, resulted in a variety of effects like negative inotropy and decreased body temperature, difficult to explain by TAAR1 and Gs activation. Therefore, we aim to identify other TAAR that are targets of 3T1AM and activate signaling pathways other than Gs. So far, only very limited information on TAAR8 exists despite the fact that TAAR8 is expressed in the rat heart.

We analyzed the human (hTAAR8) and murine (mTaar8b) orthologues of the orphan TAAR8 for different G-protein dependent signaling properties and investigated their expression.

Methods: Expression analysis of mTaar8b was carried out by locked nucleic acid in situ hybridization and qRT-PCR. Gs and Gi/0 activation was determined by AlphaScreen Technology. Gq/11, G12/13 signaling was analyzed by luciferase reporter gene assays (IP3-luc or RhoA-luc).

Results: mTaar8b is not expressed in the mouse heart. However, we identified mTaar8b expression in the liver and cerebellum. Concerning all tested signaling pathways, 3T1AM exerts only marginal effects on Gs signaling and is presumably not the endogenous ligand of hTAAR8 and mTaar8b. However, TAAR8 showed basal Gi/0 signaling.

Conclusion: TAAR8-mediated signaling is not triggered by 3T1AM in vitro, and mTAAR $8 \mathrm{~b}$ is not expressed in mouse heart. However, this study revealed a new signaling pathway for TAAR as TAAR8 it shows basal Gi/0 signaling properties which has to be further elucidated in its physiological significance.

Supported by the Deutsche Forschungsgemeinschaft, SPP 1629 “Thyroid Trans Act" BI893/5-1.

\section{P265 \\ NOVEL SYNTHETIC THYRONAMINES AS NEW POWERFUL TAAR1 AGONISTS}

Grazia Chiellini', Simona Rapposelli ${ }^{2}$, Maria Digiacomo ${ }^{2}$, Stefano Espinoza ${ }^{3}$, Martina Sabatini ${ }^{1}$, Sabina Frascarelli ${ }^{1}$, Annunziatina Laurino ${ }^{4}$, Laura Raimondi ${ }^{4}$, Riccardo Zucchi ${ }^{1}$ ${ }^{1}$ Dept. of Pathology, University of Pisa, Pisa, Italy, ${ }^{2}$ Dept. of Pharmacy, University of Pisa, Pisa, Italy, ${ }^{3}$ Italian Institute of Technology (Itt), Genova, Italy, ${ }^{4}$ Dept of Neurofarba; Section of Pharmacology, University of Florence, Italy

Objectives: Thyronamine (T0AM) and 3-iodothyronamine (T1AM) are endogenous chemical messengers identified almost a decade ago as potent trace amine associated receptors type1 (TAAR1) agonists. Functional investigations studies have shown that these compounds may be potentially useful for a wide variety of therapeutic applications, including obesity, neuro-psychiatric disorders and possibly cancer. However, exogenously administered thyronamines undergo rapid metabolism, which limits their therapeutic impact. The aim of this investigation is the production of synthetic thyronamine analogs.

Methods: We designed and efficiently synthesized a small panel of synthetic thyronamines (SG-1,2,7,8), where the introduction of selected key structural modifications is expected to improve the biopharmacological profile and pharmacokinetic properties of the parent compounds. Subsequently, we tested the agonist or antagonist properties of the newly designed compounds on the basis of the pharmacological responses that were originally described for T0AM and T1AM, i.e. cAMP production in HEK-293 cells expressing mouse TAAR1, induction of negative inotropic effect (reduced cardiac output) in isolated working rat heart preparations, and modulation of plasma glucose level in CD-1 mice.

Results: Bioluminescence Resonance Energy Transfer (BRET) based assays, indicate that all newly designed thyronamines are effective TAAR1 agonists in HEK-293 cells transfected with mouse TAAR1, and compound SG-2, which shares a close similarity to T1AM, was found to be the most potent $\left(\mathrm{EC}_{50}=240 \mathrm{nM}\right)$. Heart perfusion experiments, carried out at the present time only with SG-1 and SG-2, indicate that both compounds produced a dose-dependent reduction of cardiac output with IC(50) of $40 \mu$ icroM, and $20 \mu \mathrm{icroM}$, respectively. In addition, preliminary pharmacology experiments indicate that a single low dose of SG-1 (1.32 $\mu \mathrm{g} / \mathrm{kg}$; icv or ip) increases plasma glycaemia with potency comparable to that of T0AM and T1AM.

Conclusions: Effective synthetic thyronamines can be produced providing new tools to explore the physiological function of TAAR1, and possibly novel therapeutic agents.

\section{P266 \\ PHARMACOLOGICAL TREATMENT WITH 3-IODOTHYRONAMINE (T1AM) NORMALIZES METABOLIC SYNDROME IN POLYCYSTIC OVARY SYNDROME MOUSE MODEL}

Fariba Assadi-Porter ${ }^{1}$, Grazia Chiellini ${ }^{2}$, Marco Tonelli ${ }^{3}$, Warren Porter ${ }^{3}$, Ebru Selen ${ }^{3}$

${ }^{1}$ Texas Tech University, ${ }^{2}$ Dept. of Pathology, University of Pisa, Pisa, Italy, ${ }^{3}$ Uw-Madison

Objectives: Polycystic ovary syndrome (PCOS) is a complex metabolic and endocrine disorder, associated with weight gain and insulin resistance. PCOS affects $10-20 \%$ of adult women and is only diagnosed after puberty by the NIH criteria. Our recent metabolic studies in PCOS women, and in the PCOS-like mouse model indicated increased rates of carbohydrate to lipid synthesis and decreased lipid breakdown as common metabolic dysfunctions in PCOS. T1AM is an endogenous thyronamine that induces lipid oxidation and weight loss. We hypothesized that T1AM normalizes lipid dysfunction and associated metabolic syndromes in PCOS. The goal of this study was to examine effects of the chronic treatment with a pharmacological dose of 3-iodotyramine (T1 AM) in PCOS mice, as a possible natural treatment regime.

Methods: We screened plasma and tissues (liver, fat and muscle) from control PCOS and PCOS mice treated with T1 AM at $25 \mathrm{mg} / \mathrm{Kg} /$ Day for five days to identify changes in metabolome and transcriptome profiles associated with lipid and glucose pathways using proton nuclear magnetic resonance ( $\left.{ }^{1} \mathrm{H}-\mathrm{NMR}\right)$-based metabolomics and qRT-PCR.

Results: Statistical analysis of data revealed that T1AM treatment results in significant changes in biomarkers associated with lipid metabolism: increased lipid oxidation (3-hydroxybutyrate and acetone), decreased lipid synthesis (glycerol), increased gluconeogenesis (glucose and pyruvate), and increased antioxidant activity (glutathione and 2-hydroxybutyrate).

Conclusions: This is the first natural metabolic treatment regime in PCOS by nutrigenomics approach that may be applicable as future treatment for PCOS women.

\section{P267 \\ 3-T1AM UPTAKE IN A MURINE PANCREATIC B-CELL MODEL \\ Ina Lehmphul', Carolin Höfig ${ }^{2}$, Josef Köhrle ${ }^{1}$ \\ ${ }^{1}$ Institut Für Experimentelle Endokrinologie, Charité - \\ Universitätsmedizin Berlin, Germany, ${ }^{2}$ Karolinska Institutet, Department of Cell \& Molecular Biology, Stockholm, Sweden; Institut Für \\ Experimentelle Endokrinologie, Charité - Universitätsmedizin Berlin, Germany}

Objectives: 3-iodothyronamine (3-T1AM) is a thyroid hormone (TH) metabolite that exerts opposite effects to 3,3',5-triiodothyronine (T3), counterregulating TH action. 3-T1AM in pharmacological doses leads to hypoinsulinemia, hyperglucagonemia and hyperglycemia. The underlying mechanism 
was explained by binding to the adra2alpha receptor, as hypoinsulinemia was absent in a corresponding knock down animal model. As the pancreas expresses $\mathrm{TH}$ transporters, we tested in vitro the hypothesis, that not only plasma membrane-mediated 3-T1AM binding to G-protein coupled receptors (GPCR), but also 3-T1AM uptake and metabolism contributes to decreased glucose stimulated insulin secretion (GSIS).

Methods: MIN6 cells (murine pancreatic ß-cell line) were characterized for gene expression of TH transporters, deiodinases (DIO) and monoamineoxidases (MAO). Combined 3-T1AM uptake/binding assay and intracellular metabolism to the corresponding thyroacetic acid (TA1) was monitored and quantified by mass spectrometry at different time points after treatment with 3-T1AM ( \pm MAO-inhibitor).

Results: MIN6 cells express most of the classical TH transporters, as well as some of the proposed 3-T1AM transporters and deiodinases. 3-T1AM does not only bind to the plasma membrane, but is metabolized by intracellular MAO to TA1.

Conclusions: We conclude from these first data that MIN6 is an appropriate cell model to study $\mathrm{TH}$ metabolite effects on insulin production and the underlying biochemical mechanisms in vitro.

(Supported by DFG Graduate College 1208)

\section{P268}

NIS IS BASOLATERALY SORTED BY AP-1A AND AP-1B CLATHRIN ADAPTORS IN MDCK CELLS

Petrina Koumarianou ${ }^{1}$, Antonio De la Vieja ${ }^{2}$, Pilar Santisteban ${ }^{1}$

${ }^{1}$ Instituto de Investigaciones Biomédicas (Csic-Uam), ${ }^{2}$ Instituto de Salud Carlos III (Área de Biología Celular Y Desarrollo, Centro

Nacional de Microbiología)

Sodium Iodide Symporter (NIS) is a transmembrame protein located in the basal membrane of thyrocytes through a mechanism that is regulated by the thyroid stimulating hormone (TSH) at transcriptional and posttranscriptional levels. Despite the physiological and clinical relevance of NIS membrane localization for thyroid hormone biosynthesis and cancer treatment respectively, little is known concerning the molecular mechanisms and the traffic routes implicated in NIS basolateral sorting and plasma membrane targeting.

For this reason, we decided to study NIS basolateral traffic in the rat follicular thyroid PCC13 cells and in the MDCK-human NIS stably transfected cell line (MDCKhNIS). First, we performed time course kinetics of NIS plasma membrane targeting and intracellular compartment co-localization analysis after TSH stimulation in $\mathrm{PCCl} 3$ cells. Through membrane biotinylation we determined that NIS reaches the plasma membrane in 24 hours post-stimulation. By confocal microscopy we observed that NIS after exiting the ER passes through the Golgi apparatus where co-localizes with g-adaptin. This protein consists in a subunit of the clathrin adaptor proteins AP-1A and AP-1B that sort basolateral proteins at the trans-Golgi network and at common recycling endosomes, respectively. In order to characterize the role of AP-1 proteins in NIS basolateral traffic we used MDCKhNIS cells depleted for AP-1A, AP-1B or both, and we evaluated NIS polarized distribution through confocal microscopy and domain-selective biotinylation. We observed that knockdown of AP-1B caused a loss of NIS basolateral polarity compared to that in wildtype cells. In contrast, NIS was normally distributed after depletion of AP-1A. Strikingly, the decrease in NIS basolateral localization was more prominent in the case of the double knockdown than in AP-1B knockdown cells, indicating that AP-1A also participates in NIS basolateral sorting.

In conclusion, both AP-1A and AP-1B mediate NIS basolateral sorting; nevertheless, the interaction between NIS and the clathrin-coated machinery is still under study.

\section{P269}

Poster has been withdrawn

\section{P027 Paediatrics and Pregnancy}

\section{P270 \\ PREVALENCE OF SHORT STATURE IN JUVENILE HYPOTHYROIDISM AND THE IMPACT OF TREATMENT ON GROWTH VELOCITY- EXPERIENCE FROM A TERTIARY CARE HOSPITAL IN MEERUT, INDIA \\ Manish Gutch ${ }^{1}$, Sukriti Kumar ${ }^{2}$, Sanjay Saran ${ }^{3}$, Keshav Kumar Gupta ${ }^{1}$ ${ }^{1}$ Department OF Endocrinology, LIrm Medical College, Meerut, India, ${ }^{2}$ Department OF Radiodaignosis, Sgpgi, Lucknow, India, ${ }^{3}$ Department OF Endocrinology, LIrm Medical College, Garh Road, Meerut, India}

Background: Juvenile hypothyroidism is very common problem in developing parts of world, and produces various skeletal manifestations. One of them is short stature and it is the most common reason for referral to endocrinologist.

Aim and Objectives: To study the prevalence of short stature in juvenile hypothyroidism, to study the various radiological manifestations of juvenile hypothyroidism and to study the impact of treatment on short stature and various skeletal manifestations.

Material and Methods: Out of total nine hundred hypothyroid patients, eighty seven patients found to be of juvenile hypothyroidism were enrolled in the study that were $6-18$ years of age with newly diagnosed or on follow in the endocrine clinic over a period of $1 \frac{1}{2}$ years were evaluated clinically and by laboratory tests. Serial assays of TSH, T4, and skeletal X rays and anthropometry were done at regular interval and clinical and radiological outcome of patients were analyzed.

Statistical Analysis: Data were analyzed by SPSS version 17, the $P$ value of $<0.05$ was considered significant.

Result: The mean age of diagnosis of juvenile hypothyroidism was 11.2 years, and the females had twice the incidence than that of males, the mean TSH value were $118 \pm 24.3 \mu \mathrm{IU} / \mathrm{ml}$. Prevalence of short stature was found to be $45 \%$ while delayed bone age was found to be $72 \%$ in juvenile hypothyroid populations. Height SDS increased from $-2.9 \pm 0.9$ at the start of thyroxine therapy to $-1.8 \pm 0.8$ after 12 months later $(\mathrm{P}<0.001)$. Bone age SDS increased from $8.9 \pm 2.5$ at the start of thyroxine therapy to $10.8 \pm 2.7$ after 12 months later. Height velocity increased from $4.9 \pm 0.8 \mathrm{~cm} /$ year in the year before treatment to $8.7 \pm 1.3$ during treatment $(\mathrm{P}<0.001)$.

Conclusion: The presentations of juvenile hypothyroidism may be varied; prompt recognition of the findings can lead to early and effective treatment, and improving the skeletal defects.

\section{P271 \\ ATTENTION-DEFICIT HYPERACTIVITY DISORDER IN WOMEN WITH SUBCLINICAL HYPOTHYROIDISM \\ Mariya Petrova $^{0}$, Kiril Hristozov ${ }^{1}$, Gergana Momova $^{1}$, \\ Rinaldo Shishkov \\ ${ }^{1}$ Medical University - Varna}

Attention-deficit hyperactivity disorder/ADHD/ usual begins in childhood but excessive activity, impulsivity and academic failure are a hallmark of social behaviour of the person throughout his/her entire life. Test of clinicbased voluntary patients monitor less expressed impulsivity and hyperactivity of women in comparison with those of men. Among the wide spectrum of psychic syndromes ADHD is a manifestation of thyroid deficiency. Molecular markers for ADHD are still under research, but the prevailing opinion is that the diagnosis is clinical.

Aim: To investigate the frequency of ADHD in women and to assess the influence of subclinical hypothyroidism/SH/ on ADHD. 
Method: 2591 patients have been examined in the Clinic of Psychiatry and Medical Psychology during 2010. 35 of them responded to the diagnostic criteria of ADHD according to DSM-IV (verbal and non verbal tests). Functional test of the thyroid include TSH, FT4, FT3/RIA/, US/7, $5 \mathrm{MHz}$. Controul group-20 healthy nonpregnant women.

Results:_From all 2591 subjects 35 have ADHD/frequency 1, 35\%/. 22 patients have increased TSH and they are subjects of psychlogical and endocrinological tests. The clinical manifestations of the 22 tested subjeects are grouped as follows: A/ Predominant hyperactivity and impulsivity: $\mathrm{n}=7$ / femals/, age 22.45; score from psychic tests =110; TSH $6.77 \mathrm{mU} / 1$; FT4 20.54 $\mathrm{pmol} / \mathrm{l}$; index of correlation between IQ and TSH- $/ \mathrm{r}=-0.24 /$; B /Predominant inattention $\mathrm{n}=11 /$, age 23.15 ; score from psychic tests $=114$; TSH $6.98 \mathrm{mU} / 1$; FT4 $23.14 \mathrm{pmol} / \mathrm{l}$; index of correlation between IQ and TSH- $/ \mathrm{r}=-0.31 /$; $/$ Combined symptoms $\mathrm{n}=4$, age 21.18; score 98, TSH $5.98 \mathrm{mU} / \mathrm{l}$; FT4 18.32 $\mathrm{pmol} / \mathrm{l}$; index of correlation between IQ and TTH- $/ \mathrm{r}=-0.09 /$;

Conclusion:_The frequency of ADHD among women with $\mathrm{SH}$ is $1.35 \%$. Attention tests monitor prevailing impairment in women with $\mathrm{SH}$.

\section{P272 \\ CAN HCG CONCENTRATIONS EXPLAIN GESTATIONAL THYROID DYSFUNCTION AND ITS ASSOCIATIONS WITH ADVERSE PREGNANCY AND CHILD OUTCOMES?}

Tim Korevaar ${ }^{1}$, Marco Medici ${ }^{1}$, Yolanda de Rijke ${ }^{1}$, Edward Visser ${ }^{1}$, Albert Hofman ${ }^{1}$, Vincent Jaddoe ${ }^{1}$, Eric Steegers ${ }^{1}$, Henning Tiemeier ${ }^{1}$, Theo Visser ${ }^{1}$, Robin Peeters ${ }^{1}$

${ }^{1}$ Erasmus University Medical Center, Rotterdam, The Netherlands

Objective:High TSH and/or low FT4 levels during pregnancy have been associated with adverse outcomes such as preterm delivery, decreased birth weight, impaired child neurocognitive development and autism. hCG has thyroid-stimulating activity and has been associated with the same outcomes. However, it is unclear to what extent hCG levels are associated with thyroid dysfunction. Moreover, it has been hypothesized that the associations between gestational thyroid (dys)function and adverse outcomes may be a hCG effect. Therefore, we studied the associations between hCG, thyroid (dys)function and adverse clinical outcomes.

Methods: Serum hCG, TSH and FT4 levels were determined during early pregnancy in 5550 women from the Generation R study. hCG concentrations (per 10.000IU/L; $\beta \pm \mathrm{SE}$ ) or gestational age specific multiple of median (MoM) values were analyzed. Analyses were adjusted for various covariates (including maternal age, ethnicity, BMI, smoking and fetal gender).

Results: hCG had a log-linear relationship with TSH $(\beta-0.130 \pm 0.008)$ and FT4 ( $\beta 0.222 \pm 0.023$; both $\mathrm{P}<0.0001$ ), this relationship differed according to TPOAb status, urinary iodine, BMI, smoking and age.

Low gestational age specific hCG values were associated with an increased risk of hypothyroxinemia (OR 3.53 [1.5-8.4]) but not with subclinical hypothyroidism (OR 0.87 [0.2-3.6]). High gestational age specific hCG values were associated with an increased risk of hyperthyroidism (OR 10.9 [4.5-26]) and subclinical hyperthyroidism (OR 2.3 [1.0-5.2]).

Previous associations between thyroid dysfunction and preterm birth, birth weight, child IQ and autism were verified. Correction for hCG levels (continuous as IU/L/MoM or dichotomous using high/low) did not change these associations.

Conclusion: hCG is strongly related to TSH and FT4 levels, and with the risk of thyroid dysfunction. However, the associations of maternal thyroid dysfunction and adverse pregnancy or child outcomes are not mediated/confounded by hCG

\section{P273}

\section{LONGITUDINAL PILOT STUDY ON THE} EFFECTS OF L-THYROXINE AND/OR IODINE PROPHYLAXIS ON NEUROINTELLECTUAL PERFORMANCES IN CHILDREN BORN TO MODERATELY IODINE DEFICIENT MOTHERS

\section{Mariacarla Moleti', Giovanna llardo ${ }^{1}$, Maria Boncoddo ${ }^{1}$,}

Alice Candia Longo ${ }^{1}$, Silvia Presti ${ }^{1}$, Beatrice Di Bella ${ }^{1}$,

Giacomo Sturniolo ${ }^{1}$, Gaetano Tortorella ${ }^{1}$, Francesco Trimarchi ${ }^{1}$

Francesco Vermiglio ${ }^{1}$

${ }^{1}$ University of Messina

Objectives: To verify the effects of iodine prophylaxis by iodized salt on neurointellectual outcome of children born to moderately iodine deficient mothers.

Subjects: Sixty children (6-12 years) born to: i. mothers regularly using iodized salt prior to pregnancy (Is group, $\mathrm{n}=15$ ); ii. mothers on LT4 treament and regularly using iodized salt prior to pregnancy (Is-LT4+ group, $\mathrm{n}=15$ ); iii. mothers who had never used iodized salt (no-Is group, $\mathrm{n}=15$ ); iv. unsupplemented mothers on LT4 treatment prio to pregnancy (no-Is-LT4+ group, $\mathrm{n}=$ 15).

Methods: Wechsler Scale of Intelligence Scale for Children 4th ed. (WISC-IV), 13 subscales.

Results: Children born to Is/IsLT4+ mothers had similar total IQ scores (tIQ), which were 12 and 15 points higher than that of unsupplemented mothers (no-IS and no-ISLT4+). This finding was irrespective of maternal serum FT4 at early gestation ( $1^{\text {st }}$ trimester). Indeed, no differences were observed in the proportion of children with defective tIQ $(<1 \mathrm{SD})$ born to mothers with serum FT4 levels at early gestation above or below the lower gestational FT4 quartile $\left(c^{2} 0.23 ; p=0.62\right)$. Conversely, the proportion of children with defective tIQ was more than threefold higher (6/30 vs 20/30) in unsupplemented (no-Is and no-IsLT4+ groups) than in supplemented mothers (Is and Is-LT4 groups) $\left(\mathrm{c}^{2} 10.85 \mathrm{P}<0.0001\right)$.

Conclusions: Neurointellectual outcome seems to be affected by maternal iodine status rather than by maternal thyroxine levels, likely because of a more adequate fetal thyroid function. Nonetheless, the high rate of cognitive deficit also observed in children born to mothers who regularly used iodized salt indicates this mean of iodine prophylaxis to be insufficient during pregnancy.

\section{P274}

MILD IODINE DEFICIENCY DURING PREGNANCY IN EUROPE AND ITS CONSEQUENCES FOR COGNITIVE AND PSYCHOMOTOR DEVELOPMENT OF CHILDREN:

\section{A REVIEW}

Caroline Trumpff', Jean De Schepper ${ }^{2}$, Stefanie Vandevijvere ${ }^{3}$

${ }^{1}$ Unit of Public Health and Surveillance, Scientific Institute of Public

Health, Brussels, Belgium; Faculty of Psychology and Educational

Sciences, Université Libre de Bruxelles, Brussels, Belgium,

${ }^{2}$ Department of Paediatric Endocrinology, Uz Brussel, Brussels,

Belgium, ${ }^{3}$ Unit of Public Health and Surveillance, Scientific Institute of

Public Health, Brussels, Belgium

Background and Objectives: Several European countries still suffer from mild iodine deficiency (MID) despite introduction of salt iodinization programs. Pregnant women are the most at risk of iodine deficiency due to their increased physiological needs of iodine, this is important because MID during pregnancy may affect the neurodevelopment of the foetus. This review aims to assess the importance of iodine intake during pregnancy by reviewing European studies about the impact of MID during pregnancy on cognitive and or psychomotor development of children.

Methods: European studies about the cognitive or psychomotor outcomes of European children exposed to MID during pregnancy were reviewed.

Results: MID during pregnancy may lead to hypothyroxinaemia in the mother and/or elevated thyroid-stimulating hormone levels in the foetus. Hypothyroxinaemia during pregnancy may affect cognitive development of children when appearing before or at 12 weeks of pregnancy. Moderate cognitive impairments have been observed in children from the age of 3 weeks up to 5 years. Elevated thyroid-stimulating hormone levels in the newborn are 
related with intellectual or psychomotor delay in early childhood. However, no direct link is yet established between MID during pregnancy and maternal hypothyroxinaemia, as well as between MID during pregnancy and elevated neonatal thyroid-stimulating hormone levels at birth. Finally, iodine supplementation of pregnant women when taking place from the first trimester until the end of pregnancy may decrease the risk of developmental delay of the offspring.

Conclusions: There is some evidence that MID appearing during pregnancy may adversely affect cognitive and psychomotor development of the offspring. Iodine supplementation during pregnancy may be beneficial to avoid the risk of developmental delay.

Key words: Iodine, Pregnancy, Europe, Psychomotor development, Cognitive development.

\section{P275 \\ METABOLIC AND ATHEROSCLEROTIC EFFECTS OF AUTOIMMUN THYROIDITIS IN CHILDREN AND ADOLESCENTS}

Betul Ersoy', Seniha Kiremitci ${ }^{1}$, Turker Borucu', Gonul Dinc Horasan ${ }^{3}$

${ }^{1}$ Celal Bayar University School of Medicine, ${ }^{2}$ Celal Bayar University

School of Medicine; Pediatric Endocrinology, ${ }^{3} \mathrm{Celal}$ Bayar University

School of Medicine; Department of Epidemiology

Objectives: The aim of this study was to evaluate the relationships between lipid-carbohydrate metabolism parameters and thyroid hormones and autoantibodies and carotid intima-media thickness (CIMT) in children and adolescents with autoimmun thyroiditis (AIT) during the follow up.

Methods: We examined 60 patients with the diagnosis of AIT aged range between 9-19 years (10 male, 50 female), based on examination of autoantibodies (anti-TPO and anti-Thyroglobulin) and thyroid ultrasonography (USG). Oral glucose tolerance test (OGTT) was performed to all patients. Hepatic steatosis and CIMT using B-Mode USG, and lipid profile were evaluated in all patients. All procedures were performed to patients during the follow-up. Body mass index (BMI) was calculated as a $\mathrm{kg} / \mathrm{m}^{2}$. Insulin sensitivity was evaluated by homeostasis model assessment (HOMA-IR).

Results: Mean duration of diagnosis was $24.76 \pm 22.38$ months. Frequency of overweight and obesity were $22.4 \%$. Hepatic steatosis is only present in $3.2 \%$ of the patients. BMISDS at the diagnosis, insulin levels at 30th minute and totally insulin levels in OGTT were higher in overweight and obese children and adolescents with AIT compared with non-obese subjects with AIT ( $p=0.003,0.026$ and 0.04 , respectively). The Pearson correlation coefficient indicated that anti-TPO level at the time diagnosis was positively correlated with HOMA-IR $(\mathrm{r}=0.37 \mathrm{p}=0.008)$.

Conclusion: Overweight and obesity are common among children and adolescents with AIT. Elevated insulin levels are highly prevalent in overweight and obese children and adolescents with AIT. Insulin sensitivity may be related with anti-TPO levels at time of diagnosis.

\section{P276}

\section{TSH 2.5-3.4 MU/L IN THE FIRST TRIMESTER OF PREGNANCY}

Antonia Pérez-Lázaro ${ }^{1}$, Alia Maria Garcia Castells ${ }^{1}$,

Sol Navas de Solís ${ }^{2}$, Maria Isabel del Olmo Garcia ${ }^{2}$,

Susana Tenés Rodrigo ${ }^{1}$, Rosa Cámara Gomez ${ }^{1}$,

Juan Francisco Merino Torres ${ }^{1}$

${ }^{1}$ Hospital Universitario La Fe, ${ }^{2}$ Hospital Universitario LA Fe

Introduction: After the publication of the ATA recommendations we have been carrying out a study, in which we have established new TSH reference values $(0.2-3.4 \mathrm{mU} / \mathrm{L})$ in our pregnant patients.

Objective: To evaluate the progress of pregnant women with serum TSH values between 2.5 and $4.9 \mathrm{mU} / \mathrm{L}$ in the first trimester, and to consider the need to treat when values are lower than $3.4 \mathrm{mU} / \mathrm{L}$.

Method: 127 pregnant patients with high TSH levels were recruited. A unicentric and longitudinal study was done which collected demographic, clinical and analytical data. The analysis was carried out by SPSS 20 . The results are displayed as an arithmetic mean (SD) and percentage.

ResultsL 127 patients with high levels of TSH were evaluated in the first trimester of pregnancy. $103(81.1 \%)$ did not have previous thyroid pathology. The remaining $49 \%$ (51) of this group was remitted by TSH $2.5-4.9 \mathrm{mU} / \mathrm{L}$.
After an evaluation, $24(46 \%)$ of them were not treated since they presented TSH $2.5-3.4 \mathrm{mU} / \mathrm{L}$ with negative peroxidase antibodies. At diagnosis TSH values were $3.19(0.54) \mathrm{mU} / \mathrm{L}$, and $2.83(1.2) \mathrm{mU} / \mathrm{L}$ in the third trimester. In the 27 remaining patients, we initiated Eutirox 38 (12.7) $\mathrm{mcg} / \mathrm{day}$, with TSH $3.9(0.6) \mathrm{mU} / \mathrm{L}$ at diagnosis; TSH $2.5(0.84) \mathrm{mU} / \mathrm{L}$ in the third trimester, and positive peroxidase antibodies in $33.3 \%$ of them.

Conclusions:

- After the new ATA recommendations regarding the managing of hypothyroidism during pregnancy, the number of patients remitted by high levels of TSH has increased considerably

$-44.2 \%$ of patients with TSH $2.5-4.9 \mathrm{mU} / \mathrm{L}$ were not treated during the pregnancy since they presented having TSH $<3.4 \mathrm{mU} / \mathrm{L}$ and negative peroxidase antibodies, meaning final values of TSH of $2.83 \mathrm{mU} / \mathrm{L}$

- More studies are needed in our population to evaluate the consequences on the foetus when the treatment is avoided in pregnancy with TSH 2.5-3.4

\section{P277 \\ TSH ELEVATION AS FIRST LABORATORY EVIDENCE FOR PSEUDOHYPOPARATHYROIDISM TYPE IB (PHP-IB) PROMPTING THE SEARCH FOR GNAS METHYLATION CHANGES IN IDIOPATIC HYPOTHYROIDISM}

Angelo Molinaro ${ }^{1}$, Dov Tiosano ${ }^{2}$, Dionisios Chrysis ${ }^{3}$, William Russel/4, Petteri Ahtiainen ${ }^{5}$, Massimo Tonacchera ${ }^{6}$, Harald Jueppner ${ }^{7}$ ${ }^{1}$ Endocrinology Unit, Department of Internal Medicine, University of Pisa, Pisa, Italy, Endocrine Unit, Massachusetts General Hospital, Harvard Medical School, Boston, USA, ${ }^{2}$ Division of Pediatric Endocrinology, Meyer Children's Hospital, Rambam Health Care Campus, Haifa, Israel, ${ }^{3}$ Department of Pediatrics, Medical School, University of Patras, Patras, Greece, ${ }^{4}$ Division of Pediatric Endocrinology and Diabetes, Vanderbilt University School of Medicine, Nashville, USA, ${ }^{5}$ Central Finland Central Hospital, Jyväskylä, and Kuopio University Hospital, Kuopio, Finland, ${ }^{6}$ Endocrinology Unit, Department of Internal Medicine, University of Pisa, Pisa, Italy, ${ }^{7}$ Endocrine Unit, Massachusetts General Hospital, Harvard Medical School, Boston, USA

Objective: Hypothyroidism may be the presenting feature of pseudohypoparathyroidism type Ib (PHP-Ib) due to GNAS methylation changes. We therefore wanted to determine whether adult patients with mild idiopathic hypothyroidism (IH) have similar epigenetic changes in GNAS.

Patients: The study included 4 patients with hypothyroidism and PHP-Ib and 64 adults with IH. The patients with PHP-Ib developed PTH-resistant hypocalcemia 3-20 years after establishing the diagnosis of hypothyroidism in childhood and were found to have GNAS methylation changes. We subsequently searched in 64 adult IH patients for similar epigenetic changes. These patients had never developed PTH or calcium alteration during a mean followup time of 4.1 years (range: $0-13$ ).

Results: In three pediatric patients, who developed hypothyroidism before the onset of PHP-Ib, we observed broad epigenetic GNAS abnormalities, including loss of methylation at exons $\mathrm{AS}, \mathrm{XL}$ and $\mathrm{AB}$, and gain of methylation at exon NESP55; these findings are consistent with sporadic PHP-Ib or uniparental iso- or heterodisomy involving chromosome $20 \mathrm{q}$. In the fourth patient, an isolated loss of methylation at GNAS exon AB led to the identification of the well-known $3 \mathrm{~kb}$ deletion in STX16 gene as the cause of PHP-Ib. DNA from the patient's healthy mother revealed the same deletion. However, we did not find any GNAS methylation changes in the 64 patients with idiopathic TSH elevation.

Conclusions: PHP-Ib can present initially only with TSH elevation and the onset of PTH-resistance can be delayed by many years. We were unable to show any methylation GNAS changes in our unselected adult patients with isolated TSH elevation, indicating that adult-onset hypothyroidism is usually not associated with epigenetic changes at the GNAS locus. However, it may be necessary to screen pediatric patients with hypothyroidism for extended periods of time for changes in calcium, phosphate, and PTH to prevent symptomatic hypocalcemia and associated complications. 


\section{P278 \\ NEXT GENERATION SEQUENCING OF THYROIDAL GENES REVEALS UNEXPECTED POLYGENIC DEFECTS IN DYSHORMONOGENIC HYPOTHYROIDISM}

Ainhoa Iglesias ${ }^{1}$, P.S. Ventura ${ }^{2}$, J. Pozo ${ }^{3}$, M. Clemente ${ }^{4}$, M. Albisu', L. Audi' ${ }^{4}$, I. González-Casado ${ }^{5}$, E. Vallespin ${ }^{6}$, K. Ibáñez $^{7}$, M. Polak $^{8}$, A. del Pozo ${ }^{7}$, T.J. Visser ${ }^{9}$, J.C. Moreno ${ }^{1}$

${ }^{1}$ Thyroid Molecular Lab., Institute for Medical and Molecular Genetics (Ingemm), La Paz University Hospital, ${ }^{2}$ Paediatric Endocrinology S. Nens Hospital, ${ }^{3}$ Paediatric Endocrinology S. Niño Jesús Children University Hospital, ${ }^{4}$ Paediatric Endocrinology S. Vall Dhebron Hospital, ${ }^{5}$ Paediatric Endocrinology S. La Paz University Hospital, ${ }^{6}$ Structural and Fuctional Genomics, Institute for Medical and Molecular Genetics (Ingemm), La Paz University Hospital, ${ }^{7}$ Bioinformatic Dept. Institute for Medical and Molecular Genetics (Ingemm), La Paz University Hospital, ${ }^{8}$ Paediatric Endocrinology S. Hospital NekerEnfants Malades Hospital, ${ }^{9}$ Erasmus Medical Center

Dyshormonogenic hypothyroidism is classically a monogenic disease with recessive inheritance. Thyroid dysgenesis showed a multigenic origin in a mouse model of double-heterozygous deletions of $N k \times 2.1 /$ Pax 8 genes, suggesting a possible polygenic nature of certain cases of human hypothyroidism.

Objective: To investigate genetic traits of polygenic involvement in dyshormonogenic hypothyroidism, using next generation sequencing (NGS).

Methods: Endocrine and genetic characterization of 4 pedigrees with thyroid dyshormonogenesis. NGS of 18 thyroidal genes including $T G, T P O$, DUOX2/DUOXA2 and DEHAL1 and PCR-Sanger sequencing confirmation. Pathogenicity prediction and $\mathrm{H}_{2} \mathrm{O}_{2}$-generation testing of mutants.

Results: Three families (F1-F3) were consanguineous. All patients showed positive perchlorate discharge (PD). In F1, two siblings with congenital hypothyroidism (TSH $>200 \mathrm{mU} / \mathrm{L}$ ), had elevated thyroglobulin (>1600 ng/ $\mathrm{ml}$ ) and $100 \%$ PD. NGS revealed a homozygous frameshift mutation in TPO (p.S756fsX75) in both brothers. Additionally, one presented a heterozygous nonsense mutation in DUOX2 (p.K530X). In F2, two sisters were born with increased neonatal TSH (200 and $800 \mathrm{mU} / \mathrm{L})$. Both were homozygotes for a nonsense $T G$ mutation (p.R296X) but also were respectively heterozygote and homozygote for a pathogenic TPO mutation (p.G667S). In F3, index patient presented neonatal $\mathrm{TSH}>800 \mathrm{mU} / \mathrm{L}$, thyroglobulin $>300 \mathrm{ng} / \mathrm{ml}$ and $30 \%$ PD. NGS revealed a yet described pathogenic homozygous TPO mutation (p.G493S) in the three affected siblings plus a rare heterozygous $T G$ change (p.G653D) predicted as highly pathogenic in $2 / 3$ brothers.

In non-consanguineous F4, two sisters had pubertal euthyroid/hyperthyrotropinemic goiters and $25 \%$ PD. Both were heterozygotes for a known pathogenic TPO mutation (p.N425S), but one also presented a heterozygous DUOXA2 mutation (p.G294E) which reduced $50 \%$ the in vitro capacity of $\mathrm{H}_{2} \mathrm{O}_{2}$ generation by DUOX2-DUOXA2 pair.

Conclusion: Targeted NGS reveals unexpected mutations additional to typical homozygous defects in consanguineous children with thyroid dyshormonogenesis. Additional mutations had not been identified by standard "one-gene-approach", occur in genes within the same iodination pathway and may account for intra-familial phenotypic variability of dyshormonogenic hypothyroidism.

\section{P279 \\ SELECTIVE PITUITARY RESISTANCE TO THYROID HORMONES}

Corin Badiu', Alexandra Gabur ${ }^{2}$, Dan Hortopan ${ }^{2}$, Simona Jercalau ${ }^{2}$ ${ }^{1}$ National Institute of Endocrinology; "Carol Davila" University of

Medicine \& Pharnacy, ${ }^{2}$ National Institute of Endocrinology

Introduction: Selective pituitary resistance to thyroid hormones is a syndrome that is characterized by reduced pituitary responsiveness to elevated levels of free T4 and free T3 with non-suppressed serum TSH and intact TSH responsiveness to TRH, associated with symptoms and signs of thyrotoxicosis, caused by a mutation in the TR $\beta$.

We report the case of a man, who was first considered a cardiac and psychiatric patient, after a normal TSH value ruled out thyrotoxicosis. Repeated thyroid tests showed: $\mathrm{TSH}=3.67 / 4 \mu \mathrm{UI} / \mathrm{ml}(0.27-4.2), \mathrm{FT} 4=29.26 / 29.45$ $\mathrm{pmol} / \mathrm{l}(12-22), \mathrm{FT}=9.5 / 8.83 \mathrm{pmol} / \mathrm{l}(3.9-6.7)$.
Case: A 29 years old man who presented with laboratory tests suggestive for a syndrome of inappropriate TSH secretion. The clinical examination revealed a thyrotoxic patient, with a small goiter. Thyroid profile was as follows: $\mathrm{TSH}=3.51 \mu \mathrm{UI} / \mathrm{ml}(0.5-4.5), \mathrm{FT} 4=37.25 \mathrm{pmol} / \mathrm{l}(12-22), \mathrm{T} 3=248.1$ $\mathrm{ng} / \mathrm{dl}(80-200)$. This suggested either a TSH-secreting adenoma or the syndrome of resistance to thyroid hormones $(\mathrm{RTH}) . \mathrm{ATPO}=5.4 \mathrm{UI} / \mathrm{ml}$ (negative $<35$ )- ruled out an autoimmune disorder. The pituitary CT scan revealed a microadenoma $(7 / 4 \mathrm{~mm})$. Pituitary hormones were in the normal range. We performed a stimulation test by administering $200 \mu \mathrm{g}$ TRH iv with a significantly increase of TSH. This finding helped to distinguish the RTH from TSH-secreting adenoma. The patient was thyrotoxic so we considered a PRTH rather than GRTH. We used the SHBG which was high, $65.51 \mathrm{nmol} / 1$ (14.548.4), to confirm our suspicion.

We initiated treatment with antithyroid drugs, $\beta$-adrenergic antagonist and an anxiolytic drug.

Conclusions: A thyrotoxic patient should have both TSH and FT4 measured. In order to complete our investigations we would need to evaluate $\alpha$-subunit serum concentration, to see the TSH response to T3 and to search for mutations in TR $\beta$ gene.

\section{P280 \\ IODINE INTAKE IN WOMEN OF REPRODUCTIVE AGE AND RELATED FACTORS \\ María Riestra ${ }^{1}$, Edelmiro Menéndez ${ }^{2}$, Elías Delgado ${ }^{2}$, \\ Juan Carlos Fernández ${ }^{2}$, Francisco Díaz Cadórniga ${ }^{2}$ \\ ${ }^{1}$ Hospital de Cabueñes, Gijón (Spain), ${ }^{2}$ Hospital Universitario Central de Asturias, Oviedo (Spain)}

Introduction: Iodine deficiency is a public health problem, being the leading preventable cause of infant brain damage. Nutritional status of iodine in women of childbearing age is important in view of a future pregnancy and its impact on fetal brain development.

Objective:To determine current nutritional status of iodine in women of childbearing age and related factors.

Methods: We performed a cross-sectional study. We randomly selected a sample of women of childbearing age, across Asturias, in Northwest Spain. A survey concerning food consumption was done. A urine sample was collected to determine urinary iodine concentration (UIC), carried out by HPLC. For the statistical processing we used SPSS version 15.0.

Result: We studied 127 women, mean age 37.9 years (SD 5). Median UIC was $170.6 \mu \mathrm{g} / \mathrm{L}$ (mean 208. 5, range 37.8-798 $\mu \mathrm{g} / \mathrm{L}$ ). 21.3\% had UIC below $100 \mu \mathrm{g} / \mathrm{L}$ and only $2.4 \%$ below $50 \mu \mathrm{g} / \mathrm{L} .18 .9 \%$ had UIC $>300 \mu \mathrm{g} / \mathrm{L}$.

$72.8 \%$ of all used iodized salt. There were no statistically significant differences in UIC of women who reported using iodized salt (mean $207.2 \mu \mathrm{g} / \mathrm{L}$ ) versus those that did not use (mean $217.9 \mu \mathrm{g} / \mathrm{L}$ ). We also found no significant UIC differences in eggs, vegetables, fish or milk consumption.

Conclusions: These results confirm adequate iodine nutrition in women of childbearing age in our community, although no relation was found between UIC and common sources of iodine, as iodized salt or milk. It is necessary to control the possible silent iodization in food industry to prevent deficiency or excess of this micronutrient.

\section{P281 \\ CLASSICAL THYROID HORMONE RESISTANCE VERSUS PITUITARY RESISTANCE TO LEVOTHYROXINE}

Nerea Lacamara $^{0}$, Belen Roldan ${ }^{1}$, Francisco Morales $^{2}$,

Beatriz Lecumberri ${ }^{3}$, Beatriz Barquie ${ }^{3}$, Aranzazu Escribano ${ }^{4}$, Manel Sahún ${ }^{5}$, Isabel Gonzalez-Casado ${ }^{6}$, Jose Carlos Moreno ${ }^{7}$ ${ }^{1}$ Ramon Y Cajal University Hospital, Madrid, ${ }^{2}$ Virgen del Rocío University Hospital, Sevilla, ${ }^{3}$ La Paz University Hospital, Madrid, ${ }^{4}$ Virgen de la Arrixaca University Hospital, Murcia, ${ }^{5}$ Moises Broggi Hospital, Barcelona, ${ }^{6} \mathrm{La}$ Paz University Hospital, ${ }^{7}$ Molecular Thyroid Laboratory, Institute for Medical and Molecular Genetics (Ingemm), La Paz University Hospital, Madrid, Spain

Resistance to thyroid hormone (RTH) is a polygenic syndrome of low cellular sensitivity to T3. Defects in THRB lead to elevated FT4 and inappropriate high or normal TSH secretion, with variable symptoms of hyperthyroidism. Such hormone profile is identified in a proportion of cases with primary 
hypothyroidism requiring abnormally elevated doses of levo-thyroxine (causing hyperthyroid symptoms) to control TSH within normal range, suggesting pituitary resistance to exogenous T4. Dio2 knockout mice express pituitary resistance exclusively to $\mathrm{T} 4$.

Objective: Molecular investigation of patients with elevated FT4 and $\mathrm{TSH}$, including those receiving exogenous T4.

Methods: PCR and sequencing of the complete coding region of TRHB. Patients under L-T4 were additionally screened for DIO2 (including genotyping of Thr92Ala and $-258 \mathrm{~A} / \mathrm{G}$ functional polymorphisms compared to 46 local controls and 1109 individuals from 1000-Genomes database) and TSHR.

Results: From a total cohort of 48 patients $(60 \%$ women), $13(27 \%)$ were patients resistant to L-T4 at the pituitary, 3 having thyroiditis, 4 thyroid agenesis and 6 thyroidectomized because of MNG or Graves's disease. One patient with resistance to exogenous T4 could fully control TSH levels under combined L-T4-liothyronine therapy. In classical RTH, heterozygous missense mutations in THRB were identified in 17 patients (35.4\%), including R438H (n:5), R243W (n:3), P453R (n:2) and R243Q, P453T, C309Y, R338W, M442V, K288R, F451S. No mutations were identified in DIO2 or TSHR. DIO2 SNPThr92 was equally frequent in populations compared, but $-258 \mathrm{~A}$ was more frequent in patients under L-T4 than in 46 local controls $(\mathrm{P}<0.09)$ and 1109 1000-Genomes individuals $(\mathrm{P}<0.1)$.

Conclusion: THRB mutations are present in $35.4 \%$ of the classical RTH cohort, the rest remaining genetically orphan. No mutations in DIO2 or TSHR were identified in patients with pituitary resistance to exogenous L-T4, but implications of -258A DIO2 promoter SNP, reportedly decreasing enzyme expression, requires testing in larger cohorts with this phenotype.

\section{P028 Thyroid Cancer Diagnostics 3}

\author{
P282 \\ IS IT ENOUGH TO PERFORM FNAB FROM \\ DOMINANT NODULE? \\ Omer Engin ${ }^{1}$, Volkan Tekin², Mehmet Yilmaz ${ }^{3}$, Enis Unluturk ${ }^{3}$ \\ Ulas Urganci ${ }^{3}$ \\ ${ }^{1}$ Buca Seyfi Demirsoy State Hospital, Surgery Department, ${ }^{2}$ Aydın \\ State Hospital, ${ }^{3}$ Buca Seyfi Demirsoy State Hospital
}

Objectives: FNAB (Fine Needle Aspiration Biopsy) is performed before deciding surgical intervention in cases with thyroid nodules.If the result biopsy is malign, we perform surgery. If the biopsy result is benign, we can follow up our patients. From which nodule should FNAB be performed?Is it enough to perform from the dominant nodüle? We'll look for answers to these questions in our study.

Methods: 315 patients whom bilateral total thyroidectomy was performed and diagnosed as throid cancer between the years 2007 and 2014 were included in the study.The pathology reports of dominant nodules and the sizes of malign nodules were investigated.

Results: In 315 thyroid cancer cases; the number of dominant nodules that were benign in nature was 173 and the number of malign dominant nodules was found as 142 .The average size of maling nodules was $14.35(1-80) \mathrm{mm}$.In 142 cases, the size of malign nodüle was smaller than $8 \mathrm{~mm}$.

Conclusions: FNAB help us to decide surgery in multinodular goiter. From which nodule should FNAB be performed? Is it enough to perform from the biggest nodule?Should biopsy be performed from the second biggest nodüle, too?

FNAB can't be performed in nodules smaller than $8 \mathrm{~mm}$ because of technical inefficacy.FNAB is a useless technique for the diagnose since an important part of our patients $(45 \%)$ had malign nodules smaller than $8 \mathrm{~mm}$.It's wrong to perform biopsy only from the dominant nodüle since dominant nodule was benign in natüre in more than half of our patients.Since the histopathology of one nodule doesn't reflect the whole thyroid tissue and the histopathology of each nodule may be different;if possible;we claim to perform biopsy from all nodules that can be reached.Since performing biopsy from more than one nodüle in the same seance may increase the risk of bleeding, we think that biopsies should be performed at different seances.

\section{P283 \\ COMPARATIVE ANALYSIS OF THYROID NODULE ULTRASOUND-GUIDED FINE-NEEDLE ASPIRATION CYTOLOGY RESULTS AND POST- OPERATIVE MATERIAL HISTOMORPHOLOGIC STUDY}

Tamar Dundua ${ }^{1}$, Tamar Kaloiani², Lali Javashvili', Maia Kobulia', Meri Rekvava ${ }^{1}$, Nino Jakhaia ${ }^{1}$, Medea Papava ${ }^{3}$

${ }^{1}$ Clinic Cortex, ${ }^{2}$ National Centre of Oncology, ${ }^{3}$ Research Institute of

Clinical Medicine; Tbilisi, Georgia

Objectives: The aim of this research was to compare the results of ultrasound-guided fine-needle aspiration cytology (FNAC) and post-operative material histomorphologic analysis and evaluate the effectiveness of fineneedle aspiration cytology method. Our goal is to show the significance of FNAC (which is often neglected) in planning of the extent of the operation beforehand and avoiding the need of reoperations.

Materials: we have performed FNAC on 151 patients (102 female 49 male, the age -24 to 75 ). Patients were selected based on "AACE/AME/ETA Guideline for the Diagnosis and Management of Thyroid Nodules" criterions.

The ultrasound-guided FNAC revealed colloid nodules in $56.9 \%$ of patients, nodular hyperplasia in $9.9 \%$, adenomatous nodule in $9.2 \%$, results were consistent with the Bethesda System for Reporting Thyroid Cytopathology (TBSRTC) diagnostic categories (DC) II - benign. The nodule suspicious for a follicular neoplasm in $4.6 \%$ consistent with TBSRTC DC IV - follicular neoplasm/suspicion for a follicular neoplasm. Nodule suspicious for papillary cancer in $4 \%$ of patients corresponding to TBSRTC DC VSuspicious for Malignancy. Papillary cancer was found in $15.2 \%$ consistent with TBSRTC DC VI - malignant.

Results: In patients consistent with TBSRT DC II histomorphology was not performed. They were treated with conservative therapy, according to their thyroid functions and prescribed regular ultrasound control of existing nodules.

Patients with TBSRT DC IV, V, VI were refered to surgical treatment, but $38.2 \%$ refused. In $61.8 \%$ of patients surgery was performed and histomorphologic study of the post-operative material was done. The results were in complete accordance with cytologic study results.

Conclusions: the analysis of results of ultrasound-guided FNAC and operative material histomorphologic study revealed that ultrasound-guided FNAC is a reliable, trustworthy and effective method based on which the patient treatment methods can be selected.

\section{P284 \\ THE CLINICAL SIGNIFICANCE OF TUMOR SUPPRESSOR GENE MRNA AND PROTEIN EXPRESSION, METHYLATION CHANGES IN NODULAR THYROID DISEASE}

Wei Feng

Department of Endocrinology, the First Affiliated Hospital of Baotou

Medical College, Inner Mongolia University of Science and Technology, Baotou China

Objective:The tumor suppressor gene methylation lead to gene silencing, it plays an important role in thyroid tumor occurrence and development.This study examined the tumor suppressor gene (PTEN, DAPK) DNA methylation, mRNA and protein expression status in the patients with nodular thyroid disease. Discuss its clinical significance.

Method: Select 140 cases of nodular thyroid disease diagnosed (Including 66 cases of nodular goiter, 50 cases of thyroid adenoma, 24 cases of thyroid cancer)and 60 cases of normal control group in September 2009 - December 2013, Mining fasting peripheral venous blood collected, by the MSPPCR to detect PTEN, DAPK Methylation, by RT-PCR to detect mRNA expression. By Western Blotting to detect the protein expression in thyroid nodules tissue. Using SPSS 17.0 statistical software.

The result: 1) In the high methylation status of DAPK, PTEN gene in the peripheral blood and thyroid nodule tissue of thyroid cancer, And to compare the methylation rate of thyroid cancer $>$ adenoma $>$ nodular goiter. 
2) DAPK, PTEN mRNA and protein expression in thyroid cancer and adenoma reduce or even missing, And was negatively correlated with two genes methylation.

3) DAPK, PTEN gene promoter methylation and mRNA and protein expression was realated with lymph node metastasis in thyroid cancer patients. No relationship between Nodule size, calcification and ECT.

Conclusion:

1) PTEN, DAPK genes promoter methylation and the low mRNA, protein expression or missing are common in molecular biological events in the thyroid tumors.Closely related to the occurrence and development of thyroid tumors.

2) For the benign and malignant thyroid nodules early identification of molecular diagnosis is based.

\section{P285 \\ COMPARISON OF SERUM THYROGLOBULIN LEVEL AT 24 HR AND 72 HR AFTER RHTSH INJECTION}

Shin Young Jeong ${ }^{1}$, Sang-Woo Lee ${ }^{2}$, Byeong-Cheol Ahn' ${ }^{2}$, Jaetae Lee ${ }^{2}$

${ }^{1}$ Kyungpook National University School of Medicine; Kyungpook

National University Medical Center, ${ }^{2}$ Kyungpook National University

School of Medicine

Purpose: Measurements of thyroglobulin (Tg) levels $72 \mathrm{~h}$ after administration of recombinant human thyrotropin (rhTSH) are recommended by the manufacturer in the follow-up of patients with differentiated thyroid carcinoma (DTC). When I-123 diagnostic whole body scan and TSH stimulated Tg measurement are performed for follow-up, patients should pay additional visit to the hospital for measurements of Tg levels $72 \mathrm{~h}$ after administration of rhTSH. To evaluate the diagnostic usefulness of Tg level at $24 \mathrm{hr}$ (Tg24) and $72 \mathrm{hr}$ (Tg72) after rhTSH injection, we compared Tg values that measured both $24 \mathrm{~h}$ and $72 \mathrm{~h}$ after administration of rhTSH

Patients and Methods: For this study, 72 DTC patients underwent total thyroidectomy and I-131 ablation were included. Tg24 and Tg72 after rhTSH were done in all patients. The lower detection limit of Tg was $0.24 \mathrm{ng} / \mathrm{mL}$.

Results: In comparison between $\operatorname{Tg} 24$ and $\operatorname{Tg} 72$, there is no significant difference between two values. In 64 patients with Tg24 lower than $1 \mathrm{ng} / \mathrm{mL}$, 61 patients show that $\operatorname{Tg} 72$ is lower than $1 \mathrm{ng} / \mathrm{mL}$. $\mathrm{Tg} 72$ of other 3 patients $(2.9 \%)$ is between 1 and $2 \mathrm{ng} / \mathrm{mL}$. In 4 patients with Tg24 between 1 and 2 $\mathrm{ng} / \mathrm{mL}, 2$ patients show that $\mathrm{Tg} 72$ is greater than $2 \mathrm{ng} / \mathrm{mL}$. One patient shows $\mathrm{Tg} 72$ is lower than $1 \mathrm{ng} / \mathrm{mL}$ and 1 patient shows Tg72 is between 1 and $2 \mathrm{ng} /$ $\mathrm{mL}$. In 4 patients with $\mathrm{Tg} 24$ greater than $2 \mathrm{ng} / \mathrm{mL}$, all patients show that $\mathrm{Tg} 72$ is greater than $2 \mathrm{ng} / \mathrm{mL}$.

However, $\operatorname{Tg} 72$ is slightly greater than $\operatorname{Tg} 24$.

Conclusion: There is no significant difference between $\operatorname{Tg} 24$ and $\operatorname{Tg} 72$, and most patients shows no changes between two values. However, Tg 72 reveals slightly higher than $\operatorname{Tg} 24$, especially in patient with high $\operatorname{Tg} 24$ level. A large scale study is needed for evaluation of clinical usefulness of Tg24.

\section{P286 \\ DIFFERENTIATED THYROID CARCINOMA IN CHILDREN AND ADOLESCENTS: AN INSTITUTION'S EXPERIENCE BETWEEN 1970 AND 2013}

Raquel Guerrero Vázquez ${ }^{1}$, Noelia Gros Herguido ${ }^{1}$ Juan Ignacio Cuenca Cuenca ${ }^{2}$, Juan Manuel Martos ${ }^{3}$, Elena Navarro González ${ }^{1}$

${ }^{1}$ Endocrinology, Virgen del Rocio University Hospital, ${ }^{2}$ Nuclear Medicine Unit, Virgen del Rocio University Hospital, ${ }^{3}$ Surgery Unit, Virgen del Rocio University Hospital

Aims:

1. To describe the clinical evolution of differentiated thyroid carcinoma (DCT) in children and adolescents.

2. To analyze the long-term survival and disease free survival (DFS), identifying predictive factors for DFS.

Methods: We studied 79 DCT patients younger than 21 years diagnosed between 1970 and 2013 in a single Institution. The patients were aged 4-21 years old (median 17.5). The mean follow -up was $20.7 \pm 10.7$ years. We cal- culated overall survival and DFS. DFS was presented using Kaplan Meier survival curves and groups were compared using long rank test. The factors associated with DFS were examined using Cox multivariate analysis.

Results: 65 had papillary cancer and 14 had follicular tumor. $33(41.8 \%)$ presented with lymph nodes metastasis and 24 (30.4\%) with lung metastasis at diagnostic. All patients received ablative radioiodine treatment after surgery with a delay of 8.1 months (median). The recurrence rate was $11.4 \%$. At the time of the analysis: $64.6 \%$ were free of disease and $35.4 \%$ had persistent disease. The time to remission was 60 months (median). The overall survival at 20 years was $100 \%$. The probability to achieve the remission increased over time: $5 \%$ at 5 years and $40 \%$ at 20 years of follow-up. By Cox univariate analysis: DFS was lower in younger patients, larger tumor size, lymph nodes metastasis or lung metastasis at diagnosis and longer delay of radioiodine treatment. By Cox multivariate analysis: the delay of ablative radioiodine treatment was independently associated with DFS.

Conclusions:

1. In this series the overall survival was $100 \%$ despite $30 \%$ of patients presented with lung metastasis.

2. The probability to achieve the remission status increased over time. Only the delay of radioiodine treatment was independently associated with DFS. Early treatment with radioiodine was a key point in the prognosis of this age group.

\section{P287}

\section{ACTUAL EPIDEMIOLOGICAL SITUATION OF THYROID CANCER IN ALBANIA}

\section{Enalda Demaj ${ }^{1}$, Agron Ylli ${ }^{1}$, Marieta Kermaj ${ }^{1}$, Violeta Hoxha ${ }^{1}$,}

Endri Ahmetaj ${ }^{1}$, Gerond Husi ${ }^{1}$, Arjola Zeqollari ${ }^{1}$, Thanas Fureraj ${ }^{1}$,

Zamira Ylli ${ }^{1}$, Mariola Kapia ${ }^{1}$

${ }^{1}$ Mother Teresa Hospital of Tirana

Objective: The aim: to determine whether thyroid cancer frequency in Albania has stabilized or not; secondary to the National Thyroid Cancer Register

Material and Methods: We studied patients diagnosed with thyroid cancer during January 2013-March 2014.All medical records, demographic findings were registered in the Nuclear Medicine Department of Mother Theresa Hospital of Tirana. The definitive diagnosis was made by histologic findings postoperatively.

Results: During this period 45 patients were diagnosed of Thyroid Cancer. Mean age at diagnosis was:49 yo. According to the gender:females 33(73.3\%), males $12(26.7 \%)$. The ultrasound finding at admission was:a multinodular goiter In 34 (75.5\%)patients, solitary nodule in $11(25.6 \%)$. Cytological evaluations were done only in 5 cases: negative 1 case, suspicious 2 cases, positive 2 cases. Total thyroidectomy was the primary treatment in 33 patients with differentiated thyroid cancer(DTC), 8 patients with DTC the near total thyroidectomy was the first approach and then completion of thyroidectomy(after biopsy evaluation).According to the histopathologic form we found:DTC $41(91.2 \%)$ patients $\{28(68.3 \%)$ of them papillary form,3(7.3\%) folliculary and $10(24.4 \%$ ) papillary/follicular $\}$, medullary cancer $2(4.4 \%)$, medullary and papillary cancer $1(2.2 \%)$, primary lymphoma $1(2.2 \%)$. According to the age group:20-30 years old $3(6.7 \%)$, 30-40 years old $8(17.8 \%), 40-50$ years old $11(24.4 \%), 50-60$ years old $14(31.1 \%),>60$ years old $9(20.0 \%)$.

Conclusions: During the last few years, the frequency of thyroid cancer in Albania is increased: 36 cases in 2006(the highest registered) and nowdays, only in 14 months, we have 45 new patients (may be epidemic diagnosis). There is a female predominance $(73.3 \%)$. The papillary is the predominant histologic form $(62.2 \%)$, but the prevalence of mixed form is increasing $(19 \%)$. The most frequent presurgical ultrasound finding was a multinodular goiter(74.4\%). Most of our patients belongs to the age group of 50-60 years old(31.1\%). A total thyroidectomy was the primary surgical approach in most of our patients with DTC(33).More efforts need to be done in cytology evaluation as a first line diagnostic procedure, prior to surgical thyroid removal . 


\section{P288 \\ RISK STRATIFICATION OF PATIENTS WITH DIFFERENTIATED THYROID CANCER AT A DISTRICT GENERAL HOSPITAL 2002-2013, AND THE IMPLICATIONS FOR LONG TERM FOLLOW. $\mathbf{U P}$ \\ Martin Taylor ${ }^{1}$, Anthony Skene \\ ${ }^{1}$ Royal Bournemouth Hospital}

The Royal Bournemouth Hospital is a District General Hospital in the South of England serving a population of approximately 400,000. All patients with the diagnosis of thyroid cancer are managed in a Joint Thyroid Clinic.

In 2014, the British Thyroid Association (BTA) published its latest draft guidelines for the management of thyroid cancer. In line with American and European Guidelines, the 2014 Guideline now recommends that patients with differentiated thyroid cancer be risk-stratified into low, intermediate and high risk groups, and that 9-12 months after treatment undergo further Dynamic Risk Stratification.

This concept of Risk Stratification has evolved since the 2007 BTA Guideline; some patients are at low risk of recurrence, and for some patients total thyroidectomy and ablative radio-iodine may not be required. For low risk groups over-zealous replacement with L-thyroxine and full suppression of TSH $(<0.1 \mathrm{mu} / \mathrm{l})$ with higher doses of L-thyroxine may increase cardiovascular risk and risk of osteoporosis.

At the Royal Bournemouth Hospital patients, Clinical Practice has followed the BTA 2007 Guideline but over the last few years has moved to an ATA risk-stratification approach. All patients are registered with the Somerset Cancer Registry and management of patients is discussed at the local Endocrine Network MDT.

Between 2002 and 2013, 101 patients (Men $\mathrm{n}=19$, Women $\mathrm{n}=82$ ) with thyroid cancer have been treated and followed. Additional patients are under follow-up at the neighbouring Poole General Hospital. Patients are discussed jointly at the Regional Endocrine Network MDT.

This audit presents data on histology (papillary $n=63$, follicular/hurthle $\mathrm{n}=29$, medullary $\mathrm{n}=7$, Insular $\mathrm{n}=1$, anaplastic $\mathrm{n}=1$ ), initial treatment modalities, degree of TSH suppression. As many patients were diagnosed prior to the 2007 BTA Guidelines we look at the feasibility of retrospective risk stratifying patients to enhance patient follow-up.

\section{P289 \\ PREVALENCE AND OUTCOME OF THYROID MICROCARCINOMA IN A MILD IODINE DEFICIENCY AREA}

Mario Cappagli ${ }^{0}$, Prospero Magistrelli ${ }^{1}$, Antonella Montepagani ${ }^{2}$,

Franco Fedeli ${ }^{3}$, Donatella Intersimone ${ }^{3}$, Pierfrancesco Bonfanti ${ }^{4}$

${ }^{1}$ Surgery Department S.Andrea Hospital La Spezia, ${ }^{2}$ Endocrinology

S.Andrea Hospital La Spezia, ${ }^{3}$ Pathology S.Andrea Hospital La Spezia,

${ }^{4}$ Surgery S.Andrea Hospital La Spezia

Aim: evaluate prevalence and outcome of microcarcinoma (Mca) in a mild iodine deficiency area of about 220.000 people.

Results: between 1973-2013 were detected 964 differentiated thyroid carcinoma (DTC) (24 cases/year [yr]);236 were incidental Mca(5.9 cases/ yr).Among Mca $81.8 \%$ were female and $18.2 \%$ male. Median(M) age was 50 yrs. Patients(Pts) underwent to surgery for non toxic goiter(84\%), toxic nodular goiter(14\%), Basedow( $0.5 \%)$, larynx carcinoma $(0.5 \%)$, Thyr4 cytol$\operatorname{ogy}(0.5 \%)$, extrathyroidal lesion $(0.5 \%) .207 \mathrm{pts}$ had a total thyroidectomy, 13 a subtotal and 16 a lobectomy. 233/236 were papillary(P) [54 multifocal];1/236 hoxiphylic cell type follicular; 2/236 medullary. Follow-up (F.U.) was based on physical examination, measurement of free T4, TSH, AbTPO, AbTg, Tg, neck US. 229/236 Pts were classified Stage I, 6/236 Stage III and 1/236 Stage IVA. 98 Pts were submitted to ${ }^{131} \mathrm{I}$ ablation $(1.11-7.4 \mathrm{GBq})$; in 5 a second dose was required, in 1 a third and in 2 a fourth. The F.U. lasted from 3 to 40 yrs. In $67 \mathrm{Pts}$ a rhTSH test was performed; Tg was $<0.5 \mathrm{ng} / \mathrm{ml}$ in 60/67 Pts and from 0.8 to $13 \mathrm{ng} / \mathrm{ml}$ in 7 . At diagnosis in $27 \mathrm{Pts} \mathrm{AbTg}$ were elevated (EL), in 198 not detectable (ND) and in 11 not available (NA). During F.U., ND AbTg were 96.7\%, EL 0.8\% and NA 2.5\%.All Pts, including Stage III and IVA, were free of disease at the last FU.

Conclusions: our data show a good prognosis of Mca, also in advanced stages; this could authorize a postponed F.U. without any risk for Pts. From a critical point of view our results show an excess of radioiodine therapy, but we must consider that some data derive from seventies and eighties, when guidelines for the management of DTC were not so mandatory regarding radioiodine therapy.

\section{P290}

\section{THYROGLOBULIN AS SENTINEL IN DIFFERENTIATED THYROID CANCER IN ALBANIA}

Agron Ylli', Enalda Demaj ${ }^{1}$, Joana Rustemaj ${ }^{1}$, Marjeta Kermaj ${ }^{1}$, Endri Ahmetaj ${ }^{1}$, Violeta Hoxha ${ }^{1}$, Gerond Husi ${ }^{1}$, Zamira Ylli ${ }^{1}$,

Thanas Fureraj ${ }^{1}$

${ }^{1}$ Mother Teresa Hospital of Tirana

Objective: To assess the prognostic value of thyroglobulin(TG) measured postoperatively, before and after radioiodine therapy in patients with differentiated thyroid cancer(DTC) in Albania.

Methods: This retrospective study describes the medical records ( $\mathrm{Tg}, \mathrm{Tg}$ antibodies(AbTG)ultrasound, whole body scan(WBS)) and demographic findings of 71 patients diagnosed with DTC, followed and treated in the Nuclear Medicine Department of Mother Teresa Hospital of Tirana, between 2011 and 2013.All patients had a total or near total thyroidectomy followed by radioiodine therapy with $100 \mathrm{mCi}$ I131.

Results: Among patients $91.5 \%$ were women and $8.5 \%$ men.The mean age was 47.13 yo. In $63.16 \%$ of patients the cancer was detected in a multinodular goiter and $36.84 \%$ in a solitary nodule (ultrasound findings). Papillary carcinoma represented $57.6 \%$ of DTC, folliculary $11.3 \%$, papillary/follicular $21.1 \%$.The mean level of $\mathrm{Tg}(<5 \mathrm{ng} / \mathrm{ml})$ before radioiodine therapy, under suppression therapy was $2.99 \mathrm{ng} / \mathrm{ml}$; one month after withdrawal treatment of levothyroxine, Tg was $7.73 \mathrm{ng} / \mathrm{ml}$; the mean level of Tg after 6 months of radioiodine therapy was $1.572 \mathrm{ng} / \mathrm{ml}$, and after a year $0.163 \mathrm{ng} / \mathrm{ml}$.The $\mathrm{AbTg}$ were positive in $37.25 \%$ of patients and negative in $62.75 \%$.In most of the patients with positive $\mathrm{AbTg}, \mathrm{Tg}$ was undetectable.Patients with pulmonary metastasis in WBS, had high level of Tg $(47.37 \mathrm{ng} / \mathrm{ml})$.

Conclusions: In our study we confirmed that the serum thyroglobulin level is important in the follow-up of patients with DTC.We shown that Tg progressively decreased after radioactive iodine therapy $(1.52 \mathrm{ng} / \mathrm{ml}$ after $6 \mathrm{mo}$ and $0.163 \mathrm{ng} / \mathrm{ml}$ a year after)- indicating reduced tumore burden or the absence of disease; confirmed by ultrasound and WBS examinations. The mean level of $\mathrm{Tg}$ under suppression therapy was undetectable, $2.99 \mathrm{ng} / \mathrm{ml}$ (after surgery and before $100 \mathrm{mCi}$ I131 therapy); but after withdrawal treatment, Tg was stimulated, $7.73 \mathrm{ng} / \mathrm{ml}$-indicating persistence of disease.In $37.25 \%$ of our patients AbTg were positive and Tg undetectable.Patients in advanced stages had high levels of $\mathrm{Tg}$.

\section{P291 \\ PREDICTIVE FACTORS AND OUTCOMES IN PATIENTS WITH DIFFERENTIATED THYROID CARCINOMA: OUR EXPERIENCE}

Sonsoles Guadalix ${ }^{1}$, Maria Soledad Librizzi ${ }^{1}$, Jorge Ferrero ${ }^{1}$,

Eduardo Ferrero1, Guillermo Martínez Díaz-Guerra1,

Federico Hawkins ${ }^{1}$

${ }^{1}$ Hospital Universitario Doce de Octubre

Objectives: The aim of this study was to analyze the clinical, histopathological features and predictive factors of patients with differentiated thyroid carcinoma (DTC) in our center and to evaluate the utility of three current staging systems for predicting disease-specific mortality.

Patients and Methods: We reviewed 213 patients treated in our hospital from 1992 up to 2012 (184 females, 29 males, mean age 48.8 years, range 19-86) with DTC (192 papillary, 21 follicular carcinoma). Mean follow-up period was 6.83 years (1-20 years) and patients were ranked by the pTNM system. Risk was calculated according to MACIS, AGES and AMES staging systems. Survival probability was calculated using Kaplan-Meier analyses. Prognostic factors were analized using a univariate log rank test and a multivariate Cox regression analysis model.

Results: The factors affecting 10-year-disease-free survival were T4a, soft tissue invasion, lymph node metastasis and involvement of more than 5 nodes. The factors that better predicted the DTC recurrence were nodal involvement (HR 4.86 CI 1.42-16.54 p0.01) and soft tissue invasion (HR 4.49 CI 1.21- 
$16.65 \mathrm{p} 0.02)$. The only factor that affected overall survival was vascular invasion. In AGES and MACIS systems, high-risk patients showed lower overall survival $(\mathrm{P}<0.001)$. Significant differences were not found for AMES system.

Conclusions: In our series, lymph node metastases and soft-tissue involvement were associated with an increased risk of recurrence while vascular invasion was related to a higher risk of death. AGES and AMES were useful systems for risk assignment.

\section{P292 \\ THYROID AUTOIMMUNITY AND THYROID CANCER RISK}

Belen Perez-Pevida1, Gala Gutiérrez-Buey², Francisco Guillen-Grima², Juan Carlos Galofre ${ }^{2}$

${ }^{1}$ Clinica Universidad de Navarra; Endocrinology and Nutrition, ${ }^{2}$ Clínica

Universidad de Navarra

Background: The prognostic significance of the presence of thyroid autoantibodies (TA) in differentiated thyroid cancer (DTC) is controversial. We design a retrospective study analyzing the association of TA and differentiated thyroid cancer (DTC).

Methods: We selected the patients who were referred for thyroid surgery to our Hospital from 2010 to February 2014. We review the pathological result and the determination of thyroid peroxidase antibody (TPOAb) and/or thyroglobulin antibody $(\mathrm{TgAb})$ prior surgery. We excluded for the analysis those patients with the diagnosis of Graves' disease. Data were analyzed by an exact non-conditional logistic regression model with SPSS v.20.

Results: We select 119 patients (28 male). DTC and benign disease (BD) were found in $36(30.25 \%)$ and $83(69.7 \%)$ subjects respectively. TAs were positive in $10(27.7 \%)$ and $16(19.2 \%)$ of subjects with DTC and BD, respectively. The comparison showed a non-significant statistical difference between them. Additional analysis on the type of TAs showed that TPOAbs were present in $9(25 \%)$ seropositive DTC individuals, whereas this proportion decreased to $19 \%$ (only in 16 subjects) in seropositive BD individuals. TgAbs were present in $5(50 \%)$ seropositive DTC individuals whereas this proportion was lower $(25 \%)$ in seropositive BD individuals. The presence of TAs increased risk for DTC but the difference did not reach statistical significance $(\mathrm{OR}=2.460 ; \mathrm{p}=0.232)$.

Conclusion: The presence of thyroid autoimmunity, especially when the $\mathrm{TgAb}$ are positive indicates a positive trend of finding malignancy in the thyroid specimen after surgery. However we did not find a significant association between TAs and DCT in our study, probably because of the small sample size.

\section{P293}

\section{SURVIVAL OF PATIENTS WITH METASTATIC} DIFFERENTIATED THYROID CARCINOMA

Edelmiro Menendez ${ }^{1}$, Marta Diéguez Felechosa²,

Emma Anda Apiñániz ${ }^{3}$, Javier Santamaría Sandi ${ }^{4}$

${ }^{1}$ Hospital Universitario Central de Asturias, ${ }^{2}$ Hospital de Cabueñes,

${ }^{3}$ Complejo Hospitalario de Pamplona, ${ }^{4}$ Hospital Universitario de Cruces

To analyze clinical features and assess survival rates in all the patients with differentiated thyroid carcinoma and distant metastases from 3 general hospitals with the same protocol of treatment and follow-up wem ade a retrospective review of 131 patients with metastatic thyroid differentiated carcinoma identified from the database of three hospitals of the North of Spain diagnosed between 1988 and 2008. Disease specific survival was analyzed by the Kaplan-Meier method.

The median follow-up was $58 \pm 63$ months . Metastases were initially present in $58.8 \%$ of patients. Patients with poorly differentiated papillary carcinomas and metastases were diagnosed initially in the majority of cases $(64.3 \%$ and $77.8 \%$ respectively), but in patients with follicular carcinoma late diagnosis was more frequent (52.6\%). We observed lung metastases (LM) in $67.5 \%$ of patients, bone metastases (BM) in $15.3 \%$, CNS metastases in $2.3 \%$ and multisystemic metastases in $15.3 \%$.

In the multivariate analysis, survival was dependent on age, location and extent of metastases as seen in the table, and radiodine uptake.

Conclusions: Distant metastases are frequently the initial presentation in differentiated thyroid carcinoma, but later diagnosis during follow up is about as common.
Table 1. (for Abstract P293)

\begin{tabular}{lcccc}
\hline Disease specific survival & $5 \mathrm{yr}$ & $10 \mathrm{yr}$ & $15 \mathrm{yr}$ & $\begin{array}{l}\text { Median } \\
\text { survival } \\
\text { time } \\
\text { (months) }\end{array}$ \\
\hline Total & $57 \%$ & $33 \%$ & $28 \%$ & 82 \\
Macronodular lung metastases & $44 \%$ & $23 \%$ & $23 \%$ & 64 \\
Micronodular lung metastases & $93 \%$ & $71 \%$ & $57 \%$ & 162 \\
Bone metastases & $49 \%$ & $16 \%$ & - & 72 \\
Multiorganic metastases & $31 \%$ & $6 \%$ & - & 39 \\
\hline
\end{tabular}

Pulmonary metastases occur in all cases of papillary carcinomas in our series, whereas bone metastases occur in $50 \%$ of follicular carcinoma.

Age, location and extent of metastases and radioiodine uptake are the most important prognostic factors in this group of patients

Metastatic differentiated thyroid carcinoma can have a long survival with proper treatment

\section{P029 Thyroid Cancer Diagnostics 4}

\section{P294 \\ ENHANCER OF ZEST HOMOLOG 2 OVEREXPRESSION AS A PREDICTIVE FACTOR IN THYROID CANCER \\ Nobuyasu Suganuma ${ }^{1}$, Akira Yoshida ${ }^{2}$, Yohei Miyagi ${ }^{3}$, Junko Akaishi', Kenichi Matsuzu ${ }^{4}$, Takashi Uruno ${ }^{4}$, Kiminori Sugino ${ }^{4}$, Koichi Ito ${ }^{4}$, Tetsuo Kondo ${ }^{5}$, Munetaka Masuda \\ ${ }^{1}$ Yokohama City University; Department of Surgery, ${ }^{2}$ Kanagawa Cancer Center; Department of Breast and Endocrine Surgery, ${ }^{3}$ Kanagawa Cancer Center Reserch Institute; Division of Molecular Pathology and Genetics, ${ }^{4}$ Ito Hospital; Department of Surgery, ${ }^{5}$ University of Yamanashi; Department of Pathology}

Introduction: Enhancer of zeste homolog $2(\mathrm{EZH} 2)$ is a member of the polycomb group of genes, which are key factors in regulation of cell proliferation and differentiation through histone modification, chromatin remodeling, and interaction with other transcription factors. EZH2 is overexpressed in many malignancies, and thus has been proposed as a candidate prognostic factor and treatment target. In this study, we analyzed EZH2 expression levels in different histological subtypes of thyroid cancer to examine the utility of EZH2 as a prognostic factor for thyroid cancer.

Materials and Methods: Immunohistochemical analysis of EZH2 expression was performed in tissue samples from 67 cases of poorly differentiated thyroid cancer (PDTC) and 48 cases of undifferentiated (anaplastic) thyroid cancer (UTC), and in samples from adjacent normal and differentiated thyroid cancer (DCT) tissue.

Results: Expression of EZH2 protein was found in 17.9\% (12/67) of PDTC cases and $87.5 \%(42 / 48)$ of UTC cases, but was not found in tissue samples from normal thyroid gland and DCT. Higher expression levels of EZH2 correlated with poorer survival in PDTC ( $p=0.004$, log-rank test) and a similar trend was observed in UTC ( $p=0.166, \log$-rank test). However, in multivariate analysis, there was no difference in survival between cases with higher and lower EZH2 expression.

Conclusion: Our results suggest that immunohistochemical analysis of EZH2 overexpression might be associated with the malignant potential of thyroid cancer and that EZH2 is useful as a prognostic factor for PDTC. 
P295

DIFFERENCES BETWEEN TALL VARIANT AND TALL CELL FEATURES IN PAPILLARY THYROID CARCINOMA

Ja Seong Bae ${ }^{1}$, Chan Kwon Jung ${ }^{2}$, Dong Jung Lim³ ${ }^{3}$ Sohee Lee ${ }^{1}$, Byung Joo Song ${ }^{1}$, Sang Seol Jung ${ }^{1}$

${ }^{1}$ General Surgery, Catholic University of Korea, ${ }^{2}$ Hospital Pathology, Catholic University of Korea, ${ }^{3}$ Internal Medicine, Catholic University of Korea

Background: The tall cell variant of papillary thyroid carcinoma (TCVPTC) is more aggressive than classic PTC, but the percentage of tall cells needed to diagnose TCVPTC remains controversial. In addition, little is known about the clinicopathologic features of classic PTC with tall cell features (TCF).

Methods: Between Jan, 2012 and Dec, 2013, we retrospectively reviewed the clinicopathologic features of 1,268 classic PTCs, 149 classic PTCs with TCF, and 97 TCVPTCs, which were defined as PTCs having $<10 \%, 10-50 \%$, and $\geq 50 \%$ tall cells, respectively.

Results: TCVPTCs and classic PTCs with TCF did not vary significantly in clinicopathologic characteristics such as tumor size, pNstage, lateral lymph node metastasis, and BRAF mutations. TCVPTCs were significant extrathyroidal extension than classic PTCs with TCF. TCVPTCs and classic PTCs with TCF show more aggressive clinicopathologic features than classic PTCs; larger tumor size, high frequency of node metastasis, extrathyroidal extension and BRAF mutation rate. Similar results were obtained in a subanalysis in patients with microcarcinomas $(\leq 1.0 \mathrm{~cm}$ in size).

Conclusion: PTCs with more than $10 \%$ tall cells showed a similar $B R A F$ mutation rate and clinicopathologic features regardless of proportions of tall cells and tumor size. We suggest that a cut-off value of $10 \%$ tall cells may be sufficient for the diagnosis of TCVPTC.

\section{P296 \\ EVALUATION OF THE LEVEL OF THYROGLOBULIN AUTOANTIBODIES INTERFERING WITH THYROGLOBULIN MEASUREMENT IN PATIENTS WITH DIFFERENTIATED THYROID CARCINOMA}

Francesco Latrofa', Debora Ricci', Eleonora Sisti', Lucia Montanelli', Paolo Piaggi ${ }^{1}$, Teresa Rago ${ }^{1}$, Emilio Fiore ${ }^{1}$, Paolo Vitti', Michele Marinó $^{1}$

${ }^{1}$ Endocrinology Unit, University Hospital, University of Pisa

The level of $\mathrm{TgAb}$ that interferes with $\mathrm{Tg}$ measurement is unsettled. Sera were obtained from 177 patients with papillary thyroid carcinoma after total thyroidectomy, before thyroid remnant ablation. Tg was measured by an IMA, $\mathrm{TgAb}$ by three IMAs and three radioimmunoassays (RIA)s [positive cut-offs: $30 \mathrm{IU} / \mathrm{ml}$ (IMA1), 40 (IMA2), 4 (IMA3), 150 (RIA1), 60 (RIA2), 70 (RIA3)]. $\mathrm{TgAb}$ levels that best correlated with undetectable $\mathrm{Tg}$ values were calculated by ROC curve analysis. The recovery of $\mathrm{Tg}$ was calculated incubating 67 sera with borderline or positive TgAb (by IMA1) with 4 sera with high levels of $\mathrm{Tg}$, diluted to final Tg concentrations between 0.1 and $300 \mathrm{ng} / \mathrm{ml}$.

Among 49 patients with undetectable $\mathrm{Tg}, \mathrm{TgAb}$ were undetectable in $14.3-61.2 \%$, borderline in $8.2-24.5 \%$ and positive in $30.6-63.3 \%$ of subjects $(\mathrm{P}<0.001)$. Tg values were higher in subjects with undetectable $\mathrm{TgAb}$ (from 7.0-19.1 to $10.9-21.4 \mathrm{ng} / \mathrm{ml}$ ) then in those with borderline TgAb (from 0.0 0.0 to $5.3-14.2 \mathrm{ng} / \mathrm{ml}$ ) and positive $\mathrm{TgAb}$ (from $0.0-0.0$ to $0.0-0.8 \mathrm{ng} / \mathrm{ml})(\mathrm{P}<$ $0.001)$. An undetectable $\mathrm{Tg}$ value correlated with the following $\mathrm{TgAb}$ cutoff levels: $>9.3 \mathrm{IU} / \mathrm{ml}$ (IMA1), $>41.0$ (IMA2), $>1.3$ (IMA3), $>52.0$ (RIA1), $>0.0$ (RIA2) and $>0.0$ (RIA 3) ( $<0.001$ for all). TgAb interfered significantly with Tg recovery (Spearman correlations from -0.47 to -0.69$)(P<0.001)$. The interference (underestimation in most samples) was more common for lower Tg concentration (in 62/67 samples of Tg 0.1 and $0.33 \mathrm{ng} / \mathrm{ml}$ ). Positive, but not borderline $\mathrm{TgAb}$ levels interfered significantly with $\mathrm{Tg}$ recovery. An underestimation of $\mathrm{Tg}$ was always observed in presence of $\mathrm{TgAb} \geq 100$ and in most cases of $\mathrm{TgAb}<100$, an overestimation of $\mathrm{Tg}$ in few cases with $\mathrm{TgAb}$ $<100$. The recovery of $\operatorname{Tg} 1-4$ was similar.

In conclusions, borderline $\mathrm{TgAb}$ levels interfere with $\mathrm{Tg}$ measurement to a lesser degree than positive TgAb levels. The interference is higher for low Tg concentrations.

\section{P297 \\ IMMUNOHISTOCHEMICAL EXPRESSION OF ESTROGEN RECEPTOR ALPHA (ERALPHA) AND PROGESTERONE RECEPTOR (PR) IN TWO SERIES OF PATIENTS WITH PAPILLARY THYROID CANCER}

Giacomo Sturniolo ${ }^{1}$, Carlos Zafon ${ }^{2}$, Josep Castellvi , Gabriel Obiols², Mariacarla Moleti' ${ }^{1}$, Francesco Vermiglio ${ }^{1}$, Jorge Mesa ${ }^{4}$

${ }^{1}$ Department of Clinical and Experimental Medicine - Section of Endocrinology; University of Messina, Italy, ${ }^{2}$ Department of Endocrinology, Hospital Universitari Vall D'hebron, Universitat Autònoma de Barcelona, Barcelona, Spain, ${ }^{3}$ Department on Pathology, Hospital Universitari Vall D'hebron, Universitat Autònoma de Barcelona, Barcelona, Spain, ${ }^{4}$ Department of Endocrinology, Hospital Universitari Vall D'hebron, Universitat Autònoma de Barcelona, Barcelona, Spain

Introduction: Papillary thyroid cancer (PTC) prevalence is nearly threetimes higher in females than in males, decreasing after menopause thereafter. The gender difference suggests that growth and progression of PTC might be influenced by female sex hormones.

Objective: To analyze the expression of both estrogen receptor alpha (ERalpha) and progesterone receptor (PR) by immunohistochemistry in 203 patients from Catalonia (Spain) and Sicily (Italy).

Material and Methods: Both ERalpha and PR expression was evaluated in paraffin-embedded tumoral tissues of 45 males (M) and 158 females (F) aging $48.2 \pm 14.3 \mathrm{yrs}$ and followed up for $7.1 \pm 4$ yrs.

Results: ERalpha was expressed in $52(25.6 \%)$ patients (41 F, $11 \mathrm{M})$, PR in $94(46.3 \%)$ patients $(75 \mathrm{~F}, 19 \mathrm{M})$. ERalpha and PR were co-expressed in $31(15.3 \%)$ patients $(27 \mathrm{~F}, 4 \mathrm{M})$. There was no gender difference in terms of ERalpha and PR expression (F 25.9\% vs M 24.4\%; p = NS, and F $47.5 \%$ vs M $42.2 \% \mathrm{p}=\mathrm{NS}$, respectively). The positivity of the Eralpha expression (Eralpha+) was associated, only in women, to a higher PTC size: $22.8 \pm 11.8$ $\mathrm{mm}$ in positive cases versus $15.1 \pm 12.4 \mathrm{~mm}$ in negative cases; $\mathrm{p}=0.02$ ). Among the 148/158 women and the 44/45 men still followed up in our reference centers, 30 women $(20.3 \%)$ and 9 men $(20.4 \%)$ had persistence/recurrence $(\mathrm{P} / \mathrm{R})$ PTC. Eralpha + was associated, again only in women, to a lower risk of $\mathrm{P} / \mathrm{R}$ risk. Thus, only 2/30 (7\%) patients with $\mathrm{P} / \mathrm{R}$ were Eralpha+, while $37 / 118(31 \%)$ of those who did not have P/R were Eralpha + (Chi-square $7.5, \mathrm{P}<0.01)$. PR expression was not associated with any of the parameters analyzed.

Conclusion: Eralpha expression was found to be increased in tumor in larger diameter but was related with a reduced risk of persistence and/or recurrence of the disease. 


\section{P298 \\ EVALUATION OF A NEW EPIGENETIC BIOMARKER FOR DIAGNOSTIC AND PROGNOSTIC ASSESSMENT OF THYROID CANCER}

Raquel Buj Gómez ${ }^{1}$, Izaskun Mallona ${ }^{1}$, Anna Díez-Villanueva', Veronika Mancikova ${ }^{2}$, Esmeralda Castelblanco ${ }^{3}$, Didac Mauricio ${ }^{4}$, Manel Puig-Domingo ${ }^{5}$, Xavier Matias-Guiu ${ }^{6}$, Javier Maraval ${ }^{\beta}$, Mercedes Robledo ${ }^{7}$, Jordi Lluís Reverter ${ }^{5}$, Miquel A. Peinado Mireia Jordà ${ }^{1}$

${ }^{1}$ Institute of Predictive and Personalized Medicine of Cancer (Imppc), ${ }^{2}$ Hereditary Endocrine Cancer Group, Spanish National Cancer Research Centre (Cnio), ${ }^{3}$ Department of Endocrinology and Nutrition, University Hospital Arnau de Vilanova, Irblleida, ${ }^{4}$ Hospital Germans Trias Pujol; Dept. of Endocrinology, ${ }^{5}$ Germans Trias I Pujol Health Sciences Research Institute (Igtp); Department of Endocrinology and Nutrition, University Hospital Germans Trias I Pujol, Department of Medicine, Autonomous University of Barcelona, ${ }^{6}$ Department of Pathology, University Hospital Arnau de Vilanova, University of Lleida, Irblleida, ${ }^{7}$ Hereditary Endocrine Cancer Group, Spanish National Cancer Research Centre (Cnio); Isciii Center for Biomedical Research on Rare Diseases (Ciberer), ${ }^{8}$ Institute of Predictive and Personalized Medicine of Cancer (Imppc),

Thyroid Cancer is the most common endocrine neoplasia and although most patients survive the disease, there is still a need of new markers for early diagnosis and prognostic assessment. In the last few years, several (epi-)genetic biomarkers (mutations, promoter hypermethylation, differential miRNA expression, etc.) have been proposed, but none of them has been established in clinical routine. DNA hypomethylation is a landmark of most cancers and recent studies have proposed the analysis of methylation in DNA repeat elements as a risk and prognostic biomarker in different cancer types as colorectal, prostate, pancreatic carcinoma or glioblastoma. Moreover, unlike other biomarkers, the decrease in methylation of repetitive elements is a widespread genomic alteration.

Although a few studies have unveiled candidate epigenetic markers in thyroid cancer by characterization of DNA methylation profiles, no investigations to date have analyzed DNA methylation of repeat elements in thyroid cancer in a consistent manner. Our goal is to determine whether DNA hypomethylation of repetitive elements is also a feature of thyroid cancer and to evaluate its potential use as a prognostic and diagnostic biomarker of the disease. For this purpose, we have developed a technique for Quantification of Unmethylated Alu elements (QUAlu), that can be applied to samples routinely obtained for pathological examination (fresh tissue, FNABs, blood, etc).

QUAlu performance has been evaluated for reproducibility and accuracy. We have applied it to analyze Alu repeat demethylation in a series of thyroid carcinomas and normal thyroid tissue. Preliminary analysis of the data demonstrates a decrease of Alu methylation in thyroid carcinomas and suggests its potential applicability as biomarker, although further studies are required to evaluate its usefulness. In summary QUAlu appears as a specific, cheap and simple technique that can enhance the prognostics and diagnostics of thyroid carcinomas.

\section{P299 \\ IMPACT OF INTEGRATED MOLECULAR \\ DIAGNOSTICS OF AIR-DRIED FINE NEEDLE \\ ASPIRATION (FNA) SMEARS OF PATIENTS WITH NODULAR THYROID DISEASE IN A ROUTINE DIAGNOSTIC SETTING}

Ralf Paschke ${ }^{1}$, Maja Neustadt ${ }^{1}$, Ilka Ruschenburg ${ }^{2}$, Anna Neumann ${ }^{3}$, Christiane Franzius ${ }^{4}$, Christian Landvogt ${ }^{5}$, Sabine Adam ${ }^{6}$, Regina Hammoser ${ }^{7}$, Thomas Molwitz ${ }^{8}$, Anja Hach ${ }^{9}$, Berit Feldmann ${ }^{10}$, Stefan Gratz ${ }^{11}$, Wolfgang Braun ${ }^{12}$, Dieter Graf ${ }^{13}$, Bassam Amro ${ }^{14}$, Rainer Niemann ${ }^{15}$, Reinhard Santen ${ }^{16}$, Markus Eszlinger ${ }^{1}$ ${ }^{1}$ Division of Endocrinology and Nephrology, University of Leipzig, Leipzig, Germany, ${ }^{2}$ Mvz Wagnerstibbe Für Gynäkologie, Reproduktionsmedizin, Zytologie, Pathologie und Innere Medizin GmbH, Einbeck, Germany, ${ }^{3}$ Amedes Medizinisches Versorgungszentrum Für Laboratoriumsmedizin, Mikrobiologie und Genetik Hannover GmbH, Hannover, Germany, ${ }^{4}$ Zentrum Für Nuklearmedizin und Pet/Ct, Bremen, Germany, ${ }^{5}$ Praxis Für Nuklearmedizin und Pet/Ct-Zentrum in der Deutschen Klinik Für Diagnostik, Wiesbaden, Germany, ${ }^{6}$ Radiologie Hoheluft, Nuklearmedizin, Hamburg, Germany, ${ }^{7}$ Schilddrüsenpraxis Kantstraße, Berlin, Germany, ${ }^{8}$ Klinik Dr. Hancken, Stade, Germany, ${ }^{9}$ Institut Für Radiologie und Nuklearmedizin Bremerhaven, Bremerhaven, Germany, ${ }^{10}$ Praxis Endokrinologie und Diabetologie Im Zentrum, Stuttgart, Germany, ${ }^{11}$ Radiologie Zentrum Stuttgart, Stuttgart, Germany, ${ }^{12}$ Schilddrüsenpraxis Nuklearmedizin Augsburg, Augsburg, Germany, ${ }^{13}$ Praxisgemeinschaft Auf Dem Meere, Lüneburg, Germany, ${ }^{14}$ Praxis Für Nuklearmedizin, Oldenburg, Germany, ${ }^{15}$ Praxis Steen und Partner, Radiologie Oldenburg, Oldenburg, Germany, ${ }^{16}$ Endokrinologische Gemeinschaftspraxis, Frankfurt/Main, Germany

Objectives: After a first retrospective study we now report the first 12 month results of molecular diagnostics analyzing BRAF, RAS, RET/PTC, and PAX8/PPARG mutations in routine air-dried FNA smears.

Methods: RNA and DNA was extracted from 321 consecutive FNA cytology smears (75 FNAs with a cytological diagnosis of follicular neoplasia (FN), 195 FNAs with a cytological diagnosis of microfollicular proliferation (MFP), 48 FNAs with the cytological diagnosis of papillary carcinomas (PTC) and 3 FNAs with the cytological diagnosis suspicious for malignancy (MAL). PAX8/PPARG, RET/PTC1 and RET/PTC3 rearrangements were detected by qPCR, while BRAF and RAS mutations were detected by pyrosequencing.

Results: The total failure rate for the mutation screening was $8.8 \%$ in contrast to $61 \%$ for HBME1 and Galectin3 immunocytochemistry. NRAS mutations could be detected in nine MFP samples, in two FN samples, and in one PTC sample. HRAS mutations were detected in two MPF and one FN sample. A KRAS mutation was only detected in one FN sample, whereas BRAF mutations were detected in 29 samples with a cytological PTC diagnosis, in three MFP, and in one FN sample. PAX8/PPARG was detected in three MFP samples, while RET/PTC was detected in three MFP samples and in two PTC samples. In total, 10.3\% MFP samples, 6.7\% FN samples, and 66.7\% PTC samples harbored a mutation.

Conclusions: These results confirm the feasibility of molecular diagnostics of air-dried FNA smears in a routine diagnostic setting. BRAF positive FNAs with the cytological diagnosis PTC can result in more primary total thyroidectomies instead of diagnostic lobectomies followed by completion thyroidectomy after histologic diagnosis. The molecular analysis of borderline cases with MFP can lead to important additional information with likely clinical consequences, whereas the molecular analysis of FN samples provided further arguments for a diagnostic surgery for mutation positive samples. 


\section{P300}

CLINICAL VALUE OF VISUALLY IDENTIFIABLE 18F-FLUORODEOXYGLUCOSE UPTAKE IN PRIMARY PAPILLARY THYROID MICROCARCINOMA

Jin Koo Kang ${ }^{1}$, Seung Ook Hwang ${ }^{1}$, Jin O Baek', Jeeyeon Lee ${ }^{1}$, Wan Wook Kim ${ }^{1}$, Jin Hyang Jung ${ }^{1}$, Ho Yong Park ${ }^{1}$

${ }^{1}$ Kyungpook National University, School of Medicine

Objectives: This study evaluates the relationship between visually identifiable ${ }^{18} \mathrm{~F}$-fluorodeoxyglucose (FDG) uptake in primary papillary thyroid microcarcinoma (PTMC) and tumor aggressiveness.

Methods: Clinicopathological factors and PET/CT findings of 219 PTMC surgical patients who underwent preoperative ${ }^{18} \mathrm{~F}$-FDG-positron emission tomography/computed tomography (PET/CT) were retrospectively reviewed.

Results: ${ }^{18} \mathrm{~F}-\mathrm{FDG}$ uptake was observed in the tumors of $124(56.6 \%)$ patients. Tumor size (odds ratio [OR] 1.774, 95\% confidence interval [CI] 1.416-2.223, $P<0.0001$ ) and Hashimoto thyroiditis (OR 2.815, CI 1.237$6.404, P=0.014)$ independently predicted ${ }^{18} \mathrm{~F}-\mathrm{FDG}$ uptake. Tumor size (OR 1.495 , CI $1.217-1.835, P<0.0001$ ) and BRAF ${ }^{\mathrm{v} 600 \mathrm{E}}$ mutation (OR 3.320, CI $1.056-10.432, P=0.040$ ) independently predicted extrathyroidal invasion. Multiplicity (OR 2.375 , CI $1.278-4.415, P=0.006$ ) independently predicted central lymph node metastasis.

Conclusion: ${ }^{18} \mathrm{~F}-\mathrm{FDG}$ uptake in PTMC depends on tumor size and Hashimoto thyroiditis. Therefore, preoperative PET/CT for PTMC may not help in evaluating tumor aggressiveness.

\section{P301 \\ PAPILLARY THYROID MICROCARCINOMA OR MICROTUMOR: A PATHOLOGICAL, IMMUNOHISTOCHEMICAL AND BRAFV600E DIFFERENTIAL STUDY}

Elvin Aliyev ${ }^{1}$, María Jesús Ladra ${ }^{2}$, Barreiro Francisco Morandeira ${ }^{2}$, Clara Alvarez Villamarín ${ }^{3}$, José Cameselle-Teijeiro ${ }^{4}$

${ }^{1}$ Centre for Medical Investigations (Cimus-Idis), Clinical University Hospital, University of Santiago de Compostela, ${ }^{2}$ Department of

Surgery, Clinical University Hospital, ${ }^{3}$ Molecular Medicine and Chronic Diseases (Cimus), University of Santiago de Compostela, ${ }^{4}$ Department of Pathology, Clinical University Hospital of Santiago de Compostela, Sergas, University of Santiago de Compostela

Objectives: Papillary thyroid microcarcinomas (PTmC) are papillary thyroid carcinomas (PTC) measuring $\leq 1 \mathrm{~cm}$. PTmC have usually an excellent prognosis but a small subset may behave aggressively. Papillary thyroid microtumor (PTmT) was proposed ("Porto proposal" 2003) to describe a benign subgroup PTmC detected in adults, lacking multicentricity and without features of aggressive behaviour. We collected a group of PTmC, and studied BRAF gene status and Estrogen Receptor alpha (ERa). We classified these microneoplasms in 2 groups: a) PTmC (exluding PTmT), and b) PTmT. The hypothesis was that BRAF gene status and/or the expression of ERa may participate in the progression of the PTmC to clinical PTC.

Methods: We studied a series of 26 patients ( 20 women and 6 men), aged 34-75 years (median: 55). Immunoexpression of ERa was studied both in the carcinomas and normal tissue. Laser microcapture was used to obtain DNA for BRAF analysis. Pearson's correlations with a two tail test were performed.

Results: We found a weak but significant positive correlation of ERa expression in the microneoplasms with the female gender (Pearson: $0.43 \mathrm{P}<$ 0.01 lesion vs. $0.34 \mathrm{P}<0.045$ normal tissue); in contrast, negative correlation was detected for the male gender. Follicular variant of PTmC positively correlated with thyroiditis (Pearson $=0.49, \mathrm{P}<0.003$ ) while classical variant negatively correlated with thyroiditis (Pearson $=-0.40, \mathrm{P}<0.017$ ). BRAF was negatively correlated with size of the microneoplasms while ERa was positive correlated. Seventeen PTmC and 9 PTmT were studied. A weak significant correlation of the age with PTmC and negative correlation with PTmT was found. We could not find any association of the BRAF or the ERa expression with either PTmC or PTmT.

Conclusions: Neither BRAF status nor ERa expression seem to be different in PTmC and PTmT. More cases should be analyzed to definitively evaluate the importance of both criteria in these type microscopic neoplasms.

\section{P302}

ANALYSIS OF THE CLINICAL, EPIDEMIOLOGICAL AND PATHOLOGICAL FEATURES OF ANAPLASTIC THYROID CANCER (ATC) FOLLOWED IN A SINGLE NATIONAL REFERRAL CENTER

Elena Sabini', Eleonora Molinaro', Agnese Biagini', Carlotta Giani', Paolo Piaggi', Laura Agate', Virginia Cappagli', Fulvio Basolo², Clara Ugolini ${ }^{2}$, Aldo Sainato ${ }^{3}$, Gabriele Materazzi ${ }^{2}$, Salvatore Mazzeo ${ }^{4}$, Rossella Elisei ${ }^{1}$

${ }^{1}$ Department of Clinical and Experimental Medicine - Section of Endocrinology, ${ }^{2}$ Department of Surgery, Medical, Molecular, and Critical Area Pathology, ${ }^{3}$ Department of Oncology, Transplantations and New Technologies, ${ }^{4}$ Department of Translational Research on New Technologies in Medicine and Surgery

We collected epidemiological, clinical and pathological data of 110 cases of ATC (61 females, 49 males) followed in our Department between 1972 and 2013. The mean age at diagnosis was 63.92 years (range $33-82$ yrs). The histological diagnosis was obtained in 103/110 cases, while the diagnosis was cytological in 7/110 cases: pure ATC was found in 83/110 cases, ATC with areas of differentiated or poorly differentiated carcinoma in $27 / 110$ cases. The mean diameter was $5.63 \pm 1.97 \mathrm{~cm}$ (range 1-12 cm). At diagnosis, we found lymph-node metastases in $88 / 110$ cases, distant metastasis in $72 / 110$ cases, infiltration of the trachea and esophagus in 47/110 and 28/110 cases, respectively. No therapy could be performed in 6/110 very advanced cases, surgery (TX) was performed in 80/110 patients, radiotherapy (RT) in 48/110 patients, chemotherapy (CT) in 53/110 patients, tyrosine kinase inhibitors (TKI) in 11/110 patients. In 96/110 we could calculate the length of survival: mean 17.16 months, median: 5 months, range: 1-258 months. Regardless of surgery, patients who performed CT+RT+TKI showed a longer survival. Single therapies that had the greatest impact on prognosis were CT and RT. Distant metastases, tracheal infiltration and a bigger tumor size significantly increased the risk of death. Patients were then divided into two groups based on the time of diagnosis: group 1 (1972-1992) and group 2 (1993-2013). The only significant differences between group 1 and 2 was an increased percentage of lymph-node metastases and a bigger number of patients who underwent TX in group $2: 80.5 \%$ vs $19.5 \%$ and $20.3 \%$ vs $79.7 \%$, respectively. In conclusion, clinical and pathological features of ATC are highly variable. Mode of presentation was slightly worse in group 2 but survival did not change over the years. Multimodal treatment correlated with an increased survival. Advanced features of the tumor are bad prognostic factors for survival.

\section{P030 Thyroid Cancer Pathogenesis 2}

\section{P303 \\ CLINICOPATHOLOGIC FEATURES AND BRAF MUTATION IN PATIENTS WITH PAPILLARY THYROID CARCINOMA WITH AND WITHOUT CHRONIC LYMPHOCYTIC THYROIDITIS}

Yong Sang Lee ${ }^{1}$, Taeil Yoon ${ }^{2}$, Hang-Seok Chang ${ }^{3}$, Cheong Soo Park ${ }^{3}$ ${ }^{1}$ Gangnam Severance Hospital, Yonsei University College of Medicine, ${ }^{2} 21$ st Surgical Clinic, ${ }^{3}$ Thyroid Cancer Center, Gangnam Severance Hospital, Yonsei University College of Medicine

Background: Chronic lymphocytic thyroiditis (CLT) in patients with papillary thyroid carcinoma (PTC) has been associated with less aggressive disease at the time of surgery. BRAF mutation is a marker of aggressiveness of conventional PTC. The aim of this study was to assess whether PTC with CLT has a different pattern of BRAF mutation, and investigated the clinicopathological features of PTC with CLT.

Methods: Medical records of 2,015 PTC patients who underwent thyroid surgery between January 2010 and December 2012 were reviewed retrospectively. Of these patients, 670 (33.3\%) had coexisting CLT. The clinicopatho- 
logic characteristics of the two groups, including BRAF mutations, were compared.

Results: Age, primary tumor size, and capsular invasion were similar in the two groups. The proportions of patients with central node $(38.4 \%$ vs. $34.3 \% ; p=0.04)$ and lateral node $(11.9 \%$ vs. $9.0 \% ; p=0.026)$ metastases were significantly lower in patients without than with CLT. Mean metastatic node ratio $(0.25 \pm 0.34$ vs. $0.13 \pm 0.22 ; \mathrm{P}<0.001)$ and the proportion of patients with BRAF mutations $(83.2 \%$ vs. $70.0 \%$; $\mathrm{P}<0.001)$ were significantly higher in patients without than with CLT.

Conclusion: CLT is associated with lower frequencies of the BRAF mutation and of lymph node metastasis in patients with PTC.

\section{P304 \\ GENETIC EXPRESSIONS ALTERATION AFFECTS ON LATERAL NECK NODE METASTASIS OF THYROID PAPILLARY MICROCARCINOMA}

Sohee Lee ${ }^{1}$, Byung Joo Chae ${ }^{1}$, Ja Seong Bae ${ }^{1}$, Byunh Joo Song ${ }^{1}$,

Sang Seol Jung ${ }^{1}$, Woong Youn Chung ${ }^{2}$

${ }^{1}$ Department of Surgery, Catholic University of Korea College of

Medicine, Seoul St. Mary's Hospital Seoul, Republic of Korea,

${ }^{2}$ Department of Surgery, Yonsei University College of Medicine

Background: Papillary thyroid cancer has a mild biological behavior, especially the papillary thyroid micrcarcinoma(PTMC) shows a very favorable prognosis. The cervical lymph node metastasis of PTMC is the most significant prognostic factor for loco-regional recurrences. In this study, we compared the gene expression of PTMC which showed lateral neck node metastasis at initial diagnosis with PTMC without node metastasis and validated the functional evidences of over- or under expressed genes which correlated with early extensive lymph node metastasis.

Methods and Materials: We performed the oligonucleotide microarray analysis with 8 PTMCs and paired normal thyroid tissues using Illumina HumanHT-12 v4.0 Expression Beadchip. Of these, three were PTMC without cervical lymph node metastases, and others were PTMC with lateral neck node metastasis. Statistical significance of the differentially expressed genes (DEGs) was determined using independent T-test with two-sided $\mathrm{P}<0.05$ and median fold change cut off $>1.5$. The quantitative real-time PCR of differentially expressed genes were used to confirm the microarray data.

Results: In microarray analysis, PTMC with lateral node metastasis shows different expression in 732 probes, including 520 up-regulation and 212 down-regulation. Different gene expression implicated in the biological process of signaling, multicellular organism process, response to stimulus, developmental process, cell proliferation, death, locomotion, biological regulation in GO profile analysis. Epithelial-to-mesenchymal transition and stem cell marker-related gene including IL1RL1, ALDH1A3, FGFBP1, TM4SF1, PROM1, CAV1, CCL18, TGIF1, SMAD3, CDCP1, CXCR1 was significantly up regulated in PTMC with lateral neck node metastasis. The quantitative realtime PCR confirmed the data of microarrays.

Conclusion: Genes which plays a role in epithelial mesenchymal transition and thyroid cancer stemness are up-regulated in early extensive lymphatic spread of PTMC.

\section{P305 \\ SIMULTANEOUS MEDULLARY AND PAPILLARY THYROID CARCINOMAS SHOW INDEPENDENT GENETIC ORIGIN}

Raffaele Ciampi ${ }^{1}$, Cristina Romei ${ }^{1}$, Alessia Tacito ${ }^{1}$, Clara Ugolini ${ }^{2}$,

Fulvio Basolo², Gabriele Materazzi ${ }^{2}$, Rossella Elisei

${ }^{1}$ Department of Clinical and Experimental Medicine, University of Pisa,

${ }^{2}$ Department of Surgical, Medical, Molecular Pathology and the Critical Area, University of Pisa

Papillary (PTC) and Medullary (MTC) thyroid carcinomas have distinct embryonic origins although they share the activation of common oncogenes (RET, RAS). The occurrence of these tumors in the same gland is not a rare event. Aim of this study was to investigate the hypothetical involvement of common genetic alterations in the symultaneous occurrence of the PTC and MTC.
We studied 24 patients presenting simultaneous PTC and MTC. DNA was obtained from both tumor components and healthy tissue and sequenced for mutations in codons 12,13 and 61 of $\mathrm{H}-, \mathrm{K}-$ and N-RAS, 600 of BRAF and 634 and 918 of RET.

Two/24 patients $(8.3 \%)$ were affected by MEN2A and carrying germline mutations of the RET gene (S981A, V804M). None of the patients showed mutations in the healthy tissue and $8 / 24$ patients $(33.4 \%)$ had no mutations either in the tumor components. In the other cases, considering the MTC component: 8/24 (33.3\%) harboured the RET/M918T mutation; 3/24 (12.5\%) mutations in the H-RAS gene (G13R, Q61R, D69N) and 1/24 (4.2\%) showed simultaneously the H-RAS/Q61K and the RET/M918T mutations. In the PTC component: 1/24 (4.2\%) harboured the BRAF/V600E mutation, 1/24 (4.2\%) the H-RAS/T58A mutation and 1/24 (4.2\%) the K-RAS/M1T mutation. In one case, we found the RET (M918T) mutation in the MTC component and the $\mathrm{BRAF} / \mathrm{V} 600 \mathrm{E}$ in the PTC component. None of the mutations found were present in both tumors.

These data suggest independent genetic causes in the development of simultaneous PTC and MTC. Confirmation of this hypothesis is that one of the patients showed simultaneous mutation of RET in MTC and the BRAF mutation in PTC. In this study we also found novel H-RAS and K-RAS mutations suggesting that alterations outside the classic hot spots may play a role in the pathogenesis of these tumors.

\section{P306 DOWNREGULATION OF FUCA1 (ALPHA- L-FUCOSIDASE-1) IN HUMAN THYROID ANAPLASTIC CARCINOMAS}

Giancarlo Vecchio ${ }^{1}$, Alessia Parascandolo ${ }^{2}$, Chiara Allocca ${ }^{2}$, Andrea Strazzulli ${ }^{3}$, Marco Moracci ${ }^{3}$, Clara Ugolini ${ }^{6}$, Fulvio Basolo, Maria Domenica Castellone ${ }^{4}$, Massimo Santoro ${ }^{2}$, Nobuo Tsuchida ${ }^{5}$ ${ }^{1}$ Istituto Superiore DI Oncologia (I.S.O.); Dipartimento DI Medicina Molecolare E Biotecnologie Mediche, Università DI Napoli Federico II, Naples, ${ }^{2}$ Dipartimento DI Medicina Molecolare E Biotecnologie Mediche, Università DI Napoli Federico II, Naples, ${ }^{3}$ sstituto DI Bioscienze e Biorisorse del C.N.R., Naples, ${ }^{4}$ Istituto DI Endocrinologia Ed Oncologia Sperimentale del C.N.R., Naples, ${ }^{5}$ Department of Cellular Oncology \& Microbiology, Tokyo Medical \& Dental University, Tokyo, Japan; ${ }^{6}$ Dipartimento di Patologia Chirurgica, Medica,

Molecolare e dell'Area Critica, University of Pisa, Italy

It has been reported that alpha-L-Fucose, participates in several interaction processes between cells and between cells and extracellular matrix. It has also been reported that high levels of fucose are often related to neoplastic progression. The lysosomal enzyme a-L-fucosidase (FUCA1) is involved in the removal of fucose from glycans. The FUCA1 gene has recently been shown to be down-regulated in highly aggressive and metastatic histotypes of human tumors. The role of FUCA1 in tumor progression remains unclear. It is speculated that its inactivation perturbs the glycosylation of proteins involved in cell adhesion and promotes cancer. We compared by real time PCR the expression of FUCA1 in 15 papillary (PTC), 10 anaplastic (ATC) thyroid cancer biopsies and in 6 biopsies obtained from non tumoral thyroid tissues and found that FUCA-1 mRNA in anaplastic cancer biopsies was decreased about half with respect to papillary and normal thyroid biopsies. We also investigated the expression of FUCA-1 in human thyroid cancer cell lines. Proteins extracted from human PTC and ATC cell lines have been analyzed by Western blot using a polyclonal anti-FUCA1 antibody. Enzyme assays for FUCA1 were carried out by using the fluorescent substrate 4-methylumbelliferyl-alpha-Lfucopyranoside. We found that two ATC cell lines (8505C and CAL-62) had a lower expression of the FUCA1 - specific $51 \mathrm{~K}$ and $56 \mathrm{~K}$ protein bands and had a lower enzyme activity compared with two PTC cell lines (TPC-1 and $\mathrm{BcPAP}$ ) and one non transformed cell line (Nthy-ori 3.1). The results obtained so far have shown therefore that FUCA-1 is downregulated in ATC compared to PTC and to non transformed cell lines. As shown for other human cancers, the downregulation of FUCA-1 correlates with the increased aggressiveness of the cancer type.

Work partly supported by PON01_02782, Ministero dell'Istruzione, dell’Università e della Ricerca, Italy. 
P307

IMPACT OF MINIMAL EXTRATHYROIDAL EXTENSION IN PATIENTS WITH PAPILLARY THYROID MICROCARCINOMA TREATED WITH TOTAL THYROIDECTOMY

Sung Won Kim ${ }^{1}$, Hyoung Shin Lee ${ }^{2}$, Kang Dae Lee ${ }^{2}$, Jun Woong Song ${ }^{1}$, Taejung Park ${ }^{1}$, Jong Chul Hong ${ }^{3}$

${ }^{1}$ Kosin University College of Medicine, ${ }^{2}$ Department of OtolaryngologyHead and Neck Surgery, Kosin University College of Medicine,

${ }^{3}$ Dong-A University College of Medicine

Objective: We analyzed the clinicopathologic characteristics of patients with papillary thyroid microcarcinoma (PTMC) presenting minimal extrathyroidal extension (ETE) and investigated whether minimal ETE is correlated to the oncologic outcomes in PTMC.

Methods: A retrospective study was conducted, based on 178 patients who underwent total thyroidectomy for PTMC between April 1998 and December 2009. Patients with locally invasive tumor involving adjacent structures other than the sternothyroid muscle were excluded. The patients were divided into two groups as group A (without minimal ETE, $n=97$ ) and group B (with minimal ETE, $\mathrm{n}=80$ ). The clinicopathologic factors were compared between the groups and correlation of the factors to tumor recurrence were analyzed.

Results: Tumor size larger than $5 \mathrm{~mm}(\mathrm{p}=0.004, \mathrm{OR}=2.875)$ and radioiodine ablation therapy $(\mathrm{p}=0.013, \mathrm{OR}=2.460)$ showed significant correlation to patients of with minimal ETE (group B). During the median follow-up period of 57 months (range 36 to 176 months), recurrence occurred in 4 patients $(4.1 \%)$ of group A and 1 patient $(1.3 \%)$ of group B with no significant difference $(\mathrm{p}=0.381)$. All recurrent cases were lateral lymph node (LN) metastasis. Male $(\mathrm{p}=0.044, \mathrm{OR}=7.945)$ and lateral $\mathrm{LN}$ metastasis $(\mathrm{p}=0.016$, $\mathrm{OR}=11.901)$ were independently correlated to recurrence in patients of group $\mathrm{B}$.

Conclusion: Minimal ETE had no impact on recurrence in patients with PTMC while male and lateral LN metastasis at initial presentation were risk factors for recurrence in PTMC with $\mathrm{mETE}$.

\section{P308 \\ DIFFERENTIAL MIRNAS EXPRESSION IN PAPILLARY THYROID CANCER IS ASSOCIATED WITH CLINICO-PATHOLOGICAL FEATURES AND BRAF MUTATION \\ Esmeralda Castelblanco ${ }^{1}$, Veronica Rosado ${ }^{1}, M^{a}$ Dolores Santos ${ }^{1}$, Montserrat Martinez ${ }^{2}$, Veronica Mancikova ${ }^{3}$, Mercedes Robledo ${ }^{3}$, \\ Xavier Matias-Guiu ${ }^{4}$, Javier Maravall ${ }^{1}$, Didac Mauricio ${ }^{5}$ \\ ${ }^{1}$ Department of Endocrinology and Nutrition, University Hospital \\ Arnau de Vilanova, Irblleida, ${ }^{2}$ Biomedical Research Institute of Lleida \\ / University of Lleida, Lleida, Spain, ${ }^{3}$ National Cancer Research \\ Centre, Madrid, Spain, ${ }^{4}$ Service of Pathology and Molecular Genetics, University Hospital Arnau de Vilanova, Lleida, Spain, ${ }^{5}$ Department of \\ Endocrinology and Nutrition, Hospital Germans Trias I Pujol, Badalona, Spain}

Introduction: MicroRNAs (miRNAs) are short non-coding RNAs that regulate translation or degradation of target mRNAs. Therefore, miRNAs control gene expression in many biological processes, including proliferation, apoptosis, and differentiation. Deregulation of miRNAs expression is an important contributor to tumour development and progression. Even though several genetic and epigenetic lesions have been identified in human thyroid cancer, particularly in the papillary histotype (PTC), they lack conclusive prognostic value. The present study was undertaken to examine whether a differential expression of miRNAs could provide a tool to improve prognosis in PTC.

Design: The miRNAs expression profiles were examined using next-generation deep sequencing in 35 snap-frozen tissues from surgically removed PTC and 9 normal thyroid samples. First, TMM and Benjamini-Hochberg's methods validated the miRNAs sequences used in the final statistical analysis. The Neyman type A gene-wise modelling was used to detect the existence of statistical significance differences in miRNA expression levels between PTCs and a) normal thyroid tissue, b) PTC carrying a BRAF mutation and c) PTCs outcomes. The latter was analyzed comparing cases of poor prognosis (recurrence/metastases) vs. those with a recurrence free-time of at least 4 years.

Results: Out of the 670 miRNAs examined, 168 miRNAs were differentially expressed in PTC compared with normal thyroid tissue. In $B R A F$ mutated-PTC, there were 47 miRNAs significantly deregulated compared with non mutated-PTC. More significantly, only 8 miRNAs were differentially expressed in cases with a recurrence-free time of at least 4 years compared to cases with a poor prognosis (adjusted $\mathrm{P}$-value $<0.05$, fold change $\leq 0.5-\geq 1.5$ ).

Conclusion: These findings suggest that miRNAs expression profiles could provide a useful tool to discriminate not only PTC from normal thyroid tissue, but also cases with poor prognosis.

Funding

This work was supported by Instituto Carlos III (FIS PI11/1354).

\section{P309 \\ FAMILIAL NON-MEDULLARY THYROID CANCER IS NOT MORE AGGRESSIVE THAN SPORADIC THYROID CANCER: A MATCHED-CASE CONTROL STUDY}

Yon Seon Kim

Ulsan University Hospital

Background: It is controversial whether the clinical features of FNMTC are more aggressive and have a worse prognosis than sporadic non-medullary thyroid cancer. The goals of this study were to determine the prevalence and to evaluate the clinicopathologic features of FNMTC at a single institution.

Methods: From March 2007 to March 2014, a retrospective review of 1939 patients with differentiated thyroid cancer that underwent primary thyroidectomy. Two hundred seventeen FNMTC cases were identified among them and 217 FNMTC patients were matched to 434 control subjects based on sex and age. The clinicopathologic results were reviewed between familial and sporadic thyroid cancer.

Results: The 217 patients (11.2\%) representing 163 families have family history of thyroid cancer. In the FNMTC, the patient relationship of 2 relatives is $81.6 \%, 3$ or more relatives are $18.4 \%$, and a sibling relationship is $58.1 \%$, a parent-child relationship is $41.9 \%$. There was no significant difference in sex, mass size, extrathyroid extension, lymph node metastasis in the FNMTC and sporadic thyroid carcinoma, respectively. The FNMTC patients were more likely to have benign nodules than the patients with sporadic disease (44.2\% vs. $35.7 \%, p=0.035)$. The more number of affected patients have more tumor multiplicity $(p=0.015)$. Two male patients with FNMTC recurred of thyroid cancer and no patients with FNMTC died of disease during follow-up. Among the parent-child FNMTC cases, the second generation patients had a small tumor size than the first generation patients.

Conclusion: The FNMTC is not more aggressive than sporadic nonmedullary thyroid cancer. However, the treatment of modality should be total thyroidectomy, because the more number of affected patients have more tumor multiplicity. 


\section{P310 \\ BRAFV600E MUTATION AND THE GENE SIGNATURE OF PAPILLARY THYROID CARCINOMA- TRANSGENIC MOUSE MODEL BASED STUDY}

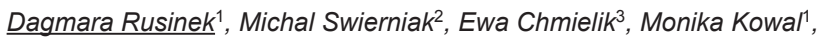
Malgorzata Kowalska ${ }^{1}$, Agnieszka Czarniecka ${ }^{4}$, Cezary Przeorek ${ }^{5}$, Elżbieta Gubała ${ }^{1}$, Michal Jarzab ${ }^{6}$, Wieslawa Widlak ${ }^{7}$, Barbara Jarzab ${ }^{1}$ ${ }^{1}$ Msc Memorial Cancer Center and Institute of Oncology, Gliwice Branch, Poland; Department of Nuclear Medicine and Endocrine Oncology, ${ }^{2} \mathrm{Msc}$ Memorial Cancer Center and Institute of Oncology, Gliwice Branch, Poland; Department of Nuclear Medicine and Endocrine Oncology; Genomic Medicine; Department of General, Transplant and Liver Surgery; Medical University of Warsaw, Poland, ${ }^{3}$ Msc Memorial Cancer Center and Institute of Oncology, Gliwice Branch, Poland; Department of Tumor Pathology;, ${ }^{4}$ Msc Memorial Cancer Center and Institute of Oncology, Gliwice Branch, Poland; Department of Oncological and Reconstructive Surgery, ${ }^{5} \mathrm{Msc}$ Memorial Cancer Center and Institute of Oncology, Gliwice Branch, Poland; Pet Diagnostics Department, ${ }^{6} \mathrm{Msc}$ Memorial Cancer Center and Institute of Oncology, Gliwice Branch, Poland; III Radiotherapy Clinic, ${ }^{7} \mathrm{Msc}$ Memorial Cancer Center and Institute of Oncology, Gliwice Branch, Poland; II Radiotherapy Clinic and III Radiotherapy Clinic

Objectives: Papillary thyroid carcinoma (PTC) harboring the BRAFV600E mutation is described as more aggressive one with higher incidence of lymph node metastasis, extrathyroidal invasion and diagnosed in more advanced disease stage. Although there were studies pointing on the significant influence of BRAFV600E on the PTC gene signature, the human material disables distinction of molecular causes of cancer from its effects. In the reported study we used data obtained from the analysis of transgenic mouse model of BRAF-induced PTC in order to find genes deregulated in a BRAFV600E-dependent manner in human PTC.

Material and Methods: Mice material consisted on 10 PTCs, 10 borderline lesions (BL), 8 borderline hyperplastic lesions (BHL; $4 \mathrm{BRAF}(+)$ and $4 \mathrm{BRAF}(-))$ and 10 asymptomatic thyroids (AT; $5 \mathrm{BRAF}(+)$ and 5 BRAF()). The human material included Polish cohort of patients (31 PTCs, 18 AT) and data made available by Giordano et al. (41 PTCs). The microarray platform used was Affymetrix. In the bioinformatical analysis the BRAFV600Ederegulated genes obtained from the mice material were verified on the human PTCs with distinct molecular events and than validated with the QPCR. Earlier data were already presented on ETA meeting, this abstract presents results of further analysis.

Results and conclusions: From 862 genes selected on the mice dataset as $B R A F \mathrm{~V} 600 \mathrm{E}-$ specific 532 were identified on the human HG-U133A microarray. When hierarchical clustering was performed the BRAF(+)PTCs and RET(+)PTCs gene signatures were more similar to each other than to RAS $(+)$ PTCs with the later being closer in their gene expression profile to WT-PTCs and AT. Altogether 18 genes were deregulated in BRAF(+)PTCs in comparison to each of the analyzed group and 57 next genes were differentially expressed between $\mathrm{BRAF}(+) \mathrm{PTCs}$ and other groups except of RET $(+)$ ones. The function of selected genes is analyzed and will be discussed.

Polish National Science Center project NN401612440

\section{P311 \\ RELEVANCE OF THE ONCOGENE DJ-1 IN FOLLICULAR THYROID CARCINOGENESIS}

Julia Badziong ${ }^{1}$, Denise Zwanziger ${ }^{1}$, Kerstin Krause ${ }^{2}$, Saskia Ting ${ }^{3}$, Kurt Werner Schmid ${ }^{3}$, Dagmar Führer ${ }^{1}$

${ }^{1}$ University Duisburg-Essen, Department of Endocrinology and Metabolism, ${ }^{2}$ University Hospital Leipzig, Department of Endocrinology and Nephrology, ${ }^{3}$ University Hospital Essen, Department of Pathology and Neuropathology

Introduction: Follicular thyroid carcinoma (FTC) is the second most common thyroid malignancy. However, FTCs show no specific genotype. We have previously reported that the $21-\mathrm{kDa}$ signaling protein DJ-1 is upregulated in FTC. We hypothesize that DJ-1 overexpression could activate signal transduction pathways like PI3K/Akt and thereby contribute to neoplastic transformation of FTC. Therefore, we investigated how DJ-1 is regulated in the thyroid and if DJ-1 inactivation leads to re-expression of phosphatase and tensin homolog (PTEN) with functional consequences in FTC.

Methods: Follicular thyroid carcinoma cells (FTC133) were transfected either with siRNA specific for DJ-1 or with siRNA control as well as DJ-1 cDNA or an empty vector control. Western blot analysis was used to confirm successful transfection and to determine DJ-1 related alteration in protein expression of e.g. PTEN or pAkt. Migration of transfected cells was analyzed by wound healing assays and effects on proliferation and apoptosis were studied. Furthermore, growth curves were performed. In addition, the localization of DJ-1 was studied by immunofluorescence.

Results: An upregulation of PTEN was observed while DJ-1 expression was downregulated by siRNA transfection. Migration experiments showed that siRNA treated cells migrate significantly slower than negative control. We also found that DJ-1 is located in the cytoplasm in FTC133 cells like previously shown in FTC tissue.

In conclusion: Differences between DJ-1 knockdown cells and normal expressing DJ-1 cells were found on protein levels by western blot analysis and by performing functional assays. Further investigations of DJ-1 in follicular thyroid carcinogenesis are in process.

\section{P312 \\ THE DETECTION OF GENETIC CHANGES IN POORLY DIFFERENTIATED THYROID CARCINOMA AND ANAPLASTIC THYROID CARCINOMAS BY NEXT-GENERATION SEQUENCING \\ Vlasta Sykorova', Sarka Dvorakova', Eliska Vaclavikova ${ }^{1}$, Josef Vcelak', Tereza Halkova', Roman Kodet'2, Daniela Kodetová2, Jan Betka ${ }^{3}$, Petr VIcek' ${ }^{4}$, Bela Bendlova ${ }^{1}$ \\ ${ }^{1}$ Institute of Endocrinology, Dept. of Molecular Endocrinology, ${ }^{2} 2$ nd Faculty of Medicine and Faculty Hospital Motol, Department of Pathology and Molecular Medicine, ${ }^{3} 1$ st Faculty of Medicine and Faculty Hospital Motol, Charles University, Department of Otorhinolaryngology and Head and Neck Surgery, ${ }^{4}$ 2nd Faculty of Medicine, Charles University, Department of Nuclear Medicine and Endocrinology}

Objectives: Poorly differentiated thyroid carcinoma (PDTC) and anaplastic thyroid carcinoma (ATC) are rare tumors with aggressive behavior. The genetic background is still unclear. The molecular pathogenesis includes some of the main genetic changes of differentiated thyroid carcinomas as mutations in $B R A F$ and $R A S$ genes as well as changes in genes like CTNNB1, PIK3CA, TP53, AXIN1, PTEN or APC leading to the dedifferentiation of the tumors.

Methods: DNAs from fresh frozen thyroid tissues of 3 PDTC and 5 ATC were extracted. The approach of next-generation sequencing (NGS) panel targeted to 94 genes involved in cancer was used. The samples were prepared using TruSight Cancer panel (Illumina) and sequenced by MiSeq sequencer (Illumina). The detected variants were analyzed by Illumina VariantStudio software. Uncommon missense variants inside coding conserved sequence with altered allele depth at least 50 reads were selected. Variants called both tolerated and benign in Sift and PolyPhen software were excluded.

Results: Several variants in PDTC as well as in ATC were detected by NGS. Besides of the genes mentioned before (HRAS, TP53) other genetic changes especially in genes included into DNA damage response and DNA repair (ATM, FANCD2, CHEK2, ERCC5, NBN, MUTYH FANCM), in genes encoding transcription factors $(H O X B 13, H N F 1 A)$, transmembrane receptors for growth factors (MET,EGFR), cell-to-cell adhesion proteins (CDH1) and inhibitors of signaling pathways (NF1, TSC2) were found.

Conclusions: Next-generation sequencing (NGS) is a useful tool for searching of new variants and genes involved in PDTC and ATC. It seems that each this rare tumor has its own specific genetic background. Additional analysis of detected genetic changes is needed.

Supported by IGA MH CR NT/13901-4 and MH CR RVO 00023761. 


\section{P313}

\section{ABI3, A NOVEL PUTATIVE TUMOR SUPPRESSOR GENE, IS SILENCED THROUGH PROMOTER DNA HYPERMETHYLATION IN FOLLICULAR THYROID CARCINOMAS}

Lais Moraes ${ }^{1}$, Ana Luiza Galrão ${ }^{1}$, Ilena Rubio ${ }^{2}$, Janete Maria Cerutti ${ }^{1}$ Genetic Bases of Thyroid Tumors Laboratory, Division of Genetics, Department of Morphology and Genetics, Federal University of São Paulo, ${ }^{2}$ Department of Biological Science, Federal University of São Paulo

We recently reported that the expression of $A B I 3$ is diminished in thyroid carcinomas, compare to benign lesions. Ectopic expression of $A B I 3$ in a follicular cancer cell line (WRO) led to a significant decrease in invasiveness, migration, cell growth and viability in vitro and tumor growth in vivo, while increased senescence. To further define the mechanism responsible for loss of $A B I 3$ expression in carcinomas, we investigated if methylation of $\mathrm{CpG}$ islands contained within promoter region of $A B I 3$ could contribute for loss of $A B I 3$ expression in follicular carcinomas and thyroid carcinoma cell lines. We initially evaluated the methylation status of the $A B I 3$ promoter and level of $A B I 3$ expression in cell lines following treatment with the demethylating agent, 5-azacytidine(5-AZA). Using quantitative RT-PCR, we observed an increased expression of $A B I 3$ after 5-AZA treatment $(\mathrm{P}<0.001)$. Although $\mathrm{CpG}$ islands were not identified in the $A B I 3$ promoter, the analysis revealed three individual regions with $\mathrm{CpGs}$ sites. Although after bisulfite-sequencing PCR, we observed that all regions were methylated. However, in a specific region (R1) the methylation degree was significantly correlated with $A B I 3$ expression $(\mathrm{P}<0.05)$. We next investigated whether expression of $A B I 3$ correlated to the methylation degree of R1 in follicular thyroid adenoma $(n=6)$ and carcinoma $(n=6)$. Hypermethylation in R1 was associated with reduced expression of $A B I 3$ in follicular thyroid carcinoma, when compare to adenomas $(\mathrm{P}<0.05)$. These findings not only substantiate $A B I 3$ as a tumor suppressor gene but also pointed out that hypermethylation of its promoter is the mechanism associated with the loss of $A B I 3$ expression in follicular thyroid carcinomas. These data contribute to the understanding of the molecular basis involved in thyroid tumorigenesis, and the potential of ABI3 as a new target for epigenetic therapeutic strategies.

\section{P314}

\section{THE MOLECULAR LINK BETWEEN BRAF MUTATION AND SODIUM IODIDE SYMPORTER (NIS) UNDEREXPRESSION}

Catarina Tavares ${ }^{1}$, Catarina Salgado ${ }^{2}$, Rui Batista ${ }^{2}$, Helena Populo²,

Paula Soares

${ }^{1}$ Institut of Pathology and Molecular Immunology of the University of Porto; Medical Faculty of the University of Porto, ${ }^{2}$ Institut of Pathology and Molecular Immunology of the University of Porto

Introduction: NIS is a glycoprotein present in thyroid follicular cells' membrane, responsible for iodide uptake from blood stream. Thyroid cancer diagnosis and therapy is based on surgery followed by radioiodine $\left({ }^{131} \mathrm{I}\right)$ ablation of tumor remnants and distant metastases. Some thyroid tumors lose NIS expression and consequently, the ability to incorporate iodide, becoming resistant to therapy. So far, treatments have failed in restore NIS expression and complete functionality.BRAF ${ }^{\mathrm{V} 600 \mathrm{E}}$ is the most frequent mutation in papillary thyroid carcinoma (PTC), its prognostic value is still controversial, but its relation with NIS under-expression is well established, yet not explained ${ }^{1}$.

Objectives: Our objective is to explore the molecular mechanisms underlying BRAFV600E induced NIS under-expression.

Methodology: K1 cell line (derived from a primary papillary carcinoma), harboring BRAF ${ }^{\mathrm{V} 600 \mathrm{E}}$ mutation and with no NIS expression, was used in this study. We performed BRAF silencing by siRNA, and evaluated, by westernblot or qRT-PCR, the levels of BRAF, pERK and NIS.

Results: BRAF was efficiently silenced by siRNA at all time points (24, 36,48 and 72 hours). NIS mRNA and protein levels were significantly higher in cells with BRAF silencing. Although BRAF remained silenced at all time points, pERKs did not followed the same pattern and at time point $48 \mathrm{H}$, they increased in silenced cells compared to scramble.

Conclusions: NIS expression seems to be related with BRAFstatus, but not necessarily with MAPK down regulation. Future studies are required to understand which mechanisms are involved, in order to develop a pharmacological strategy to improve NIS expression and subsequently a better patients' outcome.

\section{Reference}

1 Riesco-Eizaguirre G, Gutiérrez-Martínez P, García-Cabezas MA, Nistal M, Santisteban P: "The oncogene BRAF V600E is associated with a high risk of recurrence and less differentiated papillary thyroid carcinoma due to the impairment of $\mathrm{Na}+/ \mathrm{I}-$ targeting to the membrane". Endocr Relat Cancer 2006;13:257-269.

\section{P315 \\ ROLE OF OSTEOPONTIN ISOFORMS IN THYROID CANCER CELLS}

Luciana Ferreira ${ }^{1}$, Catarina Tavares ${ }^{1}$, Ana Pestana ${ }^{1}$ Ricardo Celestino ${ }^{1}$, Patricia Castro ${ }^{1}$, Rui Batista ${ }^{1}$, Hugo Prazeres ${ }^{1}$, Etel Gimba ${ }^{2}$, Paula Soares ${ }^{3}$

${ }^{1}$ Institute of Molecular Pathology and Immunology of the University of Porto (Ipatimup), Cancer Biology Group, Porto, Portugal, ${ }^{2}$ National Cancer Institute/Molecular Carcinogenesis Program and Stricto Sensu Graduate Program in Oncology, Rio de Janeiro, Brazil; Fluminense Federal University, Rio de Janeiro, Brazil, ${ }^{3}$ Institute of Molecular Pathology and Immunology of the University of Porto (Ipatimup), Cancer Biology Group, Porto, Portugal; Institute of Molecular Pathology and Immunology of the University of Porto (Ipatimup), Cancer Biology Group, Porto, Portugal

Thyroid cancer (TC) is the most common malignancy of the endocrine system. Osteopontin (OPN) is one of the gene products aberrantly expressed in TC tumors, but the contribution of each of the OPN three splicing isoform (OPNsi), named as OPNa, OPNb and OPNc, is currently unknown. The present study aims to investigate the expression profile of OPNsi in TC tissue samples and correlate its expression with molecular and clinic-pathologic features. In order to address the putative roles of OPNsi, we overexpressed OPN isoforms in a TC derived cell line. The expression profile of total OPN and OPNsi in TC cell lines and in thyroid tissue samples was evaluated by q-RT-PCR. c643 cells were transfected with vectors containing OPNsi (A, B, C) as well as empty vector, and stable overexpressing clones were selected with geneticin treatment. Cell proliferation was evaluated by BrDU assay and migration by wound-healing assay. We found that OPNa and OPNb isoform transcripts are expressed in higher levels in papillary thyroid carcinoma (PTC) samples than non-tumoral, adenomas and follicular thyroid carcinoma tissues. Conversely, OPNc isoform transcript levels are similar among these samples. In PTC tissues, OPNa and OPNb expression levels were higher in TC tumors harboring $\mathrm{BRAF}^{\mathrm{V} 600 \mathrm{E}}$ mutation than in wild-type BRAF tumors samples $(\mathrm{p}=$ 0.013 and $p=0.034$, respectively). Tumor size was significantly correlated with OPNa expression levels $(\mathrm{p}=0.031)$. In nine distinct tested TC cell lines, we observed differential expression of the three OPNsi, of which c643 cell line expressed the lower levels of OPNsi. Higher proliferation and migration rates have been observed for c643 cells overexpressing OPNa, when compared to empty vector control clone. Taken together, our data indicates that OPNa and $\mathrm{OPNb}$ are overexpressed in PTC samples, among which OPNa variant promotes cell growth and migration advantages to TC c643 cells. 


\section{P031 Thyroid Hormone and Gene Expression}

\section{P316 \\ THYROID HORMONE ACTION IN CEREBROCORTICAL CELLS INVOLVES THE TRANSITION FROM FETAL TO ADULT PATTERN OF GENE EXPRESSION}

Pilar Gil', Francisco García-García ${ }^{2}$, Joaquín Dopazo ${ }^{3}$, Juan Bernal4, Beatriz Morte ${ }^{5}$

${ }^{1}$ Intituto de Investigaciones Biomédicas Uam-Csic, Center for Biomedical Research on Rare Disease Ciberer, ${ }^{2}$ Centro de Investigación Principe Felipe Cipf; Functional Genomics Node, ${ }^{3}$ Centro de Investigación Principe Felipe Cipf; Center for Biomedical Research on Rare Disease Ciberer, ${ }^{4}$ Instituto de Investigaciones Biomédicas Csic; Center for Biomedical Research on Rare Diseases Ciberer; Center for Biomedical Research on Rare Diseases, ${ }^{5}$ Instituto de Investigaciones Biomedicas Csic-Uam; Center for Biomedical Research on Rare Diseases

Thyroid hormone (T3) is important during development of the mammalian brain acting through regulation of gene expression. T3 is involved in neuronal and glial cell differentiation and migration, axonal myelination, and synaptogenesis. To get further insight on the genes and pathways regulated by T3 in the developing brain at the cellular level, we used mouse cerebrocortical cells in primary culture. This culture maintains, to some extent, the original phenotypic cell diversity and therefore, reflects the action of $\mathrm{T} 3$ on the cortex in vivo. For example, $10 \%$ of the neurons are calbindin positive and $\mathrm{T} 3$ reduced calbindin expression without changing neuronal number. To identify thyroid hormone dependent genes we performed RNA-seq assays (Illumina). We have identified 619 genes that are upregulated by T3 as well as 526 genes that are downregulated by $\mathrm{T} 3(\mathrm{FRD}<0.05)$. The technical and biological procedures were validated measuring the expression of 32 differentially express genes by RT-PCR. The Gene Ontology analysis revealed T3 upregulated genes involved in signal transduction such as synaptic transmission and ion transport and T3 downregulated genes involved in cellular proliferation. The upregulated genes contain a high proportion of genes enriched in the adult cortex. Conversely, the downregulated genes contain a high proportion of genes enriched in the embryonic brain.

In this study we have identified many genes regulated at the cellular level by T3 in cerebrocortical primary cells. The results underscore the importance of T3 in the regulation of gene expression in stage-specific processes for a proper development and function of the brain.

\section{P317 \\ MOLECULAR MECHANISMS OF SEX- DEPENDENT THYROID HORMONE ACTION ON TARGET TISSUES}

Helena Rakov ${ }^{1}$, Kathrin Engels ${ }^{1}$, Denise Zwanziger ${ }^{1}$, Maren Rehders ${ }^{2}$, Klaudia Brix ${ }^{2}$, Sebastian Hoenes ${ }^{1}$, Lars Christian Moeller ${ }^{1}$, Dagmar Fuehrer ${ }^{1}$

${ }^{1}$ University Duisburg-Essen, Department of Endocrinology and Metabolism, Essen, Germany, ${ }^{2}$ Jacobs University Bremen, School of Engineering and Science, Bremen, Germany

Introduction: Thyroid dysfunction leads to diverse and wide spread symptoms in both sexes. However, a higher prevalence of thyroid diseases has long been known for females and from a clinical point of view presentation of thyroid dysfunction may differ between men and women. The causes for this sex disparity are still unknown. We aimed to investigate molecular mechanisms of sex-dependent thyroid hormone action on target tissues a study in mice.

Methods: Hypo- or hyperthyroidism was induced in female and male C57BL/6N mice at age of 5 months. Hyperthyroidism was induced by i.p. injections of $1 \mu \mathrm{g} / \mathrm{g}$ per body weight L-thyroxine (L-T4) every 48 hours while hypothyroidism was induced by low iodine diet and water supplemented with

Poster Presentations
$0.02 \%$ methimazole (MMI) and $0.5 \%$ perchlorate $(\mathrm{n}=16$ animals $[8$ females and 8 males] each for hyperthyroidism, hypothyroidism and euthyroidism).

Results: Male and female mice showed weight gain, increased food consumption, water intake and rise in body temperature during hyperthyroidism. Hypothyroid males, but not females, consumed more water than the control group, but less than hyperthyroid mice. Male and female mice showed significantly increased and decreased heart rate in hyper- and hypothyroid states, respectively. Thyroid hormone levels showed an approximately 5-fold increase of TT4 in hyperthyroid male mice and up to 7-fold in female mice after 3 weeks of treatment. In both sexes the hypothyroid state was defined by TT4 serum levels below the limit of detection.

In conclusion: Similar symptoms of thyroid dysfunction were observed for both sexes (body weight change, food intake and heart rate), but differences were noted for water intake and TT4 levels. Causes for the observed sex disparity and tissue specific thyroid hormone action are under investigation.

Funded by DFG SPP1629 FU 356/7-1

\section{P318 \\ HORMONAL REGULATION OF FOXE1 EXPRESSION IN THYROID EPITHELIAL CELLS Arístides López Márquez ${ }^{1}$, Pilar Santisteban ${ }^{1}$ \\ ${ }^{1}$ Instituto de Investigaciones Biomédicas (Csic-Uam)}

Differentiation of thyroid follicular cells is regulated by hormones and growth factors. Among these signals, TSH, IGF1 and TGF $\beta$ play a predominant role. These three ligands transcriptionally regulate markers of thyroid differentiation such as Thyroglobulin (Tg) Thyroperoxidase (TPO) and sodium iodide symporter (NIS). This effect takes place, at least in part, through a responsive element to where the FoxE1 binds, opening chromatin and creating an environment that facilitate the binding of other transcription factors. Taking in consideration the proposed role of FoxE1 as a mediator of the hormone/ growth factor transcriptional action, the main objective of this work was to study the regulation of FoxE1 expression by TSH, IGF1 and TGF $\beta$.

Mouse FoxE1 promoter was cloned and transient transfections were performed in PCCL3 in presence or absence of TSH, IGF1 and TGF $\beta$. Forskolin and $\mathrm{H} 89$ were used to study the cAMP/PKA pathway. Co-transfections in Hela cells were performed to elucidate the role of other transcription factors. FoxE1 protein and mRNA levels in different conditions were evaluated by westernblot and RT-qPCR.

The in silico analysis of FoxE1 promoter reveals the presence of a CRE and several Smad binding sites in its sequence. TSH and IGF1 induce the transcription of FoxE1 by the activation of its promoter. The TSH effect is cAMP/ PKA dependent and therefore CREB positively regulates FoxE1 transcription. IGF1 cooperates with TSH while TGF $\beta$ inhibits the TSH induction on FoxE1 gene expression. Not effect was found of TGF $\beta$ on IGF1 action. We conclude that there is a complex network of signals and transcription factors involved in FoxE1 expression that are essential to maintain a fine regulation of this important gene that control thyroid cell differentiation.

\section{P319 \\ EFFECTS OF THYROID HORMONES T3 AND T4 ON MESENCHYMAL STEM CELL DIFFERENTIATION AND MIGRATION}

Kathrin A. Schmohl', Andrea M. Mueller ${ }^{1}$, Nicole Salb ${ }^{2}$,

Alexandra Wechselberger ${ }^{2}$, Kerstin Knoop ${ }^{1}$, Nathalie Schwenk ${ }^{1}$,

Peter J. Nelson ${ }^{2}$, Christine Spitzweg ${ }^{1}$

${ }^{1}$ Department of Internal Medicine II, University Hospital of Munich, Germany, ${ }^{2}$ Clinical Biochemistry Group, Medical Policlinic IV, University Hospital of Munich, Germany

Mesenchymal stem cells (MSCs) are actively recruited into tumour stroma where they differentiate to carcinoma-associated fibroblast (CAF)-like cells. Thyroid hormones $\mathrm{T} 3$ and $\mathrm{T} 4$ play a critical role in the formation of the stroma through non-genomic mechanisms via integrin $\alpha v \beta 3$. To improve our understanding of specific effects of thyroid hormones on tumour stroma formation, we examined the effects of T3, T4 and the integrin-specific inhibitor tetrac on MSC biology in vitro.

Primary human bone marrow-derived MSCs were grown in tumour cellconditioned medium with $1 \mathrm{nM} \mathrm{T} 3$ or T4 with or without $100 \mathrm{nM}$ tetrac for 14 days. Changes in expression levels of genes associated with CAF-like differ- 
entiation were analysed by qPCR and ELISA. Moreover, MSCs were treated with $100 \mathrm{nM}$ T3 for 24 hours and their ability to migrate towards tumour cellconditioned medium was monitored by time-lapse microscopy.

Treatment of MSCs with T3 or T4 resulted in increased mRNA levels for genes associated with CAF-like differentiation: (1) surface marker fibroblast activating protein; (2) indicator of tissue remodelling/invasion tenascin C; (3) markers of neovascularisation $\alpha$-smooth muscle actin, desmin and vascular endothelial growth factor; (4) tumour promoting growth factors interleukin-6, transforming growth factor $\beta 1$, stromal cell-derived factor 1 , basic fibroblast growth factor, epidermal growth factor and hepatocyte growth factor. All the increases in expression levels were found to be tetrac-dependent and therefore integrin $\alpha v \beta 3$-mediated. Data on tumour promoting growth factors were also confirmed by ELISA. Pre-treatment of MSCs with T3 resulted in a 51\% increase in MSC migration towards tumour cell-conditioned medium, compared to untreated cells.

Our data suggest that $\mathrm{T} 3$ and $\mathrm{T} 4$ regulate the expression of MSC genes associated with CAF-like differentiation and enhance tumour signal-directed chemotaxis. These studies will increase our understanding of the mechanisms that regulate MSC differentiation and migration as a critical part of tumour stroma formation.

\section{P320}

COMPARISON OF TWO PROTOCOLS FOR INDUCTION OF HYPERTHYROIDISM IN MICE

Engels Kathrin ${ }^{1}$, Helena Rakov ${ }^{1}$, Maren Rehders ${ }^{2}$, Klaudia Brix²,

Sebastian Hönes ${ }^{1}$, Denise Zwanziger ${ }^{1}$, Lars Möller ${ }^{1}$, Dagmar Führer ${ }^{1}$

${ }^{1}$ University Duisburg-Essen, Department of Endocrinology and

Metabolism, Essen, Germany, ${ }^{2}$ Jacobs University Bremen, School of

Engineering and Science, Bremen, Germany

Introduction: We aimed to evaluate the efficacy of L-T4 exposure via the oral or parenteral route to induce hyperthyroidism in male and female mice. Thyroid hormone $\mathrm{TH}$ ) serum levels and $\mathrm{TH}$ responsive gene expression levels in liver and heart were investigated with a focus on the lasting efficacy of L-T4 administration.

Methods: C57BL/6NTac mice received either $1 \mu \mathrm{g} / \mathrm{g}$ BW L-T4 via i.p. injection or $3.75 \mu \mathrm{g} / \mathrm{ml} \mathrm{L}$-T4 in drinking water over 6 weeks. For dose calculation differences in water consumption, resorption and increased BW during growth were considered. During treatment changes in TH serum levels (FT3/FT4/TT4), BW and drinking pattern of the mice were determined. After 6 weeks organs were collected at 48,72 and $144 \mathrm{~h}$ post L-T4 treatment $(\mathrm{n}=$ 4) and alterations in $\mathrm{TH}$ mediated gene transcription in liver and heart were investigated by RT-PCR.

Results: Highest TH serum levels were found in i.p. injected mice (TT4: i.p. 6-8.4 fold, drinking water 2.5-6.4fold elevated). Mice exposed to L-T4 in the drinking water showed no significant change in gene expression. In liver of i.p. injected mice Spot14 and DIO 1 expression decreased while TBG expression increased with time post treatment. In heart of i.p. treated mice Myh7 expression increased whereas ATP2A2 and Myh6 expression decreased with time post treatment. We detected a hyperthyroid state at $48 \mathrm{~h}$, an almost euthyroid state at $72 \mathrm{~h}$ and a hypothyroid state at $144 \mathrm{~h}$ post treatment.

Conclusions: A severe hyperthyroid state in the mice could only be induced via i.p. injection of L-T4. Since this condition lasted for up to $48 \mathrm{~h}$ post L-T4 treatment, injection and investigation intervals in hyperthyroid mice should not exceed $48 \mathrm{~h}$.

Funded by DFG SPP1629 FU 356/7-1

\section{P321 \\ PROTOCOLS FOR INDUCTION OF HYPOTHYROIDISM IN MICE VALIDATED BY GENE EXPRESSION IN LIVER AND HEART}

Sebastian Hönes ${ }^{1}$, Anita Boelen ${ }^{2}$, Sigrun Horn ${ }^{3}$, Eddy Rijntjes ${ }^{4}$, Josef Köhrle ${ }^{4}$, Dagmar Führer ${ }^{5}$, Heike Heuer ${ }^{6}$, Lars Moeller ${ }^{1}$

${ }^{1}$ University Duisburg-Essen, Department of Endocrinology and

Metabolism, ${ }^{2}$ Academic Medical Centre, ${ }^{3}$ Leibniz Institute for Age

Research/Fritz Lipmann Institute (Fli), ${ }^{4}$ Institut Für Experimentelle

Endokrinologie, Charité - Universitätsmedizin Berlin, Germany,

${ }^{5}$ Department of Endocrinology and Metabolism and Division of

Laboratory Research, University of Duisburg-Essen, Essen, Germany,

${ }^{6}$ Leibniz-Institut Für Umweltmedizinische Forschung; Leibniz Institute

for Age Research/Fritz Lipmann Institute (Fli)

Introduction: Hypothyroidism is often a prerequisite to study thyroid hormone $(\mathrm{TH})$ action in mice. We aimed to evaluate various protocols to induce hypothyroidism by measuring not only serum TSH and TH levels, but also TH dependent gene expression in liver and heart.

Methods: Mice were rendered hypothyroid with either low iodine diet (LoI) $+0.15 \%$ propylthiouracil (PTU), addition of $0.5 \%$ perchlorate and $0.02 \%$ methimazole (MMI) to the drinking water, LoI diet $+0.15 \%$ PTU + perchlorate/MMI/sacharine or MMI/perchlorate treatment alone. TSH, FT4, TT4 and FT3 levels were measured by (radio)immunoassays. Gene expression was measured by real-time PCR after three weeks of treatment and compared to untreated mice.

Results: With all treatments hypothyroidism could be induced as TSH increased more than hundredfold. FT4 was reduced to levels below our limit of detection $(<6 \%$ of control). TT4 levels also decreased to less than $10 \%$ with all treatments compared to untreated mice. In contrast, FT3 was reduced to only $50-25 \%$ and TT3 levels were even less reduced (72-116\% of control mice). With all treatments hepatic expression of DIO 1 was reduced to $1 \%$ compared to untreated mice. Conversely, mRNA expression of TBG, normally repressed by TH in adult mice, was significantly increased in hypothyroidism (MMI: 558\%, LoI+MMI: 1086\%, LoI+PTU+MMI: 783\%, LoI+PTU: 689\%). In the heart, Myh6 mRNA expression was significantly reduced (MMI: $12 \%$, LoI+MMI: $1 \%$, LoI+PTU+MMI: $2 \%$, LoI+PTU: $2 \%$ ).

Conclusion: All protocols work well as demonstrated by markedly elevated serum TSH levels. Furthermore, reduced expression of TH-responsive genes and induction of TBG, negatively regulated by $\mathrm{TH}$, demonstrate tissue hypothyroidism in liver and heart. Interestingly, fT3 was less reduced than fT4 and TT3 levels remained normal with all treatments. This finding may be explained by physiology defending T3 levels as well as increased TBG expression in hypothyroidism.

\section{P322 \\ RELATIVE ROLES OF THYROXINE AND TRI- IODOTHYRONINE IN AVIAN MOLTING}

Atul Kathait ${ }^{1}$, Anjana Faraswan ${ }^{2}$, Asha Chandola-Saklani ${ }^{1}$

${ }^{1}$ Centre for Biosciences and Clinical Research, School of Biosciences,

Apeejay Stya University, ${ }^{2}$ Govt Pg College, Augatamuni, Uttarakhand

There is general consensus among thyroidologists that $\mathrm{T} 4$ is a prohormone that must be converted to its mono-deiodinated form T3, before biological activity is elicited. It seems that in non-mammalian vertebrates T4 is often equally, if not more, active than T3 in eliciting a response.

In this paper we have investigated the relative roles of $\mathrm{T} 4$ and $\mathrm{T} 3$ in the process of molt which involves loss of old feathers and regeneration of new ones.

Method: Spotted munia, a finch, was maintained in laboratory conditions.

Seven groups of 10-12 birds each were established.GroupI, II, III received 0.37.0.74 and $1.48 \mathrm{nM}$ of L-T4 per day (sodium salt, Sigma) respectively, and IV, V, VI received the same equimolar doses of T3 in $0.1 \mathrm{ml} 0.9 \%(\mathrm{w} / \mathrm{v})$ alkaline saline over14 days. Control GroupVII received vehicle. The head and right breast of birds was examined every third day and proportion of feather loss/regeneration assessed. A thin wire ring of known diameter divided into six equal sections was used. The ring was placed along the centre of the feather tract and the areas within the six sections examined for feather loss/ regeneration. 
Result: T3 lead to significant feather loss from head region with almost no effect on regeneration. T4 had no effect on feather loss but significantly stimulated regeneration in head and body.

Conclusion: Results indicate varied sites of action of T4 and T3 in the process of molt. The dose responsive effect of T4 on head and bodyfeather regeneration is not mimicked by $\mathrm{T} 3$ and may involve specific expression of receptors. T3 effect on feather loss may involve a general loosening of the feather.

\section{P032 Case Reports 3}

\section{P323 \\ POORLY DIFFERENTIATED THYROID CARCINOMA WITH PREDOMINANT CLEAR CELL FEATURES: A CASE REPORT \\ Alexander Abrosimov ${ }^{1}$, Aleksei Sidorin ${ }^{2}$, Anna Shinkarkina ${ }^{2}$, \\ Pavel Isaev² \\ ${ }^{1}$ Medical Radiological Research Center; Endocrinological Research \\ Center, ${ }^{2}$ Medical Radiological Research Center}

Background: In accordance with the Turin proposal Poorly Differentiated Thyroid Carcinoma (PDTC) should be diagnosed on the basis (i) of the presence of solid/trabecular/insular growth pattern, (ii) absence of conventional nuclear features of papillary thyroid carcinoma, and (iii) the presence of at least one of the following features: convoluted tumor cell nuclei, mitotic activity greater than 3 mitotic figures per 10 high-power fields, or tumor necrosis (M.Volante et al., 2007).

We present a case of thyroid tumor with histopathological features of PDTC composed of clear cells.

Case Report: A 69-yr-old woman was admitted to the Clinic of Medical Radiological Research Center with complaints of neck mass. An ultrasonographic examination revealed multiple nodules measuring up to $2.0 \mathrm{~cm}$ in both thyroid lobes and neck lymph nodes (LN) up to $3.0 \mathrm{~cm}$ in diameter. Fine needle aspiration biopsy diagnosed thyroid carcinoma. Total thyroidectomy with neck LN dissection was performed. The postoperative course was uneventful. Surgery is followed by the radioiodine ablation procedure ( 80 $\mathrm{mCi}$ ). The patient remains alive and well without evidence of tumor recurrence 7 months after treatment. Microscopically, focuses of widely invasive tumor solid in architecture resembled metastatic clear cell carcinoma. Mitotic figures and coagulative necrosis were detected in all multiple thyroid and LN metastatic tumors. Immunohistochemically, the tumor tissue was positive for thyroglobulin, TTF-1, vimentin, CK7, CK19, and negative for CK20, CK34ßE12, calcitonin, chromogranin A, CD10, RCC, and parathyroid hormone. Immunoexpression of $\mathrm{Ki}-67$ was detected in $50 \%$ cells. Molecular study revealed no BRAF (V600E), HRAS (codon 12 or 61), and NRAS (codon 12) genes alterations. No RET/PTC1, and RET/PTC3 rearrangements were detected.

Conclusion: A rare case of PDTC with predominant clear cell features should be differentially diagnosed from metastatic carcinoma of another localization (renal, parathyroid etc.). Molecular genetic alterations in clear cell PDTC should be aslo determined.

Table 1. (for Abstract P323)

\begin{tabular}{|c|c|c|c|c|c|}
\hline \multicolumn{2}{|c|}{ Treatment } & \multicolumn{2}{|c|}{ Head feathers (Mean \pm SE) } & \multicolumn{2}{|c|}{ Body feathers $($ Mean \pm SE) } \\
\hline & & Loss & Regeneration & Loss & Regeneration \\
\hline \multirow[t]{3}{*}{$\mathrm{T} 3$} & $0.37 \mathrm{nM}$ & $2.5 \pm 0.17$ & $0.6 \pm 0.22$ & 0.00 & 0.00 \\
\hline & $0.74 \mathrm{nM}$ & $2.8 \pm 0.13$ & 0.00 & 0.00 & 0.00 \\
\hline & $1.48 \mathrm{nM}$ & $3.8 \pm 0.13$ & 0.00 & 0.00 & 0.00 \\
\hline \multirow[t]{3}{*}{$\mathrm{T} 4$} & $0.37 \mathrm{nM}$ & 0.00 & $4.00 \pm 0.10$ & 0.00 & $4.40 \pm 0.22$ \\
\hline & $0.74 \mathrm{nM}$ & 0.00 & $4.10 \pm 0.10$ & 0.00 & $5.00 \pm 0.10$ \\
\hline & $1.48 \mathrm{nM}$ & 0.00 & $4.00 \pm 0.10$ & 0.00 & $5.00 \pm 0.10$ \\
\hline
\end{tabular}

Poster Presentations

\section{P324}

SERIOUS ALLERGIC REACTION ASSOCIATED WITH FAVISTAN (THIAMAZOLE) A CASE REPORT

Marjeta Kermaj ${ }^{1}$, Anisa Zeqja ${ }^{1}$, Ermira Muco ${ }^{2}$, Violeta Hoxha ${ }^{1}$, Gerond Husi ${ }^{1}$, Klotilda Resuli ${ }^{3}$, Ersida Xhaferi ${ }^{1}$, Enalda Demaj ${ }^{1}$, Agron $\mathrm{YIII}^{1}$

${ }^{1}$ University Hospital Center "Mother Tereza"; Department of Endocrinology, ${ }^{2}$ University Hospital Center "Mother Tereza";

Department of Infectious Diseases, ${ }^{3}$ Hospital Center Vlora

Introduction: Minor allergic reactions such as a limited minor rush, may occur in up to $5 \%$ of patients taking either propylthiouracil or methimazole Some people may have a skin rash and difficulty breathing.In the case of a serious allergic reaction, prescribing the alternative drug is not recommendet. We report a case with severe allergic reaction associated with Favistan (thiamazole) and review the literature.

Case Report: A 27 year- old female was admitted in emergency witht hives over whole body, rash, difficulty breathing, palpitations. She was diagnosed with hyperthyroidism (Graves' diseases) two months ago and received treatment with unimazole $30 \mathrm{mg}$ per/ day. Her doctor changed unimazole to favistan another thionamid drug .As she took the first dose of favistan just after 30 minutes, she developed severe allergic reaction with elements described before. She passed the situation after supportive measures but the same serious reaction developed in the evening after she took the second pill of thiamazole. Favistan was suspected to cause allergic reaction since there was no other drug given at that time that could cause this situation. The drug was stopped. The laboratory tests revealed free $\mathrm{T} 4=60(7-18)$, free $\mathrm{T} 3=$ 22.1 (2-4.25), TSH=0.01 (0.17-4), TSH receptor antibody=20.1 $(<1)$. We decided to normalize the thyroid function with propiltiouracil secondary to desensitization protocol and under protection of an antihistamine drug per/os Fenofexodine. In progress there was no an advers effect of drug. When euthyroid, She subsequently underwent a total thyroidectomy. Now she continous to be euthyroid on levothyroxine treatment.

In conclusion Although Thiamasole serious allergic reaction rarely happens, it could be life threatening. If antithyroid hormone synthesis must be used, an alternative drug under the protection of an antihistamine and a desensitization protocol could be a solution.

\section{P325}

GRAVES' DISEASE WITH OPHTHALMOPATHY OCCURRING ELEVEN YEARS AFTER SUBACUTE THYROIDITIS. A CASE REPORT

Bengt Hallengren ${ }^{1}$, Tereza Planck ${ }^{1}$, Peter Åsman² ${ }^{2}$ Mikael Lantz ${ }^{1}$

${ }^{1}$ Department of Endocrinology, Skåne University Hospital, Malmö, Sweden, ${ }^{2}$ Department of Ophthalmology, Skåne University Hospital, Malmö, Sweden

Introduction: The development of Graves' disease after subacute thyroiditis (SAT) is very rare indicating that a certain susceptibility to the disease probably exists in these instances.

Case Report: A 43-years old non-smoking female, whose grandmother had thyrotoxicosis, developed SAT in March, 1998. The diagnosis was verified by fine-needle aspiration with cytological examination. She was given glucocorticoids and needed these for one year. In September 1998 serum TSH level was elevated to $41 \mathrm{mIU} / 1$ (reference range 0.4-4.0), decreased without thyroxine treatment but biochemical subclinical hypothyroidism remained during 1999. TPO-antibodies were negative. In May 1999 serum TSH receptor antibodies were detected, 35 arbitrary units (arbU; reference range $<10$ ). The antibodies decreased during the autumn and in December 1999 measured 11 arbU. This month the patient was admitted to the Department of Endocrinology. She had a biochemical subclinical hypothyroidism and due to hypothyroid symptoms thyroxine substitution was instituted. In March 2000 she was feeling well, TSH was $0.89 \mathrm{mIU} / 1$ and TSH receptor antibodies had normalized (7 arbU). During the years 2004-2008 she felt healthy on thyroxine substitution. TSH receptor antibody (TRAK) levels were measured with a more sensitive method than before and were elevated, 2-2.6 IU/1 (reference range $<1$ ). January 2010 she appeared with a history of hyperthyroid symptoms since one month. On physical examination she was sweating, had tremor and signs of endocrine ophthalmopathy. TRAK was $28 \mathrm{IU} / 1, \mathrm{TSH}<0.02 \mathrm{mIU} / 1$, 
Graves' disease with ophthalmopathy was diagnosed and methimazole was instituted.

Conclusion: The case illustrates the appearance of TSH-receptor antibodies in a female one year after SAT and the development of Graves' disease with ophthalmopathy eleven years after SAT. The occurrence of SAT and Graves' disease may be coincidental but SAT may have induced the appearance of TSH-receptor antibodies and later Graves' disease in a susceptible patient.

\section{P326}

\section{THYREOTOXICOSIS FOLLOWING CHANGED EPILEPSY MEDICATION}

\section{Esa Sopp}

Eira Hospital, Helsinki, Finland

Introduction: It is well known that some drugs affect the absorption of thyroid medication. Increased clearance of levothyroxine by the liver is a much rarer phenomenon, associated mainly with phenytoin and carbamazepine. It is not known if this takes place in all patients. This case report describes the findings of how a change in epilepsy medication resulted in clinical thyrotoxicosis

Case Report: Three years ago a female aged 46 yrs was diagnosed with central hypothyreosis. Levothyroxine medication was stabilized to a dose of $0.2 \mathrm{mg} / \mathrm{d}$. About a year ago she was diagnosed with epilepsy and oxycarbazepine $1050 \mathrm{mg} / \mathrm{d}$ was instituted. While on this medication, her levothyroxine dose was increased to $0.4 \mathrm{mg} / \mathrm{d}$ to maintain euthyreosis. Four months before the patient visited my office, her epilepsy medication had been changed from oxcarbazepine to levetiracetam $2000 \mathrm{mg} / \mathrm{d}$. Also, the dose of tramadol, which she used to treat chronic pain following spinal surgery, was reduced from 400 $\mathrm{mg} / \mathrm{d}$ to $200 \mathrm{mg} / \mathrm{d}$. She also took etoricoxib $90 \mathrm{mg} / \mathrm{d}$ and pregabalin $450 \mathrm{mg} / \mathrm{d}$ at stable doses.

The patient visited my office since she had lost $8 \mathrm{~kg}$ weight in the last four months, had poor endurance, muscle weakness and hair loss. The clinical status was uneventful except for mild acral tremor. Her blood pressure was $138 / 88 \mathrm{mmHg}$ and pulse rate was $71 / \mathrm{min}$. Her free thyroxine level was 45 pmol/1, confirming the suspected hyperthyreosis. Levothyroxine was stopped for 7 days and was thereafter continued at a dose of $0.15 \mathrm{mg} / \mathrm{d}$. Since then, the follow-up of the patient has been uneventful.

The change of the medication from oxycarbazepine to levetiracetam caused overt thyreotoxicosis, since the enhanced hepatic clearance of levothyroxine induced by oxcarbazepine was reverted when levetiracetam was introduced. This led to an increased exposure of the patient to levothyroxine.

\section{P327}

\section{PAPILLARY MICROCARCINOMA IN GRAVE'S DISEASE - ABOUT A CLINICAL CASE}

\section{Ana Wessling ${ }^{1}$, José Maria Aragüés ${ }^{1}$, Mário Mascarenhas ${ }^{1}$}

${ }^{1}$ Hospital de Santa Maria

Introduction: The prevalence of thyroid nodules in Graves' disease (GD) is estimated between 15 and 33\%; several studies support a higher risk of thyroid cancer in this condition. Although the majority (88\%) coexisting carcinomas are microcarcinomas, according to some authors, thyroid ultrasound scan should be considered in the initial evaluation of GD, as nodules are not always detectable at clinical or radionuclide evaluation. The presence of nodules may justify further evaluation with important clinical and therapeutic consequences.

Case Report: A 61-year-old Caucasian male was referred to our clinic with a relapse of GD, treated medically 7 years before. He had no oftalmopathy; his thyroid was globally enlarged without palpable nodules. Blood tests showed suppressed TSH, elevated free T3 and T4, TRABs level of $23.5 \mathrm{U} / \mathrm{L}$ $(<1.5)$. Thyroid ultrasound showed a $10 \mathrm{~mm}$ heterogeneous single nodule in the right lobe, with reduced radionuclide uptake in the scintigraphy. Ultrasound guided fine needle aspiration was performed, with cytology suggestive of papillary carcinoma. After becoming euthyroid with methimazole therapy, total thyroidectomy was performed, without complications. Histology confirmed a papillary microcarcinoma, follicular variant, with tumor capsule invasion alone. The patient started levothyroxine therapy and has remained asymptomatic.

Conclusions: Most cases of thyroid carcinoma in patients with GD are diagnosed incidentally after thyroidectomy is chosen as definitive treat- ment, with prevalence comparable with patients with nontoxic or toxic goiter. Incidental thyroid carcinomas in patients with Graves' disease are mostly papillary microcarcinoma, usually considered of no clinical consequence. In this case the diagnosis and clinical evaluation of a solitary nodule, led to the decision to choose total thyroidectomy as the definitive treatment for GD relapse. Histology confirmed the nodule was a papillary microcarcinoma. The management of nodules and their malignancy risk in a patient with GD still remains some controversial.

\section{P328 \\ CERVICAL PARAGANGLIOMA MIMICKING THYROID NODULE: A RARE CLINICAL CASE}

Berna Imge Aydoğan ${ }^{1}$, Sevim Güllü

${ }^{1}$ Ankara University School of Medicine, Department of Endocrinology and Metabolism Diseases

Background: Paraganglioma is a rare neuroendocrine tumor. When it is located in the neck, it is commonly misdiagnosed as other thyroid neoplasm.

Case Report: We report a case of cervical paraganglioma in a 55 year-old female. Patient was admitted to our clinic with multinodular goiter and neck pain. Thyroid ultrasonography revealed three nodules smaller than $10 \mathrm{~mm}$ and a $20 \mathrm{~mm}$ solitary, heterogeneous nodule located between the upper pole of left thyroid lobe and trachea. She described tenderness during the palpation of the nodule. Fine needle aspiration biopsy was performed but the cytology was non-diagnostic. Because of the suspicious ultrasonographic features of the nodule, she underwent total thyroidectomy . Histopathology and immunohistochemistry showed paraganglioma. Tumor was attached to the left lobe and isthmus of the thyroid and separated from the thyroid tissue with a fibrous band . Neoplastic cells expressed CD56, chromogranin and synaptophysin. Sustentacular cells were highlighted by S100 protein. Tumor stained negative for thyroglobulin, calcitonin, TTF 1, HBME1, CK19, Galectin3, PTH and desmin.

Conclusion: Paraganglioma is an extremely rare tumor of thyroid and difficult to diagnose. Paragangliomas must be taken under consideration for the differential diagnosis of atypical thyroid nodules.

\section{P329 \\ DIAGNOSED GRAVE'S DISEASES WITH MEDIASTINAL MASS: ANOTHER THYMUS HYPERPLASIA REMOVED}

Teik Joo Quah

General Medicine, Jalan Tan Tock Seng, Singapore

Background: Many case reportings have been done for thymus hyperplasia seen with graves diseases since 1914. A review on 91 patients in 2011 reemphasized this association and suggested for watchful observation to avoid unnecessary invasive investigation and surgical removal. However, some of us still practice to remove it

Case: 23-year-old lady who has diagnosed Graves' disease clinically and biochemically with persistent dysphagia which later found to have thymus and multiple lymph nodes enlargement by Computed Tomography of the neck, thorax and abdomen. However, the reporting radiologist was unable to comment further on the fat content of the thymomegaly. A repeated CT after a month of anti-thyroid therapy did not show shrinkage of thymus enlargement and hence raised a concern of malignancy which further necessitate the referral to cardiothoracic surgeon for diagnostic and therapeutic removal by robotic assisted surgery despite improvement in dysphagia and thyrotoxicosis biochemically. The removed thymus was confirmed histologically to be thymus hyperplasia. Discussion: Fat content of thymus is crucial to tell the likelihood of the enlargement being just reactive hyperplasia. Therefore, imaging modality of choice should have ability to detect and differentiate fat and non-fat content of the thymomegaly.

Conclusion: Despite majority of the cases reported association of Graves' disease with thymus hyperplasia, up to date there are 4 cases of thymic neoplasia reported in patients with Graves' disease. Perhaps, in replacement for Computed Tomography of the neck and thorax, chemical shift MRI and/or 18F-fluorodeoxyglucose Positron emission tomography (18F-FDG PET) can be used to differentiate them as these are used for post-chemotherapy or post-resection of thymus to identify rebound thymus hyperplasia or recurrence of neoplasm. These imaging will further reduce 
worries during watchful observation of thymus enlargement hence avoid unnecessary invasive measures.

\section{P330 \\ INTRATHYROIDAL METASTASES OF A SMALL CELL NEUROENDOCRINE CARCINOMA OF THE LUNG: A NEW OBSERVATION \\ Jeudy Claire ${ }^{1}$, Buffet Alexandre ${ }^{1}$, Rouquette Isabelle ${ }^{2}$, \\ Vergez Sebastien ${ }^{3}$, Staub Anne ${ }^{4}$, Caron Philippe ${ }^{5}$ \\ ${ }^{1}$ Department of Endocrinology, Chu Larrey, ${ }^{2}$ Department of Pathology, \\ Chu Rangueil, ${ }^{3}$ Department of Otolaryngology, Chu Larrey, ${ }^{4}$ Institut \\ Claudius Regaud, ${ }^{5}$ Department of Endocrinology, Chu Larrey,}

Introduction: Intrathyroidal metastases of systemic cancers are not uncommon, but they are sometimes difficult to diagnose.

Case Report: We report herein a 49 year-old man, referred for painful and compressive goiter with a left jugular vein thrombosis. Clinical examination confirms a multinodular thyroid goiter, firm and mobile upon swallowing. The initial evaluation was compatible with the diagnosis of Riedel's thyroiditis (calcitonin $3.8 \mathrm{pg} / \mathrm{ml}$, TSH $8.7 \mathrm{mU} / \mathrm{L}$ ) without anti-Tg and anti-TPO antibodies. Unfortunately the goiter rapidly increases, and a left lung tumor with mediastinal lymph nodes was observed on the CT scan. All those lesions are positive on TEP ${ }^{18} \mathrm{FDG}$ scan.

Thus, a neoplastic diagnosis is evocated and surgical biopsies of the thyroid are performed. Histologic examination shows massive infiltration of the thyroid by carcinomatous proliferation (CK7+, CK20-, TTF1+), compatible with anaplastic thyroid carcinoma or with thyroidal metastases of a lung adenocarcinoma.

A systemic chemotherapy (cisplatin, vepeside) is started, and follow-up shows a rapid and spectacular response with a $50 \%$ decrease of the goiter, mediastinal lymph nodes and lung tumor after the first two cycles. In regard with this unusual response, and after a second look of histologic blades, the tumor seems to correspond to a neuroendocrine carcinoma (TTF1+, synaptophysin+, chromogranin-, CD56+), which would be a thyroidal metastasis of a neuroendocrine small cell lung carcinoma. The patient will consequently benefit of a cervical and encephalic radiotherapy.

Conclusion: Thyroid metastases from small cell neuroendocrine carcinoma of the lung are rare but it is important to keep in mind this possibility at the time of diagnosis and during treatment of some nodular goiters.

\section{P331 \\ SPORADIC MEDULLARY THYROID CARCINOMA IN GRAVE'S DISEASE}

Carsten Koerber ${ }^{0}$, Nicole Körber-Hafner

${ }^{1}$ Gemeinschaftspraxis

The patient presented due to hyperthyroidism because of Graves disease.

Under the medication of thiamazol and after the normalisation of the peripheral thyroid hormones an elevation and increase of the serum level of calcitonin could be observed. After an increase to $150 \mathrm{pg} / \mathrm{ml}$ a pestagastrin test was performed and the peak value of calcitonin reached the level of $850 \mathrm{pg} / \mathrm{ml}$ 2 minutes after the injection of pentagastrin.

Therefore thyroidectomy was intended proofing the diagnosis of a sporadic thyroid carcinoma in a nodule of the right thyroid lobe. Further treatment was performed according to the current guide lines.

\section{P332 \\ PRIMARY THYROID LYMPHOMA PRESENTING AS ANAPLASTIC THYROID CARCINOMA - CASE REPORT}

Catarina Roque ${ }^{1}$, Filipa Serra ${ }^{1}$, Helder Simões ${ }^{1}$, Catarina Saraiva ${ }^{1}$ Clotilde Limbert ${ }^{1}$, Celina Afonso ${ }^{2}$, Carlos Vasconcelos ${ }^{1}$, F. Lima ${ }^{3}$

A. Machado Saraiva ${ }^{4}$

${ }^{1}$ Endocrinology Department - Centro Hospitalar de Lisboa Ocidental, ${ }^{2}$ Hematology Department - Centro Hospitalar de Lisboa Ocidental, ${ }^{3}$ Director - Hematology Department - Centro Hospitalar de Lisboa Ocidental, ${ }^{4}$ Director - Endocrinology Department - Centro Hospitalar de Lisboa Ocidental

Introduction: Primary thyroid lymphoma is a rare entity accounting for $2 \%$ of all primary thyroid malignancies and $2 \%$ of all extranodal lymphomas. Hashimoto's thyroiditis is often associated.

Case Report: An 83 year old woman presented to the emergency department complaining of hoarseness, enlarging neck mass and dysphagia over the last 3 weeks. Examination revealed a large anterior-bilateral cervical mass, stony-hard, lobulated, fixed and painful. Bilateral jugular vein distention was evident and cyanosis developed progressively as she was speaking. Severe dyspnea without stridor was elicited by the supine position and Pemberton sign was present. Cervical ultrasound confirmed an adherent multinodular goiter and multiple large bilateral hypervascularized adenopathies, with no enhanced posterior echoes. CT-scan delineated the necrotic center of these superficial and deep cervical adenopathies and the mass encircling the esophagus, trachea and major vascular structures; filling of the right internal jugular vein was not visible. Fine needle aspiration cytology (FNA) of the thyroid lesions resulted benign (Bethesda) with features of lymphocytic thyroiditis. TSH $30 \mathrm{mU} / \mathrm{mL}, \mathrm{f}-\mathrm{T} 48 \mathrm{pmol} / \mathrm{l}$ and Thyroid autoantibodies were negative. Nodal FNA showed no epithelial related cells and lymphocyte morphology was unspecific. The patient refused biopsy. Immunophenotyping of the nodal sample enlightened the presence of $88 \%$ of B cells, suggestive of Diffuse Large B Cell Non Hodgkin Lymphoma. Staging showed disease localized to the neck and mediastinum - Stage IIA. Chemotherapy was started on a low intensity regimen with cyclophosphamide, vincristine and prednisolone with reduction of tumor mass after 3 sessions.

Conclusion: In the presence of a rapidly enlarging neck mass a lymphoproliferative disorder should be suspected in the differential with anaplasic carcinoma, especially if there is lymphocytic thyroiditis even when autoantibodies are negative. FNA sensitivity in the differentiation of pathologic vs reactive lymphadenopathies and thyroiditis renders mandatory the realization of a confirmatory diagnostic procedure as immunophenotyping.

\section{P333 \\ METHIMAZOLE-INDUCED AGRANULOCYTOSIS IN A PATIENT WITH GRAVES' DISEASE}

Filipe Manuel Cunha ${ }^{1}$, Pedro Rodrigues ${ }^{1}$, Joana Oliveira ${ }^{1}$, Ana Varela ${ }^{1}$, Elisabete Rodrigues ${ }^{1}$, Davide Carvalho ${ }^{1}$

${ }^{1}$ Serviço de Endocrinologia Diabetes E Metabolismo Centro Hospitalar de São João; Faculdade de Medicina Universidade Do Porto

Introduction: Agranulocytosis is a rare and sometimes fatal complication of methimazole (MMI) treatment with an estimated prevalence of 0.2 to $0.5 \%$. An immune-mediated neutrophil destruction has been proposed. We present a case of methimazole-induced agranulocytosis in a women with Graves disease.

Case Report: A 40-year-old woman presented with weight loss, increased appetite, fatigue, anxiety, palpitations, heat intolerance and tremor in the lasting 4 months. At physical examination: tachycardia (111 bpm), normal blood pressure $(110 / 70 \mathrm{mmHg})$. Palpable, non tender thyroid without discernible nodules and no audible bruits. Fine tremor at rest. No eye involvement nor peripheral oedema. Analysis: haemoglobin $(\mathrm{Hb}) 12.3 \mathrm{~g} / \mathrm{dL}$, white blood count (WBC) $5440 / \mu \mathrm{L}$, neutrophils $2010 / \mu \mathrm{L}$. Normal liver panel. TSH: $0.001 \mu \mathrm{U} /$ $\mathrm{mL}$ (0.35-4.94), FT4 $1.82 \mathrm{ng} / \mathrm{dL}(0.70-1.48)$, FT3 $4.11 \mathrm{pg} / \mathrm{mL}$ (1.71-3.71). Anti-TSH receptor antibodies $2.8 \mathrm{U} / \mathrm{L}(<1.8)$. Thyroid ultrasound: enlarged gland, heterogeneous structure, with a solid hypoechoic $6 \mathrm{~mm}$ nodule in the left lobe. I-131 scintigraphy: high diffuse radioiodine uptake. Initiated treatment with MMI $15 \mathrm{mg} /$ day and propanolol $30 \mathrm{mg} /$ day. Two months later, the patient was admitted in the emergency department with fever, odynophagia 
and erythema of the oropharynx. Analyses: Hb $12.2 \mathrm{~g} / \mathrm{dL}$, WBC $1150 / \mu \mathrm{L}$, neutrophil count $0 / \mu \mathrm{L}$, normal platelets. Normal liver panel. C-reactive protein: $107.1 \mathrm{mg} / \mathrm{L}(<3.0)$, TSH: $0.42 \mu \mathrm{U} / \mathrm{mL}$, FT4 $0.87 \mathrm{ng} / \mathrm{dL}$. HIV negative. Myelogram: erythroid hyperplasia and myeloid hypoplasia. Suspended MMI and started piperacillin-tazobactam and filgastrim. Increase of neutrophil count 7 days after MMI suspension, reaching 5830/ $\mu \mathrm{L}$. Sustained apyrexia. Progressive decrease of the acute phase reactants. Negative blood and urine cultures. Negative viral serology. Remained euthyroid. Discharged with propanolol $30 \mathrm{mg} /$ day. Treatment with I-131 (12 mCi) 6 weeks later. At 1-month follow-up, the patient presented normal thyroid function.

Conclusion: Agranulocytosis is a serious complication of MMI treatment. Differential diagnosis with viral infections can be difficult. After MMI discontinuation, neutrophil count recovery occurs usually within 2 weeks. The role of routine white blood cell count monitoring is controversial.

\section{P033 Thyroid Cancer Therapeutics 3}

\section{P334 \\ DOES CORTICOSTEROID HAVE ANY BENEFICIAL EFFECT ON VOICE CHANGE AFTER THYROIDECTOMY?}

Shirzad Nasiri', Nassim Sodagari ${ }^{1}$

${ }^{1}$ Tehran University of Medical Sciences

Introduction: Voice alteration is one of the most common complications after thyroidectomy. It has a serious effect on social communication and economic and psychosocial status of patients. It has been hypothesized that inflammation and edema in the surgery site has a major role in voice change after thyroidectomy. This randomized clinical trial study was design to evaluate the effect of a single preoperative dose of steroid on voice change after thyroidectomy. This is a prospective randomized clinical trial with registration no. IRCT201106306925N

Methods and Material: From all definitive candidates for total thyroidectomy, eligible patients were selected after exclusion of 12 criteria. Selected cases were randomly allocated to two groups. One group received intravenous dexamethasone preoperatively. Other group received placebo. Voice change was evaluated by Voice Impairment Score (VIS) postoperatively.

Results: Sixty-four patients were entered in the study and divided into two groups (dexamethasone and placebo). VIS was significantly different at the first day after surgery between dexamethasone (5) and controls (13; $\mathbf{P} \backslash 0.001$ ). This difference was seen after 7 days but without statistically significance (one vs three; P 5 0.397). VIS score significantly decreased onthe seventh day related to the first day $(\mathbf{P} \backslash 0.001)$ in both groups. There were no significant dexamethasone complications in either group.

Conclusion: Preoperative dexamethasone may decrease voice change after thyroidectomy.

\section{P335 \\ MEASUREMENT OF I-131 UPTAKE IN THE THYROID BED AFTER I-131 RADIOIODINE REMNANT ABLATION}

Joji Kawabe ${ }^{1}$, Shigeaki Higashiyama ${ }^{1}, K^{\prime}$ Kohei Kotani ${ }^{1}$, Atsushi Yoshida ${ }^{1}$, Susumu Shiomi

${ }^{1}$ Department of Nuclear Medicine, Graduate School of Medicine, Osaka City University

Objectives: I-131 accumulation in the thyroid bed is the most important factor after I-131 radioiodine remnant ablation (RRA) is performed posttotal thyroidectomy in patients with differentiated thyroid carcinoma (DTC) patients. We retrospectively evaluated thyroid-bed I-131 uptake approximately 10 days after recombinant human thyrotropin (rhTSH)- or thyroid hormone withdrawal (THW)-aided RRA.

Methods: We examined the uptake rates in thyroid beds of 29 DTC patients ( 7 men and 22 women; average age, $61.2 \pm 11.1$ years) who had undergone RRA with $1.85 \mathrm{GBq}$ of I-131 post-total thyroidectomy from November
2011 to September 2013. No visible distant or residual regional lymph node metastasis was seen in patients. For all patients, low-iodine diet was prescribed 2 weeks before and 4 days after I-131 administration. In patients who had undergone THW-aided RRA, levothyroxine was discontinued 3 weeks before and 4 days after I-131 administration. In patients who had undergone rhTSH-aided RRA, levothyroxine administration was continued. Post-therapy I-131 scintigraphy was performed approximately 10 days after I-131 administration. Whole-body and spot images of the neck were taken using the same scinti camera (ADAC FORTE). Spot images were taken for 300 s. Same-sized square regions of interest (ROIs) were determined on the thyroid bed and background area on the anterior view. For controls, the counts of $185 \mathrm{MBq}$ of I-131 capsule were determined using the same ROIs. Using these count data and time decay control, the thyroid-bed I-131 uptake was measured.

Results: Thyroid-bed I-131 uptake ranged from $0.016 \%$ to $16.639 \%$ (average uptake, $0.854 \% \pm 3.051 \%$ ). The mean uptake in 13 patients who had undergone rhTSH-aided RRA was $1.711 \% \pm 4.502 \%$, whereas that in 16 patients who had undergone THW-aided RRA was $0.159 \% \pm 0.101 \%$. A significant difference was found between these two groups.

Conclusion: Measuring thyroid-bed I-131 uptake after RRA may indicate the prognosis of RRA.

\section{P336 \\ SKIP METASTASIS TO LATERAL NECK NODE IN SOLITARY PAPILLARY THYROID CARCINOMA}

Jun Ho Kim ${ }^{1}$, Yun Beom Ryu ${ }^{1}$, Lee Su Kim ${ }^{1}$

${ }^{1}$ Division of Breast and Endocrine Surgery, Hallym University Sacred

Heart Hospital, Hallym University College of Medicine

Background: Cervical lymph node (LN) metastasis is commonly observed in papillary thyroid carcinoma (PTC) and this usually occurs in stepwise fashion. However, skip metastasis (lateral LN metastasis without central LN metastasis) can also occurs in small number of PTC patients. The goal of this study was to evaluate the clinicopathologic characteristics of skip metastasis in PTC.

Methods: We reviewed the medical records of 126 PTC patients who underwent total thyroidectomy with simultaneous central and therapeutic lateral neck dissection between 2008 and 2012. Among these, the clinicopathologic characteristics of patients with solitary tumor $(n=80)$ were analyzed according to the skip metastasis.

Results: The frequency of skip metastasis was $16.7 \%(21 / 126)$. There were no significant differences in multifocality and bilaterality between patients with and without skip metastasis. In patients with solitary tumor, the mean numbers of dissected central and lateral LNs were similar between two groups but patients with skip metastasis had fewer metastatic lateral LNs (4.6 vs. $1.9 ; \mathrm{P}<0.001)$ and mainly single metastatic $\mathrm{LN}(61.5 \%)$. The tumors with skip metastasis were more commonly located in upper pole ( $76.9 \%$ vs. $40.3 \%$; $\mathrm{p}=0.015)$ and mean tumor sizes were smaller $(1.2 \mathrm{~cm}$ vs. $2.0 \mathrm{~cm} ; \mathrm{p}=0.034)$.

Conclusions: Skip metastasis is not uncommon in PTC patients. The PTC located upper pole should be carefully evaluated for possibility of skip metastasis, especially in patients with small tumors

\section{P337 \\ REGIONAL LYMPH NODE RECURRENCE AFTER TOTAL THYROIDECTOMY AND NECK DISSECTION IN PATIENTS WITH PAPILLARY THYROID CANCER}

Sung Tae Seo ${ }^{1}$, Sungha $\mathrm{Kim}^{2}$, Bon Seok Koo ${ }^{3}$

${ }^{1}$ Department of Otolaryngology - Head \& Neck Surgery, Chungnam National University, School of Medicine, ${ }^{2}$ Chungnam National University, ${ }^{3}$ Department of Otolaryngology - Head \& Neck Surgery; Chungnam National University, School of Medicine

Background: We investigated the risk factors of the regional lymph node (LN) recurrence in papillary thyroid cancer (PTC) patients underwent thyroidectomy and neck dissection according to the clinicopathologic features, preoperative clinical nodal status and the recurrence in previously dissected or undissected compartment of the neck.

Methods: A retrospective analysis was performed on 297 patients who underwent total thyroidectomy and LN dissection between 2004 and 2010. Patients with and without regional recurrence were compared by the various 
clinicopathological factors. Recurrence-free survival rates were estimated by the Kaplan-Meier and Cox regression method.

Results: With a median follow-up of 53 months, 22 (7.4\%) patients developed regional LN recurrence. Initial LN metastasis and tumor size $>1 \mathrm{~cm}$ were independent predictive factors for regional recurrence. In patients without preoperative clinical LN, Tumor size $>1 \mathrm{~cm}$ and extrathyroidal extension were significant risk factors for regional recurrence. In cases with preoperative clinical LN, there was no specific significant factor for recurrence. Tumor size $>1 \mathrm{~cm}$, capsular invasion, extrathyroidal extension, and lymphovascular invasion were significant risk factors of regional recurrence in previously dissected compartments. Tumor size $>1 \mathrm{~cm}$ and extrathyroidal extension were significant predictive factors of regional recurrence in previously undissected compartments.

Conclusions: Tumor size and lymph node metastasis were independent predictors of regional recurrence in PTC patients who underwent total thyroidectomy and LN dissection. In addition, more carful treatment and follow-up is required for the patients with tumor size $>1 \mathrm{~cm}$ or extrathyroidal extension because of the likelihood of recurrence of previous dissected or undissected compartments though prophylactic central neck dissection was performed.

\section{P338}

\section{ROBOTIC THYROIDECTOMY LEARNING CURVE} FOR BEGINNING SURGEONS WITH LITTLE OR NO EXPERIENCE OF ENDOSCOPIC SURGERY

Jae Hyun Park' ${ }^{1}$, Jandee Lee ${ }^{2}$, Sang-Wook Kang ${ }^{2}$, Jong Ju Jeong ${ }^{2}$,

Kee-Hyun Nam ${ }^{2}$, Keum-Seok Bae ${ }^{1}$, Seong Joon Kang ${ }^{1}$,

Woong Youn Chung ${ }^{2}$

${ }^{1}$ Department of Surgery, Yonsei University Wonju College of Medicine,

${ }^{2}$ Department of Surgery, Yonsei University College of Medicine

Background: Although robotic thyroidectomy using a gasless trans-axillary approach has been shown to be safe and oncologically effective, little is known about the surgical learning curve for surgeons with little experience of endoscopic surgery. This study assessed the results of robotic thyroidectomy by fellowship-trained surgeons in their initial independent practice, and whether standard fellowship training for robotic surgery shortens the learning curve.

Materials \& Methods: This prospective cohort study evaluated outcomes in 125 patients who underwent robotic thyroidectomy by two recently graduated fellowship-trained surgeons. During the first 6-month training period, these surgeons accumulated sufficient experience to be able to competently perform conventional open thyroidectomies. During the next 6-months, candidates were then allowed to perform robotic thyroidectomy under supervision. Learning curves were analyzed by operation time, with proficiency defined as the point at which the slope of the time curve became less steep.

Results: Of the 125 patients, 113 underwent less-than-total thyroidectomy (LT), 9 underwent total thyroidectomy (TT) and 3 underwent TT with modified radical neck dissection (MRND). All operations were successfully completed using the robotic technique, with none requiring conversion to an open method. Mean total times for these three operations were $100.8 \pm 20.6$ $\mathrm{min}, 134.2 \pm 38.7 \mathrm{~min}$, and $284.7 \pm 60.4 \mathrm{~min}$ respectively. For both surgeons, the operation times gradually decreased, reaching a plateau after 20 robotic LTs.

Conclusions: The surgical learning curve for robotic thyroidectomy performed by recently graduated fellowship-trained surgeons with little or no experience in endoscopic surgery showed excellent results compared with those in a large series of more experienced surgeons. Training under the supervision of an experienced surgeon appears to minimize the learning curve for robotic thyroid surgery.

\section{P339}

\section{ANTERIOR LEVEL IV LYMPH NODE METASTASIS IN CLINICALLY LATERAL CERVICAL LYMPH NODE-POSITIVE THYROID CARCINOMA}

Jae Hoon Jang ${ }^{1}$, Jee Soo Kim ${ }^{1}$

${ }^{1}$ Division of Breast and Endocrine Surgery, Department of Surgery

Background: The indication and extent of cervical lymph node dissection in thyroid carcinoma are still being debated. Also, there have been few reports on anterior level IV cervical lymph node metastasis. The aim of this study was to analyze the pattern of cervical lymph node metastasis focused on anterior level 4 cervical lymph node metastasis and suggest the optimal extent of lateral cervical lymph node dissection for patients with clinical lateral node metastasis

Methods: We retrospectively analyzed 96 patient with thyroid carcinoma who underwent lateral neck dissection including anterior level IV cervical lymph node between November 2012 and March 2014. Anterior level IV cervical lymph node was defined as lateral cervical lymph node between sternocleidomastoid and sternohyoid muscle.

Results: The 96 patients with clinical lateral lymph node metastasis underwent 112 sides of lateral neck dissection. The metastasis rate of anterior level IV was $11.6 \%$. The mean number of lymph node removed was $2.12 \pm 1.08$ (range $0-5$ ) in anterior level IV. and the mean number of metastatic lymph node was $1.13 \pm 0.36$ (range $0-2$ ) in anterior level IV. The most frequent metastatic level was level IV (80.4\%), followed by level III (76.8\%), level $\mathrm{IIa}(51.8 \%)$, level V(10.7\%), and level IIb (8.9\%). The metastasis rate of anterior level IV was higher than level V and level IIb. The metastasis of anterior level IV was not correlated with primary tumor site, level III and level IV metastasis including other clinical parameter.

Conclusion: In thyroid carcinoma patients with clinical lateral lymph node metastasis, the optimal extent of lateral cervical lymph node dissection may include level II, III, IV, V as well as anterior level IV.

\section{P340 \\ TSH SUPPRESSION AND LEVOTHYROXINE DOSE POST RADIOIODINE ABLATION IN DIFFERENTIATED THYROID CANCERS}

Lavanya Mariappan ${ }^{1}$, Georgina Gerrard ${ }^{1}$, Emma Dugdale ${ }^{1}$,

Satiavani Ramasamy ${ }^{1}$, Katie Spencer ${ }^{1}$

${ }^{1}$ St James Institute of Oncology, Leeds, UK

Objectives: Levothyroxine dosing post radioiodine ablation is important to achieve a TSH of $<0.1 \mathrm{mIU} / \mathrm{L}$ whilst maintaining patient well being. Our aim is to determine the starting dose to achieve the above with minimum dose alterations by:

1. auditing the TSH level in patients who received $2.2 \mu \mathrm{g} / \mathrm{kg}$ of levothyroxine after 6 weeks and after 9 to 12 months.

2. comparing results with previous audits of levothyroxine dosage at $2 \mu \mathrm{g} /$ $\mathrm{kg}$ and $2.5 \mu \mathrm{g} / \mathrm{kg}$.

Methods: Retrospective study including patients who were treated with total thyroidectomy and radioiodine for thyroid cancers from 2011-2013 at our centre. Electronic medical notes were reviewed.

Results: 79 patients were included in the study.

31 of $79(39 \%)$ patients required a dose reduction at 6 weeks. In this group, 10 patients required further dose reduction at the 9 month visit.

Of the 26 patients with no dose reduction at 6 weeks, $12(46 \%)$ required dose reduction at 9 months.

The results of the $2.5 \mu \mathrm{g} / \mathrm{kg}$ dose audit showed 35 of 90 patients (39\%) needed dose reduction at 6 weeks. These results are comparable to the $2.2 \mu \mathrm{g} /$ $\mathrm{kg}$ dose study.

The results of $2 \mu \mathrm{g} / \mathrm{kg}$ study showed 64 out of 97 (66\%) patients required dose increase at 6 weeks but only 4 needed dose increase at a subsequent visit. However, the $2 \mu \mathrm{g} / \mathrm{kg}$ patients had levothyroxine withdrawal prior to radioiodine rather than recombinant TSH.

Conclusion: When patients were commenced on $2.2 \mu \mathrm{g} / \mathrm{kg}$ of levothyroxine, they needed dose reductions at 6 weeks and also at 9 to 12 months visit indicating the above starting dose is too high. This has changed our practice and we now prescribe $2 \mu \mathrm{g} / \mathrm{kg}$ dose of levothyroxine along with recombinant TSH which will be audited. The British Thyroid Asssociation guidelines on thyroid cancer recommend $2 \mu \mathrm{g} / \mathrm{kg}$ levothyroxine and our results will be useful to confirm this recommendation. 


\section{P341 \\ RECURRENT LARYNGEAL NERVE INVASION IN PATIENTS WITH PAPILLARY THYROID CARCINOMAS: PREDICTIVE FACTORS AND MANAGEMENT \\ Il-Seok Park' ${ }^{1}$, Young Soo Rho ${ }^{2}$ \\ ${ }^{1}$ Hallym University Medical Center, ${ }^{2}$ Ewha Womans University Medical Center}

Objectives: The incidence of recurrent laryngeal nerve(RLN) invasion by papillary thyroid cancer(PTC) ranges from $3.7 \%$ to $25.4 \%$. The purpose of this study was to determine the predictive factor of RLN invasion and optimal management of that in patients (pts) with PTC.

Methods: A retrospective study was performed with the medical records of 50 pts with RLN invasion among 337 pts with PTC between 1995 and 2011. After resection of involved RLN $(n=30)$, immediate reconstruction of RLN was performed on $10 \mathrm{pts}$ underwent neurorrhaphy. $20 \mathrm{pts}$ opted not to have surgical procedures. Clinical variables (preoperative vocal cord mobility, the patterns of nerve invasion, location of tumor, treatment, postoperative vocal cord function) were analyzed.

Results: Among the 50 pts with RLN invasion, RLN was involved with direct extension of PTC in 35 pts and metastatic paratracheal lymph node in 15 pts. Tumor $\operatorname{size}(p=0.002)$, extra-thyroid invasion $(p=0.025)$ and lateral lymph node involvement $(p=0.037)$ were the independent factors significantly affecting RLN invasion in multivariate analysis. Of 20 RLN of shaving off procedure, 2 showed permanent paralysis, 8 showed transient paralysis, restored normal function within 6 months, and 10 showed normal vocal cord function. Of 10 RLN neurorrhaphy, 6 showed permanent paralysis, 2 showed fully recovered mobility and 2 showed partially recovered vocal cord function after 6 months.

Conclusion: The RLN can be preserved with shaving off, if it has not been invaded directly by the tumor. And, resection of RLN at the time of thyroid cancer extirpation require RLN reconstruction, because it offers a good postoperative vocal cord function.

\section{P342 \\ INTRAOPERATIVE FROZEN BIOPSY FOR THE EVALUATION OF EXTRATHYROIDAL EXTENSION IN PAPILLARY THYROID CANCER Byungjoo Lee ${ }^{1}$, Soo Geun Wang ${ }^{1}$, Young Min Park', Jinchoon Lee ${ }^{1}$, Inju Kim², Dong Hoon Shin ${ }^{3}$, Seok-Man Son ${ }^{2}$ \\ ${ }^{1}$ Department of Otolaryngology, Pusan National University Hospital, ${ }^{2}$ Department of Internal Medicine, Pusan National University Hospital, ${ }^{3}$ Department of Pathology, Pusan Nationa University Hospital}

Objectives: We performed intraoperative frozen biopsy of surgical specimens and investigated extrathyroidal tumor extension through pathologic analysis in order to determine the clinical usefulness of frozen biopsy for intraoperative decision making in patients with papillary thyroid cancer (PTC).

Methods: Between March 2013 and May 2013, 268 patients who were diagnosed with PTC in Pusan National University Hospital were enrolled in the study. The Institutional Review Board of Pusan National University Hospital approved the study and informed consent was obtained from all patients. During the period of the study an operation was performed in all 268 patients with PTC and extrathyroidal extension was evaluated using intraoperative frozen biopsies of thyroid tissue.

Results: Extrathyroidal extension was confirmed in 54 patients (20\%) on frozen biopsy. On permanent pathologic examination, extrathyroidal extension was finally confirmed in 80 patients (30\%). Fifty-three patients among 54 patients showing extrathyroidal extension on frozen biopsy were confirmed on permanent pathologic analysis. Accordingly, frozen biopsy had a sensitivity of $66 \%$, a specificity of $99 \%$, a positive predictive value of $98 \%$, and a negative predictive value of $87 \%$. The diagnostic performance of frozen biopsy was calculated in the evaluation of ETE for micro PTC: $65 \%$ sensitivity, $99 \%$ specificity, $97 \%$ positive predictive value, and $91 \%$ negative predictive value. Tumor size (OR 4.373; CI 2.257-8.475, $\mathrm{p}=<0.001$ ) was an independent factor for predicting extrathyroidal extension on frozen biopsy by multivariate logistic analysis after controlling for age, sex and other clinical aspects.

Conclusions: Intraoperative frozen biopsy can be a useful tool in identifying the presence of extrathyroidal extension. It can also help the operator decide the extent of surgery and central neck dissection in patients with papillary thyroid carcinoma.

\section{P343 \\ THE BENEFITS AND RISKS OF PROPHYLACTIC CENTRAL NECK DISSECTION FOR PAPILLARY THYROID CARCINOMA: PROSPECTIVE ANALYSI} Kwang Jin Jung ${ }^{1}$, Soon-Young Kwon ${ }^{2}$, Jeong-Soo Woo ${ }^{2}$, Seung-Kuk Baek', Jae-Gu Jo², Min Woo Park', Si-Youn Song ${ }^{3}$, Kwang-Yoon Jung ${ }^{1}$

${ }^{1}$ Department of Otolaryngology-Head and Neck Surgery, Korea University College of Medicine; Thyroid Center, Korea University Medical Center, ${ }^{2}$ Department of Otolaryngology-Head and Neck Surgery, Korea University College of Medicine, ${ }^{3}$ Department of Otolaryngology-Head and Neck Surgery, Yeungnam University College of Medicine

Introduction: The benefits and risks of prophylactic central neck dissection(CND) in patients with papillary thyroid cancer(PTC) is controversial. The aim of study was to examine locoregional recurrence and complication rate of prophylactic CND.

Method \& materials: We reviewed 213 patients with PTC and clinical negative neck lymph nodes (cN0) between 2006 and 2008. In that group, 103 had Total thyroidectomy alone and 104 underwent total thyroidectomy with $\mathrm{CND}$. We conduct the study to compare the risk of locoregional recurrence and complications between Total thyroidectomy with central neck dissection and total thyroidectomy alone.

Results: Incidence of occult central metastasis of PTC was 34.6\%. There are no significant difference in 5-year locoregional control rates after total thyroidectomy only and total thyroidectomy with $\mathrm{CND}(96.1 \%$ vs $97.1 \%, \mathrm{P}=$ 0.388). The total thyroidectomy with CND group exhibited a significantly higher incidence of transient $(20.3 \%$ vs $36.5 \%, p=0.043)$ but no significantly different incidence of permanent hypocalcaemia $(1.9 \%$ vs $3.3 \%, \mathrm{p}=0.245)$. Two cases of transient vocal fold paralysis were developed after the operation of central neck recurrence.

Conclusion: Prophylactic CND caused more likely to have transient and permanent hypocalcaemia than total thyroidectomy alone. Total thyroidectomy alone is safe management in clinical N0 PTC and Prophylactic CND increase transient vocal fold paralysis in operation of central neck recurrence

\section{P344 \\ INITIAL TREATMENT WITH SURGERY AND RADIOIODINE ABLATION IN DIFFERENTIATED THYROID CANCER: AGREEMENT BETWEEN AMERICAN THYROID ASSOCIATION STRATIFICATION AND DELAYED RISK STRATIFICATION}

Eduarda Resende ${ }^{1}$, Liliana Violante ${ }^{2}$, Inês Lucena ${ }^{3}$,

João Pedro Teixeira ${ }^{3}$, Lúcia Costa ${ }^{3}$, Fátima Lopes ${ }^{3}$, Orlando Soares ${ }^{3}$, Hugo Duarte ${ }^{3}$

${ }^{1}$ Department of Endocrinology - Central Hospital of Funchal, ${ }^{2}$ Nuclear Medicine Department - Portuguese Institute of Oncology of Oporto, ${ }^{3}$ Department of Nuclear Medicine - Portuguese Institute of Oncology of Oporto

Introduction: The incidence of differentiated thyroid carcinoma (DTC) has been increasing over the years, and there is a rising concern on how to follow these patients. Recently is being recognized the importance of incorporating additional data of the patients available on their follow-up to their initial risk stratification. The aim of this work is to compare the agreement between the different stratification systems.

Methods: Retrospective study of 91 patients submitted to initial treatment with surgery and radioiodine ablation between 1th July of 2012 and 31th December 2012. Initially these patients were stratified using the American Thyroid Association (ATA) system; 6 to 12 months later, at the time of their first control, they were re-strafied using the Delayed Risk Stratification (DRS).

Results: 9 patients initially belong to the high risk category of ATA stratification, and 48 to the intermediary risk - in a total of 57 patients (62.6\%). Of this group, 35 patients were reclassified at their first control as being in clini- 
cal remission - they were shifted to the low risk category according to DRS. 34 patients $(37.4 \%)$ were initially classified as low risk according to ATA; of these, 12 patients were reclassified as high risk (persistent disease) according to DRS. The Cohen K coefficient to evaluate the agreement between ATA and DRS stratification systems was 0.45 (moderate agreement).

Discussion/Conclusion: Some studies show that in a long term follow-up the DRS system is more accurate in the prediction of relapse than the ATA system. In this study we need a longer follow-up to reach that conclusion; however, the re-stratification of these patients can have impact on the management of their follow-up.

\section{P345 \\ PREDICTORS OF HYPERTHYROIDISM IN PATIENTS TREATED WITH LEVOTHYROXINE SUPPRESSIVE DOSES FOR WELL- DIFFERENTIATED THYROID CANCER}

\section{Tatiana Mityukova ${ }^{1}$, Natallia Akulevich $^{2}$, Tatiana Leonova ${ }^{3}$,} Tamara Platonova ${ }^{1}$, Svetlana Mankovskaya ${ }^{4}$, Svetlana Kohan ${ }^{4}$, Vladimir Kravchuk ${ }^{5}$

${ }^{1}$ The Institute of Physiology of the National Academy of Sciences of Belarus; Belarusian Medical Academy for Postgraduate Education, Minsk, Belarus, ${ }^{2}$ The Institute of Physiology of the National Academy of Sciences of Belarus; State Center for Medical Rehabilitation, Minsk, Belarus, ${ }^{3}$ The Institute of Physiology of the National Academy of Sciences of Belarus; Clinical Oncologic Dispensary, Minsk, Belarus, ${ }^{4}$ The Institute of Physiology of the National Academy of Sciences of Belarus, ${ }^{5}$ State Center for Medical Rehabilitation, Minsk, Belarus

Recent data indicate that levothyroxine (L-T4) dose for well-differentiated thyroid cancer (WDTC) treatment requires an optimization. The purpose of our study was to search for possible predictors of hyperthyroidism in young WDTC patients.

Young adults (N-114; 90 females; mean age $28.42 \pm 0.29$ years) who are under standard L-T4 suppressive therapy for WDTC have been investigated. The control group included 73 healthy age-and sex-matched subjects. In all the participants, the height, weight and body mass index (BMI, $\mathrm{kg} / \mathrm{m}^{2}$ ) were measured and thyroid function tests (Elisa-Kits DRG, USA).

It has been demonstrated that the patients under standard suppressive therapy (median daily L-T4 dose $2.6 \mu \mathrm{g} / \mathrm{kg}$ ) had higher TT4 and FT4 levels than in the control (12.6 vs. $10.9 \mu \mathrm{g} / \mathrm{dl}$ and 1.8 vs. $1.6 \mathrm{ng} / \mathrm{dl}$, respectively; $\mathrm{P}<0.001)$. About half of the patients, had moderately suppressed TSH $(0.1-0.5 \mathrm{mIU} / \mathrm{l})$; the other part had stronger suppression (TSH $<0.1 \mathrm{mIU} / 1)$. In both subgroups, the proportions of elevated free thyroid hormones' fractions were similar (FT4 $18-19 \%$, FT3 8-12\%). In thyroid cancer patients with weight excess and obesity $\left(\mathrm{BMI}>25 \mathrm{~kg} / \mathrm{m}^{2}\right)$, TSH suppression $<0.5 \mathrm{mIU} / 1$ was achieved with lower L-T4 daily doses than in those with normal BMI $(2.2$ vs. $2.8 \mu \mathrm{g} / \mathrm{kg}$; P<0.05). Every second female patient $(51.5 \%)$ and $29 \%$ of the men had elevated T4 levels. In men, FT3 raise was found out more frequently compared to women $(20.8 \%$ vs. $7.8 \%)$. In WDTC men with normal weight, a combination of elevated FT3 levels and decreased FT4/FT3 ratio less than 5 dominated; such findings may indicate the hyperthyroidism risk under treatment with standard suppressive L-T4 doses.

The need for L-T4 in WTDC patients depends on BMI and should be individualized. An elevated FT3 level and decreased FT4/FT3 ratio (less than 5) may indicate hyperthyroidism in such patients.

\section{P034 Thyroid Cancer \\ Therapeutics 4}

\section{P346 \\ DIAGNOSTIC THYROIDECTOMY IN PATIENTS WITH CYTOPATHOLOGIC DIAGNOSIS OF AUS/ FLUS IN BETHESDA SYSTEM \\ Yong Sang Lee ${ }^{1}$, Seok Mo Kim², Hang-Seok Chang ${ }^{2}$, Cheong Soo Park ${ }^{2}$ \\ ${ }^{1}$ Gangnam Severance Hospital, Yonsei University College of Medicine, ${ }^{2}$ Thyroid Cancer Center and Department of Surgery, Gangnam Severance Hospital, Yonsei University College of Medicine}

Background: Atypia/follicular lesion of undetermined significance (AUS/FLUS) is a new category in the Bethesda System for Reporting Thyroid Cytopathology (BSRTC) for which repeat fine-needle aspiration cytology (FNAC) is recommended. The aim of this study was to find out the clinical factors to consider the diagnostic lobectomy in patients with atypia.

Patients and Methods: Between January 2011 and December 2102, 5.440 patients were underwent thyroid surgery in our hospital. Of these, 213 patients were atypia at preoperative cytopathologic diagnosis. Frequency of FNAC and ultrasonographic images were compared between patients with cancer and benign at final pathologies.

Results: Of the 213 patients, $158(74.2 \%)$ were diagnosed as thyroid carcinoma at final pathologic reports. In the univariate and multivariate analysis, frequency of FNAC was not significantly correlated with the cancer diagnosis. Hypoechogenicity (odds ratio 2.521, p-value 0.007 ) and microcalcification (odds ratio 3.247; p-value 0.005 ) were highly correlated with the cancer risk with statistical significance.

Conclusion: Although AUS/FLUS in cytopathology is recommended for repeating FNAC in BSRTC, the diagnostic lobectomy is considerable in patients with atypia showing microcalcification and hypoechogenecity in ultrasonography.

\section{P347 \\ ULTRASOUND-GUIDED PERCUTANEOUS ETHANOL ABLATION OF SELECTED METHASTATIC LYMPH NODES OF DIFFERENTIATED THYROID CARCINOMA \\ Nikolai Raikov', Diana Vyagova², Svetoslav Todorov², \\ Daniela Malcheva ${ }^{2}$, Snezhana Vicheva ${ }^{3}$, Asya Raykova $^{4}$, \\ Borislav Chaushev ${ }^{5}$, Boyan Nonchev 6 \\ ${ }^{1}$ Medical Complex "plus"; Varna, Bulgaria, ${ }^{2}$ Clinical Laboratory „status“; Varna, Bulgaria, ${ }^{3}$ Oncological Dispensary" Marko Markov"; department of Clinical Pathology; Varna, Bulgaria, ${ }^{4}$ Medical University Pleven, \\ ${ }^{5}$ Nuclear Meical Department "Saint Marina" Hospital, Varna, Bulgaria, \\ ${ }^{6}$ Clinic of Endocrinology, Medical University Plovdiv}

\section{Our experience with four patients}

Introduction: The neck recurrence of DTC occurs in about $20 \%$ of all patients in the follow up time. The traditional treatment options for neck lymph node metastases are neck dissection, radioiodine therapy, and external irradiation. But there are some contraindications, that limit the use of these methods. The ethanol ablation is reasonable alternative to the classic methods.

Objectives: To prove that ethanol ablation of single metastatic lymph nodes of differentiated thyroid carcinoma is a possible method of treatment in selected patients, that cause the reduction of their measurements and vascularization.

Methods and Results: The metastatic lymph node selection was made on:

- ultrasound check

- FNA cytology

- TGL in the needle washout.

We present four different cases $/ 8 \mathrm{LN} /$.

The initial vascularization of all nodes disappeared after the PEI sessions.

Complications: local swelling; transitory laryngeal nerve palsy in one $/ 12.5 \% /$ case. 
Table 1. (for Abstract P347)

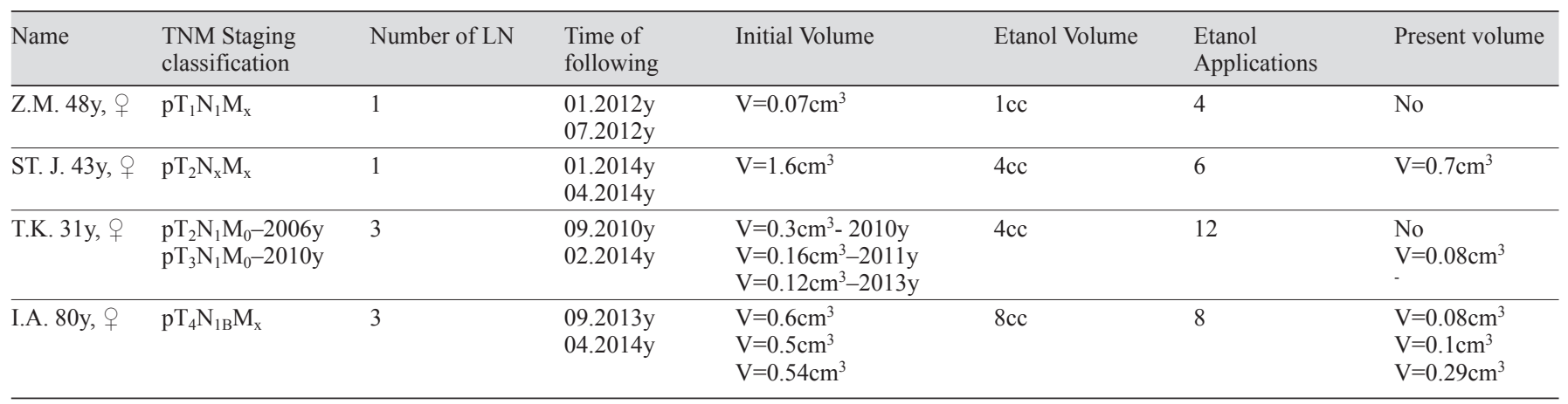

\section{Conclusions:}

- two $/ 25 \%$ / of the ablated LN disappeared.

- the rest nodes reduced their size up to $80 \%$.

- the vascularization of the nodes disappeared.

- serum TGL should not be used as a marker for a successful ethanol ablation of a single node.

The ethanol sclerotherapy of single neck metastatic lymph nodes of DTC is an alternative to surgery, causing the reduction of their size and vascularization in selected group of patients.

\section{P348}

\section{SURVIVAL OF PATIENTS WITH OSSEOUS} METASTASES AS A DEBUT OF DIFFERENTIATED THYROID CARCINOMA: OUR EXPERIENCE

\section{Carmen Caballero Loscos ${ }^{1}$, Milton Emmanuel De Jesús Acosta ${ }^{1}$,}

Astrid Lucia Santos Carreño ${ }^{1}$, Angela Ortega Manrique ${ }^{1}$,

Bernard Theillac Falcones ${ }^{1}$, Jose Manuel Castro Beiras ${ }^{1}$

${ }^{1}$ Nuclear Medicine Department - Hospital Universitario Ramon Y Cajal

Introduction: In 2013 we were asked to administer I-131 treatment to 3 patients with early bone metastases from differentiated thyroid cancer (DTC): 93 year old male, left humerus, folliculary carcinoma. 53 year old female, left femur, poorly differentiated carcinoma. 73 year old female, L4, folliculary carcinoma. Our aim is to review our previous casuistry

Case Report: From 1978 to 2014, 1391 patients with DTC have been treated with I-131. From these, 15 debuted with bone metastases, of which 3 were diagnosed in 2013 that currently have good quality of life.

The other 12 were diagnosed between 1985 and 2004; 7 follicular and 5 papillary carcinomas.

Median age: $62 \pm 10$ years

5 patients with vertebral metastases, 4 in hip, 2 in cranial vault and 1 in scapula.

Patients received an average of 4.17 $\pm 2.12 \mathrm{I}-131$ treatments and a total of $956 \pm 406 \mathrm{mCi}$ of I-131. Survival was $5.17 \pm 3.38$ years (range: between $1-12$ years) $16.6 \%$ lived 10 years or more ( 2 patients)

$58.3 \%$ lived 5 years or more (7 patients)

$91.6 \%$ lived 2 years or more (11 patients)

Only one patient did not come to control a year after beginning of treatment. It is unknown if because of death or other causes.

All patients had titles of thyroglobulin $\geq 500 \mathrm{ng} / \mathrm{ml}$.

Conclusions:

Around $60 \%$ of patients lived more than 5 years.

Longest survival was 12 -years No serious side effects were seen with I-131 treatment, despite having passed the $600 \mathrm{mCi}$ dosage in many patients. I-131 causes fibrosis in the lesion and an antialgic palliative effect in the majority of cases. Bone metastases must be treated with I-131 provided they retain the ability to capture iodine, keeping a time interval between treatments of at least 1 year.

\section{P349 \\ PROPHYLACTIC CENTRAL NECK DISSECTION IN BRAF POSITIVE PAPILLARY THYROID CANCER \\ Dmitriy Semenov ${ }^{1}$, Marina Boriskova ${ }^{1}$, Uliana Farafonova ${ }^{1}$, Irina Zinkevich ${ }^{2}$, Mikhail Bikov ${ }^{1}$ \\ ${ }^{1}$ Pavlov First Saint Petersburg State Medical University, General \\ Surgery Department, ${ }^{2} 31$ St. Petersburg City Hospital}

Routine prophylactic central neck dissection (pCND) after total thyroidectomy (TTX) in papillary thyroid cancer (PTC) does not state by majority of guidelines, but it offers the potential to decrease the disease recurrence/ persistence in BRAF positive PTC. In our previous retrospective study, we found strong correlation between BRAF mutation status and aggressive features of PTC

We aimed to determine the indication for pCND in PTC and to study effectiveness of laryngeal nerve monitoring in predicting postoperative laryngeal mobility.

Design and Patients: We undertook a prospective study of 102 patients treated for PTC from 2011 to 2014 in Pavlov First Saint Petersburg State Medical University. The BRAF V600E mutation status was analyzed in US guided fine-needle biopsy (FNAB) specimens using the allele-specific PCR method. Univariate and multivariate analyses were performed to investigate the association of the BRAF V600E mutation with clinical features. We performed pCND under laryngeal nerve monitoring in case of positive BRAF status.

Results: The occurrence of BRAF mutation in our study is 58, $8 \%$ (60 samples from 102). With use of univariate analyses, invasive growth $(\mathrm{P}=0$, 00875), multifocality $(\mathrm{P}=0.00184)$, and lymph node metastasis $(\mathrm{P}=0.000)$ were independently correlated with presence of BRAF V600E mutation. However, the frequency of each clinic-morphological feature is $40,5 \%, 33 \%$ and $75,6 \%$, accordingly. Using multivariate analyses at least one of aggressive clinic-morphological features could be detected with frequency $75,6 \%$ $(p=0.000)$ in case of BRAF positive PTC. Using the intraoperative laryngeal nerve monitoring in $\mathrm{pCND}$ let us to avoid specific complication in all cases.

Conclusion: Appropriate volume for the BRAF positive PTC is thyroidectomy with pCND. In order to avoid recurrent laryngeal nerve lesions and preserve nerve function intraoperative neuromonitoring should be used. 


\section{P350 \\ ENDOSCOPIC THYROIDECTOMY VIA RETROAURICULAR APPROACH}

Min Woo Park ${ }^{1}$, Soon-Young Kwon ${ }^{2}$, Jeong-Soo Woo ${ }^{2}$, Seung-Kuk Baek ${ }^{1}$, Jae-Gu Jo², Si-Youn Song ${ }^{3}$, Kwang Jin Jung ${ }^{2}$, Kwang-Yoon Jung ${ }^{1}$

${ }^{1}$ Department of Otolaryngology-Head and Neck Surgery, Korea University College of Medicine; Thyroid Center, Korea University Medical Center, ${ }^{2}$ Department of Otolaryngology-Head and Neck Surgery, Korea University College of Medicine, ${ }^{3}$ Department of Otolaryngology-Head and Neck Surgery, Yeungnam University College of Medicine

Introduction: There has been a surge of interest in developing variable endoscopic approaches to the thyroid gland. In the recent, endoscopic thyroidectomy via retroauricular approach was introduced to reduce the invasiveness and use familiar dissection plane for head and neck surgeons. In this study, we report our initial experience to evaluate the feasibility and safety of endoscopic thyroidectomy via a retroauricular approach.

Method \& Materials: We performed 45 cases of endoscopic thyroidectomy via a retroauricular approach from May to December 2013. The operative procedure was performed under endoscopic assistance via a retroauricular hairline incision. After making invisible retroauricular incision, subplastysmal skin flap was elevated to the midline of anterior neck. Retracting sternocleidomastoid muscle laterally, thyroid gland was exposed after elevation of omohyoid muscle and sternothyroid muscle. The thyroid lobe was dissected from upper pole to lower pole with ultrasonic shears and excised after careful identification of the recurrent laryngeal nerve and parathyroid glands.

Results: The mean age of the patient was 43.7 years. Forty three thyroid lobectomies and 3 total thyroidectomies were performed. Postoperative pathology showed 38 papillary thyroid carcinoma, 3 Hurthle cell adenoma, 2 nodular hyperplasia, 1 follicular adenoma and 1 hashimoto's thyroiditis. The mean operating time was $152 \pm 48 \mathrm{~min}$. The mean amount of postoperative drainage was $149 \pm 90 \mathrm{~mL}$, and the mean duration of drain was $4.6 \pm 1.8$ days. The postoperative hospital stay was $3.1 \pm 1.6$ days. There were no cases of conversion to open surgery. One temporary vocal fold paralysis was observed, but hypocalcemia or facial nerve palsy were not observed postoperatively. Four cases of hematoma were identified. The cosmetic outcomes were excellent and all patients were satisfied.

Conclusion: Endoscopic thyroidectomy via a retroauricular approach is a safe and feasible and less invasive option for selected patients.

\section{P351 \\ PHASE 3 STUDY OF (E7080) LENVATINIB IN DIFFERENTIATED CANCER OF THE THYROID (SELECT): RESULTS AND SUBGROUP ANALYSIS OF PATIENTS FROM EUROPE}

Kate Newbold ${ }^{1}$, Bruce Robinson ${ }^{2}$, Martin Schlumberger ${ }^{3}$, Makoto Tahara ${ }^{4}$, Marcia F. Brose ${ }^{5}$, Rossella Elisei ${ }^{6}$, Corina E. Dutcus ${ }^{7}$, Begoña de las Heras ${ }^{8}$, Junming Zhu' ${ }^{7}$, Mouhammed Amir Habra ${ }^{9}$, Manisha H. Shah ${ }^{10}$, Ana O. Hoff'1, Andrew G Gianoukakis ${ }^{12}$, Naomi Kiyota ${ }^{13}$, Matthew H. Taylor ${ }^{14}$, Sung-Bae Kim ${ }^{15}$, Monika K. Krzyzanowska ${ }^{16}$, Steven I. Sherman ${ }^{9}$, Lori Wirth ${ }^{17}$ ${ }^{1}$ The Royal Marsden Hospital National Health Service Trust, London, England, UK, ${ }^{2}$ Kolling Institute of Medical Research, University of Sydney, New South Wales 2006, Australia, ${ }^{3}$ Department of Nuclear Medicine and Endocrine Oncology, Gustave Roussy and Université Paris-Sud, Villejuif, France, ${ }^{4}$ Department of Head and Neck Medical Oncology National Cancer Center Hospital East, Kashiwa, Japan, ${ }^{5}$ Department of Otorhinolaryngology: Head and Neck Surgery, Abramson Cancer Center of the University of Pennsylvania, Philadelphia, $\mathrm{Pa}, \mathrm{USA},{ }^{6}$ Endocrine Unit, Department of Clinical and Experimental Medicine, University of Pisa, Pisa, Italy, ${ }^{7}$ Eisai Inc, Woodcliff Lake, Nj, USA, ${ }^{8}$ Eisai Ltd, Hatfield, England, UK, ${ }^{9}$ Department of Endocrine Neoplasia and Hormonal Disorders, Division of Internal Medicine, The University of Texas M. D. Anderson Cancer Center, Houston, TX, USA, ${ }^{10}$ Department of Internal Medicine, The Ohio State University Comprehensive Cancer Center, Columbus, Oh, USA, ${ }^{11}$ Instituto Do Cancer de Sao Paulo, Universidade de Sao Paulo, Sao Paulo, Brazil, ${ }^{12}$ Division of Endocrinology and Metabolism, HarborUcla Medical Center, Torrance, Ca, USA, ${ }^{13}$ Department of Medical Oncology and Hematology, Kobe University Hospital, Kobe, Japan, ${ }^{14}$ Knight Cancer Institute, Oregon Health and Science University, Portland, Or, USA, ${ }^{15}$ Department of Oncology, Asan Medical Center, University of Ulsan College of Medicine, Seoul, Korea, ${ }^{16}$ Division of Medical Oncology \& Hematology, Princess Margaret Cancer Centre, Uhn 610 University Avenue, Toronto, Ontario, Canada, ${ }^{17}$ Department of Medicine, Massachusetts General Hospital, Boston, MA, USA

Objectives: To assess the efficacy and safety of lenvatinib - an oral tyrosine kinase inhibitor of VEGFR1-3, FGFR1-4, PDGFR $\alpha$, RET, and KIT signaling - compared to placebo in patients with ${ }^{131}$ I-refractory differentiated thyroid cancer (RR-DTC), and to report treatment outcomes for the European subpopulation of the phase 3 Study of (E7080) LEnvatinib in Differentiated Cancer of the Thyroid (SELECT).

Methods: This phase 3, double-blind, placebo-controlled, multicenter study enrolled patients with documented, progressive RR-DTC (within prior 13 months), who were randomized 2:1 to lenvatinib $(24 \mathrm{mg} / \mathrm{d} ; 28-\mathrm{d}$ cycle) or placebo. The primary endpoint was progression-free survival (PFS). Secondary endpoints were overall survival (OS), objective response rates (ORR), and adverse events (AEs).

Results: Overall, 392 patients received lenvatinib $(n=261)$ or placebo $(n=131)$. Patients receiving lenvatinib demonstrated a significant PFS benefit vs placebo: median PFS was 18.3 vs 3.6 months, respectively (hazard ratio [HR] $0.21 ; 99 \%$ confidence interval [CI] $0.14-0.31 ; P<0.001)$. OS was not reached in either arm. ORR were: lenvatinib, $64.8 \%$ (4 complete, 165 partial responses); placebo, $1.5 \%$. Lenvatinib median time to response was 2.0 months. The lenvatinib PFS benefit was also evident among 195 European patients (lenvatinib, $\mathrm{n}=131$; placebo, $\mathrm{n}=64$ ); median PFS was: lenvatinib, 18.7 months; placebo, 3.7 months (HR 0.24 ; $95 \%$ CI $0.16-0.35$ ). Lenvatinib median OS was not reached (NR); placebo median OS was 20.3 months $(95 \%$ CI 15.1-NR). Treatment-related AEs (TRAEs) were experienced by $97.7 \%$ of lenvatinib-treated European patients (Grade $\geq 3,72.5 \%$ ). Dose modifications in the European subgroup comprised: dose reductions, $64.9 \%$; dose interruptions, $83.2 \%$; and discontinuations due to AEs, $17.6 \%$. The 5 most common TRAEs (any grade) in the overall lenvatinib arm were hypertension (68\%), diarrhea (59\%), decreased appetite $(50 \%)$, decreased weight $(46 \%)$, and nausea $(41 \%)$.

Conclusion: The efficacy benefit and safety profile of lenvatinib were similar in European patients with RR-DTC compared with the overall study population. 


\section{P352}

OVERALL SURVIVAL IN ANAPLASTIC CANCER GROUP OF ROMANIAN PATIENTS

\section{Chelaru lustina Cosmina ${ }^{1}$, Niculescu Dan Alexandru²,}

Ioachim Dumitru', lorgulescu Radu ${ }^{3}$, Poiana Catalina

1"ci Parhon" National Institute of Endocrinology, Bucharest, Romania,

2"ci Parhon" National Institute of Endocrinology, Bucharest, Romania;

Department of Endocrinology, Carol Davila University, Bucharest,

Romania, ${ }^{3} \mathrm{Sf}$ loan" Emergency Hospital Surgery Department

Bucharest, Romania, ${ }^{4}$ Department of Endocrinology, Carol Davila

University, Bucharest, Romania; "ci Parhon" National Institute of

Endocrinology, Bucharest, Romania

Introduction: Anaplastic thyroid cancer (ATC) is a highly lethal form of thyroid neoplasia, accounting for $1-3 \%$ of all thyroid cancers. It contributes to $14-50 \%$ of the annual mortality associated with thyroid cancer.

Subjects and Methods: We retrospectively reviewed all the ATC cases diagnosed on $\mathrm{FNAB} /$ pathology examination in a university clinic between 2007-2013(16 cases). We collected data on demographics, laboratory tests, imaging studies, FNAB/pathology reports, treatment and survival time. Fourteen patients underwent FNAB. Pathology reports were available for 5 and IHC for 3 patients. Six patients had total thyroidectomy. Chemotherapy ( 3 patients) and radiotherapy ( 4 patients) were performed in tertiary oncology centers.

Results: The median patient's age at diagnosis was 72 years $(61.5,73.25)$ old; men/woman 3:5. Weight loss was a complaint in $37.5 \%$ (average 6.83 $\mathrm{kg}$ ). TSH levels ranged between undetectable- $22.3 \mathrm{uUI} / \mathrm{L}$, with a median of 1. Besides the large neck mass, the presentation complaints were dyspnea in 6 and dysphagia in 8 patients; vocal cords were affected in 7 cases. $43.76 \%$ of the patients presented with unresectable primary tumors, with a median size of the tumor $6.8 \mathrm{~cm}$. Nodal involvement was found in 13 patients at presentation. Distant metastases were present in $81.25 \%$.

7 patients had surgery, 4 received radiation therapy ( $40 \mathrm{~Gy}$ ), and 3 patients received chemotherapy. The overall survival rate had an average of 7.968 months median 2.375 months $\left(25^{\text {th }}\right.$ percentile $=1.062,75^{\text {th }}$ percentile $\left.=13.25\right)$, with a minimal 0.25 and a maximum of over 22 months survival.

Discussion: Radiation therapy turned out to be useful, our three patients having survival times of 12, 14 and 27 months. Chemotherapy probably increased the survival rate given that the three patients that were treated lived after the diagnosis 13 to 30 months, surpassing the median survival $(2.325$ months). Given the very different survival rates we must search forward the missing piece of this puzzle.

\section{P353 \\ PAPILLARY THYROID CARCINOMA IN CHILDREN AND ADOLESCENTS - A 34-YEAR EXPERIENCE IN 227 PATIENTS - \\ Kiminori Sugino $^{1}$, Mitsuji Nagahama ${ }^{1}$, Wataru Kitagawa ${ }^{1}$, Hiroshi Shibuya ${ }^{1}$, Keiko Ohkuwa ${ }^{1}$, Takashi Uruno ${ }^{1}$, Akifumi Suzuki $^{1}$, Junko Akaishi ${ }^{1}$, Chie Masaki ${ }^{1}$, Koichi Ito \\ ${ }^{1}$ Ito Hospital}

Background: The aim of this study was to analyze clinical features and clinical outcomes of papillary thyroid carcinoma (PTC) in the pediatric and adolescent population treated in our institution.

Methods: From 1979 to 2012, 227 patients aged 20 years or younger (201 females, female: male ratio of $8: 1$ ) with PTC who underwent initial surgery in our institute, were the subjects of this study. The mean age at diagnosis was 18 year old (range 7-20 year). There were 52 children (aged 15 or younger) and 175 adolescents (aged 16 or over). Cause-specific survival (CSS) and diseasefree survival (DFS) were calculated by Kaplan-Meier method for the following variables: age at surgery, gender, tumor size, preoperatively diagnosed lymph node metastasis (LNM), histological LNM, initial distant metastasis $(\mathrm{DM})$, and extrathyroidal invasion. Differences between groups were analyzed for significance by the log-rank test. Multivariate analysis was performed by using the Cox proportional hazard model.

Results: Two patients died of the disease and 45 patients had recurrent disease (36 lymph node, 7 remnant thyroid, 11 distant metastases). The 10-year, 20 -year and 30 -year CSS were $99.3 \%, 99.3 \%$ and $96.5 \%$, respectively and the 10 -year, 20-year and 30-year DFS were $83.6 \%, 70.7 \%$ and $64.0 \%$, respectively.
Thirty one patients had DM, all of which were lung metastases. Radioactive iodine therapy was performed for 29 patients with DM and response rate was $69 \%$. Significant factors related to DFS in the univariate analyses were gender, preoperative diagnosed LNM, histological LNM, and extrathyroidal invasion. Gender and preoperative LNM were significant factors related to DFS in the multivariate analysis.

Conclusion: Prognosis of juvenile PTC was fair, but recurrences were frequently observed. Conservative therapy is considered acceptable for patients without risk factors while total thyroidectomy and neck dissection should be considered for more extensive tumors.

\section{P354 \\ LONG-TERM FOLLOW-UP RESULTS AFTER LOBECTOMY FOR THYROID PAPILLARY CARCINOMA}

Seung Ook Hwang ${ }^{1}$, Jin O Baek', Jin Koo Kang ${ }^{1}$, Jeeyeon Lee', Wan Wook Kim ${ }^{1}$, Jin Hyang Jung ${ }^{1}$, Ho Yong Park ${ }^{1}$

${ }^{1}$ Kyungpook National University, School of Medicine

Purpose: The necessity and gold standard of completion thyroidectomy after lobectomy in patients with papillary thyroid cancer is still controversial. This study analyzed long-term follow-up results after lobectomy and searched for adequate criteria of completion thyroidectomy

Method: Retrospectively, 281 patients who had lobectomy as the initial surgery for low risk papillary thyroid carcinoma in our institution between 2001 and 2008 were enrolled. The follow-up interval was 1 year at least. Age, sex, surgical method, histologic type, tumor size, extrathyroidal extension, lymph node metastasis, recurrence, survival, follow-up interval were investigated

Results: Mean tumor size was $0.69 \pm 0.43$ (range, $0.05-4.0) \mathrm{cm}$. Follicular variant type was $5 \%$ and classical type was $95 \%$. Two hundred and twenty (78.3\%) patients had lobectomy with ipsilateral central lymph node dissection and the others had only lobectomy. The rate of lymph node metastasis was $25.5 \%(56 / 220)$ in lymph node dissection group. Ninety-one (32.4\%) patients had extrathyroidal extension. Mean follow-up interval was $79.7 \pm 28.8$ (range, 12.0-153.6) month. There were $9(3.2 \%)$ recurrences; $4(1.4 \%)$ in remnant thyroid, $2(0.7 \%)$ in ipsilateral paratracheal lymph node, $3(1.1 \%)$ in ipsilateral lateral lymph node. There was no death. Five-years cumulative recurrence rate (CRR) was about $1.8 \%$ and ten-years CRR was about $3.1 \%$. There was no difference in CRR according to lymph node dissection or lymph node metastasis. Extrathyroidal extension ( $\mathrm{p}=0.020$, OR $6.506,95 \%$ CI $1.336-31.67$ ) was only significant risk factor for recurrence by Cox proportional hazard analysis.

Conclusion: Extrathyroidal extension was a risk factor for recurrence after lobectomy for low risk papillary thyroid carcinoma. However, recurrence was rare and regional. High risk group of recurrence might be manageable with close follow-up and surgery after recurrence rather than immediate completion surgery.

\section{P355 \\ THYROID REMNANT ABLATION WITH DIFFERENT METHODS OF PATIENT PREPARATION}

Sanja Kusacic Kuna ${ }^{1}$, Martina Ciglar Hlasc ${ }^{1}$, Marija Despot ${ }^{1}$, Gordana Horvatic Herceg ${ }^{1}$, Tatjana Samardzic ${ }^{1}$, Drazen Huic ${ }^{1}$ ${ }^{1}$ Clinical Department of Nuclear Medicine and Radiation Protection, Clinical Hospital Centre Zagreb, Croatia

Objective: Succesfull radioablation of thyroid remnants after total thyreoidectomy due to thyroid carcinoma requires an elevated thyroid-stimulating hormone (TSH) level achieved either by endogenous stimulation after withdrawal of replacement therapy or by administration of human recombinant thyrotropin (rhTSH). The aim of study was to compare the rate of ablation with two modalities of patient preparation with same activity of radioiodine $(1850 \mathrm{MBq}, 50 \mathrm{mCi})$.

Patients and Methods: The study included 77 patients (median age 49 years; range 16-78 years) with intrathyroid papillary carcinoma without any sign of metastatic disease at the moment of ablation. Patients were divided into two groups according to method of preparation. The first group included 42 patients who received ablation doses in hypothyroid state. Second group $(n=35)$ were placed on thyroxine after surgery and were treated in euthyreoid 
state after preparation with rhTSH. The outcome of ablation was assessed by conventional whole body-scan, and by serum thyroglobulin testing performed in hypothyroid state 6-9 months after ablation and finally 12 months after. Results: The great majority of patients (almost 99\%) had accumulation of activity in thyroid bed on postablative whole body scan. There was no significant difference between rates of ablation in these two groups neither at first nor at the second control. The radioablation was considered successful in $88.6 \%$ of patients prepared by recombinant human TSH, and $88.1 \%$ of those prepared by hormone withdrawal. No serious adverse events were related to recombinant TSH administration.

Conclusion: The study demonstrates comparable remnant ablation rates in patients prepared by either administering rhTSH or withholding thyroid hormone. In both cases $1850 \mathrm{MBq}(50 \mathrm{mCi})$ dose of radioiodine was sufficient for a satisfactory thyroid ablation rate.

\section{P356 \\ BONE MINERAL DENSITY AND BONE TURNOVER MARKERS IN PATIENTS WITH THYROID CANCER AND L-T4 SUPPRESSIVE THERAPY AFTER 25 YEARS OF FOLLOW UP \\ Maria Luisa De Mingo ${ }^{0}$, Sonsoles Guadalix ${ }^{1}$, \\ Guillermo Martinez Diaz Guerra ${ }^{1}$, Federico Hawkins ${ }^{1}$ \\ 112 de Octubre Hospital}

Background: Differentiated thyroid cancer (DTC) is treated with L-thyroxine (LT4) in relatively hight doses to suppress endogenous thyroidstimulating hormone (TSH) levels. Very long-term effect of thyroid hormones supplementation on bone is controversial.

Objetive: To study the effects on bone mineral density (BMD) and bone markers in DTC patients followed.

Methods: 46 postmenopausal women(age $62.85 \pm 11.29$ years) with DCT on TSH suppressive treatment for $27.72 \pm 4.04$ years were classified in: Group $1=$ premenopausal and Group $2=$ postmenopausal. BMD was measured by DXA at baseline (year 1992) and at the present time at lumbar spine (LS), Total hip (TH), femoral neck (FN) and ultradistal radius (UDR). Serum bone turnover markers ( $\beta$-CTX, osteocalcin), PTH and $25 \mathrm{OH}$ vitaminD were measured.

Results: Group 1: $\mathrm{n}=27$, age 56.3 \pm 8.4 ; Group 2: $\mathrm{n}=19$, age 72.1 \pm 7.8 . Compared with baseline, we found a statistically significant decrease in BMD at $\operatorname{LS}(p=0.000)$ and $\mathrm{FN}(\mathrm{p}=0.000)$ in group 1. No significant changes were found in group 2. \% of BMD change were: Group 1: LS -6.23 $\pm 11 \% ; \mathrm{FN}$ $-8 \pm 12 \%$; TH $3.2 \pm 12 \%$;UDR $2.7 \pm 7.7 \%$. Group 2:LS 3.6 $\pm 19 \%$; FN $-8.5 \pm 12.2 \%$; TH $-5 \pm 16.8 \%$; UDR $7.5 \pm 3.5 \%$. At the second DXA, group 2 had lower BMD than group 1 at all localizations (at TH and UD statistically significant). Osteoporotic women were $39.1 \%$ (total), $22.2 \%$ (group 1 ) and $63.2 \%$ (group 2 ). No correlation was found between BMD and TSH, LT4 dose, PTH, $\beta$-CTX or vitamin D. Only osteocalcin showed a negative correlation with BMD at $\operatorname{UDR}(\mathrm{r}=-0.58, \mathrm{P}<0.01)$ and $\mathrm{TH}(\mathrm{r}=-0.51, \mathrm{p} 0.04)$ in group 1 .

Conclusion: Very long-term LT4 suppressive therapy in women with DTC does not induce severe bone loss. Patients that began therapy in premenopausal status had lower BMD compared to postmenopausal women at the end of the study.

\section{P357 \\ PREDICTION OF THE SUCCESS OF THYROID REMNANT ABLATION USING PRE-ABLATIVE TC-99M PERTECHNETATE SALIVARY GLAND SCINTIGRAPHY AND POST-ABLATIVE DUAL I-131 SCINTIGRAPHY \\ Sang Mi Lee ${ }^{1}$, Jeong Won Lee ${ }^{2}$, Jo Sung Jung ${ }^{3}$ \\ ${ }^{1}$ Department of Nuclear Medicine, Soonchunhyang University \\ Hospitall, ${ }^{2}$ Department of Nuclear Medicine, Yonsei University College of Medicine, ${ }^{3}$ Department of Radiology, Soonchunhyang University Hospital}

Purpose: The aim of this study was to predict the success of I-131 ablation according to the results of pre-ablative Tc- $99 \mathrm{~m}$ pertechnetate salivary scintigraphy and post-ablative dual I-131 whole-body scan in patients with differentiated thyroid cancer (DTC).
Methods: Among DTC patients who underwent I-131 ablation after total thyroidectomy, 168 DTC patients who underwent pre-ablative salivary scintigraphy and post-ablative dual (early and delayed) I-131 scan were retrospectively enrolled. The success of thyroid ablation was assessed after 12 months. For salivary scintigraphy, the thyroid remnant uptake was visually assessed. In the dual I-131 scan, the pattern of thyroid remnant uptake was classified as increased, equal, or decreased after comparing the early and delayed thyroid remnant uptake.

Results: Of the168 patients, $69(41 \%)$ had thyroid remnant uptake on salivary scintigraphy. The success rate of ablation was significantly higher in patients with negative thyroid remnant uptake on salivary scintigraphy $(86 \%)$ than in patients with positive thyroid remnant uptake $(58 \% ; \mathrm{p}=0.0001)$. The success rate of ablation was significantly higher in patients with increased or equal thyroid remnant uptake pattern than in patients with decreased uptake pattern on the dual I-131 scan, irrespective of the remnant uptake on salivary scintigraphy $(93 \%$ vs. $71 \%, \mathrm{p}=0.01$ for patients without remnant uptake on salivary scintigraphy; $73 \%$ vs. $46 \%, p=0.04$ for patients with remnant uptake on salivary scintigraphy). The presence of thyroid remnant uptake on salivary scintigraphy $(p=0.0001)$, uptake pattern on dual I-131 scan $(p=0.0003)$ and pre-ablative serum $\mathrm{Tg}$ level $(\mathrm{P}<0.0001)$ were significant predictive factors for the success of ablation.

Conclusion: The success of thyroid remnant ablation in DTC patients can be predicted by the presence of thyroid remnant uptake on pre-ablative salivary scintigraphy and pattern of thyroid remnant uptake on post-ablative dual I-131 scan.

\section{P358 \\ LARYNGEAL PRESERVATION BY VERTICAL HEMILARYGOPHARYNGECTOMY (VHLP) IN LOCALLY ADVANCED THYROID CANCER \\ Min Sik Kim ${ }^{1}$, Young Hoon $\mathrm{Joo}^{2}$, Kwang Jae $\mathrm{Cho}^{2}$, Sang Yun Kim², \\ Byung Jun Chun ${ }^{2}$ \\ ${ }^{1}$ The Catholic University of Korea; Seoul St. Mary's Hospital, ${ }^{2}$ The \\ Catholic University of Korea}

Background: The surgical treatment of advanced hypopharynx or thyroid cancer with infiltration of the laryngeal skeleton often needs total laryngectomy for oncologic safety and functional reasons, although tumor infiltration is limited to the half of the larynx. We have performed VHLP in locally advanced laryngopharyngeal cancer to preserve the laryngeal function by the reconstruction of radial forearm free flap with tendon and we also have experienced 3 cases of locally advanced thyroid cancer.

Subjects and Methods: We have applied VHLP in locally advanced thyroid cancer with laryngeal invasion in three cases which are needs to take a total laryngectomy, and we evaluate oncological and functional results.

Case Report: All of three patients had advanced thyroid cancer with laryngeal invasion. Spindle cell carcinoma, papillary carcinoma and anaplastic carcinoma of thyroid with paraglottic space invasion. In the case of anaplastic carcinoma, the cancer was recurred locally and distant metastasis, the patient died 2 months after surgery and other two cases were NED state.

Coclusion: Vertical hemilaryngopharyngectomy is one of the good surgical procedure to preserve the laryngeal function in the surgical treatment of locally advanced head and neck cancer especially invading into the larynx. In selected cases of thyroid cancer, this method could be a useful option for preserving the function of the larynx. 


\section{P035 Biochemical and Metabolic Parameters}

\section{P359 \\ STRUCTURAL AND FUNCTIONAL STATE OF THYROID GLAND IN PATIENTS WITH METABOLIC SYNDROME}

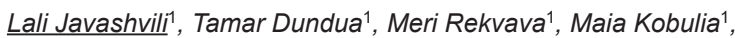

Nino Jakhaia ${ }^{1}$, Medea Papava ${ }^{2}$

${ }^{1}$ Clinic Cortex; Tbilisi, Georgia, ${ }^{2}$ Research Institute of Clinical Medicine; Tbilisi, Georgia

Objectives: The aim of this research was to evaluate functional and structural state of the thyroid gland in patients with metabolic syndrome.

Materials: We have investigated 75 patients with metabolic syndrome (48 female, 27 male, the age -22 to 65 ). All patients had BMI $>=35$. Arterial pressure was $>130 / 80 \mathrm{~mm} / \mathrm{Hg}$. C-peptide range was $2.4 \mathrm{ng} / \mathrm{dl}-7.03 \mathrm{ng} / \mathrm{dl}$. Blood glucose range $78 \mathrm{mg} / \mathrm{dl}-270 \mathrm{mg} / \mathrm{dl}$. All patients had dyslipidemia, the elevation of triglicerides quantity was the most often ( $>240 \mathrm{mg} / \mathrm{dl})$, HDL was $<30 \mathrm{mg}$ / dl. By the moment of admission 12 patients had already been diagnosed with thyroid pathologies - receiving substitutional therapy (levothyroxine 25-50 $\mu \mathrm{g})$. In the rest of the patients $(n=63)$ thyroid ultrasound was performed to evaluate thyroid structure and blood tests were done to evaluate thyroid function (TSH, FT4 levels) and Anti-thyroid antibodies levels.

Results: The study revealed that the structure was nonhomogenous in $54 \%$ of patients $(n=34)$, in the $46 \%$ the structure was normal. Thyroid nodules were found in $36.5 \%$ of patients $(n=23)$, of which in 13 patients it was a single nodule, in 10 patients 2 or more nodules. The diameter of nodules $<10 \mathrm{~mm}$ in 12 patients and $>10 \mathrm{~mm}$ in 11 . The nodule structure was complex in 4 patients, isoechoic in 6, hypoechoic in 11 and hyperechoic in 2 patients. TSH was elevated in $34.9 \%$, decresed in $3.2 \%$ and within N/L (normal limits) in the rest of the patients, (N/L 0.4-4.0 mU/1). $\mathrm{FT}_{4}$ secretion was decreased in $19 \%$ of patients, increased in $1.6 \%$, and within $\mathrm{N} / \mathrm{L}$ in the rest of the patients (N/L 9-23 pmol/1). Anti-TPO was elevated in 6.3\%, anti-TG in only $1.6 \%$.

Conclusion: According to our data a large number of patients with metabolic syndrome were diagnosed with some pathology of the thyroid gland. Based on this conclusion we consider to perform routine investigation of thyroid structure and function in patients with metabolic syndrome.

\section{P360}

SERUM FREE THYROXINE CONCENTRATION IS ASSOCIATED WITH METABOLIC SYNDROME IN EUTHYROID SUBJECTS

Ladan Mehran ${ }^{1}$, Atieh Amouzegar ${ }^{1}$, Maryam Tohidi², Fereidoun Azizi ${ }^{1}$

${ }^{1}$ Endocrine Research Center, Research Institute for Endocrine

Sciences, Shahid Beheshti University of Medical Sciences, Tehran, I.

R. Iran, ${ }^{2}$ Prevention of Metabolic Disorders Research Center, Research Institute for Endocrine Sciences, Shahid Beheshti University of Medical Sciences, Tehran, I. R. Iran

Context: The association between thyroid hormones within euthyroid range and metabolic syndrome is less clear.

Aim: This study evaluated the relationship between thyroid hormones and metabolic syndrome and its components in euthyroid subjects.
Design and Setting: This is a cross-sectional population based study conducted within the framework of the cohort of Tehran Thyroid Study.

Participants: Out of 5786 subjects aged $\geq 20$ years, 3724 euthyroid subjects without history of thyroid disorders, diabetes, steroid consumption and cardiovascular diseases were investigated.

Outcome Measures: Body weight, waist circumference (WC) and blood pressure (BP) were measured. Serum concentrations of lipids and lipoproteins, FBS, insulin, FT4 and TSH were assayed. Metabolic syndrome was determined by definition of the Joint Interim Statement (JIS) adjusted for Iranian population.

Results: After adjustment for age and sex, serum FT4 was significantly associated with HDL-C, LDL-C, TG, WC and SBP; the associations which remained significant after further adjustment for HOMA-IR except for HDLC. Serum TSH was associated only with TG after age and sex adjustment, an association which disappeared after further adjustment for HOMA-IR. Both serum FT4 $(\beta=-0.02$, 95\%CI: $-0.03,-0.01)$ and TSH $(\beta=0.03,95 \% \mathrm{CI}$ : $0.01-0.04)$ were associated with insulin resistance. The prevalence of MetS decreased from $30.4 \%$ in the lowest FT4 tertile to $22.5 \%$ in the highest FT4 tertile $(\mathrm{P}<0.001)$. The prevalence of other MetS components increased significantly from higher to lower FT4 tertile. Higher normal FT4 values were associated with lower odds of metabolic syndrome $[\mathrm{OR}=0.57,95 \%$ CI $0.36,0.92$; $\mathrm{p}=0.02]$. Second (OR 0.79, 95\% CI 0.66.0.96; $\mathrm{P}=0.02)$ and third (OR 0.77, $95 \%$ CI 0.63.0.93; P = 0.009) FT4 tertiles were also associated with lower odds of metabolic syndrome with reference to the first tertile. Sub analysis in negative TPOAb euthyroid subjects indicated similar results.

Conclusion: In euthyroid subjects FT4, rather than TSH, is associated with risk of metabolic syndrome and its components.

\section{P361 \\ NORMAL RANGES FOR THYROID HORMONES AGE-ADJUSTED \\ Piedad Santiago ${ }^{0}$, Pablo Olmedo $^{1}$, Carmen Gutierrez ${ }^{1}$, Cristina Castillo ${ }^{1}$, Maria Jose Martinez ${ }^{1}$, Manuel Gasso ${ }^{1}$ ${ }^{1} \mathrm{Ch}$ Jaen}

Objectives: To define normal ranges for thyroid hormones, age-adjusted, and the prevalence of autoimmune thyroid disease (ETA) in a healthy population.

Methods: Descriptive, cross-sectional study; population: subjects included had no any events that potentially could affect the measurement of serum thyroid hormones levels. Variables: age, sex, TSH, FT4, TPO, TSI; Statistical analysis: Descriptive statistics were used and ANOVA to correlate dependent variables.

Results: 584 women and 427 men were included in six different age groups. Hormonal study: mean TSH was $2.20 \mathrm{uUI} / \mathrm{ml}(0.01-48.02 \mathrm{uUI} / \mathrm{ml})$, mean FT4 was $0.85 \mathrm{ng} / \mathrm{dl}(0.25-1.39 \mathrm{ng} / \mathrm{dl})$. Percentile distribution according to age groups is described in the table below (subjects with positive TPO excluded). TSH was significantly higher in women $(2.25 \mathrm{uUI} / \mathrm{ml}$ versus 1.97 $\mathrm{uUI} / \mathrm{ml} ; \rho=0.009) .61$ participants $(6 \%)$ had TPO $(+)$ and 19 participants $(2.4 \%)$ positive TSI. TPO $(+)$ was significantly associated with female gender $(\rho=0.001)$, but there were no differences of TPO $(+)$ between the different age groups. Patients with TPO $(+)$, had significantly greater TSH values $(3.33 \mathrm{uU} /$ $\mathrm{ml}$ versus $2.12 \mathrm{uU} / \mathrm{ml} ; \rho=0.000)$.

Conclusions: Normal ranges of serum thyroid hormones are similar to the reference levels established by the laboratory. Autoimmune thyroid disease prevalence in this selected population is lower than the rates described in previous studies and it is significantly higher in women than in men. We confirmed that subjects with positive antithyroid antibodies have higher TSH levels.

Table 1. (for Abstract P361)

\begin{tabular}{|c|c|c|c|c|c|c|c|c|}
\hline \multirow[t]{3}{*}{ Age (years) } & \multicolumn{4}{|c|}{ men; $\mathrm{n}=401$} & \multicolumn{4}{|c|}{ women; $\mathrm{n}=518$} \\
\hline & \multicolumn{2}{|l|}{$\mathrm{TSH}$} & \multicolumn{2}{|l|}{ FT4 } & \multicolumn{2}{|l|}{$\mathrm{TSH}$} & \multicolumn{2}{|l|}{ FT4 } \\
\hline & $2.5 \mathrm{P}$ & $97 \mathrm{P}$ & $2.5 \mathrm{P}$ & $97 \mathrm{P}$ & $2.5 \mathrm{P}$ & $97 \mathrm{P}$ & $2.5 \mathrm{P}$ & $97 \mathrm{P}$ \\
\hline $20-30$ & 0.06 & 7.21 & 0.68 & 1.25 & 0.68 & 5.87 & 0.61 & 1.07 \\
\hline $31-40$ & 0.36 & 4.24 & 0.62 & 1.06 & 0.5 & 3.88 & 0.56 & 1.17 \\
\hline Older than 65 & 0.47 & 6.29 & 0.64 & 1.25 & 0.16 & 7.81 & 0.60 & 1.30 \\
\hline
\end{tabular}




\section{P362 \\ THYROID DISORDERS AND LIPID LEVELS IN A COMMUNITY BASED STUDY: TEHRAN THYROID STUDY (TTS)}

Shahram Alamdari ${ }^{1}$, Atieh Amouzegar ${ }^{1}$, Maryam Tohidi',

Safoora Gharibzadeh ${ }^{1}$, Pouyan Kheirkhah ${ }^{1}$, Parnian Kheirkhah ${ }^{1}$,

Fereidoun Azizi

${ }^{1}$ Endocrine Research Center, Research Institute for Endocrine

Sciences, Shahid Beheshti University of Medical Sciences, Tehran, I.

R. Iran, ${ }^{2}$ Prevention of Metabolic Disorders Research Center, Research

Institute for Endocrine Sciences, Shahid Beheshti University of Medical

Sciences, Tehran, I. R. Iran

Background and Purpose: There is a lack of consensus of opinions on universal reference values for thyroid volume and it is recognized that for local populations it is necessary to use and establish their specific reference ranges especially for the purpose of goiter surveys. Our objective was to describe thyroid volume measured by ultrasonography in Tehranian schoolchildren who had been living in iodine sufficient status during their whole life period.

Methods: This cross-sectional study was performed fifteen years after implementing the universal salt iodization in Iran, on 464 Tehranian schoolchildren, aged 7-15 yr. Data were collected on age, sex, weight, height and urinary iodine. Thyroid size was ascertained by palpation and ultrasonography by an expert endocrinologist.

Results: The prevalence of goiter was $4.6 \%$ by palpation. All goitrous subjects had grade 1 goiter. Median urinary iodine was $140 \mu \mathrm{g} / \mathrm{L}$. Thyroid volumes determined by ultrasonography were comparable in boys and girls of all ages. The best predictors of thyroid volume were age, weight, and body surface area. The median thyroid volumes of Tehranian schoolchildren in this study were lower in all age groups compared with our previous 2001 study and updated provisional WHO/ICCIDD reference values.

Conclusions: Tehranian schoolchildren currently living in an iodine sufficient area, where they have spent their entire lives, have smaller thyroid glands than recommended international references. Using population standards rather than universal ones in assessing thyroid volume should be considered.

\section{P363 \\ THYROID GLAND FUNCTION IS ALTERED IN PARKINSON'S DISEASE \\ Emilio Fernandez', Angel Martin de Pablos' ${ }^{1}$, Jose Chacon², Jose-Manuel Garcia-Moreno ${ }^{3}$ \\ ${ }^{1}$ Universidad de Sevilla, ${ }^{2}$ Hospital Infanta Luisa, ${ }^{3}$ Hospital Macarena Sas, Spain}

Objectives: We have detected nitrosative stress in serum of Parkinson's disease patients, and a singular version of nitrosylated serum $\alpha$-synuclein. Dysfunction of the thyroid gland, an organ where a physiological oxidative stress takes place through life, has been linked to this disease. The aim was to know if the thyroid gland is involved in idiopathic Parkinson's disease.

Study Design: We have studied patients and healthy controls. Clinical characteristics and nitrosative stress in patients and controls were studied. ELISA and immunoblotting methods were also employed.

Results: Prevalence of hypo- or hyperthyroidism was not found to be significantly higher in patients than in controls. Levels of thyroperoxydase, thyroid enzyme, were found to be elevated in early-disease patients $(\mathrm{p}<$ $0.01)$. Nitrosative stress was confirmed to be present in early-disease patients through augmented levels of serum 3-nitrotyrosine proteins $(\mathrm{p}<0.05)$, and it was found to be negatively correlated with serum levels of thyroperoxidase $(\mathrm{p}<0.04)$. The singular version of nitrosylated serum $\alpha$-synuclein of earlydisease patients was confirmed to be present. Interestingly, subjects with subtotal thyroidectomy showed lower levels of 3-nitrotyrosine proteins in serum relative to controls, and nitrosylated serum $\alpha$-synuclein was nearly absent.

Conclusions: Elevated levels of thyroperoxidase in early-disease patients and its negative correlation with serum 3-nitrotyrosine proteins support a role for the thyroid gland in Parkinsonian nitrosative stress. Finally, the thyroid gland seems to participate on nitrosylation of blood proteins, including serum $\alpha$-synuclein.

\section{P364 \\ BIOCHEMICAL THYROID DYSFUNCTION IN HOSPITALISED PATIENTS: ANALYSIS OF 270 OOO ADMISSIONS TO A LARGE CENTRE}

Barbara Torlinska', Jamie Coleman ${ }^{1}$, James Hodson², Mariam Afzal', Jayne Franklyn ${ }^{1}$, Kristien Boelaert ${ }^{1}$

${ }^{1}$ University of Birmingham, ${ }^{2}$ Univeristy Hospitals Birmingham NHS

Foundation Trust

Objectives: Routine thyroid function testing (TFT) in hospitalised patients is not recommended; however, delayed management of thyroid dysfunction may have significant consequences. We quantified the frequency of TFT during 270.000 hospital admissions and identified factors influencing the likelihood of overt thyroid dysfunction (TD).

Methods: We analysed data from a computerised system on all admissions to our large tertiary centre between 2007 and 2011.

Results: TFTs were performed during 23.026/269.388 (8.5\%) hospital admissions. Testing was more prevalent in females $(\mathrm{OR}=1.5)$, older people $(\mathrm{OR}=1.5,2.1,2.6,4.7$ [per quintile] ), longer hospital stays $(\mathrm{OR}=1.7,4.3,9.9$ $[2-7 ; 8-21,>21$ vs 1 day]), admissions under internal medicine $(\mathrm{OR}=7.7$ [vs general surgery] $)$ and in emergencies $(\mathrm{OR}=3.2)$. Admissions for general malaise, endocrine or mental disorders $(\mathrm{OR}=5.5,4.9,4.8$ [vs injuries]) were tested most often. Patients with a history of TD and those on amiodarone or lithium were more likely to be tested $(\mathrm{OR}=3.5,3.2,3.6)$. Among those tested $75 \%$ were euthyroid; hyperthyroidism was found in $1,299(5.6 \% ; \mathrm{n}=757[58.3 \%]$ subclinical) and hypothyroidism in $3,104(13.5 \% ; n=2,775[89.4 \%]$ subclinical); $7 \%$ of TFTs showed aberrant patterns with raised fT4 and normal TSH concentrations the most prevalent. Overtreatment with thyroxine or ATD contributed to $10 \%$ of abnormalities. Multivariable regression analysis identified history of TD $(\mathrm{AOR}=4.2)$, longer hospital stay $(\mathrm{AOR}=1.6[>21$ days vs 1 day]), female gender( $\mathrm{AOR}=1.6)$, endocrine or neoplasm primary diagnosis $(\mathrm{AOR}=3.7$ and 1.5 [vs injuries]) as independent factors associated with increased probability of overt TD whilst older people (AOR $=0.7,0.6[63-$ $75 y,>76 y$ vs 16-34y]) had decreased likelihood of TD.

Conclusions: Within a very large cohort, fewer than $10 \%$ of hospitalised patients underwent thyroid function testing. Abnormal TSH concentrations were evident in only a small proportion. Further studies are required to identify appropriate drivers for thyroid function testing during hospitalisation to best identify those with thyroid dysfunction who would benefit from further investigation and treatment.

\section{P365 \\ THYROID FUNCTION IN HOSPITALIZED VERY ELDERLY: ASSOCIATION WITH COGNITIVE FUNCTION, DISABILITY AND REHOSPITALIZATION}

César Esteves ${ }^{1}$, Filipa Gomes ${ }^{2}$, Francisco Cunha ${ }^{2}$, Luís Silva ${ }^{2}$ Vera Fontes ${ }^{2}$, Marta Moura², João Vilaça ${ }^{2}$, Vânia Araújón, Paulo Bettencourt ${ }^{2}$

${ }^{1}$ Endocrinology, Diabetes and Metabolism Department; Centro Hospitalar São João, ${ }^{2}$ Internal Medicine Department; Centro Hospitalar São João

Introduction: The prevalence of thyroid function disorders increases with age and there may be an association with an increased risk of cardiovascular disease and mortality. There are few studies regarding thyroid function in very elderly. Our goal was to evaluate thyroid function associated morbidity and mortality.

Methods: We conducted a prospective observational study in patients over 85 years old, admitted in an Internal Medicine ward between $1^{\text {st }}$ March and $31^{\text {st }}$ December 2010. Patients were contacted 12 months after the index hospital stay and outcomes were recorded. Statistical analysis was made with SPSS 20.0.

Results: Among 393 patients, we removed from analysis those without sufficient information on thyroid function, users of drugs that could alter thyroid function and individuals that died during hospital stay. Of the remaining 191 patients, 63 patients $(33.0 \%)$ were male and $128(67.0 \%)$ were female, with mean age $89.0 \pm 3.50$ years. Ninety three patients $(48.7 \%)$ had normal thyroid function; 3 patients $(1.6 \%)$ had hyperthyroidism; 11 patients $(5.7 \%)$ had hypothyroidism; 68 patients $(35.6 \%)$ had a pattern suggestive of euthyroid 
sick syndrome, and the remaining could not be classified in any of the groups. Mean TSH was $1.90 \pm 1.844 \mu \mathrm{U} / \mathrm{mL}$, mean free T4 was $1.17 \pm 0.414 \mathrm{ng} / \mathrm{dL}$, mean free $\mathrm{T} 3$ was $1.86 \pm 0.546 \mathrm{pg} / \mathrm{mL}$ and mean $\mathrm{T} 3 / \mathrm{T} 4$ ratio was $16.7 \pm 5.75$. There was a significant correlation between free T3 and Modified Barthel index (MBI) $(\rho=0.234 ; p=0.002)$, and between T3/T4 ratio and Mini Mental State index $(\rho=0.286 ; p=0.002)$ and MBI $(\rho=0.315 ; p=0.000)$. We found a significant correlation between the number of rehospitalizations during follow up and TSH $(\rho=0.179 ; p=0.027)$. There was no association with mortality during follow up.

Conclusions: T3 and T3/T4 ratio are correlated with Mini Mental State and MBI, suggesting a relation with cognitive function and disability in very elderly. We also found a correlation between TSH and number of rehospitalizations.

\section{P366}

\section{ASSOCIATION OF TRIIODOTHYRONINE LEVELS WITH FUTURE DEVELOPMENT OF METABOLIC SYNDROME IN EUTHYROID MIDDLE-AGED SUBJECTS: A 6-YEAR RETROSPECTIVE LONGITUDINAL STUDY}

Hye Jeong Kim ${ }^{1}$, Ji Cheol Bae ${ }^{2}$, Hyeong Kyu Park', Dong Won Byun ${ }^{1}$, Kyoil Suh ${ }^{1}$, Myung Hi Yoo', Jae Hyeon Kim ${ }^{3}$, Yong-Ki Min ${ }^{3}$, Sun Wook Kim ${ }^{3}$, Jae Hoon Chung ${ }^{3}$

${ }^{1}$ Division of Endocrinology and Metabolism, Department of Internal Medicine, Soonchunhyang University Hospital, Soonchunhyang University College of Medicine, Seoul, Korea, ${ }^{2}$ Division of Endocrinology and Metabolism, Department of Medicine, Changwon Samsung Hospital, Sungkyunkwan University School of Medicine, Seoul, Korea, ${ }^{3}$ Division of Endocrinology and Metabolism, Department of Medicine, Samsung Medical Center, Sungkyunkwan University School of Medicine, Seoul, Korea

Background: Several cross-sectional studies have been reported that thyroid hormone levels were associated with MetS even in euthyroid subjects. However, whether thyroid hormone levels were associated with the risk for incident metabolic syndrome (MetS) could not be determined. In the present study, we aimed to investigate the longitudinal effects of baseline triiodothyronine (T3) concentrations on the development of MetS in healthy subjects.

Methods: A cross-sectional and 6-year longitudinal follow-up studies were conducted in 11,832 euthyroid middle-aged subjects without MetS who participated in comprehensive health examinations. All subjects were categorized into quartiles based on $\mathrm{T} 3$ within reference range. We estimated the hazard ratios (HR) for the development of MetS according to T3 quartiles using Cox proportional hazards model.

Results: During a 6-year period, 3,510 incident cases of MetS (30\%) were identified. Incident MetS increased across the baseline T3 quartile categories ( $p$ for trend $<0.001$ ). The hazard ratio (HR) and $95 \%$ confidence intervals (CI) for the development of MetS were significantly higher in the highest than in the lowest T3 quartile group, even after adjustment for confounding variables such as sex, age, body fat percentage, smoking, and HOMA-IR (HR 1.157, 95\% CI 1.024-1.307, $\mathrm{p}=0.019$ ).

Conclusions: In euthyroid middle-aged subjects, T3 levels are strongly associated with increased risk for future development of MetS.

\section{P367 \\ FATE OF THE OLDEST IODIDE POOL IN THE THYROID GLAND AFTER HYPOPHYSECTOMY}

Vladan Bajic $^{1}$, Zorka Milicevic ${ }^{2}$

${ }^{1}$ Department for Radiobiology and Molecular Genetics, Institute for Nuclear Sciences "vinca", University of Belgrade, Belgrade, Serbia, ${ }^{2}$ Department of Molecular Biology and Endocrinology, Institute of

Nuclear Sciences "Vinca” University of Belgrade, Belgrade, Serbia

Since deiodination is greatly reduced after TSH suppression, the fate of the internal iodide pool was studied after hypophysectomy. Short-term (131I injection) or long-term (125I constant administration) kinetic studies were carried out on intact or hypophysectomized rats adapted to received $5 \mu \mathrm{g}$ iodide daily.

Short-term kinetic studies showed in control rats the labelled fraction of thyroid iodide was always greater than that of plasma iodide. This discrepancy is caused by intrafollicular deiodination of labelled iodotyrosine residues as soon as the beginning of labelling. By contrast, in hypophysectomized rats, the labelled fraction of thyroid iodide was similar to that of plasma iodide until 48 hours after label injection, becoming slightly greater thereafter. Thus, in hypophysectomized rats, deiodination of labelled iodotyrosine residues becomes significant only 48 hours after label injection. Long-term kinetic studies showed that plasma iodide was entirely renewed in 8 days, both in control and hypophysectomized rats. However, in hypophysectomized rats, the turnover of thyroid iodide was much slower than that of PBI, while in control rats these 2 turnovers were similar. This indicates in hypophysectomized rats a deiodination of the oldest molecules. Furthermore addition, in control rats thyroid iodide was entirely renewed by 50 days, while only $80 \%$ of the $\mathrm{Tg}$ is renewed at the same time. In a hypophysectomized rats, $4 \%$ of the thyroid iodide pool is renewed in 8 days and $5 \%$ in 60 days. In contrast, $4 \%$ of thyroglobulin is renewed in 14 days and $20 \%$ in 60 days. Since in hypophysectomized rats thyroglobulin is renewed more rapidly than the total thyroid iodide, only part of the total iodide pool is the unique and direct precursor for thyroglobulin. This rapidly renewed precursor compartment is iodide taken up by the gland from plasma.

It is concluded that, in hypophysectomized rats, the oldest iodotyrosine residues are selectively deiodinated. 


\section{European Thyroid Journal}

Abdulkader I. P62

Abe K. P27

Abelleira E. OP69

Abello N. P208

Abraham-Nordling M. P261

Abrahamsen B. OP74

Abrosimov A. P323

Abu-Khudir R. OP01

Açııgöz A.S. P209

Acıoğlu H.Ç. P209

Adam S. P299

Adonakis G. P218

Afonso C. P332

Afzal M. P364

Agate L. OP20, OP43, P64, OP88, OP91, P302

Agin A. OP53

Agrícola J.M.C. P115

Aguilera B.G. P81

Ahmetaj E. P287, P290

Ahn B.-C. P285

Ahn J.-C. P66

Ahn K.H. P14

Ahtiainen P. P277

Aide N. P166

Akaishi J. P189, P294, P353

Aktas A. P216

Akulevich N. P238, P345

Alamdari S. P362

Alanis P.F. P115

Albano E. P04, P07

Albisu M. P278

Alevizaki M. P174, P243

Alexandrescu D. P130

Alexandru N.D. P352

Alfano F. OP48

Alfonsín-Barreiro N. P62

Ali S.Z. P113

Alibrandi A. P234

Aliyev E. P301

Allen H.L. OP18

Allocca C. P306

Almeida N. P13, P17

Alonso-Merino E. OP96, OP99

Alves D. P173

Alves V. P120

Ambrosio R. OP48

Amendola E. OP38, P44

Amendola S. P61

Amino N. P254

Amouzegar A. P93, P360, P362

Amro B. P299

Anastasiou E. P174

Andelfinger G. OP01

Andersen S. P158

Andersen S.L. OP13, OP76

Andersson M. P25

Andrei F. P219

Andriollo V. OP37

Antonelli A. P08, P47, OP61, OP67, OP89, P150, P244

Antonini S. P149

Apellaniz-Ruiz M. P83
Apiñániz E.A. P293

Apostolakis M. P174

Aragüés J.M. P327

Aral Y. P216

Aranda A. OP96, OP99

Aranda G. P97

Araújo V. P365

Arczewska K. P247

Ares J. P208

Argatska A. P26, P35

Argirova R. P48

Arjona F.J. OP52

Arpi M.L. P123, P153

Artal A.R. P214

Askari S. P32

Aslan G. P212

Aslan H. P209

Åsman P. P06, P325

Assadi-Porter F. P266

Athanasiadou A. P174

Atzori F. P23

Audí L. P278

Aurore C. OP98

Aweimer A. P222

Aydin H. P212

Aydın Ü. P216

Aydoğan B. P209

Aydoğan B.İ. P209, P216, P328

Aygenc E. P82

Azizi F. P32, P93, P360, P362

Azouzi N. OP86

Baatout S. P157

Babenko A. P136

Babin E. OP90

Bachvarova M. P114

Badalyan M. P211

Badiong J. P180

Badiu C. P78, P106, P279

Badziong J. P203, P311

Bae J.C. P366

Bae J.S. P70, P172, P295, P304

Bae K.-S. P338

Baek J.H. P112

Baek J.O. P300, P354

Baek S.-K. P75, P343, P350

Baek S.M. OP44

Bagci E. OP83

Baghino G. P23

Bagnato G. P246(2)

Bajic V. P367

Bajuk V. P154

Bakhsh A. OP64

Bakker S.J.L. OP71

Baldari S. P117

Baldeschi L. OP59

Bandai S. P124

Bar-Andziak E. P215

Barczynski M. P91

Bardet S. OP90, P166

Barez-Lopez S. P202

Barquiel B. P281

Bartalena L. OP55, P134
Bartolazzi A. P61

Bartolome L. P36

Basile M. OP38

Basolo F. OP88, P176, P178, P302, P305

Bastholt L. OP87

Batista R. P314, P315

Battaglia V. OP91

Baudin E. P165, P185

Bauer D.C. OP71

Baumgartner C. OP71

Bayburdian G. P211

Bednarczuk T. P215

Behets C. OP59

Beiras J.M.C. P348

Belardini V. OP46

Beleslin B. P03, P128, P259

Bell O. P177

Bella B.D. P273

Bellotti C. P61

Belykh N. P29

Bendlova B. P86, P312

Benvenga S. P146

Berberoğlu Z. P216

Berche M. P05

Berchner-Pfannschmidt U. P10

Berdelou A. P165, P185

Berenguer J. P97

Berkovich O. P148

Bernabeu I. P104

Bernal J. P163, P202, P316

Berta E. P11, P131, P224

Betivoiu M. P219

Betka J. P312

Bettencourt P. P365

Biagini A. OP20, OP43, P64, P302

Bianchi F. P64, P125

Bianchini A. P237

Bidault S. P165

Biebermann H. P16, P43, P264

Bienvenu M. OP41, P89

Bigorgne C. OP41, P89

Bikov M. P349

Bilezikci B. P82

Bilz S. P252

Bisogno G. OP89

Bisschop P. OP35

Bizzarri G. P237

Bjergved L. P18, P96

Bjørner J.B. P98, P110, P260

Bjøro T. OP92

Blanca M. P36

Blanchard D. OP90

Blanco J.A. P85, P214

Blanco-Vaca F. P177

Bloigu B.A. P140

Bloise F. OP57

Blum M.R. OP71

Boas M. P156

Bocci G. OP67

Bocheva Y. P94

Bodor M. P11, P131, P210, P224

Boelaert K. OP63, OP66, P364
Boelen A. P19, OP32, OP35, OP54, P321

Boesenberg E. OP40

Boi F. P23

Boichard A. OP86

Boiko J. P238

Boncoddo M. P273

Bonfanti P. P223, P289

Bonichon F. OP94

Bonnema S. P60

Bonnema S.J. OP78, P98, P110, P260

Bony-Trifunovic H. OP08

Borda J.G. P163

Borges F. P103

Borget I. P165

Boriskova M. P349

Borissova A.-M. P52

Borojevic R. OP57

Borrego S. OP12

Borson-Chazot F. OP04

Borucu T. P275

Borysewicz-Sańczyk H. P250

Bossowska A. P250

Bossowski A. P250

Botoula E. P72

Bottici V. OP20, OP43, P64, OP91, P181

Bourgeois N. OP84, P201

Bowers J. P204

Brabant G. OP58, OP72

Brandáriz L. P163

Brandt F. P145, P256

Braun D. OP03, OP51

Braun W. P299

Brenmer A. OP71

Britvin T. P45, P102

Brix K. P203, P206, P264, P317, P320

Brix T.H. OP74, P145, P256

Brorson M.M. P156

Brose M.F. OP02, P351

Brose M.S. OP87

Brozzi F. P64, P125

Bruhnke G. P109

Brusca N. P230

Brzac H.T. P227

Bueno F. OP69

Buffet A. P330

Bulfamante G. OP07, OP68

Burch H.B. OP55

Burns R. P25

Buset J. P157

Busonero G. OP06

Buy X. OP94

Buziak-Bereza M. P215

Byström K. P261

Byun D.W. P366

Caballero M.G.R. P208, P214

Cabezas-Agrícola J.-M. P62, P171

Cadórniga F.D. P280

Caff A. P153 
Cailloux J. OP86

Çakar E. P209

Cakir M. P129

Caldas A.R. P103

Calissendorff J. P06, P261

Calvo A. OP47

Cameselle-Teijeiro J. P42, P62, P171, P301

Cammarata I. OP37

Campennì A. P117

Campi I. OP25, OP29, OP61, P127

Campos S. OP28

Can I. P129

Canipari R. OP37

Cantara S. OP06

Cappagli M. P223, P289

Cappagli V. OP91, P302

Cappola A.R. OP71

Caravia D. P85

Carbotta G. P90

Cardianale S. OP06

Cardoso L. P46, P65, P149

Carlé A. P18, P141

Carles Z. P58, P188

Carlsen J. OP09

Carlsson T. OP17

Carmela I. P188

Carmen S.G. OP47

Carnicelli V. P263

Caron P. P330

Carreño A.L.S. P348

Carrera E. P97

Carrilho F. P65

Carsote M. P107

Carvalho D. P108, P120, P137, P139, P225, P228, P333

Casanueva-Freijo F. P62

Cascón A. P83

Casella F. OP20, P176

Casey M. P25

Casini G. P07

Castagna M.G. OP21, OP46

Castelblanco E. OP12, P298, P308

Castellone M.D. P306

Castells A.L.G. P276

Castellvì J. P297

Casterás A. P79, P87

Castilho E. P186

Castillo C. P361

Castro P. P315

Caswell R. OP18

Catalina P. P352

Cazzato R.L. OP94

Ceausu I. P106

Cecchetto G. OP89

Celestino R. OP92, P315

Çelik A. P209

Cellini M. P230

Centanni M. OP27, OP37, P230

Ceresini G. OP71

Cerqueira C. P24

Certo R. P117, P246

Cerutti J.M. P313

Cevenini G. OP21, OP46

Chacon J. P363

Chae B.J. P304

Chaker L. OP71

Chakera A. OP18

Chandola-Saklani A. P33, P322

Chang H.-S. P303, P346

Chapman M. OP75

Chartoumpekis D. OP95

Chatzipavlidou V. P183

Chatzitomaris A. P101, P222
Chaushev B. P347

Chengot P. P59

Cheung J.Y. P28

Chew C.K. P105

Chiamolera M. P21

Chiarella G. P242

Chiellini G. OP97, P263, P265, P266

Chikh I. P45, P53, P248

Chiti A. OP25

Chmielik E. OP93, P310

Cho A. P121

Cho K.I. P14, P132

Cho K.J. P358

Choe J.-H. P67, P164

Choi Y.D. P161

Choi Y.J. P112

Chong S. P159

Choong E.S. P194

Chougnet C. OP11

Christiansen P. P60

Chrysis D. P277

Chueca M. OP08

Chun B.J. P358

Chung E.-J. P184, P192, P195

Chung J. OP87

Chung J.H. P366

Chung P.S. P66

Chung W.Y. P304, P338

Ciampi R. OP20, P176, P181, P305

Ciappuccini R. OP90, P166

Cibas E. OP45

Cirello V. OP07, OP29, P170

Ciric J. P03, P128, P259

Ciric S. P03

Clarke I. OP75

Clemente M. P278

Clerget-Froidevaux M.-S. OP31, P204

Coen L. OP36

Cold F. OP78

Cold S. OP78

Coleman J. P364

Coles D. P106

Colin I. P22, P157

Collet T.-H. OP71

Colombo C. OP07, OP29, OP68

Comino-Mendez I. P83

Conde J.B. P167

Contillo B.P. OP91

Cordeiro A. P13

Corrado A. P47, OP67, P150

Correia R. P120

Corssmit E. P76

Coscio G.D. OP88

Cosmina C.I. P352

Costa G. P65

Costa L. P344

Costagliola S. OP01

Costante G. P242

Cöster M. P16

Cotelli F. OP82

Couto-Carvalho A. P103

Covelli D. OP21, OP25, OP61, P127, P217

Cramon P. P98, P110

Craps J. P22, OP59

Credendino S. P44

Crippa M. OP29

Criscuolo C. P198

Cristobal B. P163

Crock P. OP08

Cubas A.D. OP12

Cucinotta M. P117
Cuenca I.C. P81

Cuenca J.I.C. P286

Cunha F. P365

Cunha F.M. P333

Curras-Freixes M. P83

Currò N. OP61, P127

Curtò L. P234

Cyplinska R. OP70

Czarniecka A. OP15, P71, OP93, P310

Czarnocka B. P247

Dacou-Voutetakis A. P193

Dahlberg J. OP17

Dakovska L. P52

D’Alessandri M. P90

D’Ambrosio C. OP38

Dambrova M. P3

D'Andrea B. P44

Dane B. P209

Danesi R. OP67

D’Angelo F. OP38

Daniel H. OP79

Darias-Garzón R. P100

Darras V.M. OP83, OP84, P201

Daumerie C. OP59, P213

Davcheva D. P26, P35

Day C. P19

Dayan C. OP14, OP60, OP62, P245

Dazzi D. OP61

de Angelis M.H. OP53

De Cubas A.A. P83

De Deken X. P38

de Filippis T. OP05

De Francesco G. P61

De Jesús Acosta M.E. P348

de la Fouchardiere C. OP87

de la Fuente H. OP28, P122

De la Vieja A. OP101, P268

de las Heras B. OP02, P351

de Leiva A. P177

de Lloyd A. OP64

de Mello-Coelho V. OP57

de Mena R.M. P200, P202

De Mingo M.L. P356

de Rijke Y. P272

de Santos Iglesias F.J. P233

de Segura I.L.-G. P100

de Solís S.N. P276

De Stefano M.A. OP48

de Vries E. OP32

Deandrea M. OP42, P92

Deandreis D. P165, P185

Debrabant B. OP78

Decherf S. P204

Dehghan A. OP71

Dehnfeld M. P158

Deichsel A. OP34

Dekkers O. P76

del Carmen Suarez M. P42, P170

Del Duca S.C. OP27, P230

del Olmo García M.I. P276

del Pozo A. P278

Dela Paz A.G.S. P220

Deladoëy J. OP01

Delbaere J. OP84, P201

Delgado E. P280

Delgado L. P137, P139

Delshad H. P93

Demaj E. P287, P290, P324

Demeneix B. OP31, OP36, P204

Demir A. OP17

den Elzen W.J.P. OP71

Dentice M. OP48, OP54

de Pablos A.M. P363

Derradji H. P157

Désilets V. OP01

Despot M. P355

Dessanti P. P223

Dhandapani P. OP100

Di Domenicoantonio A. OP61

Di Paolo V. OP37

Diana T. OP16, OP23, OP26, OP56

Dias-da-Silva M.R. P21

Díaz L. P85

Díaz-Guerra G.M. P291

Dierick-Gallet A. P165

Dietrich J.W. P101, P222

Díez-Villanueva A. P298

Digiacomo M. P265

Dimova M. P133

Dinter J. P16, P264

Diosdado M.A. P118

Dirk A.-L. OP58, OP72

Dobrescu R. P78, P106

Dold S. P25

Domenicantonio A.D. P47, OP67, P150, P244

Donadi E. P173

Donzelli R. OP97, P198

Dopazo J. P316

Dora S. P148, P152, P251

Dörr M. P15

dos Santos Valença S. OP57

Dotta F. OP06

Dottore G.R. P07

Dragicevic M. P128

Draman M.S. OP60, OP62, P126

Drechsler C. OP71

Dreval A. P45, P102, P248

Du Pasquier Fediaevsky L. P05

Duarte A. P103

Duarte A.M. P177

Duarte D.G. P118

Duarte H. P344

Dubois G.M. OP31

Ducena K. P31

Dugdale E. P194, P340

Dullaart R.P. OP71

Dumitrascu A. P130

Dumitru I. P352

Dumnicka P. P215

Dumont J. P39, P175

Dumont J.E. OP08

Dundua T. P283, P359

Duntas L. P155

Dupayrat J. OP04

Dupuy C. OP86, OP98

Dutcus C.E. OP02, P351

Duvernois-Berthet E. OP31

Dvorakova S. P86, P312

Dygun O. P148

Easter T. P126

Eckstein A. P10

Eftychiou N. P168

Ekiz A. P209

El Hassani Rabii A. OP86, OP98

Elgadi A. P126

Elisei R. OP02, OP20, OP43, P64, OP87, OP88, OP91, P176, P181, P302, P305, P351

Ellard S. OP18

Elmansori I. P126

Encinas M. OP12

Endo K. P27 
Engels K. P317, P320

Engin O. P282

Erdei A. P11, P131, P210, P224

Erdoğan M.F. P209, P216

Erenel H. P209

Erkenekli K. P216

Ersoy B. P275

Escola J.C. P177

Escribano A. P281

Espinoza S. P265

Esteves C. P137, P139, P365

Eszlinger M. OP40, OP45, P299

Expósito M.R.A. P236

Faber J. P144, P147

Fadeyev V. OP77, P142

Fagman H. OP17, OP80

Falcones B.T. P348

Fallahi P. P08, P47, OP61, OP67, OP89, P150, P244

Falzacappa C.V. OP37

Fandrey J. P10

Fanjul Rodríguez L.F. OP99

Farafonova U. P349

Faraswan A. P322

Fatemi S. P169

Fattori B. P242

Faustino L. OP85

Fedeli F. P289

Fedorowicz A. P215

Feijoo L.C. P167

Feldkamp J. OP16

Feldmann B. P299

Feldthusen A.-D. P95

Feldt-Rasmussen U. P98, P110, P156, P260

Felechosa M.D. P293

Felice M.D. OP38

Felipe-Pérez N.C. P100

Feng W. P160, P284

Fernández A.F. OP12

Fernandez Em. P363

Fernandez Ev. P104

Fernández J.C. P280

Fernandez L.P. P40

Fernández T. P163

Ferrandino G. OP38

Ferrari S.M. P08, P47, OP67, P150, P244

Ferrario M. P134

Ferreira L. P315

Ferrero E. P291

Ferrero J. P291

Ferrero S. OP29

Fiaño R.S. P167

Fica S. P219

Figueiredo-Feitosa N. P173

Filipsson H. P261

Finelli P. OP29

Fioravanti C. OP46

Fiore E. P125, P178, P296

Fischer J. P43

Fletcher R. OP63

Fliers E. OP32, OP35, OP54

Flux G. P253

Fonseca H. OP57

Fontes V. P365

Foppen E. OP35

Forga L. P79, P87

Fraga M.F. OP12

Franceschini S.S. P08

Franco O.H. OP71

Frangos S. P119, P168

Franken J. OP23
Franklyn J.A. OP63, OP66, OP71, P364

Franzius C. P299

Frascarelli S. P265

Freddi M. OP42

Frederiksen H. P156

Freitas C. P103

Freitas P. P228

Frendl D.M. P98

Frolova A. P135, P262

Fugazzola L. OP07, OP21, OP29, P42, OP68, P170, P217

Führer-Sakel D. OP22, OP64, P88, P109, P180, P203, P206, P226, P311, P317, P320, P321

Fujikawa M. P124

Fujiwara M. P254

Fukahori M. P257

Fumarola A. P90

Fureraj T. P287, P290

Furmaniak J. OP24, OP30

Furnica R.M. P213

Fusarini C.F. OP21

Fuziwara C. P17

Gaberšček S. P12, P154, P255

Gabriele C. OP06

Gabur A. P279

Gagnidze K. OP85

Gajate G.J.V. P233

Galgoczi E. P09, P210

Gallego N.V. P79, P87

Galletti M.R. P117

Gallo D. P134

Galofre J.C. P292

Galrão A.L. P313

Galuska L. P11

Gambale C. OP43, P64

Gao Y. P240

García A. P104

Garcia A.B. P115

Garcia M. OP08

García V. P163

García-Bray B.F. P100

Garcia-Burillo A. P188

García-Conde M. P163

García-Doncel L.G. P118

García-García F. P316

Garcia-Moreno J.-M. P363

García-Rendueles A.R. P42, P170

Gargano L. OP27, P230

Garino F. OP42, P92

Garzón L. OP10

Gasparri G. OP42

Gasso M. P361

Gatto I. OP27, P230

Gavars D. P31

Gazdag A. P09, P11, P131, P210, P224

Gazso A.A. P210

Gelmini G. OP05

Gérard A.-C. P22, P157

Gerenova J. P241

Gerrard G. P59, P190, P194, P340

Gessl A. P80

Gharibzadeh S. P362

Ghelardoni S. P263

Gheorghiu M.L. P130

Ghezzani C. P263

Ghuzlan A.A. OP86, P165, P185

Giani C. OP88, P245, P302

Giannakou M. P243

Giannocco G. P21

Giannoula E. P119, P168, P183
Gianoukakis A.G. OP02, P351

Gil P. P316

Gil-Díez D. P163

Gimba E. P315

Giovanella L. P117

Giovinazzo S. P117, P234

Girard E. P165

Girardot F. OP36

Girolamo D.D. OP48

Giulea C. P219

Glonti S. P50

Godbert Y. OP94

Godlewska M. P247

Goebel A. OP72

Goeke B. OP09

Goldspring R. P59

Goldstein A. P78, P106

Gomes F. P365

Gómez Á. P83

Gomez G.N. P167

Gómez R.B. P298

Gomez R.C. P276

Gómez-Alvarez B. P100

Gonzalez C. P177

González E.N. P81, P286

Gonzalez J.M.G. P233

Gonzalez O. P188

Gonzalez-Amaro R. OP28, P122

González-Casado I. OP08, P278, P281

González-Melo E. P100

González-Peramato P. OP96

Gorcheva D. P48

Górska M. P215

Görtz G.-E. P10

Goscik J. P250

Gothié J.D. OP31

Gotoh N. P257

Göttlich M. OP72

Gotzmann M. P101

Graf D. P299

Grani G. P90

Grassi E.S. OP65

Gratz S. P299

Gray M. P253

Graziano F. P237

Greenfield H. P137, P139

Grennan-Jones F. OP60, OP62, OP64, P126, P245

Grineva E. P136, P148

Groenvold M. P98, P110

Gruson D. P213

Grüters A. P16, P43, P264

Guadalix S. P291, P356

Guadaño-Ferraz A. P202

Guardiola Y.S. P118

Gubała E. P71, P310

Gudieva M. P251

Guelho D. P65

Guerra G.M.D. P356

Guglielmi R. P237

Guidi S. OP06

Guillen-Grima F. P292

Guizzardi F. OP05

Gullo D. P123, P153

Güllü S. P328

Gunnar K. OP03

Guo X. P240

Gupta K.K. P239, P270

Gursoy A. P82

Gussekloo J. OP71

Gutch M. P239, P270

Gutierrez C. P361

Gutierrez L.S. P208, P214

Gutierrez S. P36

Gutiérrez-Buey G. P292

Guttman M. P57

Györy F. P09

Habeos I. OP95

Habra M.A. OP02, P351

Hach A. P299

Hacker M. OP09, P80

Haenold R. OP32

Hakobyan V. P211

Halkova T. P86, P312

Hallengren B. P06, P261, P325

Halperin I. P97

Hammer E. OP58

Hammoser R. P299

Han W.G. P182

Handkiewicz-Junak D. OP70, P71, OP93

Hansen J.F. P156

Hanusek K. P247

Hanzu F.A. P97

Harmer C. P253

Hartikainen A.-L. P140

Hartl D. OP86, P185

Hartoft-Nielsen M.-L. P156

Har-Zahav G. P57

Hatori S. P257

Hattersley A. OP18

Hauch O. OP19

Hawkins F. P291, P356

Hazi G. P34

Hegedüs L. OP74, OP78, P98, P110, P145, P256, P260

Heijlen M. OP83

Heldmann M. OP72

Henriques de Figueiredo B. OP94

Herceg G.H. P227, P355

Herguido N.G. P81, P286

Hernández J.C. P115

Hernanz L.R. P233

Herold T. OP22, P88

Héron E. P05

Herranz A. OP10

Herrero E.F. P163

Hesselink E.K. P76

Heuer H. OP32, P321

Heutte N. OP90, P166

Higashiyama S. P335

Hinz K. OP49, P199

Hircsu I. P210

Hirokawa M. OP39

Hitz M.-P. OP01

Hlasc M.C. P355

Hodson J. P364

Hoenes S. P317

Hoff A.O. OP02, P351

Höfig C. OP79, P267

Hofman A. OP71, P272

Hofman-Bang J. P156

Hofmann P. OP34

Hojker S. P12, P154, P255

Holmberg M. P261

Homuth G. OP58, P264

Hönes S. P320, P321

Hong J.C. P73, P191, P307

Hong M.J. P112

Hong S.J. P112

Hong S.W. P161

Horasan G.D. P275

Horn S. OP32, P321

Horstmann M. P10

Hortopan D. P130, P279 
Hoxha V. P287, P290, P324

Hoyas M. P157

Hristozov K. P94, P114, P271

Huang R.J. P25

Hubalewska-Dydejczyk A. P84, P215

Huic D. P227, P355

Husi G. P287, P290, P324

Huth S. P37

Hwang S.O. P300, P354

Hyer S.L. P253

Iacoviello M. OP71

Iakovou I. P119, P168

Ibanez E. OP47

Ibáñez K. P278

Ibayashi H. P124

Ide A. P254

Iervasi G. OP71

Iglesias A. OP08, P202, P278

Iglesias P. P36

Igna C.P. P34

Ikram M.A. OP71

Ilany J. P49

Ilardo G. P273

Ilgan S. P82

İlhan G.A. P209

Iliadou P. P183

Ilias I. P218

Imaizumi M. OP71

Império G. P13, P17

Imruetaicharoenchoke W. OP63, OP66

Ingeson C. OP17

Inoue Y. P27

Inserra A. OP89

Insilla A.C. OP88

Intersimone D. P289

Ioachim D. P78, P106

Ioannidis G. P72

Ionta M.T. P23

Isabelle B. OP11, P185

Isaev P. P323

İșçi H. P209

Ito K. P189, P294, P353

Ito M. P254

Ittermann T. P15

Iwasaki H. P257

Iwen A. OP58

Jaddoe V. P272

Jaeger A. P109, P180, P206

Jaeschke H. P37

Jaime P. P175

Jakhaia N. P283, P359

Jang E.K. P74

Jang J.H. P67, P164, P339

Jansson S. OP17

Järvelin M.-R. P140

Jarzab B. OP15, OP70, P71, OP87, OP93, P310

Jarzab M. OP15, P71, OP93, P310

Javashvili L. P283, P359

Jekabsone A. P31

Jelstrup S. P95

Jeney F. P09

Jensen L.T. OP73

Jeon M.J. P74

Jeon Y.K. P14

Jeong J.J. P338

Jeong S.Y. P285

Jercalau S. P279

Jespersen M.L. P60

Jeudy C. P330
Jiménez C.M. P236

Jing W. P160

Jo J.-G. P75, P343, P350

Johannes J. OP03

Johansson E. P41

Johner S. P20

Jonas W. OP100

Joo Y.H. P358

Jordà M. P298

Jordi T. P188

Jørgensen H.L. OP74

Jørgensen T. P18, P24, P96, P141

Joris V. OP59

Jueppner H. P277

Jukema J.W. OP71

Jung C.K. P161, P172, P295

Jung J.H. P300, P354

Jung J.S. P357

Jung K. P28

Jung K.J. P75, P343, P350

Jung K.-Y. P75, P343, P350

Jung S.S. P70, P295, P304

Jürgens C. P15

Kahaly G.J. OP16, OP23, OP26, OP55, OP56, P01, P02

Kalemba M. OP70

Kalere I. P31

Kaloiani T. P283

Kalsbeek A. OP35

Kampmann E. P01, P02

Kan Ö. P212

Kanaka-Gantenbein C. P193

Kanazirev B. P114, P133

Kanellos I. P235

Kang H.-C. P179

Kang J.K. P300, P354

Kang K. P162

Kang S. P254

Kang S.J. P338

Kang S.-W. P338

Kanitz M. OP23, OP56

Kannivelu A. P113

Kanouta F. P174

Kapia M. P287

Kapiteijn E. P76

Kappeler C. OP87

Karagüzel G. P231

Karakaş H.M. P212

Karakonstantakis T. P155

Karapanou O. P193

Karger S. OP16

Kassabova L. P52

Kathait A. P33, P322

Katkó M. P09, P131, P210, P224

Kawabe J. P335

Kawano M. P189

Kayan B. P209

Kensler T. OP95

Kermaj M. P287, P290, P324

Kersseboom S. OP52, OP84

Khamisi S. P261

Khaw K.-T. OP71

Kheirkhah P. P362(2)

Khmara I. P238

Khroyan A. P138

Kiedrowski M. P247

Kihara M. OP39

Kim B.H. P14, P132

Kim B.K. P67, P164

Kim B.S. P162

Kim H.J. P366

Kim H.K. P179

Kim H.S. P162
Kim I. P342

Kim I.J. P14

Kim J.-H. P55, P67, P164

Kim J.H. P14, P187, P336, P366

Kim J.S. P67, P164, P339

Kim L.S. P187, P336

Kim M.H. P172

Kim M.S. P358

Kim S. P337

Kim S.-B. OP02, P351

Kim S.J. P159, P162, P179, P258

Kim S.K. P67, P164

Kim S.M. P346

Kim S.S. P14

Kim S.W. OP44, P73, P191, P307, P366

Kim S.Y. P358

Kim T.Y. P74

Kim W.B. P74

Kim W.G. P74

Kim W.J. P14

Kim W.W. P300, P354

Kim Y.J. P258

Kim Y.S. P309

Kimura E. P17

Kinne A. OP49, OP50, P199

Kiremitci S. P275

Kitagawa W. P189, P353

Kitazono T. P124

Kivuva E. OP18

Kiy Y. P186

Kiyota N. OP02, P351

Kizys M.M.L. P21

Klein H.H. P101, P222

Klein M. OP53

Kleinau G. P16, P43, P264

Klingenspor M. OP53

Knapen D. OP83

Knezevic M. P03

Knoop K. OP09, P319

Knudsen N. P18, P24, P96, P141, P144

Knyhala K.M. P101

Kobulia M. P283, P359

Koç G. P216

Kodet R. P312

Kodetová D. P312

Koeditz R. P101

Koerber C. P331

Kohan S. P345

Köhrle J. P16, OP49, OP51, OP53, OP79, OP100, P199, P264, P267, P321

Kolbe E. P02, OP26, OP56

Koleva V. P48

Komerdous I. P45, P102, P248

Kondo T. P294

Konrade I. P31

Konstantinidis I. P119

Konturek A. P91

Koo B.S. P337

Körber-Hafner N. P331

Korevaar T. P272

Kostareva A. P136

Kostecka-Matyja M. P215

Kostova B. P48

Kotani K. P335

Koukkou E. P218

Koumarianou P. P268

Kovalenko N. P29

Kovatcheva R. P232

Kowal M. OP70, OP93, P310

Kowalska M. OP15, OP70, P310

Krajewska J. OP15, P71, OP93

Krane J. OP45

Krasnaliev I. P94

Krasnodębska M. P215

Krasuska W. P247

Kratzsch J. OP16

Krause G. OP49, OP50, P199

Krause K. P311

Kravaritis S. P218

Kravchuk V. P345

Kreissl M. OP19

Krejbjerg A. P18, P96

Kristensen T.T. P95

Krętowski A. P250

Krude H. P16, P43, P264

Krzyzanowska M.K. OP02, P351

Kuchel T. OP75

Kudo T. P254

Kukulska A. P71

Kula D. OP70

Kumar S. P239, P270

Kuna S.K. P227, P355

Kurzynska A. P84

Kvetny J. P95

Kwak J.J. P161

Kwan P. OP63

Kwon H.J. P161

Kwon S.Y. P182

Kwon S.-Y. P75, P343, P350

Kyriazopoulou V. OP95

la Cour J.L. OP73

Lacamara N. P281

Ladas I. OP64

Ladra M.J. P301

Lai A. P134

Lalic T. P03, P128, P259

Lamartina L. P185

Landvogt C. P299

Lane C. OP60, OP62

Lantz M. P06, P261, P325

Larsen J. P95

Latina A. P123

Latrofa F. P04, P125, P296

Lau E. P108

Laulund A.S. OP74

Laurberg P. OP13, P18, P24, OP76, P96, P141, P158

Laure T. P39

Laurino A. OP97, OP102, P265

Lauro R.D. OP38, P44

Lavra L. P61

Lawton S. P59

Lazic B. P259

Leboulleux S. P165, P185

Lecumberri B. P281

Lee B. P342

Lee H. P55

Lee H.C. P67, P164

Lee H.S. OP44, P73, P191, P307

Lee J. P285, P300, P338, P342, P354

Lee J.D. P121

Lee J.H. P67, P112, P164

Lee J.W. P121, P357

Lee K. P75

Lee K.D. OP44, P73, P191, P307

Lee K.S. P28

Lee M.J. P161

Lee S. P70, P172, P295, P304

Lee S.M. P357

Lee S.-W. P285

Lee Y.S. P303, P346 
Leenhardt L. P05

Lefrandt J. P76

Lehmann M. P02, OP23

Lehmphul I. OP79, P267

Lejnieks A. P31

Lengelé B. P22, OP59

Leo M. P04, P07

Leo S.D. OP07, OP68

Leoni S. OP101

Leonova T. P345

Lepoutre C. P165

Leskela S. OP28

Letón R. P83

Levi A.M.R. OP28, P122

Liang S. OP17, OP80

Librizzi M.S. P291

Lietzow J. OP100

Lilis I. OP95

Lim D.J. P172, P295

Lim H.K. P112

Lima F. P332

Limbert C. P332

Limone P.P. OP42, P92

Links T. P76

Lisurek M. P199

Liu Z. P56

Llorente L.R. OP99

Lluch A.R. P81

Lomas M. P163

Longo A.C. P273

Lopes F. P344

López M. P54

LoPresti J. P169

Lorenzini L. P263

Loscos C.C. P348

Lothe R.A. OP92

Loukari E. P243

Lozanov B. P48

Lozanov L. P48

Luben R.N. OP71

Lubetzky A. P49

Lucchini B. P252

Lucena I. P344

Ludbrook G. OP75

Ludgate M. OP60, OP62, OP64, P126, P245

Lumbroso J. P165

Luna C. OP10

Luongo C. OP48

Luzuriaga C. OP08

Łyczkowska A. P247

McCabe C. OP63, OP66

Macchini F. OP21

McCutcheon J. P221

Maciejewski A. OP93

Maciel L. P46, P149, P173

Maciel R. P21

Madeddu C. P23

Madiyarova M. OP77, P142

Maenhaut C. P175

Maffini A. OP29

Magalhães P. P46, P149, P173

Magi F. P61

Magistrelli P. P223, P289

Magurean M. P106

Maiden M. OP75

Main K. P156

Maino F. OP21, OP46

Makarava Y. P238

Makrecka M. P31

Malandrino P. P153

Malcheva D. P347

Maldonado S.M.N. P115
Mallona I. P298

Mamali I. OP95, P218

Mambet C. P34

Mamedova T. P45, P53, P248

Mamenko M. P29

Mancikova V. OP12, P83, P298, P308

Mandanas S. P183

Mangialardo C. OP37

Mangino G. OP27

Mankovskaya S. P345

Manni M.E. OP97

Männistö T. P140

Manolakou S. OP95

Manolova I. P241

Manrique A.O. P348

Mantzou A. P243

Mantzou E. P155

Many M.-C. P22, OP59

Maravall J. P298, P308

Marazuela M. OP28, P122

Marcello R. OP25

Marchetti I. OP88

Marcocci C. P04, P07

Marcus C. P126

Marelli F. OP05, OP82

Margaritidou E. P235

Mariappan L. P59, P190, P194, P340

Marinò M. P04, P07, P125, P296

Mariotti S. P23

Markou K. P218

Marotta P. P44

Marques M. P186

Márquez A.L. P318

Marshall L. OP36

Martin S. P219

Martínez A.H. P236

Martinez M. P308

Martinez M.J. P361

Martinez R. P122

Martínez R. OP28

Martinez-Iglesias O. OP96, OP99

Martin-Orozco R. OP96, OP99

Martitz J. OP34, OP47

Martos J.M. P286

Masaki C. P189, P353

Mascarenhas M. P327

Masuda M. P189, P294

Mateo Y.A.M. P233

Materazzi G. OP67, OP88, P302, P305

Matheis N. OP56

Matias-Guiu X. OP12, P298, P308

Mato E. P177

Matrone A. OP20, OP43, P64

Matsuzu K. P189, P294

Mattheis N. OP16

Mauricio D. P58, P298, P308

Mazeto G. P186

Mazzeo S. P64, OP91, P302

Mazzi B. P04, P125

Medici B. P144, P147

Medici M. OP71, P272

Medina J.L. P137, P139

Mehran L. P32, P360

Meier R. P37

Meima M. OP50

Mekjavic P.J. P12

Melo M. P65

Memmo S. OP46

Menconi F. P04, P07

Mendes-Junior C. P173
Menéndez E. P85, P214, P280, P293

Menezes J. P225

Mesa J. P79, P87, P297

Meyer F. OP53, P197

Meyer K. P199

Mezhapuke R. P31

Miccoli P. OP67, OP88, OP89

Michaelsson L.F. P144

Michalopoulos N. P235

Michaux A. P157

Michels J.-J. OP90, P166

Miguel R.N. OP24, OP30

Mikos H. P249

Mikos M. P249

Milicevic Z. P205, P367

Min H.S. P55, P161

Min Y.-K. P366

Minyaylo N. P29

Miot F. P38

Miranda C. P46

Mirghani H. P165, P185

Misischi I. P237

Misiti S. OP37

Mittag J. OP79

Mityukova T. P77, P345

Miwa A. OP39

Miyagi Y. P294

Miyauchi A. OP39, P254

Modasia B. OP66

Moeller L.C. P109, P203, P206, P226, P317, P320, P321

Moilanen I. P140

Moledda V. P23

Moleti M. P273, P297

Molinaro A. P277

Molinaro E. OP20, OP43, P64, OP88, OP91, P178, P302

Moll A. OP40

Molnar I. OP87

Molwitz T. P299

Momotani N. P27

Momova G. P271

Montanelli L. P125, P296

Montepagani A. P223, P289

Monzani F. P242

Moon J.H. P28

Mora M. P97

Moracci M. P306

Moraes L. P313

Morales F. P281

Mora-Mendoza A. P100

Morandeira B.F. P301

Morel Á.R. P115

Moreno C. P65

Moreno J.C. OP08, OP10, P202, P278, P281

Moreno M.A.G. P236

Morgunova T. OP77, P142

Mormile A. OP42, P92

Moro M. OP05

Moroni M. P223

Morris D. OP60

Morte B. P202, P316

Mosca M. P244

Motta C.L. OP67

Motta M. OP42

Moura M. P365

Moya C.M. OP10

Mpakakis M. OP95

Muco E. P324

Muegge A. P222

Mueller A.M. OP09, P319

Muente T.F. OP72

Mühlhaus J. P16, P264

Mullan K. P25

Müller A. P43

Muller I. P245

Murray R.D. P190

Muzza M. OP07

Na D. P55

Nacci A. P242

Nagahama M. P189, P353

Nagy D.M. P131, P210

Nagy E.V. P09, P11, P131, P210

Nakamura H. OP39, P254

Nam G.Y. OP44, P191

Nam K.-H. P338

Nam Y.J. P14

Narumi S. P27

Nascimento M. P167

Nasiri S. P111, P334

Navarro E. P79, P87

Naya L.D. P208, P214

Nechaeva O. P45, P248

Negri I. OP65

Nekrasova T. P143

Nelson P.J. OP09, P319

Nenkov R. P229

Nesca A. P90

Neumann A. P299

Neustadt M. P299

Neves C. P137, P139

Newbold K. OP02, P253, P351

Nexø M.A. P260

Nicolaescu E. P34

Niederle B. P80

Niedźwiedzka B. P215

Niedziela M. P249

Nielsen V. P60

Niemann R. P299

Nilsson M. OP17, P41, OP80

Nishihara E. P254

Nogueira C. P225

Nogueira C.R. P186

Nogueiras R. P54

Nome T. OP92

Nonchev B. P26, P35, P347

Nourbakhsh S. P32

Nowak W. P91

Nunes J. P120

Núñez N. OP10

Nuñez V.P. P115, P167

Nürnberg D. P264

Nutting C. OP87

Nybo M. OP74, OP78

Nygaard B. OP73, P144, P147

Obiols G. P188, P297

Obregon M.J. OP04, P200, P202

Oczko-Wojciechowska M. OP15, OP93

O'Dowd C. P25

Ogimi Y. P189

Oh K.H. P182

Ohkuwa K. P189, P353

Okamura K. P124

Ökten A. P231

Olchovsky D. P49

Oliva-García J.G. P100

Olivares E. P17

Oliveira A. P120

Oliveira A.I. P108

Oliveira F.L. OP57

Oliveira J. P228, P333

Oliveira K.C. P21 
Oliveira M.D. P44

Olmedo P. P361

Olsen J. OP13

Opazo R.B. P236

Opitz R. OP01

Oral E. P209

Orbetzova M. P26, P35

Origlia N. P198

Ortega A.J.M. P81

Ortiga T. P17

Ortiga-Carvalho T.M. OP85

Osokina D. P30

Osokina I. P30

Ovesen L. P18, P24, P96, P141

Owen L. P190

Owens M. OP18

Ozarar M. P212

Özdemir A. P212

Özel A. P209

Pacchioni D. OP42

Pach D. P215

Pacini F. OP06, OP21, OP46, OP87

Padovani C.R. P186

Paek S.H. P162

Päkkilä F. P140

Palacios J. OP96

Palestini N. OP42

Paliczka-Cieslik E. P71

Palmares C. P137, P139

Palomino G. P173

Palop J.A. OP47

Palussiere J. OP94

Pani F. P23

Paniagua A. P36

Papachristou D. OP95

Papachristou N. OP95

Papadakis G. P174

Papakotoulas P. P183

Papassotiriou I. P155

Papava M. P283, P359

Papavramidis T. P235

Papier A. P91

Papini E. P237

Papp M. P131

Pappa T. P174

Pappas A. P174

Parado I.D. P115

Parascandolo A. P306

Pariani N. P134

Park C.S. P303, P346

Park H.J. P161

Park H.K. P366

Park H.S. OP44, P191

Park H.Y. P300, P354

Park I.-S. P341

Park J.H. P338

Park M.W. P75, P343, P350

Park S.B. P14

Park S.H. P159

Park S.J. P162

Park T. P73, P307

Park Y.M. P342

Parkhimovich R. P53

Parrinello G. P153

Pascarella A. OP38, P44

Paschke R. P37, OP40, OP45, OP87, P299

Pasqualetti G. P242

Pawlaczek A. OP15

Pazaitou-Panayiotou K. P183, P235

Pazos-Moura C.C. P13, OP57

Pedersen I.B. P18, P96, P141

Pedersen P.L. P95
Peeters R.P. OP50, OP52, OP71, P272

Pehlivanov B. P26, P35

Peinado M.A. P298

Pellegriti G. P153

Pepe G. OP25

Percik R. P49

Pereira G. P17

Pereira J. P120

Pereira M. P137, P139

Perez B. P120

Pérez J.I. P177

Pérez J.M. P118

Pérez L.I. OP12, P83

Perez S. P42, P104, P170

Pérez S. P54

Pérez-Lázaro A. P276

Perez-Pevida B. P292

Péricard L. OP36

Perrild H. P18, P24, P96, P141, P144

Perrino M. OP07, OP68, P217

Persani L. OP05, OP65, OP82

Pertierra J. P85

Pery R. P57

Pestana A. OP92, P315

Peteiro-González D. P171

Petrou M. P168

Petrov R. P48

Petrova M. P94, P271

Petrovic I. P169

Petzsch K. P206

Pezzino G. P123

Pfaff D. OP85

Philippou G. P243

Piaggi P. OP91, P125, P296, P302

Piana S. OP40

Piantanida E. P134

Piechowski C.L. P16, P264

Piekiełko-Witkowska A. OP33

Pieruzzi L. P178

Pietschmann N. OP47

Pigliacelli F. P90

Pignatti E. OP07

Pilli T. OP06

Pirags V. P31

Pirkl A. P02

Pirnat E. P12, P154, P255

Pitoia F. OP69

Pitz S. P01

Planck T. P06, P325

Platonova T. P345

Plugatarenko N. P29

Poiana C. P107

Pointillart V. OP94

Polak M. P278

Polanska J. OP70

Poltorak S. OP93

Poole V. OP63, OP66

Popławski P. OP33

Populo H. P314

Porcelli T. OP48, P61

Porillo P. P87

Portegies M.L.P. OP71

Porter R. P126

Porter W. P266

Portillo P. P79

Pouta A. P140

Pozo J. P278

Pragacz K. P91

Pratt B. P253

Prazeres H. P315

Presti S. P273

Priestley L. P194
Provenzale M.A. P178

Przeorek C. P310

Przybylik-Mazurek E. P84

Puch Z. OP70

Puig-Domingo M. P58, P298

Pujol A. P177

Puleo L. OP88

Purice M. P130

Quah T.J. P329

Quak E. OP90, P166

Quinn A. OP18

Rabska-Pietrzak B. P249

Radetti G. OP05

Radev R. P229

Radu I. P352

Ragazzoni F. OP42, P92

Rago T. P296

Raikov N. P26, P347

Raimondi L. OP97, OP102, P265

Rakov H. P317, P320

Ramasamy S. P340

Rame J.-P. OP90, P166

Ramos F. P186

Ramos I. P17

Rapposelli S. P265

Rasmussen Å.K. P98, P110, P156, P260

Rasmussen L.B. P18, P24, P96, P141, P158

Raucourt D.D. OP90

Raue F. OP16

Raykova A. P347

Razvi S. OP71

Read M. OP63, OP66

Rees A. P126

Regadera J. OP96, OP99

Rehders M. P206, P264, P317, P320

Rehn S. P180

Rekvava M. P283, P359

Remaud S. OP31

Remer T. P20

Renko K. OP47

Rentziou G. P174

Resende E. P167, P344

Resta F. OP102

Resuli K. P324

Reverter J.L. P298

Reyes B.D. P10

Reyes-Santías R. P62

Rezeberga D. P31

Rho Y.-S. P184, P192, P195, P341

Riaño I. OP08

Ribeiro C. P65

Ricci D. P125, P296

Riedl M. P02

Riesco G. OP81

Riestra M. P280

Rijntjes E. OP47, OP53, P321

Rino Y. P189

Rivero-Melián B. P100

Riviere S. P05

Rizzo R. OP29

Roberts W.N. P246

Robinson B. OP02, P351

Robledo M. OP12, P83, P298, P308

Rocchi R. P04, P07

Rodiguez M.C. P118

Rodionova T. P135, P262

Rodondi N. OP71

Rodrigo S.T. P276

Rodrigues E. P120, P333

Rodrigues J. P42

Rodrigues P. P225, P228, P333

Rodrigues R. P46

Rodríguez E.D. P104

Rodríguez M.-G. P85

Rodriguez P.C. P167

Rodríguez-Antona C. P83

Rodríguez-García J. P170

Rodriguez-Muñoz A. OP28, P122

Roeder M. P144

Roldan B. P281

Romano M.A. P21

Romano R.M. P21

Romei C. OP20, P176, P181, P305

Romeo G. OP27

Rontogianni P. P193

Roque C. P332

Rosado V. P308

Rosbach J. P01

Rosenthal M. OP26

Roskosz J. P71

Rossi S. OP07, OP68

Roth M. OP26

Rouquette I. P330

Rouxel A. OP41, P89

Royer B. OP41, P89

Rozman J. OP53

Ruas L. P65

Rubeck K. P60

Rubio I. P313

Ruchała M. P215

Rudzińska M. P247

Ruf J. P247

Ruffilli I. P47, P150, P244

Ruge F. OP62, P126

Ruggeri R.M. P117, P234, P246

Ruiz-Ponte C. P171

Ruokonen A. P140

Ruschenburg I. P299

Rusinek D. OP15, OP93, P310

Russ G. OP41, P89

Russell W. P277

Russo D. P242

Russo M. P153

Rustemaj J. P290

Ruy L. OP98

Ryu H.S. P159

Ryu J.-S. P74

Ryu Y.B. P187, P336

Saba A. OP97, P198

Sabatini M. P198, P265

Sabini E. P302

Sacher A. OP93

Sahún M. P281

Sainato A. P302

Saito F. P189

Salb N. P319

Salehi L.B. P61

Salerno M.C. OP05

Salgado C. P314

Saltiki K. P174, P243

Salvatore D. OP48, OP54

Salvetti A. P263

Salvi M. OP25, OP61, P127

Samardzic T. P355

Sampaio R. P103

Sampedro-Núñez M.A. OP28, P122

Samson L. P210

Sancak S. P209, P212

Sánchez I. P122

Sánchez I.P. P236

Sánchez L. P83 
Sanders J. OP24, OP30

Sanders P. OP24, OP30

Sandi J.S. P293

Santaguida M.G. OP27, P230

Santen R. P299

Santiago L. P17

Santiago P. P361

Santini P. P125

Santisteban P. OP10, P40, OP81, OP101, P268, P318

Santoro M. P306

Santos D. P177

Santos M.D. P308

Saraceno G. P146

Saraiva A.M. P332

Saraiva C. P332

Saraiva J. P65

Saran S. P239, P270

Sargin M. P212

Sarkheil P. P32

Sassi L. P134

Sastre-Marcos J. P83

Sastre-Perona A. OP101

Sato K. P124

Savic S. P03, P128, P259

Savitskaya D. P136

Scaloni A. OP38

Scamporrino A. OP37

Scapellato C. OP21

Scarfò M. P44

Schaarschmidt J. P37

Scheel S. OP16

Scheeler M. P101, P222

Schepper J.D. P274

Scheuba C. P80

Schildroth J. P101

Schlicker C. OP51

Schlumberger M. OP02, OP11, OP19, OP86, OP87, P165, P185, P351

Schmid K.W. OP22, P88, P203, P226, P311

Schmidt A. P46

Schmidt M. P206

Schmitz R. P20

Schmohl K.A. OP09, P319

Schomburg L. OP34, OP47

Schöneberg T. P16

Schuppan D. OP26

Schweizer U. OP03, OP51, OP53, OP79, P109, P197, P206

Schwenk N. OP09, P319

Sciacca L. P123

Sciacchitano S. P61

Scollo C. P153

Seemann P. OP34

Seffou B. P204

Selen E. P266

Selmer C. P144

Selmi-Ruby S. OP04

Semenov D. P349

Senra J.S.R.A. P170

Seo H.B. P55

Seo S.T. P337

Sepe R.M. P44

Serra F. P332

Serramito R. P104

Serrano A. OP28, P122

Serres X. P188

Sestini F. OP21, OP46

Sethugavalar B. P59, P190

Settimo F.D. OP67

Seugnet I. P204

Šfiligoj D. P12
Sgarbi J.A. OP71

Shabtai E. P57

Shabtai M. P57

Shah M.H. OP02, P351

Sharma N. OP63

Sharma V. P33

Sherman S.I. OP02, OP87, P351

Shestakova T. P45, P102, P248

Sheu S.-Y. P203

Shibasaki H. P124

Shibuya H. P189, P353

Shin D.H. P342

Shin M.K. P161

Shinkarkina A. P323

Shinkov A. P52

Shiomi S. P335

Shirayama S. P257

Shishkov R. P271

Shong Y.K. P74, OP87, P112

Shuren L.I. P80

Siderova M. P94

Sidorin A. P323

Sieli R. P242

Siena S. OP87

Siewko K. P215

Sigstad E. OP92

Silva A.M. P103

Silva L. P365

Simescu M. P34

Simioniuc A. P78

Simões H. P332

Simón R. OP10

Simoni M. OP07

Siracusa M. P117

Sisti E. P04, P07, P296

Sjölin G. P261

Skene A. P221, P288

Skotheim R.I. OP92

Smit J.W. OP87

Smith B.R. OP24, OP30

Smith J. OP66

Smith V. OP63, OP66

Smyth P. P25

Soares O. P344

Soares P. OP92, P314, P315

Soboleva D. P152

Sobrinho-Simões M. OP92

Sodagari N. P111, P334

Soe A. P19

Sokhatska O. P137, P139

Soldin O. P49

Solncev V. P136

Solnica B. P215

Somisetty S. P19

Son S.-M. P342

Song B.J. P70, P295, P304

Song D.E. P74

Song J.W. OP44, P73, P191, P307

Song S.-Y. P75, P343, P350

Soppi E. P51, P326

Sordino P. P44

Sordo M.D. P90

Souza L.L. P13

Sowiński J. P215

Spadaro O. OP38

Specchia P. P237

Spencer C. P169

Spencer K. P190, P340

Spinelli C. OP89

Spitzweg C. OP09, P319

Squarcia M. P97

Squatrito S. P123, P153

Stanilova S. P241

Staub A. P330
Stavrianos C. P243

Steegborn C. OP51

Steegers E. P272

Steenvoorden D. P76

Steiber Z. P09, P224

Steuhl K.-P. P10

Stobiecka E. OP93

Stoedter M. OP47

Stoinov J. P232

Stojkovic M. P03, P128, P259

Stopa M. P91

Strazzulli A. P306

Strele I. P31

Strongin L. P143

Sturniolo G. P273, P297

Suárez L. P85

Suganuma N. P189, P257, P294

Sugino K. P189, P294, P353

Sugisawa C. P27

Suh K. P366

Surcel H.-M. P140

Suvanto E. P140

Suzuki A. P189, P353

Svinarov D. P52

Swierniak M. OP15, P310

Sykiotis G. OP95

Sykorova V. P86, P312

Szabados L. P11

Szanto A. P224

Szelachowska M. P215

Tabury K. P157

Taccogna S. P237

Tacito A. OP20, P176, P181, P305

Tagliarini J.V. P186

Tahara M. OP02, P351

Tahmasebinejad Z. P32

Tallstedt L. P06

Tanda M.L. P134

Tandoğan B. P209

Taniyama M. P27

Tavares C. P314, P315

Taylor M. P221, P288

Taylor M.H. OP02, P351

Taylor P.N. OP14, OP60

Tazartes M. P05

Tébar J. P79, P87

Teixeira J.P. P344

Tekin V. P282

Terrien J. P204

Terroir M. P165

Terzidis K. P243

Tetere E. P31

Thamm M. P20

Thuesen B. P24

Thvilum M. P145, P256

Tiedje V. OP22, P88, P203, P226

Tiemeier H. P272

Timpson N. OP14

Ting S. OP22, P88, P203, P226, P311

Tiosano D. P277

Tirelli S. OP25, OP61

Tobias J. OP14

Todorov S. P347

Tognini S. P242

Tohidi M. P32, P360, P362

Tonacchera M. P125, P277

Tonelli M. P266

Torlinska B. P364

Torpy D. OP75

Torre E.M. P208

Torregrossa L. OP88, P176, P178

Torres J.F.M. P276

Törring O. P06, P261

Tortorella G. P273

Tosi D. OP68

Tost F. P15

Tovar S. P54

Träisk F. P06

Trbojevic B. P03

Trifanescu O.-G. P107

Trifanescu R. P107

Trimarchi F. P117, P146, P234, P246, P273

Trivizas P. P72

Trofimiuk-Müldner M. P215

Trubiroha A. OP01

Trumpff C. P274

Tsagarakis S. P72, P193

Tschöp M. P264

Tsirona S. P72

Tsuchida N. P306

Tucci M. P149

Turan E. P129

Turan Y. P129

Turnpenny P. OP18

Turrini L. P237

Tyszkiewicz T. OP15, OP70

Tzanela M. P193

Ubl P. P80

Ugolini C. P176, P302, P305, 306

Ujhelyi B. P09, P11, P131, P224

Ulivieri A. P61

Unluturk E. P282

Urciuoli C. OP69

Urdaneta J.L. P115

Urganci U. P282

Ursu H.I. P130

Uruno T. P189, P294, P353

Uyar M. P129

Uzun Ö. P209

Vääräsmäki M. P140

Vaclavikova E. P86, P312

Vaidya B. OP18

Valdés N. P208

Valerio L. OP91, P181

Valero R. P97

Vallespín E. P278

van den Akker E. OP08

van der Horst-Schrivers A. P76

van der Spek A. OP54

van Heerebeek R.E.A. OP52

Van Herck S.L.J. OP84, P201

Van Regemorter E. OP59

van Sande J. OP08, P39, P175

Vandernoot I. OP01

Vanderstraeten J. P22, P157

Vandevijvere S. P274

Vanezi A. P168

Vankova M. P48

Vannucchi G. OP25, OP29, OP61, OP68, P127, P217

Varciu M. P34

Varela A. P333

Varga Z. P210

Vargas-Uricoechea H. P207

Varo I.J. P81

Vasconcelos C. P332

Vassilopoulos H. P72

Vaur D. OP90

Vázquez R.G. P81, P286

Vazquez S.A. P115

Vcelak J. P86, P312

Vecchio G. P306 
Veisa V. P31

Velasco-Martín J.P. OP96, OP99

Velten E. P19

Ventura P.S. P278

Vergez S. P330

Vermiglio F. P273, P297

Veronesi G. P134

Vezzoli V. OP65

Vicchio T.M. P117

Vicente N. P65

Vicentini L. OP68

Vicheva S. P347

Vicuña A. P122

Vieira T. P120

Vielh P. P165

Vigo B. P217

Vigone M.C. OP05

Vilaça J. P365

Villamarín C.A. P42, P104, P170, P301

Villar F. P46

Villar-Taibo R. P171

Viola D. OP43, P64, OP88, OP91

Violante L. P344

Virili C. OP27, P230

Visser E. OP50, P272

Visser T.J. OP50, OP52, OP84, P272, P278

Visser W.E. OP52

Vita G.D. OP38, P44

Vita R. P146
Vitale G. OP65

Vitale M. P90

Vitti P. P04, P07, OP43, P64, OP88, OP91, P125, P178, P296

Vivien C. OP36

Vlahov J. P52, P232

Vlassopoulou B. P72

Vlcek P. P86, P312

Voigtländer R. P109, P206

Völker U. OP58

Volkova A. P148, P152, P251

Völzke H. P15, OP71

Vukov M. P48

Vyagova D. P347

Wachowiak-Ochmańska K. P215

Wagner E. OP09

Wallaschofski H. OP58

Wallin G. P261

Walsh J.P. OP71

Wang S.G. P342

Wanner C. OP71

Watkins R. OP63, OP66

Watt T. P98, P110, P260

Wawrusiewicz-Kurylonek N. P250

Weber G. OP05

Weber J. P203

Webster A. OP19

Wechselberger A. P319

Weih F. OP32

Wert-Lamas L. OP81
Wessling A. P327

Westendorp R.G. OP71

Widlak W. P310

Willershäuser M. OP53

Williams F. P19

Willim J. OP26

Winther K. OP78

Wirth E.K. OP53, P197

Wirth L. OP02, P351

Wittner M. OP49

Woo J.-S. P75, P343, P350

Worm K. P88

Wu C.S. OP13

Wuensch T. OP79

Xhaferi E. P324

Yadav N. P33

Yao C. P220

Yeo M.-K. P172

Yi C.-X. P264

Yilmaz M. P282

Ylli A. P287, P290, P324

Y1li Z. P287, P290

Yoldemir T. P209

Yongchao L. P160

Yoo H. P161

Yoo J. P182

Yoo M.H. P366

Yoon J.H. P179

Yoon T. P303
Yoshida A. P294, P335

Yoshioka W. P254

Youn Y. P55

Young S. OP24, OP30

Yuan S. P240

Yun M. P121

Yun W. P160

Zafon C. P297

Zaletel K. P12, P154, P232, P255

Zarkovic M. P03, P128, P259

Zebracka-Gala J. OP15

Zembala-Nozynska E. OP93

Zeqja A. P324

Zeqollari A. P287

Zevenbergen C. OP50, OP84

Zhang C. OP04

Zhang L. OP60, OP62, OP64, P126, P245

Zhang Y. P240

Zhang Z. OP35

Zhao L. P240

Zhaoxia W. P160

Zhu J. OP02, P351

Zineb E. P38

Zinkevich I. P349

Ziros P. OP95

Zucchi R. OP97, OP102, P198, P263, P265

Zwanziger D. OP64, P180, P203, P206, P311, P317, P320 